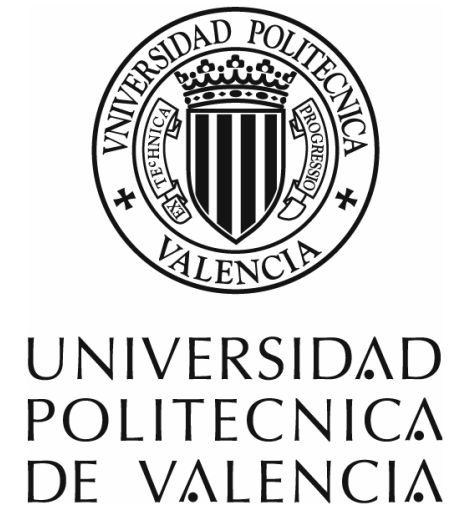

DEPARTAMENTO DE INFORMÁTICA DE SISTEMAS Y COMPUTADORES

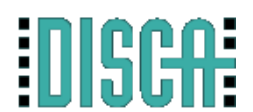

\title{
Contribución a la autolocalización de robots móviles basada en la fusión de información multisensorial
}

Tesis doctoral presentada como requisito parcial para obtener el grado de

Doctor en Automática e Informática Industrial

Danilo Alfonzo Navarro García

Director:

Dr. Ginés Benet Gilabert

Valencia, Septiembre 2009 

A mi esposa, Georgina, A mis hijas, Victoria y Diana A mi madre, Olga. 



\section{Agradecimientos}

En general, a fin de garantizar el éxito en los objetivos planteados, la investigación y los desarrollos científicos requieren del concurso de varias personas e instituciones. Es así como este trabajo se hizo realidad gracias al esfuerzo propio y a la colaboración desinteresada de terceros. En ese sentido, de manera especial quiero manifestar mi agradecimiento al director de esta tesis, Dr. Ginés Benet Gilabert. A él le agradezco las oportunas orientaciones que durante el desarrollo de este trabajo a bien tuvo brindarme, y también el apoyo pleno que me ofreció desde la misma concepción del tema de investigación hasta los asuntos académico-administrativos que surgieron. Igualmente quiero agradecer a la Universidad Politécnica de Valencia - España, por haberme acogido en su seno y por poner a mi disposición las instalaciones y equipos que requerí durante el desarrollo de este trabajo de tesis. Finalmente, no podría haber culminado este trabajo sin el apoyo de la Universidad de Oriente - Venezuela, institución a la que manifiesto mi agradecimiento por concederme los permisos y avales necesarios, así como también por concederme parte del financiamiento para mi estancia en la ciudad de Valencia - España, donde se desarrolló esta investigación.

Danilo Navarro 



\section{Resumen}

Los primeros robots operaban en entornos especialmente preparados para ellos. Cada componente de su espacio de trabajo se encontraba situado en una posición y orientación predefinidas, de modo que el robot conocía a priori y con exactitud el escenario donde se encontraba. En la actualidad muchas de las aplicaciones requieren que los robots tengan rasgos de autonomía como son la capacidad para identificar mediante sus sensores las características que tiene el entorno, la capacidad para autolocalizarse dentro del mismo, y la capacidad para navegar por su espacio de trabajo respondiendo a las variaciones temporales que vayan surgiendo. A pesar de que los resultados obtenidos en el campo de la construcción de mapas y auto-localización de robot móviles son abundantes y muy significativos, aun existen problemas por resolver sobre todo los vinculados al uso de sensores imprecisos y de bajo costo, ya que la información obtenida con estos sensores resulta poco fiable al momento de integrarla a las distintas aplicaciones en este tipo de sistemas. Este trabajo se centra en el estudio y modelado de sensores de bajo costo de uso típico en robótica móvil (codificadores rotativos, sensores de ultrasonidos e infrarrojos, compás magnético), y de cómo pueden ser utilizados en labores de auto-localización y representación del entorno.

Mediante la fusión de datos provenientes de sensores de bajo coste se logra que un robot móvil se auto localice adecuadamente de forma que pueda navegar confiablemente en entornos estructurados. Para probar y evaluar off-line los distintos mecanismos de fusión y filtrado propuestos, a lo largo de esta tesis se desarrollan modelos sensoriales así como un pseudo código para la simulación de la operación de estos sensores en un robot real. Igualmente, en esta tesis se formulan los procedimientos y se elabora el software que se insertará en un robot real para que éste ejecute las acciones de control de navegación, de adquisición y filtrado de datos sensoriales, y de fusión pertinente a la localización y el mapeado en su entorno de operación. En este sentido, aquí se han desarrollado modelos de sensores que permiten integrar los mismos a sistemas más avanzados de fusión sensorial, que a la postre sirven para construir mapas de densidad de ocupación y de características geométricas del entorno. Así mismo, a partir de los modelos sensoriales se han desarrollado dos sistemas de localización. El primero usa los odómetros de abordo para mantener la posición del robot mediante un procedimiento de seguimiento(Tracking) conocido como DeadReckoning. El segundo se fundamenta en un Filtro de Kalman Extendido que integra la información proveniente de un sensor de ultrasonidos para mantener y corregir la 
posición del robot utilizando 2 tipos de hitos(Landmark) naturales: Paredes y Esquinas.

Para la comprobación y evaluación del funcionamiento de los métodos propuestos, en esta tesis se ha utilizado un robot llamado YAIR(Yet Another Intelligent Robot), el cual es una plataforma multi-sensorial que cuenta un sistema impulsor de principio diferencial. Dado que se trata de un sistema de tiempo real que operará en un entorno real, la técnica de análisis y validación de los resultados se basó en la observación directa del comportamiento del robot cuando realizaba las acciones de navegación y exploración del entorno que le eran programadas. Los resultados obtenidos muestran que con la fusión de datos se mejora sensiblemente la calidad de los mapas de representación del entorno, y con ello se mejora también la precisión en la localización del robot. Así mismo, con el sistema de localización desarrollado en esta tesis, el cual se fundamenta en el Filtro Extendido de Kalman para la fusión de los datos de localización odométrica con los datos de observación de un sensor de ultrasonidos, se logró mejorar la autolocalización del robot, pasando de errores del orden de 3 metros en trayectos de 80 metros, a errores de pocas décimas de metros en el mismo tramo. 


\section{Abstract}

At past, robots were working in environments specially prepared for them. Every component of their work space was placed in a predefined position and orientation, in order to robots knew with precision the place where they were. At present many applications require that robots have autonomy features as for example: skill to identify by means of their sensors the environment characteristics, capability for self localization, and aptitude to move along of their work space, paying attention to the temporary variations arising from the environment. In spite of the results obtained in the robots localization and mapping area are abundant and very significant; even so exist problems for solving those that are specially linked to the use of low-cost sensors. This is because the data obtained with these kind of sensors is noisy and very uncertain. Aimed to contribute towards to solve the problem mentioned before, this thesis focus on the modeling of low-cost sensors which are typically used in mobile robotics (Optical encoder, ultrasounds and infrared sensors, magnetic compass), thus as on the studying of how they could be used in auto-location and map building problems.

By mean of information fusing, a mobile robot using low-cost sensors is capable of estimate its position adequately, and in this way navigate reliably in structured environments. In order to test off-line the different mechanisms of merging and filtering that we propose, along this thesis we develop the sensory models of three low-cost sensors which are frequently used in mobile robotics: ultrasonic and infrared range finder, and odometric dead-reckoning sensor. Furthermore we develop the pseudo code that is necessary for the embedded operation of each one of these sensors on a generic real robot. In addition we formulate the procedures and synthesize the software to be embedded in the real robot YAIR(Yet Another Intelligent Robot) for purpose of navigation control, acquisition and filtering of sensor data, and for merging sensory information related to environment mapping and robot localization. The models on this way developed allow incorporate they to another advanced systems intended for density map or featured map construction. Likewise, from the models mentioned before we have developed two localization systems: the first one uses odometric information for Dead-Reckoning, whereas the second one uses walls and corner as natural Landmarks and an Extended Kalman Filter approach for correction of the robot position.

In order to evaluate and validate the proposed methods, we carried out mapping and localization experimental test using a robot called YAIR which is a multi-sensorial 
differential-drive robot. Because this robot is a real time system operating in a real environment, the analysis and validation of the results was based on the direct observation of the robot behavior when it was exploring the environment or navigating on it. The results showed that with the information merging approach the quality of the environment maps obtained was improved significantly, likewise the precision of the robot localization process. Moreover, with the localization system developed in this thesis, the localization error was reduced from 3 meters to few centimeters in distances about 80 meters long. 


\section{Resum}

Els primers robots operaven en entorns especialment preparats per a ells. Cada component del seu espai de treball es trobava situat en una posició i orientació predefinides, de manera que el robot coneixia a priori i amb exactitud l'escenari on es trobava. En l'actualitat moltes de les aplicacions requerixen que els robots tinguen trets d'autonomia com són la capacitat per a identificar per mitjà dels seus sensors les característiques que tinga l'entorn, la capacitat per a autolocalitzar-se dins del mateix, i la capacitat per a navegar pel seu espai de treball responent a les variacions temporals que vagen sorgint. Malgrat que els resultats obtinguts en el camp de la construcció de mapes i localització de robot mòbils són abundants i molt significatius, finsitot hi ha problemes per resoldre, sobretot els vinculats a l'ús de sensors imprecisos i de baix cost, ja que la informació obtinguda amb estos sensors resulta poc fiable en el moment d'integrar-la a les distintes aplicacions en este tipus de sistemes. Este treball se centra en l'estudi i modelatge de sensors de baix cost d'ús típic en robòtica mòbil (codificadors rotatius, sensors d'ultrasons i infrarrojos, compàs magnètic), i de com poden ser utilitzats en labors d'autolocalització i representació de l'entorn.

Per mitjà de la fusió de dades provinents de sensors de baix cost s'aconseguix que un robot mòbil es localitze adequadament de manera que puga navegar confiablement en entorns estructurats. Per a provar i avaluar off-line els distints mecanismes de fusió i filtrat proposats, a través d'esta tesi es desenvolupen models sensorials així com un pseudo codi per a la simulació de l'operació d'estos sensors en un robot real. Igualment, en esta tesi es formulen els procediments i s'elabora el programari que s'inserirà en un robot real perquè este execute l'accions de control de navegació, d'adquisició i filtrat de dades sensorials, i de fusió pertinent a la localització i el mapejat en el seu entorn d'operació. En este sentit, ací s'han desenvolupat dos sistemes de localització. El primer usa els hodòmetres d'aborde per a mantindre la posició del robot per mitjà d'un procediment de seguiment(Tracking) conegut com Dead-Reckoning. El segon es fonamenta en un Filtre de Kalman Estés que integra la informació provinent d'un sensor d'ultrasons per a mantindre i corregir la posició del robot utilitzant 2 tipus de fites(Landmark) naturals: Parets i cantons.

Per a la comprovació i avaluació del funcionament dels mètodes proposats en la tesi s'ha utilitzat un robot anomenat YAIR(Yet Another Autonomous Robot), el qual és una plataforma multi-sensorial que comté amb un sistema impulsor de principi diferencial. Atés que es tracta d'un sistema de temps real que operarà en un entorn 
real, la tècnica d'anàlisi i validació dels resultats es va basar en l'observació directa del comportament de robot quan realitzava les accions de navegació i exploració de l'entorn que li eren programades. Els resultats obtinguts mostren que amb la fusió de dades es millora sensiblement la qualitat dels mapes de representació de l'entorn, i amb això es millora també la precisió en la localització del robot. 


\section{Índice general}

1. Motivación, objetivos, y organización de esta tesis 1

1.1. Motivación . . . . . . . . . . . . . . . . . . . . 1

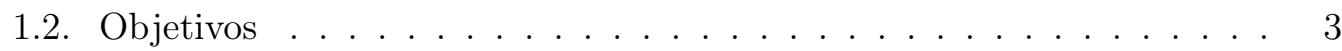

1.3. Organización de la tesis . . . . . . . . . . . . . 5

2. Introducción a la localización de robots móviles 9

2.1. Sensorización en robótica móvil . . . . . . . . . . . . . . . . . 11

2.1.1. Codificador rotativo. . . . . . . . . . . . . . . . . 12

2.1.2. Sensores de ultrasonido . . . . . . . . . . . . . . . . 13

2.1.3. Sensor de infrarrojos . . . . . . . . . . . . . . . . . . 16

2.1.4. Compás . . . . . . . . . . . . . . . . . . 17

2.2. Fusión sensorial . . . . . . . . . . . . . . . . . . . . . . 19

2.2.1. Fusión Bayesiana . . . . . . . . . . . . . . . 20

2.2.2. Fusión basada en la teoría de Dempster - Shafer . . . . . . . . . 23

2.2.3. Fusión mediante Filtros de Kalman . . . . . . . . . . . . . . . . 26

2.3. Representación del entorno. . . . . . . . . . . . . . . . . . . . . . . . . . . . . . . . . . 28

2.3.1. Mapas topológicos. . . . . . . . . . . . . . . . . . . . 28

2.3.2. Mapas de características geométricas. . . . . . . . . . . . 30

2.3.3. Mapas de Ocupación . . . . . . . . . . . . . . . . . 32

2.4. Localización relativa mediante medidas propioceptivas . . . . . . . . . 33

2.5. Localización absoluta basada en Hitos o Landmark . . . . . . . . . . . 34

2.6. Localización basada en mapas . . . . . . . . . . . . . . . . . . . . . 38

2.6.1. Localización con mapas métricos . . . . . . . . . . . . . . . . 39

2.6.2. Localización con mapas topológicos . . . . . . . . . . . . . . . . 40

2.6.3. Localización usando mapas de alta densidad de muestreo . . . . 40

2.7. Localización y Mapeado: enfoque probabilístico . . . . . . . . . . . . . 41

2.8. Localización Bayesiana o localización de Markov . . . . . . . . . . . . 44

2.8.1. Implementación de la localización Bayesiana o de Markov . . . 47

2.8.1.1. Enfoque métrico . . . . . . . . . . . . . . . . . 49

2.8.1.2. Enfoque topológico . . . . . . . . . . . . 50 
3. Modelado cinemático y localización odométrica del robot diferencial $\mathbf{5 3}$

3.1. Modelado cinemático de robot diferenciales . . . . . . . . . . . . . . 54

3.2. Análisis de los principales errores de la localización odométrica en un robot diferencial . . . . . . . . . . . . . . . . . . . . . . . 59 59

3.2.1. Errores sistemáticos . . . . . . . . . . . . . 60

3.2.1.1. Incertidumbre en el diámetro de las ruedas . . . . . . 61

3.2.1.2. Incertidumbre en la distancia entre los puntos de apoyo de ruedas . . . . . . . . . . . . . . . . 62

3.2.1.3. Sensor virtual de estado completo basado en codificadores ópticos rotativos . . . . . . . . . . . . 63

3.2.2. Errores no sistemáticos . . . . . . . . . . . . . . 66

3.3. Identificación de los parámetros cinemáticos de un robot diferencial . . 66

3.3.1. Determinación de la sensibilidad odométrica mediante el Método Directo . . . . . . . . . . . . . . . . . . 67

3.3.2. Determinación de la base de giro de un robot diferencial mediante el Método Directo . . . . . . . . . . . . . . . 70

3.3.3. Resultados experimentales en el robot YAIR . . . . . . . . . . . 74

3.3.3.1. Identificación y calibración de parámetros del modelo cinemático . . . . . . . . . . . . 74

3.3.3.2. Resultados para la localización mediante sensores de odometría . . . . . . . . . . . . . . 78

3.4. Un método automático para la identificación y calibración multisensorial directa de la cinemática de un robot diferencial. . . . . . . . . . . 82

3.4.1. Técnica multisensorial de identificación y calibración automática de la odometría en robot diferenciales . . . . . . . . . . 84

3.4.1.1. Fundamentos geométricos del movimiento de un robot diferencial . . . . . . . . . . . . . 84

3.4.1.2. Esquema y procedimiento experimental del proceso de identificación sensibilidad odométrica . . . . . . . 85

3.4.1.3. Esquema y procedimiento experimental del proceso de identificación de la base de giro . . . . . . . . . . 88

3.4.1.4. Procedimiento experimental del proceso de calibración 89

3.4.1.5. Detección de valores atípicos. . . . . . . . . . . . . 91

3.4.2. Resultados experimentales . . . . . . . . . . . . . . 92

3.4.2.1. Resultados proceso de identificación . . . . . . . . . . 92

3.4.2.2. Resultados en el proceso de calibración odométrica y detección de valores atípicos . . . . . . . . . . 9 95

3.4.2.3. Comparación de resultados autocalibración multisensorial directa vs UMBmark . . . . . . . . . . . . . . 97

4. Percepción, fusión sensorial y construcción de mapas 101

4.1. Construcción de mapas de ocupación mediante datos de ultrasonidos . 102 4.1.1. El SONAR . . . . . . . . . . . . . . . . . 103 4.1.2. Principales problemas de la medición con SONAR . . . . . . . 103 
4.1.3. Modelado del SONAR tipo rotativo . . . . . . . . . . . . 105

4.1.4. Modelo probabilista del SONAR . . . . . . . . . . . . . 108

4.1.5. Modelo posibilista del SONAR . . . . . . . . . . . . . 117

4.1.6. Mejoras al mapeado ultrasónico aplicando máscaras en las esquinas . . . . . . . . . . . . . . . . . . . . 118

4.1.7. Construcción de mapas de ocupación mediante fusión sensorial. 121 4.1.7.1. Construcción de mapas mediante fusión Bayesiana . . 123

4.1.7.2. Construcción de mapas mediante la teoría evidencial . 125

4.2. Construcción de mapas mediante el sensor infrarrojo . . . . . . . . . . 127

4.2.1. Medición de distancias basados en infrarrojos . . . . . . . . . 130

4.2.2. Modelado de los reflectores extraídos mediante el sensor de infrarrojos . . . . . . . . . . . . . . . 133

4.2.3. Representación paramétrica del entorno mediante segmentos de líneas . . . . . . . . . . . . . . . . . 137

4.2.4. Extracción de líneas mediante métodos recursivos . . . . . . . . 139

4.2.5. Construcción incremental de mapas de líneas mediante fusión de datos de infrarrojos . . . . . . . . . . . . . . 143 4.2.5.1. Detección e iniciación de los segmentos de línea . . . . 144 4.2.5.2. Agrupación de puntos y segmentación de líneas . . . . 146 4.2.5.3. Correspondencia entre segmentos de línea . . . . . . . 148

4.2.5.4. Mecanismos de fusión de segmentos de línea . . . . . . 150

4.2.6. Experimentos . . . . . . . . . . . . . . . . 151 4.2.6.1. Configuración experimental . . . . . . . . . . . 151 4.2.6.2. Resultados . . . . . . . . . . . . . . . . . 152

\section{Manejo de la incertidumbre en el sistema, en la medición y en la} $\begin{array}{lr}\text { representación } & 155\end{array}$

5.1. Introducción . . . . . . . . . . . . . . . . . . . . 155

5.2. Modelo cinemático estocástico de un vehículo de tracción diferencial . 157

5.2.1. Modelo de ruido de un vehículo diferencial . . . . . . . . . . . 158

5.2.2. Estimación del error de estado . . . . . . . . . . . . . . . 159

5.2.3. Modelado de la varianza en los sensores de odometría . . . . . 162

5.2.4. Estrategia experimental para determinar la varianza en sensores de odometría . . . . . . . . . . . . . . . . . 163

5.2.5. Resultados experimentales en la determinación de la varianza en los sensores de odometría . . . . . . . . . . . . 166

5.3. Modelo estocástico del sensor de ultrasonidos . . . . . . . . . . . . . . 168

5.3.1. Clasificación y extracción de landmark a partir de datos de ultrasonidos . . . . . . . . . . . . . . . . . . . . 169

5.3.2. Modelo ideal de un SONAR tipo rotativo . . . . . . . . . . 170

5.3.3. Modelo de ruido del SONAR tipo rotativo . . . . . . . . . . . . 171

5.3.4. Resultados experimentales en la determinación del ruido sensorial172

5.4. Representación del entorno mediante hitos o Landmarks . . . . . . . . 175

5.4.1. Modelado de esquinas como Landmarks puntuales . . . . . . . 177 
5.4.1.1. Sistemas coordenados de referencia . . . . . . . . . 177

5.4.1.2. El modelo de representación: Transformación de hitos puntuales y propagación de su covarianza al marco Global . . . . . . . . . . . . . . . . . . . 179

5.4.1.3. El modelo de Observación: Transformación de hitos puntuales y propagación de su covarianza al marco Robot . . . . . . . . . . . . . . . 180

5.4.2. Modelado de paredes y estructuras planas como landmark tipo línea . . . . . . . . . . . . . . . . . . . . 181

5.4.2.1. Transformación de coordenadas para el modelo Hessiano de línea infinita . . . . . . . . . . . . . . . . 182

5.4.2.2. El modelo de observación para líneas Hessiana . . . . 185

5.5. Métodos de correspondencia . . . . . . . . . . . . . . . 185

5.5.1. Correspondencia entre landmark tipo puntuales . . . . . . . . . 187

5.5.2. Correspondencia entre líneas . . . . . . . . . . . . . . . 189

6. Construcción de Mapas y Localización Estocástica 191

6.1. Introducción al enfoque estocástico en la robótica móvil . . . . . . . . 191

6.2. Construcción de mapas de esquinas . . . . . . . . . . . . . . . . . . . 194

6.2.1. El algoritmo de mapeado mediante el Filtro de Kalman Extendido195

6.2.2. Resultados experimentales extracción y correspondencia de landmark puntuales en la construcción de mapas de esquinas. . . . 198

6.3. Localización probabilística mediante landmark naturales tipo puntuales 201

6.3.1. El algoritmo de localización mediante hitos tipo puntuales usando el Filtro Extendido de Kalman . . . . . . . . . . . . . . . . . 203

6.3.1.1. Etapa de predicción. Modelado de la cinemática del vehículo . . . . . . . . . . . . . . 204

6.3.1.2. Etapa de medición y correspondencia. Modelado de la función de observación . . . . . . . . . . . 206

6.3.1.3. Etapa de corrección y actualización. Cálculo de la ganancia de Kalman . . . . . . . . . . . . . . . . 207

6.3.2. Resultados experimentales en la localización usando esquinas extraídas con un SONAR rotativo. . . . . . . . . . . . 208

6.4. Localización basada en mapas de líneas usando un SONAR y el EKF 214 6.4.1. Predicción de la posición del vehículo . . . . . . . . . . . . . 214

6.4.2. Observación . . . . . . . . . . . . . . . . . . 215

6.4.3. Predicción de las observaciones . . . . . . . . . . . . . . . . . 215

6.4.4. Correspondencia o asociación de datos . . . . . . . . . . 216

6.4.5. Corrección del estado. . . . . . . . . . . . . . . . . . 218

6.4.6. Experimentos . . . . . . . . . . . . . . . . . . . . 219

6.4.7. Resultados y discusión . . . . . . . . . . . . . . . . . . . 219

6.5. Localización y mapeado simultáneo basado en Datos de ultrasonido . . 220

6.5.1. El algoritmo de SLAM basado en el Filtro de Kalman Extendido223

6.5.1.1. El vector de estado . . . . . . . . . . . . . . . 223 
6.5.1.2. Predicción del estado . . . . . . . . . . . . . . . 224

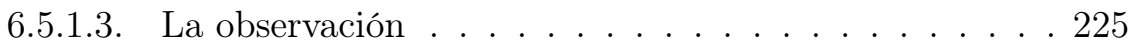

6.5.1.4. La corrección del estado . . . . . . . . . . . . . . . . . . . . . . . . 226

6.5.1.5. Integración de objetos al mapa . . . . . . . . . . 227

6.5.2. SLAM basado en hitos puntuales extraídos con un sensor de ultrasonidos . . . . . . . . . . . . . . . . . . . 228

6.5.2.1. Predicción del estado aumentado y de su covarianza . 229

6.5.2.2. Representación del entorno . . . . . . . . . . . . . 231

6.5.2.3. Asociación de datos o correspondencia entre hitos puntuales . . . . . . . . . . . . . . 233

6.5.2.4. Objeto nuevo: incorporación al vector de estado aumentado . . . . . . . . . . . . . 233

6.5.2.5. Objeto existente: Corrección del estado estimado del vehículo . . . . . . . . . . . . . . 234

6.5.3. Resultados experimentales del SLAM usando esquinas como hitos puntuales . . . . . . . . . . . . 236

6.6. Limitaciones en los métodos ensayados . . . . . . . . . . . . . . . 241

7. Conclusiones, trabajos futuros, y publicaciones $\quad 245$

7.1. Sumario y Conclusiones . . . . . . . . . . . . . . . . . . . . 245

7.2. Trabajos futuros . . . . . . . . . . . . . . . . 251

7.3. Publicaciones relacionadas a esta tesis . . . . . . . . . . . . 253

A. Localización de Markov $\quad \mathbf{2 6 1}$

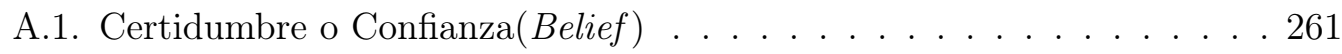

A.2. Modelo probabilístico de actuación del robot . . . . . . . . . . . . 262

A.3. Modelo probabilístico de medición . . . . . . . . . . . . . . . 263

A.4. Algoritmo de Localización de Markov . . . . . . . . . . . . . . . . . . 263

A.4.1. Confianza inicial . . . . . . . . . . . . . . . . . . . . 264

A.4.2. Predicción de la certidumbre . . . . . . . . . . . . . 264

A.4.3. Corrección de la confianza . . . . . . . . . . . . . . . 266

A.4.4. Fórmula de localización de Markov . . . . . . . . . . . . . . 267

B. Filtro de Kalman $\quad \mathbf{2 6 9}$

B.1. Conceptos y asunciones básicas del Filtro de Kalman . . . . . . . . . . 269

B.2. Algoritmo del Filtro del Kalman lineal . . . . . . . . . . . . . . . . . 271

B.2.1. Predicción . . . . . . . . . . . . . . . . 271

B.2.2. Corrección . . . . . . . . . . . . . . . . . . . 272

B.2.3. Actualización de la covarianza . . . . . . . . . . . . . . . . 273

B.2.4. Parámetros y ajuste del Filtro de Kalman . . . . . . . . . . . . 274

B.3. Filtro de Kalman Extendido - EKF . . . . . . . . . . . . . . . . . . . 274 
C. Estimación de parámetros mediante Mínimos Cuadrados

C.1. Mínimos Cuadrados Generalizados . . . . . . . . . . . . . . . . . 278

C.2. Mínimos Cuadrados Recursivos . . . . . . . . . . . . . . . . . . . . 280 


\section{Lista de algoritmos}

1. Estimación de estado basado en información odométrica . . . . . . . . 59

2. Busca el valor de $K^{2}$ que satisface la elipse de error. . . . . . . . . . . . 165

3. Determinación del estado del robot después de una secuencia de entrada.165

4. Determinación del punto de intersección de una elipse con la recta definida por el centro de la misma y un punto cualquiera. . . . . . . 166

5. Determinación de correspondencia entre landmark puntuales . . . . . . 188

6. Algoritmo de construcción de mapas de landmark puntuales mediante el Filtro de Kalman Extendido . . . . . . . . . . . . . . . . . . . . . . 199

7. Algoritmo de localización Bayesiana o de Markov . . . . . . . . . . . . 264

8. Estimación de parámetros mediante mínimos cuadrados recursivos . . 281

9. qyeweueieioe . . . . . . . . . . . . . . . . . . . . 281 



\section{Índice de Tablas}

3.1. Resumen estadístico sensibilidad odométrica de la rueda izquierda del robot YAIR . . . . . . . . . . . . . . . 75

3.2. Resumen estadístico sensibilidad odométrica de la rueda derecha del robot YAIR . . . . . . . . . . . . . . . 76

3.3. Resumen estadístico base de giro óptima del robot YAIR . . . . . . . . 77

3.4. Comparación de resultados para los parámetros cinemáticos de YAIR obtenidos por el método directo y por el UMBmark. . . . . . . . . . . 78

3.5. Estadísticos de error de estimación odométrica de la posición del robot YAIR .......................... 80

3.6. Resultados de la identificación automática de parámetros odométricos en el robot YAIR. . . . . . . . . . . . . . . . . . . . . . . . . 94

3.7. Resultados proceso de calibración de parámetros odométricos con filtrado de outliers. $C_{R}$ y $C_{L}$ : sensibilidad odométrica derecha e izquierda en $\mathrm{mm} /$ pulso, respectivamente. $B$ : base de giro en $\mathrm{mm}$. . . . . . . . . 97

3.8. Comparación de resultados para los parámetros odométricos modificados de YAIR obtenidos por el método de autocalibración directa y por el UMBmark. . . . . . . . . . . . . . . . . . . . . . . . . 98

3.9. Comparación de errores de localización después de la calibración del robot YAIR mediante la autocalibración multisisensorial directa y mediante el método UMBmark . . . . . . . . . . . . . . . . . . . . . .

5.1. Valores de $K^{2}$ óptimo para la odometría del robot YAIR. Nótese que después de hacer un análisis estadístico de los datos se determinó que el resultado del ensayo número 8 es un valor espurio u outlier. . . . . 167

5.2. Registro del error en el modelado de las esquinas de un entorno de oficina usando el SONAR rotativo del robot YAIR. . . . . . . . . . . . 173

5.3. Resumen de los estadísticos principales para el error en el radio y para el error en el ángulo al modelar esquinas con el SONAR del robot YAIR . . . . . . . . . . . . . . . . . . . . . 174 
6.1. Resumen estadístico del error de posicionamiento resultante al aplicar la técnica de localización que usa sólo odometría. De particular interés están la asimetría estandarizada y la curtosis estandarizada, las cuales pueden utilizarse para determinar si la muestra procede de una distribución normal. Valores de estos estadísticos fuera del rango de -2 a +2 indican una desviación significativa de la normalidad, que tendería a invalidar muchos de los procedimientos estadísticos aplicados habitualmente a estos datos. En este caso, el error $\varepsilon_{x}$ muestra una asimetría estandarizada y una curtosis estandarizada fuera del rango esperado. . 211

6.2. Resumen estadístico del error de posicionamiento resultante al aplicar la técnica de localización basada en el Filtro Extendido de Kalman. De particular interés están la asimetría estandarizada y la curtosis estandarizada, las cuales pueden utilizarse para determinar si la muestra procede de una distribución normal. Valores de estos estadísticos fuera del rango de -2 a +2 indican una desviación significativa de la normalidad, que tendería a invalidar muchos de los procedimientos estadísticos aplicados habitualmente a estos datos. En este caso, el error $\varepsilon_{x}$ muestra una curtosis estandarizada fuera del rango esperado. . . . . . . . . . . 211

6.3. Correlaciones, de acuerdo al momento de Pearson, entre los componentes del error de posición. a) Estimación de la posición usando sólo odometría. b) Estimación de la posición usando el Filtro Extendido de Kalman . . . . . . . . . . . . . . . . . . . . 212

6.4. Comparación del error RMS al final del trayecto de 140 metros programado en el robot. . . . . . . . . . . . . . . . . . . . . . . 212

6.5. Comparación del error de estimación de la posición al final del trayecto, obtenido usando dos estrategias de localización: Sólo odometría y localización basada en EKF. . . . . . . . . . . . . . . . . . . . . 220

6.6. Resumen de estadísticos del error en la estimación del estado. $\varepsilon_{x}, \varepsilon_{y}, \mathrm{y}$ $\varepsilon_{\theta}$ es el error en la coordenada $x, y$, y $\theta$, respectivamente . . . . . . . 240

6.7. Error de localización en varias de las esquinas extraídas en el proceso de SLAM . . . . . . . . . . . . . . . . . . . . 242 


\section{Capítulo 1}

\section{Motivación, objetivos, y organización de esta tesis}

\subsection{Motivación}

Los progresos tecnológicos han permitido ampliar los campos de aplicación de la robótica respecto a los de su interés inicial centrado principalmente en las aplicaciones de manipulación y fabricación industrial. Es así como irrumpió un nuevo foco de atención en la investigación en robótica: los robots móviles. Entre las áreas, tareas o aplicaciones donde se usan este tipo de de robots se encuentran[62]: El transporte y entrega de materiales, vehículos inteligentes (asistentes para el conductor del vehículo o vehículos sin operador humano), vigilancia e inspección en la industria (Monitoreo de áreas radioactivas de plantas nucleares), en ambientes de difícil acceso (Minas), en el espacio o bajo el agua; en la desactivación de bombas y minas, en tareas de servicio en casas (robots podadores de pasto o robots limpiadores), en tareas de ayuda para personas(i.e. sillas de ruedas con cierta autonomía), y asistentes robóticos para ancianos. En los últimos años se ha experimentado también un creciente interés por los robots con capacidad de identificar variaciones en su entorno, y con capacidad de reaccionar respecto al mismo sin la necesidad de intervención humana. O sea, robots con características de autonomía. La autonomía del robot es una de las características más difícil de conseguir sobre todo cuando se pretende lograrla en entornos dinámicos. Pero fundamentalmente, en aplicaciones de robótica móvil, el conocimiento de la posición o estado del robot es lo más importante para lograr la autonomía del mismo. El proceso de localización puede ser dividido de forma jerárquica general en Seguimiento de la posición, Localización basada en mapas ya conocidos, y Auto-Localización sin mapa previo o SLAM (Simultaneous Localization And Mapping). Cada uno de estos niveles constituye un amplio campo de investigación que se ha extendido en función a la manera como la comunidad científica ha enfocado el problema. Así vemos que usualmente los robots diseñados para operar en entornos interiores cuentan con sensores propioceptivos (codificadores y giroscopios) con los que a partir del conocimiento de la posición inicial, el robot puede estimar su posición a través del tiempo. 
Pero tal proceso de estimación se ve afectado por errores sistemáticos y por ruidos aleatorios que a la postre producen un error, entre la posición estimada y la posición real del robot, que es creciente con el tiempo [28]. De aquí la importancia de contar con sistemas o procedimientos que permitan tratar a este tipo de errores. En este sentido, la fusión sensorial es un excelente mecanismo para tratar esta problemática, y por lo tanto, el desarrollo de mecanismos y algoritmos de fusión pertinentes a la autolocalización de robots móviles sigue siendo de interés general en la comunidad investigadora.

Para las tareas de localización de un robot y modelado del entorno, las primeras investigaciones avanzaron fundamentándose en el uso de sensores bastante primitivos como por ejemplo los sensores de contacto o bumpers [147]. Posteriormente se avanzó hacia la navegación autónoma usando sensores de ultrasonidos, o también usando sensores de infrarrojos, como elementos sensoriales básicos. Aquí se desarrollaron sistemas que han resuelto el problema de localización y mapeado del entorno con métodos particulares, pero que en general adolecen de rapidez y de confiabilidad sobre todo al reconocer las características del entorno. Al día de hoy, gracias al desarrollo vertiginoso de la tecnología, los robots experimentales usan sensores láser[3] que son más rápidos, precisos y fiables en las distintas tareas de navegación, localización y reconocimiento del entorno. Otros robots usan sofisticados sistemas de visión estéreo que le permiten medir distancia, reconocer formas, y en general modelar el entorno de manera fiable a un costo computacional medianamente significativo. En el mercado tecnológico actual, estos son sistemas que suelen conseguirse ya fabricados y listo para su utilización e integración en otros sistemas de mayor jerarquía como por ejemplo los robots autónomos; pero tienen la desventaja de que su precio inicial puede ser alto como para integrarlos en robots de uso ordinario. Además de lo anterior, y a pesar de globalización tecnológica y de la tendencia actual a bajar los costes de los sensores más avanzados; una gran parte de los países en vías de desarrollo aun no tienen acceso a este tipo de tecnología de avanzada, o tienen dificultad para su adquisición debido a su coste. Esto último constituye una motivación adicional para no abandonar las investigaciones en el área de localización y reconocimiento del entorno usando sensores de bajo coste. Así, un trabajo que integre sensores de bajo costo para producir sistemas más avanzados y de mayor jerarquía que ofrezcan soluciones a niveles de mapeado, localización y control de robots móviles, debe gozar de alta importancia dentro de la comunidad de investigadores y desarrolladores de sistemas robotizados en estos países en vías de desarrollo.

Por otra parte, una motivación adicional al estudio y desarrollo de métodos y algoritmos para la fusión de datos provenientes de sensores de bajo costo, es que existen aplicaciones donde un sistema de medición rápido y preciso como el láser, no puede emplearse por la naturaleza del entorno de trabajo (paredes traslucidas, navegación submarina, etc.), o aplicaciones que simplemente no ameritan usar sistemas sensoriales muy avanzados (robot de servicios, robot asistenciales, etc.). En este tipo de aplicaciones, los sensores de ultrasonidos y los de infrarrojos se convierten en sistemas alternativos o cooperantes en las tareas de navegación, localización y modelado del entorno. No obstante, al utilizar este tipo de sensores hay que tomar en cuenta ciertas 
limitaciones como por ejemplo que el sensor de ultrasonidos tiene una resolución angular muy pobre(típicamente 25 grados)[16], o que el sensor de infrarrojos ofrece poca precisión y fiabilidad en la medición de distancias(aproximadamente 10\%)[18]. Estos razonamientos, aunado al hecho de que los sensores de bajo coste en general son poco fiables, obligan a desarrollar metodologías mediante las cuales se puedan fusionar la información proveniente de múltiples sistemas sensoriales, a fin de hacerle frente a los diversos retos de localización y modelado del entorno a los cuales se enfrenta un robot móvil con características de autonomía.

El grupo de Informática Industrial y Sistemas de Tiempo Real, perteneciente al departamento de Informática de Sistemas y Computadores de la Universidad Politécnica de Valencia, reúne más de 10 años dedicándose al estudio, análisis y desarrollo de investigación básica y aplicada para cubrir los aspectos de control, sensorización, planificación y tele-operación de sistemas industriales robotizados. Dentro de sus actividades se han construido una serie de prototipos de robots móviles que han servido como plataformas para la investigación en tópicos de sistemas distribuidos para control en tiempo real. Uno de los primeros prototipos, conocido como YAIR(Yet Another Intelligent Robot)[25], es una plataforma de tracción diferencial la cual cuenta con recursos sensoriales limitados (SONAR - SOund NAvigation and Ranging, anillo de infrarrojos y sensor de odometría) que han sido desarrollados en el laboratorio del mismo grupo de investigación. Este robot, a pesar de estar suficientemente instrumentado para la navegación autónoma, no cuenta con un sistema de localización fiable que le permita ampliar sus posibilidades de operación. De aquí surge la motivación de desarrollar en esta tesis un sistema de autolocalización que esté basado en los recursos sensoriales limitados que posee el robot YAIR, y que sea lo suficientemente fiable para que el robot opere de forma autónoma en ambientes estructurados. El objetivo final del sistema de localización es otorgarle a YAIR parte de la autonomía requerida para desplazarse por pasillos y habitaciones de entornos interiores, y realizar las tareas que le sean encomendadas. Para garantizar esto, se debe dotar a este robot de la capacidad de razonamiento y aprendizaje para la construcción de mapas y para autolocalizarse a partir de la información sensorial limitada e imprecisa con la que cuenta.

\subsection{Objetivos}

Los objetivos de esta tesis están enmarcados dentro del área de auto localización y construcción de mapas de forma simultanea en aplicaciones de robótica móvil. El objetivo general planteado es

- Diseñar y evaluar métodos de auto localización de robots móviles basándose en mapas construidos mediante la fusión de la información proveniente de sensores de bajo costo.

Los objetivos específicos se pueden enumerar según: 


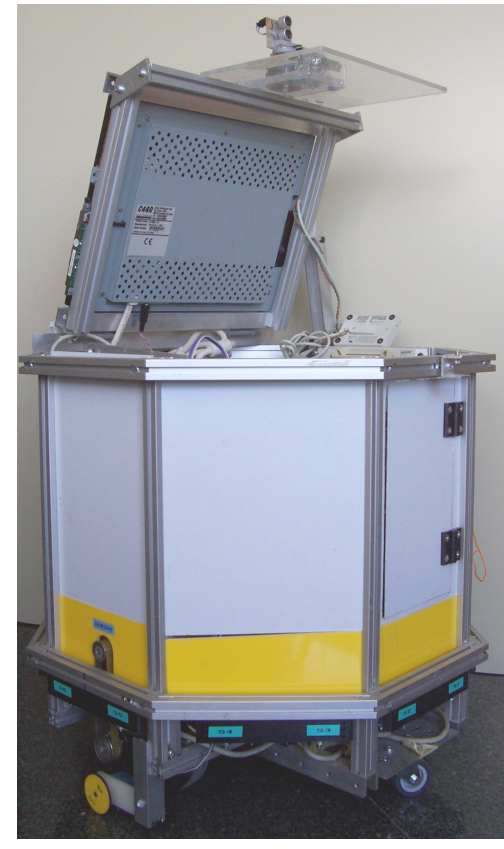

Figura 1.1: El prototipo de robot móvil de tracción diferencial conocido como YAIR(Yet Another Intelligent Robot)

1. Desarrollar modelos matemáticos de sensores de bajo costo de uso típico en la percepción de entornos interiores en aplicaciones de robótica móvil, considerando también el modelo de propagación de la incertidumbre en la medición.

2. Derivar el modelo cinemático y estocástico de localización de un robot diferencial a partir de la información proveniente de codificadores rotativos. Este modelo debe ser pertinente y poseer características que permitan incluirlos en procesos de auto-localización basadas en el Filtro Extendido de Kalman(EKF).

3. Proponer y evaluar métodos de fusión que contribuyan a mejorar la calidad de los mapas de entorno usados en la localización de robots móviles.

4. Implementar métodos de localización de robots móviles basándose en la información multi-sensorial disponible en el mismo, asegurándose que los mismos puedan ejecutarse en tiempo real.

Dado que los sistemas y algoritmos que se propongan serán validados mediante experimentos realizados con un robot real y en entornos reales, se debe garantizar que los mismos puedan ser ejecutados en tiempo real y con los recursos computacionales y sensoriales con los que cuenta el robot YAIR. En este sentido, los objetivos anteriormente descritos estarán limitados a las siguientes condiciones referidas a los sensores, al robot y al entorno donde él opera: 
Entorno interior bien estructurado, estático, y sin hitos o landmark artificiales. El robot se moverá en un entorno tipo oficina, con superficie plana. Se asume que tanto durante la construcción del mapa como durante la localización del robot, el entorno no cambia, y que el robot es el único objeto que se mueve en el mapa. También se considera que el entorno no ha sido modificado con ningún tipo de landmark artificial para ayuda a la navegación.

Robot móvil de tracción diferencial. La planta de ensayos que se utilizará es una plataforma multisensorial de tracción diferencial desarrollada en nuestro laboratorio como banco de prueba en experimentos relacionados con sistemas de tiempo real. Este tipo de configuración diferencial facilita la representación del robot, pero al mismo tiempo le confiere restricciones de holonomía que a la postre limita la posibilidad de movimiento del mismo.

Sensores de bajo costo. El robot que sirve como planta experimental en el desarrollo de esta tesis cuenta con los siguientes sensores: un sensor rotativo de ultrasonido, un anillo de infrarrojo, un compás electrónico, y un sensor de odometría basado en codificadores rotativos acoplados a las ruedas motrices del robot. La utilización de estos sistemas sensoriales, que en general son de bajo costo, no debe afectar la funcionabilidad del sistema de autolocalización, el cual debe ser capaz de manejar fiablemente las incertidumbres propias e inherentes de cualquier sistema sensorial o de actuación.

\subsection{Organización de la tesis}

El problema de la autolocalización de un robot móvil es uno de los mas difíciles de abordar debido a la cantidad de instancias, técnicas y limitaciones que se conjugan en ella. Pero en general hay tres instancias principales que siempre hay que tomar en cuenta: la sensorización, la representación del entorno, y los métodos o procedimientos para la combinación de los datos y el manejo de la incertidumbre. El capítulo 2 de esta tesis es un capítulo introductorio donde se ofrece un primer contacto con los diferentes sensores que típicamente se usan en la localización y el mapeado del entorno en el contexto de la robótica móvil. En este capítulo también se introduce los principales esquemas y métodos para la fusión de datos, los tipos de representación del entorno más usuales, y las técnicas comúnmente usadas para la localización relativa o absoluta de un robot móvil.

En robótica móvil, contar con una metodología o técnica básica de localización es muy útil al momento de ejecutar otras tareas más avanzadas o complejas. El proceso más básico de localización de un robot móvil se basa en el modelo cinemático del sistema de propulsión. Este tipo de localización conocido como Dead-Reckonig ofrece poca fiabilidad debido a factores como la imperfección en el modelado del vehículo, 
la variación en el tiempo de los parámetros de este modelo, o también debido a los errores no sistemáticos producto de la interacción entre el robot y el resto del entorno. En el capítulo 3 se obtiene el modelo cinemático de un robot diferencial prototipo, se analizan las principales fuentes de error sistemático que tienen influencias en el proceso de localización odométrica, y se proponen un método de identificación y calibración automática de los parámetros cinemáticos de un robot diferencial. Este método integra los datos sensoriales del sensor de ultrasonidos, del compás magnético y de los codificadores rotativos; para dotar al robot de la capacidad no sólo de auto ajustar sus parámetros cada vez que lo requiera, sino también con la capacidad de ajustar los mismos de acuerdo a las condiciones del plano de rodamiento donde se encuentre operando en ese momento.

La representación del entorno es otro de los pilares fundamentales que soportan al proceso de autolocalización de robots móviles. El tipo de representación que se utilice para este último fin estará condicionado a la capacidad sensorial con la que cuente el vehículo móvil. En el capítulo 4 se hace una revisión de la tecnología asociada a los sensores que forman parte del robot YAIR, y se desarrollan los modelos sensoriales necesarios para aplicar las estrategias de fusión sensorial a la construcción de mapas de entorno. Particularmente, se hace un estudio mas detallado del sensor de medición de distancia basado en ultrasonido con el que cuenta el robot YAIR, y se desarrollan los modelos probabilísticos y posibilísticos de este sensor. Esto permite insertar a este sensor en la construcción de mapas de densidad de ocupación obtenidos mediante fusión de datos basados en la teoría de Bayesiana y en la teoría de la evidencia de Dempster-Shafer respectivamente. Así mismo, se propone una metodología que mejora la calidad de estos mapas, basándose en la incorporación de una máscara que se aplica a las paredes y a las esquinas detectadas en el proceso de exploración del entorno. Por otra parte, en este mismo capítulo se introduce el sensor de infrarrojos que usa el robot YAIR, y se revisa el mecanismo de fusión bi-sensorial que permite eliminar la dependencia del ángulo de incidencia en los procesos de medición de distancias mediante este sensor. Así mismo, Se desarrollan la metodología para la extracción y construcción de mapas de características lineales usando un sensor de infrarrojos. Debido al poco alcance que ofrecen estos sensores, los mapas se deben construir incrementalmente mientras se explora el entorno. De aquí que en el capítulo 4 también se desarrollen los métodos de agrupación de datos de infrarrojo, y de segmentación y fusión de líneas extraídas de los datos proporcionados por este sensor.

Hoy en día los métodos más exitosos usados en robótica móvil son todos probabilísticos. Cuando se requiere usar alguno de estos métodos es necesario contar con modelos estocásticos que sirvan para modelar la incertidumbre y representar los errores no sistemáticos en el sistema, la observación y la representación del entorno. De aquí que en el capítulo 5 se desarrolle el modelo estocástico de un robot diferencial y se proponga un método experimental para determinar la varianza que afecta al modelo del mismo. Igualmente, se desarrolla el modelo estocástico de un SONAR rotativo que es el sensor que sirve como medio principal de observación del entorno en las tareas de extracción de características y autolocalización que cumple el robot YAIR. Por otro lado, la representación de entorno también está caracterizada por una in- 
certidumbre que generalmente se hereda de la conjugación del modelo de observación y del modelo de localización odométrico del vehículo que realiza la exploración del entorno. Así mismo, usualmente es necesario transformar esta incertidumbre entre los distintos sistemas coordenados que sirven de referencia en el proceso general de autolocalización (coordenadas del sensor, coordenadas del vehículo, coordenadas de la representación global). Por esta razón, en este mismo capítulo se desarrollan los modelos estocásticos de la representación del entorno mediante características puntuales y mediante segmentos de línea, así como también se desarrolla la metodología de transformación o propagación del error de estas características entre los distintos sistemas de referencia. Otra fuente de incertidumbre durante el proceso de autolocalización en la robótica móvil es el problema de correspondencia, también conocido como el problema de asociación de datos. Este problema consiste en determinar si las mediciones tomadas con el sensor en distintos instantes de tiempo se corresponden con algún objeto del mundo que ya haya sido observado. En la sección 5.5 de esta tesis se propone un método basado en la distribución bivariante de la varianza del sensor, que sirve para determinar la correspondencia entre Hitos puntuales o la correspondencia entre segmentos de línea. Este método se basa en determinar si existe algún objeto del entorno ya observado, que esté dentro de la elipse de incertidumbre asociada a la característica que actualmente se esté observando.

Dado que el movimiento del robot y el modelado del entorno están caracterizados por la incertidumbre y el ruido sensorial, la robótica móvil está dominada por técnicas probabilísticas. El principio básico subyacente en todo algoritmo probabilístico es la Regla de Bayes. Esta provee una manera conveniente de computar la probabilidad a posteriori de una determinada distribución, en función de la probabilidad a priori de la misma distribución. El Filtro de Bayes extiende la Regla de Bayes para tratar así problemas de estimación en el dominio del tiempo. Una de las implementaciones más usadas del Filtro de Bayes, está basada en el Filtro de Kalman. Este filtro es un estimador recursivo del método de mínimos cuadrados, que produce un estimado óptimo del estado en el sentido que la varianza resultante es mínima. Esta solución asume que el modelo del sistema y el modelo de observación están sujetos al efecto del ruido Gausiano. En el capítulo 6 se emplean los modelos probabilísticos del robot, de sus sensores y de su entorno, para junto con las ecuaciones del algoritmo del Filtro de Kalman, resolver tres problemas distintos de estimación: construcción de un mapa de entorno a partir de las posiciones perfectamente conocidas del móvil, localización del móvil asumiendo que el entorno es perfectamente conocido, construcción del mapa y localización del vehículo de forma simultanea. Así, en este capítulo se desarrollan los métodos y estrategias que permiten integrar los sensores limitados con los que cuenta el robot YAIR, en operaciones de estimación óptima del estado basada en el Filtro de Kalman.

Finalmente, en los anexos se proporciona información que puede ser útil para que lectores con poca experiencia en la robótica móvil, puedan entender con mayor facilidad los algoritmos y métodos que fueron usados o que son propuestos en esta tesis. Así, en el anexo A se proporciona información sobre un marco Bayesiano conocido como Localización de Markov. Este marco teórico sirve como base para el 
desarrollo de algoritmos probabilísticos que estiman el estado o posición de un robot móvil en presencia de incertidumbre. En el anexo B, se presentan las ecuaciones del Filtro de Kalman, el cual es un algoritmo recursivo de estimación óptima del estado de un sistema contaminado con ruido blanco. Este filtro es el que se usa en esta tesis como método fundamental de fusión de datos sensoriales para la autolocalización y mapeado del entorno usando un robot móvil. En el anexo $\mathrm{C}$ se introduce el método de Mínimos Cuadrados, el cual es uno de los métodos fundamentales usados en el algoritmo de extracción de segmentos de línea a partir de datos de distancias obtenidas mediante el sensor de infrarrojos. 


\section{Capítulo 2}

\section{Introducción a la localización de robots móviles}

El problema de la localización de robots móviles consiste en contestar, desde el punto de vista del robot, a la pregunta: ¿Donde me encuentro? (where am I?). Esto significa que el robot, valiéndose de sus sensores, debe encontrar su posición relativa al entorno donde se encuentra. El problema general de localización consta de un cierto número de instancias que se pueden clasificar según su grado ascendente de dificultad: seguimiento de posición(Tracking), posicionamiento global(Global localization), recuperación ante transferencias intempestivas(Kinapping problem), y localización en ambientes dinámicos .

En el problema de seguimiento de la posición, el robot conoce su posición inicial. El objetivo de este tipo de localización es mantener un seguimiento sobre la posición del robot mientras que este navega a través del entorno. Usualmente las técnicas que resuelven este tipo de problema se conocen como técnicas locales o de seguimiento[96]. Cuando se conoce la posición inicial del robot, basta con estimar su desplazamiento relativo para tener localizado al mismo. Dentro de las técnicas llamadas a ejecutar esta tarea, la más sencilla consiste en usar los sensores de abordo del robot (odómetros, acelerómetros, giroscopios) para medir el desplazamiento del mismo. El principal problema con esta técnica es que el error en el estimado de la posición se va integrando en el tiempo y por lo tanto crece sin límites. Para corregir este problema es necesario usar sensores externos que suministren información relativa a la posición absoluta del robot. Esto último a su vez se logra buscando la correspondencia entre las medidas sensoriales y un mapa o modelo del entorno. Ahora bien, encontrar esta correspondencia se convierte en el principal problema del proceso de estimación. Sin embargo, si se suministra un buen estimado de la posición inicial del robot, el problema de correspondencia se hace más sencillo de resolver ya que no es necesario considerar todo el espacio de trabajo para localizar al robot, sino que hay que considerar solamente un espacio prudente en los alrededores de la posición inicial suministrada.

En el problema de posicionamiento global, el robot desconoce su posición inicial, y por lo tanto él debe autolocalizarse desde cero, por lo que posiblemente necesite ser 


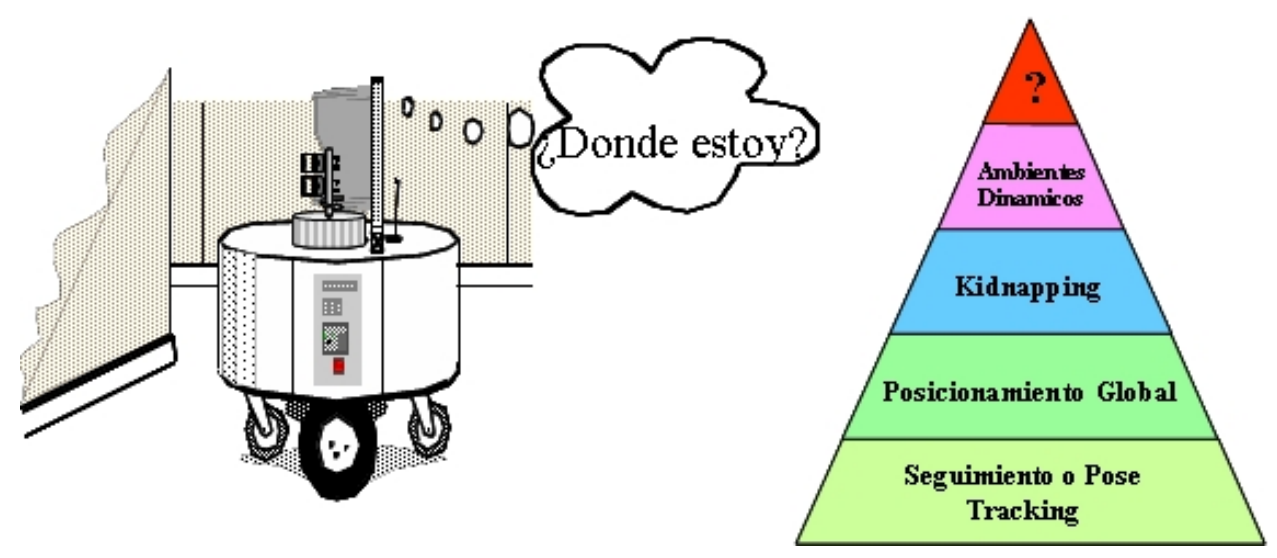

Figura 2.1: Clasificación jerárquica de la localización de robots móviles

capaz de tratar con un problema multi-hipótesis referido a su estado. Los métodos que tratan con este tipo de problemas de localización se conocen como técnicas de localización global[75]. Su dificultad es muy superior al problema de seguimiento, ya que se debe resolver el problema de correspondencia de datos sobre todo el espacio de trabajo. Así, el nivel de complejidad de esta tarea varía en función del tamaño y simetría del entorno.

Un problema aun más difícil de resolver es el de recuperar la posición después de un súbito e intempestivo traslado del robot debido a causas externas. En este problema conocido como secuestro(Kidnapping)[68], el robot conoce exactamente su posición pero súbitamente es trasladado a otra localización sin que el mismo se pueda enterar de este hecho. De esta forma, el problema para el robot es detectar que ha sido trasladado y encontrar entonces cual es su nueva posición. Las técnicas que se utilizan para localización global, también pueden ser utilizadas en este problema. De hecho, el problema de localización global es un caso especial de kinapping en el cual se le ha dicho al robot que ha sido trasladado o secuestrado.

La mayoría de las investigaciones en localización de robots se limitan a ambientes estáticos donde el robot es el único objeto que se mueve, lo que no es caso muy realista. Un factor que complica cada uno de los problemas de localización anteriormente descritos es el dinamismo que pueda caracterizar el entorno donde se mueve el robot. Los entornos dinámicos contienen objetos que se mueven y eso hace más difícil la localización es este tipo de ambientes, ya que el movimiento de estos objetos pueden generar confusion al contaminar los datos exteriores que el robot usa para su localización[76].

Para la implementación de los procesos que resuelven todos los problemas anteriormente descritos, es necesario contar con un mapa del entorno. En algunos casos se puede disponer de un mapa del entorno hecho mediante CAD (Computer Aided Design), pero el caso más general es que el mapa deba ser construido a partir de los datos suministrados por los sensores del robot, por lo que la posición del mismo debe ser previamente conocida con cierta precisión. Ya que la estimación de la posición 
requiere de un mapa, y la construcción del mapa requiere del conocimiento de la posición, surge una nueva jerarquía en el problema de localización, la cual es conocida como SLAM (Simultaneous Localization And Mapping). El problema de construir un mapa del entorno, al mismo tiempo que se estima la posición del robot, es un problema de incrementada complejidad ya que se requiere explícitamente que se modele la correlación entre la posición estimada para el robot y el mapa en si mismo.

\subsection{Sensorización en robótica móvil}

Muy estrechamente ligada a la autonomía de un robot está la capacidad de percibir el entorno y actuar sobre el mismo. Por medio de los sensores el robot obtiene datos sobre su entorno (sensación) y luego los procesa para su utilización (percepción). De esta forma, los sensores representan para el robot la fuente de datos sobre los cambios tanto en su entorno (distancias a objetos, luz ambiental) como en sí mismo (nivel de las baterías, consumo de los motores, pulsos de codificadores, etc.).

En robótica móvil se usan una gran variedad de sensores que miden distintas magnitudes. En general se puede clasificar los sensores en dos grupos: sensores internos y sensores externos. Los sensores internos son aquellos que no interaccionan con el entorno del robot. Su función es suministrar datos relacionados a las variables cinemáticas del robot y de como varían en función del accionamiento de los motores. Los sensores internos más característicos son: potenciómetros, tacómetros, codificadores ópticos rotativo, giroscopios y acelerómetros.

Los sensores externos son sistemas que interaccionan con el entorno para cuantificar alguna variables perteneciente al mismo. Entre los sensores externos cabe destacar:

Ultrasonidos Son ampliamente utilizados en aplicaciones de modelado del entorno. Los sensores de ultrasonidos permiten la medida de distancias a partir del tiempo transcurrido desde la emisión de un pulso de ultrasonido hasta que éste es recibido nuevamente por el receptor. La precisión obtenida puede ser de un centímetro pero las medidas dependen de factores como temperatura ambiente, movimientos de aire y fuentes acústicas de alta frecuencia (máquinas rotativas). Tienen un alcance entre $40 \mathrm{~cm}$ y $10 \mathrm{~m}$.

Infrarrojos Tienen mayor precisión $( \pm 2 \mathrm{~mm}$ ) pero menor alcance que los sensores de ultrasonidos (entre $10 \mathrm{~cm}$ y $80 \mathrm{~cm}$ ) por lo que son útiles mas que nada para maniobras de aproximación y de navegación por evitación de obstáculos.

Láser Puede ser utilizado para detectar medidas de distancias a objetos opacos, así como para la localización mediante triangulación determinando la distancia a puntos conocidos del entorno. Poseen buena precisión y velocidad de adquisición de datos, pero en contrapartida presenta un costo generalmente elevado.

Visión Las cámaras son ampliamente usadas en sistemas de localización y construcción de mapas del entorno. Los sistemas de visión estereoscópica, consisten en 
un par de cámaras que además de permitir la detección de contornos, también permite obtener de forma aproximada la distancia a objetos a partir de la divergencia de ambas cámaras.

Compás o Brújula, Son sensores capaces de medir el campo magnético de la tierra y expresarlo en forma de una señal eléctrica. Ofrecen una resolución alrededor de 0.1 grados, con exactitud promedio de 0.5 grados. Su tiempo de respuesta está por debajo de 1 microsegundo, lo que los hace útiles en aplicaciones de navegación automática, sobre todo en ambientes exteriores.

En la robótica móvil, los sensores que más se utilizan son los orientados a resolver el problema de la determinación de la posición. Enmarcado dentro de esta problemática, en $[69,71]$ se lleva a cabo una revisión de varias de las tecnologías y metodologías utilizadas en el ámbito de sensores y procesamiento de la información proporcionada por los mismos. Adams[3] hace una revisión algo más actualizada que incluye detalles sobre el procesamiento básico de cada sensor. A continuación se hace una introducción de los principios de los sensores que se usan en el desarrollo de esta tesis.

\subsubsection{Codificador rotativo.}

A fin de desarrollar sus estrategias de navegación, la gran mayoría de los vehículos en la robótica móvil se fundamentan en la estimación del estado. La forma más simple de implementación de la estimación del estado en robótica móvil se conoce como Odometría, ya que el termino implica que los desplazamientos del vehículo a lo largo de una trayectoria son derivados a partir de las observaciones de un odómetro de abordo. Un instrumento odométrico muy común es el codificador óptico rotativo, el cual se acopla directamente al eje del motor o al de las ruedas de tracción. El principio básico del codificador óptico esta centrado en un haz de luz que alcanza un foto detector. Este haz de luz es interceptado por áreas opacas y trasparentes que se alternan en un disco rotativo que está sólidamente unido al eje de interés.

Existen dos tipos básicos de codificadores rotativos: Los increméntales y los absolutos. La versión incremental mide la velocidad de rotación y puede inferir la posición relativa. Por su parte los modelos absolutos miden directamente la posición angular y puede inferir la velocidad. En la aplicaciones de posicionamiento se prefiere el uso de los codificadores incrementales, ya que el mayor interés está en la velocidad de las ruedas y no en su posición angular. También, la interfaz de los codificadores incrementales es en general más sencilla que la de los codificadores absolutos, y además los primeros proveen la misma resolución equivalente que los segundos, pero a menor costo. Un tipo sencillo de codificador incremental es el codificador tacométrico de un sólo canal, el cual produce a su salida un cierto numero de pulsos de onda senoidal o de onda cuadrada por cada revolución de su eje. Una limitación de este tipo de codificador es que es incapaz de resolver la dirección de rotación, por lo que no puede ser usado en aplicaciones de estimación de la posición. Los codificadores incrementales de cuadratura resuelven este problema adicionándole un segundo canal, el cual esta desplazado 90 grados del primer canal, según se muestra en la figura 2.2. Usando 

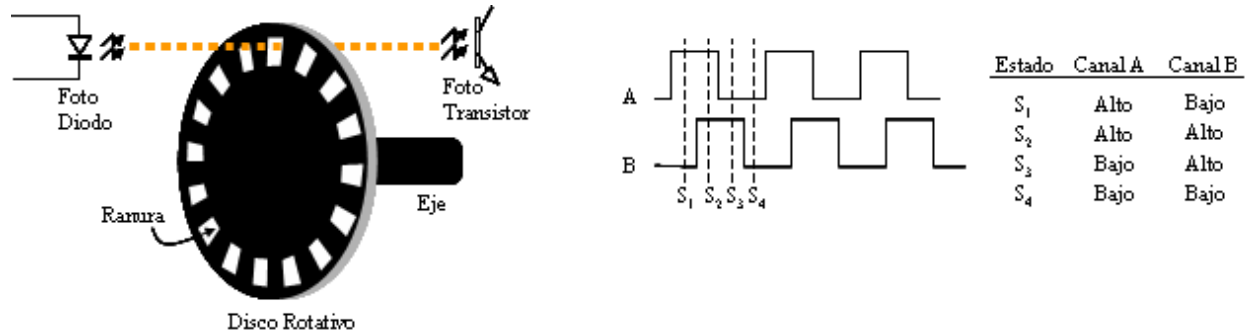

Figura 2.2: Principio del codificador óptico de cuadratura de dos canales, similar a los que usa el robot YAIR

la electrónica adecuada, mediante esta técnica se puede determinar cual canal esta adelantado respecto al otro, estableciéndose de esta manera la dirección de rotación.

\subsubsection{Sensores de ultrasonido}

Los ultrasonidos corresponden a ondas vibratorias similares a las ondas sonoras, pero a una frecuencia demasiado altas para ser percibidas por el oído humano. Aunque su rango se extiende entre 20 y $500 \mathrm{KHz}$, sus aplicaciones más típicas, como por ejemplo la medición de distancias, están ubicadas en la banda entre 40 y $125 \mathrm{KHz}[117]$.

Un transductor electro-acústico frecuentemente usado en robótica móvil es el tipo piezoeléctrico, el cual se usa típicamente en tareas de medición de distancia. La simetría circular o tipo pistón de estos transductores permite expresar el patrón de intensidad de campo como una función del ángulo $\theta[117]$,

$$
E_{T}=e^{-2 \theta^{2} / \theta_{0}^{2}}
$$

Este ángulo se mide a partir del eje del transductor o línea central del haz, como se ve en la figura (2.3). El ángulo $\theta_{0}$ representa la mitad del lóbulo de sensibilidad del transductor (ancho del haz), y se puede aproximar según Skolnik [182] como,

$$
\theta_{0}=\sin ^{-1}(0,6158 \lambda / a)
$$

donde $\lambda$ es la longitud de onda de la señal aplicada al transductor, y $a$ es el radio de apertura circular del mismo.

También, como el patrón del transductor que se usa como receptor es igual al del transmisor, entonces el patrón de señal de un sensor de ultrasonidos basado en principios piezoeléctricos puede ser modelado como,

$$
E_{T / R}=E_{T} \cdot E_{R}=e^{-4 \theta^{2} / \theta_{0}^{2}}
$$

En la robótica móvil, la aplicación básica de los sensores de ultrasonidos es la medición de distancias. La gran mayoría de estas aplicaciones se basan en la estimación del tiempo que transcurre entre la emisión de un corto tren de pulsos de ondas ultrasónicas, y su recepción después de haber sido reflejado por algún objeto del entorno. 


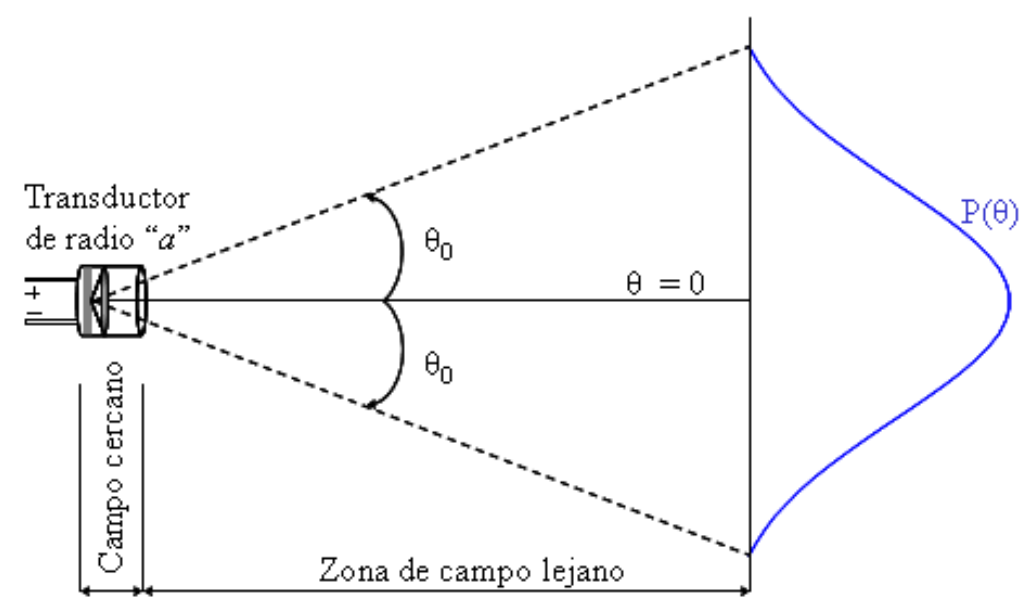

Figura 2.3: Patrón de amplitud de campo lejano para un transductor ultrasónico tipo pistón

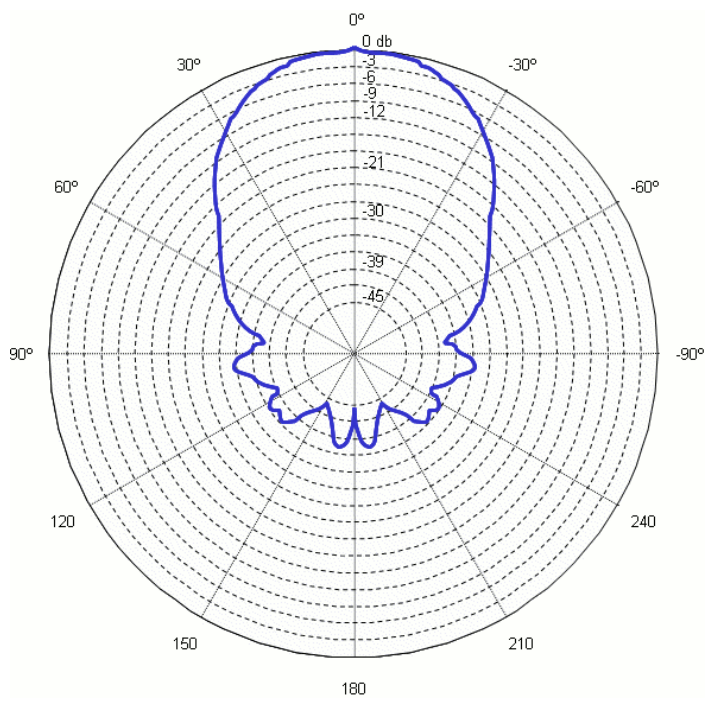

Figura 2.4: Patron de sensibilidad de los transductores ultrasónicos usados por el sensor de YAIR 


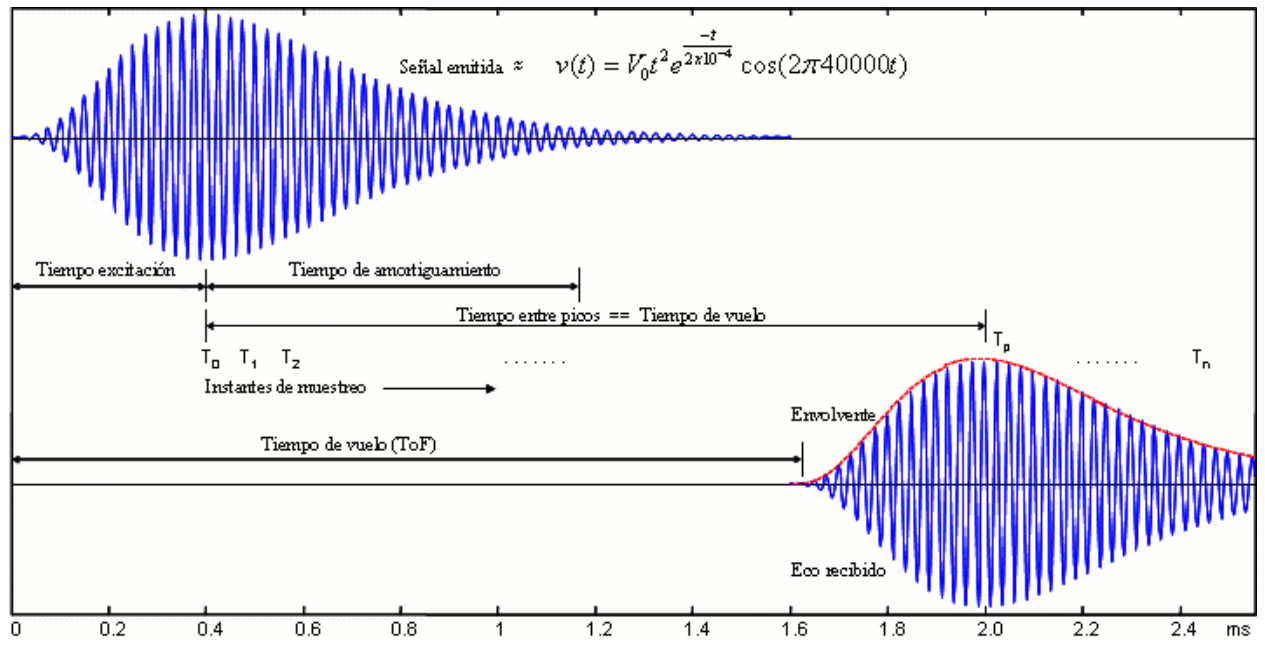

Figura 2.5: Diagrama de tiempos típico en un medidor de distancias por ultrasonidos. En este caso, el tiempo de vuelo se mide entre los máximos de la señal emitida y el máximo del eco recibido.

Este período de tiempo se conoce comúnmente como tiempo de vuelo o ToF (Time of Fly $[164,140]$. La distancia al reflector puede ser estimada mediante la fórmula

$$
d=\frac{1}{2} v \cdot t_{f}
$$

donde $v$ representa la velocidad del sonido en el medio de transmisión y $t_{f}$ el tiempo de vuelo.

Una técnica para determinar el tiempo de vuelo se basa en generar un pulso de señal de fácil identificación como la mostrada en la figura (2.5). Entonces, Una manera simple de determinar el tiempo de vuelo consiste en medir el tiempo entre el pico máximo de la señal emitida y el pico máximo del eco recibido. Esta operación se facilita si se extrae la envolvente de la señal. De esta manera basta con determinar el instante de muestreo $p$ para el pico máximo de la envolvente del eco recibido, para obtener así el tiempo de vuelo $T_{p}$. Ya que $T_{p}=p \cdot T_{s}$, con $T_{s}$ igual al periodo de muestreo y $p$ igual al índice de la muestra, entonces la distancia $d$ al reflector se obtiene mediante la ecuación,

$$
d=\frac{1}{2} v_{s} \cdot T_{p}
$$

Dado que la resolución del tiempo de vuelo $T_{p}$ se ve limitada por el período de muestreo $T_{s}$, se requiere de una mejora para poder obtener una exactitud mejor que la mitad de este período. Esta mejora puede ser obtenida mediante una interpolación parabólica sobre tres puntos: el punto de máxima amplitud estimado inicialmente y los dos puntos que lo rodean(ver figura 2.6).

El diferencial de tiempo $\Delta T_{s}$ entre el máximo de la parábola y el máximo estimado en el muestreo se puede calcular mediante la ecuación, 


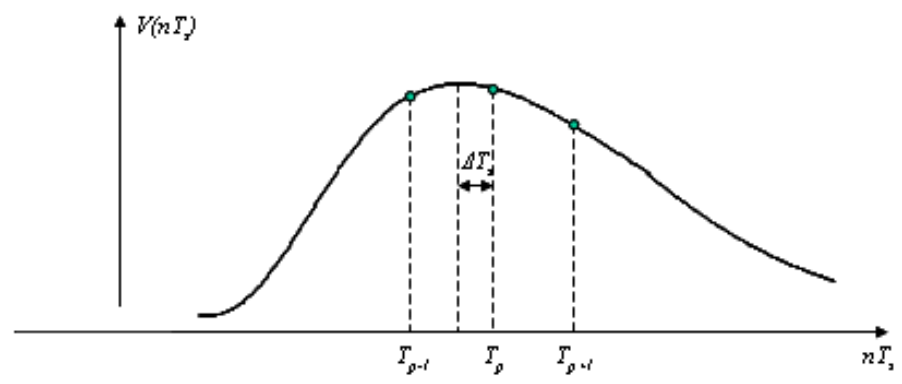

Figura 2.6: Interpolación parabólica en un eco de ultrasonidos para determinar con mayor exactitud el tiempo de pico.

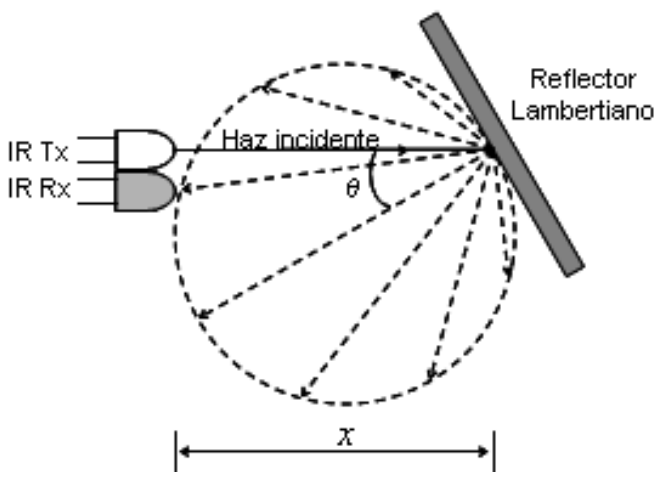

Figura 2.7: Principio de reflexión del haz infrarrojo incidente en un reflector Lambertiano.

$$
\Delta T_{s}=\frac{V\left(T_{p-1}\right)-V\left(T_{p+1}\right)}{2\left[V\left(T_{p-1}\right)-2 V\left(T_{p}\right)+V\left(T_{p+1}\right)\right]}
$$

donde $V\left(T_{p}\right)$ es el valor de amplitud medido en el instante $p$, el cual representa la máxima amplitud determinada en el proceso de muestreo(figura 2.6).

\subsubsection{Sensor de infrarrojos}

El transductor de infrarrojos es sensible a la intensidad de una señal luminosa. Por eso uno de los principales inconvenientes en las aplicaciones de medición por infrarrojos es el efecto de otras señales luminosas que no son propiamente las que se quieren medir (luz solar, luces artificiales). Un sensor de distancia por infrarrojos esta compuesto típicamente por un emisor LED y un fotodiodo encerrado en una cápsula que filtra la radiación visible. En su funcionamiento más básico el emisor es excitado mediante un pulso de corriente continua, emitiéndose así un haz de luz del espectro infrarrojo que al ser reflejado por algún objeto, entonces es captado por el fotodiodo el cual produce un pequeño voltaje de salida que depende de la cantidad de energía que alcance al receptor (ver figura 2.7). 
La energía infrarroja que refleja un objeto posee las siguientes características:

1. Decae aproximadamente en forma cuadrática con la distancia entre el reflector y el receptor.

2. Es mayor cuanto más reflectivo sea el objeto. El coeficiente de reflexión depende principalmente del color, brillo y textura del objeto.

3. Es mayor cuanto más cercano sea a $0^{\circ}$ el ángulo que forma el sensor con la normal al objeto reflector.

Considerando la ley de fotometría cuadrática-inversa y un reflector Lambertiano perfecto, se puede modelar la salida del sensor como

$$
s(d, \eta)=\frac{\alpha_{n} \alpha_{s}}{d^{2}} \cos \eta+\beta
$$

donde $\alpha_{n}$ incluye las características del transductor $n$ (intensidad de los emisores, sensibilidad al espectro de los fotodiodos y la ganancia del sensor), $\alpha_{s}$ modela la característica del reflector(coeficiente de reflexión del objeto), y $\beta$ modela el desplazamiento de la señal debido a la luz ambiental.

\subsubsection{Compás}

La integración del compás en la robótica móvil es una posibilidad ampliamente considerada por los investigadores[69]. Sin embargo en la práctica son pocos los robots equipados con compás que han sido reportados en la literatura, ya que su uso en ambientes interiores tiene una importante desventaja: la señal de salida del compás puede ser fácilmente contaminada por fuentes electromagnéticas(i.e. cables eléctricos) o por grandes estructuras ferromagnéticas(i.e. estantería metálicas). Esto hace imposible usar las brújulas electrónicas como sensores de orientación confiables en ambientes interiores. Debido a lo anterior, en general el compás es usado más que nada como un sensor de apoyo a otros sistemas sensoriales, como por ejemplo cuando se usa para compensar el efecto de los derrapes en las ruedas de odometría[90, 109, 136]. Por otra parte, de los tres estados $\langle x, y, \theta\rangle$ usualmente controlados en un robot móvil, el más significante es la orientación $\theta$ debido a la gran influencia que tiene en la acumulación de los errores de estimación de la posición. Por esta razón, los sensores que proveen una medición de la orientación absoluta o de la velocidad angular relativa son extremamente importantes en la resolución de los problemas de navegación de una plataforma móvil autónoma. De aquí que el compás siga siendo de interés en la comunidad científica en la robótica móvil.

El compás se basa en la medición de las componentes de campo magnético de la tierra para determinar la orientación del móvil donde se encuentra instalado. Los instrumentos sensibles al campo magnético son conocidos como magnetómetros, y para aplicaciones de navegación en robótica móvil, son sólo de interés aquellos que son capaces de medir el campo magnético de la tierra, y expresarla a través de una 

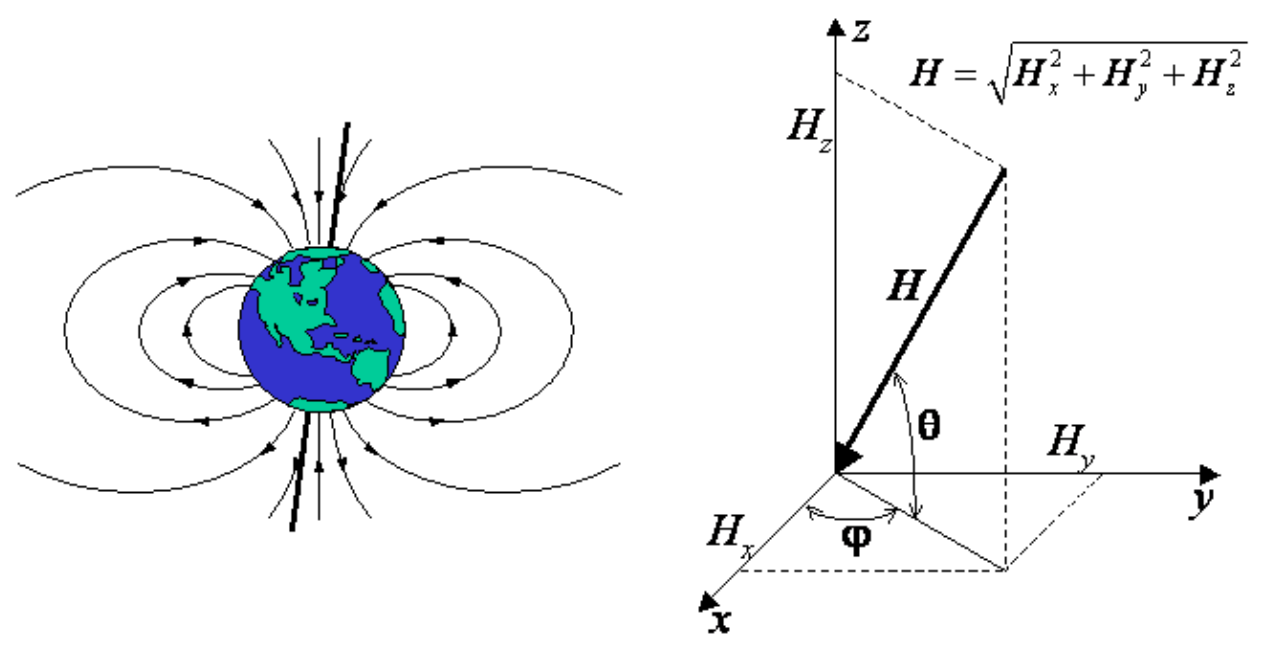

Figura 2.8: Izquierda y derecha: forma y vector del campo magnético de la tierra

señal eléctrica. Este tipo de instrumento se les conoce como compás electrónico. La intensidad del campo magnético de la tierra está entre 0.5 y 0.6 gauss, y puede ser modelado como un dipolo magnético cuyas líneas de campo se originan en un punto cerca del sur geográfico de la tierra y terminan en un punto cercano al norte geográfico de la tierra. La dirección e intensidad de campo magnético se representa por un vector de tres componentes $\left\langle H_{x}, H_{y}, H_{z}\right\rangle$ según se puede ver en la figura 2.8.

Un tipo de compás electrónico muy usado es el basado en transductores magneto resistivos, cuya resistencia eléctrica varia con los cambios del campo magnético aplicado. Estos tipos de sensores presentan sensibilidades por debajo de 0.1 miliGauss con tiempos de respuesta menor a 1 microsegundo, lo que permite su uso confiable en vehículos que se mueven a altas velocidades[38].

El compás electrónico más simple esta compuesto por dos transductores magneto resistivos alineados verticalmente y desfasados 90 grados entre sí. Estos transductores presentan su máximo valor de salida cuando su respectivo eje se encuentra alineado con el polo norte de la tierra. De esta manera, si el compás se encuentra en una zona abierta, despejada y suficientemente plana, las componentes de salida del magnetómetro $\left\langle H_{x}, H_{y}\right\rangle$ pueden ser modeladas $\operatorname{como} \cos \varphi$ y $\sin \varphi$ respectivamente, donde $\varphi$ representa el azimut referido al norte magnético. Ya que $\varphi=\tan ^{-1}\left(H_{y} / H_{x}\right)$, entonces en el caso más simple, se puede determinar la orientación de un compás mediante la medición de las componentes de campo paralelas a la superficie terrestre. En este modelo hay que tomar en cuenta que la tangente así calculada es válida sólo para el intervalo $-90^{\circ}<\varphi<90^{\circ}$, y que además no se permite la división por $H_{x}=0$. Por eso, un conjunto de ecuaciones que puede ser usado para determinar la orientación de un compás de este tipo son las mostradas en la figura 2.9. 


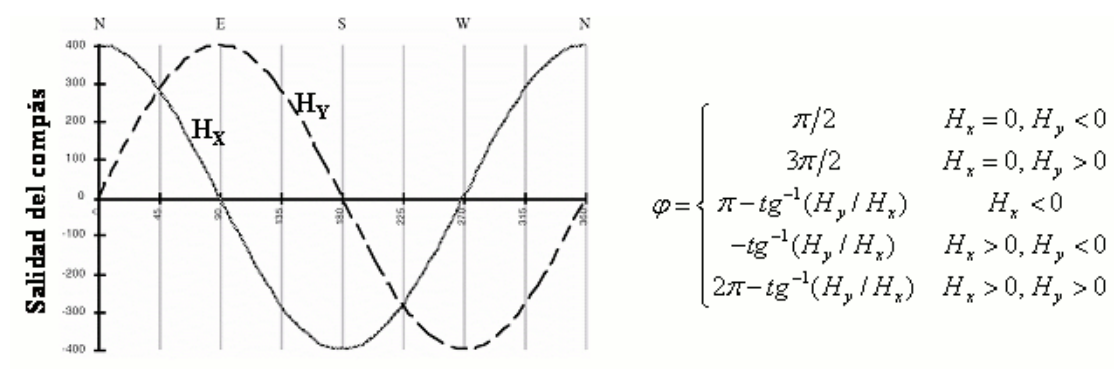

Acimut(gradas)

Figura 2.9: Izquierda: componentes de salida de un compás magnético como el usado en el robot YAIR. Derecha: Ecuación para determinar el ángulo de acimut.

\subsection{Fusión sensorial}

La fusión sensorial se refiere a la combinación de diferentes datos provenientes de un mismo sensor a diferentes instantes de tiempo(fusión en tiempo) o provenientes de múltiples sensores al mismo tiempo (fusión multisensorial). La fusión de datos persigue mejorar la exactitud y realizar inferencias más específicas que las que se podrían obtener usando un simple dato o un sólo sensor. En este sentido, la fusión de datos multisensoriales, además de proporcionar la ventaja del filtrado estadístico que se gana con la combinación de los datos provenientes de la misma fuente(observaciones redundantes), puede incrementar la exactitud del resultado del proceso gracias a las características individuales de cada sensor. Por ejemplo, un sonar tiene buena capacidad para determinar con exactitud la distancia a la cual se encuentra un determinado objeto, sin embargo tiene capacidad limitada para determinar su posición angular. Por otra parte, un sensor de infrarrojos puede determinar con buena exactitud la dirección angular en la que se encuentra el objeto, pero al mismo tiempo es menos preciso al momento de determinar la distancia a la cual se encuentra el mismo. Si estas dos observaciones son combinadas adecuadamente, entonces el estimado de la posición del objeto puede ser mejorado. La idea de este proceso se muestra en la figura (2.10).

Una forma de clasificar la fusión sensorial esta basada en el nivel de señal al cual se realice el proceso[99]: fusión a nivel de observación o de señal, a nivel de vector de estado o característica, o a nivel de decisión.

Señal Los datos a nivel de señal pueden ser combinados directamente siempre que los datos de los distintos sensores estén conmensurados. Es decir, que los sensores estén midiendo el mismo fenómeno físico, como por ejemplo dos sensores de imagen o dos sensores de ultrasonidos. La idea consiste en promediar los datos para obtener información más precisa. Ejemplo de este método es el Filtro de Kalman.

Característica A este nivel la fusión consiste en combinar varias características, tales como esquinas y líneas, en un sólo mapa de características que pueda ser 


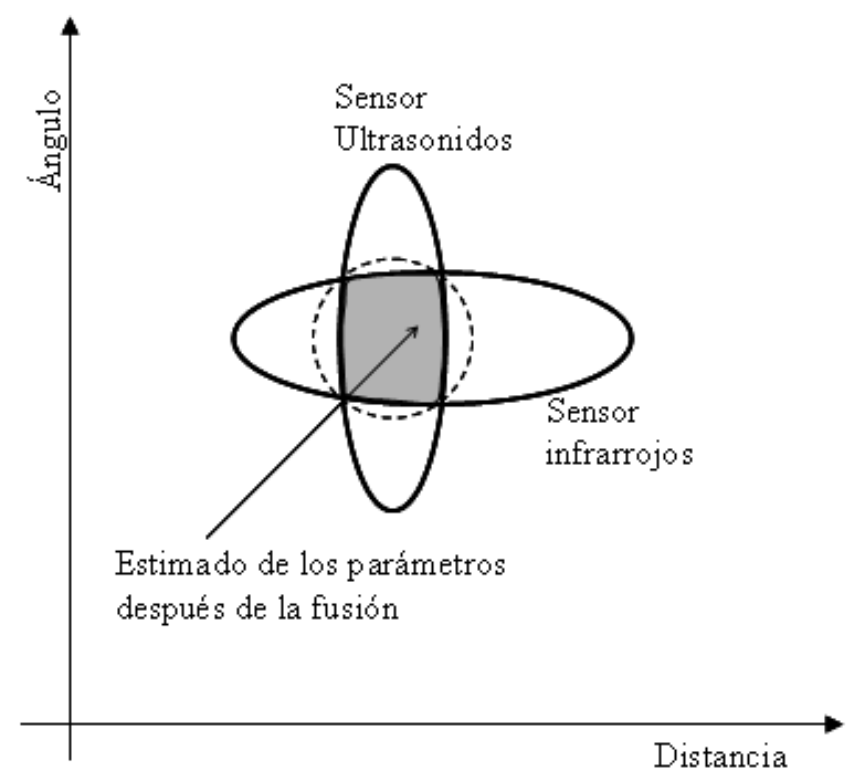

Figura 2.10: Ejemplo de fusión por intersección de elipses de covarianza.

utilizado en procedimientos de más alto nivel. Las características combinadas pueden provenir de varias fuentes de datos o de la misma fuente. Ejemplo de este método son los algoritmos de agrupamiento.

Decisión La fusión a nivel de decisión incluye la fusión de información sensorial después de que cada sensor ha hecho la determinación preliminar de la localización, atributos e identificación de cada instancia. Ejemplos de métodos de fusión a nivel de decisión incluyen el método del voto, métodos estadísticos, métodos basados en lógica difusa,etc.

Una de las áreas de aplicación más importantes dentro de la fusión e integración multisensorial es la robótica móvil. La fusión e integración de datos de sensores de visión, táctiles, térmicos, ultrasónicos, láser e infrarrojos; desempeña un papel muy importante para los sistemas robóticos, sobretodo cuando operan en ambientes dinámicos o en ambientes no del todo conocidos. En estos casos, la fusión e integración multisensorial le permite al robot obtener una rápida percepción del ambiente para propósitos de navegación o evitación de obstáculos(ver figura 2.11).

A lo largo de esta tesis se usa más que nada la fusión a nivel de señal, razón por la cual a continuación se introduce algunas de las estrategias de fusión utilizadas a este nivel.

\subsubsection{Fusión Bayesiana}

El teorema de Bayes determina la probabilidad de una causa sabiendo el efecto que ha producido[35]. Sea un espacio muestral $E$, compuesto de un determinado número 


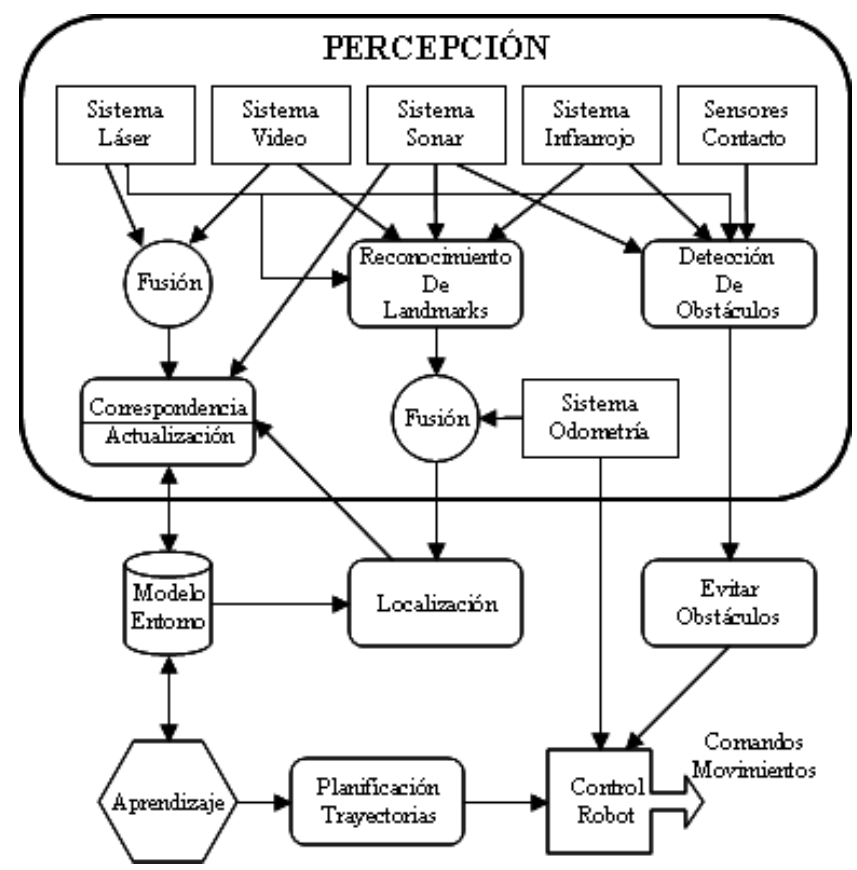

Figura 2.11: Arquitectura típica del sistema de percepción y fusión multisensorial de un robot móvil.

de sucesos disjuntos $A_{i}$, de tal manera que

$$
E=\bigcup_{i=1}^{n} A_{i}
$$

La probabilidad de que ocurra un suceso $B \mid B \subseteq E$, se puede escribir como

$$
\mathrm{P}(B)=\mathrm{P}\left(\bigcup_{i=1}^{n} B \cap A_{i}\right)=\sum_{i=1}^{n} \mathrm{P}(B \cap A)
$$

Aplicando la ecuación de reducción del espacio muestral se obtiene la siguiente ecuación, que es el enunciado del teorema de la partición.

$$
\mathrm{P}(B)=\sum \mathrm{P}\left(A_{i}\right) \mathrm{P}\left(B \mid A_{i}\right)
$$

$\mathrm{P}(B)$ probabilidad a posteriori de que ocurra el suceso $B$

$\mathrm{P}\left(A_{i}\right)$ probabilidad a priori de que ocurra la causa $A_{i}$

$\mathrm{P}\left(B \mid A_{i}\right)$ probabilidad condicional de que haya ocurra el suceso $B$, dado que existe la causa $A_{i}$ 


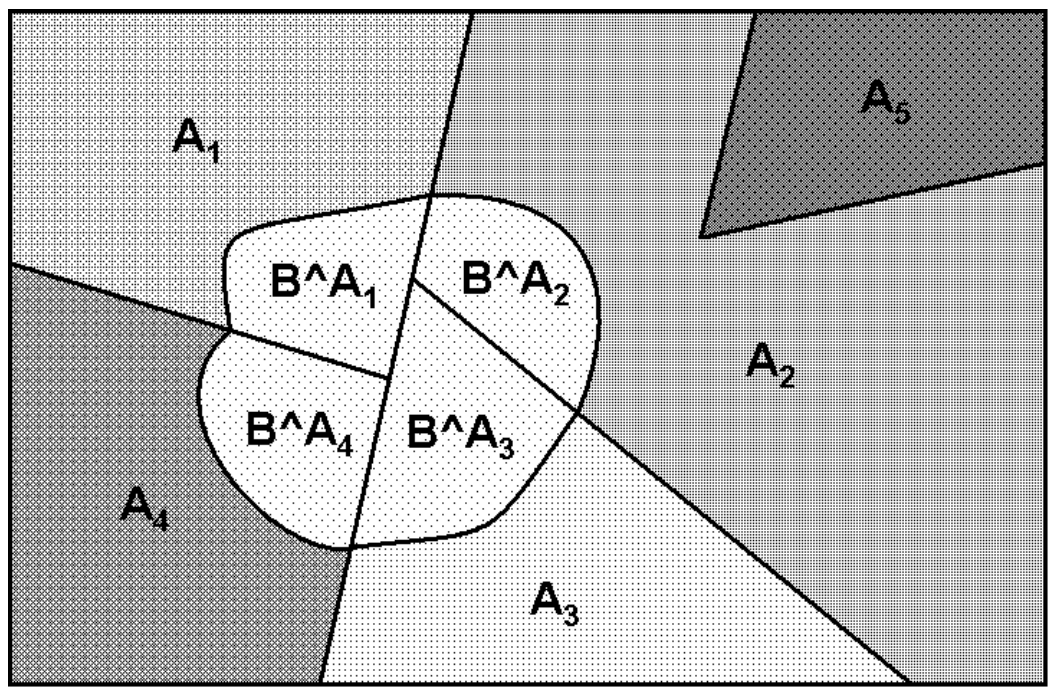

Figura 2.12: Representación de un espacio muestral

La fusión de datos consiste en combinar varias piezas de información de un mismo fenómeno pero emanadas de diferentes fuentes, con la finalidad última de tomar la mejor decisión respecto a este mismo fenómeno. La información extraída de cada sensor es representada mediante números reales como el grado de confianza en cierto evento, tomando así en cuenta la imprecisión, la incertidumbre y la naturaleza incompleta de la información [26]. En el caso de la fusión Bayesiana, el grado de confianza esta representado por probabilidades (a priori, condicional y a posteriori). Las decisiones son usualmente tomadas a partir de una probabilidad a posteriori.

Sea $A$ el evento a ser evaluado, y $x_{1}, x_{2}$ la información proveniente de dos sensores distintos. En este caso cada sensor asigna una probabilidad al evento $A$ dada cierta medida $x$ (por ejemplo, ver figura 2.13). Así, dada una cierta medida $x_{1}$, la misma es convertida a un valor de probabilidad mediante el modelo inverso del sensor $\mathrm{P}\left(x_{1} \mid A\right)$ . Este modelo se relaciona con las propiedades del sensor mediante la regla de Bayes según,

$$
\mathrm{P}\left(A \mid x_{1}\right)=\frac{\mathrm{P}\left(x_{1} \mid A\right)}{\mathrm{P}\left(x_{1}\right)} \mathrm{P}(A)
$$

De la misma forma, el modelo inverso del sensor 2 estaría relacionada con el evento $A$ a través de la ecuación,

$$
\mathrm{P}\left(A \mid x_{2}\right)=\frac{\mathrm{P}\left(x_{2} \mid A\right)}{\mathrm{P}\left(x_{2}\right)} \mathrm{P}(A)
$$

La probabilidad de observación del evento $A$ dado las mediciones $x_{1}$ y $x_{2}$, se puede derivar de la regla del producto, 


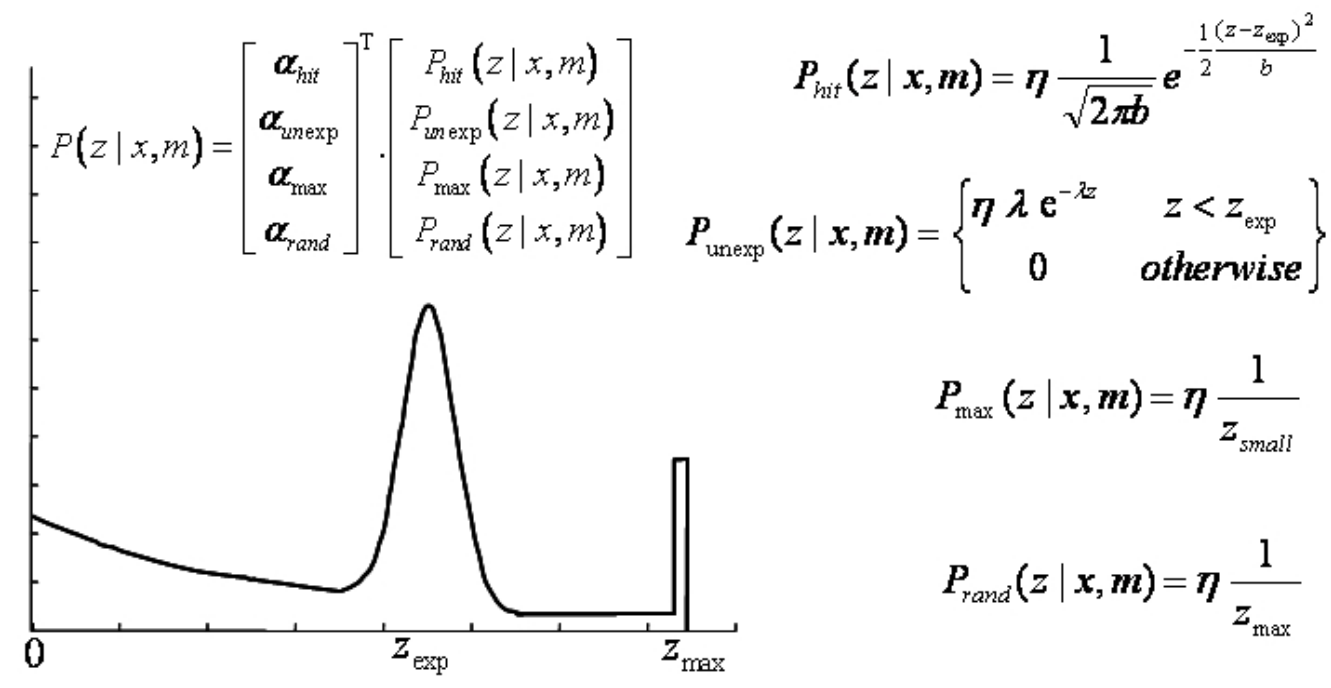

Figura 2.13: Ejemplo de modelo inverso del sensor representado mediante una función de densidad de probabilidad. Los factores $\alpha$ ponderan el grado de confianza de la zona de medición. Tomado de Thrun et al[198].

$$
\mathrm{P}\left(A \mid x_{1}, x_{2}\right)=\frac{\mathrm{P}\left(x_{1} \mid A, x_{2}\right)}{\mathrm{P}\left(x_{1} \mid x_{2}\right)} \mathrm{P}\left(A \mid x_{2}\right)
$$

Asumiendo la independencia de las mediciones $x_{1}$ y $x_{2}$, entonces $\mathrm{P}\left(x_{1} \mid A, x_{2}\right)$ y $\mathrm{P}\left(x_{1} \mid x_{2}\right)$ corresponden a $\mathrm{P}\left(x_{1} \mid A\right)$ y $\mathrm{P}\left(x_{1}\right)$ respectivamente, transformando la probabilidad conjunta anterior en

$$
\mathrm{P}\left(A \mid x_{1}, x_{2}\right)=\frac{\mathrm{P}\left(x_{1} \mid A\right)}{\mathrm{P}\left(x_{1}\right)} \mathrm{P}\left(A \mid x_{2}\right)
$$

Aplicando nuevamente la regla de Bayes a la probabilidad condicional P $\left(x_{1} \mid A\right)$, se obtiene la densidad de probabilidad conjunta,

$$
\mathrm{P}\left(A \mid x_{1}, x_{2}\right)=\frac{\mathrm{P}\left(A \mid x_{1}\right) \mathrm{P}\left(A \mid x_{2}\right)}{\mathrm{P}(A)}
$$

La función anterior representa los estimados y los valores de certidumbre del evento $A$ a partir de la fusión de las medidas $x_{1}$ y $x_{2}$ de dos sensores diferentes.

\subsubsection{Fusión basada en la teoría de Dempster - Shafer}

El razonamiento evidencial es un método de describir la creencia de eventos inciertos soportándose en la existencia de ciertas evidencias[181]. En este enfoque, la mejor representación de la probabilidad usa una función de confianza(Belief), en lugar de la distribución de probabilidad usada en los métodos Bayesianos. Aquí se le asigna valores de probabilidad a conjuntos de posibilidades en lugar de asignársela 


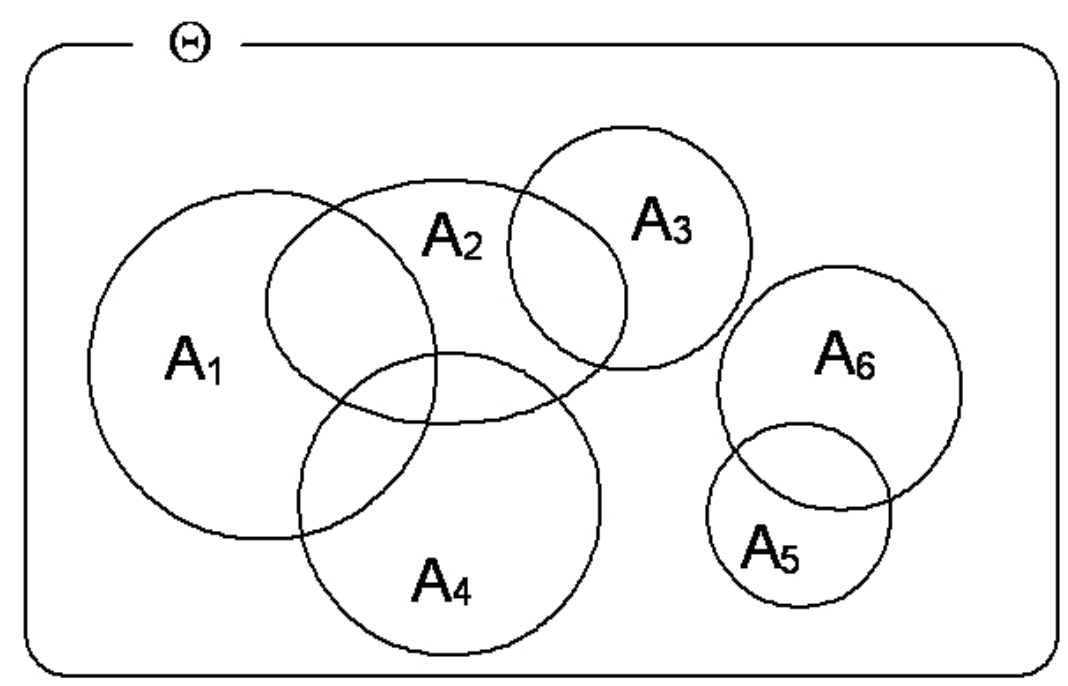

Figura 2.14: Ejemplo de un marco de discernimiento.

a un simple evento. Así, uno de sus atractivos reside en el hecho de que las funciones de confianza codifican la evidencia en favor de las proposiciones, de una manera inherentemente natural.

La teoría de Dempster-Shafer (D-S) envuelve la idea común de asignar valores entre 0 y 1 para indicar el grado de certeza de alguna hipótesis, pero en lugar de enfocar sobre como son determinados estos valores, ella se interesa por los grados de confianza de las mismas.

La teoría D-S de la evidencia está caracterizada por un marco de discernimiento(FOD : Frame of Discernment), una asignación básica de probabilidad (bpa: basic probability assignment), la función de confianza (Bel: Belief), la función de verosimilitud (Pls: plausibility) y por la combinatoria de Dempster.

El marco de discernimiento, denotado como $\Theta$, está compuesto por un conjunto finito de etiquetas que representan exhaustivamente los eventos y sus posibles combinaciones. Por ejemplo,

$$
\Theta=\left\{A_{1}, A_{2}, A_{3},\left\langle A_{1}, A_{2}\right\rangle,\left\langle A_{1}, A_{3}\right\rangle,\left\langle A_{2}, A_{3}\right\rangle,\left\langle A_{1}, A_{2}, A_{3}\right\rangle,\right\}
$$

La asignación básica de probabilidad es la función $m: \Psi \rightarrow[0,1]$, donde $\Psi$ es el conjunto de todos los subconjuntos de $\Theta$, o también conocido como el conjunto potencia de $\Theta, \Psi=2^{\Theta}$. La función $m$ puede ser interpretada como una distribución de probabilidad de todos los miembros de $\Psi$, donde se deben satisfacer lo siguientes criterios:

$$
\begin{gathered}
\sum_{\forall A \subseteq \Psi} m(A)=1 \\
m(\varnothing)=0
\end{gathered}
$$


De esta manera, a la etiqueta $A$ le es es asignada una probabilidad básica $m(A)$ que describe el grado de confianza que se le asigna exactamente a $A$. Sin embargo, la evidencia o confianza total que se le atribuye a $A$ es la suma de todas las probabilidades asignadas a $A$ y a sus subconjuntos.

$$
\operatorname{Bel}(A)=\sum_{\forall B \mid B \subseteq A} m(B)
$$

La función $\mathrm{Bel}: \Psi \rightarrow[0,1]$ es la cantidad de evidencia que le da soporte a la proposición $A$ y tiene las siguientes propiedades:

$$
\begin{gathered}
\operatorname{Bel}()=0 \\
\operatorname{Bel}(\Theta)=1 \\
\operatorname{Bel}(A)+\operatorname{Bel}(\neg A) \leq 1 \\
\operatorname{Bel}(A) \leq \operatorname{Bel}(B) \text { si } A \subset B \\
\operatorname{Bel}(A \cap B)=\min (\operatorname{Bel}(A), \operatorname{Bel}(B))
\end{gathered}
$$

La verosimilud de una proposición $A$ se puede ver como la cantidad de evidencia que no le da soporte a su negación $\neg A$. La misma está definida como la plausabilidad $P l s: \Psi \rightarrow[0,1]$. Esta última tiene las siguientes propiedades:

$$
\begin{gathered}
P l s(A)=1-\operatorname{Bel}(\neg A)=1-\sum_{\forall B \mid A \nsubseteq B} m(B) \\
P l s(A)-\operatorname{Bel}(A) \geq 0 \\
P l s(A \cup B)=\operatorname{máx}(P l s(A), P l s(B))
\end{gathered}
$$

El estado de cada componente de $\Psi$ (descrito por la bpa) se puede combinar con una nueva fuente de evidencia independiente, mediante la Regla de la Combinación de Dempster. La combinación de las dos funciones de evidencia o confianza, también se conoce como la suma ortogonal, cuyo operador es $\oplus$; por lo que la regla de la combinación se escribe como,

$$
\begin{gathered}
m_{1} \oplus m_{2}(A)=\frac{\sum_{\forall B, C \in \Psi \mid B \cap C=A} m_{1}(B) \cdot m_{2}(C)}{1-\sum_{\forall B, C \in \Psi \mid B \cap C=} m_{1}(B) \cdot m_{2}(C)} \\
m_{1} \oplus m_{2}()=0
\end{gathered}
$$


Dentro del razonamiento probabilístico, la formulación de D-S provee de un método para combinar la evidencia soportada por un número de fuentes diferentes sin la necesidad de ninguna distribución a priori. Supóngase que existen dos sensores $S_{1}$ y $S_{2}$ que proporcionan medidas de evidencia $m_{1}$ y $m_{2}$ de los eventos o subconjuntos de eventos del universo $\Theta$. Si se asume que las observaciones de los sensores son independientes y que se intersectan al menos en un cierto evento $A$, se pueden fusionar tales observaciones mediante la ecuación 2.28, a fin de obtener una nueva función de confianza para el evento en cuestión. La combinación de las dos funciones de confianza se escribe como,

$$
\operatorname{Bel}(A)=\operatorname{Bel}_{1}(A) \oplus \operatorname{Bel}_{2}(A)=\frac{\sum_{\forall a, b \subseteq \Theta \wedge a \cap b=A} m_{1}(a) \cdot m_{2}(b)}{1-\sum_{\forall a, b \subseteq \Theta \wedge a \cap b=} m_{1}(a) \cdot m_{2}(b)}
$$

En términos simples, para fusionar información de sensores distintos en el marco de la teoría de la evidencia, básicamente se calcula el producto de los valores de confianza de todos los subconjuntos que se intersectan exactamente en el evento $A, \mathrm{y}$ se divide por 1 menos el producto de los valores de confianza de todos los subconjuntos cuya intersección es vacía.

\subsubsection{Fusión mediante Filtros de Kalman}

El filtro de Kalman es un excelente mecanismo recursivo para fusionar información redundante proveniente de diferentes sensores o sistemas lineales[103]. Cuando la incertidumbre que caracteriza la información se distribuye de manera normal, el filtro de Kalman representa un filtro estadístico optimo en el sentido que se minimiza la varianza del error. La aplicación de este filtro en sistemas no lineales se hace mediante una extensión conocida como EKF (Extended Kalman Filter), la cual surge de la linealización de las ecuaciones de estado mediante la expansión en Series de Taylor de primer orden alrededor del estado estimado. Al aplicar esta operación de linealización, el Filtro de Kalman deja de ser óptimo, pero sin embargo sigue siendo cuasi óptimo sobretodo para sistemas con no-linealidades poco severas. Más detalles se encuentran en el anexo B.

El filtro de Kalman es básicamente un conjunto de ecuaciones matemáticas mediante las cuales se estima el estado de un sistema lineal en forma eficiente, a través de una solución recursiva del método de mínimos cuadrados. Estas ecuaciones permite implementar un estimador lineal, insesgado y óptimo, del estado de un proceso, basándose en la información disponible en el instante anterior, y corrigiendo este estado estimado a partir de la información disponible en el momento actual. Este estimador es óptimo en el sentido que minimiza el error cuadrático medio del estado estimado.

En general se estima el estado de un proceso lineal el cual es afectado por ruido Gaussiano, según

$$
x_{k}=\mathbf{A} x_{k-1}+\mathbf{B} u_{k-1}+w_{k}
$$




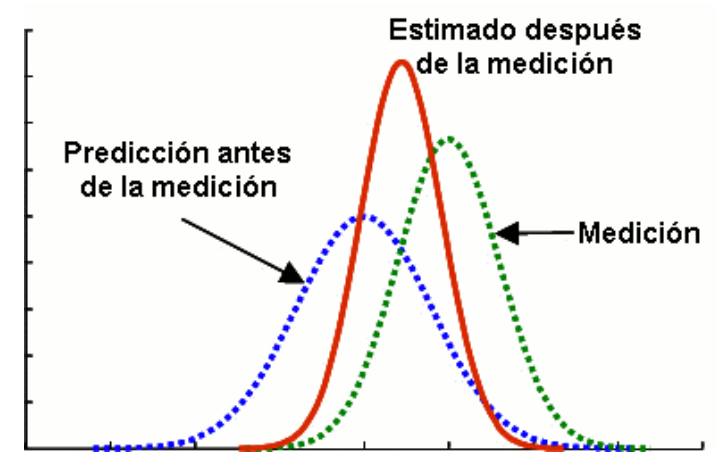

Figura 2.15: Resultado típico de la fusión mediante el Filtro de Kalman.

Esta ecuación define el modo como cambia el estado $x$, el cual está relacionado en el tiempo con el estado anterior $\left(x_{k-1}\right)$ y con la entrada de control $u$, a través de la matriz de parámetros $\mathbf{A}$ y B respectivamente.

La ecuación de salida del sistema, a la que se le ha incorporado el ruido de medida, se muestra a continuación.

$$
z_{k}=\mathbf{H} x_{k}+v_{k}
$$

Este ecuación se conoce como el modelo de medición, y en ella la lectura del sensor $z$ esta relacionada con el estado $x$ a través de la matriz de parámetros $\mathbf{H}$.

En las ecuaciones 2.31 y 2.32 , las variables $w$ y $v$ representan el ruido de sistema y de medida respectivamente, los cuales se asume que no están correlacionados y que tienen distribución de probabilidad normal o Gaussiana, con media igual a cero.

$$
\left\{\begin{array}{l}
\mathrm{P}(w) \simeq \mathrm{N}(0, \mathbf{Q}) \\
\mathrm{P}(v) \simeq \mathrm{N}(0, \mathbf{R})
\end{array}\right.
$$

El proceso de fusión a través del Filtro de Kalman consiste de los subprocesos de predicción y corrección, los cuales son repetidos recursivamente. A partir de la estimación del valor previo de la variable fusionada $\left(x_{k-1}\right)$, mediante la ecuación 2.31 el algoritmo pronostica el valor que tendrá la misma en el próximo instante $\left(x_{k}^{\text {pronosticada }}\right)$. Luego se realiza una medición sensorial $\left(z_{k}\right)$ que se utiliza para generar un término de corrección proporcional al error de predicción. Este término se añade a la variable pronosticada para generar así la variable fusionada corregida $\left(x_{k}^{\text {corregida }}\right)$.

$$
x_{k}^{\text {corregida }}=x_{k}^{\text {pronosticada }}+\mathbf{K}\left(z_{k}-\mathbf{H} x_{k}^{\text {pronosticada }}\right)
$$

El factor de corrección $\mathbf{K}$, conocido como la ganancia de Kalman, se determina a partir de las matrices $\mathbf{Q}$ y $\mathbf{R}$, la cuales representan la varianza/covarianza del ruido en el sistema (calculada a partir de la ecuación de medición indirecta) y la varianza/covarianza del ruido en la medición. 


$$
\mathbf{K}=\frac{\mathbf{Q}}{\mathbf{Q}+\mathbf{R}}
$$

De la ecuación anterior se ve que si el ruido en el sistema es más importante que el ruido en la medición $(\mathbf{Q}>>\mathbf{R})$, entonces se le da más importancia a la medición directa $(\mathbf{K} \rightarrow 1)$. También, si el ruido en la medición es más importante que el ruido en el sistema $(\mathbf{Q}<<\mathbf{R})$, entonces se le da más importancia al estimado $(\mathbf{K} \rightarrow 0)$.

\subsection{Representación del entorno.}

Existen varias formas de representar la manera como el robot percibe el entorno. El caso más general es el que clasifica los tipos de mapa como:

Topológicos Representan el entorno como un gráfico no dirigido, en donde los nodos corresponden a lugares de marcada importancia y las interconexiones o ramas representan la forma como se puede ir de un nodo a otro. El concepto de Mapa topológico fue introducido por Benjamin Kuipers a finales de los 70[118]. Un mapa topológico es una descripción concisa de la estructura de los grandes espacios, ya que describe en forma compacta una colección de lugares o adyacencias interrelacionadas por medio de los trayectos que las enlazan o unen entre sí.

Métricos Son representaciones de las propiedades métricas o de las coordenadas de los objetos del entorno(áreas, distancias, tamaño, localización, orientación, etc.). Este tipo de representación suele realizarse en el mismo sistema de coordenadas $2 \mathrm{D}$ en el cual se representa al robot, lo que facilita la fusión de los datos propioceptivos y externoceptivos que se obtienen mediante los sensores del robot. Este tipo de representación tiene dos casos particulares que son los mapas Geométricos y los mapas de Ocupación.

1. Los mapas geométricos representan el entorno mediante un conjunto de características geométricas tales como puntos, líneas y esquinas, los cuales pueden ser parametrizados en función de su longitud, diámetro, color, etc.[129]

2. Los mapas de ocupación representan el entorno mediante celdas cuadriculadas. Cada celda representa una pequeña área o volumen del entorno donde opera el robot. Normalmente se le asigna a estas celdas un valor que expresa la probabilidad de que la parte del entorno que ella representa esté o no ocupada[151].

\subsubsection{Mapas topológicos.}

Frecuentemente los mapas topológicos son definidos por medio de las estructuras presentes en el entorno. Chatila \& Laumond[45] definen el concepto de nodo o place 


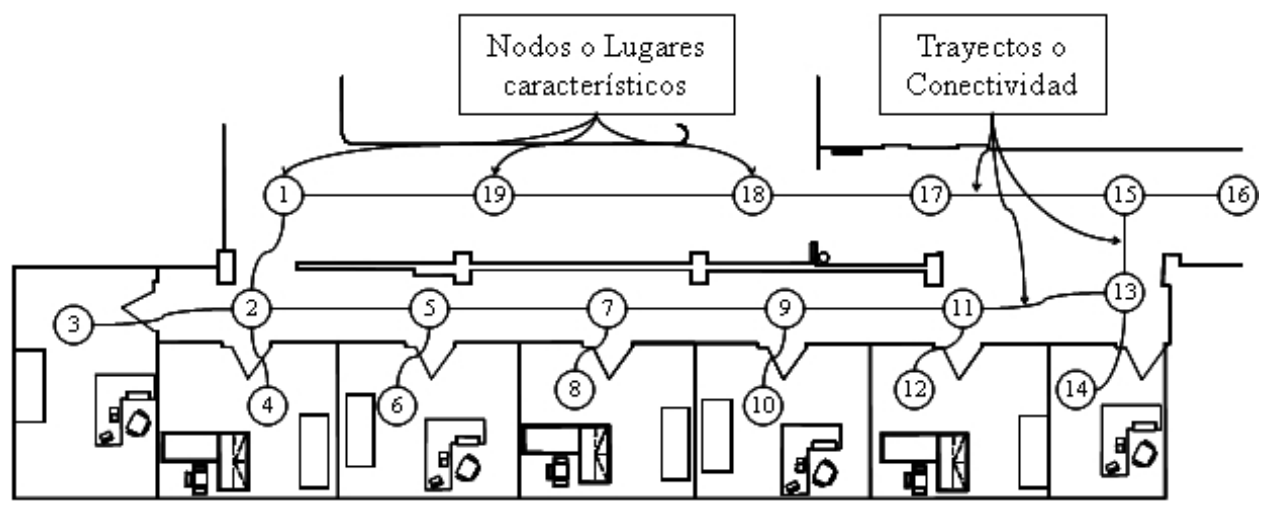

Figura 2.16: Mapa Topológico de un entorno típico de oficinas. Los números representan cada uno de los nodos, y las líneas la forma de ir de un nodo a otro.

como el área que corresponde a una unidad funcional o a una unidad topológica. Ejemplos de unidades topológicas son los corredores y salones de un ambiente de oficina, mientras que una unidad funcional puede ser por ejemplo una impresora. En un mapa topológico las ramas son usadas para interconectar los nodos. Ejemplo de ellos son las puertas, escaleras y elevadores.

Como se puede ver en la figura 2.16, la idea central de los mapas topológicos es representar las características esenciales del entorno percibidas por un robot móvil, utilizando un grafo donde los nodos representan los lugares distintivos, y las ramas representan la trayectoria directa que conectan a diferentes nodos.

Los mapas topológicos son abstractos y pueden ser construidos sin el conocimiento exacto de las relaciones geométricas entre los diferentes nodos. Mataric[148] propone una metodología en la cual el robot navega siguiendo la pared y los nodos son descubiertos en una secuencia que corresponde a su relación topológica. Kurz[120] propone la construcción de un mapa topológico en dos etapas. Primero identifica las áreas distintivas y después el robot explora el entorno, buscando las transiciones entre estas áreas distintivas. Thrun et al[197] proponen primero construir un mapa métrico probabilista y luego a partir de él, obtener un mapa topológico.

Los mapas topológicos a menudo son extendidos agregando alguna información métrica, típicamente incluyendo las longitudes estimadas de las trayectorias entre los nodos y la orientación de dichas trayectorias. Con la nueva información es posible realizar una planificación para buscar las trayectorias más cortas, así como utilizarla para resolver ambigüedades referidas a los nodos. La fuente de la información es usualmente el odómetro del robot, dado que las trayectorias son normalmente cortas y el error acumulado es pequeño[124].

Thrun[192], presenta una síntesis de las ventajas y desventajas de los sistemas que utilizan mapas topológicos:

1. Permite una planificación eficiente y tiene una baja complejidad del espacio (la resolución depende de la complejidad del entorno). 
2. No requiere de una determinación precisa de la localización del robot. Solamente requiere reconocer que ha alcanzado el lugar asociado a un nodo del mapa.

3. Representación conveniente para planificadores o solucionadores de problemas simbólicos. Teniendo en cuenta que normalmente el número de nodos es pequeño, esta representación facilita la asociación de etiquetas o significados a los nodos y las ramas del mapa.

4. Dificultad para construir y mantener en ambientes de gran escala, si la información de los sensores es ambigua.

5. El reconocimiento de lugares es a menudo difícil y sensitivo al punto de vista o lugar desde donde se enfoque el lugar.

6. El planificador puede obtener trayectorias más largas, comparadas con la longitud de las trayectorias obtenidas al utilizar mapas métricos.

\subsubsection{Mapas de características geométricas.}

En general son aquellos construidos a partir de primitivas geométricas: puntos, líneas, arcos de círculo, etc. Estos mapas representan numéricamente las coordenadas y propiedades de los objetos del entorno donde se mueve el robot. Por ejemplo, almacenan al robot mismo, a las paredes, hitos puntuales naturales o artificiales, etc. Son útiles para representar entornos estructurados en los es posible la extracción suficientemente robusta de las características que lo conforman. También son altamente eficientes en lo que a almacenamiento se refiere, debido a que grandes regiones del espacio pueden ser representadas por un modelo con pocos parámetros numéricos. Un mapa de características se puede describir a través del modelo $M=\left\{f_{i} \mid i=1, \ldots, n\right\}$, donde $f_{i}$ es uno de los elementos del entorno y $n$ es el número de elementos del entorno.

Existen multitud de tipos de modelos geométricos del entorno. Por ejemplo existen los que describen el entorno a través de un conjunto de características geométricas(segmentos, esquinas, etc.) y una serie de relaciones entre ellas(distancia, posición, etc.) $[50,155]$. Otros trabajos definen el entorno como un mapa tipo CAD, que refleja los distintos elementos a modelar, sus dimensiones y posiciones[36, 127]. Un ejemplo de este tipo de mapas puede observarse en la figura 2.17. Por último, otros trabajos [87, 133], proponen utilizar como modelo del entorno los propios datos percibidos por lo sensores del robot, aplicándoles el mínimo tratamiento posible.

Entre las desventajas de representación del entorno mediante mapas de características geométricas se puede destacar que este tipo de representación es incapaz de realizar un modelo completo del entorno. Esto es porque todo lo que no sea una característica geométrica típica, será descartado y no considerada en el mapa. Otras dificultades descritas por Dudek \& Jenkin [62] son las siguientes:

1. Pérdida de estabilidad. La representación puede cambiar en forma drástica ante pequeñas variaciones de la entrada sensorial. Por ejemplo, si una pared es de- 


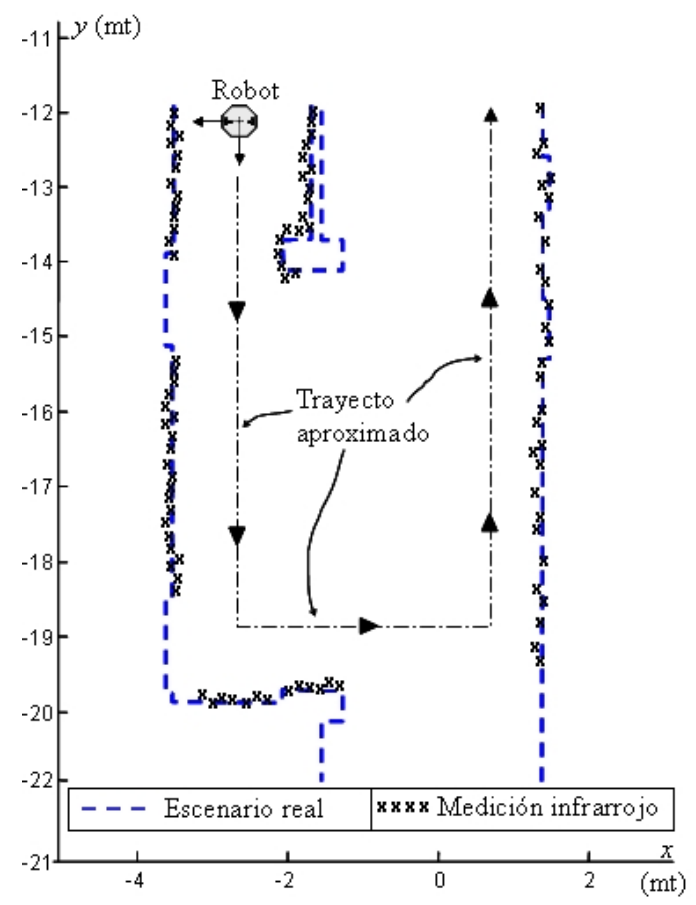

Figura 2.17: Un área de pasillos representada mediante un mapa de líneas mapeado mediante sensores de infrarrojos 
tectada por un sensor como una secuencia de puntos alineados, se podría inferir una línea recta a partir de ellos. Sin embargo con un sensor más ruidoso, la pared podría resultar modelada por una multitud de pequeñas líneas.

2. Pérdida de unicidad. Dado que los datos sensados solamente son aproximados por primitivas geométricas, es posible que la representación no sea única. Considérese, por ejemplo, un método de seguimiento de la ubicación del robot basado en la comparación de mapas. La comparación de un mapa local, generado a partir de los datos sensados, con un mapa global del ambiente, puede resultar compleja cuando se tienen varias representaciones posibles de los datos sensados.

3. Pérdida de poder expresivo. Puede ser difícil representar todas las características del entorno dadas ciertas primitivas geométricas.

\subsubsection{Mapas de Ocupación}

Los mapas de ocupación tienen su origen en un articulo seminal de Moravec \& Elfes[151]. Posteriormente, este método de mapeado que divide el ambiente en celdas a las que se les asigna una probabilidad de ocupación, será conocido como Occupancy Grid. Esta representación discretiza el entorno en celdas de igual dimensión que contendrán información acerca si el área que ella representa está ocupada o no. El método más simple consiste en dividir el espacio usando una rejilla cuadrada, como se ilustra en la figura 2.18. Cada una de estas celdas mantiene una probabilidad de que la zona del entorno que ella representa esté ocupada. Los marcos teóricos mas utilizados en el problema de actualización del contenido de la celda son el Bayesiano[151, 47] y la lógica difusa[70, 161].Si las celdas guardan solamente el estado de ocupación: libre u ocupado, la representación se denomina mapa de bits. Si guarda otra información, en general se le conoce como mapa de píxeles[62], rejilla de ocupación[66] o mapas métricos de área[124].

La principal ventaja de los mapas de ocupación es su generalidad: no se hace ninguna suposición acerca del tipo de objetos presente en el entorno. Las celdas pueden representar cualquier cosa. Comúnmente las celdas sólo guardan la probabilidad de ocupación de la celda y en este caso se habla de un mapa de ocupación probabilista. La principal desventaja es que la resolución o fidelidad es limitada por el tamaño de la celda y la representación requiere la misma cantidad de almacenamiento aún si gran parte del entorno está libre o está ocupado. La cantidad de celdas puede llegar a representar un problema, especialmente si se considera ambientes bidimensionales grandes, ya que se requerirá grandes recursos de almacenamiento.

Thrun [192] presenta la siguiente síntesis con las ventajas y desventajas de los sistemas que utilizan mapas de ocupación:

1. Fáciles de construir, representar y mantener.

2. El reconocimiento de lugares no es ambiguo y es independiente del punto de vista. 


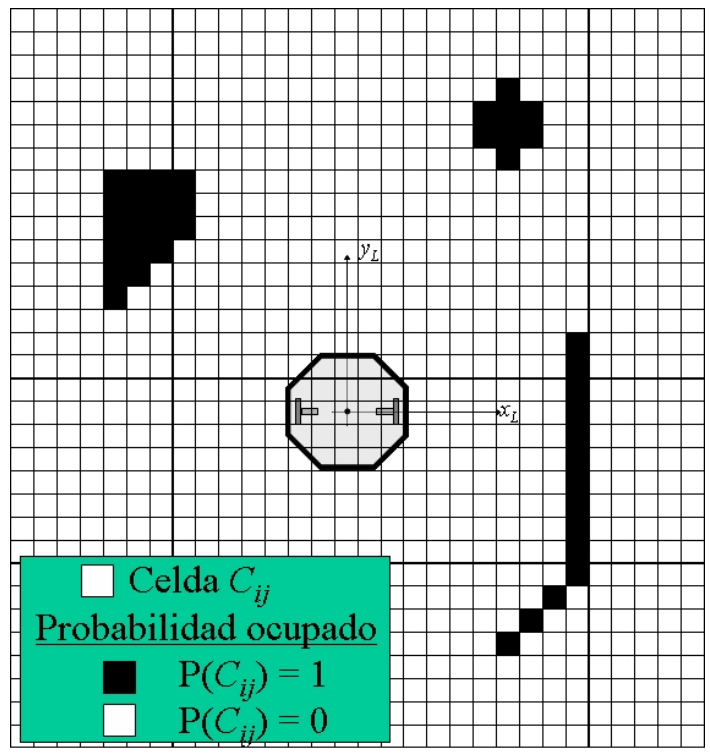

Figura 2.18: Mapa de grid: división del espacio de trabajo en celdas. Las celdas oscuras están ocupadas y las claras están vacías.

3. Se facilita el cálculo de las trayectorias más cortas.

4. Se requiere una determinación precisa de la posición del robot.

5. Debido a la gran cantidad de celdas que puede tener el mapa, esta representación presenta una interfase pobre para los solucionadores de problemas simbólicos. No se facilita la asociación de significados a celdas o a conjuntos de celdas del mapa.

\subsection{Localización relativa mediante medidas propiocepti- vas}

La localización relativa, en general es aquella que está basada en la sola observación del robot y de sus sensores de abordo; es decir, aquella donde no se usa información externa al robot. Una forma simplista de estimar la localización relativa de un robot consiste en monitorear las variables de estado del robot (i.e. $v(t)$ y $w(t)$, ver figura 3.2) usando sensores de abordo como giroscopios, acelerómetros, tacómetros y codificadores rotativos. Este tipo de localización se conoce como dead reckoning, lo que originalmente es el proceso de estimar la posición de un avión o de una embarcación, basándose en la velocidad y dirección del vehículo, y en el tiempo transcurrido desde la ultima posición conocida hasta la actual[2]. De aquí que este tipo de medidas de posición sólo da información referida al punto desde donde se inicio la navegación del robot. Un inconveniente que surge de inmediato es que el error en el estimado 


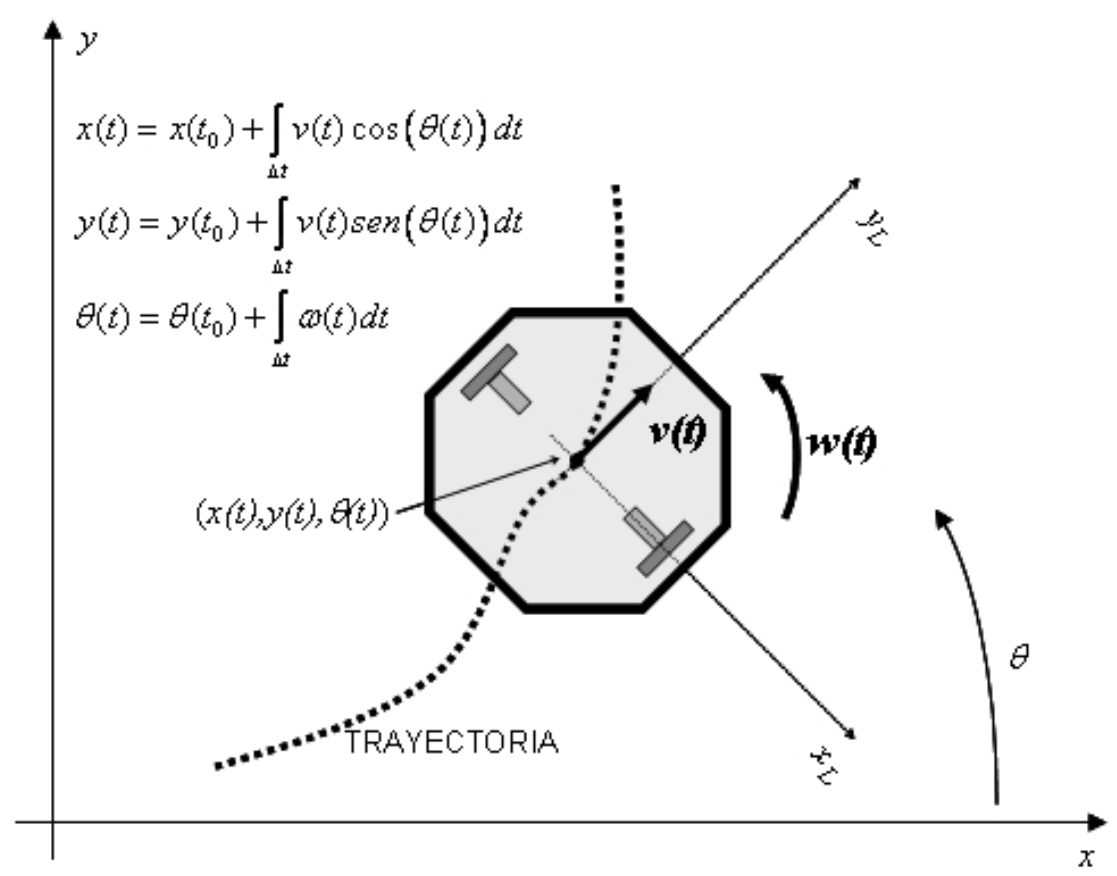

Figura 2.19: Variables cinemáticas usadas para el posicionamiento relativo de un móvil diferencial

de posición se incrementa con el tiempo ya que el mismo esta basado en el estimado anterior.

Una de las técnicas más usadas para llevar a cabo la localización relativa de un robot se basa en la integración a través del tiempo de la información proveniente de sensores de odometría acoplados al cuerpo o a las ruedas del robot. Esta técnica, conocida como localización odométrica, esta sujeta a grandes fuentes de error como por ejemplo el derrape y patinaje de las ruedas de odometría. Otra desventaja de la odometría es su sensibilidad al tipo de terreno, ya que estos sistemas no son capaces de detectar si se trata de una superficie plana o de una con muchas irregularidades[27]. A pesar de estas desventajas, la localización odométrica sigue siendo ampliamente usada porque proporciona buena exactitud, a bajos costos, para trayectos cortos.

\subsection{Localización absoluta basada en Hitos o Landmark}

Los Hitos o Landmarks son elementos del entorno que poseen características distintivas especiales, que mediante los sensores, el robot puede detectar. Una vez que estas Landmarks son detectadas, se contrastan con la información a priori que se tiene del entorno(correspondencia o matching), o también se pueden aplicar técnicas de triangulación, para determinar la posición del robot. Así, este método de localización puede dividirse en 4 fases según se ve en la figura 2.20 


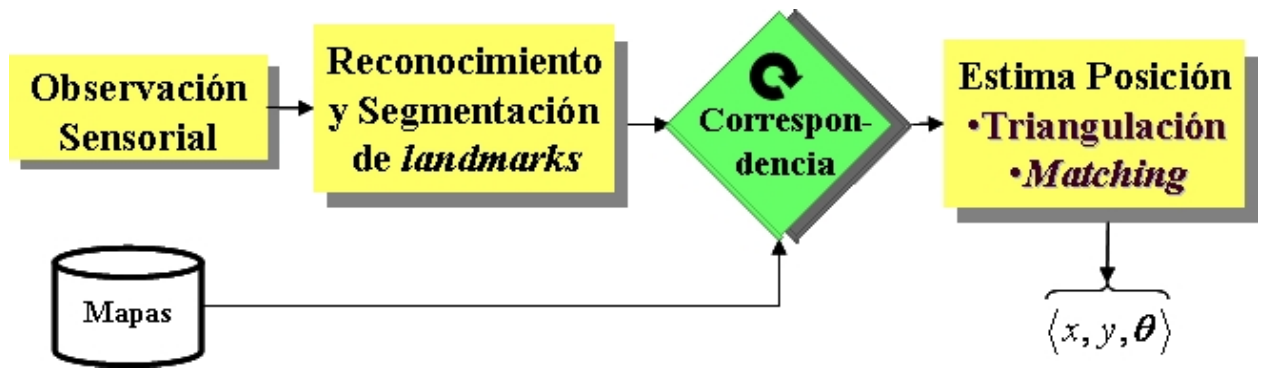

Figura 2.20: Fases de localización de robots basada en Landmarks.

La clasificación más usual de estas Landmarks es la que las divide en naturales y artificiales. Las Landmarks naturales son aquellas que de antemano forman parte del entorno donde se mueve el robot. Ejemplo de estas en ambientes interiores son las puertas, ventanas y lámparas de techo; mientras que para ambientes exteriores se pueden mencionar a los árboles, caminos y a las señales de tráfico. El problema principal con este tipo de Landmarks es que son difíciles de reconocer y en consecuencia el robot generalmente necesitará un número mayor de observaciones para poder determinar inequívocamente su posición.

Las Landmarks artificiales son colocadas intencionalmente en el entorno donde se mueve el robot, de forma tal que sean bien visibles a los sensores del mismo[79]. Este tipo de Landmarks se pueden clasificar en activas y pasivas. Las Landmarks activas, también conocidas como faros, son aquellas que emiten algún tipo de señal que informa sobre su localización. Ejemplo de ellas son los satélites de los sistemas GPS, los faros ultrasónicos, los radiofaros y dipolos magnéticos, etc. Las principales desventajas de este tipo de hitos activos, es que la señal que emiten puede verse perturbada por las condiciones geográficas o también por las condiciones atmosféricas del entorno. Otra desventaja es que en la práctica estas Landmarks no pueden enviar la señal en forma omnidireccional y por lo tanto el robot no las puedes ver desde cualquier lugar. Otra importante desventaja es que su costo de construcción y mantenimiento puede ser excesivamente elevado. Por su parte, las Landmarks pasivas son las que no emiten activamente ningún tipo de señal, y en consecuencia el robot tiene que buscarlas activamente mediante sus sensores para poder ejecutar el proceso posterior de autolocalización. Ejemplo de este tipo de Landmarks son las balizas, las figuras geométricas coloreadas, y los códigos de barra. La principal desventaja de estas Landmarks es que mientras más alejado se encuentre el robot de ellas, menos ajustado y preciso será la estimación de estado. También, al compararlas con las Landmarks activas, se pone de manifiesto que las pasivas son más difícil de detectar y que requieren de mayor cantidad de proceso para poder identificarlas.

Los métodos comúnmente usados para determinar la posición de un móvil a partir de la detección de Landmarks (activas y pasivas) y del conocimiento previo de su localización $\left(x_{i}, y_{i}\right)$ son dos: Trilateración, el cual se basa en las distancias desde el móvil a cada una de las tres o más Landmarks, y Triangulación, el cual se basa en los ángulos de vista a tres o más Landmarks[52]. 


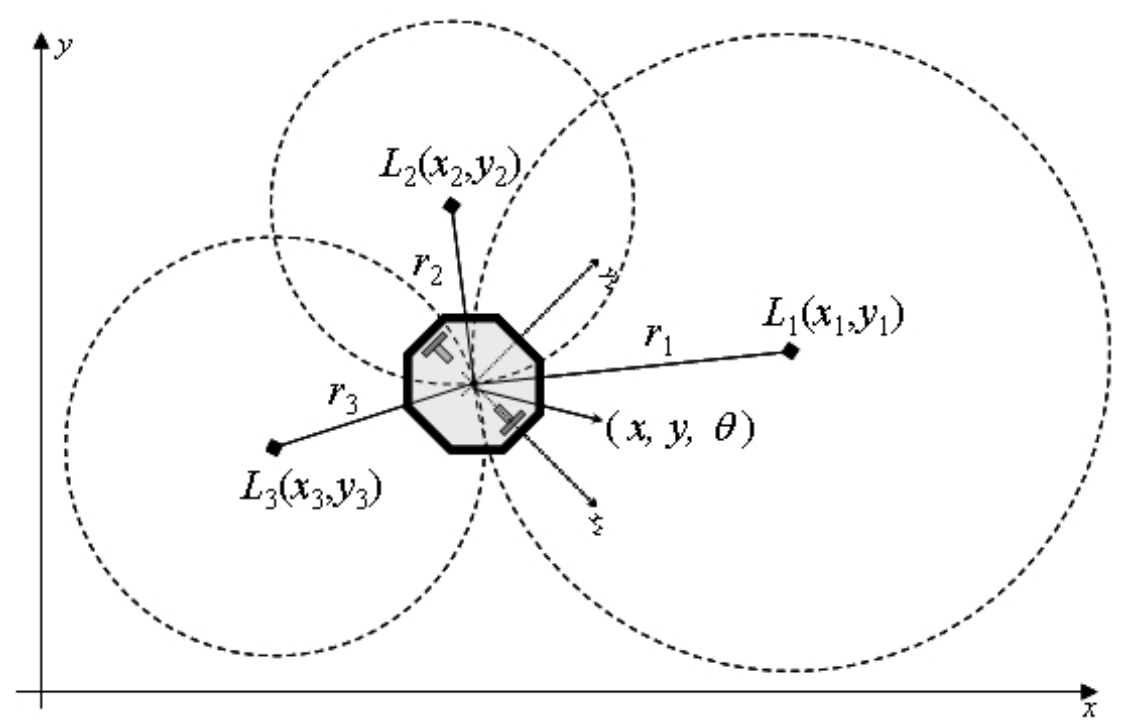

Figura 2.21: Esquema de localización por trilateración. Este método se basa en la medición de las distancias $\left\{r_{1}, r_{2}, r_{3}\right\}$ del móvil a las Landmarks.

La trilateración es la determinación de localización $(x, y)$ de un móvil basado en la medición de las distancias $\left(r_{1}, r_{2}, r_{3}\right)$ a 3 o más Landmarks cuyas coordenadas $\left\{\left\langle x_{1}, y_{1}\right\rangle,\left\langle x_{2}, y_{2}\right\rangle,\left\langle x_{3}, y_{3}\right\rangle\right\}$ son conocidas[205](ver figura 2.21). En un plano 2D, la trilateración se puede definir como el problema de encontrar la intersección de tres circunferencias. Esto es encontrar la solución al sistema de ecuaciones cuadráticas expresadas en la ecuación (2.36)[190].

$$
\left.\begin{array}{l}
\left(x-x_{1}\right)^{2}+\left(y-y_{1}\right)^{2}=r_{1} \\
\left(x-x_{2}\right)^{2}+\left(y-y_{2}\right)^{2}=r_{2} \\
\left(x-x_{3}\right)^{2}+\left(y-y_{3}\right)^{2}=r_{3}
\end{array}\right\}
$$

Una forma simple de resolver el sistema dado en (2.36), es sustrayendo la segunda y la tercera ecuación, de la primera ecuación. Así, el sistema se reduce a un sistema de primer orden con dos ecuaciones y dos incógnitas según,

$$
\left.\begin{array}{l}
x_{1}^{2}-x_{2}^{2}-2 x\left(x_{1}-x_{2}\right)+y_{1}^{2}-y_{2}^{2}-2 y\left(y_{1}-y_{2}\right)=r_{1}-r_{2} \\
x_{1}^{2}-x_{3}^{2}-2 x\left(x_{1}-x_{3}\right)+y_{1}^{2}-y_{3}^{2}-2 y\left(y_{1}-y_{3}\right)=r_{1}-r_{3}
\end{array}\right\}
$$

La implementación práctica de la trilateración, usualmente consiste en instalar 3 o más transmisores(faros) en lugares conocidos del entorno, e instalar un único receptor abordo del móvil. A pesar de la simplicidad y buena precisión que puede ofrecer el método de la trilateración, en robótica móvil este esquema de localización es insuficiente, debido a que el mismo sólo determina las coordenadas cartesianas $(x, y)$ del robot; y como es sabido la orientación del robot respecto al sistemas de coordenadas global es de muy alta importancia en las tareas de predicción de la posición durante la navegación. 


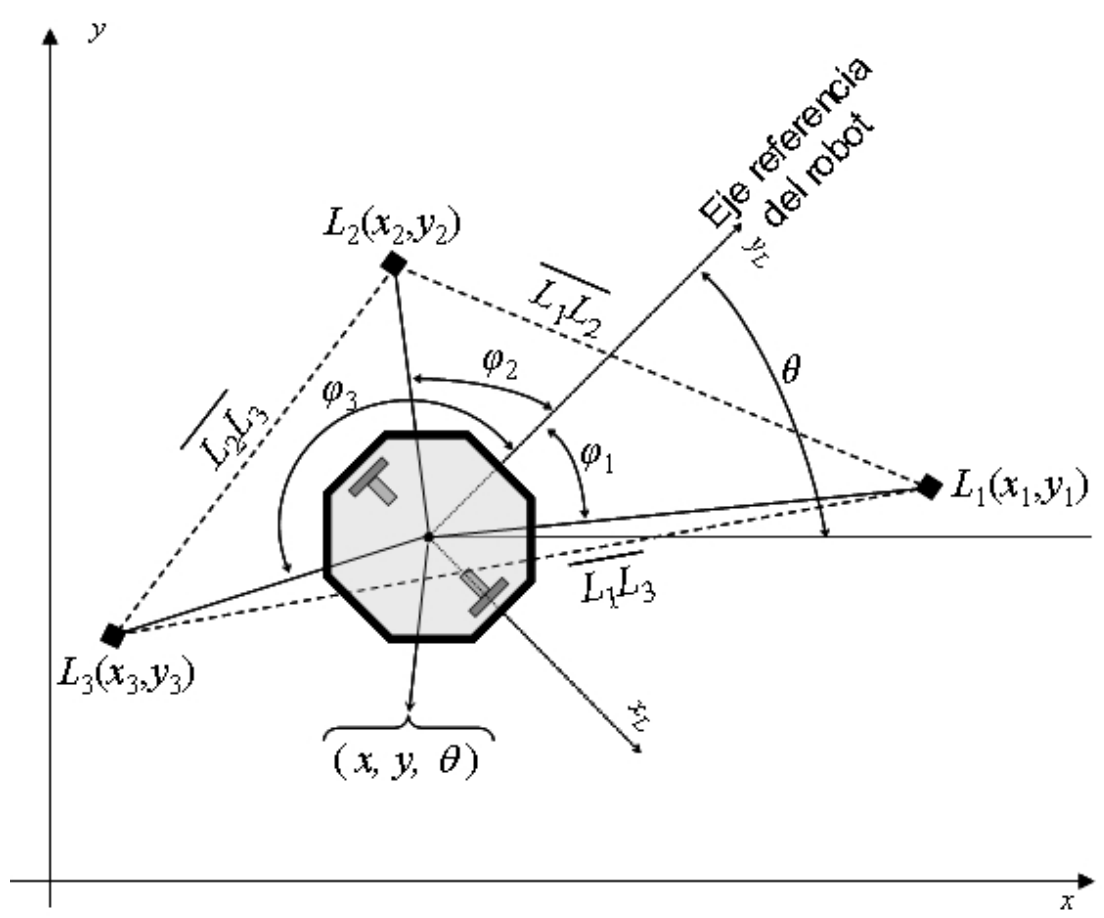

Figura 2.22: Esquema de localización por triangulación. Este método se basa en la medición de los ángulos de vista $\left\{\phi_{1}, \phi_{2}, \phi_{3}\right\}$ del móvil a las Landmarks.

Por su parte, la triangulación es el proceso de determinar la posición del robot (localización + orientación: $(x, y, \theta))$ a partir de tres $\operatorname{Landmarks}\left(L_{1}, L_{2}, L_{3}\right)$ cuyas respectivas localizaciones en el espacio cartesiano $\left(x_{i}, y_{i}\right)$ son conocidas. En general el proceso requiere que el robot tenga capacidad sensorial para detectar estas Landmarks y para medir la orientación o ángulo de vista hasta cada una de ellas $\left(\phi_{1}, \phi_{2}, \phi_{3}\right)$, con respecto al sistema de referencia de él mismo.[23] (ver figura 2.22 ). Aplicando la ley de coseno, la distancia entre dos Landmark cualquiera se puede expresar como

$$
\left.\begin{array}{rl}
r_{1}^{2}+r_{2}^{2}-2 r_{1} r_{2} \cos \phi_{12} & =\overline{L_{1} L_{2}} \\
r_{1}^{2}+r_{3}^{2}-2 r_{1} r_{3} \cos \phi_{13} & =\overline{L_{1} L_{3}} \\
r_{2}^{2}+r_{3}^{2}-2 r_{2} r_{3} \cos \phi_{23} & =\overline{L_{2} L_{3}}
\end{array}\right\}
$$

Aquí, $\left\{r_{1}, r_{2}, r_{3}\right\}$ son las distancias desconocidas desde el robot hasta las Landmarks, y $\left\{\overline{L_{1} L_{2}}, \overline{L_{1} L_{3}}, \overline{L_{2} L_{3}}\right\}$ son las distancias entre Landmarks que se consideran conocidas. Igualmente, $\left\{\phi_{12}, \phi_{13}, \phi_{23}\right\}$ surgen de sustraer los ángulos $\left\{\phi_{1}, \phi_{2}, \phi_{3}\right\}$ entre sí, por lo que también se consideran conocidos.

El sistema de ecuaciones de (2.38) puede resolverse utilizando un método de aproximación por mínimos cuadrados para obtener así las distancias desconocidas $\left\{r_{1}, r_{2}, r_{3}\right\}$. Una vez obtenidas estas distancias, se pasa a resolver la localización $(x, y)$ del móvil usando el procedimiento descrito para las ecuaciones (2.36) y (2.37). Una 


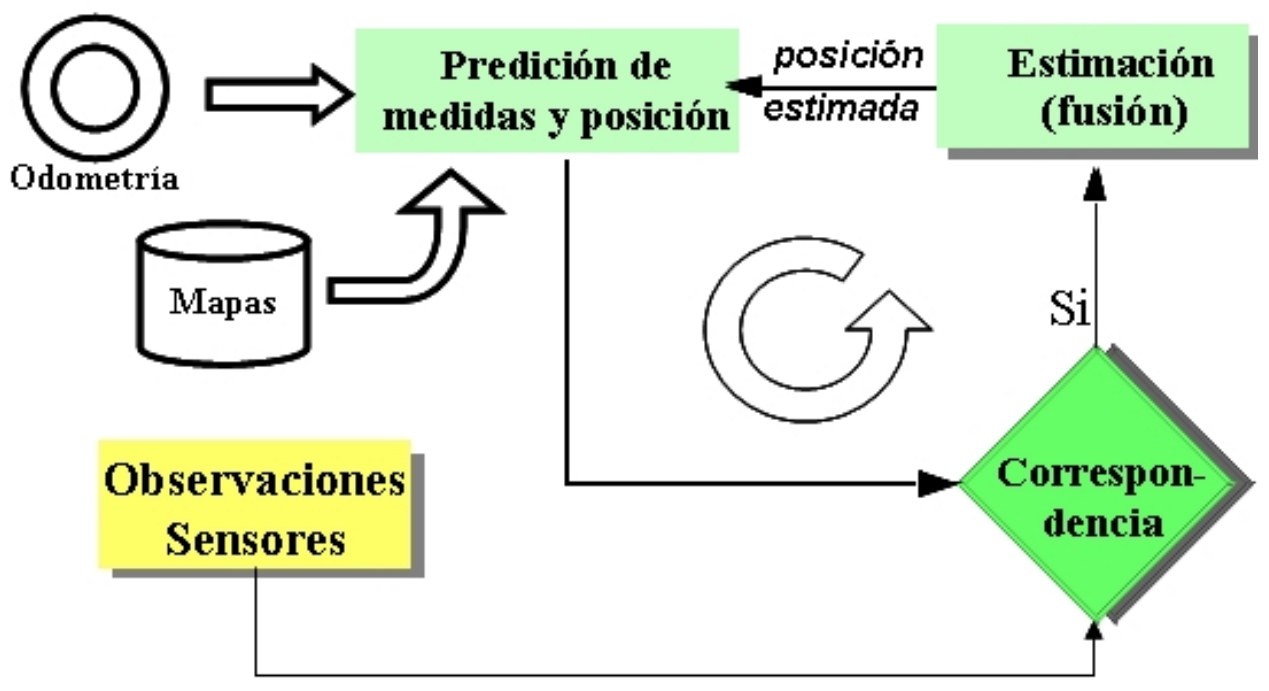

Figura 2.23: Esquema de localización basada en mapas

vez obtenida la localización del móvil, se puede computar el ángulo del segmento que va desde el punto $(x, y)$ hasta cualquiera de las Landmarks conocidas $\left(x_{i}, y_{i}\right)$; y luego calcular el ángulo $\theta$ de orientación del móvil con la ecuación,

$$
\theta=L_{\left(x_{i}, y_{i}\right)}^{(x, y)}-\phi_{i}
$$

\subsection{Localización basada en mapas}

Esta metodología se basa en la búsqueda de la correspondencia entre un mapa local que el robot construye mediante sus sensores, y un mapa global del entorno que el robot conoce con anterioridad, o que el mismo va construyendo mientras explora el entorno. De aquí el nombre general que recibe esta técnica: Correspondencia entre Modelos(Model Matching).

Partiendo de que el robot dispone de un mapa de su entorno, el cual puede ser obtenido por el mismo robot en una fase previa de exploración, o pueden ser suministrado externamente en una fase de inicialización (mapa tipo CAD), o pueden ser construidos simultáneamente mientras el robot navega(SLAM); el procedimiento general que se sigue con esta técnica de localización se divide en los siguientes pasos:

1. Predicción de la posición basada en el estimado previo y en los datos de odometría recogidos mientras el robot esta en movimiento.

2. Predicción de observaciones basado en el estado estimado de la posición y en el mapa del entorno el cual es conocido con anticipación.

3. Observación del entorno mediante los sensores del robot. 
4. Búsqueda de correspondencia entre las observaciones y el mapa.

5. Actualización de la posición real del robot.

Los distintos métodos de localización basados en mapas se diferencian en la manera de caracterizar el mapa del entorno, y en la forma como se maneja la incertidumbre en la posición que el robot ocupa dentro del mismo. A continuación se introducen varias de las metodologías usadas para la estimación de la posición de un robot móvil.

\subsubsection{Localización con mapas métricos}

Se refiere a la técnica que usa mapas que describen el entorno en términos métricos referidos a un sistema global de coordenadas, como lo son los mapas de rejillas (grid) o los de características geométricas(features).

Moravec \& Elfes [151, 66] proponen técnicas para encontrar la posición de un robot realizando la correlación entre un mapa local, construido por el robot con ayuda de sus sensores, y un mapa de grid que representa el entorno global. Esta técnica la aplican con mapas de diferentes resolución a fin de reducir los costos computacionales, sin embargo aun reportan que la rutina de actualización del mapa es computacionalmente pesada. Borenstein \& Koren[30,31] proponen un técnica de actualización más simple donde solamente las celdas que están a lo largo del eje acústico son actualizadas. Con esta técnica se reduce significativamente la carga computacional, manteniéndose una distribución de probabilidad realista.

Schiele \& Crowley[179] evalúan diferentes métodos para correlacionar el mapa de grid obtenido con los sensores del robot con un mapa global del entorno. Los métodos bajo consideración son: a) celdas locales Vs. celdas globales, b) líneas extraídas del mapa local Vs. celdas globales, c) celdas locales Vs. líneas extraídas del mapa global, d) líneas extraídas del mapa local Vs. líneas extraídas del mapa global. El resultado fué que la mejor localización se consigue cuando se correlacionan los mapas a igual nivel de abstracción. Es decir, celdas Vs. celdas, o líneas Vs. líneas.

Los mapas de características están formados por los elementos que caracterizan un determinado ambiente como por ejemplo líneas, esquinas y columnas; los cuales pueden ser parametrizados en función de su longitud, diámetro, color, etc.[44]. Para la localización se usan algoritmos de correspondencia(matching) entre los elementos característicos del entorno que va observando el robot con sus sensores, y un mapa del entorno que se conoce con anterioridad[129]; o un mapa que se construye simultáneamente mientras el robot explora el ambiente[6]. Una tendencia actual es la localización basada en mapas que incluyen Landmarks naturales como paredes y esquinas[72, 83]. Existen muchas técnicas para llevar a cabo este tipo de localización, pero usualmente todas necesitan de métodos de extracción de mucha precisión o de sensores que suministren datos de alta densidad[33, 175]. De aquí que en la actualidad, las características extraídas a partir de los datos de sensores láser sean las más usadas $[8,43,168]$. Por otra parte, la localización basada en características geométricas extraídas con sensores de ultrasonido es menos común. Esto se debe a la falta de 
precisión en la información angular proporcionada por el sonar y a la dificultad que existe para clasificar con él las distintas características del entorno[97, 112, 205].

\subsubsection{Localización con mapas topológicos}

Se refiere a la técnica que usa mapas topológicos, con los cuales se sigue una estrategia de localización no-métrica, ya que estos mapas indican la conectividad de los lugares pero no necesariamente las relaciones métricas o cuantitativas entre ellos. Para que un robot se autolocalice en un mapa topológico basta con que identifique en cual nodo se encuentra, por lo que el problema principal en la localización topológica lo constituye el reconocimiento fiable de los distintos nodos. Kuipers \& Byun[119] usan un anillo de 16 sonares y un compás para distinguir los diferentes nodos de un entorno de oficinas. Kortenkamp \& Weymouth[115] definen lugares especiales que llaman gateways, y los usan como nodos. Estas compuertas marcan la transición entre dos lugares del entorno. La idea es que aunque el sonar no da suficiente información como para marcar el lugar como una zona distintiva, sin embargo sí tiene la capacidad para detectar que el robot esta en una de estas zonas. El reconocimiento del nodo lo hacen a posteriori usando visión. Ejemplos de gateways son las puertas y las intersecciones de pasillos. Otra forma de resolver el problema de localización es basándose en el reconocimiento no de uno, sino de una secuencia de nodos. Estos sistemas aunque son efectivos, requieren que el robot haga varios movimientos con la sola finalidad de determinar su posición en el entorno[148]. Un caso particular de localización usando mapas topológicos y sensores de bajo coste es el propuesto por Duckett et al[60]. Estos investigadores presentan un método unificado, basado en un mapa topológico aumentado con características métricas (topológico-métrico), para la localización global y el seguimiento de la posición de un robot móvil. El método combina técnicas de Scan-Matching sobre mapas de densidad de ocupación locales extraídos con un SONAR, con técnicas de localización multihipótesis aplicadas sobre una representación topológica del mapa global. De esta manera el método es eficiente por su baja carga computacional en las operaciones de navegación por el entorno global, y es preciso al momento de corregir su posición en lugares particulares.

\subsubsection{Localización usando mapas de alta densidad de muestreo}

Dentro de este tipo de técnicas, el de análisis de correspondencia o Scan Matching es uno de los más usados. Aquí, el robot necesita un mapa del entorno, que puede ser suministrado previamente o puede ser una lista de exploraciones o barridos previamente ejecutados desde posiciones conocidas. En general, el estado del robot sigue una hipótesis única que se va corrigiendo a medida que se encuentran datos válidos(Pose tracking). Para determinar la posición real del robot el sistema de localización analiza dos mapas para calcular la distancia y la rotación que existe entre ellos. Esto es, el mapa adquirido en la posición actual del robot es comparado con el mapa conocido que este más cercano a la posición actual. Si el proceso de correspondencia se lleva a cabo con éxito, el resultado es la diferencia real entre la posición 


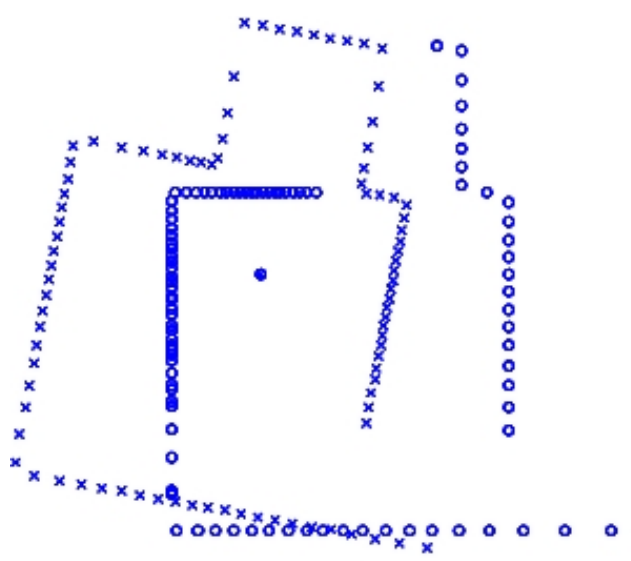

a)

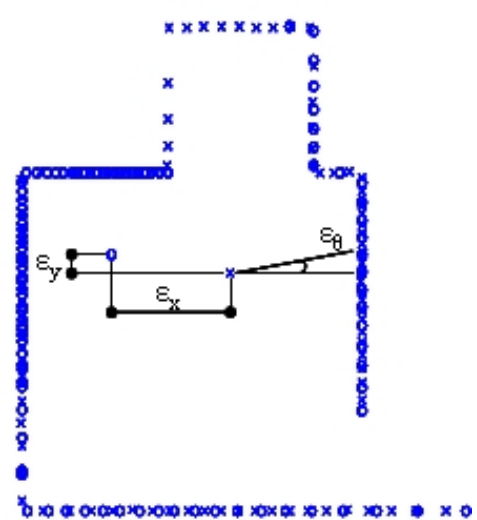

b)

Figura 2.24: a) Dos barridos láser tomados desde "o" y desde "x". b) Los mismos barridos mostrando los resultados del análisis de correspondencia[21].

desde donde fue tomado el mapa actual y la posición del mapa de referencia, lo cual es entonces usado para actualizar el estimado en la posición del robot.

Los algoritmos de análisis de correspondencia más usados son los de correlación(CCACross Correlation Algorithm)[203, 87] y el de correspondencia dual iterativa(IDCIterative dual correspondence)[134]. Más detalles sobre estos algoritmos y sobre algunos mas recientes se encuentran en [21]. Para obtener buenos resultados a partir del algoritmo de Scan Matching es fundamental que el algoritmo sea robusto frente a los cambios del entorno, porque si no, el algoritmo confiará en que se trata de mediciones ruidosas en los sensores y como resultado entregará malos resultados. También es importante que el algoritmo tenga la capacidad para juzgar sus propios resultados, es decir, que pueda indicar al robot cuando confiar o no en los resultados del análisis de coincidencias realizado. Así, los resultados buenos serán utilizados para la corrección de la estimación de posición del robot, mientras que los resultados defectuosos serán descartados y no habrá ningún cambio en la estimación de la posición del mismo. También es deseable que el algoritmo sea rápido y a la vez preciso en el sentido que pueda realizar más análisis por cada metro recorrido y que mantenga el error en la estimación de la posición dentro del error estándar del sensor utilizado.

\subsection{Localización y Mapeado: enfoque probabilístico}

En los métodos clásicos utilizados en robótica, el éxito de los algoritmos depende en gran medida del uso de sensores precisos y de la obtención de modelos exactos, tanto del robot como de su entorno. Sin embargo, estas dos condiciones no son suficientes garantía de pleno éxito, ya que los errores e incertidumbres siempre estarán presentes en cualquier sistema robótico real. Dentro de los principales factores que causan incertidumbre destacan los siguientes: 
1. Ruido en los sensores. Las observaciones realizadas por los sensores son normalmente ruidosas y la distribución estadística de este ruido no suele ser sencilla de modelar[198].

2. Ruido en la detección de la posición. Los movimientos del robot no suelen ser exactos, ni tampoco detectados de forma precisa mediante odometría. Los errores de odometría son además acumulativos. Pequeños errores en la rotación del robot pueden tener efectos importantes en la estimación de los movimientos de traslación y en la determinación de su posición final[27].

3. Entornos complejos y dinámicos. Los entornos interiores en los que se desplazan los robots suelen ser complejos y dinámicos, haciendo casi imposible mantener modelos consistentes de los mismos.

Ante esta problemática, los métodos o enfoques probabilísticos han demostrado que ofrecen resultados más robustos que los métodos clásicos. Esto último se le atribuye al hecho de que los métodos probabilísticos se apoyan en modelos que representan la información a través de funciones de probabilidad, lo que los hace más robustos de cara a las limitaciones del sensor y al ruido mismo en la cinemática del robot y en el modelo del entorno. Por otra parte, las limitaciones más citadas de los algoritmos probabilísticos son dos: la ineficiencia computacional al tener que considerar todas las distribuciones de probabilidad del espacio de posiciones del robot, y la inherente necesidad de tener que aproximar discretamente la realidad continua del contexto del robot[193].

El principio básico subyacente en todo algoritmo probabilístico es la regla de Bayes. Si $a$ es una variable que queremos inferir a partir de otra variable $b$, la probabilidad $p(a)$ se conoce como la distribución de probabilidad a priori, y la variable $b$ es conocida como los datos. La distribución $p(a / b)$ es conocida como la distribución de probabilidad a posteriori. La regla de Bayes provee una manera conveniente de computar la probabilidad a posteriori en función de la probabilidad inversa $p(b / a) \mathrm{y}$ la probabilidad a priori $p(a)$, de acuerdo a

$$
p(a / b)=\eta p(b / a) p(a)
$$

donde $\eta$ es un factor normalizador que asegura que la expresión se encuentre en el rango $[0,1]$.

La robótica probabilista buscar estimar la posición de un robot $s=\langle x, y, \theta\rangle$ y el modelo del entorno que lo rodea $\langle m\rangle$. En adelante, la composición de la posición del robot y del mapa, forma y se conoce como el estado $\mathbf{x}=\langle s, m\rangle$. La evolución del estado, el control y la sensorización de un robot móvil puede ser modelado mediante una red dinámica de Bayes. De acuerdo a la figura 2.25, el estado $\mathbf{x}_{k}$ es estocásticamente dependiente del estado anterior $\mathbf{x}_{k-1}$ y del control $\mathbf{u}_{k}$, mientras que la medición $\mathbf{z}_{k}$ depende estocásticamente del estado $\mathbf{x}_{k}$.

El Filtro de Bayes extiende la regla de Bayes de la ecuación 2.40 para tratar problemas de estimación en el dominio del tiempo. Este filtro es un estimador recursivo 


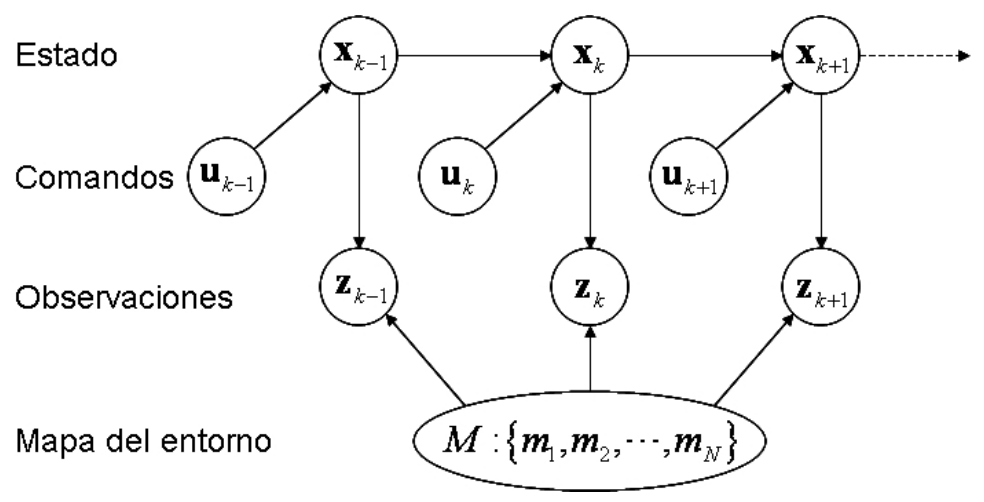

Figura 2.25: Red dinámica de Bayes que caracteriza la evolución del estado $\mathbf{x}$, la medición $\mathbf{z}$, y el control $\mathbf{u}$ en un robot móvil. El subíndice $k$ indica la dependencia temporal del modelo

que permite computar la secuencia de una distribución de probabilidad posterior que no puede ser observada directamente. Así, con un Filtro de Bayes se puede calcular la probabilidad posterior sobre el estado $\mathbf{x}_{k}$ por medio de la siguiente ecuación.

$$
\begin{aligned}
& p\left(s_{k}, m_{k} \mid z_{k}, u_{k}\right)= \\
& \eta p\left(z_{k} \mid s_{k}, m_{k}\right) \iint p\left(s_{k}, m_{k} \mid u_{k}, s_{k-1}, m_{k-1}\right) p\left(s_{k-1}, m_{k-1} \mid z_{k-1}, u_{k-1}\right) d s_{k-1} d m_{k-1}
\end{aligned}
$$

Asumiendo que el mapa del entorno es estático y que el movimiento del robot es independiente del mapa, la ecuación 2.41 se transforma en

$$
\begin{aligned}
& p\left(s_{k}, m \mid z_{k}, u_{k}\right)= \\
& \eta p\left(z_{k} \mid s_{k}, m\right) \int p\left(s_{k} \mid u_{k}, s_{k-1}\right) p\left(s_{k-1}, m \mid z_{k-1}, u_{k-1}\right) d s_{k-1}
\end{aligned}
$$

En la ecuación 2.42, la distribución $p\left(s_{k}, m \mid z_{k}, u_{k}\right)$ se conoce como la confianza o certidumbre del estado $\mathbf{x}_{k}$. La misma se denota como $\operatorname{Bel}\left(x_{k}\right)$ y representa la probabilidad del que el sistema se encuentre en el estado $\mathbf{x}_{k}$, dado las mediciones $\mathbf{z}_{1: k}$ y comandos de control $\mathbf{u}_{1: k}$ ejecutados hasta el estado $k$. En la misma ecuación, el segundo factor dentro de la integral representa la confianza del estado anterior $\operatorname{Bel}\left(x_{k-1}\right)$, por lo que esta condición le da un carácter recursivo al filtro de Bayes. Una forma más compacta de expresar la ecuación 2.42, utilizando la forma recursiva del filtro de Bayes es,

$$
\operatorname{Bel}\left(x_{k}\right)=\eta p\left(z_{k} \mid x_{k}\right) \int p\left(x_{k} \mid x_{k-1}, u_{k}\right) \operatorname{Bel}\left(x_{k-1}\right) d x_{k-1}
$$

Para implementar o poner en funcionamiento este estimador, se deben especificar dos densidades generativas: la densidad $p\left(z_{k} \mid x_{k}\right)$ conocida como el modelo perceptual, y la densidad $p\left(x_{k} \mid x_{k-1}, u_{k}\right)$ conocido como el modelo de transición. La densidad $p\left(z_{k} \mid x_{k}\right)$ describe en términos probabilísticos la manera como se generan 
las distintas observaciones $\mathbf{z}$ para diferentes localizaciones y mapas del entorno. La densidad $p\left(x_{k} \mid x_{k-1}, u_{k}\right)$ describe como afecta el control $\mathbf{u}$ a la evolución de la posición del robot. Así, esta densidad describe la probabilidad de que estando el robot en el estado $x_{k-1}$, alcance el estado $x_{k}$ si le es aplicado el control $u_{k}$. Por eso este modelo también se conoce como el modelo de actuación.

\subsection{Localización Bayesiana o localización de Markov}

La localización probabilística consiste en estimar la posición de un robot móvil a partir de la secuencia histórica de las observaciones sensoriales y de un mapa del entorno. Esto puede ser expresado como la distribución de probabilidad,

$$
p\left(\mathbf{x}_{k} / o_{0: k}, M\right)
$$

donde $\mathbf{x}_{k}=\langle x, y, \theta\rangle_{k}$ es la posición del robot en el entorno en el instante $k, o_{0: k}$ es la secuencia de percepciones sensoriales $\left\{o_{0}, o_{1}, \ldots, o_{k}\right\}$ hasta el instante $k$, y $M$ es un modelo del entorno.

Cabe mencionar que los datos $o_{0: k}$ en general pueden ser de dos tipos: datos $z$ que caracterizan la situación momentánea del robot y que provienen de la observación del entorno mediante sensores exteroceptivos como cámaras de vídeo, láser, sensores de ultrasonido, etc.; y datos $u$ relacionados al cambio de posición como consecuencia de una acción sobre el robot, como por ejemplo la señal de control de los motores o la lectura de odometría. Así,

$$
o_{0: k}=\left\{z_{0: k}, u_{0: k}\right\}
$$

donde $z_{0: k}$ representa la historia de las observaciones exteroceptivas hasta el instante $k$, y $u_{0: k}$ representa la secuencia de comandos dados al sistema o los datos propioceptivos hasta el mismo instante $k$. De esta manera, la función de posición de un robot móvil se puede expresar como la distribución de probabilidad condicional

$$
p\left(\mathbf{x}_{k} / z_{0: k}, u_{0: k}, M\right)
$$

A pesar de que las técnicas de localización probabilística surgieron para dar respuesta al problema de localización global, esta metodología también ha sido usada para resolver el problema de localización relativa. Este tipo de localización es conocida como seguimiento de la posición(Pose Tracking) ya que la nueva posición se calcula asumiendo que se posee un buen estimado de la posición o estado previo del robot. De esta forma la función de localización puede ser expresada como la probabilidad de que el robot alcance el estado $\mathbf{x}_{k}$ dadas las condiciones de observación $z_{k-1}$, control $u_{k-1}$, y estado previo $\mathbf{x}_{k-1}$.

$$
p\left(\mathbf{x}_{k} / z_{k-1}, u_{k-1}, \mathbf{x}_{k-1}, M\right)
$$

Usualmente, en las aplicaciones de seguimiento de posición, la probabilidad del estado posterior del robot se representa usando distribuciones Gausianas. De aquí que este tipo de aplicaciones sean uní-modales, y de esta forma sólo pueden seguir 
una hipótesis única. Si se escoge una hipótesis a priori muy desviada de la realidad, el estimado resultante tiene poca probabilidad de converger al valor real. En contraste con lo anterior, la localización global es capaz de tratar con situaciones ambiguas mediante la representación del estimado posterior a través de una distribución multi modal. Este tipo de representación del estado le da robustez a la aplicación, pero a cambio su complejidad computacional se ve significativamente incrementada.

En la localización de robots móviles existe una situación extrema en la que no se tiene conocimiento previo del mapa del entorno de trabajo del robot, y por lo tanto se requiere que el robot explore el ambiente para construir el mismo. Esto se conoce con el nombre de construcción de mapas[194], y consiste en determinar un mapa del entorno dada la secuencia de estados del robot, y dada la información proveniente de sensores de distancia. Analíticamente se puede expresar como la probabilidad de un conjunto de elementos del entorno $M_{k}=\left\{m_{1: n}\right\}$, dada la historia de estados $\mathbf{x}_{0: k} \mathrm{y}$ de observaciones $z_{0: k}$ del robot.

$$
p\left(M_{k} / \mathbf{x}_{0: k}, z_{0: k}\right)
$$

Es de hacer notar que la formulación anterior asume que se conoce la correcta posición del robot, y tal asunción es fundamental para que los métodos puedan conducir a resultados correctos. Esto situación de conocimiento de la posición del robot se consigue estructurando el entorno convenientemente mediante la colocación de Landmarks activas, o si es en exteriores, por ejemplo puede usarse un GPS.

Por otra parte, los sensores usados en la construcción de mapas poseen longitud de vista limitada y por lo tanto, los mapas que se pueden generar con ellos están limitados a ciertas porciones del entorno. Para modelar espacios medianamente grandes, un robot móvil se basa en sus sensores propioceptivos para estimar su posición a lo largo de la trayectoria, mientras va adquiriendo datos del entorno. Esta operación, conocida como autolocalización, conlleva a que vaya creciendo el error de posición a medida que el robot se mueve, y por lo tanto el mapa resultante perderá precisión y exactitud. Para evitar esto, la posición del robot tiene que ser corregida basándose en los mismos datos que se van observando en el entorno. Las inconsistencias detectadas deben ser entonces corregidas combinado la evidencias suministrada por las sucesivas lecturas sensoriales. Esta combinación de autolocalización y mapeado simultáneo se le conoce como SLAM[58]; lo cual consiste en estimar la posición de un robot y el mapa del entorno al mismo tiempo, dado solamente el histórico de percepciones provenientes de los sensores. Este problema se puede representar matemáticamente como la probabilidad conjunta del mapa $M_{k}$ y el estado $x_{k}$, dado las historia de las observaciones $z_{0: k} \mathrm{y}$ las acciones $u_{0: k}$.

$$
p\left(M_{k}, x_{k} / z_{0: k}, u_{0: k}\right)
$$

La solución analítica de este problema es computacionalmente compleja[194], siendo exponencial en espacio y tiempo con respecto a la longitud del trayecto viajado.

En los últimos años han comenzado a aplicarse con éxito en la robótica nuevos métodos, basados en un enfoque Bayesiano, que proporcionan un potente marco 


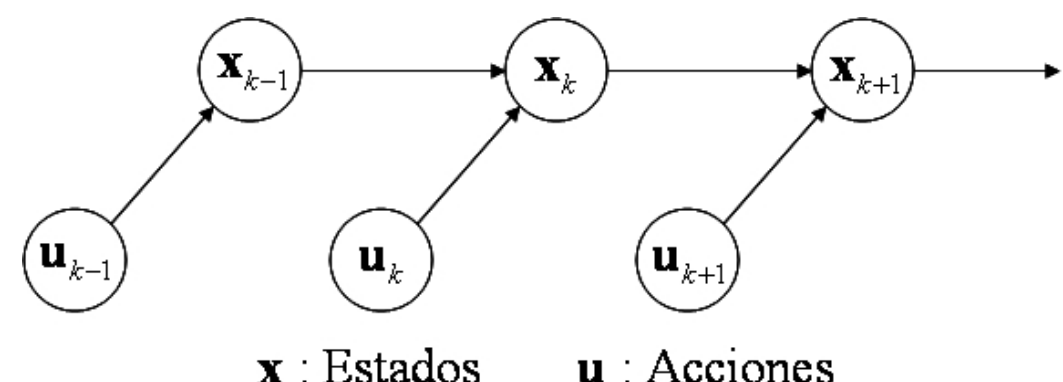

Figura 2.26: Red de Bayes dinámica donde se muestra que el estado actual depende únicamente del estado anterior y de la acción ejecutada.

probabilístico para estimar la posición de un robot móvil a partir de observaciones ruidosas y acciones inciertas. Si $\mathbf{X}$ es una variable aleatoria que representa los posibles estados $\mathbf{x}_{k}$ de un robot, la localización Bayesiana, conocida también como Localización de Markov $^{1}$, busca estimar la distribución posterior de probabilidad sobre el espacio de posiciones del robot condicionado a los datos disponibles. Esta a función de probabilidad se le conoce usualmente como Confianza o Certidumbre, y se denota como $\operatorname{Bel}(x)$.

La estimación de $\operatorname{Bel}(x)$ en cada instante se realiza mediante una formulación recursiva que se apoya en la condición de Markov. Esta condición determina que el estado del robot en cualquier instante depende únicamente del estado y la acción del instante anterior(ver figura 2.26). Es decir,

$$
\operatorname{Bel}\left(\mathbf{x}_{k} \mid \mathbf{x}_{1}, \ldots, \mathbf{x}_{k-1}, \mathbf{u}_{1}, \ldots, \mathbf{u}_{k-1}\right)=\operatorname{Bel}\left(\mathbf{x}_{k} \mid \mathbf{x}_{k-1}, \mathbf{u}_{k-1}\right)
$$

donde $\mathbf{x}_{k}$ es el estado del robot en el instante $k, \mathbf{x}_{k-1}$ y $\mathbf{u}_{k-1}$ son el estado y la acción del instante anterior, respectivamente.

El algoritmo de localización de Markov se realiza mediante dos pasos básicos: la predicción y la observación.

Predicción Cuando el robot se mueve, la distribución de probabilidad se actualiza para tomar en cuenta la incertidumbre adicionada en la posición del robot debido por ejemplo a los errores de odometría. Durante esta etapa, el modelo de propagación $P\left(\mathbf{x}_{k} / \mathbf{x}_{k-1}, u_{k}\right)$, también conocido como modelo de movimiento o de actuación, se usa para incorporar el efecto de la acción sobre un estimado o certidumbre previo denotado como $\mathrm{Bel}^{-}\left(\mathbf{x}_{k}\right)$. Esto se formula matemáticamente de acuerdo a,

$$
\operatorname{Bel}^{-}\left(\mathbf{x}_{k}\right)=\int_{\Xi} P\left(\mathbf{x}_{k} / \mathbf{x}_{k-1}, \mathbf{u}_{k}\right) B e l^{+}\left(\mathbf{x}_{k-1}\right) d \mathbf{x}_{k-1}
$$

donde $\mathrm{Bel}^{+}\left(\mathbf{x}_{k-1}\right)$ representa la confianza del instante anterior, y $\Xi$ es el espacio de las posibles localizaciones del robot.

\footnotetext{
${ }^{1}$ En el anexo A se encuentra un desarrollo más extendido sobre las bases de esta teoría
} 
Observación Cuando el robot observa su entorno, la distribución de probabilidad es recalculada para tomar en consideración los nuevos datos que aporta esta observación exteroceptiva. La observación $z_{k}$ es incorporada a la función de localización mediante la regla de Bayes[152], la cual en general permite reducir la incertidumbre en lo que a la posición del robot se refiere.

$$
\operatorname{Bel}^{+}\left(\mathbf{x}_{k}\right)=\frac{P\left(z_{k} / \mathbf{x}_{k}\right) B e l^{-}\left(\mathbf{x}_{k}\right)}{P\left(z_{k} / z_{1}, \mathbf{u}_{1}, \ldots, z_{k-1}, \mathbf{u}_{k-1}\right)}
$$

El factor $P\left(z_{k} / \mathbf{x}_{k}\right)$ que pondera la confianza previa, es el modelo de observación. Este factor depende en gran medida del sensor físico, y por ello se conoce también como el modelo generativo del sensor[198]. El denominador corresponde a una constante de normalización que asegura que la densidad de probabilidad resultante $\mathrm{Bel}^{+}\left(\mathbf{x}_{k}\right)$, como máximo sea igual a 1 .

Aplicando el teorema de probabilidad total y la asunción de Markov[193], la ecuación de localización de Markov puede transformarse en

$$
B e l^{+}\left(\mathbf{x}_{k}\right)=\eta_{k} P\left(z_{k} / \mathbf{x}_{k}\right) \int_{\Xi} P\left(\mathbf{x}_{k} / \mathbf{x}_{k-1}, u_{k-1}\right) B e l^{+}\left(\mathbf{x}_{k-1}\right) d \mathbf{x}_{k-1}
$$

donde $P\left(\mathbf{x}_{k} / \mathbf{x}_{k-1}, \mathbf{u}_{k-1}\right)$ es una generalización del modelo cinemático del robot que caracteriza el efecto de la acción a sobre la posición del robot, y $\eta_{k}$ es una constante normalizadora que asegura que el resultado general sea como máximo igual a 1.

Una descripción gráfica del algoritmo de localización de Markov se puede ver en la figura 2.27. Mayores detalles de esta técnica puede encontrarse en [75] donde se utiliza esta técnica para desarrollar un método activo para la localización de robot en ambientes dinámicos.

\subsubsection{Implementación de la localización Bayesiana o de Markov}

La implementación de la fórmula de localización 2.53 depende del espacio en donde se vaya a representar la certidumbre o Confianza $\operatorname{Bel}\left(\mathbf{x}_{k}\right)$ : discreto o continuo. De esta manera existen dos grupos de métodos que calculan la certidumbre de manera diferente[12]: Métodos que representan la certidumbre continuamente (enfoque métrico) $[6,42]$ y métodos que representan la certidumbre discretamente (enfoque topológico)[41, 56, 196]. Sobre un enfoque métrico, la variable aleatoria $\mathbf{X}$, que representa el estado del robot $\mathbf{x}_{k}$, es el conjunto de configuraciones $\langle x, y, \theta\rangle$ de posición métrica del robot dentro del mapa global del entorno. Aquí , $\langle x, y\rangle$ son las coordenadas cartesianas del centro del robot, y $\theta$ la orientación de un eje del mismo. Así mismo, las acciones son movimientos métricos medidos por ejemplo a través de los sensores de odometría del robot, mientras que las observaciones son las mediciones realizada con los sensores exteroceptivos del mismo. Sobre un enfoque topológico, la variable $\mathbf{X}$ representa posiciones topológicas dentro de un grafo del entorno (nodos), las acciones pueden ser comportamientos de navegación local del tipo "seguir pared" o "girar a la derecha" y las observaciones suelen ser información procesada de los sensores. 


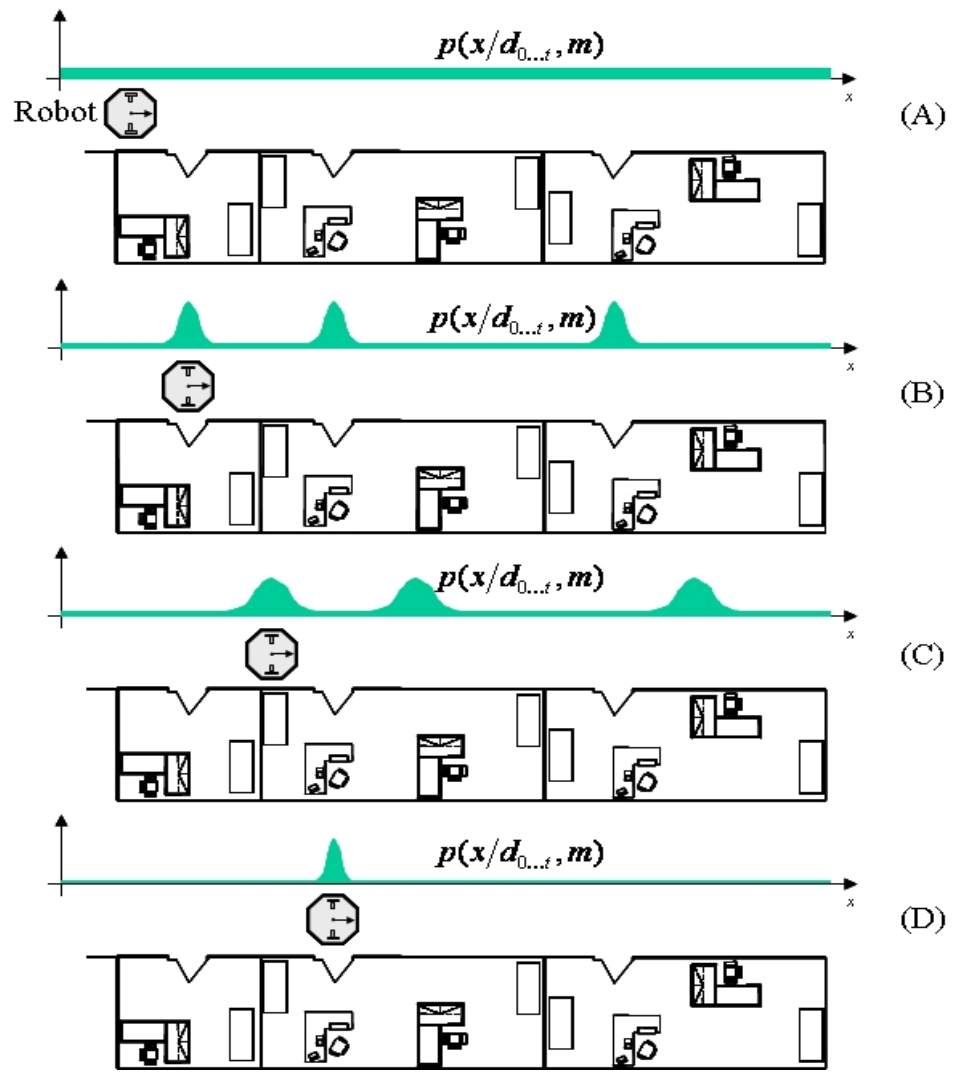

Figura 2.27: Secuencia típica en localización de Markov. A) El robot desconoce su estado inicial. La distribución de probabilidad o creencia del estado es uniforme. B) El robot consulta sus sensores y se observa una puerta. Se utiliza el modelo de observación en conjunto con un mapa de puertas y pasillos, y se determina que hay tres zonas que tienen igual probabilidad de corresponder al estado actual del robot. C) El robot se desplaza aplicando el modelo de predicción. Ahora las tres zonas posibles de la ubicación actual del robot también se desplazan y su distribución de probabilidad se aplana un poco al tomar en cuenta el modelo de incertidumbre en el movimiento del robot. D) El robot consulta nuevamente sus sensores y determina que esta frente a una puerta. De esta manera se determina un único máximo en la distribución de probabilidad del estado, finalizando así el proceso de localización global y comenzando el proceso de seguimiento o tracking. 


\subsubsection{Enfoque métrico}

Localización unimodal o de hipótesis única La implementación más sencilla del método Bayesiano sobre un enfoque métrico es aquélla en la que $\operatorname{Bel}\left(\mathbf{x}_{k}\right)$ es una distribución Gaussiana unimodal sobre el espacio de movimiento. En este caso la estimación de los parámetros de $\operatorname{Bel}\left(\mathbf{x}_{k}\right)$ se realiza mediante un filtro de Kalman. Los métodos basados en el filtro de Kalman pueden ser considerados como un caso especial de localización probabilística donde la distribuciones de probabilidad están limitadas a ser sólo del tipo normal. Sin embargo, esta metodología ha sido usada con satisfacción en los problemas de robótica móvil(distribuciones no gausianas) mediante la implementación del filtro de Kalman extendido(EKF- Extended Kalman Filter), el cual es una herramienta de estimación que representa cualquier tipo de distribución mediante el primer y segundo momento de la misma[13, 10, 54]. La localización es formulada como un problema de filtrado, en el cual el mapa consiste de un conjunto de objetos $\left\{m_{1}, \ldots, m_{i}\right\}$ conocidos como Landmarks, el vector de estado es el vector de posicionamiento del robot, y el vector de observación consiste de las localizaciones de las Landmarks , vistas desde el marco de referencia del robot. Los trabajos realizados en esta línea[127, 87, 9] demuestran que esta solución es un método robusto para resolver el problema de localización local (tracking), pero debido a que sólo puede representar una única hipótesis, su tendencia es a no trabajar bien con los problemas de localización global y de Kidnapping. Por lo tanto, estos métodos requieren que la posición inicial del robot sea conocida (al menos de forma aproximada) y no son capaces de recuperarse por sí solos ante fallos generales de localización.

Localización multimodal o de hipótesis múltiple Si el conjunto de Landmarks que modelan el entorno pueden ser inequívocamente identificadas, entonces la localización unimodal o de hipótesis única trabajaría bien; pero si no se puede resolver convenientemente la correspondencia de datos, entonces es necesario adoptar una técnica de hipótesis multimodal o de hipótesis múltiple[154]. Así por ejemplo, un escenario donde es particularmente relevante la localización multihipótesis con sensores de bajo coste es la ROBOCUP[166]. En el dominio de la localización global, hay varios enfoques que representan el estado a posteriori mediante una distribución multimodal. Una forma de representar el estado del robot que admite el planteamiento multimodal son las rejillas de probabilidad [36, 77]. En este caso, el espacio de estados viene dado por una rejilla regularmente espaciada, en la que la resolución espacial suele oscilar entre 10 y 40 centímetros y la angular entre 2 y 5 grados. Este enfoque usa un filtro de Bayes que representa la distribución a posteriori en términos de la suma de gausianas. Cada gausiana es actualizada de acuerdo a un filtro de Kalman, pero se usa un marco externo de inferencia Bayesiana (filtro de partícula) para asignar el peso que tendrán las gausianas. Fox [75] usa este filtro discreto de Bayes para hacerle seguimiento a una serie de filtros de Kalman, que a la postre generan o suprimen la hipótesis única, dependiendo de la evolución que en estos últimos se suceda. Una nueva hipótesis se genera cada vez que la observación actual no puede ser explicada de acuerdo a la sucesión de eventos actuales. Una hipótesis anterior es 
eliminada cuando presenta baja probabilidad en comparación con las hipótesis actuales. Un inconveniente evidente de este método es el tamaño del espacio de estados necesario cuando el entorno de movimiento es grande.

\subsubsection{Enfoque topológico}

En este enfoque, el entorno es representado mediante una discretización más gruesa basada en lugares distinguibles (pasillos, habitaciones, puertas, etc.). El método de localización es formalizado matemáticamente como un Proceso de Decisión de Markov Parcialmente Observable (POMDP - Partially Observable Markov Decision Process)[40].

Un POMDP es un modelo matemático del problema de decisión secuencial en tiempo discreto en donde los estados son parcialmente observables. En el caso de la robótica móvil, la observabilidad parcial implica que no existe una relación inequívoca entre las observaciones del robot y su estado. De aquí que el robot no pueda conocer con exactitud su estado real. En este sentido, uno de los elementos de un POMDP, conocido como estimador de estados, es la implementación directa del método de localización de Markov que usa como espacio de estados los nodos de un grafo topológico del entorno. La aplicación de este método en unión de las observaciones sensoriales del entorno, le permiten al robot tener cierta confianza o certidumbre de cuál puede ser su estado real.

Las observaciones del entorno se modelan también mediante una función que indica la probabilidad de realizar cada observación en cada estado que alcanza el robot. Puesto que varios estados pueden producir la misma observación (condición que se conoce como solapamiento perceptual), es necesario mantener el registro de las acciones y observaciones pasadas de tal manera que se pueda reducir la ambigüedad que exista con respecto al estado más probable que tenga el robot en un momento determinado. En la localización modelada mediante un POMDP, esto puede realizarse de forma sencilla manteniendo una distribución de probabilidad sobre los estados del robot.

Al igual que en los enfoques métricos de la localización de Markov, en la localización mediante POMDP la confianza de la ubicación del robot se mantienen a través de una distribución de probabilidad que se actualiza usando la información de los sensores propioceptivos y exteroceptivos. Sin embargo, en la metodología basada en POMDP se agrega la noción de recompensa al efectuar una acción en cierto estado. Estas recompensas se utilizarán para calcular la política óptima de movimiento para que el robot navegue hacia cierto estado o meta.

La estructura de un POMDP para aplicaciones de robótica móvil consiste de:

1. Un conjunto de posibles estados $\mathbf{x}$ que el robot puede tomar dentro de su espacio de trabajo. Estos estados generalmente se corresponden a los diferentes nodos de un mapa topológico.

2. Un conjunto finito de acciones $\mathbf{u}$ que el robot toma para pasar de un estado a otro. Estas acciones corresponden a comportamientos de navegación local como 


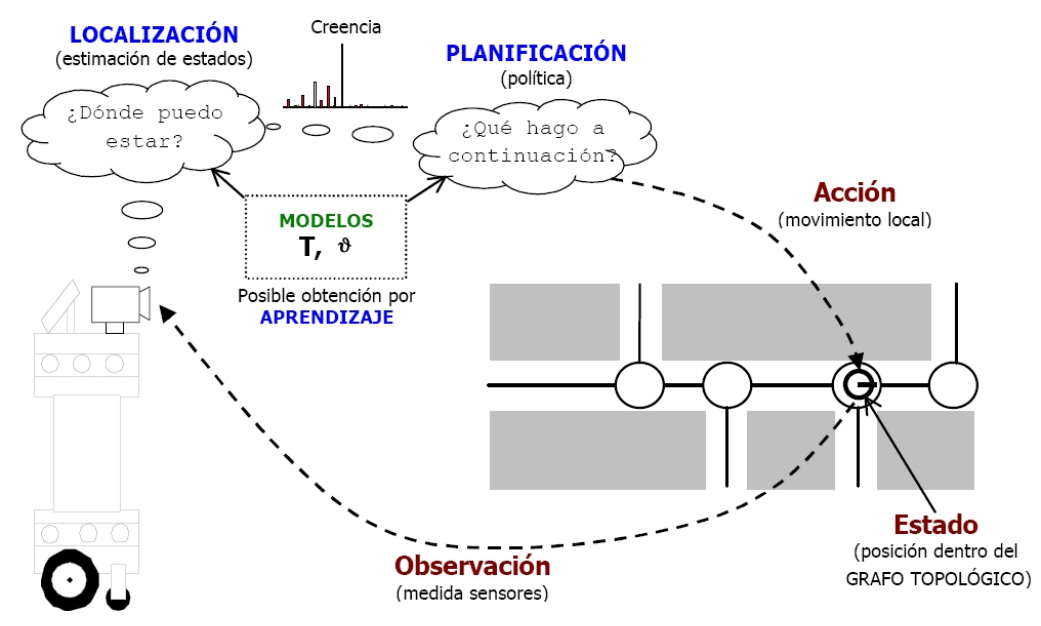

Figura 2.28: Modelo de interacción robot-entorno en un POMDP para la navegación[132].

por ejemplo: girar a la derecha, avanzar recto, atravesar puerta, etc.

3. La función de transición de estado $\mathbf{T}$ que modela la probabilidad de que el nuevo estado del sistema sea $\mathbf{x}_{k}$, dado que estando en $\mathbf{x}_{k-1}$ se tomó la acción $\mathbf{u}_{k-1}$. Esto es $P\left(\mathbf{x}_{k} \mid \mathbf{x}_{k-1}, \mathbf{u}_{k-1}\right)$.

4. La función de recompensa $\mathbf{R}$, que para cada estado en $\mathbf{x}$ y acción en $\mathbf{u}$, determina la recompensa obtenida por el robot en la búsqueda del objetivo de navegación.

5. Un conjunto de observaciones $\mathbf{Z}$ que el robot puede extraer al percibir el entorno mediante sus sensores.

6. La función de observación condicional $\vartheta$ que asocia a cada estado en $\mathbf{x}$ la probabilidad de percibir cada observación en $\mathbf{Z}$. Esto es $\vartheta=P\left(z_{k} \mid \mathbf{x}_{k}\right)$.

Aunque la teoría de POMDPs se desarrolló en los años 60, su aplicación al campo de la robótica móvil es mucho más reciente. Los primeros robots guiados mediante este tipo de modelos no aparecieron sino hasta mediados de los años 90. Las primeras publicaciones sobre la aplicación de la teoría de POMDPs a la navegación de robots móviles en entornos interiores surgen en 1994, destacando las realizadas por Kaelbling y Cassandra[39, 41, 101]. En estos trabajos se realiza la traslación de los conceptos teóricos a la aplicación de navegación, y se proponen algoritmos para resolver los problemas de localización (estimación de estados) y planificación (resolución del POMDP). Un trabajo más reciente realizado por E. López en la Universidad de Alcalá, aborda el desarrollo de una arquitectura robótica destinada a la asistencia personal en hospitales, residencias para mayores o casas particulares[132]. El sistema desarrollado, basado en una representación topológica y probabilística de entornos 
estructurados en habitaciones y pasillos, utiliza un Proceso de Decisión de Markov Parcialmente Observable (POMDP) como modelo de razonamiento. El sistema de localización utiliza un estimador de estados basado en el Método de Localización de Markov para mantener una distribución de probabilidad sobre los posibles nodos del grafo topológico en los que puede ubicarse el robot. De esta forma es posible localizar globalmente el robot con posición inicial desconocida, utilizando hitos naturales del entorno (puertas y pasillos) detectadas mediante ultrasonidos y visión. El autor demuestra que la incorporación de información visual robustece y acelera la localización global durante el desplazamiento por los pasillos. 


\section{Capítulo 3}

\section{Modelado cinemático y localización odométrica del robot diferencial}

El proceso más básico para la localización de un robot móvil se basa en el modelo cinemático del sistema de propulsión. Este sistema es el que permite al robot moverse alrededor de un determinado entorno. El sistema más usual se basa en el uso de simples ruedas de tracción, ya que el mismo es adecuado para la navegación en los entornos de desarrollo típico de las actividades del ser humano, y además es un sistema poco complejo. De la distintas configuraciones basadas en ruedas de tracción, la más popular ha sido la configuración diferencial. La posición de un robot de este tipo puede ser estimada a partir de las ecuaciones geométricas que surgen de la relación entre los componentes del sistema de propulsión y de la información de los codificadores rotativos que usualmente llevan acoplados a sus ruedas[110]. Este método de localización se conoce como estimación odométrica(Odometric Dead-Reckoning). El problema de este método es que por pequeña que sea la constante de error en el sensor, el error de localización del robot crecerá sin límites porque el mismo se va integrando a través del tiempo. Varios investigadores han estudiado este problema, tratando de reducir el error en la localización por medio de mejoras en el modelado del mismo. Uno de los primeros trabajos es el de Borenstein y Feng quienes analizaron las fuentes de error y propusieron un modelo de error para un robot tipo diferencial[32]. Otra forma de atacar el problema de la acumulación del error es ir corrigiendo la posición del robot a intervalos regulares. Esto último usualmente se consigue mediante la colocación de sistemas de Landmarks o de sistemas de señalización activos[71]. Estos sistemas tienen altos costos de instalación y quizás de mantenimiento, y su costo se incrementa con el numero de Landmarks necesarias. Por esta razón, los sistemas de localización odométrica siguen siendo de interés, porque además de producir resultados aceptables para la localización en trayectos cortos, también ayudan a reducir los costos de otros sistemas de localización ya que hacen que la actualización de la posición absoluta del robot sea menos frecuente. Además, los resultados de la localización odométrica 


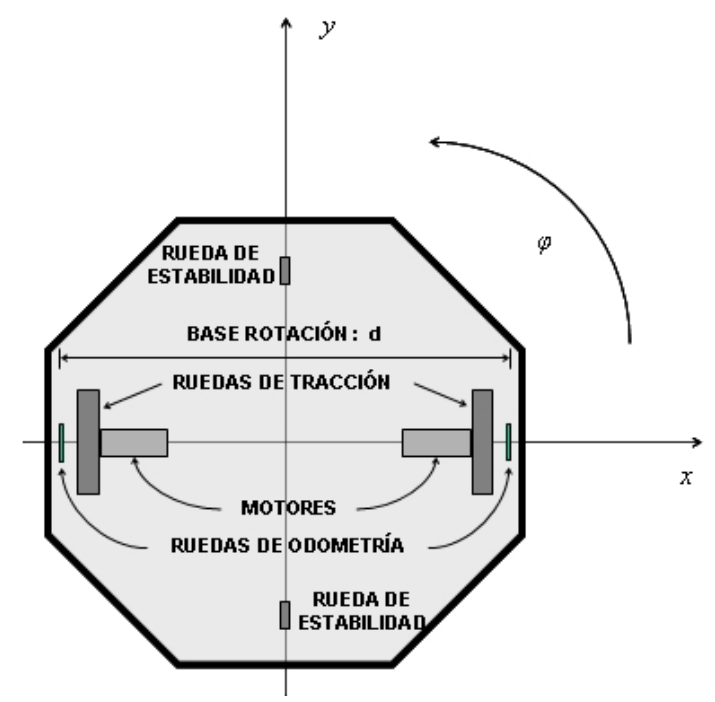

Figura 3.1: Vista de planta inferior del robot YAIR

pueden ser fusionados con mediciones de posición absoluta para producir así mejores resultados en la localización global del vehículo.

En este capítulo se aborda el problema del modelado cinemático de los robot móviles del tipo diferencial, y se hace un análisis exhaustivo de los errores que afectan a la estimación de la posición de este tipo de vehículos, cuando se utiliza el método de localización odométrica basada en codificadores rotativos. Como resultado de este análisis, se propone un sensor virtual de estado completo, que en general es de bajo costo y que además disminuye el efecto de algunas de las causas de error que afectan a la localización odométrica. Igualmente, se hace una revisión de los métodos para identificación y calibración de los parámetros cinemáticos que han sido utilizados por otros investigadores, y se propone una nueva metodología orientada a desacoplar los efectos de los distintos diámetros de las ruedas y de la longitud efectiva de la base de giro, que aparecen durante la ejecución en otros métodos de calibración tradicionales como el UMBmark[27]. Finalmente, en base al método de identificación y calibración que se propone en esta tesis, hacia final del capitulo se propone y desarrolla la metodología de un método multisensorial para la identificación y calibración automática de los parámetros cinemáticos de un robot diferencial.

\subsection{Modelado cinemático de robot diferenciales}

Típicamente una plataforma móvil de tracción diferencial cuenta con dos pares de ruedas: dos ruedas de tracción que tienen acoplados sendos motores DC, dos ruedecillas de estabilización que mantienen el balance del vehículo(figura 3.1).

La traslación y la rotación de este tipo de plataformas diferenciales están determinados por el movimiento independiente de cada una de las ruedas de tracción. Así, 


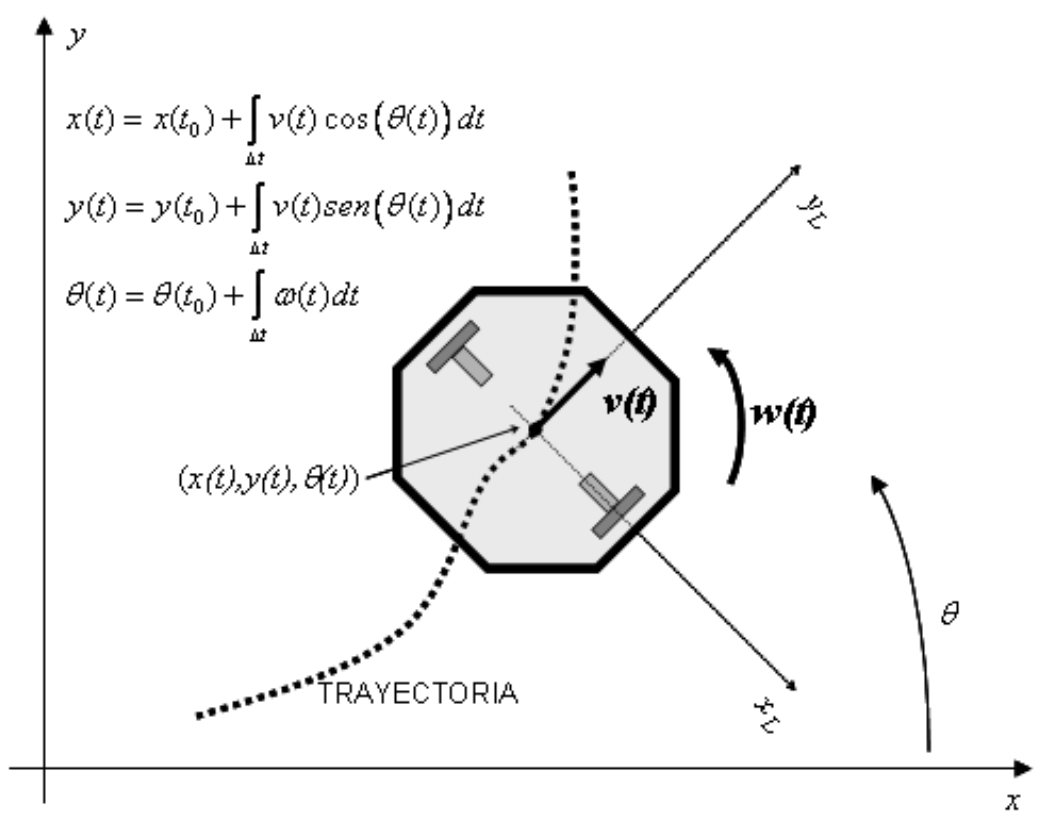

Figura 3.2: Variables cinemáticas globales de una móvil diferencial

las ecuaciones cinemáticas del punto medio del eje entre las ruedas activas, referidas a un sistema de referencia global son (ver figura 3.2):

$$
\begin{aligned}
\dot{x} & =v(t) \cos (\theta(t)) \\
\dot{y} & =v(t) \sin (\theta(t)) \\
\dot{\theta} & =\omega(t)
\end{aligned}
$$

donde $\dot{x}, \dot{y}$, y $\dot{\theta}$ se refieren a la derivada respecto al tiempo de $x, y$, y $\theta$ respectivamente. Es decir, se refiere a la velocidad lineal en la dirección de $x$ e $y$, y a la velocidad angular del robot respectivamente.

La posición y orientación del móvil se obtienen integrando las velocidades del móvil en un periodo de tiempo $\Delta t$.

$$
\begin{aligned}
x & =x_{0}+\int_{\Delta t} v(t) \cos (\theta(t)) d t \\
y & =y_{0}+\int_{\Delta t} v(t) \sin (\theta(t)) d t \\
\theta & =\theta_{0}+\int_{\Delta t} \omega(t) d t
\end{aligned}
$$


Si el período de observación tiende a ser infinitesimalmente pequeño $(\Delta t \rightarrow 0)$, entonces las integrales anteriores pueden ser remplazadas por los desplazamientos diferenciales. Esto es,

$$
\begin{aligned}
\lim _{\Delta t \rightarrow 0}\left[\int_{\Delta t} v(t) \cos (\theta(t)) d t\right] & \approx \Delta x \\
\lim _{\Delta t \rightarrow 0}\left[\int_{\Delta t} v(t) \sin (\theta(t)) d t\right] & \approx \Delta y \\
\lim _{\Delta t \rightarrow 0}\left[\int_{\Delta t} \omega(t) d t\right] & \approx \Delta \theta
\end{aligned}
$$

Así, la ecuación (3.2) se puede rescribir como

$$
\begin{aligned}
& x=x_{0}+\Delta x \\
& y=y_{0}+\Delta y \\
& \theta=\theta_{0}+\Delta \theta
\end{aligned}
$$

Por lo que si se mantiene una frecuencia de muestreo constante y elevada sobre la odometría de un móvil, se puede estimar la posición y orientación del mismo mediante las siguientes ecuaciones en diferencia:

$$
\begin{aligned}
& x_{k}=x_{k-1}+\Delta x_{k} \\
& y_{k}=y_{k-1}+\Delta y_{k} \\
& \theta_{k}=\theta_{k-1}+\Delta \theta_{k}
\end{aligned}
$$

donde $k$ representa los índices de tiempo discreto.

Por otra parte, dado que se está considerando una alta frecuencia de muestreo, entonces también se puede considerar que la velocidad angular en cada una de las ruedas se mantiene constante durante el período de intermuestreo. Esto lleva a que el movimiento del robot pueda ser aproximado a través de pequeños desplazamientos de curvatura constante, según se muestra en la figura 3.3.

En la figura (3.3), $\Delta S_{k}$ es la distancia recorrida por el centro del robot en el intervalo $[k-1, k]$, mientras que $\Delta \theta_{k}$ es el cambio de orientación durante el mismo intervalo. Estas relaciones se pueden expresar según,

$$
\begin{aligned}
\Delta \theta_{k} & =\frac{\Delta S_{k, R}-\Delta S_{k, L}}{d} \\
\Delta S_{k} & =\frac{\Delta S_{k, R}+\Delta S_{k, L}}{2}
\end{aligned}
$$



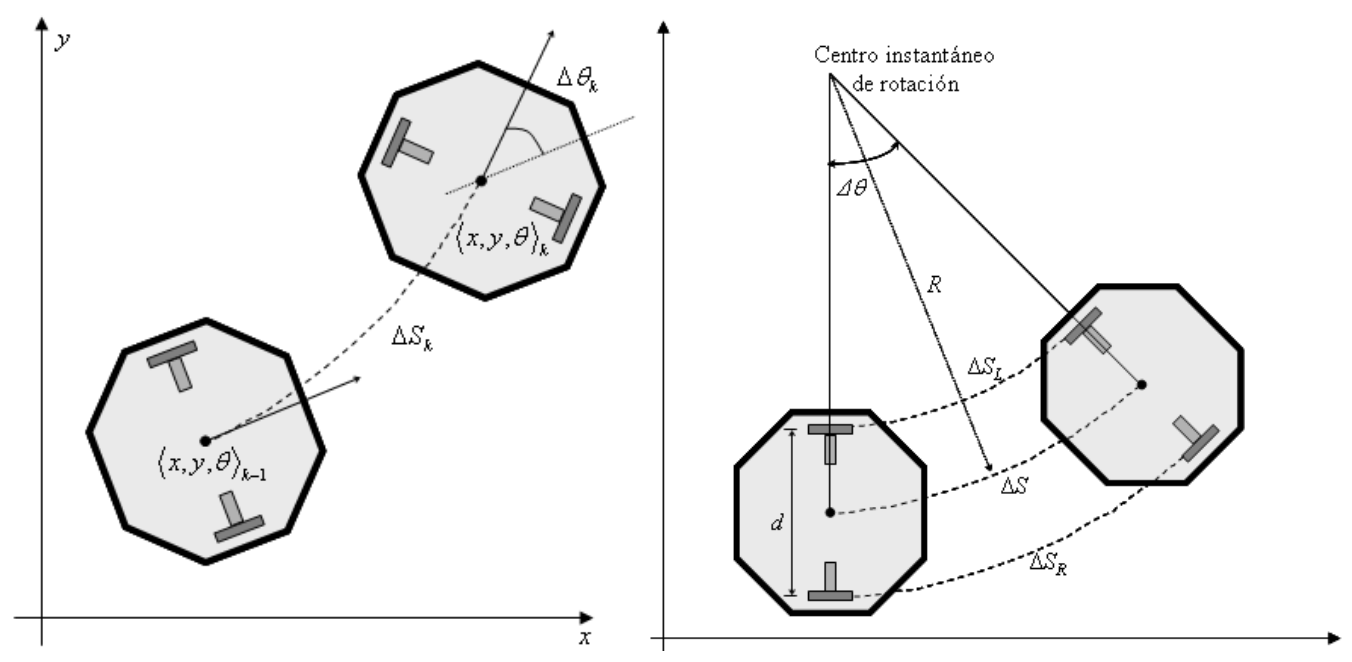

Figura 3.3: Desplazamiento relativo entre instantes de muestreo(derecha) y relaciones geométricas definidas por el movimiento(izquierda).

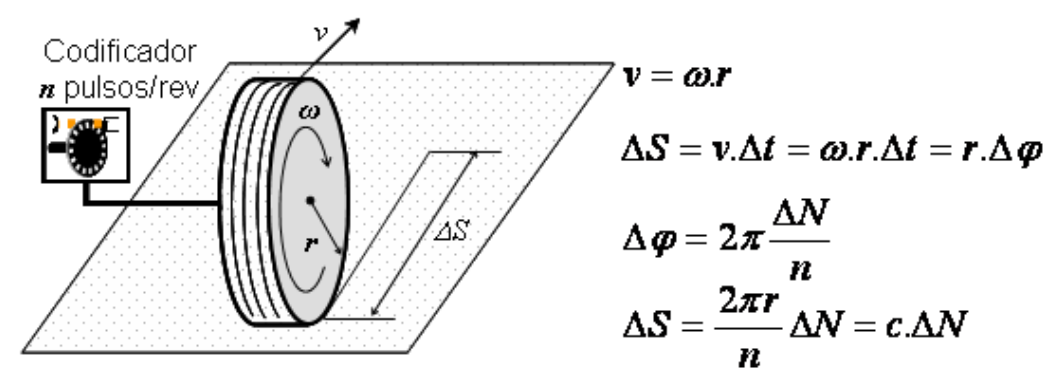

Figura 3.4: Esquema del sistema de medición de desplazamiento lineal mediante codificadores rotativos

donde $\Delta S_{k, R}$ y $\Delta S_{k, L}$ son los arcos recorridos en el intervalo $[k-1, k]$ por las ruedas derecha e izquierda respectivamente. Estos arcos pueden ser calculados a partir de la cadena cinemática ruedas-sensor según como se muestra en la figura 3.4.

En el caso de que el sensor sea un codificador rotativo incremental, el arco recorrido por la rueda se calcula según,

$$
\Delta S=\frac{2 \pi r}{n} \Delta N=C \cdot \Delta N
$$

Donde,

$r$ es el radio de la rueda.

$n$ resolución del codificador o número de pulsos por vuelta.

$C$ es la constante de conversión de pulsos de codificador a unidades de longitud. 


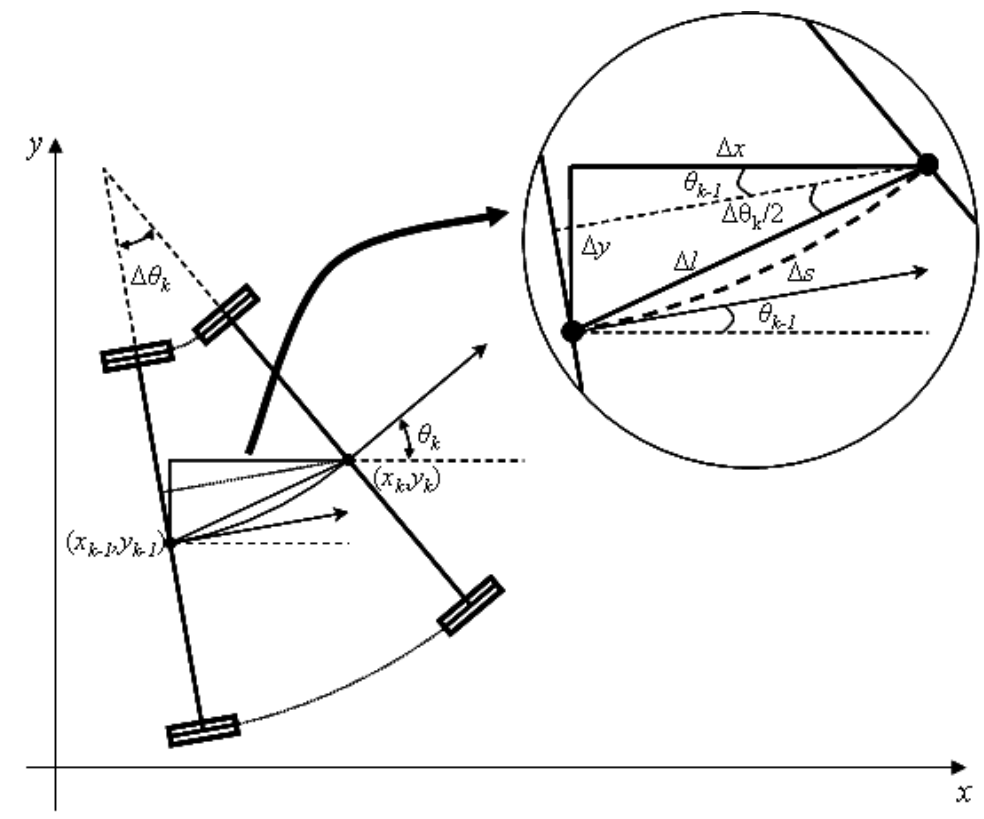

Figura 3.5: Relaciones de transformación trayecto circular a cartesianas

$\Delta N$ es la cantidad de pulsos observados durante el período $\Delta t$.

$\Delta S$ es el desplazamiento lineal de la rueda durante el período $\Delta t(\mathrm{~mm})$.

Considerando que los pulsos suministrados por cada sensor odométrico representan la única entrada del sistema, el vector de excitación o de comandos se puede escribir como

$$
\mathbf{u}_{k}=\left[\begin{array}{c}
u_{k, R} \\
u_{k, L}
\end{array}\right]=\left[\begin{array}{c}
\Delta N_{k, R} \\
\Delta N_{k, L}
\end{array}\right]
$$

Ahora bien, el diferencial de giro $\Delta \theta$ que sufre el robot en el intervalo $[k-1, k]$ es exactamente igual al calculado en la ecuación 3.6, mientras que los desplazamientos cartesianos $\Delta x$ y $\Delta y$ deben calcularse a partir de las relaciones mostradas en la figura 3.5 , de donde resulta

$$
\begin{gathered}
\Delta x_{k}=\Delta S_{k} \cos \left(\theta_{k-1}+\Delta \theta_{k} / 2\right) \\
\Delta y=\Delta S_{k} \sin \left(\theta_{k-1}+\Delta \theta_{k} / 2\right)
\end{gathered}
$$

Considerando que en un robot diferencial las ruedas motrices suelen tener las mismas características físicas, y por lo tanto la constante de conversión $C$ es igual para las dos, entonces las ecuaciones que modelan el movimiento de un robot diferencial en el plano se pueden reescribir según, 


$$
\begin{aligned}
& x_{k}=x_{k-1}+\frac{C}{2}\left(u_{k, R}+u_{k, L}\right) \cos \left(\theta_{k-1}+C \cdot\left(u_{k, R}-u_{k, L}\right) / 2 d\right) \\
& y_{k}=y_{k-1}+\frac{C}{2}\left(u_{k, R}+u_{k, L}\right) \sin \left(\theta_{k-1}+C \cdot\left(u_{k, R}-u_{k, L}\right) / 2 d\right) \\
& \theta_{k}=\theta_{k-1}+\frac{C}{d}\left(u_{k, R}-u_{k, L}\right)
\end{aligned}
$$

La implementación de la localización del robot mediante datos odométricos es el mostrada en algoritmo 1.

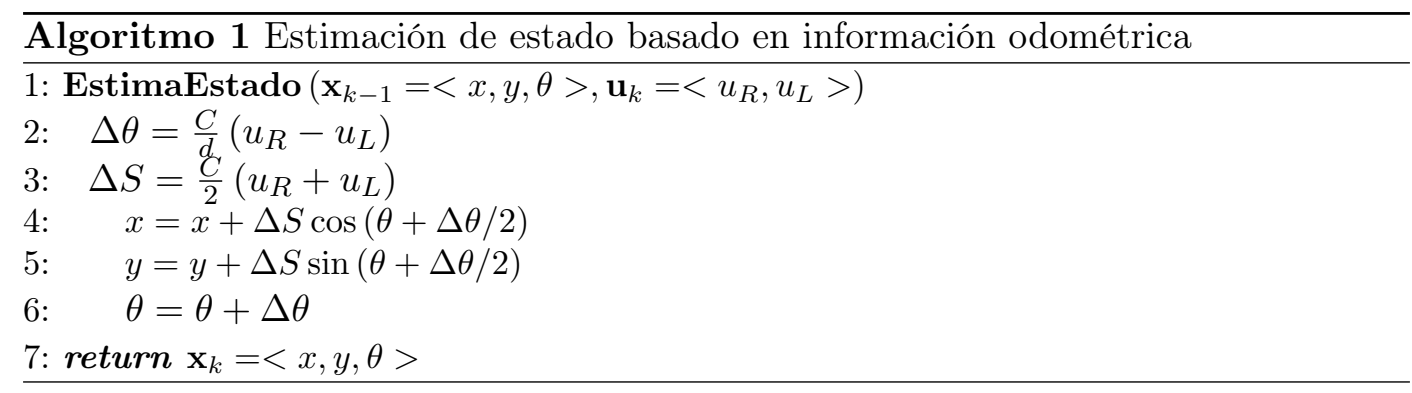

Para mostrar la validez de este modelo, se diseño un experimento en el cual se le suministró un perfil de velocidades al control de motores del robot YAIR, para que el mismo realizará una trayectoria cuadrada 4x4 metros. A lo largo de esta trayectoria se registró el estado real del centro del robot, así como también se estimó su estado a partir del algoritmo presentado anteriormente. Los parámetros cinemáticos del modelo (radio de las ruedas y distancia entre ellas) corresponden a los valores nominales los cuales fueron medidos directamente sobre el hardware del robot. Los resultados se muestran gráficamente en la figura 3.6, donde se observa que el modelo de estimación de posición se ajusta al patrón de la trayectoria real, lo cual valida la forma del mismo. Sin embargo, también se observan discrepancias de trayecto entre las posiciones absolutas registradas para el trayecto real y para el trayecto estimado. Estas discrepancias se deben más que todo a la falta de calibración de los parámetros cinemáticos ya antes mencionado. En la sección 3.4 de esta tesis abordamos esta problemática.

\subsection{Análisis de los principales errores de la localización odométrica en un robot diferencial}

El método de localización relativa más ampliamente utilizado es la odometría. Esta consiste en medir las revoluciones en las ruedas para estimar el desplazamiento del móvil a partir de una posición conocida. Ya se mostró en la sección 3.1 que el desplazamiento horizontal y los cambios de orientación de un robot diferencial no holomónico, pueden ser calculados en función de los desplazamientos diferenciales en 


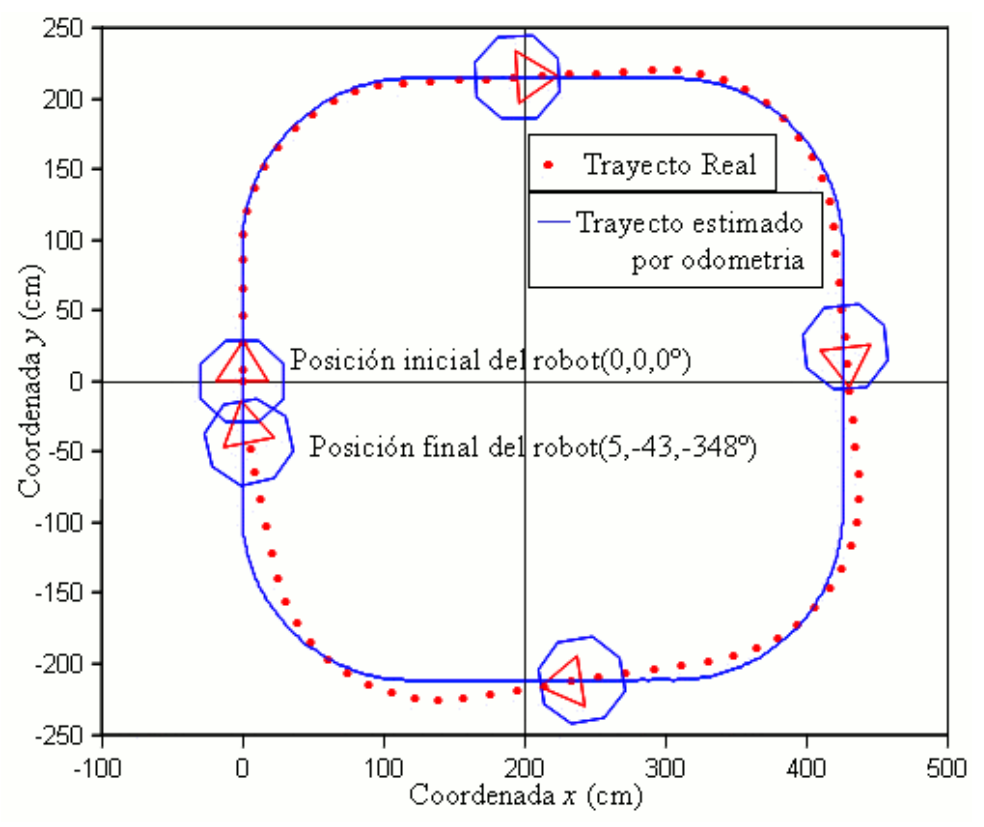

Figura 3.6: Trayecto real y trayecto estimado mediante el modelo odométrico del robot YAIR

cada una de las ruedas motrices. Este procedimiento de estimación de la posición conocido como odometric dead-reckoning, tiene validez limitada debido a que el proceso se ve influenciado por varios factores que afectan sobretodo a la conversión de las lecturas de los codificadores en desplazamiento lineal del móvil. La clara distinción de los errores que afectan al sistema es muy importante en la corrección de los errores en la odometría de un vehículo. Estas fuentes de error se dividen en forma general en dos categorías: errores sistemáticos y errores no-sistemáticos[32]. Para reducir los errores de odometría es necesario distinguir los errores sistemáticos de los no sistemáticos, ya que el efecto y los procedimientos de medición y corrección de los mismos son distintos. Por ejemplo, en superficies rugosas con irregularidades importantes, los errores no sistemáticos son la fuente dominante de errores de odometría, en cambio que en las superficies lisas, los errores sistemáticos son los que más contribuyen a este tipo de errores.

\subsubsection{Errores sistemáticos}

Los errores sistemáticos son debidos a imperfecciones en el diseño y en la implementación mecánica del sistema mismo. Son especialmente graves, ya que se acumulan constantemente. Dentro de los agentes causantes de los errores sistemáticos se encuentran:

1. Distinto diámetro de las ruedas.

2. Diferencia entre el diámetro teórico y el diámetro real de las ruedas. 
3. Diferencia entre la distancia entre ruedas teórica y la real.

4. Resolución finita de los codificadores.

5. Frecuencia finita en el muestreo de datos de los codificadores.

6. Mala alineación de las ruedas.

Las dos fuentes principales de error sistemático son:

- La incertidumbre sobre el diámetro real de las ruedas, que influyen dominantemente sobre los largos trayectos rectilíneos, aunque también es un factor que afecta directamente a la estimación de la orientación del móvil.

- La incertidumbre en la base de giro, la cual influye directamente en la estimación del ángulo de orientación del móvil

\subsubsection{Incertidumbre en el diámetro de las ruedas}

Esta incertidumbre puede surgir debido a dos hechos independientes: el primero tiene que ver con el tipo de rueda y su proceso de manufactura, mientras que el segundo forma parte de la integración de los parámetros de las ruedas al control de navegación del robot. La mayoría de los robots llevan incorporadas en las ruedas bandas de caucho para mejorar la tracción, y estas bandas además de que están sujetas a defectos sutiles durante su fabricación, se deforman en función de la carga sobre ella aplicada o también debido al desbalance de cargas en el cuerpo del robot, conduciendo definitivamente a una variación de su diámetro nominal. Por otro lado, cuando se mide el diámetro de las ruedas para incorporarlas al control de navegación del robot, existe incertidumbre sobre el diámetro real y el diámetro teórico o el que se pueda medir con un instrumento ad hoc. Independientemente de su origen, este error que refleja la incertidumbre que existe entre el diámetro real y el diámetro teórico de las ruedas, influye directamente sobre el factor de conversión mm/pulsos de cada una de las ruedas, o lo que es lo mismo, sobre su sensibilidad de medición de trayecto definida como $s=\pi D / n$, donde $D$ es el diámetro de la rueda y $n$ es la resolución del codificador. De aquí que este error definido como $\varepsilon_{s}=D_{\text {teorico }} / D_{\text {real }}$ tenga dos efectos claros: uno de ellos es sobre la longitud del trayecto, el cual seria por ejemplo menor que la longitud del trayecto programado; mientras que el segundo y más importante de los efectos sería sobre la forma del trayecto, el cual seria un trayecto curvilíneo en lugar de uno rectilíneo como el programado; o en el caso de que el trayecto programado sea curvilíneo, el robot por ejemplo girará menos de lo que se le ha programado. Estos efectos se muestran en la figura 3.7.

Cuantitativamente también se puede observar el efecto de este tipo de error. Por ejemplo, la propagación del error $\varepsilon_{s}$ a través de la ecuaciones 3.7 y 3.6 se expresan respectivamente como,

$$
\varepsilon_{\Delta S}=\frac{\varepsilon_{s, R}+\varepsilon_{s, L}}{2}
$$




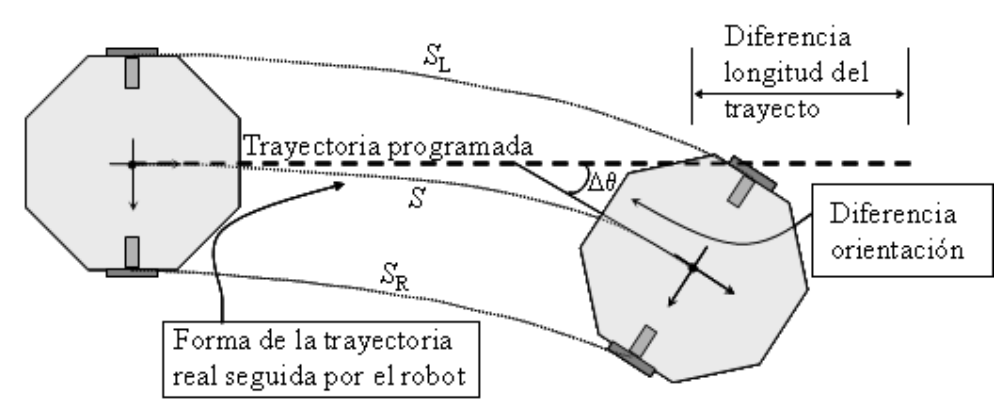

Figura 3.7: Efecto del error en el diámetro de ruedas: efecto en la longitud y forma del trayecto del robot

$$
\varepsilon_{\Delta \theta}=\frac{\varepsilon_{s, R}+\varepsilon_{s, L}}{d}+\frac{\left|\Delta S_{R}-\Delta S_{L}\right|}{d^{2}} \varepsilon_{d}
$$

donde $\varepsilon_{\Delta S}$ y $\varepsilon_{\Delta \theta}$ son los errores de cómputo de la distancia recorrida y ángulo de giro del robot, respectivamente. También $\varepsilon_{s, R}$ y $\varepsilon_{s, L}$ son las componentes de error, en la traducción de la distancia recorrida, de los sensores derecho e izquierdo respectivamente. Finalmente $\varepsilon_{d}$ es el error debido a la diferencia entre el valor nominal y el valor real de la distancia entre los puntos de apoyo de las ruedas o base de giro.

Como se ve en 3.13, el error en los diámetros de las ruedas influye de manera directa en el computo de la distancia recorrida por el robot. Con respecto a la influencia de este tipo de error en el cómputo de la orientación del robot, la ecuación 3.14 muestra que la misma se ve escalada por la dimensión de la base de giro. Esto último sugiere que en el diseño de un robot diferencial se debe dar la máxima longitud a la base de giro. Sin embargo en la práctica, debido a los espacios de navegación disponibles, esto esta limitado a unos 50 centímetros.

\subsubsection{Incertidumbre en la distancia entre los puntos de apoyo de ruedas}

A partir de la ecuación 3.14 parece inminente que una vez que se corrige el error en los diámetros de las ruedas, la suficiente corrección mediante ensayos prácticos del valor de la base de giro $d$, corregiría en gran medida el error en la estimación del ángulo. Sin embargo, en la mayoría de los casos no se cumple la condición ideal de un contacto puntual entre la rueda y el plano de desplazamiento, sino más bien que se trata de un área de contacto que generará en forma directa una incertidumbre en la base de giro real. La figura 3.8 muestra una idea de como afecta el corrimiento del punto de contacto al ángulo de giro real del robot. A partir de esta misma figura se pueden establecer que el ángulo de giro real $\Delta \theta^{\prime}$ es

$$
\Delta \theta^{\prime}=\frac{\Delta S_{R}^{\prime}-\Delta S_{L}}{d+\delta}
$$

Si se asume que se cumple la restricción de no holonomía y que además $\Delta S_{R}^{\prime} \cong$ $\Delta S_{R}$, entonces considerando la forma de la ecuación 3.6, se puede establecer que 


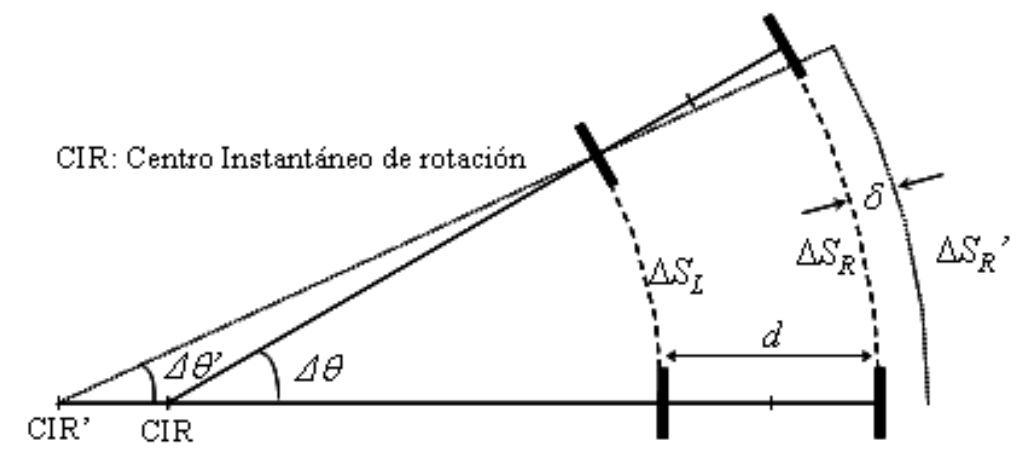

Figura 3.8: Efecto de la incertidumbre de la base de giro en el cómputo del ángulo de giro

$$
\Delta \theta^{\prime}=\frac{d}{d+\delta} \Delta \theta
$$

Aquí $\delta$ es la deriva del punto de contacto que causa efectivamente un aumento o una disminución de la base real de giro. Este tipo de incertidumbre depende mucho del mecanizado de las ruedas y del balance dinámico que tenga el móvil al realizar los giros. Suele ocurrir que si para un sentido de giro la deriva es hacia afuera(aumenta la base efectiva de giro), en el sentido de giro contrario la deriva sería hacia adentro(disminuye la base real de giro). Este efecto combinado tiende a empeorar el estimado general de la orientación del móvil. Para corregir la problemática anterior, en principio se escoge el mejor diseño de ruedas posible, como por ejemplo las del tipo "filo de cuchillo"; y luego se determina, mediante la experimentación con giros en ambos sentidos, el valor de la base de giro $d$.

\subsubsection{Sensor virtual de estado completo basado en codificadores ópti- cos rotativos}

Un sistema de localización odométrica basada en sensores acoplados a las ruedas motrices del vehiculo presenta varios inconvenientes entre los que se encuentran principalmente el derrape de las ruedas, las imperfecciones del acoplamiento de las ruedas a la superficie de rodamiento, y las no linealidades por desgaste de los engranajes. Un sistema de bajo costo que ha mostrado superar estos inconvenientes es aquel formado igualmente por 2 codificadores rotativos pero acoplados a unas ruedas de diseño especial que van a su vez acopladas al cuerpo de la plataforma móvil. Este sensor resuelve en mucho los problemas presentes en la localización odométrica clásica, gracias a las siguientes características propias del sensor descrito:

1. El sistema no va acoplado a las ruedas motrices, sino al cuerpo del móvil. Esto disminuye los errores por deslizamientos y patinazos. Los sensores solo registran datos cuando hay movimiento efectivo de la plataforma. 


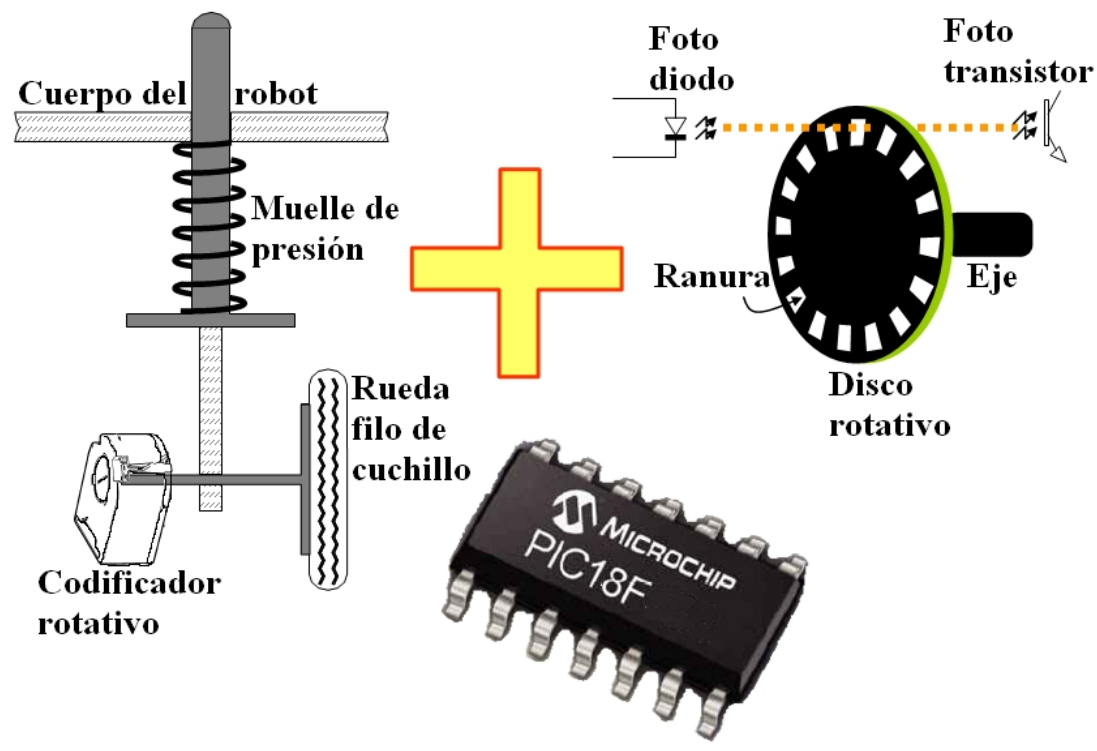

Figura 3.9: Hardware básico del sensor odométrico mejorado propuesto en esta tesis. Las figuras son sólo referenciales. Nótese el sistema de muelle propuesto, el cual trata de garantizar el acoplamiento continuo con la superficie de rodamiento

2. Las ruedas de odometría no soportan carga. Esto permite que las mismas sean del tipo filo de cuchillo sin sufrir deformación elástica. A su vez esto garantiza que los parámetros del modelo cinemático (diámetros de ruedas y base de giro) se mantengan constantes.

3. El sistema de eje deslizante y muelle, le confiere la propiedad de absorber las cargas y desbalances dinámicos, sin perder la traza y el contacto con la superficie de movimiento.

El sistema sensorial propuesto está formado por 2 codificadores ópticos incrementales tipo HDES-5500, que alimentan sus señales de cuadratura a un PIC 18F2480. Este circuito integrado se encarga de estimar el estado del vehículo basándose en el grupo de ecuaciones (3.12). Además de lo anterior, este microcontrolador le da a este sensor capacidad de integración con otros sistemas, a través del módulo CAN (Controller Area Network) o de la interfaz serial USART(Universal Synchronous Asynchronous Receiver Transmitter).

Para validar el sensor propuesto, se realizo un experimento con la ayuda de un prototipo de robot diferencial llamado YAIR (Yet Another intelligent Robot)[25]. Las ruedas motrices del YAIR cuentan con un codificador rotativo acoplado al eje de cada motor, con el cual se suministra al sistema la información odométrica necesaria para tareas de control de cada uno de los motores. Al mismo tiempo, esta información odométrica se aprovecha para el sistema básico de estimación del estado del robot. Por otra parte, el sensor odométrico propuesto en este trabajo se acopló al cuerpo del 


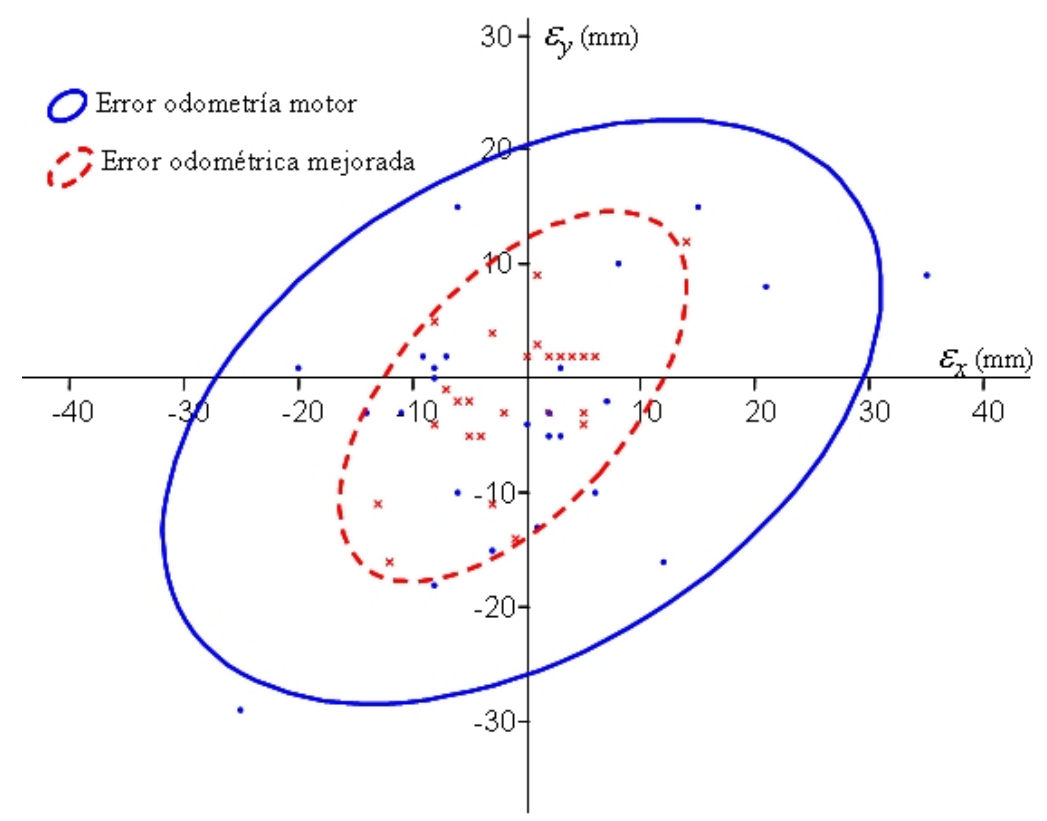

Figura 3.10: Comparación de las elipses de error de odometría para el $95 \%$ de confianza.

robot en lugares simétricos y muy cercanos al eje de tracción del robot. Para controlar los ensayos, las pruebas experimentales se realizaron en un escenario interior cuyo plano de desplazamiento fue adecuadamente demarcado para constituir así un plano cartesiano donde el robot pudiera moverse. De esta forma, para cualquier posición que adoptará el robot dentro de este plano de trabajo, se pudo obtener las coordenadas del centro del mismo. Durante el experimento, el robot ejecutó un trayecto aleatorio de aproximadamente 4 metros, a una velocidad máxima de $250 \mathrm{~mm} / \mathrm{seg}$. Este proceso se repitió 25 veces y se midió la posición real del robot al final de cada trayecto, para compararla con la estimada por el sistema odométrico acoplado a las ruedas motrices y con el sensor de odometría propuesto en esta tesis.

En la figura 3.10 se pueden observar las elipses de error para la localización basada en la odometría acoplada al motor y para la odometría mejorada a coplada al cuerpo del robot. En ella se observa la evidente mejora que produce esta última, lo que permite concluir que el sistema de localización odométrica desarrollado muestra ser adecuado para procesos de localización de robots móviles en entornos interiores. Sin embargo, mediante un experimento adicional se mostró que a pesar de que el sistema muestra tener una significativa mejoría cuando se le compara con un sistema odométrico convencional, en trayectos por encima de los 15 metros, ejecutados a velocidades por encima de $100 \mathrm{~mm} / \mathrm{seg}$, el sistema disminuye en cuanto a la exactitud y precisión del estimado del estado del móvil. Como trabajo futuro se propone fusionar los datos de los dos sistemas odométricos con los que cuenta el robot YAIR, para verificar si se puede obtener una mejora adicional. 


\subsubsection{Errores no sistemáticos}

Son aquellos que no son causados directamente por las propiedades cinemáticas del vehículo, sino por la interacción del móvil con características impredecibles del entorno por donde este se desplaza. No se producen constantemente (depende de la aplicación y del entorno) por lo que son más difíciles de medir y corregir. La principal fuente de error no sistemático en los sistemas odométricos es el deslizamiento de las ruedas, lo cual causa que las revoluciones de las ruedas no se correspondan al desplazamiento real del móvil. Los errores no sistemáticos esta relacionados con:

1. Suelos Irregulares. Muchas superficies de hormigón o asfalto son rugosas y además pueden tener baches, grietas, y cualquier otro tipo de irregularidades que dificultan el correcto acoplamiento de las ruedas.

2. Choque con objetos o desplazamiento a través de ellos.

3. Deslizamiento de ruedas debido a:

a) Superficies resbaladizas.

b) Sobre aceleración. o Giros rápidos.

c) Falta de contacto con la superficie de desplazamiento.

d) Bloqueos externos(obstáculos) o internos(ruedas estabilizadoras).

La consideración de este tipo de errores se trata más adelante en el capítulo 5.

\subsection{Identificación de los parámetros cinemáticos de un robot diferencial}

Dado que la odometría es una parte importante del sistema de localización de un robot móvil, mejorar la exactitud y precisión de los parámetros que modelan la misma conduce a una importante mejora del sistema general de navegación. Borenstein y Feng determinaron que las fuentes dominantes de error en los sistemas de localización odométrica en un robot diferencial son la diferencia entre los diámetros de las ruedas y la incertidumbre con respecto a la base de giro real[32]. A continuación se desarrolla un método general mediante el cual se determina, para un robot tipo diferencial, la sensibilidad odométrica y la base de giro (para su definición ver sección 3.1). Ahora bien, dado que estas relaciones se obtienen por medición directa de los pulsos de codificadores acoplados a las ruedas y de la medición directa de las variables que estos parámetros relacionan(desplazamiento en las ruedas y giro diferencial del cuerpo del robot), el método que describiremos a continuación lo llamamos Método Directo. 


\subsubsection{Determinación de la sensibilidad odométrica mediante el Mé- todo Directo}

La sensibilidad odométrica corresponde a un factor de escala $C=2 \pi r / n$ cuyos parámetros ya fueron definidos en la sección 3.1. No obstante, en forma práctica ésta sensibilidad es igual a la relación desplazamiento/pulsos de cada una de las ruedas. De aquí que una manera simplista de determinar esta relación sea programar el robot para que se mueva sobre un trayecto rectilíneo corto, medir la distancia recorrida por cada rueda, registrar la cantidad de pulsos generados en el trayecto por el codificador correspondiente, y entonces determinar el cociente ( $\mathrm{mm} /$ pulsos). Sin embargo, esta sensibilidad en general no es adecuada para el seguimiento fiable de la posición de un robot, y por lo tanto es usual determinar la sensibilidad real o efectiva mediante métodos experimentales mas exhaustivos[27, 5, 176, 143].

En esta tesis el modelo que proponemos para la sensibilidad efectiva es

$$
C^{\text {efectiva }}=\left(1+\alpha_{C}\right) C^{\text {nominal }}+\varepsilon_{C}
$$

$C$ es la sensibilidad odométrica de la rueda en (unidades de longitud)/pulso.

$\alpha_{C}$ es el factor de corrección que recoge las imperfecciones del hardware.

$\varepsilon_{C}$ es el termino que modela los efectos aleatorios debido a los derrapes, tipo de suelo, etc.

Si se asume que los efectos aleatorios se distribuyen de manera normal con media cero y varianza $\sigma_{C}^{2}$, entonces la sensibilidad odométrica también se distribuirá de manera normal según,

$$
C^{\text {efectiva }} \sim N\left(\bar{C}, \sigma_{C}^{2}\right)
$$

donde $\bar{C}$ es la media y $\sigma_{C}^{2}$ su varianza.

Para determinar la sensibilidad odométrica la estrategia general consiste en excitar los parámetros odométricos del robot a través de un trayecto rectilíneo a fin de minimizar el efecto que tiene la base de giro en el estimado de la posición del robot.

A partir de la figura 3.11 se pueden deducirse las siguientes relaciones,

$$
\left\{\begin{array}{l}
S_{R}^{\prime}=\frac{L_{R}}{\sin c(\Delta \theta / 2)} \\
S_{L}^{\prime}=\frac{L_{L}}{\sin c(\Delta \theta / 2)}
\end{array}\right.
$$

donde $\sin c($.$) es la función seno cardinal o más popularmente conocida como la fun-$ ción de muestreo, la cual es definida como,

$$
\sin c(x)=\left\{\begin{array}{c}
1 \text { si } x=0 \\
\frac{\sin (x)}{x} \forall x \neq 0
\end{array}\right.
$$

En estas ecuaciones, 


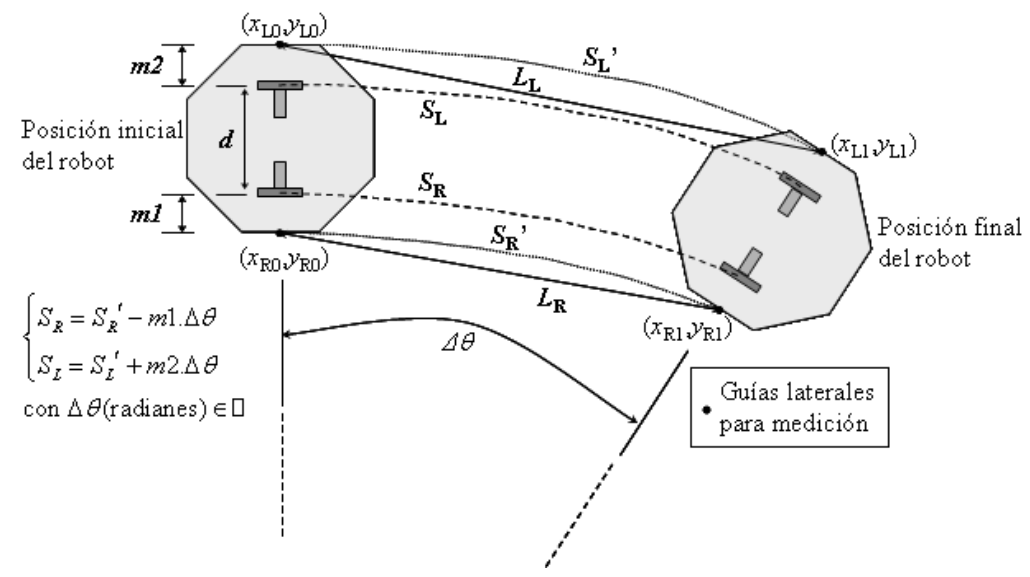

Figura 3.11: Esquema experimental para determinación de la sensibilidad odométrica en un robot diferencial

$L_{R}, L_{L}$ corresponden a la distancia en línea recta que recorre el punto de medición derecho e izquierdo respectivamente.

$S_{R}, S_{L}$ corresponden a los arcos recorridos por los puntos de medición derecho e izquierdo respectivamente.

$\Delta \theta$ es el ángulo que gira el robot debido a velocidades desiguales en ambas ruedas, o a que los diámetros de las mismas son diferentes entre sí.

Otro par de ecuaciones que pueden deducirse de la figura 3.11 son,

$$
\left\{\begin{array}{l}
S_{R}=S_{R}^{\prime}-m_{1} \Delta \theta \\
S_{L}=S_{L}^{\prime}+m_{2} \Delta \theta
\end{array}\right.
$$

donde

$S_{R}, S_{L}$ son los arcos recorridos por los puntos de apoyo de las ruedas derecha e izquierda respectivamente.

$m_{1}, m_{2}$ corresponden a la distancia que existe entre el punto de apoyo de la rueda derecha e izquierda respectivamente y el punto de medición de su correspondiente lado.

Aquí $m_{i} \Delta \theta$ se puede ver como el factor de corrección que toma en cuenta la diferencia de realizar las mediciones en una referencia externa o exactamente en el punto de apoyo de las ruedas motrices.

Combinando la ecuación 3.20 con la ecuación 3.21 se obtiene la expresión para la distancia recorrida por las ruedas de odometría. 


\subsection{IDENTIFICACIÓN DE LOS PARÁMETROS CINEMÁTICOS DE UN}

$$
\left\{\begin{array}{c}
S_{R}=\frac{L_{R}}{\sin c(\Delta \theta / 2)}-m_{1} \Delta \theta \\
S_{L}=\frac{L_{L}}{\sin c(\Delta \theta / 2)}+m_{2} \Delta \theta
\end{array}\right.
$$

Como se aprecia, la expresión para el arco recorrido tiene una importante dependencia del ángulo de rotación del robot; y ya que este ángulo no es muy fácil de medir, es conveniente que el desplazamiento del robot sea perfectamente rectilíneo, o lo que es lo mismo, que $\Delta \theta \rightarrow 0$.

Si la velocidad en las ruedas motrices son iguales, el movimiento curvilíneo del centro robot se debe principalmente a la diferencia de diámetros entre las ruedas, las cuales usualmente no resultan ser tan diferentes como para producir este tipo de curvaturas tan pronunciadas a cortas distancias. En ese sentido, una acotación que se debe hacer en la determinación de los parámetros del modelo odométrico del robot, es que la distancia entre dos puntos consecutivos de medición sea lo más corta posible, para así disminuir el efecto de cualquier curvatura existente. Una regla empírica es que el robot se desplace una longitud correspondiente a una revolución de sus ruedas odométricas, es decir, que basta con que se mueva una longitud equivalente a $\pi D$, donde $D$ es el diámetro nominal de las mencionadas ruedas.

Cuando no es posible lograr una trayectoria suficientemente rectilínea como para asumir $\Delta \theta \approx 0$, si se cuenta con un escenario susceptible de ser normalizado o si se puede fijar un marco de referencia(p.ejemplo dos paredes es escuadra), se pueden determinar las coordenadas absolutas de los puntos de medición del robot. Posteriormente, siempre que durante el trayecto la velocidad en las ruedas del móvil se hayan mantenido constantes, se puede determinar el ángulo de giro y las longitudes de las trayectorias recorridas por las ruedas del robot basándose en el siguiente conjunto de ecuaciones,

$$
\begin{aligned}
\Delta \theta & =\sin ^{-1}\left[\frac{\left(x_{L 1}-x_{R 1}\right)\left(y_{R 0}-y_{L 0}\right)+\left(y_{L 1}-y_{R 1}\right)\left(x_{L 0}-x_{R 0}\right)}{\left(d+m_{1}+m_{2}\right)}\right] \\
S_{R}^{\prime} & =\frac{\sqrt{\left(x_{R 1}-x_{R 0}\right)^{2}+\left(y_{R 1}-y_{R 0}\right)^{2}}}{\sin c(\Delta \theta / 2)} \\
S_{L}^{\prime} & =\frac{\sqrt{\left(x_{L 1}-x_{L 0}\right)^{2}+\left(y_{L 1}-y_{L 0}\right)^{2}}}{\sin c(\Delta \theta / 2)}
\end{aligned}
$$

Aquí $d$ es la distancia nominal entre los puntos de apoyo de las ruedas de odometría. $\left\langle x_{R 0}, y_{R 0}\right\rangle$ y $\left\langle x_{L 0}, y_{L 0}\right\rangle$ son las coordenadas de los puntos de medición derecho e izquierdo respectivamente, medidas al inicio del movimiento. $\left\langle x_{R 1}, y_{R 1}\right\rangle$ y $\left\langle x_{L 1}, y_{L 1}\right\rangle$ son las coordenadas de los puntos de medición derecho e izquierdo respectivamente, medidas al finalizar el movimiento(ver figura 3.11).

Finalmente, por vía de la aproximación rectilínea o por el método de las coordenadas absolutas, la sensibilidad odométrica se obtiene mediante el cociente, 


$$
\left\{\begin{array}{l}
C_{R}=\frac{S_{R}}{\Delta N_{R}} \\
C_{L}=\frac{S_{L}}{\Delta N_{L}}
\end{array}\right.
$$

donde $\Delta N_{R}$ y $\Delta N_{L}$ corresponden al número de pulsos registrados por los codificadores rotativos a través de los trayectos $S_{R}$ y $S_{L}$ respectivamente.

\subsubsection{Determinación de la base de giro de un robot diferencial me- diante el Método Directo}

En un robot diferencial, la distancia entre los dos puntos de contacto de las ruedas de odometría con el plano de desplazamiento, se conoce como base de giro $B$. Las ecuaciones cinemáticas para la estimación de la posición mediante odometría, se basan en el caso ideal donde las ruedas se apoyan sólo en su punto de tangencia con el ya mencionado plano de desplazamiento. Sin embargo debido a que normalmente lo que las ruedas ofrecen es un área de contacto, entonces la distancia entre los puntos de apoyo suele variar ligeramente dependiendo de factores como el desbalance dinámico a causa de movimiento giratorios, a la deformación elástica que sufren las ruedas por el peso muerto sobre el robot, etc. De aquí que sea necesario determinar el valor práctico de esta distancia.

El carácter diverso de los factores que afectan la base de giro, sugieren que la misma puede modelarse como

$$
B^{\text {efectiva }}=\left(1+\alpha_{B}\right) B^{\text {nominal }}+\varepsilon_{B}
$$

$B$ es la distancia entre los puntos de apoyo de la rueda derecha e izquierda.

$\alpha_{B}$ es un factor de corrección debido a imperfecciones del hardware.

$\varepsilon_{B}$ es el componente aleatorio debido sobre todo al desbalance de carga y a la deformación elástica.

Si se asume que los efectos aleatorios se distribuyen de manera normal con media cero y varianza $\sigma_{B}^{2}$, entonces la base de giro también se distribuirá de manera normal según,

$$
B^{\text {efectiva }} \sim N\left(\bar{B}, \sigma_{B}^{2}\right)
$$

donde $\bar{B}$ es la media y $\sigma_{B}^{2}$ su varianza.

Distintos que otros métodos que proponen trayectorias de excitación en la cual está acoplado el efecto de la incertidumbre en la base de giro y la incertidumbre en el diámetro de las ruedas[32, 201], aquí se propone un método que minimiza este efecto ya que se trata de una trayectoria de excitación estrictamente angular, la cual se muestra en la figura 3.12. En la mencionada figura se muestra el lugar geométrico que describen los puntos de apoyo y el centro del robot cuando el móvil se hace girar 

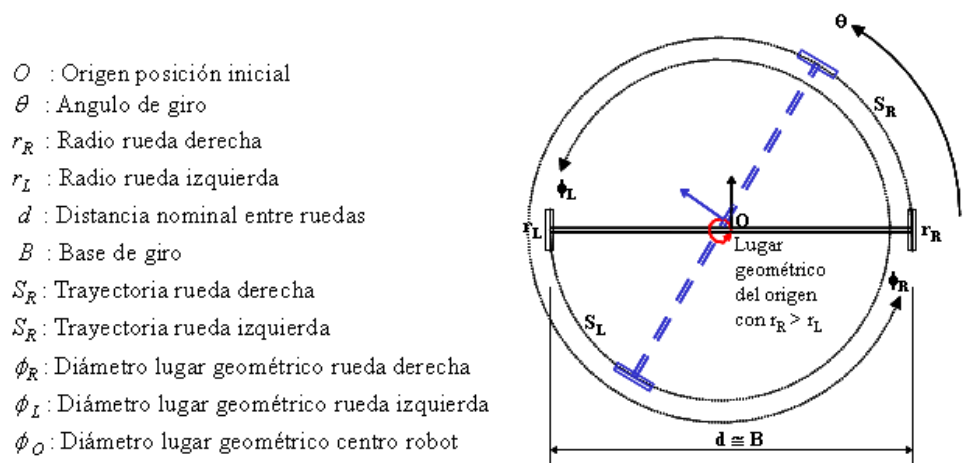

Figura 3.12: Lugar geométrico puntos de apoyo de la base de giro en móvil diferencial.

sobre su mismo eje a velocidad constante y con diámetros de ruedas distintos entre si. En el caso de la figura anterior, $-V_{L}=V_{R}$ y $r_{R}>r_{L}$.

Un primer detalle que se observa es que el centro robot describe una circunferencia cuyo diámetro está relacionado a su vez con los diámetros de los lugares $S_{R}$ y $S_{L}$ mediante la siguiente fórmula,

$$
\phi_{\text {origen }}=\frac{\phi_{R}-\phi_{L}}{2}
$$

Donde $\phi_{R}$ y $\phi_{L}$ son los diámetros de la circunferencia descrita por el punto de contacto de la rueda derecha e izquierda respectivamente. De lo anterior, y después de algunas operaciones, se puede deducir que la base de giro $B$ es el promedio de los diámetros de los lugares geométricos $S_{R}$ y $S_{L}$.

$$
B=\frac{\phi_{R}+\phi_{L}}{2}
$$

Un segundo detalle es que a pesar de que el centro robot hace un giro excéntrico, los puntos de apoyo mantienen la misma trayectoria consistentemente ciclo tras ciclo. Esto permite afirmar que al término de un giro de $360^{\circ}$, el centro robot y los puntos de apoyo coincidirán con el punto del cual partieron originalmente. Esto es especialmente conveniente porque permitirá entonces determinar las coordenada $\langle x, y\rangle$ de los puntos de medición dentro de un marco de referencia local. Así, el procedimiento para determinar $S_{R}$ y $S_{L}$ se inicia programando el robot para que gire aproximadamente $360^{\circ}$ sobre su mismo eje, al tiempo que se registran los pulsos $\left(N_{R}, N_{L}\right)$ proveniente de los codificadores derecho e izquierdo respectivamente. Entonces, conociendo el ángulo de giro total $\theta$, se determinan los diámetros derecho e izquierdo de acuerdo a lo siguiente

$$
\left\{\begin{array}{l}
\phi_{R}=2 \frac{\bar{C}_{R} \Delta N_{R}}{\theta} \\
\phi_{L}=2 \frac{\bar{C}_{L} \Delta N_{L}}{\theta}
\end{array}\right.
$$




\section{CAPÍTULO 3. MODELADO CINEMÁTICO Y LOCALIZACIÓN ODOMÉTRICA DEL ROBOT DIFERENCIAL}

Donde $C_{R}$ y $C_{L}$ corresponden a la sensibilidad odométrica ya determinada en la ecuación 3.24, $\Delta N_{R}$ y $\Delta N_{L}$ corresponden al número de pulsos registrados por los codificadores rotativos a través de los trayectos $S_{R}$ y $S_{L}$ respectivamente, y $\theta$ es el giro total que se determina mediante la ecuación,

$$
\theta=2 \pi+\sin ^{-1}\left[\frac{\left(x_{L 1}-x_{R 1}\right)\left(y_{R 0}-y_{L 0}\right)+\left(y_{L 1}-y_{R 1}\right)\left(x_{L 0}-x_{R 0}\right)}{\left(d+m_{1}+m_{2}\right)}\right]
$$

Aquí $d$ es la distancia nominal entre los puntos de apoyo de las ruedas de odometría, $\left\langle x_{R 0}, y_{R 0}\right\rangle$ y $\left\langle x_{L 0}, y_{L 0}\right\rangle$ son las coordenadas de los puntos de medición derecho e izquierdo respectivamente, medidas al inicio del movimiento. $\left\langle x_{R 1}, y_{R 1}\right\rangle$ y $\left\langle x_{L 1}, y_{L 1}\right\rangle$ son las coordenadas de los puntos de medición derecho e izquierdo respectivamente, medidas al finalizar el movimiento. Igualmente $m_{1}$ y $m_{2}$ corresponden a la distancia que existe entre el punto de apoyo de la rueda derecha e izquierda respectivamente y el punto de medición de su correspondiente lado.

Finalmente, al sustituir las ecuaciones 3.30 y 3.29 en la ecuación 3.28, se obtiene la base de giro según

$$
B=\frac{\bar{C}_{R} \Delta N_{R}+\bar{C}_{L} \Delta N_{L}}{\theta}
$$

El método anterior es útil para determinar la distancia entre ruedas cuando se desconoce del todo la misma. Sin embargo el mismo no recoge los posibles efectos aleatorios que pueden surgir cuando el robot ejecuta un trayecto típico o más real. Una opción mas general consiste en excitar al robot con una trayectoria aleatoria que recoja los factores dinámicos no modelados, como el efecto de los derrapes, como los desbalances de carga durante el giro, o como las no linealidades en los acoplamientos y engranajes del conjunto motor-ruedas de tracción.

Para desarrollar una metodología que contemple trayectos aleatorios es necesario considerar la cinemática del robot a través de la ecuación 3.5, donde se modela la evolución de la orientación del mismo mediante la ecuación

$$
\hat{\theta}_{k}=\hat{\theta}_{k-1}+\Delta \theta_{k}
$$

Expandiendo esta ecuación, se ve que la misma se relaciona con los parámetros odométricos de la siguiente forma,

$$
\hat{\theta}_{k}=\hat{\theta}_{k-1}+\frac{1}{B}\left[\bar{C}_{R}-\bar{C}_{L}\right]\left[\begin{array}{c}
\Delta N_{R} \\
\Delta N_{L}
\end{array}\right]
$$

Si el parámetro $B$ permanece constante, la orientación del robot en cualquier instante $k$ se puede estimar según,

$$
\hat{\theta}_{k}=\theta_{0}+\frac{1}{B} \sum_{i=1}^{k}\left(\bar{C}_{R}\left(\Delta N_{R}\right)_{i}-\bar{C}_{L}\left(\Delta N_{L}\right)_{i}\right)
$$

Ahora bien, el ángulo de orientación real del robot puede modelarse como 


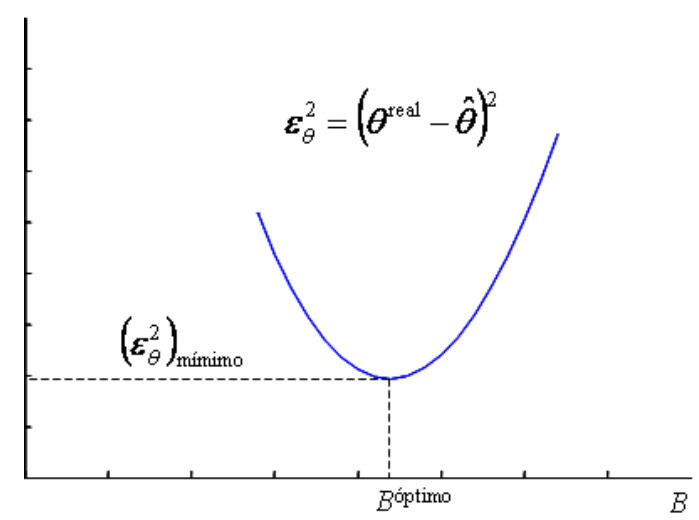

Figura 3.13: Forma de la función del error cuadrático del ángulo en función de la distancia entre ruedas $B$

$$
\theta_{k}^{\text {real }}=\hat{\theta}_{k}+\varepsilon_{\theta}
$$

donde $\varepsilon_{\theta}$ modela el ruido aleatorio que afecta al proceso de estimación del ángulo, y el cual se asume que se distribuye de forma normal con media cero y varianza $\sigma_{\theta}^{2}$. De acuerdo a lo anterior, la función del error se expresa según

$$
\varepsilon_{\theta}=\theta_{k}^{\text {real }}-\theta_{0}-\frac{1}{B} \sum_{i=1}^{k}\left(\bar{C}_{R}\left(\Delta N_{R}\right)_{i}-\bar{C}_{L}\left(\Delta N_{L}\right)_{i}\right)
$$

Una opción para determinar el parámetro $B$ óptimo, es estimar el valor que minimiza la función cuadrática del error. Esto es,

$$
B^{\text {óptimo }}=\arg \operatorname{mín} \varepsilon_{\theta}^{2}=\arg \operatorname{mín}\left(\theta_{k}^{\text {real }}-\theta_{0}-\frac{1}{B} \sum_{i=1}^{k}\left(\bar{C}_{R}\left(\Delta N_{R}\right)_{i}-\bar{C}_{L}\left(\Delta N_{L}\right)_{i}\right)\right)^{2}
$$

Dado la características de la función de error, una posibilidad para determinar $B$ óptimo es derivar la misma según,

$$
\begin{aligned}
\frac{\partial\left(\varepsilon_{\theta}^{2}\right)}{\partial B}= & 2\left(\theta_{k}^{\text {real }}-\theta_{0}-\frac{1}{B} \sum_{i=1}^{k}\left(\bar{C}_{R}\left(\Delta N_{R}\right)_{i}-\bar{C}_{L}\left(\Delta N_{L}\right)_{i}\right)\right) \\
& x\left(\frac{1}{B^{2}} \sum_{i=1}^{k}\left(\bar{C}_{R}\left(\Delta N_{R}\right)_{i}-\bar{C}_{L}\left(\Delta N_{L}\right)_{i}\right)\right)
\end{aligned}
$$

Descartando toda solución trivial, se obtiene que una solución para $B$ óptimo es

$$
B^{\text {óptimo }}=\frac{\sum_{i=1}^{k}\left(\bar{C}_{R}\left(\Delta N_{R}\right)_{i}-\bar{C}_{L}\left(\Delta N_{L}\right)_{i}\right)}{\theta_{k}^{\text {real }}-\theta_{0}}
$$




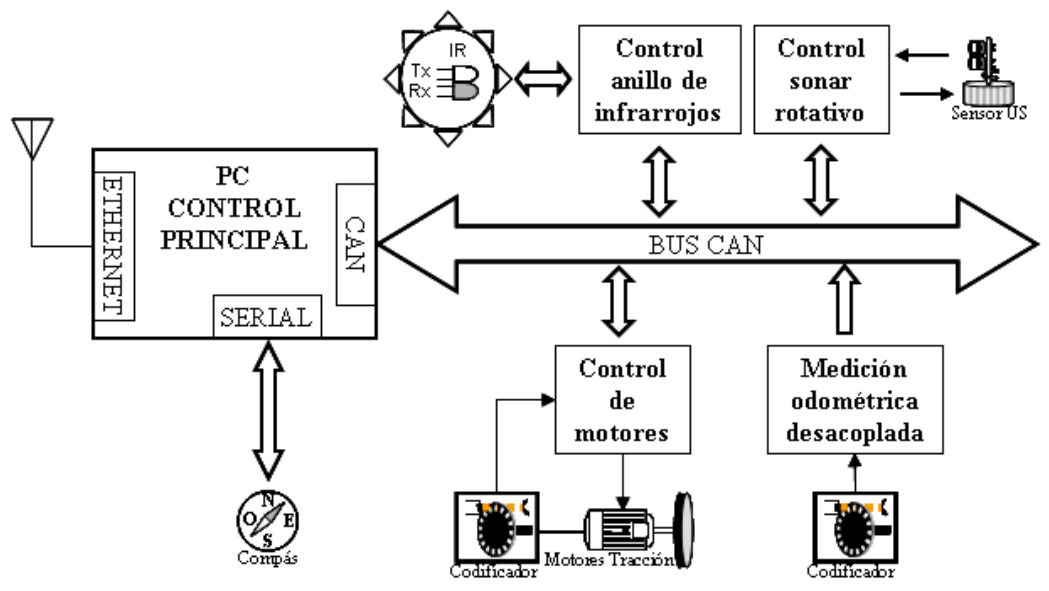

Figura 3.14: Arquitectura de distribución multi-sensorial del robot YAIR

Con este último resultado queda claro que la estrategia propuesta requiere de la medición de la orientación inicial y la final del robot (i.e. mediante un compás), así como también requiere de que se estimen los desplazamientos diferenciales de las ruedas de odometría en cada instante de muestreo a través del trayecto de excitación.

\subsubsection{Resultados experimentales en el robot YAIR}

\subsubsection{Identificación y calibración de parámetros del modelo cinemático}

El robot YAIR es un prototipo multisensorial desarrollado como banco de pruebas para la investigación de varios problemas relacionados con los sistemas distribuidos de tiempo real[25]. El bloque motor de este robot esta formado básicamente por un par de motores DC controlados por sendos integrados HCTL1100 de Hewlett Packard, y por una tarjeta microprocesada que sirve como interfase de comando y medición con el procesador principal. Las ruedas motrices del YAIR tienen un diámetro nominal de 160 milímetros y una separación entre ellas de 363 milímetros. Igualmente, estas ruedas motrices cuentan con un codificador rotativo acoplado al eje de cada motor, con el cual se suministra al sistema la información odométrica necesaria para tareas de control de cada uno de los motores. Al mismo tiempo, esta información odométrica se aprovecha para el sistema básico de estimación del estado del robot. Además de estos sensores, YAIR también cuenta con un sensor de distancias basados en un SONAR rotativo, un sensor de distancia tipo anillo basado en tecnología de infrarrojos, un compás electrónico, y un sensor odométrico independiente de la parte motriz, el cual esta basado en un par de codificadores rotativos acoplados a los lados del cuerpo del robot y que están alineados con el eje motor del mismo. Según como se puede ver en la figura 3.14, los sistemas sensoriales de YAIR están interconectados mediante un bus CAN (Controller Area Network).

Para validar los procedimientos aquí descritos, se realizaron pruebas experimentales en un escenario interior cuyo plano de desplazamiento fue adecuadamente de- 

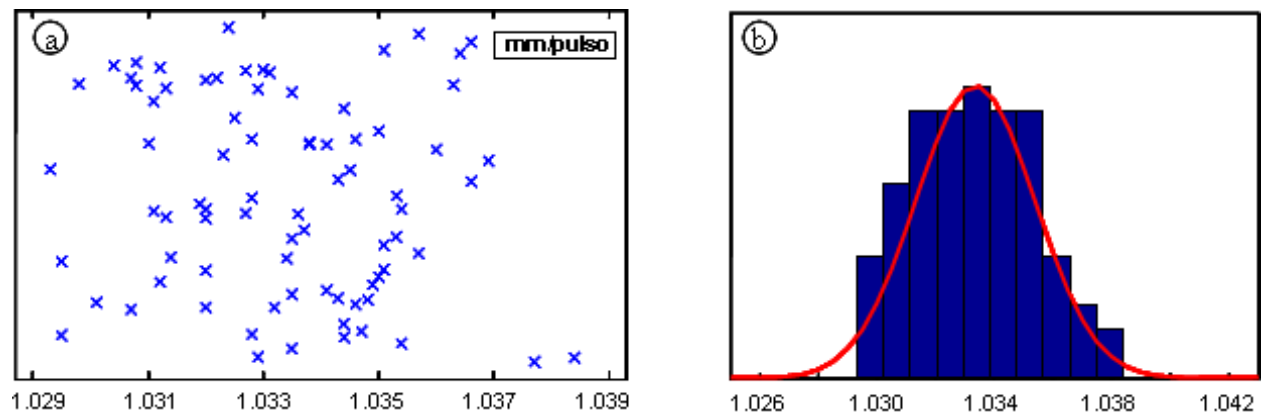

Figura 3.15: Sensibilidad odométrica rueda izquierda. a) Datos obtenidos experimentalmente. b) Histograma y distribución probabilística aproximada.

Tabla 3.1: Resumen estadístico sensibilidad odométrica de la rueda izquierda del robot YAIR

\begin{tabular}{|c|c|}
\hline Frecuencia & 79 \\
\hline Media & 1.0334 \\
\hline Varianza & $4,13 \times 10^{-6}$ \\
\hline Desv. Típica & $2,03 \times 10^{-3}$ \\
\hline Mínimo & 1.0293 \\
\hline Máximo & 1.0384 \\
\hline Rango & 0.0091 \\
\hline Asimetría & 0.2420 \\
\hline Curtosis & -0.8991 \\
\hline
\end{tabular}

marcado para constituir así un plano cartesiano donde el robot pudiera moverse. De esta forma, para cualquier posición que adoptará el robot dentro de este plano de trabajo, se pudo obtener las coordenadas de los puntos de medición situados a ambos lados del eje odométrico-motor del robot YAIR. Para la medición de la sensibilidad odométrica, se programaron trayectos rectilíneos de 500 milímetros, los cuales fueron ejecutados indistintamente en la dirección de avance o en la dirección de retroceso.

La figura 3.15 muestra la distribución y la dispersión de los datos de sensibilidad odométrica de la rueda izquierda de YAIR; mientras que la tabla 3.1 muestra los principales estadísticos correspondiente a los datos de esta sensibilidad. Entre ellos, la asimetría tipificada junto a la curtosis tipificada muestran que los datos se distribuyen de manera normal.

En la tabla 3.1 se aprecia que el valor más probable para $C_{L}$ es 1.0334 , por lo que considerando este como el valor real de la sensibilidad odométrica, entonces el valor para el factor de corrección $\alpha$ es igual a 0.0281. También, dado que las evidencias anteriores muestran que los datos para la sensibilidad odométrica se distribuyen de manera normal, entonces este parámetro puede modelarse como,

$$
C_{L}^{\text {efectiva }} \sim N\left(1,0334,4,13 \times 10^{-6}\right)
$$



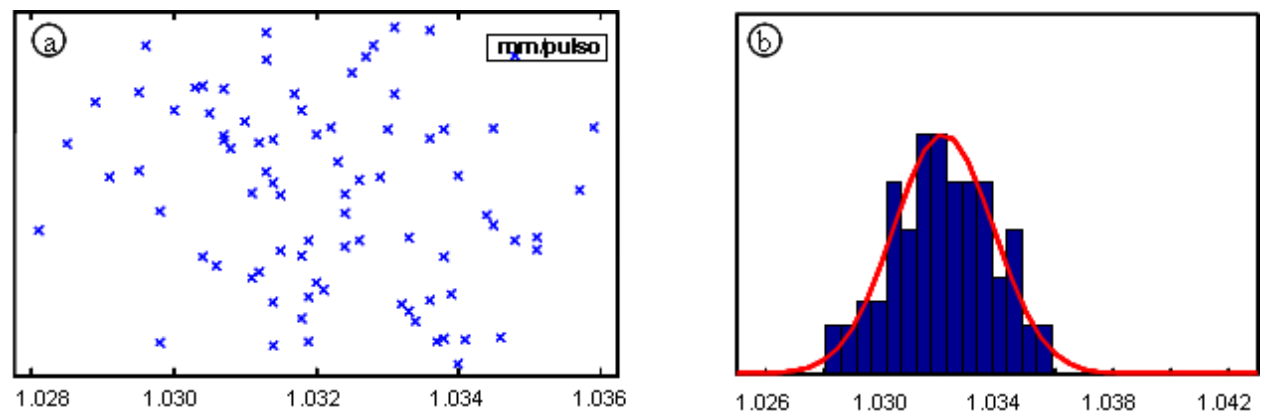

Figura 3.16: Sensibilidad odométrica rueda derecha. a) Datos obtenidos experimentalmente. b) Histograma y distribución probabilística aproximada.

Tabla 3.2: Resumen estadístico sensibilidad odométrica de la rueda derecha del robot YAIR

\begin{tabular}{|c|c|}
\hline Frecuencia & 82 \\
\hline Media & 1.0322 \\
\hline Varianza & $2,97 \times 10^{-6}$ \\
\hline Desv. Típica & $1,72 \times 10^{-3}$ \\
\hline Mínimo & 1.0281 \\
\hline Máximo & 1.0359 \\
\hline Rango & 0.0078 \\
\hline Asimetría & -0.1426 \\
\hline Curtosis & -0.8436 \\
\hline
\end{tabular}

La figura 3.16 muestra la distribución y la dispersión de los datos de sensibilidad odométrica de la rueda derecha de YAIR; mientras que la tabla 3.16 muestra los principales estadísticos correspondiente a los datos de esta sensibilidad. Entre ellos, la asimetría tipificada junto a la curtosis tipificada muestran que los datos se distribuyen de manera normal.

En la tabla 3.2 se aprecia que el valor más probable para $C_{R}$ es 1.0322 , por lo que considerando este como el valor real de la sensibilidad odométrica, entonces el valor para el factor de corrección $\alpha$ es igual a 0.0269. También, dado que las evidencias anteriores muestran que los datos para la sensibilidad odométrica se distribuyen de manera normal, entonces este parámetro puede modelarse como,

$$
C_{R}^{\text {efectiva }} \sim N\left(1,0322,2,97 \times 10^{-6}\right)
$$

Para la determinación de la base de giro se programó el robot para que ejecutase un conjunto de trayectos arbitrarios. En estos trayectos se midió la orientación real del robot al principio y al final del movimiento, lo que permitió determinar la base de giro óptima basándose en el procedimiento descrito en la subsección 3.3.2. La figura 3.17 muestra la distribución y la dispersión de los datos de la base de giro óptima del robot 

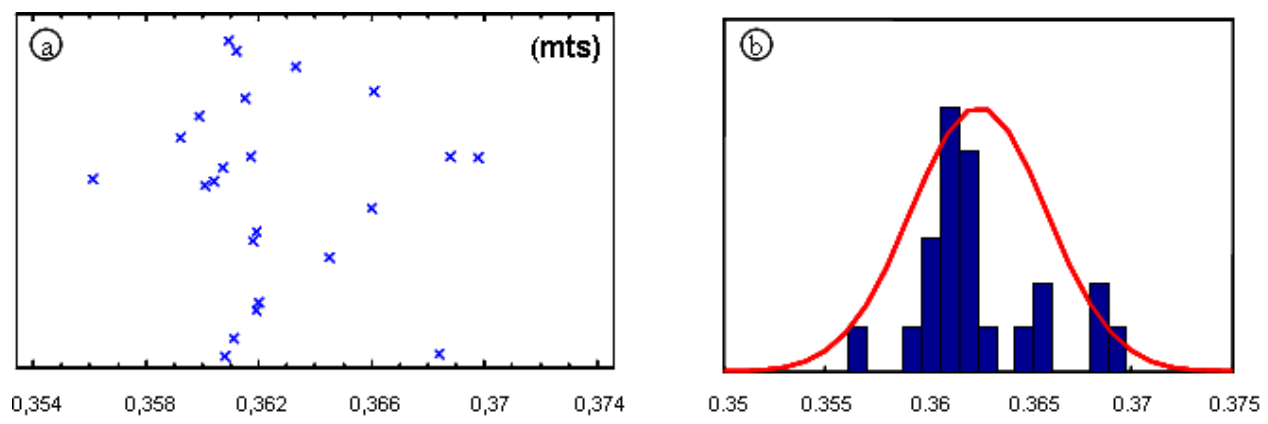

Figura 3.17: Distancia entre ruedas. a) Datos obtenidos experimentalmente. b) Histograma y distribución probabilística aproximada..

Tabla 3.3: Resumen estadístico base de giro óptima del robot YAIR

\begin{tabular}{|c|c|}
\hline Frecuencia & 23 \\
\hline Media & 0.3625 \\
\hline Varianza & $11 \times 10^{-6}$ \\
\hline Desv. Típica & $3,32 \times 10^{-3}$ \\
\hline Mínimo & 0.3561 \\
\hline Máximo & 0.3698 \\
\hline Rango & 0.0137 \\
\hline Asimetría & 1.5046 \\
\hline Curtosis & 0.4051 \\
\hline
\end{tabular}

YAIR así calculada; mientras que la tabla 3.3 muestra los principales estadísticos correspondiente a los datos de esta base de giro. Entre ellos, la asimetría tipificada junto a la curtosis tipificada muestran que los datos se distribuyen de manera normal.

En la tabla 3.3 se aprecia que el valor más probable para $B$ es $0.3625 \mathrm{mts}$, por lo que considerando este como el valor real de la base de giro, entonces el valor para el factor de corrección $\alpha$ es igual a 0.0005. También, dado que las evidencias anteriores muestran que los datos para la base de giro óptima se distribuyen de manera normal, entonces este parámetro puede modelarse como,

$$
B^{\text {óptima }} \sim N\left(0,3625,11 \times 10^{-6}\right)
$$

Como experimento de contraste se ejecutó la técnica de calibración propuesta por Borenstein y Feng conocida como UMBmark(University of Michigan Benchmark). Este experimento requiere que el robot repita varias veces un trayecto en forma de cuadrilátero cuyos lados son de longitud L, y que al mismo tiempo estime su

posición final usando para ello los parámetros cinemáticos nominales y la información provista por los sensores de odometría. En este caso, se programó al robot para que ejecutara trayectos cuadrados de 4 metros por lado.

La tabla 3.4 muestra la comparación de los valores nominales con los resulta- 
Tabla 3.4: Comparación de resultados para los parámetros cinemáticos de YAIR obtenidos por el método directo y por el UMBmark.

\begin{tabular}{cccccc}
\hline YAIR & Valores & \multicolumn{2}{c}{ Método directo } & \multicolumn{2}{c}{ UMBmark } \\
& nominales & Result. & $\sigma$ & Result. & $\sigma$ \\
\hline$C_{R}(\mathrm{~mm} /$ pulso $)$ & 1.0 & 1.0322 & 0.0017 & 0.9990 & - \\
$C_{L}(\mathrm{~mm} / \mathrm{pulso})$ & 1.0 & 1.0334 & 0.0020 & 1.0010 & - \\
$B(\mathrm{~mm})$ & 363.0 & 362.5 & 3.3 & 360 & - \\
\hline
\end{tabular}

dos obtenidos mediante el método de calibración directa aquí propuesto, y con los resultados obtenidos mediante el método del UMBmark.

A pesar de que los resultados de sensibilidad odométrica arrojados por el UMBmark están más cercanos a los valores nominales, los mismos distan de los valores reales para estos parámetros. Para comprobar estos resultados se programó al YAIR para que siguiera un trayecto de aproximadamente 40 metros de longitud que incluía 8 giros sobre su mismo eje de aproximadamente 90 grados cada uno. Cada 0.5 metros se midió la posición real del robot, así como también se registró la posición estimada mediante los parámetros calibrados con el método directo aquí propuesto, y con el método UMBmark.

Los resultados mostrados en la figura 3.18 evidencian una notable mejora de la exactitud del estimado de la posición del sistema calibrado mediante el método directo respecto al sistema calibrado mediante el método UMBmark. Igualmente también se muestra la capacidad del robot de navegar en interiores manteniendo un buen seguimiento de su posición real.

\subsubsection{Resultados para la localización mediante sensores de odometría}

El modelo de estimación del estado o de localización odométrica del robot YAIR puede ser expresado según,

$$
\left[\begin{array}{l}
x_{k} \\
y_{k} \\
\theta_{k}
\end{array}\right]=\left[\begin{array}{l}
x_{k-1} \\
y_{k-1} \\
\theta_{k-1}
\end{array}\right]+\left[\begin{array}{c}
\Delta S_{k} \cos \left(\theta_{k-1}+\Delta \theta_{k} / 2\right) \\
\Delta S_{k} \sin \left(\theta_{k-1}+\Delta \theta_{k} / 2\right) \\
\Delta \theta_{k}
\end{array}\right]
$$

con

$$
\begin{aligned}
& \Delta S_{k}=\frac{1}{2}\left(\bar{C}_{R} \Delta N_{k, R}+\bar{C}_{L} \Delta N_{k, L}\right) \\
& \Delta \theta_{k}=\frac{1}{B}\left(\bar{C}_{R} \Delta N_{k, R}-\bar{C}_{L} \Delta N_{k, L}\right)
\end{aligned}
$$

donde $\Delta N_{k, R}$ y $\Delta N_{k, L}$ corresponden a la cantidad de pulsos de codificador que se reciben en el intervalo de tiempo comprendido entre $(k-1)$ y $k$, en los sensores izquierdo y derecho respectivamente. 


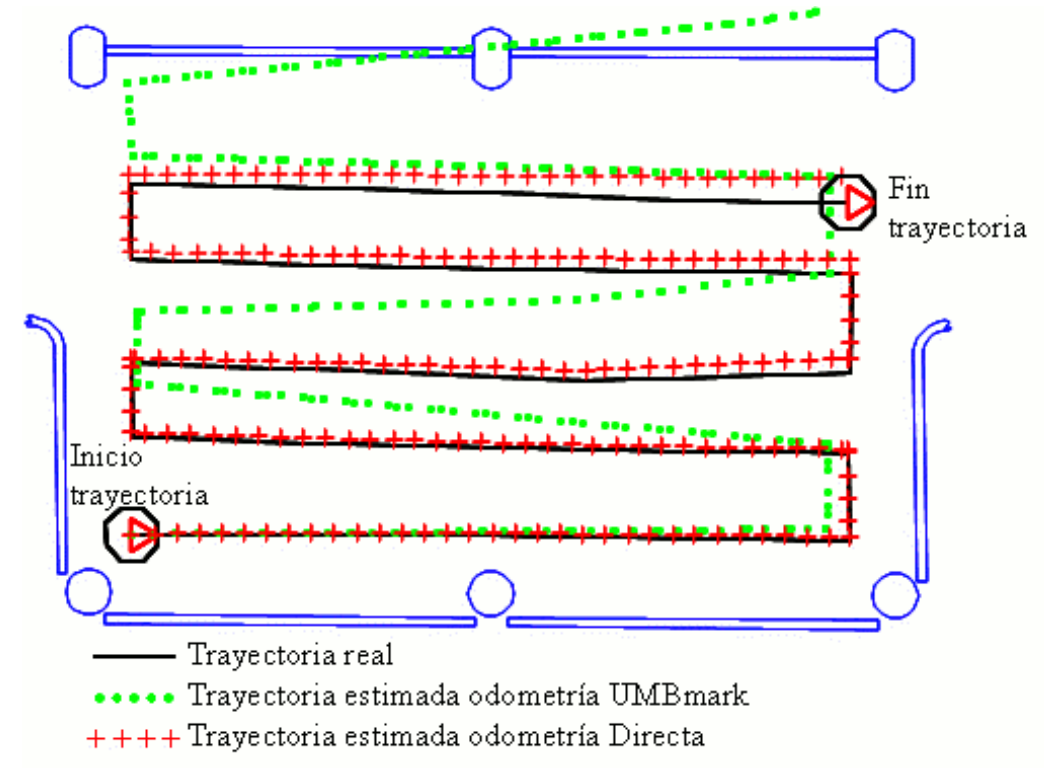

Figura 3.18: YAIR: comparación de la localización odométrica estimada con dos conjuntos de parámetros distintos.

Para evaluar el desempeño del sistema de localización odométrica, se introdujo el conjunto de ecuaciones (3.43), (3.44) y (3.45) en el software operativo del robot YAIR, y se programó al mismo para que ejecutase trayectos aleatorios con longitud total aproximada a 4 metros. El proceso se repitió 25 veces y se midió la posición real del robot al final de cada trayecto, para compararla con la estimada por el robot mediante su modelo cinemático directo. En la tabla (3.5) se muestra los valores de error resultantes, así como los estadísticos que lo describen.

Observando los estadísticos que describen los datos, y en particular a la asimetría y a la curtosis tipificada, se puede determinar que el error tabulado proviene de una distribución normal. De esta manera, el error de localización puede ser tratado como una distribución Gausiana bivariante en un espacio cartesiano, la cual tiene la forma

$$
\varepsilon_{x y}=\frac{1}{2 \pi \sqrt{\operatorname{det}\left[\begin{array}{cc}
\sigma_{x}^{2} & \sigma_{x y} \\
\sigma_{x y} & \sigma_{y}^{2}
\end{array}\right]}} e^{-\frac{1}{2}\left[\begin{array}{c}
x-\bar{x} \\
y-\bar{y}
\end{array}\right]^{\mathrm{T}}\left[\begin{array}{cc}
\sigma_{x}^{2} & \sigma_{x y} \\
\sigma_{x y} & \sigma_{y}^{2}
\end{array}\right]^{-1}\left[\begin{array}{c}
x-\bar{x} \\
y-\bar{y}
\end{array}\right]}
$$

En la figura 3.19 se muestra la dispersión del error de localización, así como la elipse de concentración para un nivel de confianza de $95 \%$. 
Tabla 3.5: Estadísticos de error de estimación odométrica de la posición del robot YAIR

\begin{tabular}{cccc}
\hline \multicolumn{4}{c}{ Error (Estado estimado - Estado real) } \\
\hline Num & Error $x(\mathrm{~mm})$ & Error $y(\mathrm{~mm})$ & Error $\theta$ (grados) \\
\hline 1 & -7 & 2 & 0.35 \\
2 & 3 & -5 & -0.58 \\
3 & 21 & 8 & -1.28 \\
4 & -11 & -3 & 0.54 \\
5 & -8 & -18 & -0.69 \\
6 & -8 & 1 & 0.75 \\
7 & -8 & 0 & 1.41 \\
8 & -14 & -3 & 0.18 \\
9 & -20 & 1 & -0.88 \\
10 & -3 & -15 & -0.66 \\
11 & 6 & -10 & -1.68 \\
12 & 12 & -16 & -1.02 \\
13 & 35 & 9 & -1.11 \\
14 & 7 & -2 & 2.30 \\
15 & -25 & -29 & 1.31 \\
16 & 1 & -13 & -0.58 \\
17 & 3 & 1 & 0.60 \\
18 & 8 & 10 & 1.31 \\
19 & 15 & 15 & 1.13 \\
20 & 0 & -4 & 0.31 \\
21 & -9 & 2 & 0.60 \\
22 & 2 & -3 & -1.12 \\
23 & -6 & -10 & 0.76 \\
24 & -6 & 15 & -1.10 \\
25 & 2 & -5 & -0.47 \\
\hline & & & \\
Varianza & 165 & 109 & 0.02 \\
Resv. típica & 12.8 & 10.4 & 1.09 \\
Mínimo & -25 & -29 & -1.68 \\
Máximo & 35 & 15 & 2.30 \\
Rango & 60 & 44 & 3.98 \\
\hline Errosis & 1.42 & -0.83 & 0.60 \\
\hline & & 0.45 & -0.84 \\
\hline & & &
\end{tabular}




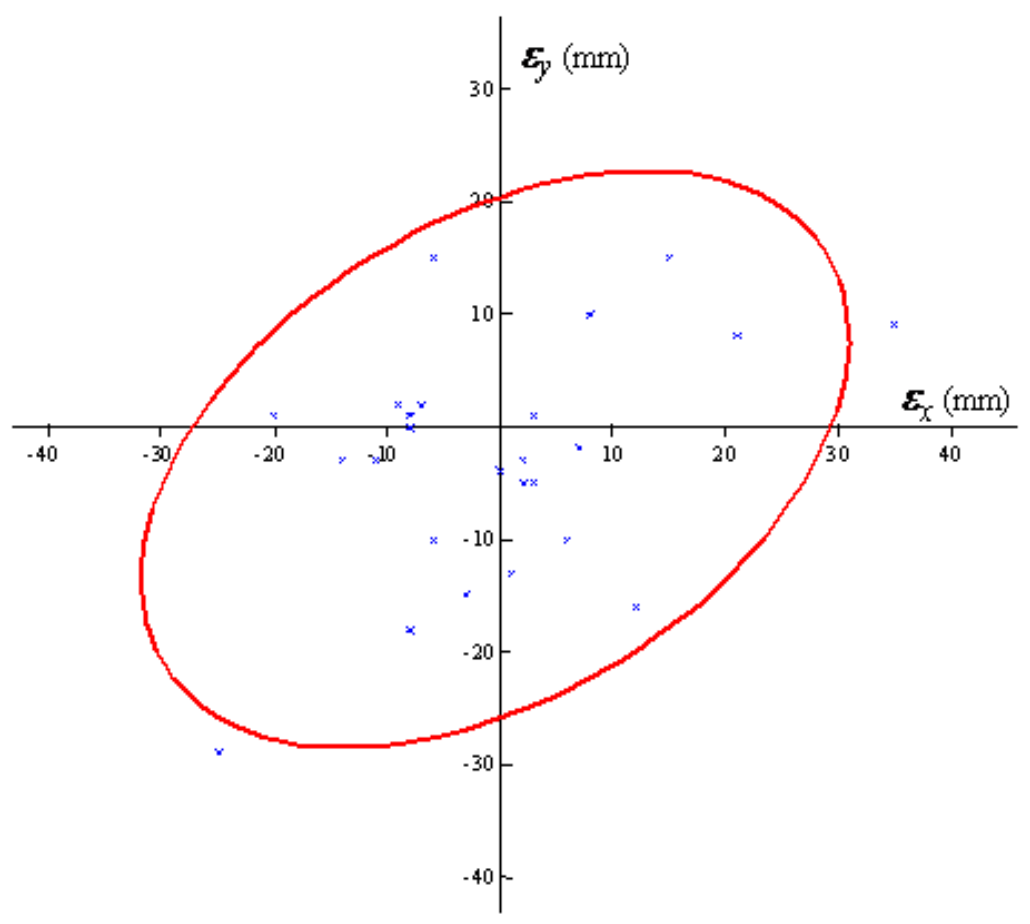

Figura 3.19: Elipse de concentración del error de localización mediante odometria al $95 \%$ de confianza. 


\subsection{Un método automático para la identificación y ca- libración multisensorial directa de la cinemática de un robot diferencial.}

La acumulación de errores es la mayor desventaja del sistema de localización mediante odometría. La calibración ocasional del sistema de odometría incrementa la exactitud y reduce los costos operacionales ya que se requieren menos actualizaciones de la posición absoluta del robot. Son pocos los trabajos científicos orientados a la cuantificación de los errores de odometría. Un método de calibración simple consiste en la medición directa de los parámetros del modelo cinemático del móvil. Este método consiste en medir los parámetros cinemáticos del robot (diámetros de ruedas y distancias entre ellas) de manera directa; o también en determinar las relaciones de conversión cinemática (por ejemplo $\mathrm{mm} /$ pulsos, etc.) a través de ensayos especiales. Goel et al[84] usan un procedimiento de calibración bastante simple para compensar los errores sistemáticos. El método usado sólo requiere de un tacómetro de precisión el cual se usa para medir la velocidad real en las ruedas motrices. Ellos trabajaron sobre un robot diferencial Pioneer AT, en el cual midieron la velocidad real en las ruedas motrices al tiempo que medían la velocidad registrada por los codificadores. De esta manera encontraron la relación efectiva entre la velocidad indicada por los codificadores y la velocidad real en las ruedas. Debido a que cuando el robot cambia de dirección las ruedas no se apoyan en su respectivos centros, la longitud de la base de giro efectiva no se corresponde al de las especificaciones nominales. Por esta razón estos autores aplicaron un método ad hoc (el cual no describen) para medir la longitud efectiva de este eje de giro. Finalmente los factores así determinados fueron incorporados al modelo del sistema que a su vez es usado por un filtro de Kalman en la corrección de la localización del robot. En mi opinión, a este método falta complementarlo con ensayos de rodamiento real del robot, que por la naturaleza de las pruebas que ellos describen, parece que no ejecutaron.

La falta de procedimientos bien definidos para la cuantificación de los errores de odometría, llevan a los investigadores a seguir métodos ad hoc para la calibración de las distintas plataformas móviles. En general, esto último conlleva a la falta de compatibilidad al comparar los reportes sobre odometría de distintos autores. Con la idea de establecer un método sistemático para la medición y corrección de la odometría en robot diferenciales, Borenstein y Feng han desarrollado una técnica de calibración que llamaron UMBmark (University of Michigan Benchmark) que es una herramienta para la calibración de los errores sistemáticos que típicamente afectan a las plataformas móviles diferenciales[27]. Este método es usado con mucha frecuencia en la determinación de los errores en los parámetros cinemáticos de un robot diferencial, pero al mismo tiempo es relativamente difícil de realizar y es muy sensitivo a los errores no sistemáticos. El método requiere que el robot se mueva en ambos sentidos (CW - clockwise (CW) y CCW - counter-clockwise), siguiendo una trayectoria cuadrada de longitud igual a $4 \mathrm{~L}$. Durante los ensayos es necesario registrar la posición cartesiana absoluta $\langle x, y\rangle$ al final de cada trayecto realizado, al tiempo que 


\subsection{UN MÉTODO AUTOMÁTICO PARA LA IDENTIFICACIÓN Y CALIBRACIÓN MULTISENSORIAL DIRECTA DE LA CINEMÁTICA DE UN ROBOT DIFERENCIAL.}

se permite que el sistema de control del robot estime la localización del mismo mediante el modelo odométrico y los codificadores acoplados a las ruedas de odometría. Una desventaja que presenta el UMBmark es que requiere de un espacio de experimentación relativamente grande en comparación con los entornos interiores donde se realizan; y esto no siempre es posible. Este requerimiento de espacio aumenta cuando la diferencia entre los diámetros de las ruedas es significativo, ya que ello desviaría al robot de la trayectoria cuadrada perfecta (ver figura 3.26). Por último, el test no es adecuado para identificación automática de los parámetros cinemáticos del robot, ya que el algoritmo requiere que se le suministre el valor inicial de los parámetros buscados.

El método de Goel y el método de Borenstein requieren de un movimiento específicamente diseñado para excitar la contribución de los parámetros de la cinemática del robot. Sin embargo existen otras técnicas de calibración orientadas al procesamiento, que les basta con conocer un conjunto de valores del estado previo, y posterior a un movimiento arbitrario del robot, para entonces estimar los parámetros odométricos desconocidos. Dentro de las técnicas orientadas al procesamiento, Roy y Thrun sugieren un algoritmo que usa los sensores del robot para automáticamente calibrar el mismo durante su operación[176]. Los autores enfocan el problema como uno de estimación de máxima verosimilitud, donde proponen un estimador exponencial que determina el valor más probable de los parámetros a través de la comparación iterativa de pares de lecturas sensoriales. Una desventaja clara de esta propuesta es la necesidad de usar sensores especialmente construidos para tal fin. Igualmente, $A n$ tonelli et al desarrollan un método basado en la técnica de estimación de mínimos cuadrados[4]. Para ello reescriben las ecuaciones cinemáticas de un robot tipo uniciclo con la finalidad de representar el estado mediante un conjunto de ecuaciones lineales que dependen de los parámetros odométricos desconocidos. De esta forma, mediante una regresión por mínimos cuadrados los autores determinan los parámetros del modelo cinemático del robot. Pero al igual que la metodología de Roy, En esta metodología se propone el uso de sistemas de posicionamiento basados en cámaras de video, lo cual no siempre es posible.

Independientemente de cual sea el sistema o el método de calibración usado, es interesante que el mismo se pueda realizar de manera automática, y sobre el mismo escenario de trabajo del robot. Esto es necesario porque además que con el tiempo, los parámetros cinemáticos pueden cambiar ligeramente, también puede ocurrir que el robot cambie del área donde usualmente trabaja, y con ello cambien también las condiciones de acoplamiento de las ruedas sobre el suelo. En esta sección se desarrollan las ecuaciones y la metodología necesaria para la identificación y calibración automática de los parámetros cinemáticos de un robot diferencial dotado de sensores de bajo costo(sonar, compás, codificador rotativo). El método es sistemático, muy simple de realizar e insensitivo a los errores no sistemáticos ya que utiliza un mecanismo de filtrado estadístico para rechazar los valores atípicos u outliers. Además de lo anterior, el método propuesto utiliza trayectos que desacoplan el efecto de la incertidumbre en los diámetros de las ruedas, del efecto de la incertidumbre en la base de giro. 


\subsubsection{Técnica multisensorial de identificación y calibración auto- mática de la odometría en robot diferenciales}

Apoyándose en los sensores exteroceptivos de un robot móvil, este procedimiento usa la metodología basada en la medición directa para identificar y posteriormente calibrar los parámetros de la cinemática del robot. La idea es que un robot diferencial dotado de codificadores rotativos en cada una de sus ruedas motrices, puede auto determinar los parámetros de su modelo odométrico con la ayuda de un sensor de distancias y de un compás magnético electrónico. Este proceso se lleva a cabo en dos etapas: identificación y calibración. La identificación persigue determinar los parámetros aproximados de la sensibilidad odométrica $C_{R}$ y $C_{L}$ medidas en $\mathrm{mm} / \mathrm{pulso}$, así como también busca determinar un valor aproximado para la base de giro $B$. Por su parte, la calibración usa los resultados previos junto al modelo cinemático directo del robot, para determinar de forma más precisa los valores de los ya mencionados parámetros. La calibración se lleva a cabo a través de una serie de ensayos sistemáticos que incluyen el uso de técnicas de filtrado estadístico para eliminar los valores atípicos que puedan surgir en el proceso.

\subsubsection{Fundamentos geométricos del movimiento de un robot diferencial}

En el caso generalizado de una trayectoria curvilínea de un robot diferencial, como la mostrada en la figura 3.20, se pueden establecer la siguiente relación

$$
L=S \sin c(\Delta \theta / 2)
$$

Aquí $S$ es el arco que describe el centro del robot y $\Delta \theta$ es el diferencial de giro desde la posición inicial a la posición final.

Igualmente se puede ver que $\Delta L$ corresponde al diferencial de longitud desde la posición inicial a la posición final del robot, y se puede calcular como,

$$
\Delta L=L \cos (\Delta \theta / 2)
$$

Combinando las ecuaciones 3.47 y 3.48 , y resolviendo para $S$ se obtiene

$$
S=\frac{\Delta L \cdot \Delta \theta}{\sin (\Delta \theta)}
$$

Igualmente, definiendo $R$ como el radio de curvatura a velocidad constante, el mismo se relacionaría con el trayecto $S$ mediante el diferencial de giro $\Delta \theta$ según,

$$
R=\frac{\Delta L}{\sin (\Delta \theta)}
$$

Otro par de relaciones importantes que se sacan de la geometría del movimiento curvilíneo general son,

$$
\left\{\begin{array}{l}
S_{R}=(R-d / 2) \Delta \theta \\
S_{L}=(R+d / 2) \Delta \theta
\end{array}\right.
$$




\subsection{UN MÉTODO AUTOMÁTICO PARA LA IDENTIFICACIÓN Y \\ CALIBRACIÓN MULTISENSORIAL DIRECTA DE LA CINEMÁTICA DE UN ROBOT DIFERENCIAL.}

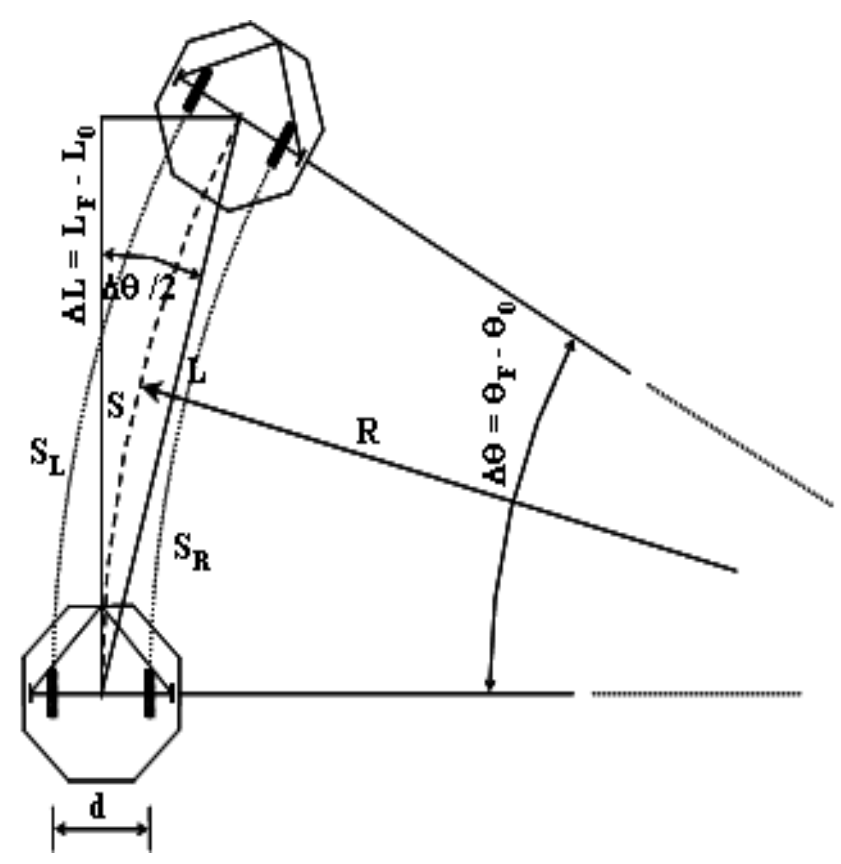

Figura 3.20: Relaciones geométricas en un trayecto curvilíneo generalizado de un robot diferencial

donde $S_{R}$ y $S_{L}$ corresponden a la longitud de arco recorrida por la rueda derecha e izquierda respectivamente, y $d$ es la distancia nominal entre los puntos de apoyo de las mencionadas ruedas.

Finalmente, sustituyendo 3.50 en 3.51 se resuelve el arco recorrido por cada rueda como

$$
\left\{\begin{array}{l}
S_{R}=\frac{\Delta L}{\sin c(\Delta \theta)}-\frac{d . \Delta \theta}{2} \\
S_{L}=\frac{\Delta L}{\sin c(\Delta \theta)}+\frac{d . \Delta \theta}{2}
\end{array}\right.
$$

\subsubsection{Esquema y procedimiento experimental del proceso de identifi- cación sensibilidad odométrica}

El proceso se inicia cuando el robot, sin conocimiento previo de sus parámetros odométricos, ni del entorno que lo rodea, identifica mediante sus sensores de distancia, una estructura que le servirá como referencia. Por ejemplo, en el caso del robot YAIR, el cual cuenta con un medidor rotativo de distancias por ultrasonidos, la referencia que se debe buscar es un buen reflector de señales ultrasónicas, como por ejemplo una pared. Una vez localizado el reflector de referencia, se realizan movimientos de rotación sobre su mismo eje para que el robot "encare" el reflector, es decir para $\operatorname{lograr} \mu_{\mathrm{x}} \approx \mu_{r}$, donde $\mu_{\mathrm{x}}$ es el vector unitario normal al eje motriz del robot y $\mu_{r}$ es el vector unitario de la superficie reflectora según se aprecia en la figura 3.21. La figura muestra la trayectoria típica de un robot diferencial cuando es comandado a 


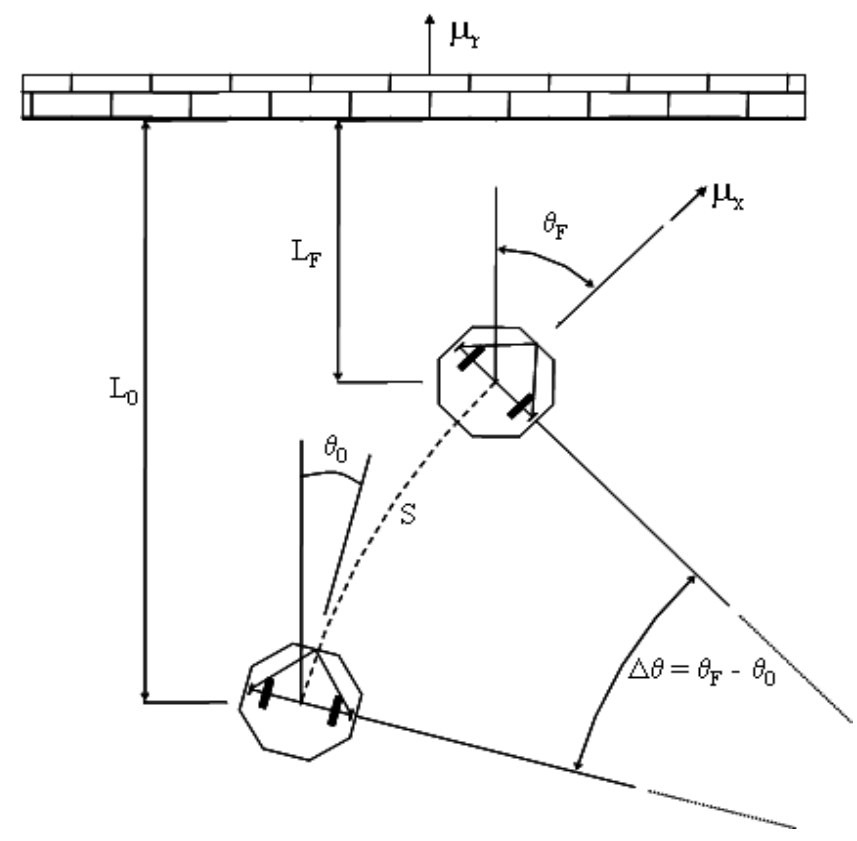

Figura 3.21: Esquema del ensayo y definición de variables en la auto-calibración de un robot diferencial. Para efectos de claridad, en esta figura se han exagerado tanto el ángulo inicial $\theta_{0}$ como la longitud y curvatura de la trayectoria S.

velocidad en ruedas constante.

En el proceso de identificación se obtienen mejores resultados si se condiciona a que $\mu_{\mathrm{x}} \approx \mu_{r}$, o sea, que el ángulo inicial $\theta_{0}$ tienda a cero. Igualmente los resultados mejoran si disminuye la curvatura de la trayectoria $S$. Ahora bien, si la velocidad en las ruedas motrices son iguales, el movimiento curvilíneo del centro $\operatorname{robot}\left\langle x_{\mathrm{c}}, y_{\mathrm{c}}\right\rangle$ a lo largo de $S$ se debe principalmente a la diferencia de diámetros entre las ruedas. Afortunadamente en general estos diámetros no resultan ser tan diferentes como para que se produzca curvaturas pronunciadas si longitud de trayectoria se mantiene acotada y corta. De ahí que para el proceso de identificación de los parámetros se recomienda que se realice un recorrido corto, pero que a la vez permita excitar el rango pleno de los codificadores rotativos de las ruedas de odometría. Por tanto, aquí estaríamos hablando de una longitud equivalente a $\pi D$, donde $D$ es el diámetro nominal de las ruedas de odometría.

Así, una vez completada la anterior etapa de alineación, para la determinación de la sensibilidad odométrica del modelo cinemático del robot, basta con que el mismo realice un movimiento rectilíneo cuya longitud aproximada corresponda a una revolución completa de sus ruedas odométricas. También, durante la ejecución del movimiento se deben registrar los pulsos de odometría provenientes de los codificadores acoplados a las ruedas odométricas, e igualmente determinar, mediante el medidor de distancias, a $L_{0}$ y $L_{F}$ que son las distancias desde el robot al reflector de referencia, 


\subsection{UN MÉTODO AUTOMÁTICO PARA LA IDENTIFICACIÓN Y CALIBRACIÓN MULTISENSORIAL DIRECTA DE LA CINEMÁTICA DE UN ROBOT DIFERENCIAL.}

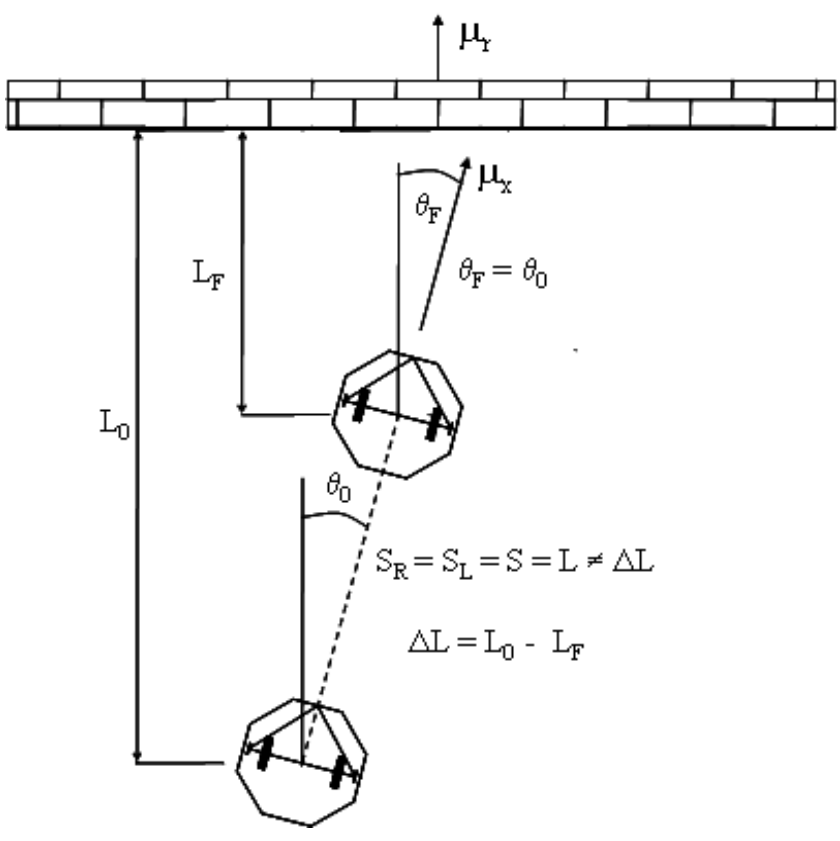

Figura 3.22: Error por desplazamiento sesgado al reflector de referencia

antes y después de ejecutar el movimiento, respectivamente.

Ahora bien, dada la naturaleza automática del proceso de identificación propuesto, el parámetro $d$ se supone desconocido. De aquí que en este proceso será necesario garantizar que el diferencial de giro $\Delta \theta$ sea lo suficientemente pequeño como para despreciar el segundo termino de la ecuación (3.52). Esto se logra haciendo el recorrido lo suficientemente corto como para minimizar $\Delta \theta$, y que al mismo tiempo se pueda registrar suficiente data confiable. Es decir, una distancia suficientemente grande en comparación con el error estándar del medidor de distancias, y que al mismo tiempo se registren suficientes pulsos de los codificadores como para estar también alejado del ruido promedio de los mismos.

Sin embargo, aun logrando la situación ideal donde $\Delta \theta=0$, la estimación inicial se vera afectada por un error importante si no se corrige el ángulo inicial con la que el robot esta viendo a su reflector de referencia. Este problema se ilustra en la figura 3.22 donde nuevamente se ha exagerado la longitud del recorrido con propósitos de claridad. De acuerdo a esta figura, el problema es que la longitud de trayecto que se puede medir mediante $\Delta L$, en general será menor que la longitud de trayecto real $L$. Este problema se agudiza a medida que el producto punto $\mu_{\mathrm{x}} \cdot \mu_{r}$ se aleja de la unidad. De aquí que sea deseable que los vectores $\mu_{\mathrm{x}} \mathrm{y} \mu_{r}$ se encuentren lo más alineado posible.

Para lograr la alineación de los vectores $\mu_{\mathrm{x}} \mathrm{y} \mu_{r}$ hay que contar con las bondades que pueden ofrecer los distintos medidores de distancia. Este problema no se presenta en caso de que el medidor de distancias este basado en tecnología láser, pero en este 


\section{CAPÍTULO 3. MODELADO CINEMÁTICO Y LOCALIZACIÓN ODOMÉTRICA DEL ROBOT DIFERENCIAL}

trabajo no se considera su posibilidad de uso debido a limitaciones de costo. No obstante, en el caso del robot YAIR es sencillo lograr la alineación de $\mu_{\mathrm{x}} \mathrm{y} \mu_{r}$, ya que el mismo cuenta con un sensor de ultrasonido tipo rotativo, y que además tienen la capacidad de medir la amplitud del eco ultrasónico[167]. De aquí que basta con buscar la orientación del máximo eco para maximizar el producto punto $\mu_{\mathrm{x}} \cdot \mu_{r}$. Esta metodología es fácilmente extensible al uso de sensores de infrarrojos, siempre y cuando los mismos midan la distancia a través del modelo fotométrico del sensor[17], ya que se obtendría la máxima amplitud de la señal(mínima distancia), cuando el sensor este enfocando el reflector de forma perpendicular.

Si se logran establecer las condiciones anteriores, la estimación inicial de la sensibilidad pulso-métrica de cada rueda es directa, ya que la ecuación 3.52 se simplifica según,

$$
\lim _{\Delta \theta \rightarrow 0}\left[\begin{array}{c}
S_{R}=\frac{\Delta L}{\sin c(\Delta \theta)}-\frac{d . \Delta \theta}{2} \\
S_{L}=\frac{\Delta L}{\sin c(\Delta \theta)}+\frac{d . \Delta \theta}{2}
\end{array}\right]=\left[\begin{array}{c}
S_{R}=\Delta L \\
S_{L}=\Delta L
\end{array}\right]
$$

Entonces, para determinar las sensibilidades aproximadas en las ruedas $\left[\tilde{C}_{R}, \tilde{C}_{L}\right]$ basta con registrar los pulsos de los codificadores derecho e izquierdo $\left[N_{R}, N_{L}\right]$ y aplicar las siguientes ecuaciones:

$$
\left\{\begin{array}{l}
\tilde{C}_{R}=\frac{\Delta L}{N_{R}} \\
\tilde{C}_{L}=\frac{\Delta L}{N_{L}}
\end{array} \quad \text { unidades de longitud } /\right. \text { pulso }
$$

\subsubsection{Esquema y procedimiento experimental del proceso de identifi- cación de la base de giro}

Ya se mostró en la sección 3.3.2 que la base de giro puede determinarse mediante la ecuación 3.31, la cual se muestra a continuación.

$$
B=\frac{\bar{C}_{R} \Delta N_{R}+\bar{C}_{L} \Delta N_{L}}{\theta}
$$

También se mostró en la sección (3.3.2) que un robot diferencial que gira sobre su mismo eje a velocidad en ruedas constante, mantiene una trayectoria monótona ciclo tras ciclo aunque posea diámetros de ruedas distintos. Esto permite afirmar que al término de un giro de $360^{\circ}$, el centro robot y los puntos de apoyo coincidirán con el punto del cual partieron originalmente. Esto es especialmente conveniente ya que de esta manera se puede utilizar un compás como elemento sensor para determinar cuando el robot a completado una vuelta. En este sentido no hará falta hacer ningún tipo de compensación ni calibración sobre el compás ya que debido a la buena precisión que el mismo ofrece, con mucha garantía indicará la misma lectura que inicialmente registró en ese mismo punto.

Así, el procedimiento para determinar $B$ se inicia programando el robot para que gire aproximadamente $360^{\circ}$ sobre su mismo eje, al tiempo que se registran los pulsos de los codificadores derecho e izquierdo $\left[N_{R}, N_{L}\right]$. Entonces, conociendo el ángulo de 


\subsection{UN MÉTODO AUTOMÁTICO PARA LA IDENTIFICACIÓN Y CALIBRACIÓN MULTISENSORIAL DIRECTA DE LA CINEMÁTICA DE UN ROBOT DIFERENCIAL.}

giro total $\theta_{\text {c }}$ proporcionado por el compás, y la sensibilidad odométrica aproximada

$\left[\tilde{C}_{R}, \tilde{C}_{L}\right]$ determinada en la sección anterior, se puede determinar la base de giro aproximada de acuerdo a

$$
\tilde{B}=\frac{\tilde{C}_{R} N_{R}+\tilde{C}_{L} N_{L}}{\theta_{\mathrm{c}}}
$$

\subsubsection{Procedimiento experimental del proceso de calibración}

El proceso de identificación esta sujeto a la condición de que el producto punto entre el vector director del robot y el vector del plano del reflector de referencia sea aproximadamente igual a $1\left(\mu_{\mathrm{x}} \cdot \mu_{r} \approx 1\right)$. Esto es necesario ya que al inicio del proceso de identificación se desconocen los valores nominales de los parámetros odométricos del robot, y se requiere hacer una aproximación de la ecuación 3.52. Sin embargo, ahora que se conocen una aproximación de estos parámetros, es conveniente aplicar un proceso de calibración paramétrica que considere el modelo cinemático completo del robot, y que aplique técnicas de filtrado estadístico para rechazar los valores atípicos o outliers que suelen aparecer en todo proceso de ensayo experimental.

El proceso de calibración consiste en repetir una serie de movimientos sistemáticos que excitan los parámetros del modelo cinemático del robot; al mismo tiempo que se registra su configuración inicial y final, valiéndose para ello de los sensores exteroceptivos y propioceptivos del mismo. Fundamentalmente el proceso de calibración es similar al de identificación ya antes descrito, excepto que ahora se toma en cuenta el ángulo de curvatura de la trayectoria del robot, a través de la ecuación 3.52. Para este caso, es necesario contar con un mecanismo que pueda medir el ángulo de desviación que sufre el móvil en la ejecución de la trayectoria de excitación. Este mecanismo puede ser por ejemplo un sensor láser, o un sensor rotativo basado en ultrasonidos[25], o cualquier otro mecanismo sensorial ad hoc. No obstante, si tal mecanismo no existe o es de poca precisión, siempre se puede estimar la desviación angular basándose en los parámetros odométricos que ya se conocen del proceso de identificación previo. Esto es, que de acuerdo a la cantidad de pulsos recibidos y de acuerdo a los parámetros previamente estimados, se puede hacer una mejor aproximación de la curvatura ejecutada. Estimar de manera aproximada el arco recorrido por cada una rueda de las ruedas de odometría mediante la ecuación 3.52, tiene su validez siempre y cuando se guarden ciertos límites que reduzcan el error de aproximación del trayecto curvilíneo al trayecto rectilíneo.

El error relativo que se comete al aproximar el trayecto curvilíneo como si fuera un trayecto rectilíneo, se puede representar de acuerdo a la gráfica mostrada en la figura 3.23. En la mencionada figura se aprecia que el error crece de manera rápida e importante con el ángulo de desviación $\Delta \theta$. No obstante también se aprecia que este error se suaviza si se selecciona una longitud de trayecto igual o superior a 2 veces la separación entre ruedas. Por ejemplo, en un móvil diferencial cuya separación nominal entre ruedas sea $d$, se puede mostrar que si se programa una longitud de trayecto $\Delta L$ igual a $2 d$, entonces el error de aproximación del trayecto $S$, permanecerá por debajo 


\section{CAPÍTULO 3. MODELADO CINEMÁTICO Y LOCALIZACIÓN ODOMÉTRICA DEL ROBOT DIFERENCIAL}

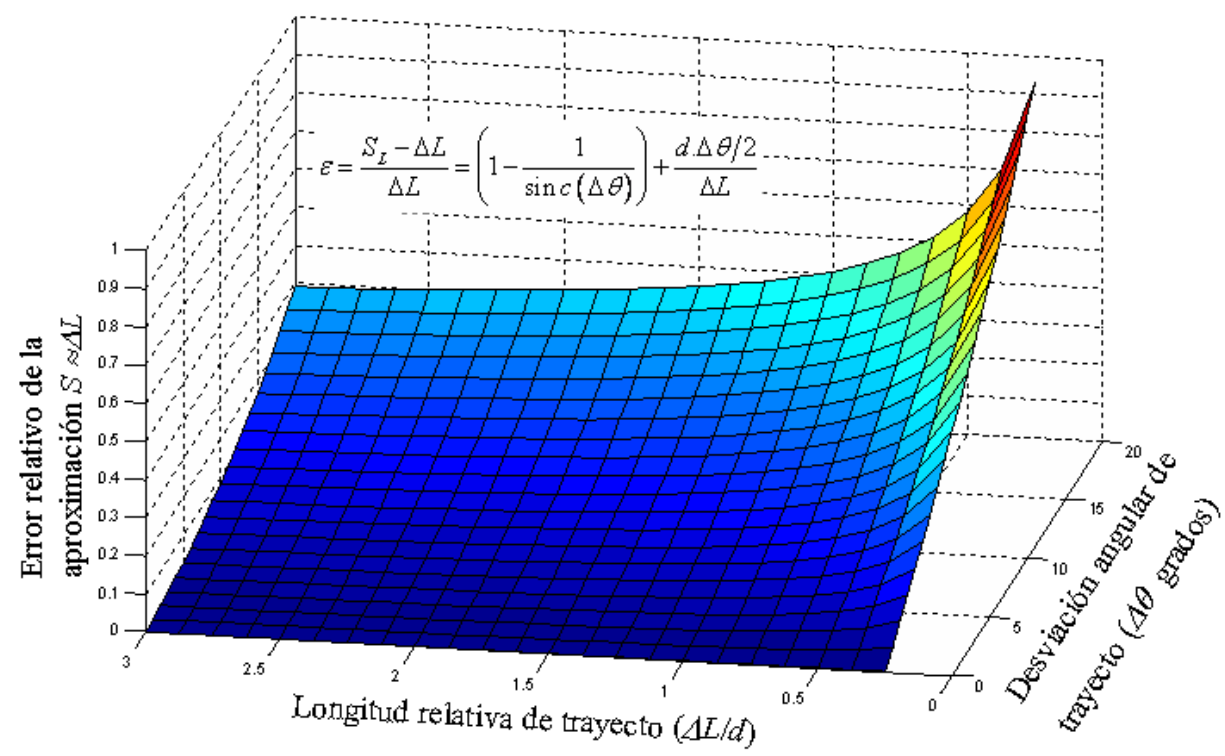

Figura 3.23: Error relativo entre el trayecto real y el trayecto rectilíneo aproximado

del $1 \%$ siempre que el ángulo de desviación $\Delta \theta$ no sobrepase los 2 grados; o también que permanecerá por debajo del $2 \%$ siempre que no se desvié más de 3 grados. No obstante la ventaja anterior de aumentar la longitud de trayecto para disminuir la sensibilidad al ángulo de desviación, en términos prácticos no se puede hacer sin cuidado porque se corre el riesgo de que el robot sufra una mayor desviación por curvatura, y de esa forma aumente el error de aproximación al aumentar el ángulo de desviación.

Considerado los detalles anteriores, el procedimiento de calibración consiste en repetir de forma alternada un movimiento de traslación cuya longitud $\Delta L$ aproximada sea igual a $2 d$, y un movimiento de rotación sobre su mismo eje, de arco aproximado a 360 grados. Este operación se repite por lo menos hasta completar 10 ensayos de cada movimiento, durante los cuales se van registrando los datos proveniente de el sensor de distancia, del compás, y de los codificadores rotativos de las ruedas de odometría.

$$
\left\{\Delta L, \Delta \theta, \Delta \theta_{\mathrm{c}}, N_{R}, N_{L}\right\}^{i} \text { con } i=1 \ldots n
$$

donde

$\Delta L$ es la longitud del trayecto medida mediante el sensor de distancias.

$\Delta \theta$ es el ángulo de desviación medido o estimado.

$\Delta \theta_{\mathbf{c}}$ es el arco angular total medido por el compás durante la rotación.

$N_{R}$ es el número de pulsos recibidos por el codificador derecho. 


\subsection{UN MÉTODO AUTOMÁTICO PARA LA IDENTIFICACIÓN Y CALIBRACIÓN MULTISENSORIAL DIRECTA DE LA CINEMÁTICA DE UN ROBOT DIFERENCIAL.}

$N_{L}$ es el número de pulsos recibidos por el codificador izquierdo.

$n$ es el número de repeticiones de una misma trayectoria o movimiento.

Con estos datos, y mediante la ecuación 3.52 se obtiene el conjunto de arcos de trayectoria,

$$
\left\{S_{R}, S_{L}\right\}^{i} \operatorname{con} i=1 \ldots n
$$

y mediante la ecuación (3.54) se obtiene el conjunto de valores de la sensibilidad odométrica,

$$
\left\{C_{R}, C_{L}\right\}^{i} \operatorname{con} i=1 \ldots n
$$

Por otra parte, el procedimiento de calibración de la base de giro es similar al descrito en el apartado 3.4.1.3, sin presentar ventaja adicional por el hecho de conocer a priori un valor estimado de la base de giro.

\subsubsection{Detección de valores atípicos.}

Los outliers o valores espurios son observaciones que parecen ser inconsistentes con el resto de los datos colectados. Las principales causas de su aparición son los errores en la medición y en el registro de datos. También, la incorrecta asunción de la distribución de datos lleva a la aparición de falsos outliers[15]. Para datos que están distribuidos aproximadamente en forma normal, existen varias técnicas de detección que en general están basadas en métodos de regresión y que clasifican como outliers a todos aquellos valores que estén fuera de cierto rango o de cierto umbral[93] : método del coeficiente de variación o regla $\pm 3 \sigma$ de Huber, z-score y el test de Grubb[85] , z-score modificado(indicador de Hampel), y el método del grafico de dispersión o box-plot.

Estas técnicas tienen un procedimiento general de detección en el cual intervienen tres componentes,

1. Un valor de referencia $x_{0}$, el cual se determina a partir de los datos recolectados.

2. Una medida de la variación de los datos o coeficiente de variación $\tau$.

3. Un umbral de clasificación $t$.

De esta manera, cada componente $x_{i}$ del conjunto de datos, debe ser probado para determinar si es un outlier de acuerdo a la siguiente regla:

$$
\text { si }\left|x_{i}-x_{0}\right|>t \cdot \tau \Rightarrow x_{i} \text { es un outlier }
$$

Los métodos más simples usan como valor de referencia $x_{0}$ a la media de la muestra de datos, y como medida de variación $\tau$ a la desviación estándar de los mismos. Sin embargo estos métodos de detección y filtrado son poco robustos ya 


\section{CAPÍTULO 3. MODELADO CINEMÁTICO Y LOCALIZACIÓN} ODOMÉTRICA DEL ROBOT DIFERENCIAL

que sus parámetros de clasificación están fuertemente influenciados por los propios outliers; y de esa forma al ir eliminando datos clasificados como atípicos, entonces también se va moviendo la ventana de clasificación y filtrado. Debido principalmente a esta razón, en esta tesis se usa el z-score modificado ya que el mismo es más robusto porque usa como valor de referencia $x_{0}$ a la mediana en lugar de la media, y como medida de dispersión a la desviación absoluta de la mediana (MAD) en lugar de la desviación estándar.

La desviación absoluta de la mediana se obtiene de acuerdo a,

$$
M A D=\text { mediana }\left|x_{i}-\tilde{x}\right| \operatorname{con} i=1 \ldots n
$$

donde $\tilde{x}$ representa la mediana del conjunto de $n$ datos.

El método z-score de detección de outliers requiere que se calcule el valor de relación de cada observación, según

$$
z_{i}=\left(x_{i}-\tilde{x}\right) / M A D \operatorname{con} i=1 \ldots n
$$

Comúnmente, si el valor de relación $z_{i}$ es superior a 5 , entonces $x_{i}$ es clasificado como outliers y entonces es eliminado del conjunto de observaciones válidas.

\subsubsection{Resultados experimentales}

La implementación de la formulación de identificación y calibración automática aquí propuesta fue probada en el robot YAIR. Las ruedas motrices de este robot tienen un diámetro nominal de 160 milímetros y una separación entre ellas de 363 milímetros. Los codificadores rotativos acoplados a las ruedas tienen una resolución de 500 pulsos por revolución. Esto último indica que la sensibilidad odométrica nominal en este caso es de $1 \mathrm{~mm} /$ pulso. Sin embargo ya se mostró en la sección 3.3.3.1 que el valor más probable para esta sensibilidad es $1.033 \mathrm{~mm} /$ pulso. Con el objetivo de probar el método de identificación y calibración en condiciones menos ideales o más severas, se modificó el diámetro de la rueda izquierda del robot añadiéndole varias vueltas de cinta adhesiva. De aquí que el valor más probable para la sensibilidad odométrica de esta rueda haya alcanzado un valor de $1.052 \mathrm{~mm} /$ pulso.

\subsubsection{Resultados proceso de identificación}

En la figura 3.24 se muestra un diagrama conceptual de todo el proceso de identificación automática de los parámetros del modelo cinemático directo de un robot diferencial. En esa figura se distinguen las siguientes variables y parámetros:

$\mu_{r}$ es la dirección normal a la superficie del reflector de referencia.

$\mu_{\theta}$ es la orientación del robot.

$\theta_{\mathbf{c}}$ es el ángulo de acimut suministrado por el compás. 


\subsection{UN MÉTODO AUTOMÁTICO PARA LA IDENTIFICACIÓN Y}

CALIBRACIÓN MULTISENSORIAL DIRECTA DE LA CINEMÁTICA DE UN ROBOT DIFERENCIAL.

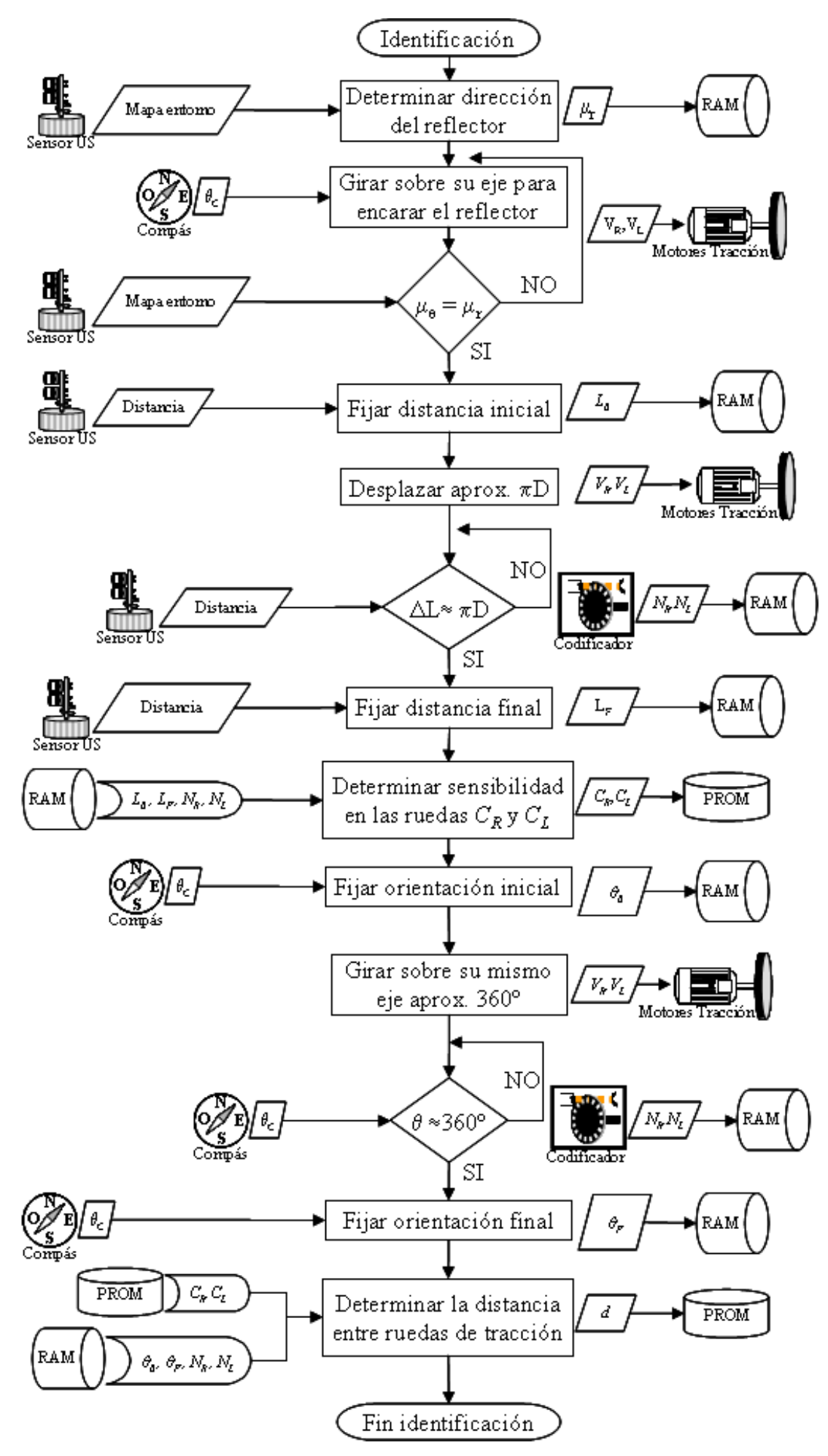

Figura 3.24: Esquema del proceso de identificación de parámetros odométricos en el robot YAIR. 


\section{CAPÍTULO 3. MODELADO CINEMÁTICO Y LOCALIZACIÓN ODOMÉTRICA DEL ROBOT DIFERENCIAL}

Tabla 3.6: Resultados de la identificación automática de parámetros odométricos en el robot YAIR.

\begin{tabular}{|l|c|c|c|}
\hline & \multicolumn{2}{|c|}{ Sensibilidad odométrica } & Dist. entre ruedas \\
\hline & $C_{R}(\mathrm{~mm} / \mathrm{pulso})$ & $C_{L}(\mathrm{~mm} / \mathrm{pulso})$ & $d(\mathrm{~mm})$ \\
\hline Valor más probable & 1.033 & 1.052 & 363 \\
\hline Prueba $\mathrm{N}^{\mathrm{O}} 1$ & 1.032 & 1.038 & 361 \\
\hline Prueba $\mathrm{N}^{\mathrm{O}} 2$ & 1.039 & 1.043 & 363 \\
\hline Prueba $\mathrm{N}^{\circ} 3$ & 1.039 & 1.044 & 363 \\
\hline
\end{tabular}

$V_{R}, V_{L}$ son los comandos de velocidad aplicado a las ruedas derecha e izquierda respectivamente.

$D$ es el diámetro nominal de las ruedas de odometría.

$L_{0}, L_{F}$ es la distancia medida desde el robot al reflector de referencia, antes y después de realizar el desplazamiento, respectivamente.

$\Delta L=L_{F}-L_{0}$ es la distancia recorrida por el robot sin tomar en cuenta la curvatura del trayecto.

$N_{R}, N_{L}$ son los pulsos de codificador acumulados durante el trayecto recorrido por las ruedas de odometría derecha e izquierda respectivamente.

$C_{R}, C_{L}$ son las sensibilidades odométricas de las ruedas derecha e izquierda respectivamente, estimadas mediante el proceso de identificación.

$\theta_{0}, \theta_{F}$ es el acimut del robot antes y después del movimiento de rotación sobre su mismo eje, respectivamente.

$d$ es la distancia entre ruedas estimada mediante el proceso de identificación.

Una vez que se programó el robot para que ejecutará tres veces el proceso de identificación de los parámetros odométricos, se obtuvieron los resultados de la tabla 3.6. A modo de comparación, en esta tabla se muestran también los valores más probables que toman los parámetros odométricos después de la modificación ya antes mencionada.

Como se aprecia en la tabla 3.6, mediante el método de identificación propuesto se logró detectar el desbalance de diámetros entre las ruedas, y además se obtuvieron valores bastantes ajustados a los valores reales. De esta manera se concluye que el método arroja resultados suficientemente satisfactorios; sobretodo si se considera que las condiciones en la que se realizó la prueba eran extremas, y que el robot no tenia conocimiento alguno de los valores nominales de sus parámetros odométricos originales. 


\subsection{UN MÉTODO AUTOMÁTICO PARA LA IDENTIFICACIÓN Y CALIBRACIÓN MULTISENSORIAL DIRECTA DE LA CINEMÁTICA DE UN ROBOT DIFERENCIAL.}

\subsubsection{Resultados en el proceso de calibración odométrica y detección de valores atípicos}

Una vez que el robot ha identificado sus parámetros odométricos, realiza el proceso de calibración el cual consiste en repetir una serie de movimientos sistemáticos que excitan los parámetros de su modelo cinemático, al tiempo que registra su configuración inicial y final, valiéndose de sus sensores exteroceptivos y propioceptivos. Los datos obtenidos los analiza en busca de posibles valores atípicos, para filtrar estos antes de determinar el primer y segundo momento estadístico de los mismos. Un valor agregado a este proceso es que el robot usa su propio estado estimado mediante los parámetros identificados previamente, para controlar más finamente su motores, y ejecutar así los movimientos de excitación necesarios. En la figura 3.25 se muestra un diagrama conceptual del proceso de calibración automática. En esa figura se distinguen las siguientes variables y parámetros:

$\mu_{r}$ es la dirección normal a la superficie del reflector de referencia.

$\mu_{\theta}$ es la orientación del robot.

$\theta_{\mathbf{c}}$ es el ángulo de azimut suministrado por el compás.

$V_{R}, V_{L}$ son los comandos de velocidad aplicado a las ruedas derecha e izquierda respectivamente.

$N_{R}, N_{L}$ son los pulsos de codificador acumulados durante el trayecto recorrido por las ruedas de odometría derecha e izquierda respectivamente.

$C_{R}, C_{L}$ son las sensibilidades odométricas de las ruedas derecha e izquierda respectivamente, previamente estimadas durante el proceso de identificación.

$\left(C_{R}, C_{L}\right)_{i}$ son las sensibilidades odométricas de las ruedas derecha e izquierda respectivamente, estimadas en el ciclo i-esimo del proceso de calibración.

$d$ es la distancia entre ruedas previamente estimada mediante el proceso de identificación.

$d_{i}$ es la distancia entre ruedas estimada en el ciclo i-esimo del proceso de calibración.

$\langle X, Y, \theta\rangle$ es el estado en cada instante de muestreo, estimado usando los parámetros obtenidos en el proceso de identificación y el modelo cinemático del robot.

$n$ es el número de veces que se ejecuta el proceso de estimación de parámetros.

La tabla 3.7 muestra los resultados obtenidos después que el robot realizará el procedimiento de calibración en tres oportunidades distintas. A través de estos resultados se muestra la capacidad del robot de detectar el desbalance introducido en las ruedas, y la capacidad de identificar con buena exactitud sus parámetros odométricos. Igualmente, el método de clasificación y filtrado de valores espurios funciona adecuadamente, lo que se puso de manifiesto en la detección y filtrado del valor atípico que aparece marcado con un asterisco $(*)$. 


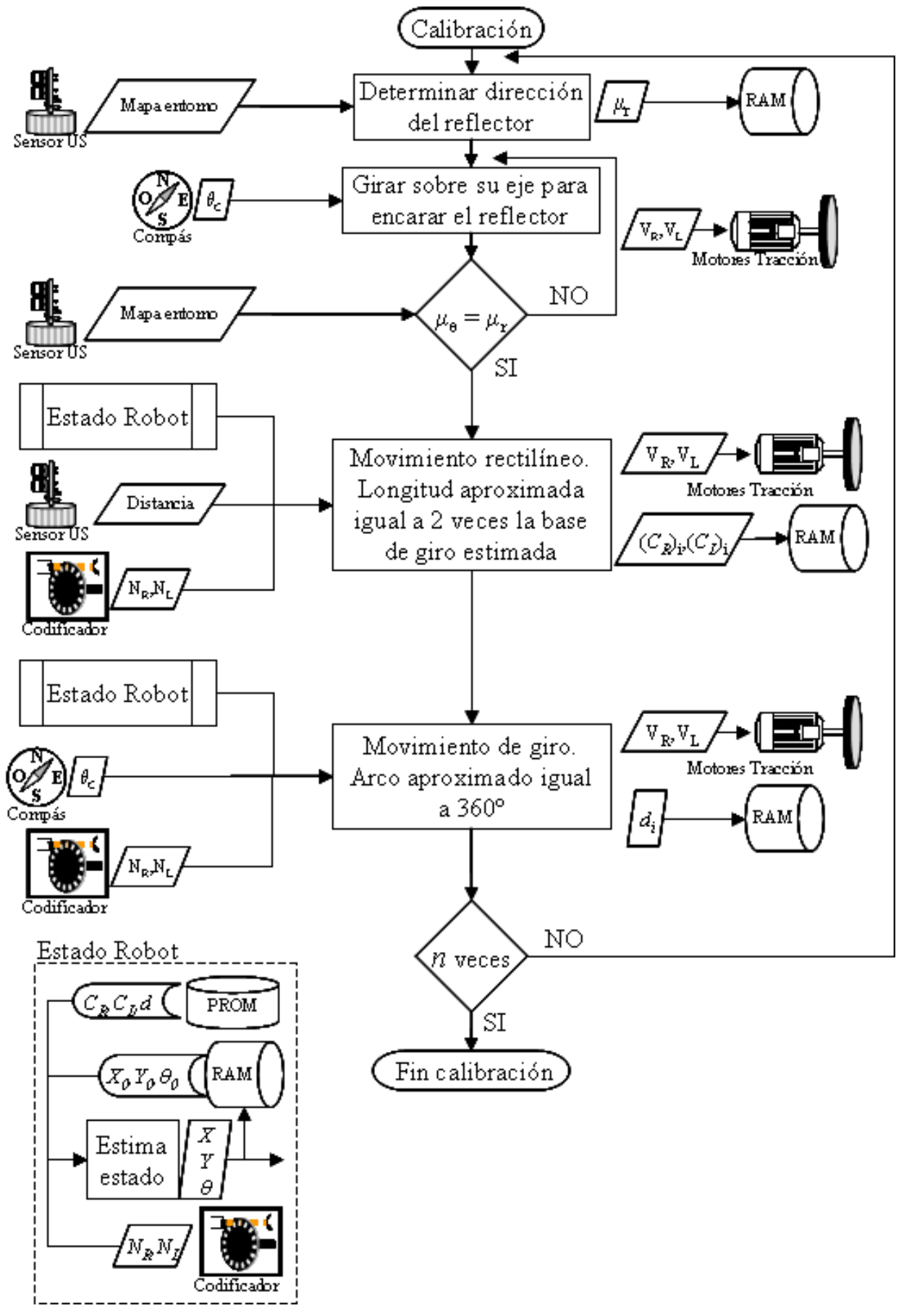

Figura 3.25: Esquema del proceso de calibración de parámetros odométricos del robot YAIR. 


\subsection{UN MÉTODO AUTOMÁTICO PARA LA IDENTIFICACIÓN Y CALIBRACIÓN MULTISENSORIAL DIRECTA DE LA CINEMÁTICA DE UN ROBOT DIFERENCIAL.}

Tabla 3.7: Resultados proceso de calibración de parámetros odométricos con filtrado de outliers. $C_{R}$ y $C_{L}$ : sensibilidad odométrica derecha e izquierda en $\mathrm{mm} / \mathrm{pulso}$, respectivamente. $B$ : base de giro en $\mathrm{mm}$.

\begin{tabular}{cccccccccc}
\hline$n$ & \multicolumn{3}{c}{ Prueba No 1} & \multicolumn{3}{c}{ Prueba No 2} & \multicolumn{3}{c}{ Prueba No 3} \\
& $C_{R}$ & $C_{L}$ & $B$ & $C_{R}$ & $C_{L}$ & $B$ & $C_{R}$ & $C_{L}$ & $B$ \\
\hline 1 & 1.049 & 1.051 & 363 & 1.039 & 1.035 & 361 & 1.044 & 1.048 & $356^{*}$ \\
2 & 1.020 & 1.066 & 364 & 1.038 & 1.031 & 360 & 1.036 & 1.076 & 366 \\
3 & 1.065 & 1.018 & 363 & 1.008 & 1.053 & 360 & 1.063 & 1.038 & 365 \\
4 & 1.032 & 1.055 & 364 & 1.006 & 1.069 & 360 & 1.049 & 1.047 & 365 \\
5 & 1.051 & 1.026 & 363 & 1.024 & 1.066 & 361 & 1.016 & 1.065 & 365 \\
6 & 1.030 & 1.075 & 363 & 1.049 & 1.026 & 361 & 1.032 & 1.055 & 364 \\
7 & 1.051 & 1.033 & 363 & 1.034 & 1.036 & 361 & 1.045 & 1.025 & 364 \\
8 & 1.036 & 1.059 & 363 & 1.043 & 1.037 & 361 & 1.061 & 1.040 & 364 \\
9 & 1.044 & 1.042 & 363 & 1.025 & 1.003 & 360 & 1.042 & 1.044 & 363 \\
10 & 1.030 & 1.081 & 364 & 1.019 & 1.043 & 360 & 1.071 & 1.026 & 364 \\
\hline & & & & & & & & & \\
Media & 1.041 & 1.051 & 363 & 1.029 & 1.040 & 361 & 1.046 & 1.046 & 364 \\
Varianza & $182 \mathrm{E}-6$ & $433 \mathrm{E}-6$ & 0.400 & $212 \mathrm{E}-6$ & $377 \mathrm{E}-6$ & 0.277 & $263 \mathrm{E}-6$ & $254 \mathrm{E}-6$ & 0.777 \\
\hline
\end{tabular}

\subsubsection{Comparación de resultados autocalibración multisensorial direc- ta vs UMBmark}

En distintos trabajos se ha demostrado que el método de UMBmark es capaz de corregir los errores de odemetría en un robot diferencial, obteniéndose así mejoras significativas en la estimación de la posición del robot. Sin embargo, los creadores de este método establecen una serie de limitaciones y aproximaciones que hacen que el método pierda robustez para sistemas con pequeñas diferencias en los diámetros de las ruedas.

Con el objetivo de contrastar los resultados obtenidos mediante el método automático de calibración directa, con el método del UMBmark, se modificó el diámetro de la rueda izquierda del robot YAIR añadiéndole varias vueltas de cinta adhesiva. De esta forma, la sensibilidad odométrica de esta rueda alcanzó un valor aproximadamente igual a $1.052 \mathrm{~mm} /$ pulso. A continuación se fijó una velocidad de navegación máxima de $250 \mathrm{~mm} / \mathrm{seg}$, y se procedió a realizar el protocolo de calibración establecido en el UMBmark. Debido a limitaciones de espacio físico, para los ensayos con el YAIR se seleccionó una longitud nominal $\mathrm{L}=3 \mathrm{mts}$.

Ejecutada la trayectoria que se propone en el método UMBmark, pero ajustado a las condiciones particulares para el robot YAIR ya antes descritas, se obtuvo las formas de trayectoria real mostrada en la figura 3.26.

La tabla 3.8 muestra la comparación de los valores nominales modificados por la incorporación de las vueltas de cinta adhesiva, con los resultados obtenidos mediante el método de autocalibración multisensorial directa aquí propuesto y con los 


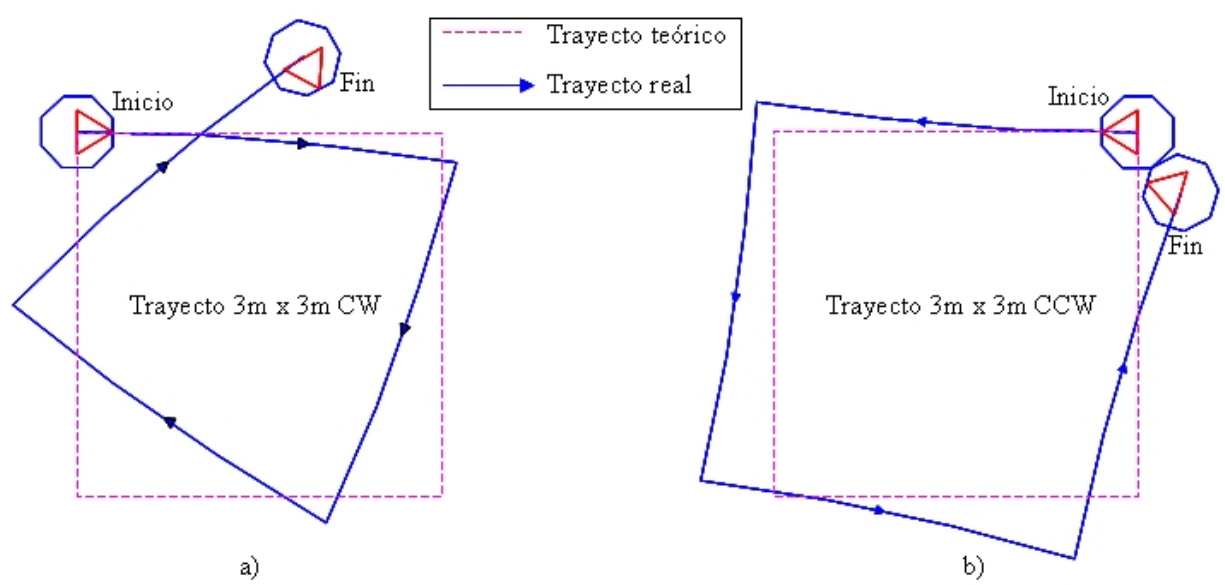

Figura 3.26: Trayectorias en la pruebas UMBmark realizadas. a) Sentido de las manecillas del reloj. b) Sentido contrario a la manecillas del reloj

Tabla 3.8: Comparación de resultados para los parámetros odométricos modificados de YAIR obtenidos por el método de autocalibración directa y por el UMBmark.

\begin{tabular}{cccccc}
\hline YAIR & Valores & \multicolumn{2}{c}{ Autocalibración directa } & \multicolumn{2}{c}{ UMBmark } \\
& reales & Result. & $\sigma$ & Result. & $\sigma$ \\
\hline$C_{R}(\mathrm{~mm} / \mathrm{pulso})$ & 1.0330 & 1.0384 & 0.0003 & 0.994 & \\
$C_{L}(\mathrm{~mm} / \mathrm{pulso})$ & 1.0520 & 1.0456 & 0.0004 & 1.006 & \\
$B(\mathrm{~mm})$ & 363.0 & 362.6 & 3.2 & 363 & \\
\hline
\end{tabular}

resultados obtenidos mediante el método del UMBmark.

En la tabla 3.8 se puede apreciar que aunque el método del UMBmark logra detectar que existe un desbalance entre los diámetros de las ruedas, sin embargo no es capaz de ofrecer un resultado ajustado a los valores reales. Esto no sucede con el método de la autocalibración multisensorial directa, que además de detectar el marcado desbalance entre los diámetros de las ruedas, también es capaz de aproximarse bastante bien a los valores reales. Para verificar esto, se introdujo los valores estimados por ambos métodos en el modelo cinemático directo del robot, y con los valores odometricos registrados durante los distintos ensayos del UMBmark, se procedió a estimar la posición final del robot. Los resultados de la estimación se compararon con la posición real medida.

En la tabla 3.9 se compara el error de localización cuando se usan los parámetros calibrados mediante el UMBmark, y cuando se usan los parámetros calibrados mediante el método de autocalibración directa. Aquí se observa que en este caso el segundo método ofrece resultados significativamente mejores que el primero.

De la tabla 3.9 se concluye que el error máximo con el método de calibración directa es mucho menor que si se usará el UMBmark. No obstante, algo mas importante en la desmesurada proporción entre los errores medido en ambas direcciones cuando 
3.4. UN MÉTODO AUTOMÁTICO PARA LA IDENTIFICACIÓN Y

CALIBRACIÓN MULTISENSORIAL DIRECTA DE LA CINEMÁTICA DE UN ROBOT DIFERENCIAL.

Tabla 3.9: Comparación de errores de localización después de la calibración del robot YAIR mediante la autocalibración multisisensorial directa y mediante el método UMBmark

\begin{tabular}{|c|c|c|c|c|c|}
\hline & \multicolumn{2}{|c|}{ Dirección CW } & \multicolumn{2}{|c|}{ Dirección CCW } & Error máximo \\
\hline & $\varepsilon_{x}(\mathrm{~mm})$ & $\varepsilon_{y}(\mathrm{~mm})$ & $\varepsilon_{x}(\mathrm{~mm})$ & $\varepsilon_{y}(\mathrm{~mm})$ & $\varepsilon_{\max }(\mathrm{mm})$ \\
\hline Autocalibración & -5 & 57 & -23 & 25 & 57 \\
\hline UMBmark & -116 & 1021 & 23 & 19 & 1027 \\
\hline
\end{tabular}

se aplica el UMBmark. A partir de ahí se evidencia que después de aplicar el método del UMBmark, la odometría permanece descalibrada, ya que el error mínimo que se observa en la dirección CCW se debe a la cancelación mutua de los efectos del error debido al desbalance en los diámetros de ruedas y a la falta de exactitud en el valor real del diámetro nominal de las ruedas. 



\section{Capítulo 4}

\section{Percepción, fusión sensorial y construcción de mapas}

La construcción de mapas en robótica móvil aborda el problema de representar un modelo espacial del entorno físico que rodea a un robot. Este modelo es comúnmente utilizado para operaciones de localización y navegación del mismo. Muy estrechamente ligado a esta representación del entorno está la capacidad de percibir el mismo. Por medio de los sensores el robot obtiene datos sobre su entorno (sensación) y luego los procesa para su utilización (percepción). De esta forma, los sensores representan para el robot la fuente de datos sobre los cambios tanto en su entorno (distancias a objetos, luz ambiental) como en sí mismo (nivel de las baterías, consumo de los motores, pulsos de codificadores, etc.). Por otra parte, las tareas fundamentales de un robot móvil son la representación del entorno y la localización. Para realizar estas tareas el robot se basa en los datos proporcionados por sus sensores. Debido a las limitaciones de cada tipo de sensor, resulta conveniente combinar la información proveniente de uno o de múltiples sensores, sean del mismo tipo o no. Este proceso, conocido como fusión de Datos, persigue mejorar la información disponible mediante la combinación de diferentes entradas que pueden provenir de un único sensor a diferentes instantes de tiempo(fusión en tiempo), o provenientes de múltiples sensores que entregan información al mismo tiempo(fusión multisensorial). En este capítulo se hace una revisión de la tecnología asociada a los sensores que forman parte del robot YAIR. Igualmente se desarrollan los modelos sensoriales necesarios para aplicar las estrategias de fusión sensorial a la construcción de mapas de ocupación tipo probabilístico y tipo posibilísticos, basados en datos de ultrasonidos. También, se desarrollan modelos y estrategias tendientes a construir mapas de líneas a partir de los datos de distancia suministrados por sensores de infrarrojos. Finalmente se presentan los resultados experimentales obtenidos. 


\subsection{Construcción de mapas de ocupación mediante datos de ultrasonidos}

En la robótica móvil, el proceso de construcción de mapas se refiere a la obtención de información del entorno a través del uso de los sensores del robot, y al procesamiento de dicha información con la finalidad de actualizar un mapa disponible. Este problema consiste en construir un modelo espacial del entorno físico donde opera un robot móvil. Este modelo es comúnmente utilizado para operaciones de navegación del robot, como por ejemplo la localización del mismo. Normalmente el mapa es construido por el mismo robot, por lo que éste necesita poseer sensores que le permitan a su vez percibir el mundo exterior. Los sensores comúnmente utilizados incluyen cámaras de video y medidores de distancia que utilizan tecnología basada en Ultrasonidos, Láser, o Infrarrojos.

Actualmente todos los algoritmos de mapeo actualmente desarrollados se apoyan en mayor o menor grado en métodos probabilísticos. Una primera clasificación que de ellos se puede hacer corresponde a que algunos son algoritmos incrementales por lo tanto operan en tiempo real, mientras que otros requieren acumular cierta cantidad de datos para poder construir el modelo del entorno. Por otro lado, algunos algoritmos requieren el exacto y explicito conocimiento de la posición del robot respecto al mapa global para poder construir el modelo de su entorno, mientras que otros pueden hacerlo apoyándose en datos provenientes de su odometría. Independientemente de la instrumentación y del método utilizado, una clasificación preponderante es la que divide los mapas de entorno en mapas de Grid, mapas de objetos y características geométricas básicas, y mapas topológicos.

El caso más simple de construcción de mapas es el que genera mapas de áreas ocupadas por objetos que están en el entorno. Estos mapas conocidos como mapas de rejilla (OGM: Occupancy Grids Maps), se generan a partir de los datos obtenidos por sensores tales como cámaras de video y medidores de distancia que utilizan tecnología basada en ultrasonidos, Láser, o Infrarrojos. El algoritmo básico fue desarrollado por Elfes y Moravec [151, 66] a mediados de los ochenta, y el problema principal se enfocaba en la generación de un mapa métrico a partir de datos contaminado con ruido o datos incompletos provenientes de un sensor de ultrasonidos. El algoritmo representaba los mapas mediante una rejilla dividida en pequeñas celdas que modelan el espacio libre y el espacio ocupado del entorno del robot, mediante una distribución de probabilidad que va desde 0 para el espacio vacío hasta 1 para el espacio ocupado.

Desde la aparición de OGM, el proceso de construcción de mapas de ocupación ha consistido generalmente de 3 pasos básicos:

Percepción. Los sensores del robot se activan en una secuencia adecuada, y entonces un paquete de $n$ mediciones de distancia son registradas. Lo más común es usar sólo sensores de ultrasonidos, pero algunas veces los mismos también se usan en combinación con un sensor Láser, o con uno de infrarrojos, o con cámaras de vídeo. 
Procesamiento. Las medidas registradas son procesadas con la finalidad de construir una representación local del entorno en términos de espacios libres u ocupados. Esta etapa requiere de la discretización previa del espacio y del desarrollo de modelos sensoriales que puedan describir el estado del espacio discreto.

fusión. La representación local es integrada en la representación global mediante el filtrado de la información incompleta o contradictoria. Usualmente se utilizan uno de los siguientes marcos teóricos: La teoría Bayesiana, La teoría Evindencial de Dempster-Shafer, La teoría posibilística o Lógica Fuzzy.

En adelante, se desarrollan cada uno de estas etapas ajustadas a las condiciones particulares del sensor ultrasónico o SONAR usado en esta tesis.

\subsubsection{El SONAR}

El SONAR es un sistema de medición de distancias basado en ultrasonido que opera usando el principio del tiempo de vuelo. En su forma más común se trata de un único transductor que transmite al entorno un corto tren de pulsos, y que luego cambia a modo recepción a fin de esperar durante un determinado tiempo la llegada de la onda acústica, después de que esta última haya sido reflejada por algún objeto del entorno. Si un eco reflejado es detectado, la distancia $r$ al objeto reflector puede ser estimada mediante la ecuación $r=\frac{1}{2} v_{s} \cdot t_{f}$, donde $v_{s}$ es la velocidad del sonido en el medio, la cual se asume que es constante, y $t_{f}$ es el tiempo medido entre la emisión y la recepción de la señal. En aplicaciones de robótica móvil, es usual aplicar el proceso de estimación de distancias alrededor de un eje vertical fijo, con el objetivo último de obtener un mapa de distancias 2D como el mostrado en la figura 4.1. En esa figura, las líneas discontinuas muestran el modelo simplificado del entorno real, y las líneas punteadas representan las distancias estimadas mediante el proceso básico del sonar. Igualmente, la figura octogonal representa el robot que a su vez lleva integrado el sensor de ultrasonidos. Aunque en principio parece una representación del entorno muy poco apropiada, después se verá que con el adecuado procesamiento e interpretación de estos datos, se puede obtener información muy útil a un costo bastante bajo.

\subsubsection{Principales problemas de la medición con SONAR}

Como se puede ver en la figura 4.2, un primer problema de la medición con SONAR es la reflexión tipo espejo, la cual es causa de que objetos del entorno con cierto tamaño y orientación no sean visto por el sensor, o que en general aparezcan más lejos de lo que realmente están.

Debido a la reflexión tipo espejo, sólo las lecturas que se tomen de manera perpendicular a la superficie reflectora serán correctas. El ángulo de incidencia se mide entre el eje del sensor y la normal a la superficie, por lo que si las lecturas se toman con un ángulo de incidencia superior a cierto ángulo crítico $\alpha_{\mathrm{c}}$, el objeto reflector quedará oculto o en general se sobreestimará su distancia. Esto ocurre sobretodo en 


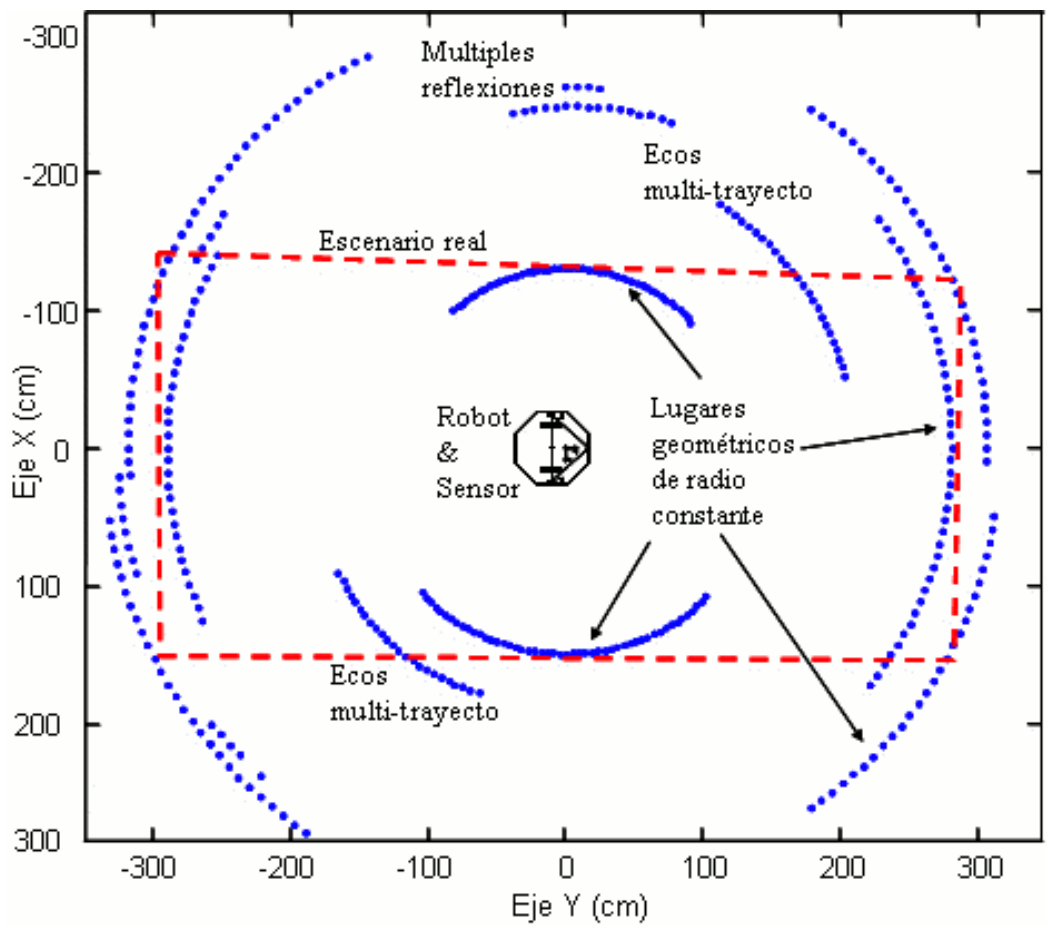

Figura 4.1: Datos de distancias tomados mediante SONAR en un ambiente de laboratorio. Líneas discontinuas: modelo simplificado del entorno real. Líneas punteadas: distancias basadas en el tiempo de vuelo.

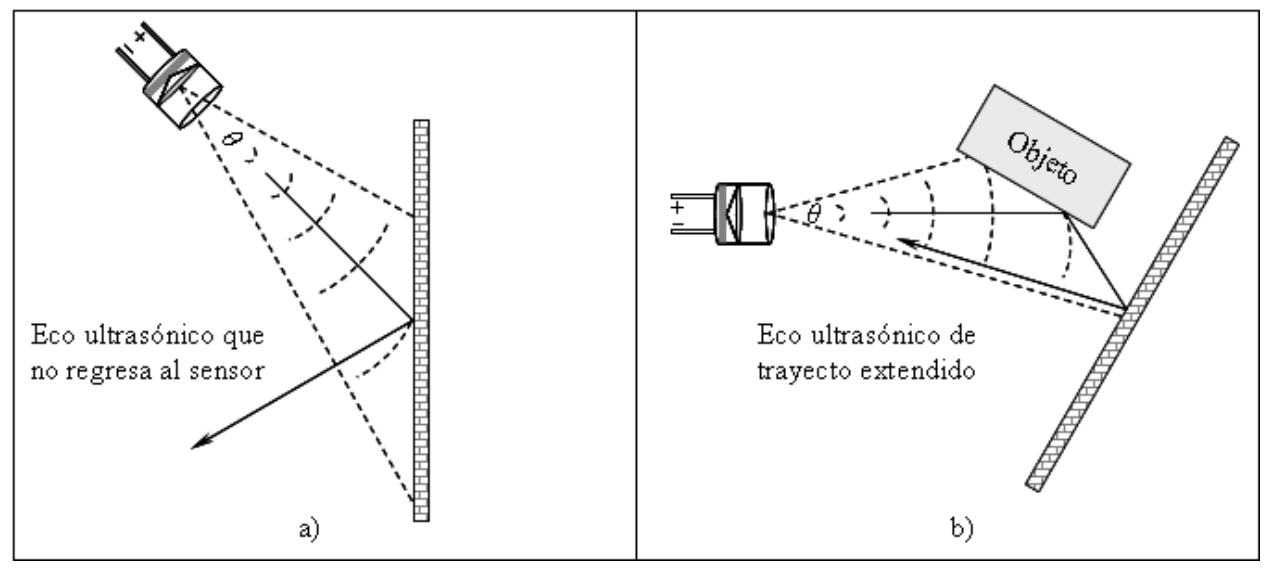

Figura 4.2: Principales efectos de la reflexión tipo espejo de la señal ultrasónica. 


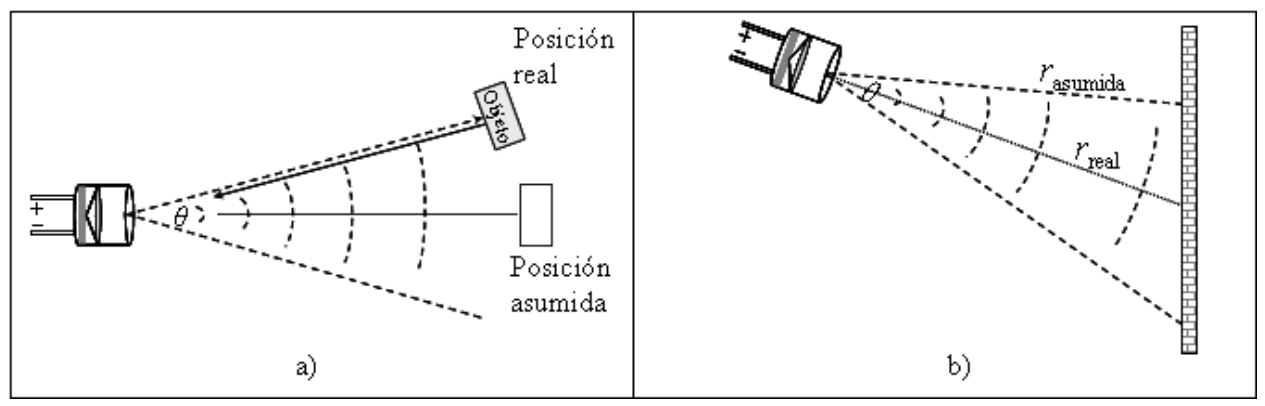

Figura 4.3: Principales efectos de la apertura acústica del sensor de ultrasonidos. a) Incertidumbre en la posición angular del reflector. b) Incertidumbre en la distancia radial del reflector.

las esquinas y se debe a que la onda ultrasónica sufre varias reflexiones antes de regresar al sensor. En la figura 4.1 se puede observar como los segmentos de pared en la vecindad de las esquinas aparecen a una distancia superior a la que realmente están.

Otro problema importante en los sistemas SONAR tiene que ver con su amplio ancho de haz. En general los sistemas SONAR no son altamente enfocados o directivos. Esto se debe a que los transductores de ultrasonido poseen cierta región cónica de sensibilidad que en general depende del tamaño físico del elemento vibratorio y de la frecuencia a la que este oscile[117]. Este cono de sensibilidad, también conocido como apertura acústica del sensor, introduce incertidumbre tanto en la posición como en la distancia del objeto reflector.

La incertidumbre en la posición se refiere a que un objeto que este fuera del eje del sensor, aun podrá ser detectado siempre y cuando presente una orientación normal a la trayectoria de alguna de las componentes del frente de ondas incluido dentro de la apertura acústica del sensor. La incertidumbre en la distancia se refiere a que si un objeto reflector que se encuentre dentro del cono de sensibilidad, es observado por el sensor a un ángulo de incidencia distinto de cero pero menor al ángulo crítico, entonces la distancia que se computará no será la del punto que este en la orientación del sensor sino la del punto del objeto que este más cerca al mismo. Como ejemplo de este último efecto, en la figura 4.1 se puede observar la forma de arco en que se mapean las paredes cuando el sensor las enfoca con ángulos de incidencia distintos de cero.

\subsubsection{Modelado del SONAR tipo rotativo}

El SONAR (Sound Navigation and Ranging) ha sido utilizado con frecuencia en robot móviles que operan en ambientes de oficinas para la construcción del mapa del entorno. En la literatura se describen diversas formas de utilización del sonar, desde un sólo sonar con capacidad de rotar[130, 124], hasta uno o más arreglos de sonares situados a diferentes alturas. Un arreglo común es en forma de anillo, con los sonares uniformemente espaciados y orientados radialmente. Esta configuración presenta el 

MAPAS

inconveniente de tener dificultad para detectar obstáculos que están arriba o abajo del plano del anillo, por ejemplo mesas o sillas[158]. Pero tal vez el mayor problema de los sensores ultrasónicos lo representa su gran ancho de haz, cuya consecuencia inmediata es la incertidumbre para determinar la orientación azimutal de un objeto reflector ubicado dentro de la apertura acústica del transductor. Los sistemas SONAR rotativos son una modificación de los sistemas pulso-eco ordinarios, los cuales surgen como uno de los mecanismos más sencillos para atacar la problemática de la incertidumbre angular. En efecto, la ventaja principal de los sistemas SONAR rotativos es la resolución angular mejorada que ellos ofrecen. Con los arreglos de transductores estacionarios, la resolución del barrido de la escena esta limitada al número de transductores que puedan ser dispuestos dentro de un arreglo circular. Por su parte, el modelo de SONAR rotativo se puede hacer girar de forma muy fina obteniéndose así las prestaciones de un anillo ultrasónico de muy alta densidad de transductores. Esto permite la determinación más precisa de los objetos de la escena, con la consecuente mejor representación del entorno a un costo notablemente muy bajo. No obstante, como contrapartida, el SONAR rotativo presenta una baja frecuencia de actualización debido a la gran cantidad de tiempo que emplea para realizar un barrido de la escena (en el caso del SONAR de YAIR, el tiempo promedio es de 22 segundos).

Uno de los primeros sistemas rotativo fue reportado por J. L. Crowley quien usó una especie de corneta rotativa para enfocar el haz de ultrasonido alrededor del eje acimutal de una plataforma móvil[53]. J. Leonard y H. Durrant-Whyte usaron barridos de SONAR a pasos muy finos(0.588 grados por paso) para localizar lugares geométricos de distancia constante a las que llamaron RCD (Regions of Constant Depth)[127]. Estos investigadores definieron un RCD como el conjunto contiguo de los ecos de SONAR cuya distancia no difería por más de $1 \mathrm{~cm}$. Limitando el ancho mínimo de un RCD a unos $10^{\circ}$, ellos garantizaban que el eco detectado provenía del lóbulo principal del haz del transductor. De aquí que en este caso la utilidad principal del SONAR rotativo y las RCD sea la eliminación de los ecos débiles y las múltiples reflexiones; aunque sin embargo también es posible delimitar a un rango más estrecho la dirección en la que se encuentra un objeto. A pesar de la utilidad que ha demostrado este sistema, se ha perdido el interés en él ya que el mismo tardaba aproximadamente 2 minutos para realizar un barrido de 360 grados, lo que evidentemente no es práctico en las tareas de navegación de un robot móvil. La solución a esta limitación la propusieron los mismos autores, y consistía en el uso de varios de estos sensores con su campo de acción acotado a una determinada región del entorno; lo que también es bastante impráctico.

Una propuesta que utiliza un sólo SONAR rotativo y que reduce el tiempo de barrido a 3 segundos, manteniendo prestaciones similares al método de las RCD, es la propuesta por Lee en su trabajo con el robot ARNE[124]. Este investigador considera la reducción de la incertidumbre angular mediante la realización de un barrido de 360 grados a pasos discretos de $18^{\circ}$; y el posterior procesamiento e interpretación de los ecos provenientes de la escena. Si un objeto es detectado desde dos o más orientaciones angulares consecutivas del sensor rotativo, y además la estimación de las distancias respectivas no difieren de $3 \mathrm{~cm}$, entonces se podrá reducir la incertidumbre angular ya 


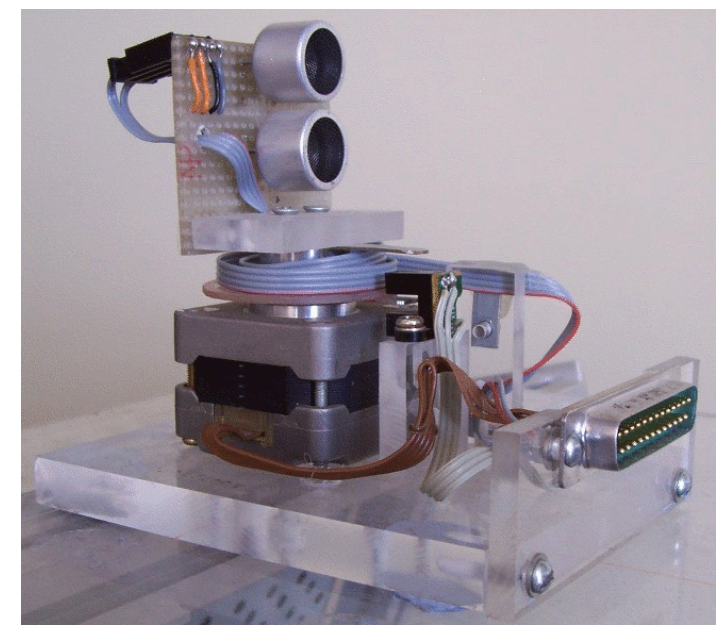

Figura 4.4: Aspecto del sensor de ultrasonidos de YAIR. Se muestran el motor a pasos y los transductores piezoeléctrico.

que el objeto reflector estará en el área donde se superponen los haces emitidos en cada una de las direcciones angulares involucradas en la detección. Con esta metodología Lee logra reducir la incertidumbre angular y el tiempo de barrido de la escena a valores comparables a los 10 grados y 3 segundos respectivamente.

En esta tesis se considera un SONAR rotativo que además de medir distancias basado en la técnica del pulso-eco, también presenta la ventaja de recuperar la envolvente del eco ultrasónico[25]. Este SONAR es capaz de reducir la incertidumbre acimutal a un arco de 1.8 grados basándose en un sólo sensor que realiza un barrido de 360 grados en aproximadamente 20 segundos. Esta velocidad puede ser mejorada a cambio de desmejorar la resolución angular[24].

Por otra parte, el sensor rotativo usado por YAIR posee una característica adicional que le permite atacar el problema de incertidumbre angular de una manera novedosa. Se trata de que el mencionado sensor es capaz no sólo de indicar la medida de distancia desde el sensor a un obstáculo del entorno, sino que además también recoge la amplitud del eco reflejado por el mismo.

Como es sabido, la amplitud de un eco de ultrasonidos depende del ángulo de incidencia según como se describe en el modelo de modulación angular o patrón angular para sensores de ultrasonidos tipo polaroid propuesto por Kuc et al en 1991[117],

$$
A(\theta)=e^{-4 \theta^{2} / \theta_{0}^{2}}
$$

donde el ángulo $\theta$ se mide a partir del eje del transductor o línea central del haz, y $\theta_{0}$ depende de las características del transductor. En el caso del sensor de YAIR, $\theta_{0}$ se determinó experimentalmente ajustándose al valor de $50^{\circ}$, lo que lleva a que su cono sensible sea de aproximadamente $40^{\circ}\left( \pm 20^{\circ}\right)[145]$.

Cuando el haz ultrasonido es perpendicular a la superficie reflectora es mucho más probable obtener un eco de mayor amplitud que cuando el haz alcanza la superficie 


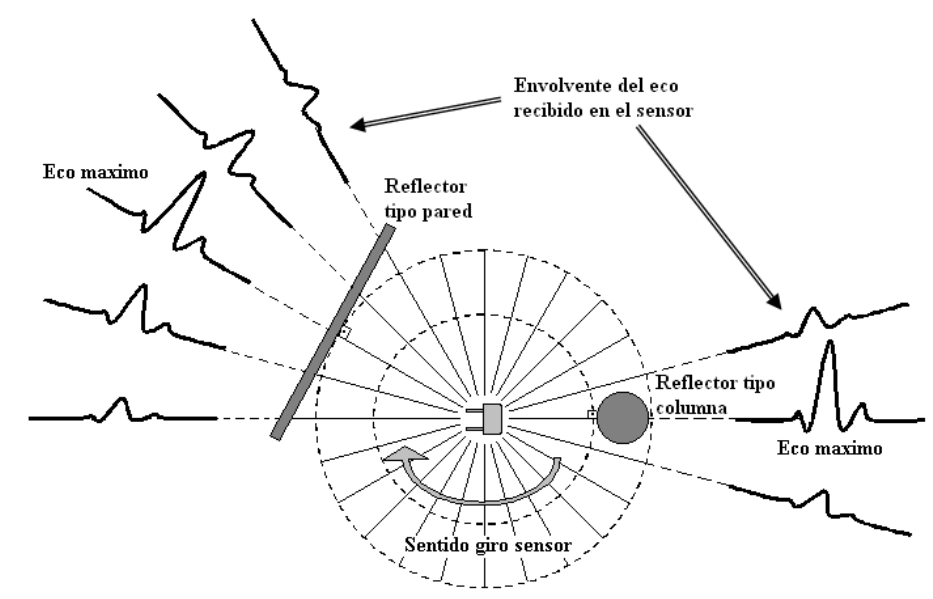

Figura 4.5: Estrategia para determinar el enfoque perpendicular del sensor de ultrasonidos con respecto al reflector. Cuando el sensor está enfocando perpendicularmente al reflector, la amplitud recibida es máxima.

con cualquier otro ángulo. Ahora bien, como el sensor rotativo de YAIR realiza un barrido de 360 grados a pasos angulares de 1.8 grados, si se utiliza una estrategia de seguimiento de los máximos del barrido(ver figura 4.5), se puede determinar en cual paso angular el sensor está orientado perpendicularmente a un cierto obstáculo; quedando reducido así el arco de incertidumbre de detección a sólo 1.8 grados.

Una vez determinada la dirección del reflector, se procede a determinar la distancia a la cual se encuentra el mismo, aplicando la técnica basada en el tiempo de vuelo que ya fue descrita en la sección 2.1.2.

La estrategia de filtrado anterior se aplicó de manera experimental al escenario mostrado en la figura 4.1, obteniéndose mayor exactitud en lo que a la ubicación y distancia a los reflectores se refiere. Estos resultados se muestran en la figura 4.6, donde se pueden ver la segmentación del escenario en regiones de profundidad constante $(\mathrm{RCD})$, así como también los puntos reflectores perpendiculares mapeados por el sonar rotativo.

\subsubsection{Modelo probabilista del SONAR}

Como ya se ha discutido en la sección 4.1.2, debido más que nada al ancho del haz y a las reflexiones múltiples, un sensor de distancias basado en ultrasonidos dista de ser un sensor ideal. Dada la dificultad para modelar estos efectos, la tendencia actual es construir modelos sensoriales tipo probabilísticos. Dentro de las ventajas de un modelo probabilista están:

- Los modelos probabilísticos son adecuados para describir la característica de incertidumbre que es inherente a los procesos de observación del entorno real.

- Existen metodología suficientemente probadas para el análisis de grupos de 


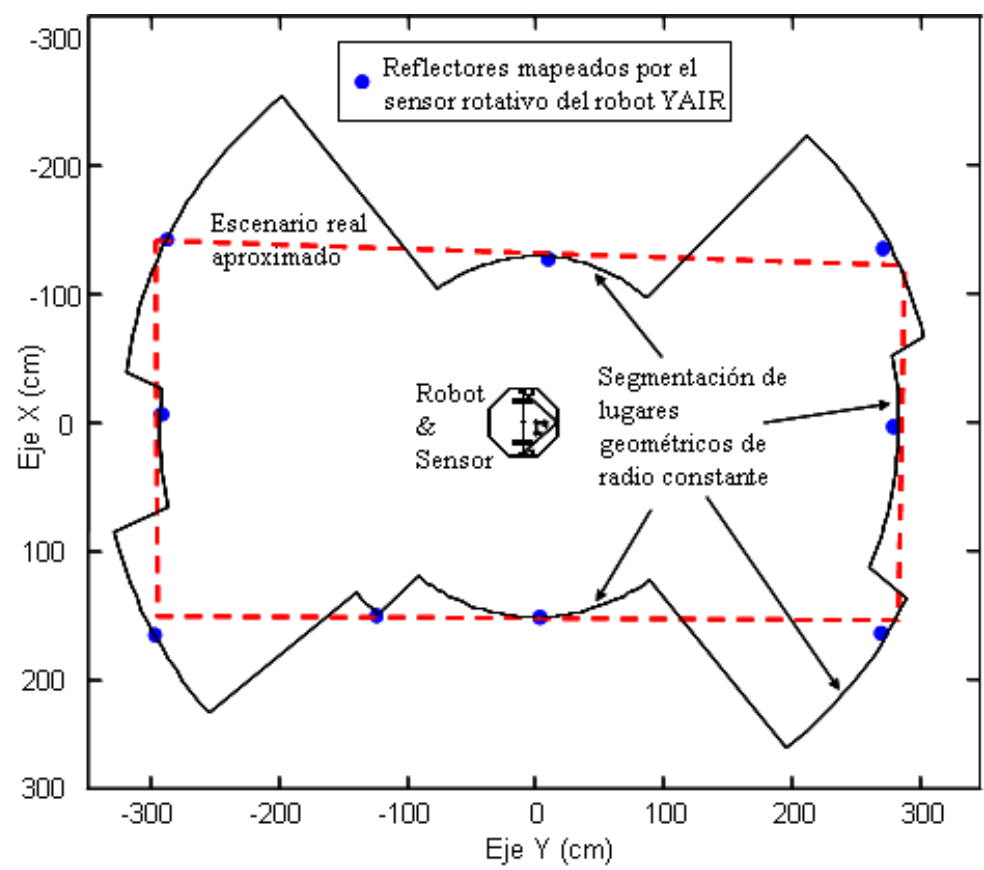

Figura 4.6: Mapa de regiones de radio constante donde se muestran los puntos reflectores mapeados por el sonar de YAIR después de aplicar la estrategia de filtrado por seguimiento de máximos perpendiculares. 


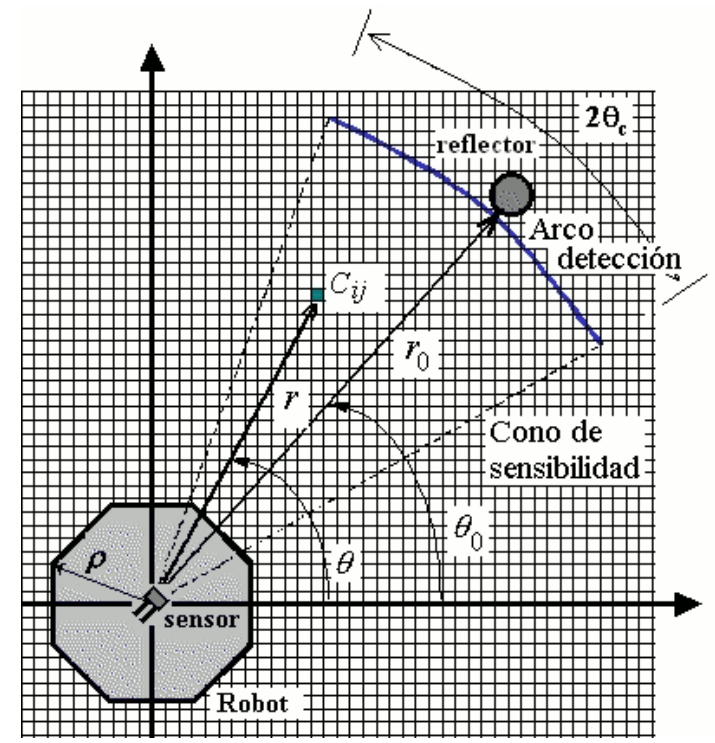

Figura 4.7: Representación de variables del modelo del SONAR. Se considera que dada una medición de distancia $r_{0}$ a un ángulo de orientación $\theta_{0}$, cada celda $C_{i j}$ ubicada en el cono de sensibilidad $\left(2 \theta_{c}\right)$ o en el arco de detección $\left(r_{0} \pm \sigma_{r}\right)$ tendrá una probabilidad de ocupación expresada como $\mathrm{P}(O \mid r, \theta)$.

datos descritos a través de distribuciones probabilísticas.(teoría de Bayes)

- La descripción de datos sensoriales de diferente naturaleza en un mismo marco probabilista, permite que las diversas observaciones sean comparadas e integradas de una manera consistente.

- Si se usa una política de modelado común, se facilita la extensión o mejora del sistema de percepción mediante incorporación de nuevos sensores.

Muchos de los algoritmos de fusión sensorial y de localización de robots, requieren que el sensor represente las observaciones, de forma probabilista, a través de una función de verosimilitud expresada como $\mathrm{P}(\mathrm{z} \mid \mathbf{x})$. En esta investigación, para el caso del SONAR se propone un modelo para estimar la ocupación $O$ de una celda de grid dada la lectura de la distancia $r$ a cierta orientación $\theta$, lo cual expresamos como $\mathrm{P}(O \mid r, \theta)$. La figura 4.7 ayuda a entender los argumentos de modelado.

Para el modelado del sensor se considera lo siguiente:

1. La probabilidad de que un obstáculo sea detectable depende solamente de si está o no dentro del cono de sensibilidad $\left(2 \theta_{c}\right)$ y del alcance máximo fiable del sensor $\left(r_{\max }\right)$. En el caso del robot YAIR, se determinó experimentalmente que el cono de sensibilidad es de aproximadamente $42^{\circ}$, y que el alcance radial máximo es de 4.4 metros. De aquí que toda celda de grid fuera del rango mencionado tenga de principio una probabilidad de ocupación de 0.5. 


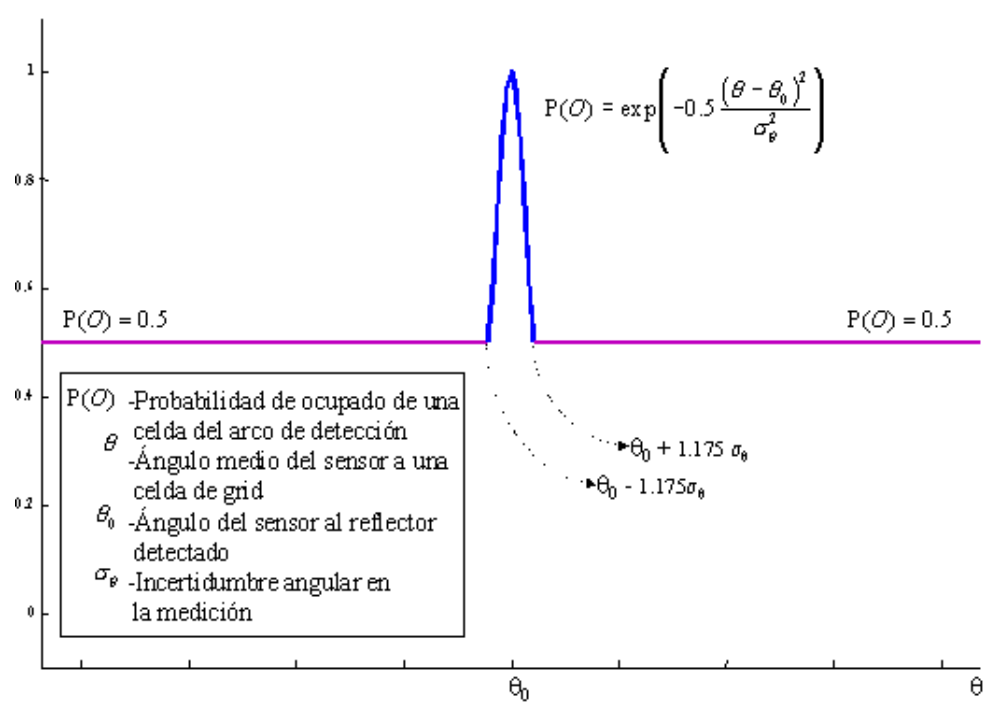

Figura 4.8: Forma unidimensional de la funcion de distribución de probabilidad de ocupación en el arco de detección con respecto al ángulo de vista.

2. El objeto del modelo se trata de un sensor rotativo que es capaz de medir la amplitud del eco reflejado cada $1.8^{\circ}$. También, gracias a una estrategia de seguimiento de la evolución angular de la amplitud del eco (figura 4.5), se puede asegurar que una vez detectado un obstáculo a una distancia $r_{0}$ y con orientación angular del sensor igual a $\theta_{0}$; es porque el mencionado obstáculo se encuentra dentro de un arco de incertidumbre $\theta_{0} \pm \sigma_{\theta}$ y a una distancia radial a partir de la ubicación del transductor de $r_{0} \pm \sigma_{r}$. Una forma funcional que modela la incertidumbre angular en el arco de detección es la que se muestra en la figura 4.8, mientras que una que modela la incertidumbre radial se puede ver en el tramo $\left[r_{0}-1,175 \sigma_{r}, r_{0}+1,175 \sigma_{r}\right]$ de la función mostrada en figura 4.10. Experimentalmente se determinó que en el arco de detección la incertidumbre angular sigue un patrón de distribución normal cuya varianza es aproximadamente igual a 9 grados, y que la incertidumbre radial también se distribuye de forma normal con una varianza aproximadamente igual a $4 \mathrm{~cm}$. De aquí que los limites de incertidumbre definidos anteriormente se pueden aproximar según $\sigma_{\theta} \approx 3^{\mathrm{o}}$ y $\sigma_{r} \approx 2 \mathrm{~cm}$.

3. De acuerdo a la naturaleza del haz ultrasónico, la posición angular de un reflector dentro del cono sensible no está exactamente determinada. Por ello, lo normal es suponer que en el área cónica entre el transductor y todo el arco de sensibilidad, la probabilidad de encontrar un obstáculo en nula. Esto en cierto sólo para un área reducida y muy próxima al eje que forman el transductor y el punto real de reflexión. De aquí que un modelo más realista corresponda a suponer una distribución que asigne probabilidad de ocupación mínima a lo largo 


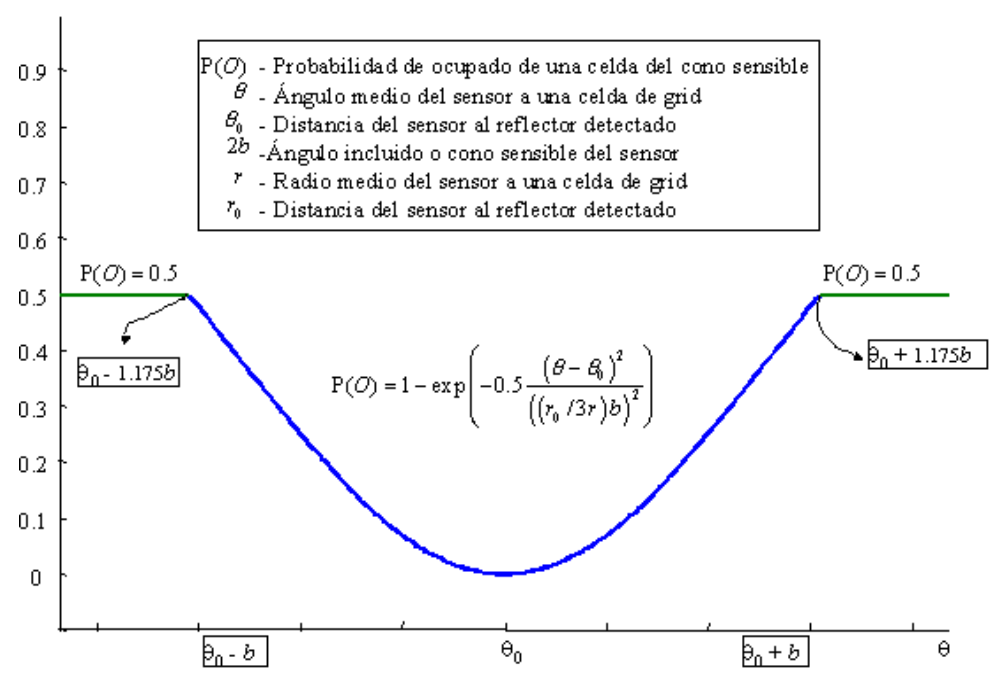

Figura 4.9: Forma unidimensional de la función de distribución de probabilidad de ocupación en el cono de sensibilidad con respecto al ángulo de vista.

del eje transductor-punto de reflexión mas probable, la cual debe ir creciendo suavemente hacia los extremos del cono. Por ejemplo, lo usual es asumir que el reflector se encuentra ubicado en el centro del cono de sensibilidad, por lo que a lo largo del eje bisectriz se puede asumir que la probabilidad de ocupación debe ser igual a cero $(\mathrm{P}(O)=0)$, mientras que en los bordes del cono se debe fijar una probabilidad de ocupación de $50 \%(\mathrm{P}(O)=0,5)$. Una función que puede modelar este efecto es la mostrada en la figura 4.9. Por otra parte, en el cono de sensibilidad también hay que tomar en cuenta que los reflectores pueden quedar ocultos debido a que el ángulo de incidencia del haz ultrasónico es mayor a cierto ángulo crítico. Este ángulo depende de las características de la superficie, variando entre 7-8 grados para superficies lisas y casi 90 grados para superficies en extremo rugosas. Se puede demostrar que el ángulo de incidencia sobre los reflectores ubicados a ambos lados del eje del sensor, disminuye con la distancia radial. Una forma de modelar este efecto es hacer que la varianza de la función disminuya con la distancia, logrando así que la campana se estreche otorgando mayor probabilidad de ocupación a las celdas que están más alejadas del sensor.

4. La atenuación de la señal ultrasónica es cuadrática con la distancia. De aquí que la incertidumbre que se tiene al procesar el eco recibido, en general crece cuando aumenta la distancia al reflector. En el arco de detección la entropía de la incertidumbre es absorbida normalmente por la resolución en la discretización del espacio, sin embargo en el cono de sensibilidad no pasa lo mismo. En el cono de sensibilidad la probabilidad de ocupación crece con la distancia. Así, en las cercanías del sensor la probabilidad de ocupación dentro del cono debe ser 


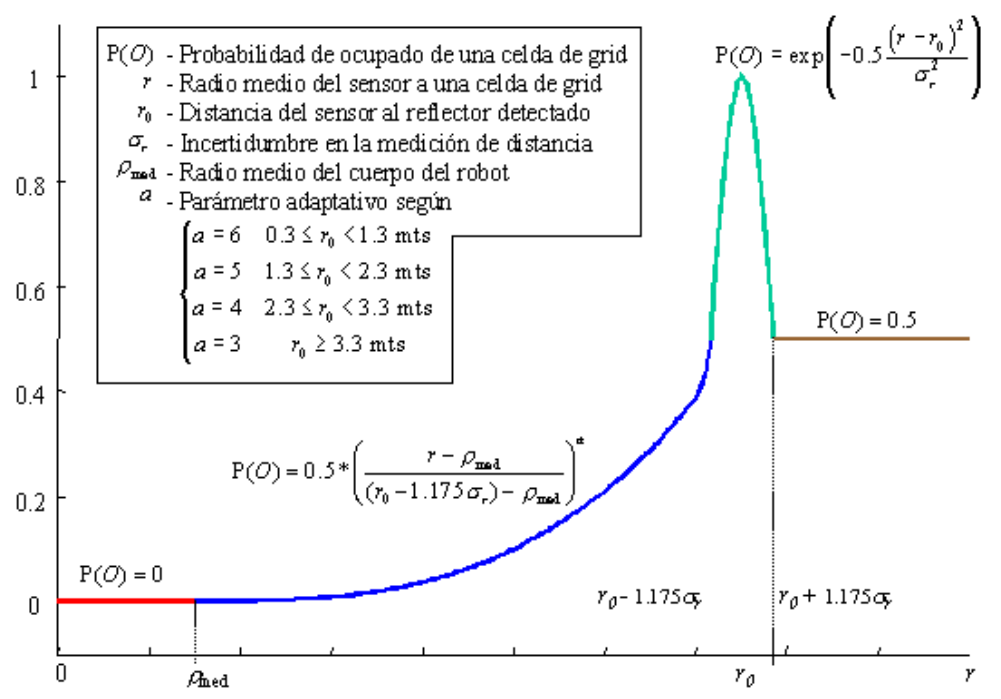

Figura 4.10: Forma unidimensional de la función de distribución de probabilidad de ocupación respecto a la distancia medida desde el sensor.

mínima, mientras que la misma crece suavemente hacia las cercanías del arco de detección. Para modelar este efecto, una posibilidad es fusionar la función que modela la probabilidad debido a la incertidumbre angular en el cono con una que modele la probabilidad de ocupación en función de la distancia, como la mostrada en la sección $\left[\rho_{\text {med }}, r_{0}-1,175 \sigma_{r}\right]$ mostrada en la figura 4.10.

5. Típicamente los sensores de ultrasonidos están instalados en plataformas que tienen capacidad de moverse por el entorno. De aquí que cada vez que se hace una medición con el SONAR es de suponer que el espacio en las cercanías del mismo está vacío. Si el sensor esta instalado en el centro del vehículo, se puede afirmar que habrá cierto espacio alrededor del SONAR cuya probabilidad de estar ocupado será cero. Independientemente de la forma del vehículo, lo usual es definir una región circular de radio medio igual $\rho_{\text {med }}$. Esto se puede ver en la figura 4.11, donde se aprecia que la probabilidad de ocupación es cero en los alrededores del sensor.

Las consideraciones anteriores conducen a la formulación de un modelo cuyo mapa probabilístico se muestra en la figura 4.11. Un conjunto de ecuaciones que modelan la distribución de probabilidad propuesta es el siguiente, 


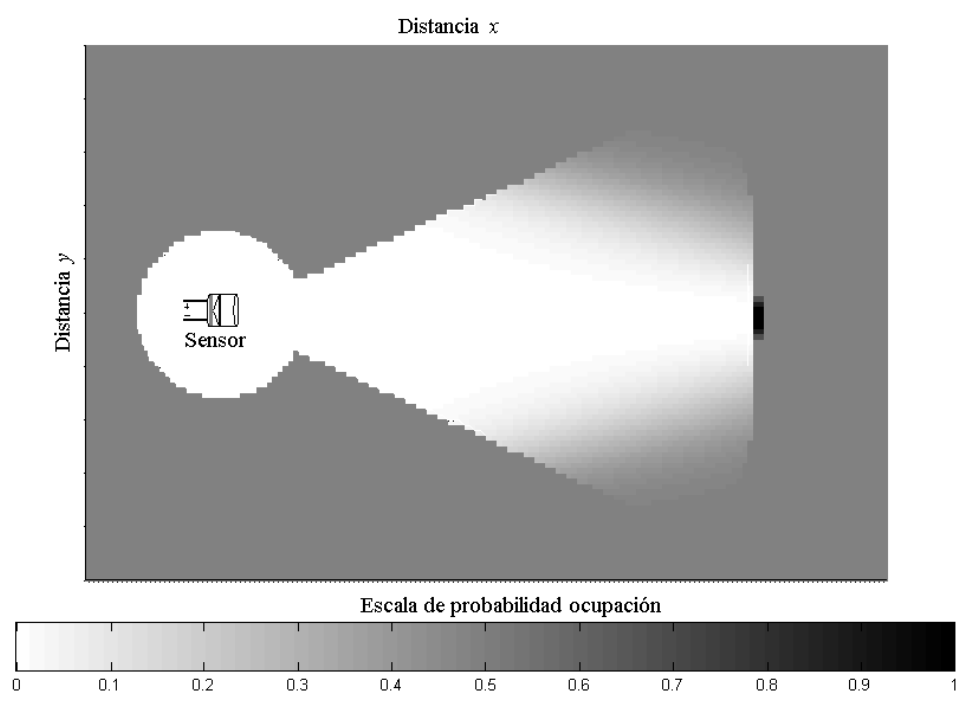

Figura 4.11: Mapa de probabilidades para la detección de un sólo reflector mediante un sensor de ultrasonidos.

$$
\mathrm{P}(O \mid r, \theta)= \begin{cases}0 & 0 \leq r \leq \rho_{\text {med }} \\
\mathrm{P}_{\mathrm{C}}(O \mid \theta) \oplus \mathrm{P}_{\mathrm{C}}(O \mid r) & \left\{\begin{array}{c}
\rho_{\text {med }} \leq r<r_{0}-1,175 \sigma_{r} \\
\theta_{0}-1,175 b \leq \theta \leq \theta_{0}+1,175 b
\end{array}\right. \\
0,5+0,5 \mathrm{P}_{\mathrm{A}}(O \mid \theta) \mathrm{P}_{\mathrm{A}}(O \mid r) \quad\left\{\begin{array}{l}
r_{0}-1,175 \sigma_{r} \leq r \leq r_{0}+1,175 \sigma_{r} \\
\theta_{0}-1,175 \sigma_{\theta} \leq \theta \leq \theta_{0}+1,175 \sigma_{\theta}
\end{array}\right. \\
0,5 & \text { para cualquier otro caso }\end{cases}
$$

donde

$r$ es el radio medio del sensor a una celda de grid

$\theta$ es el ángulo medio del sensor a una celda de grid

$r_{0}$ es la distancia del sensor al reflector que devuelve el eco

$\theta_{0}$ es el ángulo del sensor al reflector que devuelve el eco

$\sigma_{r}$ es la incertidumbre del sensor en la medición de distancia

$\sigma_{\theta}$ es la incertidumbre del sensor en la medición del ángulo

$b$ es la mitad el ángulo incluido o cono sensible del sensor 
$\rho_{\text {med }}$ es el radio medio del cuerpo del robot

P. $(O \mid \cdot)$ corresponde a las distribuciones de probabilidad involucradas que son las siguientes:

$$
\begin{gathered}
\mathrm{P}_{\mathrm{C}}(O \mid \theta)=0,5-\exp \left[-0,5 \frac{\left(\theta-\theta_{0}\right)^{2}}{\left(\frac{r_{0}}{3 r} b\right)^{2}}\right] \\
\mathrm{P}_{\mathrm{C}}(O \mid r)=0,5\left(\frac{r-\rho_{\text {med }}}{r_{0}-1,175 \sigma_{r}-\rho_{\text {med }}}\right)^{a} \\
\mathrm{P}_{\mathrm{A}}(O \mid \theta)=\exp \left[-0,5 \frac{\left(\theta-\theta_{0}\right)^{2}}{\sigma_{\theta}^{2}}\right] \\
\mathrm{P}_{\mathrm{A}}(O \mid r)=\exp \left[-0,5 \frac{\left(r-r_{0}\right)^{2}}{\sigma_{r}^{2}}\right]
\end{gathered}
$$

donde $a$ es un parámetro adaptativo que toma sus valores según,

$$
\left\{\begin{array}{cc}
a=6 & \text { si } 0,3 \leq r_{0}<1,3 \mathrm{mts} \\
a=5 & \text { si } 1,3 \leq r_{0}<2,3 \mathrm{mts} \\
a=4 & \text { si } 2,3 \leq r_{0}<3,3 \mathrm{mts} \\
a=3 & \text { si } r_{0} \geq 3,3 \mathrm{mts}
\end{array}\right.
$$

En el conjunto de ecuaciones 4.2, el símbolo $\oplus$ se refiere al operador de suma simétrica no asociativa[26], el cual es una función de combinación definida por

$$
g(x, y)=\frac{x+y-x . y}{1+x+y-2 . x . y}
$$

En la figura 4.12 se muestra el modelo probabilístico bivariante en 3D para el caso en el que se detecta un sólo reflector.

La validez del modelo propuesto para el sensor de ultrasonidos se probó en un ambiente estacionario de aproximadamente $580 \times 280 \mathrm{cms}$, el cual fue dividido en celdas cuadradas de $2 \mathrm{cms}$ por lado. El entorno mencionado estaba totalmente libre de obstáculos y limitado por paredes lisas con propiedades de difracción ultrasónica no significativas. Con un escaner ultrasónico, construido con un sólo sensor montado sobre el eje de un motor de pasos con resolución de 1.8 grados, se realizó un barrido de 360 grados sobre el entorno ya descrito. En la figura 4.13 se muestra el resultado obtenido, donde se puede observar que el mapa en general es satisfactorio en lo que respecta a los bordes del área explorada, con excepción de los alrededores de las esquinas donde las celdas correspondientes son afectadas adversamente. Este problema particular lo solucionamos mas adelante mediante la aplicación de mascaras en las esquinas, para lo cual hace falta aplicar previamente un mecanismo de diferenciación de paredes y esquinas[146]. 


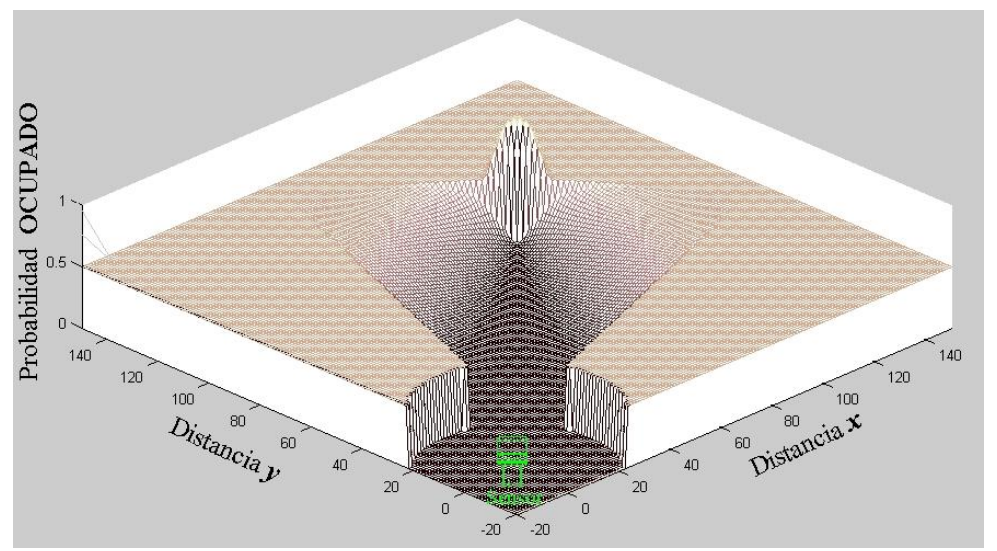

Figura 4.12: Modelo probabilístico para un reflector detectado a $150 \mathrm{~cm}$, visto desde el sensor a $45^{\circ}$. El sensor está localizado en las coordenadas $(0,0)$.

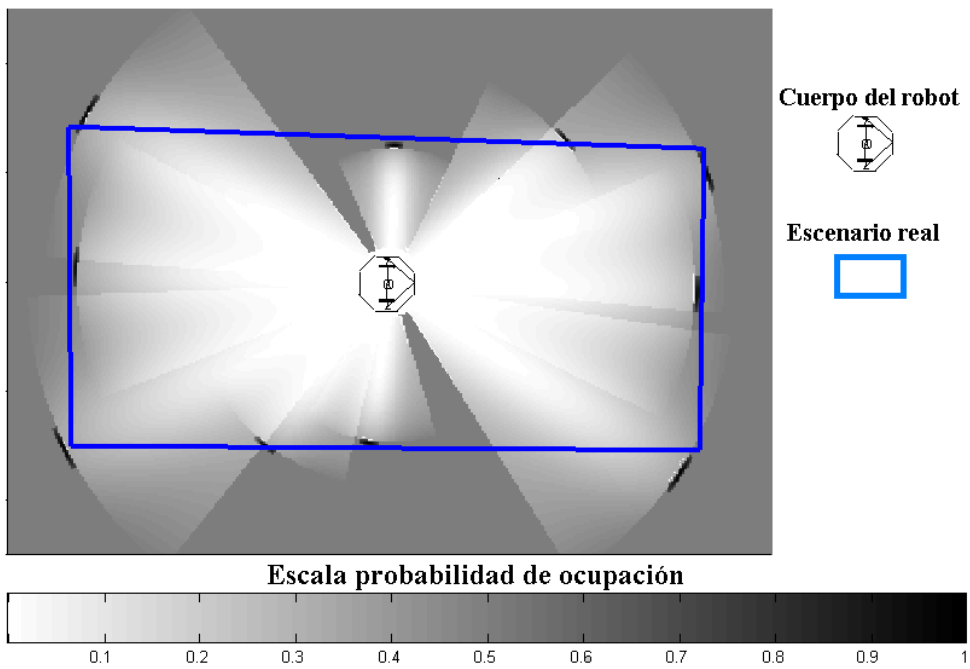

Figura 4.13: Mapa probabilístico de ocupación construido a partir de los datos adquiridos con el sensor de ultrasonidos del robot YAIR. 


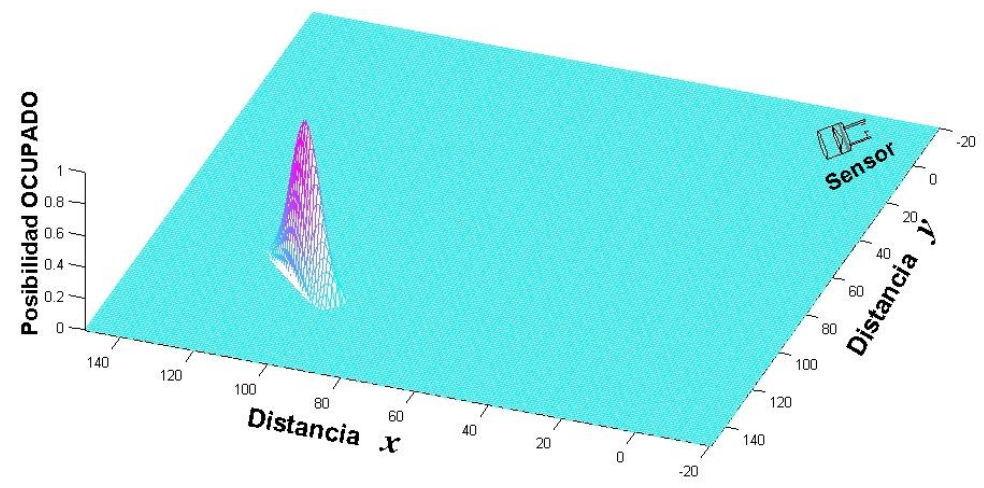

Figura 4.14: Forma de la función de proposición de ocupación usada en el método evidencial o en la lógica difusa para interpretar las medidas de distancias obtenidas con un SONAR. Se supone que un reflector fue detectado a una distancia radial de $150 \mathrm{~cm}$, y a un ángulo de vista de 45 grados.

\subsubsection{Modelo posibilista del SONAR}

La percepción del entorno a través de los sensores de un robot móvil es de por si un proceso caracterizado por la imprecisión y la incertidumbre. Aparte de la aproximación Bayesiana la cual es ampliamente usada para el tratamiento y fusión de datos inciertos[67, 141, 195], existen otras dos metodologías que también son usadas para los mismos fines: la lógica difusa[160] y el razonamiento evidencial[163, 78]. Para manejar la incertidumbre sensorial en la construcción de mapas de grid de espacios bivalentes $\{E, O\}$, en estas dos últimas metodologías se utilizan dos modelos sensoriales diferentes: un modelo que le da soporte a la proposición de que el espacio esté vacío $f_{E}(\cdot)$ y otro que le da soporte a la proposición de que el espacio este ocupado $f_{O}(\cdot)$. La descripción cualitativa del sensor en la sección 4.1.4, permite modelar las funciones que darán soporte al estado de las celdas, pero distinto que en el razonamiento Bayesiano donde la información sensorial respecto a un estado de las celdas debe ser complementaria (i.e $\mathrm{P}(O)=1-\mathrm{P}(E)$ ), en los modelos posibilísticos la información sensorial no tienen que cumplir necesariamente con este axioma. En la aproximación posibilista, las funciones de proposición no son en extremo complementarias por lo que se permite solapar parcialmente las mismas a fin de representar aquellas áreas donde la información del sensor es conflictiva, insuficiente o no concluyente.

Un conjunto de ecuaciones que permiten formular las proposiciones de ocupación y de vacío necesarias para la aplicación del razonamiento evidencial o la teoría difusa en la construcción de mapas de grid es el que se muestra a continuación.

$$
f_{O}(r, \theta)=\exp \left[-0,5 \frac{\left(r-r_{0}\right)^{2}}{\sigma_{r}^{2}}\right] \exp \left[-0,5 \frac{\left(\theta-\theta_{0}\right)^{2}}{\sigma_{\theta}^{2}}\right]
$$



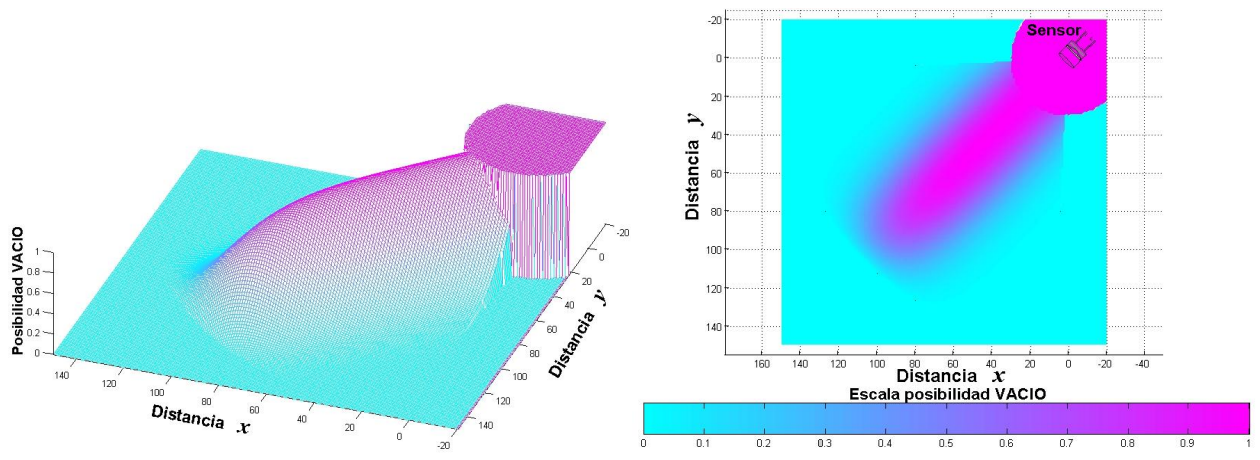

Figura 4.15: Forma de la función de proposición de VACÍO usada en el método evidencial o en la lógica difusa para interpretar las medidas de distancias obtenidas con un SONAR. Se supone un reflector fue detectado a una distancia radial de 150 $\mathrm{cm}$, a un ángulo de vista de 45 grados.

$$
f_{E}(r, \theta)= \begin{cases}1 & 0 \leq r \leq \rho_{\text {med }} \\
f_{E}(r) f_{E}(\theta) & \left\{\begin{array}{c}
\rho_{\text {med }}<r \leq r_{0} \\
\theta_{0}-2 b \leq \theta \leq \theta_{0}+2 b \\
0
\end{array}\right. \\
\text { para cualquier otro caso }\end{cases}
$$

donde las variables involucradas ya fueron definidas en la sección 4.1.4, y las funciones de proposición son las siguientes:

$$
\begin{gathered}
f_{E}(\theta)=\exp \left[-0,5 \frac{\left(\theta-\theta_{0}\right)^{2}}{\left(\frac{r_{0}}{3 r} b\right)^{2}}\right] \\
f_{E}(r)=1-\left(\frac{r-\rho_{\text {med }}}{r_{0}-\rho_{\text {med }}}\right)^{a}
\end{gathered}
$$

La forma de la función que asigna la posibilidad de ocupación se puede ver en la figura 4.14, mientras que la que asigna la posibilidad de celdas vacías se muestra en la figura 4.15 .

Este modelo posibilístico fue probado en las mismas condiciones descritas en la subsección 4.1.4, obteniéndose los resultados mostrados a través de los mapas de las figuras 4.16 y 4.17 .

\subsubsection{Mejoras al mapeado ultrasónico aplicando máscaras en las esquinas}

Hasta en el momento, en la construcción de mapas de ocupación mediante ultrasonidos, el modelo sensorial que se ha venido utilizando es el que está basado en la característica de sensibilidad del transductor ultrasónico. En algunos trabajos[173, 113] 


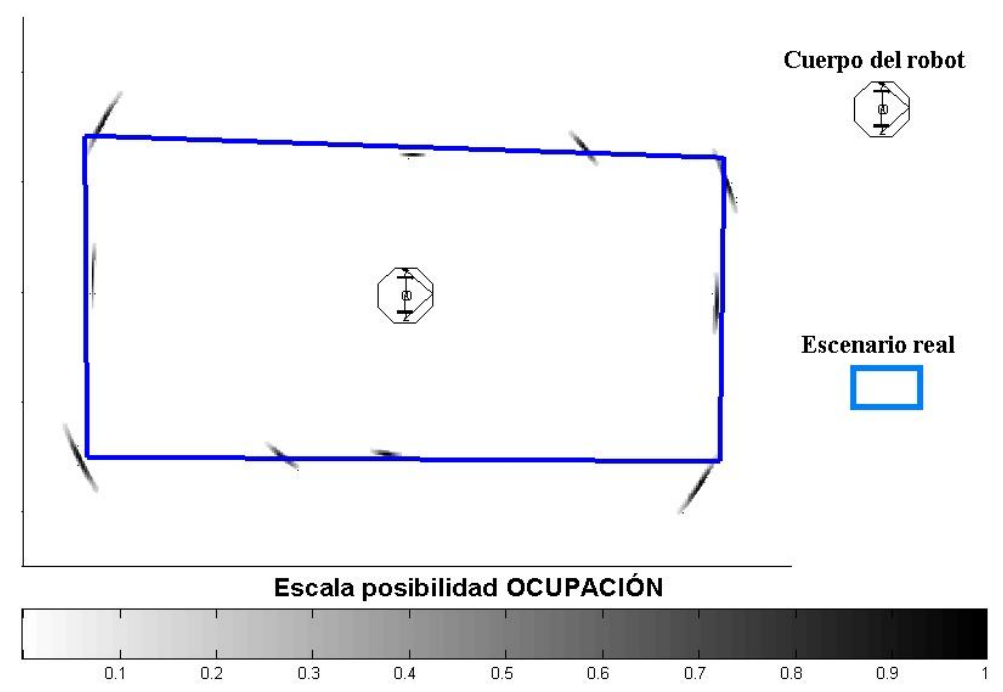

Figura 4.16: Mapa de un salón mostrando la asignación básica de probabilidad para las celdas OCUPADAS obtenida en un simple barrido del SONAR de YAIR.

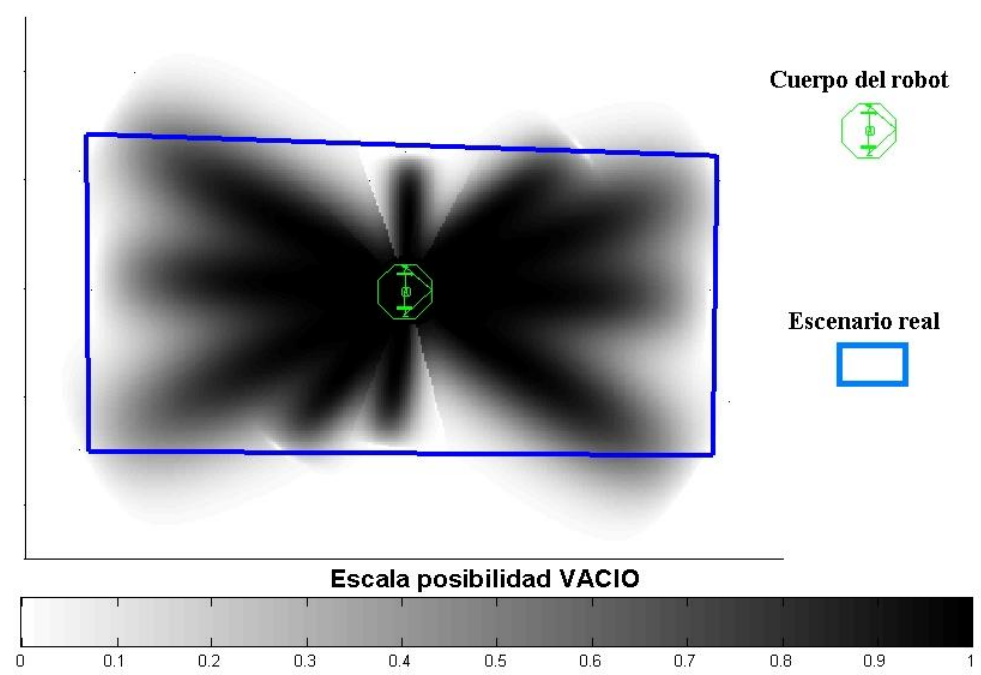

Figura 4.17: Mapa de un salón mostrando la asignación básica de probabilidad para las celdas VACÍAS obtenida en un simple barrido del SONAR de YAIR. 


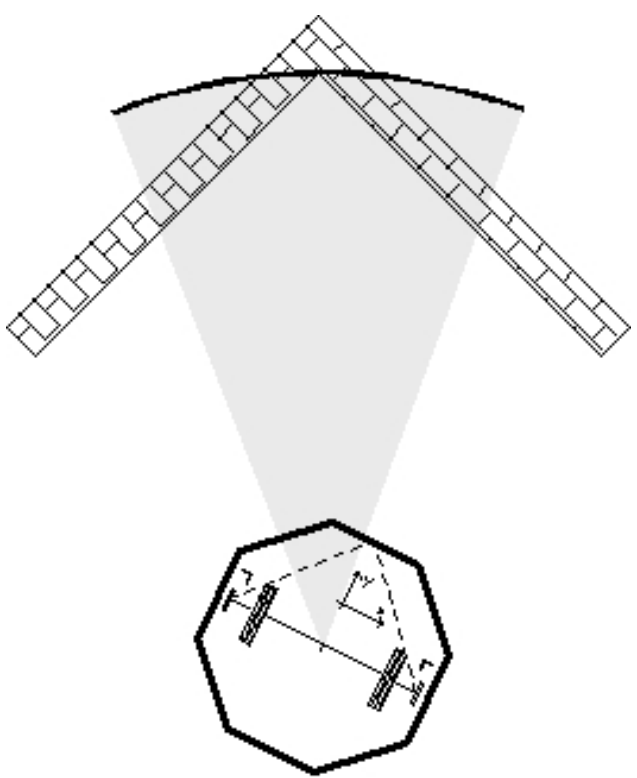

a)

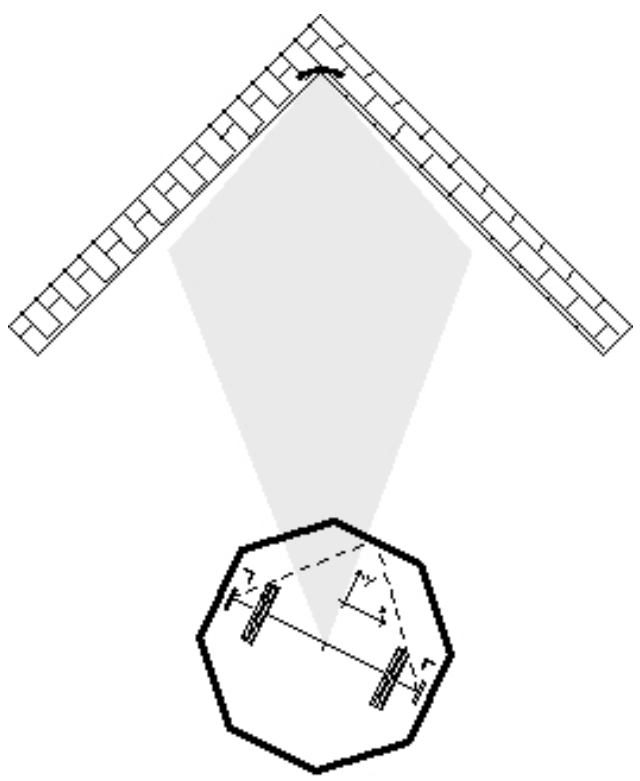

b)

Figura 4.18: a) Situación típica donde la aplicación del modelo sensorial a un reflector tipo esquina deja ocultos objetos de la escena. b) Aplicación del modelo sensorial modificado mediante una mascara de esquinas que protege los objetos que conforman la misma

se han propuestos modelos más elaborados como los desarrollados en la secciones 4.1.4 y 4.1.5 de esta tesis, obteniéndose buenos resultados. Sin embargo, todos estos modelos tienen un inconveniente en común, y es que mapean incorrectamente los alrededores de las esquinas. Esta problemática se presenta cuando el sensor no puede distinguir entre los ecos reflejados por paredes y los ecos reflejados por esquinas. Esta incapacidad causa que el espacio que es ocupado por las paredes que conforman las esquinas, sea tratado como un espacio vacío, deteriorándose así la calidad del mapa generado. Este efecto puede observarse en la figura 4.18-a). Igualmente, más allá del simple error de entrada perceptual, está el hecho de que algunos algoritmos de mapeado (los basados en técnicas probabilísticas) no tienen capacidad para regenerar fácilmente una zona que ha sido marcada como plenamente vacía, por lo que el problema de la calidad del mapa persistirá en el tiempo. Aunque los algoritmos basados en técnicas posibilísticas, como los basados en lógica difusa (ver [160] ), están es capacidad de superar este tipo de situaciones, nosotros proponemos a continuación un modelo sensorial orientado a la resolución del problema en el marco probabilísticos y a la posible mejora en el marco posibilístico.

La propuesta que hacemos consiste en utilizar un modelo sensorial modificado que considere la diferencia entre paredes y esquinas, y que cuando se trate de estas últimas aplique un enmascaramiento que proteja los objetos laterales que conforman las mismas. La descripción gráfica de esta propuesta se puede ver en la figura 4.18-b). 
Obviamente, para aplicar esta estrategia es necesario que el sensor tenga capacidad de extraer esquinas de una escena, y en este sentido el SONAR que se usa en esta tesis es capaz de extraer este tipo de características basándose en la amplitud y el tiempo de vuelo del eco ultrasónico devuelto[146]. El modelo de amplitud del eco que se propone para este sensor es el siguiente[20]

$$
A(r, \theta)=\mathrm{A}_{0} \mathrm{C}_{r}^{\mathrm{N}} \frac{e^{-2 \alpha r}}{2 r} e^{-4 \theta^{2} / \theta_{0}}
$$

donde $A$ es el pico de amplitud obtenido en el receptor de ultrasonidos, $\mathrm{A}_{0}$ es una constante del transductor, $\alpha$ es el coeficiente de atenuación en el aire, $r$ es la distancia desde el sensor al reflector, y $\mathrm{C}_{r}$ es el coeficiente de reflexión acústica de la superficie reflectora, el cual varia entre 0 y 1 . Adicionalmente, $\mathrm{N}$ es un parámetro que depende del tipo de reflector y representa el número de reflexiones antes de que el eco alcance el receptor. De esta manera, $\mathrm{N}=1$ para paredes, $\mathrm{y} N=2$ cuando se trata de esquinas. Si en la ecuación 4.13 se resuelve para $\mathrm{N}$, entonces es posible determinar que tipo de reflector fue el que devolvió el eco ultrasónico. Los resultados experimentales obtenidos para este estrategia de clasificación muestran un porcentaje de aciertos entre el 80 y el $90 \%$ cuando se trata de paredes, y del $80 \%$ cuando se trata de esquinas que forman ángulos rectos. Igualmente, los resultados mostraron que las esquinas pueden ser detectadas siempre y cuando el sensor no este apartado más allá de los 30 grados con respecto a la bisectriz del cuadrante que forman las paredes que constituyen la esquina.

Para mostrar la mejora general que introduce el uso de máscaras en las esquinas en la construcción de mapas de ocupación, se aplicó el modelo sensorial modificado al mismo escenario descrito en la sección 4.1.4. La máscara aplicada consistió en un cono cuyo vértice se localiza en el punto más probable de reflexión $\left(r_{0}, \theta_{0}\right)$, y cuyo ángulo incluido es igual a 60 grados. Este ángulo es un valor conservativo que surge heuristicamente después de realizar suficientes ensayos para determinar la capacidad de extracción de esquinas del sensor del robot YAIR. A manera de comparación, en la figura 4.19 se muestran el mapa de un barrido ultrasónico en un salón vacío obtenidos con y sin aplicación de las máscara de esquina para el caso de modelo probabilístico del SONAR del YAIR.

\subsubsection{Construcción de mapas de ocupación mediante fusión senso- rial.}

El problema de la construcción de mapas a partir de datos de ultrasonidos se hace difícil debido a la gran cantidad de incertidumbre presente en el proceso de la percepción. En particular, el gran ángulo de apertura que posee el SONAR lo inhabilita para localizar fiablemente un reflector dentro de su cono sensible y además lo hace más susceptible a la detección de objetos falsos debido a las reflexiones múltiples. Para considerar la incertidumbre inherente a este sensor, la investigaciones se ha enfocado en métodos probabilísticos de modelado del SONAR. Así, los investigadores típicamente aplican mayores probabilidades al eco generado cerca del eje del sensor 
CAPÍTULO 4. PERCEPCIÓN, FUSIÓN SENSORIAL Y CONSTRUCCIÓN DE MAPAS

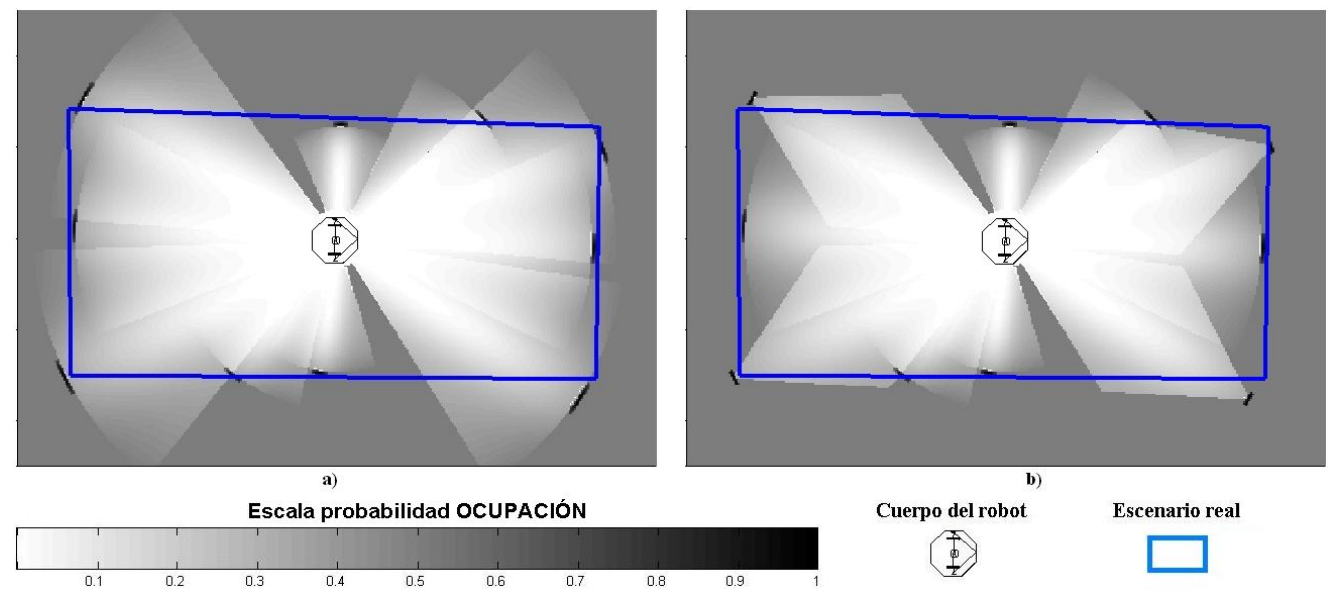

Figura 4.19: Comparación de mapas probabilísticos de ocupación. a) Sin aplicar máscara en las esquinas. b) Aplicando una máscara cónica de 60 grados en las esquinas

y bajas probabilidades a los lados del mismo[65, 149, 29, 131]. Otros investigadores han enfocado el problema mediante la combinación de múltiples lecturas de un mismo sensor. Así, J. Leonard y H. Durrant-Whyte usaron barridos de un SONAR rotativo a pasos muy finos(0.588 grados por paso) para localizar lugares geométricos de distancia constante a las que llamaron RCD (Regions of Constant Depth)[127]. Una RCD la definieron como una región donde las lecturas contiguas del sonar no difieren más de $1 \mathrm{~cm}$ y tiene al menos un ancho mínimo (típicamente de 10 grados). Con la creación de las RCD se mostró una forma efectiva para el garantizar que las lecturas provienen del lóbulo central de emisión del sonar. La limitación práctica del método es el tiempo empleado en realizar las lecturas. Lee también utilizó un SONAR rotativo, realizando las lecturas cada 18 grados, y construyendo un mapa geométrico basado en características puntuales y líneas rectas. En la metodología de Lee, las lecturas del SONAR que indican el valor máximo de distancia $(2.53 \mathrm{~m}$.) son desechadas. En las lecturas restantes se buscan grupos de lecturas adyacentes cuyos valores no difieran por más de $3 \mathrm{~cm}$. La lectura del grupo se calcula como la mínima de las lecturas involucradas y la dirección como el promedio de direcciones. Otra solución orientada a reducir la incertidumbre angular en las lecturas del SONAR, es la que ya se presentó en la sección 4.1.3. Esta última metodología se basa en el seguimiento de la máxima amplitud del eco en una misma RCD, y con ella se logra reducir la incertidumbre angular a $\pm 3^{\circ}$, a costa de hacer un barrido refinado de $360^{\circ}$ a pasos de $1.8^{\circ}$.

A pesar de que las soluciones descritas anteriormente proporcionan resultados aceptables, una solución más generalizada que puede ser adoptada sin excluir las mejoras ya alcanzadas, es utilizar la fusión sensorial. Esta técnica implica que la mediciones de un mismo sensor que han sido tomadas en distintos instantes de tiempo, o las mediciones sobre la misma variable que proporcionan distintos sensores, deben ser combinadas para acentuar o disminuir las posibilidades de ocupación que se le 


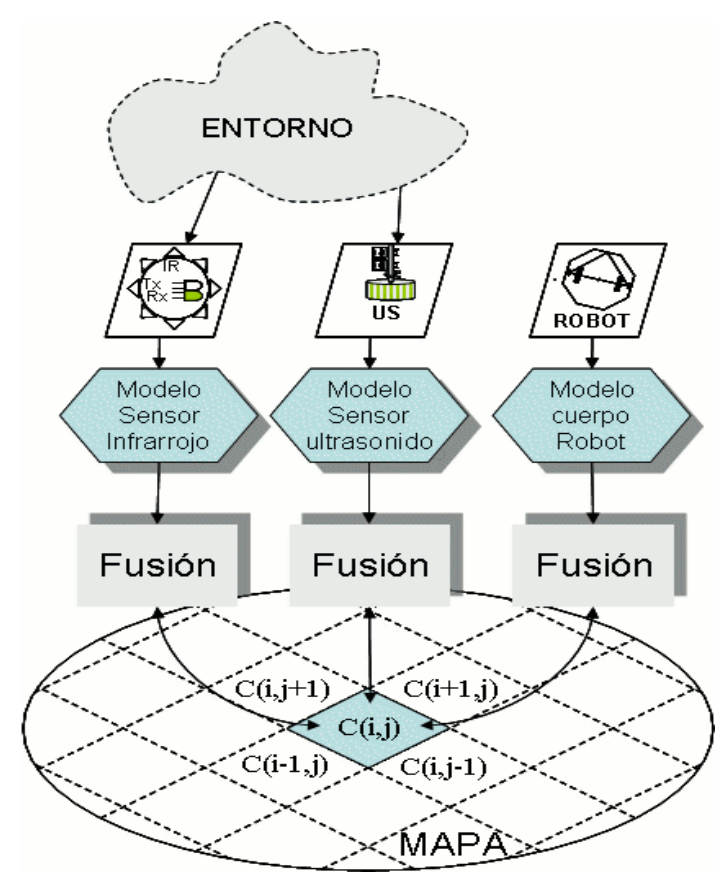

Figura 4.20: Esquema de fusión multisensorial para la construcción de mapas de grid.

ha otorgado a un área en especifico. En esta tesis se propone el uso del esquema de fusión representado en la figura (4.20). Este esquema permite la fácil inserción de sensores de distinta naturaleza y es compatible con cualquiera de las metodologías existentes para la fusión de datos a nivel de señal. Particularmente, en la construcción de mapas de grid mediante datos de ultrasonido, son tres las técnicas básicas usadas para la fusión de datos a nivel de señal: fusión Bayesiana[67, 141], fusión Combinatoria Evidencial[163], y fusión Posibilistica basada en lógica Fuzzy[160]. En adelante se desarrolla la aplicación de las dos primeras metodologías antes mencionadas, y se muestran los resultados con ellas obtenidos.

\subsubsection{Construcción de mapas mediante fusión Bayesiana}

La fusión Bayesiana es un método probabilístico muy atractivo usado en la construcción de mapas de grid. Esto se debe a que la metodología facilita la actualización recursiva e incremental de las probabilidades condicionales involucradas en el proceso. Asumiendo un espacio de trabajo que ha sido dividido mediante celdas de grid bidimensionales $C_{i j}$, las cuales pueden tener uno de dos estados (E: vacío, O: ocupado), el objetivo de esta metodología es determinar la probabilidad que tiene cada celda de estar ocupada $\mathrm{P}\left(C_{i j}=\mathrm{O}\right)$.

En el método Bayesiano, se asume que el estado de las celdas son independientes entre sí, y que en una misma celda se cumple que, 


$$
\mathrm{P}\left(C_{i j}=\mathrm{O}\right)+\mathrm{P}\left(C_{i j}=\mathrm{E}\right)=1
$$

Igualmente, cuando no se tiene información sobre el estado de la celda, se asume que su probabilidad de ocupación es 0.5. De aquí que el algoritmo de mapeado se inicia poniendo todas las celdas a este valor

$$
\mathrm{P}\left(C_{i j}=\mathrm{O}\right)=0,5 \quad \forall i, j
$$

Luego, después de activar los sensores y recibir una lectura $r$, la probabilidad de ocupación de la celda es actualizada utilizando el teorema de Bayes según,

$$
\mathrm{P}\left(C_{i j}=\mathrm{O} \mid r\right)=\frac{\mathrm{P}\left(r \mid C_{i j}=\mathrm{O}\right)}{\mathrm{P}(r)} \mathrm{P}\left(C_{i j}=\mathrm{O}\right)
$$

Aquí, la probabilidad condicional $\mathrm{P}\left(C_{i j}=\mathrm{O} \mid r\right)$ representa la probabilidad de que una determinada celda $C_{i j}$ este ocupada dado que se ha recibido una medida de distancia $r$; mientras que $\mathrm{P}\left(C_{i j}=\mathrm{O}\right)$ es el conocimiento o la evidencia a priori que se tiene sobre el estado de ocupación de la misma celda. Ahora bien, dado que se esta trabajando en un espacio muestral bivaluado $\{\mathrm{E}, \mathrm{O}\}$, de acuerdo al teorema de la probabilidad total se cumple que,

$$
\mathrm{P}(r)=\sum_{\mathrm{X} \in\{\mathrm{E}, \mathrm{O}\}} \mathrm{P}\left(r \mid C_{i j}=\mathrm{X}\right) \mathrm{P}\left(C_{i j}=\mathrm{X}\right)
$$

Esta última ecuación permite rescribir la ecuación 4.16 de acuerdo a

$$
\mathrm{P}\left(C_{i j}=\mathrm{O} \mid r\right)=\frac{\mathrm{P}\left(r \mid C_{i j}=\mathrm{O}\right) \mathrm{P}\left(C_{i j}=\mathrm{O}\right)}{\mathrm{P}\left(r \mid C_{i j}=\mathrm{O}\right) \mathrm{P}\left(C_{i j}=\mathrm{O}\right)+\mathrm{P}\left(r \mid C_{i j}=\mathrm{E}\right) \mathrm{P}\left(C_{i j}=\mathrm{E}\right)}
$$

En la ecuación anterior, la probabilidad condicional P $\left(r \mid C_{i j}=\mathrm{O}\right)$ es la función de verosimilitud de los datos de distancia. Esto normalmente se corresponde con el modelo sensorial, ya que el mismo representa la probabilidad de registrar la distancia $r$ dado que la celda $C_{i j}$ está ocupada. En esta tesis se modela esta función de verosimilitud mediante las ecuaciones desarrolladas en la sección 4.1.4.

También, en el denominador de la ecuación 4.18 aparece la probabilidad condicional $\mathrm{P}\left(r \mid C_{i j}=\mathrm{E}\right)$ la cual es conocida como un falso positivo, ya que la misma representa la probabilidad de registrar la distancia $r$ dado que la celda está vacía. Esta probabilidad es difícil de modelar, pero en general se acepta que una buena aproximación de la misma se consigue con la ecuación

$$
\mathrm{P}\left(r \mid C_{i j}=\mathrm{E}\right)=1-=1-\mathrm{P}\left(r \mid C_{i j}=\mathrm{O}\right)
$$

Con esta última aproximación, la ecuación de actualización de la probabilidad de ocupación de las celdas de grid se convierte en,

$$
\mathrm{P}\left(C_{i j}=\mathrm{O} \mid r\right)=\frac{\mathrm{P}\left(r \mid C_{i j}=\mathrm{O}\right) \mathrm{P}\left(C_{i j}=\mathrm{O}\right)}{\mathrm{P}\left(r \mid C_{i j}=\mathrm{O}\right) \mathrm{P}\left(C_{i j}=\mathrm{O}\right)+\left(1-\mathrm{P}\left(r \mid C_{i j}=\mathrm{O}\right)\right)\left(1-\mathrm{P}\left(C_{i j}=\mathrm{O}\right)\right)}
$$


Esta ecuación es fácilmente transformable en una fórmula recursiva si se considera que la probabilidad de ocupación a priori del momento actual, es la probabilidad de ocupación a posteriori que se obtuvo en el instante de tiempo anterior. Esto es,

$$
\mathrm{P}_{k}\left(C_{i j}=\mathrm{O}\right)=\mathrm{P}_{k-1}\left(C_{i j}=\mathrm{O} \mid r\right)
$$

De esta manera, la fórmula recursiva para la actualización de la probabilidad de ocupación de un celda de grid es

$$
\mathrm{P}_{k}\left(C_{i j}=\mathrm{O} \mid r\right)=\frac{\mathrm{P}_{k}\left(r \mid C_{i j}=\mathrm{O}\right) \mathrm{P}_{k-1}\left(C_{i j}=\mathrm{O} \mid r\right)}{\mathrm{P}_{k}\left(r \mid C_{i j}=\mathrm{O}\right) \mathrm{P}_{k-1}\left(C_{i j}=\mathrm{O} \mid r\right)+\left(1-\mathrm{P}_{k}\left(r \mid C_{i j}=\mathrm{O}\right)\right)\left(1-\mathrm{P}_{k-1}\left(C_{i j}=\mathrm{O} \mid r\right)\right)}
$$

En la figura 4.21 se muestra el resultado experimental obtenido al implementar esta técnica de fusión junto con el modelo sensorial desarrollado en la sección 4.1.4. El entorno explorado es un pasillo de aproximadamente 40 metros de largo, en el cual hicimos que el robot navegara deteniéndose en algunos sitios para realizar un barrido de 360 grados con su sensor de ultrasonidos. La localización del robot fue medida manualmente a fin de anular la incertidumbre que introduce el sistema de localización. A través de esta experiencia se observó que la fusión Bayesiana no sólo permite reforzar la evidencia de ocupación o de vacío de las distintas zonas del entorno, sino que también permite la construcción de mapas de ocupación de forma incremental, lo que viene a resolver el problema del alcance limitado que poseen los sensores. Igualmente, la utilidad del modelo sensorial propuesto en esta tesis se pone de manifiesto cuando se observa que en el mapa resultante se conservan las características esenciales que definen las esquinas del entorno presentes en el entorno.

\subsubsection{Construcción de mapas mediante la teoría evidencial}

Esta metodología usa la formulación establecida en la teoría de la evidencia de Dempster-Shafer para determinar el soporte a las proposiciones de ocupación (O) y de vacío (E) de un área del entorno de trabajo de un robot móvil. En este caso el entorno hay que discretizarlo en celdas que estarán caracterizadas por dos estados: ocupado y vacío. De esta manera el universo de discernimiento $\Theta$ estará definido por el conjunto,

$$
\Theta=\{\mathrm{O}, \mathrm{E}\}
$$

donde $\mathrm{O}$ y E corresponden a la posibilidad de que la celda este ocupada o vacía, respectivamente. También, los posibles subconjuntos del universo de discernimiento queda definido por,

$$
\Lambda=2^{\Theta}=\{\emptyset, \mathrm{O}, \mathrm{E},\{\mathrm{O}, \mathrm{E}\}\}
$$

El estado de cada celda $C_{i j}$ se describe mediante la asignación básica de probabilidad $m(\cdot)$ de cada una de los miembros de $\Lambda$; tal que si se asume que $m_{i j}(\emptyset)=0$, entonces 

MAPAS

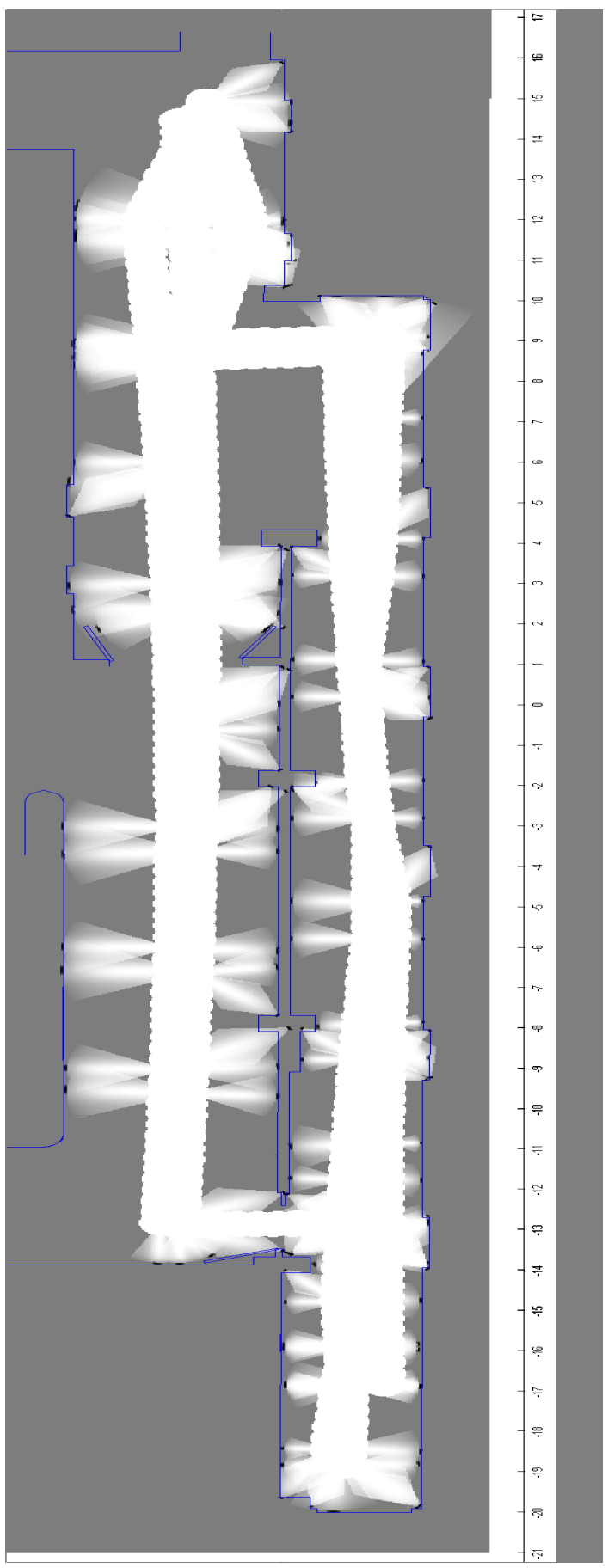

Figura 4.21: Mapa probabilístico de ocupación construido mediante fusión Bayesiana. Los lugares más claros indican espacios vacíos, mientras que los más oscuros representan los espacios ocupados. La escala está en metros. 


$$
m_{i j}(\mathrm{O})+m_{i j}(\mathrm{E})+m_{i j}(\{\mathrm{O}, \mathrm{E}\})=1
$$

Considerando esta dependecia lineal, es suficiente con registrar la asignaciones básica de probabilidad $m_{i j}(\mathrm{O})$ y $m_{i j}(\mathrm{E})$, para representar el estado de la celda $C_{i j}$. Estas asignaciones son determinadas con las ecuaciones del sensor desarrolladas en la sección 4.1.5.

Finalmente, en el proceso de construcción de un mapa de grid mediante la teoría de la evidencia, se combinan las distintas mediciones sensoriales con la evidencia previa existente en el mapa, para actualizar el mismo mediante la regla de la combinación de Dempster. Esta regla establece que para un universo $\Theta$, el soporte a una proposición $A$ dado dos fuentes de información se puede calcular según,

$$
m(A)=m_{1}(A) \oplus m_{2}(A)=\frac{\sum_{\forall a, b \subseteq \Theta \wedge a \cap b=A} m_{1}(a) \cdot m_{2}(b)}{1-\sum_{\forall a, b \subseteq \Theta \wedge a \cap b=} m_{1}(a) \cdot m_{2}(b)}
$$

Designando el supraescrito $M$ para la evidencia previa almacenadada en el mapa, y el supraescrito $S$ para la evidencia aportada por el sensor, la nueva asignación de probabilidad para las proposiciones de ocupación $(\mathrm{O})$ y de vacío(E) son las siguientes[163]:

$$
\begin{aligned}
& m(\mathrm{O})=m_{i j}^{M}(\mathrm{O}) \oplus m_{i j}^{S}(\mathrm{O})=\frac{m_{i j}^{M}(\mathrm{O}) m_{i j}^{S}(\mathrm{O})+m_{i j}^{M}(\mathrm{O}) m_{i j}^{S}(\{\mathrm{O}, \mathrm{E}\})+m_{i j}^{M}(\{\mathrm{O}, \mathrm{E}\}) m_{i j}^{S}(\mathrm{O})}{1-m_{i j}^{M}(\mathrm{O}) m_{i j}^{S}(\mathrm{E})-m_{i j}^{M}(\mathrm{E}) m_{i j}^{S}(\mathrm{O})} \\
& m(\mathrm{E})=m_{i j}^{M}(\mathrm{E}) \oplus m_{i j}^{S}(\mathrm{E})=\frac{m_{i j}^{M}(\mathrm{E}) m_{i j}^{S}(\mathrm{E})+m_{i j}^{M}(\mathrm{E}) m_{i j}^{S}(\{\mathrm{O}, \mathrm{E}\})+m_{i j}^{M}(\{\mathrm{O}, \mathrm{E}\}) m_{i j}^{S}(\mathrm{E})}{1-m_{i j}^{M}(\mathrm{O}) m_{i j}^{S}(\mathrm{E})-m_{i j}^{M}(\mathrm{E}) m_{i j}^{S}(\mathrm{O})}
\end{aligned}
$$

El algoritmo de actualización de la evidencia en el mapa de grid se inicia asignando $m_{i j}^{M}(\mathrm{O})=m_{i j}^{M}(\mathrm{E})=0$ para designar así la total ignorancia del estado de cada celda del mapa. La aplicación de este algoritmo en el mismo escenario y condiciones descritas en la sección 4.1.7.1, pero utilizando el modelo posibilístico del SONAR, se muestra en la figura 4.22. En esta figura nuevamente se puede observar la conveniencia del modelo sensorial con mascaras en las esquinas que se propone en esta tesis. De acuerdo al resultado, este modelo es útil para preservar las estructuras u objetos del mapa que conforman las esquinas.

\subsection{Construcción de mapas mediante el sensor infrarrojo}

La representación geométrica del entorno juega un papel importante en las aplicaciones de robótica móvil ya que la misma le da soporte a tareas como la localización, planificación de trayectorias y el control general de navegación de este tipo de vehículos. Una forma popular de representar un entorno es mediante características geométricas tales como puntos, círculos y líneas. La representación del entorno mediante líneas es una alternativa muy popular al uso de los mapas de grid. En la 


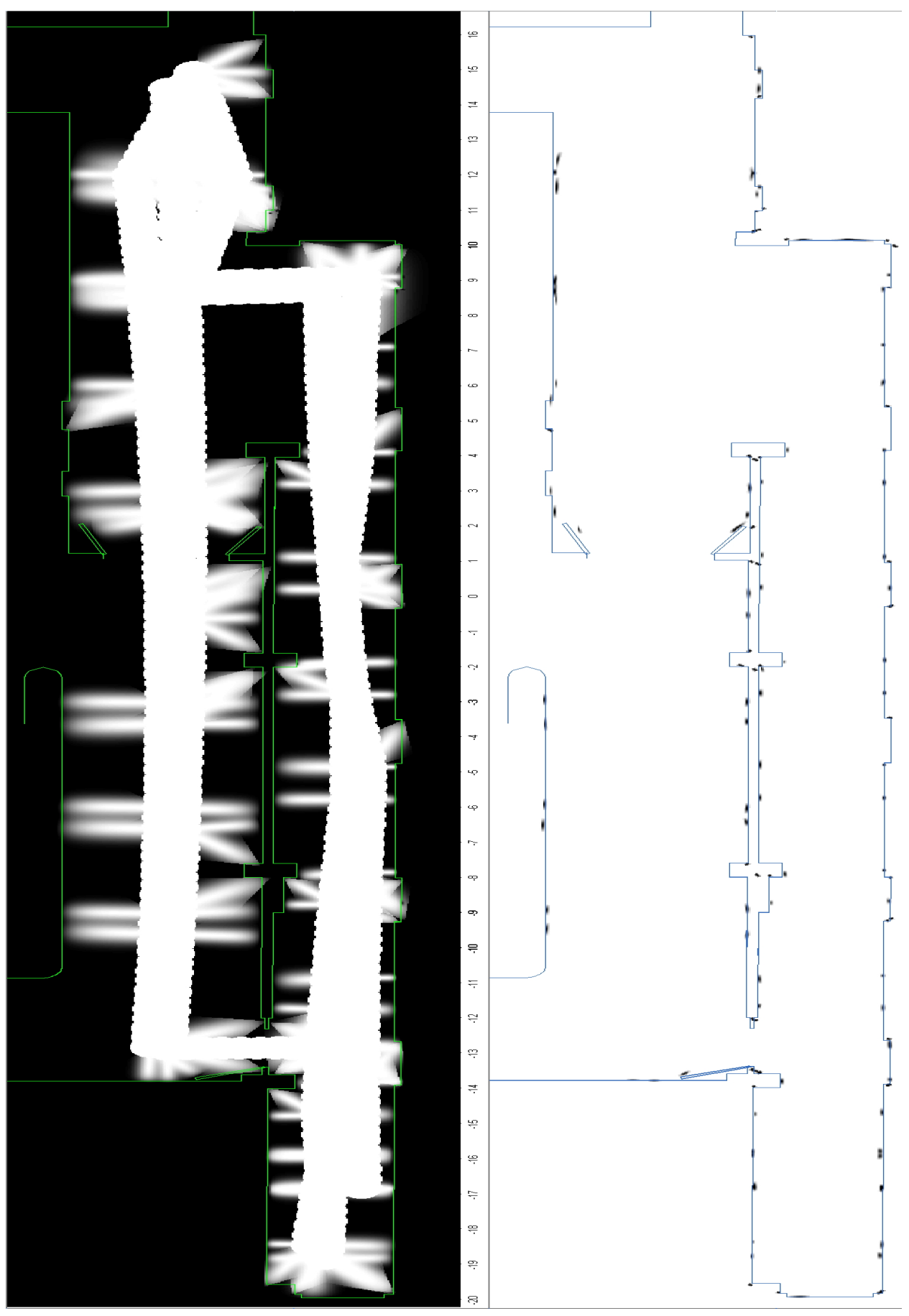

Figura 4.22: Mapas posibilísticos obtenidos mediante la fusión de medidas sensoriales provenientes de un SONAR. Izquierda: Mapa de evidencia de zonas vacías. Derecha: Mapa de evidencia de zonas ocupadas. La escala está en metros. 
representación de grandes espacios, los mapas de líneas requieren significativamente menos memoria que los mapas de ocupación, y además ofrecen mayor exactitud ya que no sufren el problema de discretización que existe con los mapas de rejilla. Los mapas de líneas están basados en la asunción de que todos los espacios interiores están conformados por estructuras planas como paredes, puertas, etc. Estos mapas son más compactos que lo tradicionales mapas de grid, ya que los objetos pueden ser efectivamente representados por un pequeño número de segmentos de línea.

El problema de la construcción de mapas de líneas a partir de la distancia medida a los objetos del entorno ha sido estudiada intensivamente. Sack y Burgard[177] hacen una breve revisión de las distintas metodologías que han sido utilizadas, al mismo tiempo que analizan y comparan tres de éstas: la incremental con procesamiento en línea, la del procesamiento por lotes fuera de línea, y una tercera basada en el algoritmo EM de máxima verosimilitud (EM - Expectation-Maximization). Desde el punto de vista sensorial, el problema de construcción de mapas de líneas se puede resolver usando varios tipos de sensores exteroceptivos. D. Lee construye un mapa de características basado en líneas y puntos, usando solamente un SONAR giratorio [124]. Leonard et al usan la Transformada Hough para extraer líneas a partir de una secuencia de mediciones ultrasónicas consecutivas[128]. Nguyen et al[157] presentan la evaluación experimental de 6 algoritmos de extracción de líneas que usan datos de distancia obtenidos con un sensor láser, y determinan, desde el punto de vista de desempeño global, que el algoritmo de segmegtación\&fusión(Split-and-Merge) y el algoritmo incremental son los que ofrecen mayores prestaciones para aplicaciones de tiempo real. Por otra parte Veeck y Burgard[200], así como también Arras y Siegwart[7], usan técnicas de agrupamiento para construir mapas del entorno basados en líneas extraídas de los datos obtenidos mediante barridos de un medidor de distancia láser. Una posibilidad poco explorada es el uso de sensores de distancia por infrarrojos. A pesar de que estos sensores tienen amplia disponibilidad, son económicos, proporcionan alta velocidad en la medición distancias y sus algoritmos de procesamiento de señal son computacionalmente ligeros; su uso en la robótica móvil ha estado orientado más que todo al proceso de la navegación con evitación de obstáculos $[135,139,86]$. El uso de los sensores de infrarrojos en procesos de construcción de mapas ha sido desatendido por impráctico, debido a que este sensor posee un alcance muy corto (alrededor de 1 metro)[73, 159]. Una solución a esta problemática consiste en adoptar metodologías de mapeado incremental, en las cuales el robot va construyendo el mapa mientras se va moviendo por el entorno. Benet et al usan una metodología de fusión basada en la teoría Bayesiana para construir mapas de ocupación con los datos de distancia adquiridos mediante un sensor de infrarrojos[18]. A partir de los resultados obtenidos por Benet y su grupo, en esta sección se extiende este trabajo y se desarrolla una metodología para la extracción y seguimiento de líneas. Aquí se comparan dos posibles estrategias: Extracción mediante Mínimos Cuadrados Recursivos y extracción mediante Filtrado de Kalman. Aunque las dos estrategias permiten fusionar datos ruidosos de forma óptima, en este caso el Filtro de Kalman es mas robusto ya que toma en cuanta la incertidumbre en los dos ejes del plano donde se representan las características del entorno. 

MAPAS

\subsubsection{Medición de distancias basados en infrarrojos}

Los sensores infrarrojos han sido ampliamente usados como detectores de proximidad binarios en aplicaciones de robótica móvil, sin embargo se han hecho pocos intentos de utilizar la característica de amplitud de la señal reflejada para propósitos de estimación de distancia o para la construcción de mapas del entorno. Esto debido sobretodo a su comportamiento no lineal y a la fuerte dependencia de la manera en que las superficies dispersan, absorben o reflejan el haz de infrarrojos. Existen modelos de infrarrojos comerciales que integran técnicas opto-electrónicas para determinar la distancia en rangos hasta de $10 \mathrm{~m}$, pero en general presentan la desventaja de poseer un alto costo. Korba et al[114] evalúan varios de estos dispositivos comerciales a fin de aplicarlo en robótica móvil. Novotny y Ferrier [159] hacen uso de un anillo de sensores de infrarrojos y del modelo de luminancia de Phong para determinar la distancia y la orientación de una superficie reflectora. En esta tesis se usa un novedoso sistema de medición de distancias basado en infrarrojos propuesto por Benet et al[17]. El sistema se basa en la fusión de la información proveniente de un arreglo de dos sensores infrarrojos que poseen la misma orientación (ver figura 4.23). Con este arreglo se logra cancelar o disminuir la dependencia del ángulo de incidencia sobre la superficie, que afecta a los sensores de este tipo.

Los sensores infrarrojos del robot YAIR están basados en la medición directa de la magnitud de la luz infrarroja que es reflejada desde una superficie que se encuentre frente a ellos. Si se asume una superficie difusa perfecta, la señal de amplitud $(V)$ que se recibe es no lineal con la distancia, y sigue una ley cuadrática inversa que depende de la distancia al reflector, de el ángulo de incidencia, y del coeficiente de reflexión de la superficie. Esta relación ya se introdujo en la ecuación (2.7) del capitulo 2. De a cuerdo a esta ecuación, para conocer la distancia entre el sensor y el objeto reflector es primordial conocer a priori el ángulo de incidencia $\eta$ y la característica de reflexión $\alpha_{s}$ de la superficie del objeto, según

$$
d=\sqrt{\frac{\alpha}{V}} \sqrt{\cos \eta}
$$

donde se ha sustituido $V=s(d, \eta)-\beta$, y $\alpha=\alpha_{n} \alpha_{s}$.

Dando por hecho que se puede determinar a priori la característica de reflexión típica $\alpha_{s}$ del entorno de trabajo del un robot, es entonces el ángulo de incidencia $\eta$ el que juega el papel más importante en la determinación de la distancia de un objeto.

Aunque en un entorno real el ángulo de incidencia $\eta$ es desconocido, como una primera aproximación se podría suponer que la incidencia es cercana a $0^{\circ}$. Esto supondría una sobreestimación de la distancia cuando lo anterior no sea estrictamente cierto. De aquí que si se puede estimar mejor el ángulo de incidencia, se podría corregir la estimación de la distancia previamente hecha.

El ángulo de incidencia $\eta$ se puede estimar mejor si se fusiona la información proporcionada por un par de sensores de infrarrojos, que aunque estén separados una cierta distancia $L$, tengan la misma orientación. 


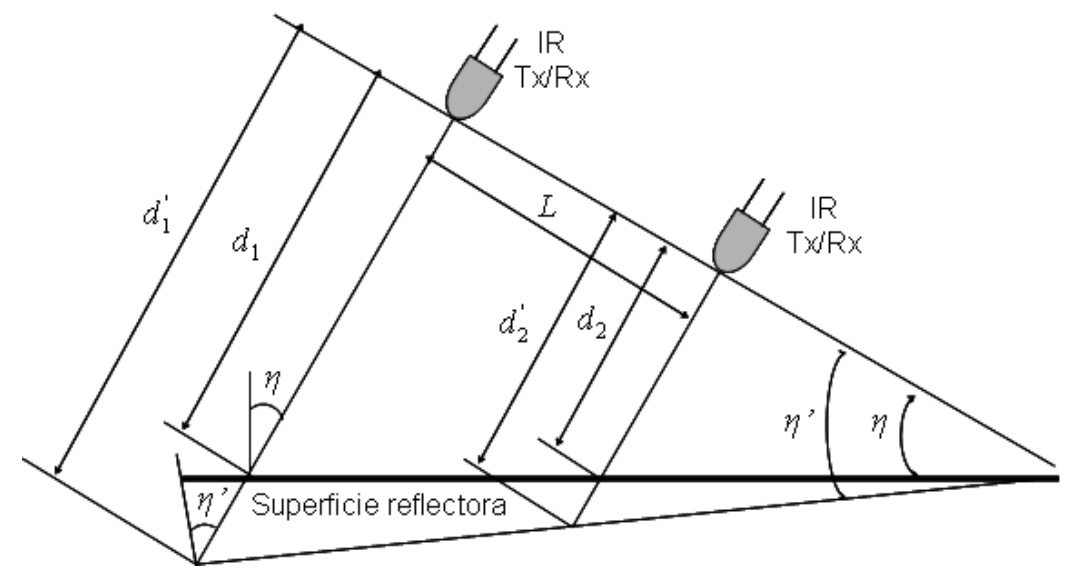

Figura 4.23: Incidencia del haz infrarrojo de dos sensores con igual orientación respecto a un único reflector.

Asumiendo que el ángulo de incidencia $\eta^{\prime}$ en la figura 4.23 es muy cercano a cero, las distancias erróneas estimadas en cada sensor corresponden a

$$
\left\{\begin{array}{l}
d_{1}^{\prime}=\sqrt{\frac{\alpha_{1}}{V_{1}}} \\
d_{2}^{\prime}=\sqrt{\frac{\alpha_{2}}{V_{2}}}
\end{array}\right.
$$

mientras que las distancias reales son,

$$
\left\{\begin{array}{l}
d_{1}=\sqrt{\frac{\alpha_{1}}{V_{1}}} \sqrt{\cos \eta}=d_{1}^{\prime} \sqrt{\cos \eta} \\
d_{2}=\sqrt{\frac{\alpha_{2}}{V_{2}}} \sqrt{\cos \eta}=d_{2}^{\prime} \sqrt{\cos \eta}
\end{array}\right.
$$

También de la figura 4.23 se puede extraer,

$$
\tan \eta=\frac{d_{1}-d_{2}}{L}=\left(\frac{d_{1}^{\prime}-d_{2}^{\prime}}{L}\right) \sqrt{\cos \eta}=R \sqrt{\cos \eta}
$$

por lo que para obtener el valor exacto de $\eta$ es necesario resolver una ecuación cúbica de la forma $R^{2} \cos ^{3} \eta+\cos ^{2} \eta-1=0$.

Una alternativa a la solución de esta ecuación cúbica, es la construcción de una tabla de pares $(R, \eta)$ que pueda ser usada para obtener un valor de $\eta$ aproximado mediante interpolación lineal. Por ejemplo, en la figura 4.24 se muestra la gráfica de 100 puntos $(R, \eta)$ obtenidos en el intervalo $\left[-45^{\circ},+45^{\circ}\right]$.

Por otra parte, una opción a la interpolación propuesta es ajustar los pares $(R, \eta)$ para obtener una expresión algebraica de la forma $\eta=a R+b R^{3}$, lo cual arroja como resultado $a=53,345441$ y $b=-11,905434$, con una desviación estándar de $0.5830191^{\circ}$ y un coeficiente de correlación de 0.9997581 . 

MAPAS

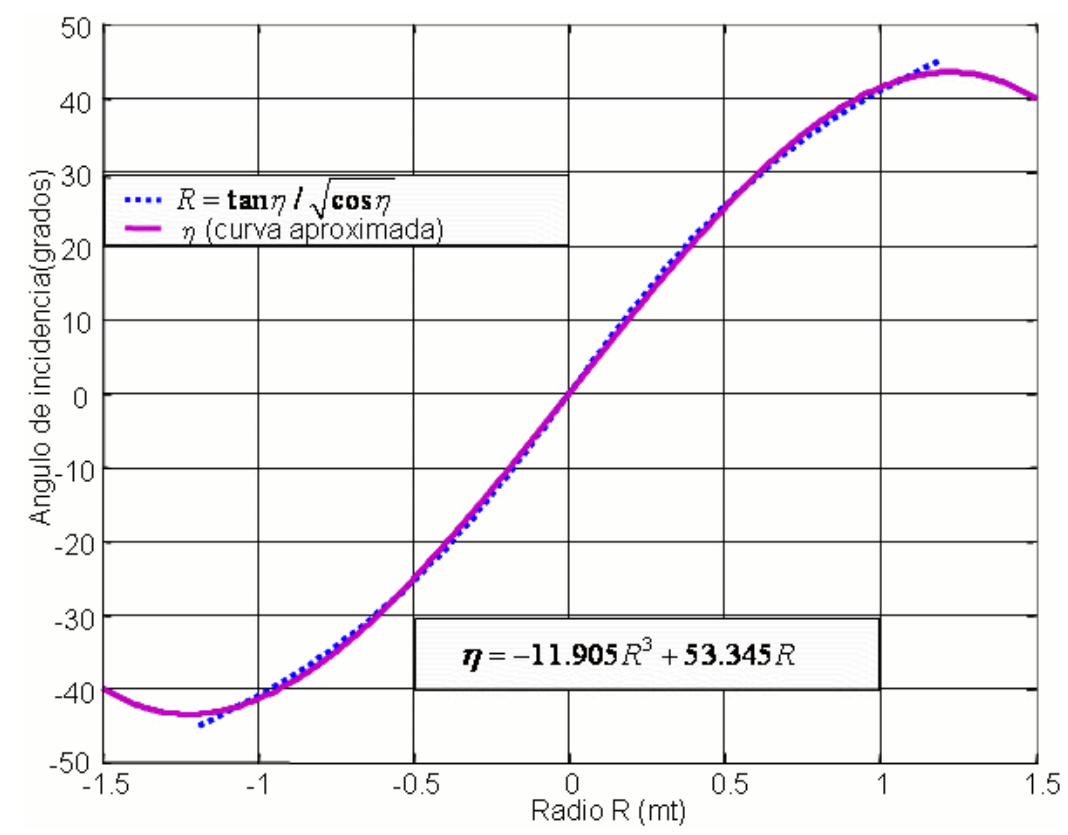

Figura 4.24: Función de interpolación gráfica del ángulo de incidencia $\eta$ como una función de $R$.

Una vez obtenido una mejor aproximación del ángulo de incidencia $\eta$, se puede calcular la distancia compensada $d_{1}$ y $d_{2}$ usando las ecuaciones en 4.31 , y la distancia media puede ser calculada de acuerdo a

$$
d=\frac{d_{1}^{\prime}+d_{2}^{\prime}}{2} \sqrt{\cos \eta}
$$

A partir de la ecuación 4.29, Benet et al[17] demuestran que la desviación estándar de la incertidumbre total en la medición de distancias mediante la metodología descrita anteriormente puede ser expresada como

$$
\sigma_{d_{i}}=\sqrt{\left(\frac{d_{i}^{3}}{2 \alpha_{i} \cos \eta}\right)^{2} \sigma_{V}^{2}+\left(\frac{d_{i} \tan \eta}{2}\right)^{2} \sigma_{\eta}^{2}}
$$

mientras que la desviación estándar de la incertidumbre en la determinación del ángulo puede ser estimada de acuerdo a

$$
\sigma_{\eta}=\sqrt{\left(\frac{\cos \eta}{L\left(\sin ^{2} \eta+2\right)}\right)^{2}\left(\frac{d_{1}^{6}}{\alpha_{1}^{2}}+\frac{d_{2}^{6}}{\alpha_{2}^{2}}\right) \sigma_{V}^{2}}
$$

En las ecuaciones 4.34 y $4.35, \sigma_{V}$ representa el ruido en la conversión de la señal recibida, mientras $\alpha_{i}=\alpha_{n} \alpha_{s}$ incluye la intensidad radiante de los emisores de 


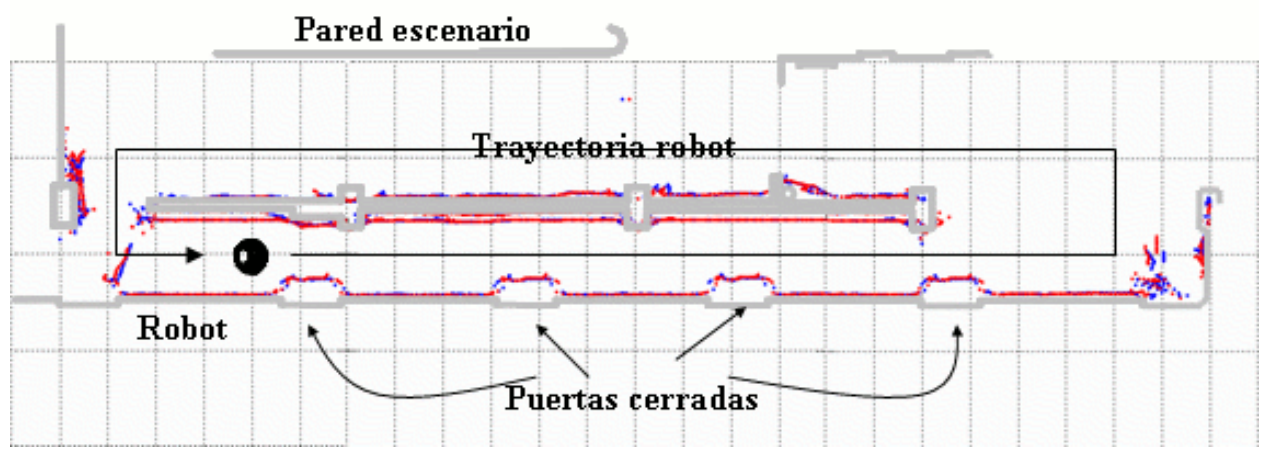

Figura 4.25: Mapa de infrarrojos obtenido por simple proyección en el plano cartesiano de las medidas de distancia resultantes de la fusión sensorial entre el par de sensores instalados en cada cara del octágono que forman el robot.

infrarrojos, la sensibilidad espectral de los fotodiodos receptores, la ganancia del amplificador, y el coeficiente de reflexión de la superficie refleclectora. Aquí el indice $i$ se usa para discriminar entre un sensor y otro.

Para validar este modelo de medición de distancias por infrarrojos, se programó al robot YAIR para que navegara a través de un pasillo, mientras recogía datos provenientes de su sensor de distancias por infrarrojos. Como resultado se obtuvo el mapa de la figura 4.25, el cual muestra ser bastante ajustado a la realidad. Sin embargo, también aparecen unos errores prominentes en las puertas, que se deben a que las mismas estaban recubiertas con un material con un coeficiente de reflectividad mayor al coeficiente de reflectividad típico que se fijó para hacer el ensayo.

Visto el problema que ocasiona el desconocimiento del coeficiente de reflectividad del entorno explorado, como trabajo futuro se pretende desarrollar una configuración multisensorial, que fusione la información provista por un sensor de ultrasonidos con uno de infrarrojos, para lograr la identificación automática del coeficiente de reflexión de la superficie observada.

Otro problema que se observa es la dispersión de los datos de distancia en algunos lugares del mapa. Esto le resta calidad, definición y exactitud al mapa. Para resolver este problema, en la sección 4.2.5 de esta tesis, se aplican, técnicas de agrupamiento, extracción y fusión de segmentos de líneas.

\subsubsection{Modelado de los reflectores extraídos mediante el sensor de infrarrojos}

El robot YAIR cuenta con un anillo de 16 sensores infrarrojos compuestos por dos LED emisores altamente direccionales $( \pm 10$ grados $)$ y por un fotodiodo PIN de alta velocidad que está rodeado por un encapsulamiento plástico negro que sirve como filtro a la luz ambiental. Los sensores se agrupan en 8 pares ubicados en cada una de las caras del cuerpo octogonal del robot, según se muestra en la figura (4.26). La separación entre sensores de un mismo par es de $15 \mathrm{cms}$. El LED emisor de 


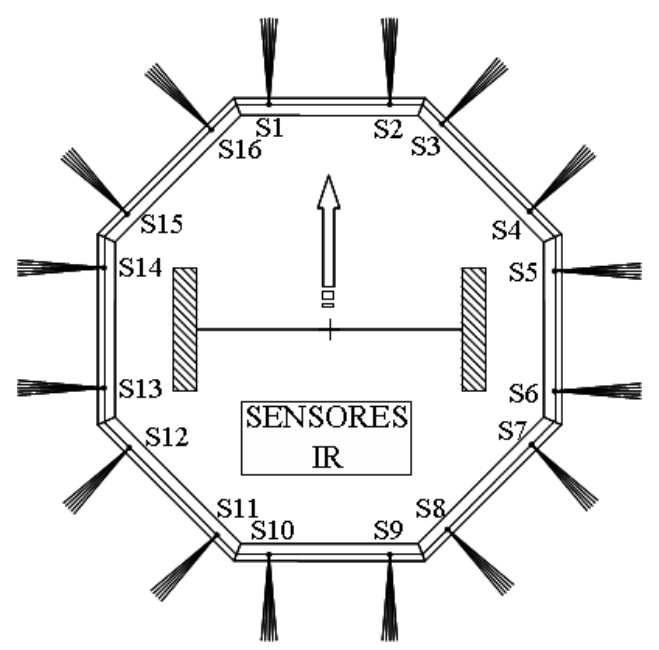

a)

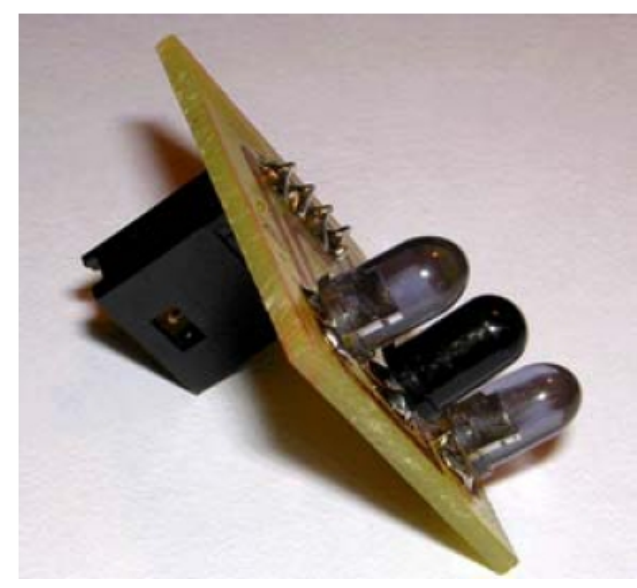

b)

Figura 4.26: a) Configuración del anillo de infrarrojos del robot YAIR. Existen 16 sensores agrupados en pares y distribuidos en el perímetro del cuerpo de robot. b) Detalle de un sensor de infrarrojos del robot YAIR. Nótese que esta compuesto por dos diodos emisores de color claro, y un diodo receptor de color oscuro.

infrarrojos usado tiene una intensidad radiante de $80 \mathrm{~mW} / \mathrm{sr} @ 100 \mathrm{~mA}$. El fotodiodo receptor tiene una sensitividad espectral de $50 \mathrm{microA} /(\mathrm{mW} / \mathrm{cm} 2) @ 900 \mathrm{~nm})$, con un lóbulo de sensibilidad de \pm 20 grados. Esta combinación le otorga al sensor un rango máximo de detección de aproximadamente 1 metro.

Los datos básicos de distancia registrados por cada uno de los sensores $S_{i}$ (ver figura 4.26), normalmente son adquiridos y almacenados continuamente mientras el robot se está moviendo, pero también se pueden registrar de manera selectiva y asincrónica en cualquier estado del robot.

La posición y la orientación de cada sensor $S_{i}$ con respecto al origen de coordenadas del marco ROBOT $\{\mathrm{R}\}$ son almacenadas en una tabla de configuración, la cual servirá para realizar cualquier operación de transformación que involucre los datos adquiridos por los sensores de infrarrojos. Así, para cada uno de los sensores se almacena lo siguiente(ver figura 4.27):

$\Delta x_{\mathbf{i}}$ Traslación en el eje $\mathrm{x}$ del origen de coordenadas del marco SENSOR $\{\mathrm{Si}\}$ con respecto al origen del marco $\operatorname{ROBOT}\{\mathrm{R}\}$

$\Delta y_{\mathbf{i}}$ Traslación en el eje y del origen de coordenadas del marco SENSOR $\{\mathrm{Si}\}$ con respecto al origen del marco ROBOT

$\beta_{\mathbf{i}}$ Rotación del marco SENSOR $\{\mathrm{Si}\}$ respecto al marco ROBOT $\{\mathrm{R}\}$

donde $i=[1, \ldots, 16]$ indica el numero del sensor.

En la figura 4.27 se puede observar que un punto perteneciende a un reflector se mapea en el marco $\operatorname{ROBOT}\{\mathrm{R}\}$ según, 


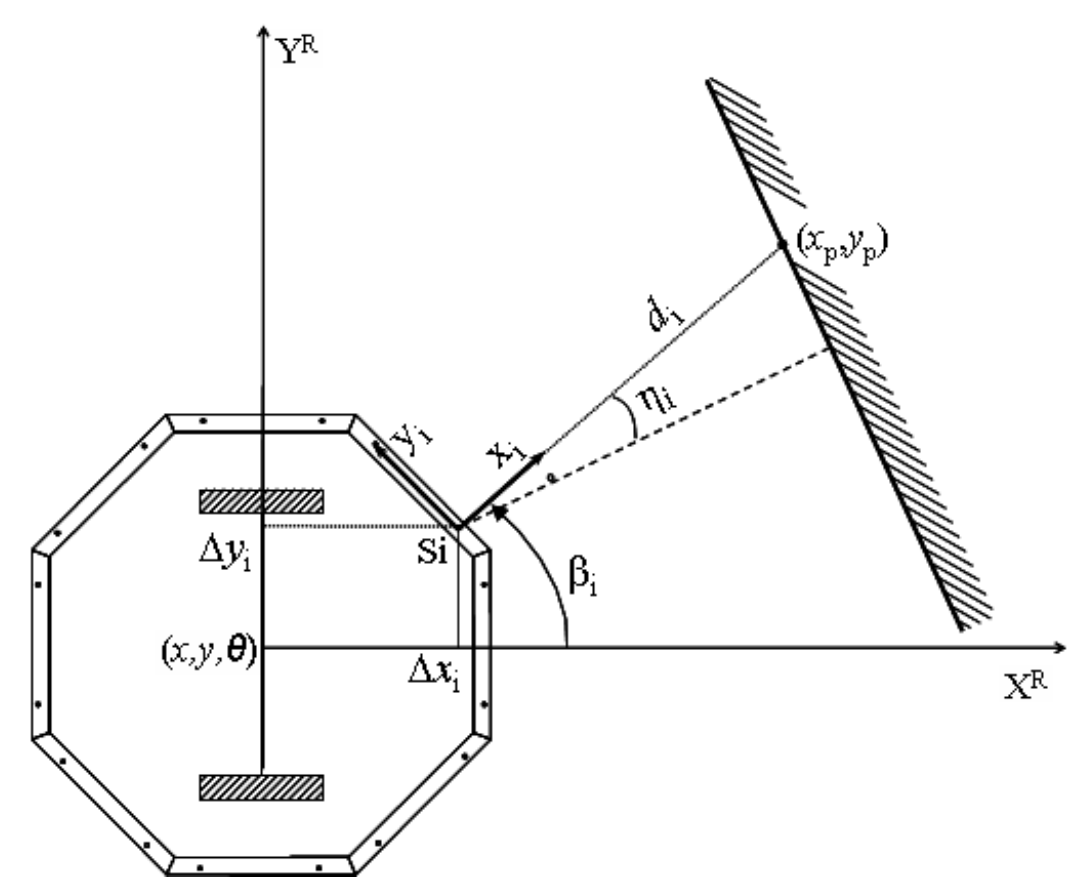

Figura 4.27: Relaciones geométricas entre el marco SENSOR $\left\{S_{i}\right\}$ y el marco ROBOT $\{R\}$. Nótese que de aquí surgen las relaciones necesarias para transformar entre estos dos marcos, los datos de distancia registrados por cualquiera de los sensores del anillo de infrarrojos. 


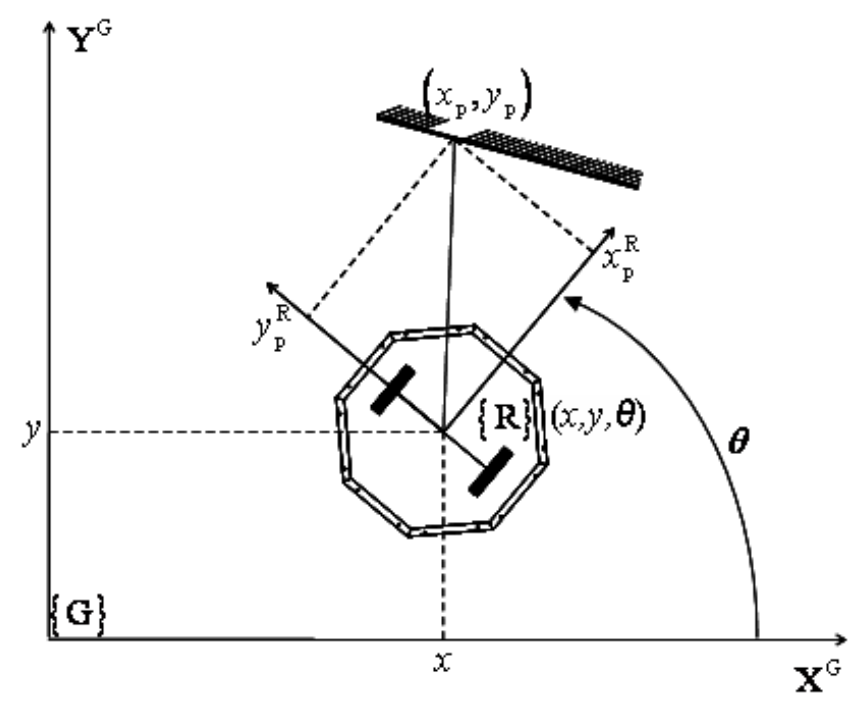

Figura 4.28: Relaciones geométricas entre el marco ROBOT $\{\mathrm{R}\}$ y el marco GLOBAL $\{G\}$. Nótese que de aquí surgen las relaciones necesarias para transformar al mapa global los reflectores observados por el robot.

$$
\mathbf{p}^{\mathrm{R}}=\left[\begin{array}{l}
x_{\mathrm{p}} \\
y_{\mathrm{p}}
\end{array}\right]^{\mathrm{R}}=\left[\begin{array}{c}
d_{i} \cos \beta_{i}+\Delta x_{i} \\
d_{i} \sin \beta_{i}+\Delta y_{i}
\end{array}\right]
$$

con una matriz de covarianza vista en el sistema $\{R\}$ igual a

$$
\mathbf{C} x y^{\mathrm{R}}=\mathbf{J}\left[\sigma_{d}^{2}\right] \mathbf{J}^{\mathrm{T}}
$$

donde $\sigma_{d}^{2}$ es la varianza en la medición de distancias del sensor de infrarrojos (ver ecuación 4.34), y $\mathbf{J}$ es el Jacobiano de la función de transformación, el cual está descrito por la ecuación

$$
\mathbf{J}=\frac{\partial \mathbf{p}^{\mathrm{R}}}{\partial d}=\left[\begin{array}{c}
\cos \beta_{i} \\
\sin \beta_{i}
\end{array}\right]
$$

Con la ecuación 4.38, la expresión para la covarianza del punto reflector visto en el marco ROBOT sería,

$$
\mathbf{C} x y^{\mathrm{R}}=\left[\begin{array}{cc}
\sigma_{x_{p}}^{2} & \sigma_{x y_{p}} \\
\sigma_{x y_{p}} & \sigma_{y_{p}}^{2}
\end{array}\right]^{\mathrm{R}}=\sigma_{d}^{2}\left[\begin{array}{cc}
\cos ^{2} \beta_{i} & \cos \beta_{i} \sin \beta_{i} \\
\cos \beta_{i} \sin \beta_{i} & \sin ^{2} \beta_{i}
\end{array}\right]
$$

Basándose en la figura 4.28, se determina que a partir de los datos de posición del robot $\langle x, y, \theta\rangle$ y de los datos de distancia de los sensores de infrarrojos $\left\langle d_{1}, d_{2}, \cdots, d_{16}\right\rangle$, la proyección de cada uno de los puntos reflectores $\mathbf{p}=\left\langle x_{\mathrm{p}}, y_{\mathrm{p}}\right\rangle$ en el plano cartesiano global, se puede calcular de acuerdo a las ecuaciones 


$$
\mathbf{p}^{\mathrm{G}}=\left[\begin{array}{c}
x_{\mathrm{p}} \\
y_{\mathrm{p}}
\end{array}\right]^{\mathrm{G}}=\left[\begin{array}{c}
x_{\mathrm{p}}^{\mathrm{R}} \cos \theta-y_{\mathrm{p}}^{\mathrm{R}} \sin \theta+x \\
x_{\mathrm{p}}^{\mathrm{R}} \sin \theta+y_{\mathrm{p}}^{\mathrm{R}} \cos \theta+y
\end{array}\right]
$$

Con la finalidad de combinar los datos desde diferentes puntos de vista, o con los datos de otros sensores, es necesario obtener una expresión para la incertidumbre de los puntos proyectados en el marco GLOBAL. La propagación del error en las coordenadas $\left\langle x_{\mathrm{p}}, y_{\mathrm{p}}\right\rangle$ a través de la transformación de coordenadas descritas en la ecuación 4.40, se pueden obtener por aproximación lineal mediante la ecuación

$$
\mathbf{C} x y^{\mathrm{G}}=\mathbf{J}_{1} \mathbf{C} x y^{\mathrm{R}} \mathbf{J}_{1}^{\mathrm{T}}+\mathbf{J}_{2} \mathbf{C}_{\text {Robot }} \mathbf{J}_{2}^{\mathrm{T}}
$$

En la ecuación 4.41 el operando $\mathbf{C}_{\text {Robot }}$ representa la covarianza del error en el estado estimado del robot, la cual se extrae a partir del modelo cinemático del mismo y es de la forma, ( ver sección 5.2)

$$
\mathbf{C}_{\text {Robot }}=\left[\begin{array}{ccc}
\sigma_{x}^{2} & \sigma_{x y} & \sigma_{x \theta} \\
\sigma_{x y} & \sigma_{y}^{2} & \sigma_{y \theta} \\
\sigma_{x \theta} & \sigma_{y \theta} & \sigma_{\theta}^{2}
\end{array}\right]
$$

Por su parte $\mathbf{J}_{1}$ y $\mathbf{J}_{2}$ son matrices Jacobianas relacionadas a las coordenadas del marco ROBOT y al estado del robot respectivamente, de acuerdo a

$$
\begin{gathered}
\mathbf{J}_{1}=\frac{\partial \mathbf{p}^{\mathrm{G}}}{\partial x_{\mathrm{p}}^{\mathrm{R}} \partial y_{\mathrm{p}}^{\mathrm{R}}}=\left[\begin{array}{cc}
\cos \theta & -\sin \theta \\
\sin \theta & \cos \theta
\end{array}\right] \\
\mathbf{J}_{2}=\frac{\partial \mathbf{p}^{\mathrm{G}}}{\partial x \partial y \partial \theta}=\left[\begin{array}{ccc}
1 & 0 & -(\sin \theta+\cos \theta) \\
0 & 1 & (\cos \theta-\sin \theta)
\end{array}\right]
\end{gathered}
$$

De lo anterior, cada lectura de cualquiera de los sensores de infrarrojos se modela mediante las coordenadas de un punto en el plano cartesiano GLOBAL, acompañado por una elipse de incertidumbre cuyo eje mayor y menor corresponden a los valores propios de la matriz de covarianzas de la ecuación 4.41 .

\subsubsection{Representación paramétrica del entorno mediante segmentos de líneas}

Los mapas basados en líneas son muy adecuados para representar los entornos interiores ya que estos entornos poseen muchas estructuras planas, que al ser representadas como líneas, derivan en representaciones muy convenientes y de baja demanda computacional y de almacenamiento. Este tipo de mapas, en su mayoría son extraídos a partir de mapas de puntos obtenidos con sensores Láser[7, 168], a los cuales se les aplican métodos de fusión como el Método Iterativo de División Recurrente (RIEPFA - Recursive Iterative End Point Fit Algoritm) [61], o como la Transformado Hough[94]. Otros investigadores han usado sensores de ultrasonidos, y a cuenta de la gran incertidumbre que estos presentan, han utilizados métodos de 


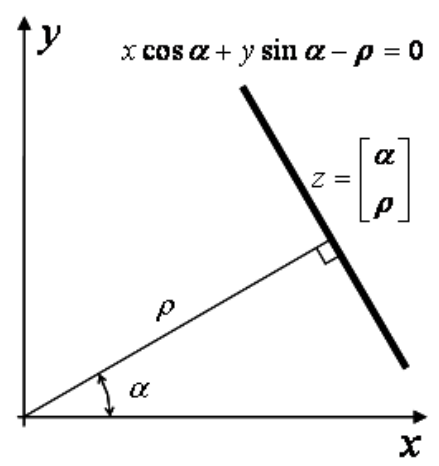

Figura 4.29: Representación paramétrica de líneas infinitas.

fusión basados en técnicas más relajadas como por ejemplo la fusión de puntos colineales mediante conjuntos difusos[80]. Independientemente de la técnica de fusión o del sensor utilizado, el punto común de estos trabajo ha sido representar las líneas o segmentos de línea de forma compacta. Los mapas basados en líneas son muy adecuados para representar los entornos interiores ya que estos entornos poseen muchas estructuras planas. Una forma conveniente representar segmentos de línea es mediante los puntos de inicio $\left(\mathbf{p}_{0}=\left\langle x_{0}, y_{0}\right\rangle\right)$ y de fin $\left(\mathbf{p}_{1}=\left\langle x_{1}, y_{1}\right\rangle\right)$ de los mismos, ya que estos puntos contienen toda la información requerida para caracterizar el segmento de línea que ellos forman. Sin embargo, una manera más compacta de representar las líneas es mediante el modelo Hessiano, el cual modela una línea infinita como $z=[\alpha, \rho]^{\mathrm{T}}$, donde $\alpha$ y $\rho$ representa la orientación y la magnitud del vector que se extiende desde el origen de coordenadas hasta el punto donde se intersecta de forma perpendicular a la recta(ver figura 4.29). Esta forma de representación conduce a un modelo paramétrico que es igual a

$$
x \cos \alpha+y \sin \alpha-\rho=0
$$

De esta forma, el conjunto de parámetros mínimos requeridos para representar un línea son:

$\rho$ Distancia perpendicular desde el origen de coordenadas a la línea

$\alpha$ Orientación del segmento representado por $\rho$

$\sigma_{\rho}$ Incertidumbre en la distancia perpendicular $\rho$

$\sigma_{\alpha}$ Incertidumbre en la orientación $\alpha$

Si se quiere representar segmentos de recta, hay que definir adicionalmente el punto inicial y el punto final del segmento. 


\subsubsection{Extracción de líneas mediante métodos recursivos}

En las aplicaciones de robótica móvil, la mayoría de los métodos para la extracción de líneas se basa en procesamiento por lotes que requieren que se cuente con una cantidad importante de datos antes de que se pueda extraer la característica[157]. Para esto, los investigadores han venido usando sensores como el Láser, que puede suministrar datos densamente muestreados, con tiempos de latencia que no rebasan el segundo. No obstante, hay aplicaciones y sistemas que están orientados a la utilización de sensores de bajo costo, que no cuentan con este tipo de sensores Láser o con sensores y sistemas como cámaras de video. Es el caso del robot YAIR, el cual sólo cuenta con sensores de bajo costo que en general tienen poco alcance o son de baja velocidad, y que a la postre no pueden suministrar la alta densidad de datos que requieren los métodos como la transformada de Hough, ni pueden suministrar suficientes datos por barrido como lo requieren los métodos de ajuste por regresión y sus variantes como el RIEPFA.

Las limitaciones anteriores conducen a la utilización de métodos de fusión que trabajen de forma incremental, y que en ese sentido puedan ir extrayendo la característica a medida que se vayan obteniendo datos válidos, mientras el robot se mueve por el entorno. Navarro \& Benet, comparan el desempeño de tres algoritmos que permiten la extracción de líneas de forma incremental[153]. Estos algoritmos, el de Minimos Cuadrados Recursivos o RLS, el de Minimos Cuadrados Recursivos Totales o RTLS (usa los auto-vectores), y el del Filtro de Kalman Extendido o EKF, en general requieren que se les suministre el modelo paramétrico de la característica a extraer. Un modelo lineal que permite representar superficies planas de un entorno es la forma canónica de la recta.

$$
y=\mathrm{m} x+\mathrm{c}
$$

Un modelo discreto de observación de la función anterior corresponde a

$$
y_{k}=\left[\begin{array}{ll}
x_{k} & 1
\end{array}\right]\left[\begin{array}{c}
\mathrm{m} \\
\mathrm{c}
\end{array}\right]+\varepsilon_{k}=\mathbf{X}_{k} \mathbf{A}+\varepsilon_{k}
$$

donde

$y_{k}$ es una medida escalar en el instante $k$

$\mathbf{X}_{k}$ es el vector de coeficientes de observación en el instante $k$

A es vector de parámetros de la función

$\varepsilon_{k}$ es el error de observación de $y_{k}$

En el supuesto que se conocen con exactitud en cada instante los vectores $\mathbf{X}_{1: k}$, entonces a partir de los valores medidos de la variable $y_{1: k}$ se puede estimar el vector de parámetros $\hat{\mathbf{A}}$, que minimizan el error cuadrático, mediante la ecuación de ajuste por Minimos Cuadrados (Para más detalles ver sección C.1) 


$$
\hat{\mathbf{A}}=\left[\mathbf{X}_{1: k}^{\mathrm{T}} \mathbf{X}_{1: k}\right]^{-1} \mathbf{X}_{1: k}^{\mathrm{T}} \mathbf{Y}_{1: k}
$$

El problema con esta forma de estimar los parámetros es que hace falta tener la historia total del vector de coeficiente de observación y de la observación misma, para poder estimar los parámetros de la función. Un conjunto de ecuaciones que permiten hacer la estimación en forma recursiva son

$$
\begin{gathered}
\mathbf{P}_{k}=\frac{1}{\lambda}\left(\mathbf{P}_{k-1}-\frac{\mathbf{P}_{k-1} \mathbf{X}_{k} \mathbf{X}_{k}^{\mathrm{T}} \mathbf{P}_{k-1}}{\lambda+\mathbf{X}_{k}^{\mathrm{T}} \mathbf{P}_{k-1} \mathbf{X}_{k}}\right) \\
\mathbf{K}_{k}=\frac{\mathbf{P}_{k-1} \mathbf{X}_{k}}{\lambda+\mathbf{X}_{k}^{\mathrm{T}} \mathbf{P}_{k-1} \mathbf{X}_{k}} \\
\hat{\mathbf{A}}_{k}=\hat{\mathbf{A}}_{k-1}+\mathbf{K}_{k}\left(y_{k}-\mathbf{X}_{k}^{\mathrm{T}} \hat{\mathbf{A}}_{k-1}\right)
\end{gathered}
$$

donde

$\mathbf{P}_{k}$ es la matriz de covarianza del error en la estimación de los parámetros de la línea en el instante $k$

$\mathbf{K}_{k}$ es la matriz de ganancias de corrección de los parámetros de la línea en el instante $k$

$\hat{\mathbf{A}}_{\mathbf{k}}$ es vector de parámetros de la línea estimados en el instante $k$

$\lambda$ es conocido como el factor de olvido, el cual previene la caida a cero de la matriz de ganancia de corrección.

El método anterior, conocido como Mínimos Cuadrados Recursivos, asume que todo el error de medición lo tiene la variable dependiente, y que además es constante en todos los ciclos de medición; lo que claramente representa una aproximación poco fiable. Otros dos problemas de consideración con este algoritmo son su ineficiencia computacional proveniente de la inversión de matrices que es necesaria en cada ciclo de propagación de la covarianza, y su incapacidad para tratar problemas con relaciones no lineales. Un conjunto de ecuaciones que proveen una solución recursiva eficiente del método de mínimos cuadrados, es el conocido como Filtrado de Kalman. Esta solución permite calcular un estimador lineal, insesgado y óptimo del estado de un proceso en cada instante de tiempo, con base a la estimación hasta el instante de tiempo anterior y a la información adicional disponible en el momento actual.

El Filtro de Kalman es una herramienta que permite estimar el estado de un sistema dinámico a partir de mediciones ruidosas. El filtro trabaja recursivamente a través del tiempo y suministra el estado estimado cuya varianza es mínima. En el anexo B se desarrollan las ecuaciones que se utilizan en este algoritmo para el caso general de un sistema dinámico lineal. También, y para el caso donde se desconocen los parámetros que describen el modelo lineal del sistema, si se considera a estos parámetros como un estado más a estimar dentro del vector de estados, el sistema se 
convierte en uno no lineal y se puede determinar el valor óptimo de dichos parámetros desconocidos utilizando el Filtro de Kalman Extendido.

En el caso de la estimación recursiva de los parámetros de una línea recta, el sistema es estático y responde al modelo

$$
y=\mathrm{c}+\mathrm{m} u
$$

donde c y m son parámetros que caracterizan al sistema y que en este caso se suponen desconocidos. Si se incluyen los parámetros desconocidos como variables de estado, y se define el siguiente vector de estados

$$
\mathbf{x}=\left[\begin{array}{lll}
y & c & m
\end{array}\right]^{\mathrm{T}}
$$

el sistema podría remodelarse como un sistema discreto no lineal de la forma

$$
\left[\begin{array}{l}
y_{k} \\
c_{k} \\
m_{k}
\end{array}\right]=\left[\begin{array}{l}
c_{k-1}+m_{k-1}\left(u_{k}+w_{k}^{u}\right) \\
c_{k-1} \\
m_{k-1}
\end{array}\right]
$$

donde se asume que $w_{k}^{u}$ es una función de ruido aleatorio de distribución normal con media igual a cero y varianza U. En forma compacta, el valor esperado para el estado del sistema en el instante $k$, se expresa como

$$
\hat{\mathbf{x}}_{k}=f\left(\hat{\mathbf{x}}_{k-1}^{+}, \hat{u}_{k}\right)
$$

de donde surgen de inmediato que la matrices Jacobianas son

$$
\begin{gathered}
\mathbf{A}=\frac{\partial f(\mathbf{x}, u)}{\partial \mathbf{x}}=\left[\begin{array}{lll}
0 & u & 1 \\
0 & 1 & 0 \\
0 & 0 & 1
\end{array}\right] \\
\mathbf{B}=\frac{\partial f(\mathbf{x}, u)}{\partial u}=\left[\begin{array}{c}
m \\
0 \\
0
\end{array}\right]
\end{gathered}
$$

Ahora bien, asumiendo que en este caso se puede medir la salida del sistema en forma directa, el modelo de observación sería

$$
\mathbf{z}_{k}=f\left(\mathbf{x}_{k}\right)=y_{k}+v_{k}
$$

donde $v_{k}$ es el ruido en la medición, el cual se asume que se distribuye en forma normal con media igual cero y varianza $\mathbf{R}$. De aquí se obtiene la matriz Jacobiana de observación, la cual es igual a

$$
\mathbf{H}=\frac{\partial h(\mathbf{x})}{\partial \mathbf{x}}=\left[\begin{array}{lll}
1 & 0 & 0
\end{array}\right]
$$

A continuación, para ejecutar el algoritmo de identificación recursivamente, primero hay que asumir los valores estimados iniciales $\hat{\mathbf{x}}_{k-1}$ de los parámetros a estimar, 
así como también la matriz de covarianzas asociada $\mathbf{P}_{k-1}$. Usualmente se suele inicializar $\mathbf{P}_{k-1}=\alpha \mathbf{I}_{n \times n}$, donde $\mathbf{I}$ es la matriz identidad y $\alpha$ una constante muy grande. Por otra parte, si no se tiene ningún conocimiento acerca del valor de los parámetros a estimar, lo más frecuente es inicializarlos en cero. A partir de aquí, lo restante es aplicar recursivamente las siguientes ecuaciones (Ver detalles en la sección B.3)

$$
\begin{gathered}
\hat{\mathbf{x}}_{k}^{-}=f\left(\hat{\mathbf{x}}_{k-1}^{+}, \mathbf{u}_{k}\right) \\
\mathbf{P}_{k}^{-}=\mathbf{A}_{k} \mathbf{P}_{k-1} \mathbf{A}^{\mathrm{T}}+\mathbf{B}_{k} \mathbf{U}_{k} \mathbf{B}^{\mathrm{T}}+\mathbf{Q}_{k} \\
\mathbf{K}_{k}=\mathbf{P}_{k}^{-} \mathbf{H}_{k}^{\mathrm{T}}\left(\mathbf{H}_{k} \mathbf{P}_{k}^{-} \mathbf{H}_{k}^{\mathrm{T}}-\mathbf{R}_{k}\right)^{-1} \\
\hat{\mathbf{x}}_{k}^{+}=\hat{\mathbf{x}}_{k}^{-}+\mathbf{K}_{k}\left(\mathbf{z}_{k}-h\left(\hat{\mathbf{x}}_{k}^{-}\right)\right) \\
\mathbf{P}_{k}^{+}=\left(\mathbf{I}-\mathbf{K}_{k} \mathbf{H}_{k}\right)^{-1} \mathbf{P}_{k}^{-}
\end{gathered}
$$

Con la finalidad de comprobar la consistencia entre los parámetros estimados y los parámetros que modelan al sistema, se puede aplicar la prueba chi-cuadrado. Esta prueba es considerada como una prueba no paramétrica que mide la discrepancia entre una distribución observada y otra teórica (bondad de ajuste), indicando en que medida las diferencias existentes entre ambas, de haberlas, se deben al azar. Ya ha sido señalado[14] que dentro del algoritmo del Filtro de Kalman, una buena prueba de consistencia entre los datos se lleva a cabo mediante el test de la innovación también conocido como Validation Gate. Típicamente, la innovación normalizada al cuadrado

$$
\chi^{2}=(\mathbf{z}-h(\mathbf{x}))^{\mathrm{T}} \mathbf{P}^{-1}(\mathbf{z}-h(\mathbf{x}))
$$

la cual se calcula on-line, sirve como una medida del grado de innovación contenido en la medición sensorial. Si se asume que el vector de residuales se distribuye conjuntamente en forma normal o gausiana, entonces $\chi^{2}$ se conoce como la Distancia de Mahalanobis, la cual es una función que se distribuye de acuerdo a la distribución chi-cuadrado. De ahí el nombre que recibe esta prueba. Mientras más pequeña sea la distancia de Mahalanobis, mayor será la probabilidad de que los parámetros estimados pertenezcan a la distribución de los parámetros reales. El valor límite para el rechazo del modelo estimado lo impone el valor dado en la tabla de distribución $\chi_{\alpha, \mathrm{M}}^{2}$, con $\mathrm{M}$ grados de libertad determinado por el rango de $\mathbf{P}$, y confianza $\alpha$ que suele ajustarse entre el 90 y $99 \%$. Si el valor $\chi^{2}$ calculado es menor que el valor $\chi_{\alpha, \mathrm{M}}^{2}$ tabulado, entonces se acepta el modelo, de lo contrario se rechaza.

Una vez obtenido el modelo del segmento de línea por cualquiera de los métodos descritos, el modelo Hessiano se consigue fácilmente mediante las ecuaciones

$$
\rho=\left|\frac{\mathrm{c}}{\sqrt{1+\mathrm{m}^{2}}}\right|
$$




$$
\begin{gathered}
\alpha^{\prime}=\arctan \left(-\frac{1}{\mathrm{~m}}\right) \\
\alpha= \begin{cases}\alpha^{\prime}+\pi & \text { si }\left(\alpha^{\prime}<0\right) \&(\mathrm{c}>0) \text { o si }\left(\alpha^{\prime}>0\right) \&(\mathrm{c}<0) \\
\alpha^{\prime} & \text { para cualquier otro caso }\end{cases}
\end{gathered}
$$

El error en los parámetros estimados se consigue mediante la propagación lineal de los mismos a través de las ecuaciones de transformación 4.66 y 4.67. Esto es que, si se considera a $\mathbf{C}_{\mathrm{m}, \mathrm{c}}$ como la covarianza en los parámetros estimados a partir de los métodos recursivos antes descritos(ecuación 4.49 o 4.64), entonces la covarianza de los parámetros referidos al modelo Hessiano $\mathbf{C}_{\alpha, \rho}$, corresponde a

$$
\mathbf{C}_{\alpha, \rho}=\mathbf{J C}_{\mathrm{m}, \mathrm{c}} \mathbf{J}^{\mathrm{T}}
$$

donde las covarianzas son sendas matrices cuadradas y $\mathbf{J}$ es la matriz Jacobiana definida para este caso como,

$$
\begin{gathered}
\mathbf{C}_{\mathrm{m}, \mathrm{c}}=\left[\begin{array}{cc}
\sigma_{\mathrm{m}}^{2} & \sigma_{\mathrm{mc}} \\
\sigma_{\mathrm{mc}} & \sigma_{\mathrm{c}}^{2}
\end{array}\right] \\
\mathbf{C}_{\alpha, \rho}=\left[\begin{array}{cc}
\sigma_{\alpha}^{2} & \sigma_{\alpha \rho} \\
\sigma_{\alpha \rho} & \sigma_{\rho}^{2}
\end{array}\right] \\
\mathbf{J}=\frac{\partial f(\mathrm{~m}, \mathrm{c})}{\partial \mathrm{m} \partial \mathrm{c}}=\frac{\partial}{\partial \mathrm{m} \partial \mathrm{c}}\left[\begin{array}{c}
\arctan \left(-\frac{1}{\mathrm{~m}}\right) \\
\mathbf{J}=\left[\frac{\mathrm{c}}{\sqrt{1+\mathrm{m}^{2}}} \mid\right.
\end{array}\right] \\
\frac{1}{\frac{\mathrm{c}}{\mathrm{m}^{2}+1}} \frac{|\mathrm{c}| \mathrm{m}}{\left(\mathrm{c} \mid \sqrt{\mathrm{m}^{2}+1}\right.} \\
\frac{\left(\mathrm{m}^{2}+1\right)^{3 / 2}}{]}
\end{gathered}
$$

\subsubsection{Construcción incremental de mapas de líneas mediante fusión de datos de infrarrojos}

Los datos de distancia suministrado por el sensor de infrarrojos son bastante imprecisos debido a que la cantidad de energía que devuelven las diversas superficies reflectoras depende de sus características retroflectivas y del ángulo de incidencia del haz de infrarrojos. Para resolver el problema de la imprecisión del sensor infrarrojos en la construcción de mapas de entorno, en esta tesis usamos redundancia en la medición y además aplicamos técnicas de ajuste de datos a funciones paramétricas.

La primera fuente de redundancia en la medición es la alineación. Los puntos de reflexión rara vez están alineados, lo que implica que si aparecen 2 o más puntos alineados, lo más probable es que pertenezcan a una misma superficie. La segunda fuente de redundancia es la movilidad. Dado que el robot se mueve al tiempo que va observando su entorno, las medidas que se obtienen representan los objetos del 

MAPAS

mismo visto desde distintas perspectivas. Esto último permite implementar técnicas de fusión mediante las cuales una medición refuerza la verosimilitud de otra.

Los datos de distancia suministrado por los sensores de infrarrojos son proyectados al plano cartesiano GLOBAL mediante la combinación de las ecuaciones 4.36 y 4.40. Estos puntos cartesianos son utilizados para extraer segmentos de línea modeladas por la ecuación

$$
y=\mathrm{m} x+\mathrm{c}
$$

La formación de los segmentos de línea en un marco cartesiano global, facilita los mecanismos de correspondencia y de fusión entre los distintos segmentos detectados.

A continuación se describe una metodología para construir mapas de líneas de manera incremental, utilizando los datos de distancia suministrado por el sensor de infrarrojos y la técnica de medición ya descrita en la sección 4.2.1. Dada esta limitación, la formulación está especialmente adaptada a las características particulares del sensor de infrarrojos del robot YAIR, pero en general la metodología se puede extender fácilmente para usarla en otras arquitecturas mas generales.

\subsubsection{Detección e iniciación de los segmentos de línea}

Para iniciar el algoritmo de extracción de algún segmento de línea en particular, se parte de la superficie reflectora que se puede extraer de la medición instantánea en cualquiera de los 8 pares de sensores que forman el anillo de infrarrojos de YAIR(ver figura 4.30). De acuerdo a lo que se describió en la sección 4.2.1, cuando los dos sensores que conforman un mismo par detectan un reflector al mismo tiempo, lo más probable es que se trate de una superficie reflectora común, de la cual se puede extraer tanto la distancia $d$ como la orientación $\eta$ que tiene respecto al marco SENSOR; lo que además se puede extrapolar al marco ROBOT. Sin embargo, en este trabajo se prefirió proyectar las mediciones $d s_{i}$ y $d s_{i+1}$ al marco GLOBAL y estimar ahí los parámetros iniciales de la recta $y=\mathrm{m} x+\mathrm{c}$ con las ecuaciones

$$
\begin{gathered}
\mathrm{m}=\frac{y p_{i}-y p_{i+1}}{x p_{i}-x p_{i+1}} \\
\mathrm{c}=y p_{i}-\mathrm{m} x p_{i}
\end{gathered}
$$

También, la incertidumbre en el estimado inicial de los parámetros de la recta, calculados de esta forma, se pueden aproximar mediante la propagación lineal del error de acuerdo a las ecuaciones

$$
\begin{gathered}
\sigma_{\mathrm{m}}^{2}=\mathbf{J}_{\mathrm{m}} \mathbf{C} \mathbf{J}_{\mathrm{m}}^{\mathrm{T}} \\
\sigma_{\mathrm{c}}^{2}=\mathbf{J}_{\mathrm{c}} \mathbf{C} \mathbf{J}_{\mathrm{c}}^{\mathrm{T}}
\end{gathered}
$$

Aquí, $\mathbf{C}$ es la matriz de covarianza ampliada que incluye la covarianza del punto $\mathbf{p}_{i}=\left(x p_{i}, y p_{i}\right)$ y la del punto $\mathbf{p}_{i+1}=\left(x p_{i+1}, y p_{i+1}\right)$, según 


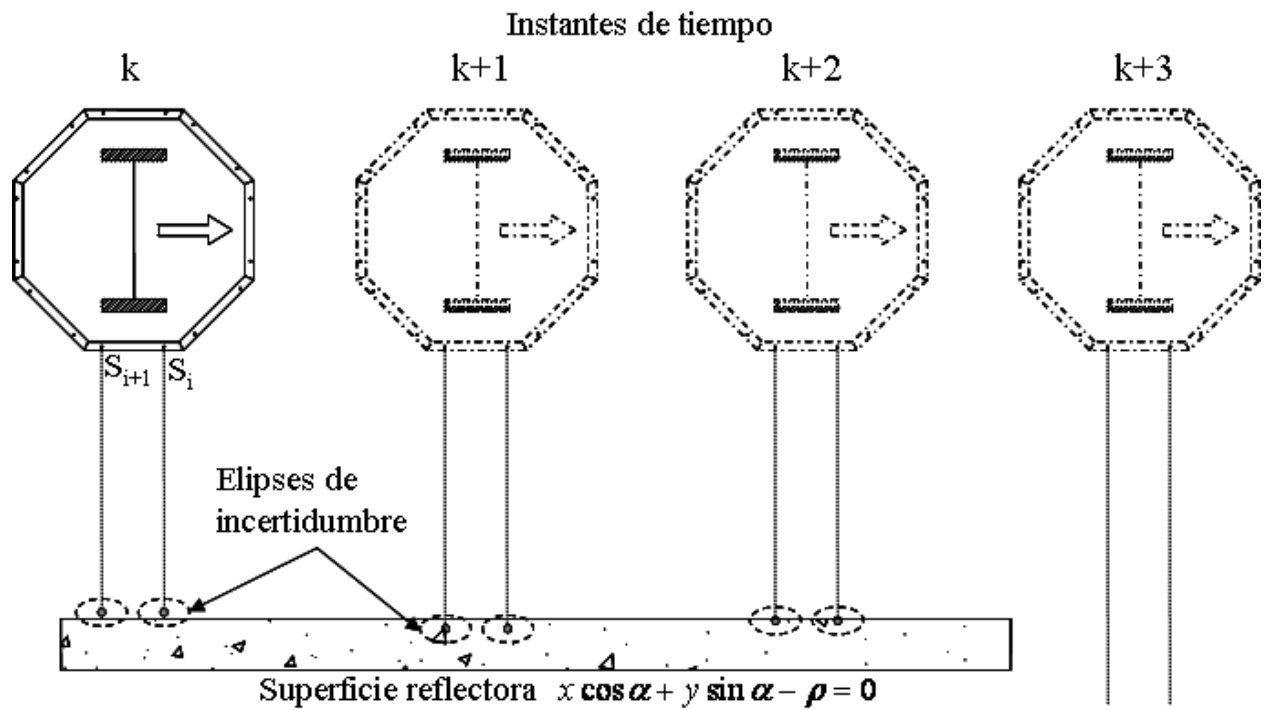

Figura 4.30: Mapeado incremental mediante sensores de infrarrojos. En el caso mostrado, la recursión se inicia en el instante $k$ cuando detecta la superficie, y finaliza en $k+3$ cuando deja de detectar la superficie.

$$
\mathbf{C}=\left[\begin{array}{cc}
\mathbf{C} x y_{i}^{\mathrm{G}} & \cdots \\
\cdots & \mathbf{C} x y_{i+1}^{\mathrm{G}}
\end{array}\right]
$$

donde $\mathbf{C} x y^{\mathrm{G}}$ ya se definió en la ecuación 4.41, por lo esta matriz tiene la forma

$$
\mathbf{C}=\left[\begin{array}{cccc}
\sigma x_{i}^{2} & \sigma x y_{i} & 0 & 0 \\
\sigma x y_{i} & \sigma y_{i}^{2} & 0 & 0 \\
0 & 0 & \sigma x_{i+1}^{2} & \sigma x y_{i+1} \\
0 & 0 & \sigma x y_{i+1} & \sigma y_{i+1}^{2}
\end{array}\right]
$$

donde $\sigma y_{i}^{2}$ y $\sigma x_{i}^{2}$ son las varianzas, medidas en el marco global, correspondiente al punto $\left(x p_{i}, y p_{i}\right)$.

También, $\mathbf{J}_{\mathrm{m}}$ y $\mathbf{J}_{\mathrm{c}}$ son las matrices Jacobianas correspondientes a

$$
\begin{aligned}
& \mathbf{J}_{\mathrm{m}}=\frac{\partial \mathrm{m}}{\partial \mathbf{p}_{i} \partial \mathbf{p}_{i+1}}=\left[\begin{array}{llll}
\frac{-\left(y p_{i}-y p_{i+1}\right)}{\left(x p_{i}-x p_{i+1}\right)^{2}} & \frac{1}{\left(x p_{i}-x p_{i+1}\right)} & \frac{\left(y p_{i}-y p_{i+1}\right)}{\left(x p_{i}-x p_{i+1}\right)^{2}} & \frac{-1}{\left(x p_{i}-x p_{i+1}\right)}
\end{array}\right] \\
& \mathbf{J}_{\mathrm{c}}=\frac{\partial \mathrm{c}}{\partial \mathbf{p}_{i} \partial \mathbf{p}_{i+1}}=\left[\begin{array}{llll}
\frac{x p_{i+1}\left(y p_{i}-y p_{i+1}\right)}{\left(x p_{i}-x p_{i+1}\right)^{2}} & \frac{-x p_{i+1}}{\left(x p_{i}-x p_{i+1}\right)} & \frac{-x p_{i}\left(y p_{i}-y p_{i+1}\right)}{\left(x p_{i}-x p_{i+1}\right)^{2}} & \frac{x p_{i}}{\left(x p_{i}-x p_{i+1}\right)}
\end{array}\right]
\end{aligned}
$$

Finalmente, para evitar iniciar nuevos segmentos de línea con datos erróneos o poco fiables, un nuevo segmento no se dá como válido hasta tanto no se detecten consecutivamente al menos 2 segmentos que posean pendientes similares. En esta 


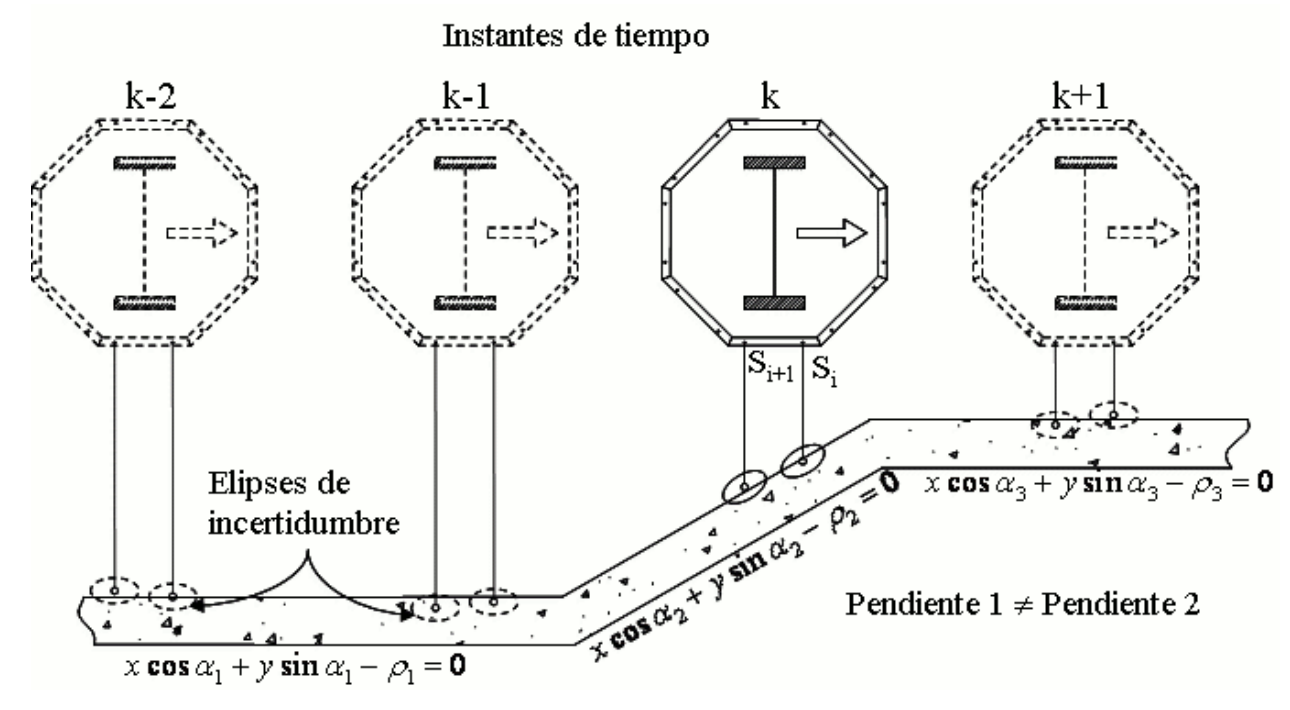

Figura 4.31: Extracción recursiva e incremental de líneas mediante sensores de infrarrojos. Condiciones típicas para segmentación debido a diferencia de pendientes

tesis se admite que dos segmentos tienen igual pendiente cuando su diferencia no excede el rango de $\pm 15^{\circ}$. Este valor heurístico proviene del error estándar obtenido en la medición de distancias mediante infrarrojos en un entorno típico de oficinas.

Por otro lado, la iniciación de un segmento de línea requiere que se defina el punto inicial y el punto final del mismo. En esta tesis se adopta el criterio simple de fijar como punto inicial aquel cuya abscisa está más cercana al extremo negativo del eje horizontal del plano cartesiano. Este mismo criterio fija como punto final aquel cuya abscisa está más cercana al extremo positivo del mismo eje.

\subsubsection{Agrupación de puntos y segmentación de líneas}

Sea el segmento de línea $L$ definido en el plano cartesiano $\langle x, y\rangle$ y modelado paramétricamente como $L:\langle\mathrm{m}, \mathrm{c}, \mathbf{p} 1, \mathbf{p} 2\rangle$, donde $\mathbf{p} 1=\left[x_{1}, y_{1}\right]$ y $\mathbf{p} 2==\left[x_{2}, y_{2}\right]$ son los puntos extremos del segmento perteneciente a la recta de pendiente $\mathrm{m}$ y que cruza con el eje de las ordenadas en el punto $(0, c)$. La segmentación consiste en determinar cuales puntos pertenecen al modelo que describe al segmento $L$. Así, un par de puntos de reflexión $\left(x p_{i}, y p_{i}\right)$ y $\left(x p_{i+1}, y p_{i+1}\right)$, que han sido extraídos de los datos de distancia $d s_{i}$ y $d s_{i+1}$ suministrados por el par sensorial $S_{i}$ y $S_{i+1}$, pueden ser incluidos en el segmento de línea $L$ siempre que acumulen la siguiente evidencia:

1. Que el segmento de recta que definen $\left(x p_{i}, y p_{i}\right)$ y $\left(x p_{i+1}, y p_{i+1}\right)$ tenga una pendiente similar al segmento de línea $L$. Si la pendiente del segmento de recta extraído en el instante $k$ ( ver figura 4.31), no se aparta mas allá de $\pm 15^{\circ}$ respecto a la pendiente del segmento extraído o actualizado en el instante anterior $(k-1)$, entonces los puntos $\left(x p_{i}, y p_{i}\right)$ y $\left(x p_{i+1}, y p_{i+1}\right)$ se agregan al conjunto de puntos que forman al segmento de recta $L$. 
Instantes de tiempo

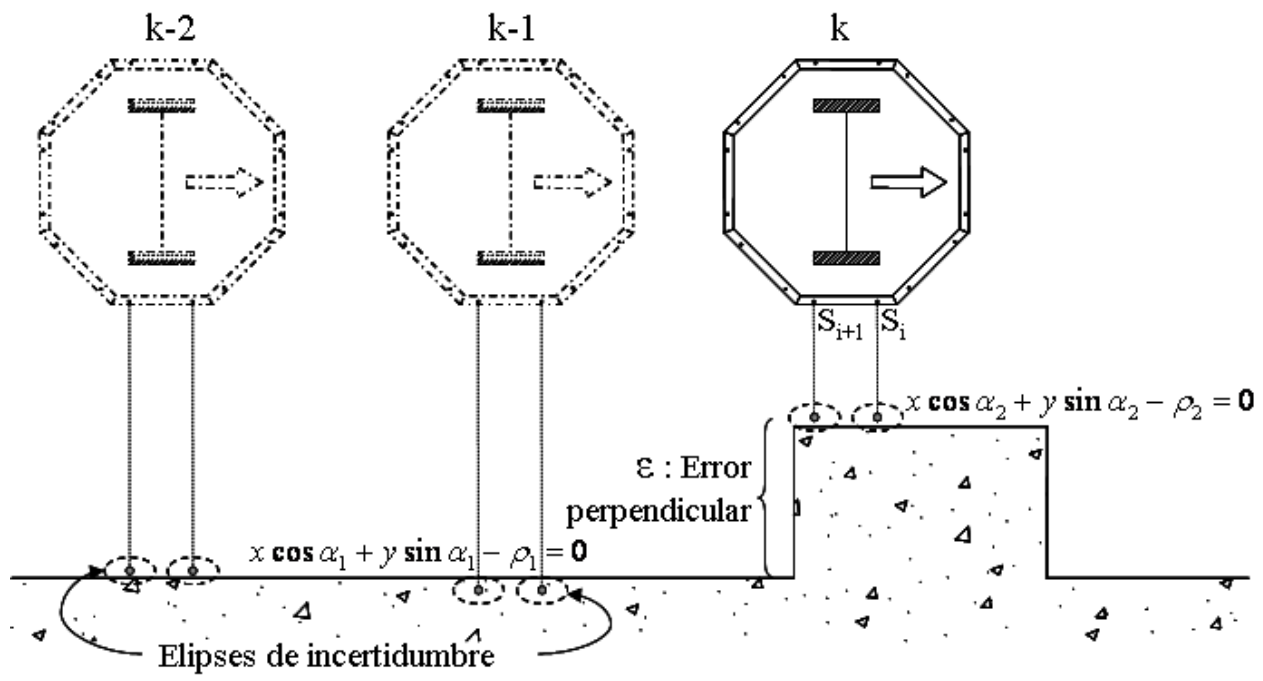

Figura 4.32: Extracción recursiva e incremental de líneas mediante sensores de infrarrojos. Condiciones típicas para segmentación debido a que la distancia perpendicular excede la distancia de tolerancia.

2. Que la distancia perpendicular $(\varepsilon)$ medida desde el punto medio $\left(x_{\mathrm{m}}, y_{\mathrm{m}}\right)$ del segmento definido por $\left(x p_{i}, y p_{i}\right)$ y $\left(x p_{i+1}, y p_{i+1}\right)$, hasta la recta que contiene al segmento $L:\langle\mathrm{m}, \mathrm{c}, \mathbf{p} 1, \mathbf{p} 2\rangle$, sea menor que cierta distancia de tolerancia (ver figura 4.32). Las paredes representan una de las superficies de reflexión más típicas en los entornos interiores. Generalmente estas paredes pueden tener un espesor aproximado de $10 \mathrm{~cm}$. De aquí que dos rectas paralelas a mas de $10 \mathrm{cms}$ y a menos de unos 15 o $20 \mathrm{cms}$, pudiera ser la otra cara de una misma pared. Con esto último, se pudiera establecer heurísticamente que la distancia de tolerancia para discriminar dos segmentos de rectas sea de $0.1 \mathrm{mts}$. La distancia perpendicular $\varepsilon$ entre un punto y una línea recta puede ser calculada mediante.

$$
\varepsilon=\frac{1}{\sqrt{1+\mathrm{m}^{2}}}\left|y_{m}-\mathrm{m} x_{\mathrm{m}}-\mathrm{c}\right|
$$

A partir de la estimación inicial de los parámetros del segmento de recta $L$ : $\langle\mathrm{m}, \mathrm{c}, \mathrm{p} 1, \mathrm{p} 2\rangle$ mediante las ecuaciones 4.74 y 4.75 , a cada instante $k$ se van agregando los puntos de reflexión que cumplan con las condiciones descritas en los apartados 1 y 2 de la sección 4.2.5.2. Si por el contrario las coordenadas de los puntos de reflexión no cumplen con alguna de estas condiciones, entonces se detiene el mecanismo recursivo de extracción de la línea y la recta es entonces segmentada asignándole un punto de inicio y un punto de fin al segmento extraído. La idea subyacente de este mecanismo de segmentación es mostrada en las figuras 4.31 y 4.32. En estas figuras se puede ver que si la proyección de las mediciones $d s_{i}$ y $d s_{i+1}$ no cumplen con la condiciones 

MAPAS

impuesta de pendiente y de distancia perpendicular, entonces la recta es segmentada hasta el instante $k-1$ y se reinicia una nueva línea con los puntos extraídos en el instante $k$.

La extracción de líneas mediante los métodos descritos en la sección 4.2.4, conlleva a que los puntos de reflexión $\left(x p_{i}, y p_{i}\right)$ no necesariamente pertenezcan a la línea extraída. En este sentido se requiere de un mecanismo que actualice y ajuste los puntos de inicio y de fin del segmento extraído. Así, a cada instante $k$ se va actualizando el punto inicial y el punto final del segmento siguiendo el criterio ya expuesto en la sección 4.2.5.1. Una vez que se detiene el proceso recursivo de extracción de la línea, los puntos inicial y final son proyectados perpendicularmente sobre la recta extraída para así finalizar con el proceso de segmentación. La proyección perpendicular de un punto sobre una línea recta se puede calcular mediante las ecuaciones

$$
\begin{aligned}
& x=\frac{x p+\mathrm{m}(y p-\mathrm{c})}{1+\mathrm{m}^{2}} \\
& y=\frac{\mathrm{m} x p+\mathrm{m}^{2} y p+\mathrm{c}}{1+\mathrm{m}^{2}}
\end{aligned}
$$

donde $(x p, y p)$ son las coordenadas del punto que se desea proyectar y $(x, y)$ son las coordenadas del punto proyectado sobre la línea de pendiente $\mathrm{m}$ y punto de corte con el eje vertical igual a $(0, c)$.

\subsubsection{Correspondencia entre segmentos de línea}

El propósito general de la correspondencia es determinar cual segmento de línea tiene mayor parecido con otro segmento de línea dado. En esta tesis, para el caso de segmentos de línea extraídos a partir de datos de infrarrojos, la prueba de correspondencia se organiza como una secuencia de pruebas que están basadas en una función de costo dependiente de: la pendiente de los segmentos, la distancia entre segmentos y la posición relativa de los mismos. La secuencia del test de correspondencia es mostrado en la figura 4.33 .

En esta tesis se han fijado los siguientes valores heurísticos relacionados a las pruebas de correspondencia:

1. La correspondencia es positiva si el ángulo entre los segmentos no se aparta más allá de $\pm 15^{\circ}$.

2. La correspondencia es positiva si la distancia perpendicular desde el centro de uno de los segmentos a la línea que incluye al otro es menor a los 0.1 metros.

3. La corespondencia es positiva si alguno de los extremos de uno de los segmentos se encuentra ubicado dentro de la elipse de incertidumbre que circunscribe al otro. Los distintos tipos de situaciones que pueden ocurrir se muestran en la figura 4.34. En este trabajo se ha establecido una longitud de 0.1 metros para el semieje de la elipse que es perpendicular al segmento, mientras que la longitud 

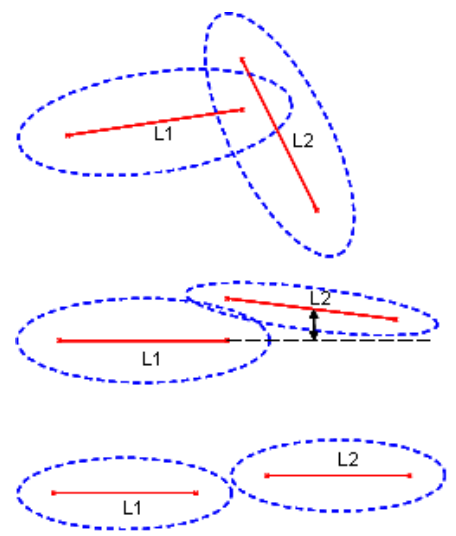

Diferencia entre las

pendientes de los segmentos

b) Distancia perpendicular desde el centro de un segmento a la línea que contiene al otro

Segmento fuera de la elipse de
incertidumbre del segmento base

Figura 4.33: Secuencia de la prueba de correspondencia entre segmentos

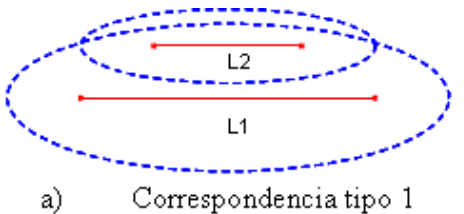

a) Correspondencia tipo 1

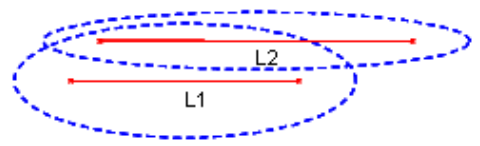

c) Correspondencia tipo 3
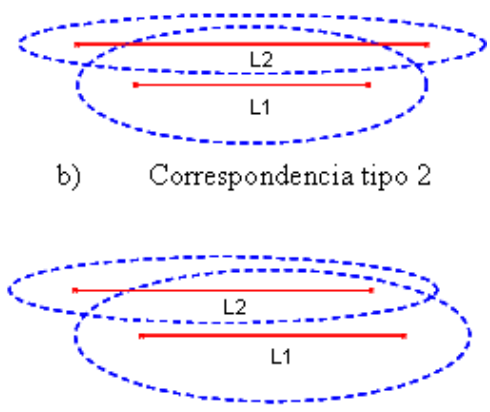

d) Correspondencia tipo 4

Figura 4.34: Tipos de correspondencia en función de la posición relativa de los extremos del segmento.

del semieje axial a la recta es igual a la mitad de la longitud del segmento, más 0.1 metros.

Para ejecutar la prueba de correspondencia, se supone que se conoce previamente el modelo del entorno $M:\left\{L_{1}, L_{2}, \ldots, L_{i}\right\}$ el cual estará igualmente descrito mediante segmentos de recta. Este modelo puede obtenerse mediante una exploración previa del robot, en cuyo caso los segmentos estarán también caracterizados por cierta incertidumbre que se debe más que nada al error odométrico en la localización del robot; o también puede obtenerse mediante sistemas tipo CAD, en cuyo caso el error será despreciable.

La prueba de correspondencia se inicia una vez se extrae el segmento $L:\langle\mathrm{m}, \mathrm{c}, \mathbf{p} 1, \mathbf{p} 2\rangle$, el cual servirá de segmento base para contrastarlo con cada uno de los segmentos $L_{i}$ pertenecientes al modelo del entorno. El algoritmo prueba secuencialmente cada segmento $L_{i}$, y si el segmento bajo prueba falla en cualquiera de las pruebas de la figura 4.33 , simplemente se pasa al siguiente segmento de recta. El algoritmo se detiene cuando alguno de segmentos $L_{i}$ cumple con las tres pruebas de correspondencia, o 

MAPAS

cuando se alcanza el máximo índice de los segmentos. Si se logra la correspondencia plena con alguno de los segmentos, se pasa al proceso de fusión el cual es explicado más adelante. Si no se consigue la correspondencia plena, entonces se trata de un nuevo elemento y por lo tanto se agrega al mapa $M$.

\subsubsection{Mecanismos de fusión de segmentos de línea}

En esta tesis, el proceso de fusión corresponde al mecanismo mediante el cual los segmentos de línea extraídos de los datos sensoriales de infrarrojos, son combinados entre sí para obtener un mapa del entorno más refinado y menos segmentado. En este sentido, las funciones principales del proceso de fusión son:

1. Adicionar al mapa del entorno los nuevos segmentos de línea que se vayan observando.

2. Incrementar la confianza de los segmentos existentes cuando se consigue correspondencia con un segmento observado.

Una vez que se extrae un segmento de línea $L:\langle\mathrm{m}, \mathrm{c}, \mathbf{p} 1, \mathbf{p} 2\rangle$, y se consigue una correspondencia plena con algún otro segmento $L_{i}:\langle\mathrm{m}, \mathrm{c}, \mathbf{p} 1, \mathbf{p} 2\rangle_{i}$ perteneciente al mapa del entorno, entonces se fusionan los dos segmentos mediante el método de Minimos Cuadrados Totales . Este método se implementa mediante las ecuaciones que siguen. Aquí la matriz con los datos que se requieren ajustar corresponde a

$$
\mathbf{D}=\left[\begin{array}{c}
\mathbf{p} 1 \\
\mathbf{p} 2 \\
\mathbf{p} 1_{i} \\
\mathbf{p} 2_{i}
\end{array}\right]
$$

donde cada punto $\mathbf{p}$ corresponde a la tupla $[x, y]$ que representa los extremos de los segmentos a fusionar. A partir de aquí, el centro de gravedad o media de los datos se puede definir como

$$
\mathbf{g}=\left[g_{x}, g_{y}\right]
$$

de aquí resulta que el desplazamiento o error de los datos respecto al centro de gravedad es

$$
\mathbf{E}=\mathbf{D}-\mathbf{g}
$$

Por lo que la matriz de covarianza de los datos es

$$
\mathbf{Q}=\mathbf{E} \cdot \mathbf{E}^{\mathrm{T}}
$$

Por otra parte, para una matriz 2x2 (por ejemplo $\mathbf{Q}$ ), los valores propios $\lambda$, y los vectores propios $\mathbf{v}$ pueden obtenerse de forma, 


$$
\begin{gathered}
\mathbf{Q}=\left[\begin{array}{ll}
q_{11} & q_{12} \\
q_{21} & q_{22}
\end{array}\right] \\
\lambda_{p}=\frac{\operatorname{tr}(\mathbf{Q}) \pm \sqrt{\operatorname{tr}(\mathbf{Q})^{2}-4 \operatorname{det}(\mathbf{Q})}}{2} \\
\mathbf{v}_{p}=\left\langle\frac{q_{12}}{\lambda_{p}-q_{11}}, 1\right\rangle
\end{gathered}
$$

donde $p=1, \cdots, 2$

Los vectores propios de la matriz de covarianza $\mathbf{Q}$ corresponden a la dirección de los semiejes de la elipse que circuncribe a los datos, mientras que los valores propios de la misma matriz corresponden a la longitud de estos semiejes. Así pues, el menor de los semiejes corresponde a una recta, que pasando por el centro de gravedad, es perpendicular a la recta cuya distancia perpendicular a cada uno de los puntos de la matriz de datos, es mínima. De acuerdo a esto, la pendiente de la recta que mejor se ajusta a los datos se puede calcular como,

$$
\mathrm{m}=-\frac{q_{12}}{\lambda_{\min }-q_{11}}
$$

donde $\lambda_{\min }$ es el más pequeño de los valores propios de $\mathbf{Q}$.

Dado que el centro de gravedad $\mathbf{g}$ pertenece al modelo de la recta ajustada con los datos $\mathbf{D}$, entonces el corte con la ordenada de esa misma recta se puede calcular mediante

$$
\mathrm{c}=g_{y}-\mathrm{m} g_{x}
$$

La segmentación de la recta resultante se consigue proyectando perpendicularmente hacia la misma los 2 puntos más distantes del centro de gravedad. Esta operación se muestra en la figura 4.35, y se consigue mediante las ecuaciones 4.83 y 4.84 .

\subsubsection{Experimentos}

\subsubsection{Configuración experimental}

Los experimentos han sido conducidos con el robot YAIR el cual ya ha sido descrito en la sección 3.3.3.1. Como se dijo, este robot en forma de octágono cuenta con un anillo de 16 sensores infrarrojos agrupados en pares dispuestos en cada una de las caras del octágono (ver figura 4.26). Los sensores de infrarrojos han sido construidos en nuestro laboratorio. Su información general ya se expuso en la sección 4.2.2. Para una información más detallada puede consultarse [18].

Para colectar los datos y así poder comprobar la validez de los algoritmos aquí propuestos, se programó al YAIR para que ejecutará un trayecto de aproximadamente 50 metros a través de un pasillo que conecta con nuestro laboratorio y con algunos despachos de profesores (ver figura 4.36a). Este área de aproximadamente 30x4 m 


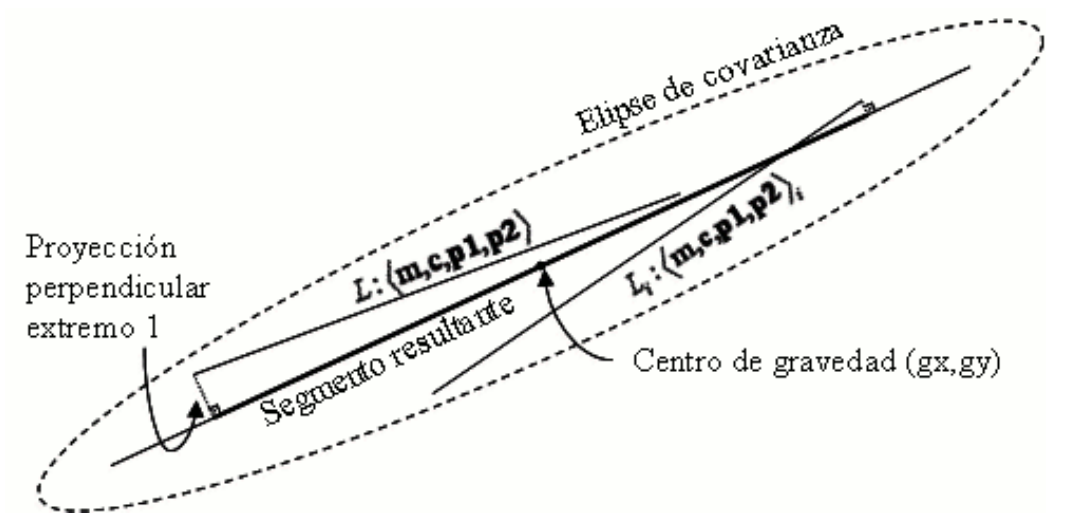

Figura 4.35: Esquema de fusión de dos segmentos de rectas. Nótese que los puntos de segmentación de la recta resultante se obtienen proyectando los extremos exteriores del conjunto de segmentos fusionados

representa un entorno de oficinas típico que está formado por distintas superficies que son idóneas para representarlas mediante líneas.

Para simplificar los ensayos, los datos de posición del robot se registraron mediante el sistema de localización odométrica del mismo, y se asumió que el error introducido era cero. Por otra parte, los datos de distancia de los reflectores fueron colectados y procesados con el robot en movimiento, por lo que para disminuir la carga computacional durante el ensayo se usaron solamente los pares sensoriales $S 5 \& S 6$ y $S 13 \& S 14$. Previo al ensayo, estos sensores fueron calibrados para trabajar en un entorno con coeficiente de reflectividad típico $\alpha_{s}=0,60$.

\subsubsection{Resultados}

En la figura $4.36 \mathrm{~b}$ se observa un mapa de densidad que representa los datos de distancia a los reflectores colectados por los sensores de infrarrojos $S 5$ y $S 14$. Ahí se puede observar que el entorno es representado con bastante precisión, con excepción de que las hendiduras inferiores de figura del entorno real(representan los marcos de las puertas) aparecen mapeadas a una menor distancia de la que realmente se encuentran. Este fenómeno se debe a que las puertas están recubiertas con un material cuyo coeficiente de reflectividad $\alpha_{s}$ es notablemente superior al valor que fueron calibrados los sensores del YAIR.

En la figura 4.36c se muestran gráficamente los resultados del proceso de extracción y segmentación de líneas. En esa misma figura se observa que el mapa esta sobresegmentado. Esto se debe a que la naturaleza recursiva y el procesamiento en línea del algoritmo no permite hacer un filtrado sofisticado del ruido sensorial. Este filtrado se deja para ejecutarlo en un procesamiento posterior (fusión) que se ejecuta durante los períodos de baja carga computacional de la aplicación.

La figura 4.36d muestra los segmentos de rectas resultantes después de aplicar el proceso de fusión a los segmentos de líneas extraídas previamente. En esta figura se 


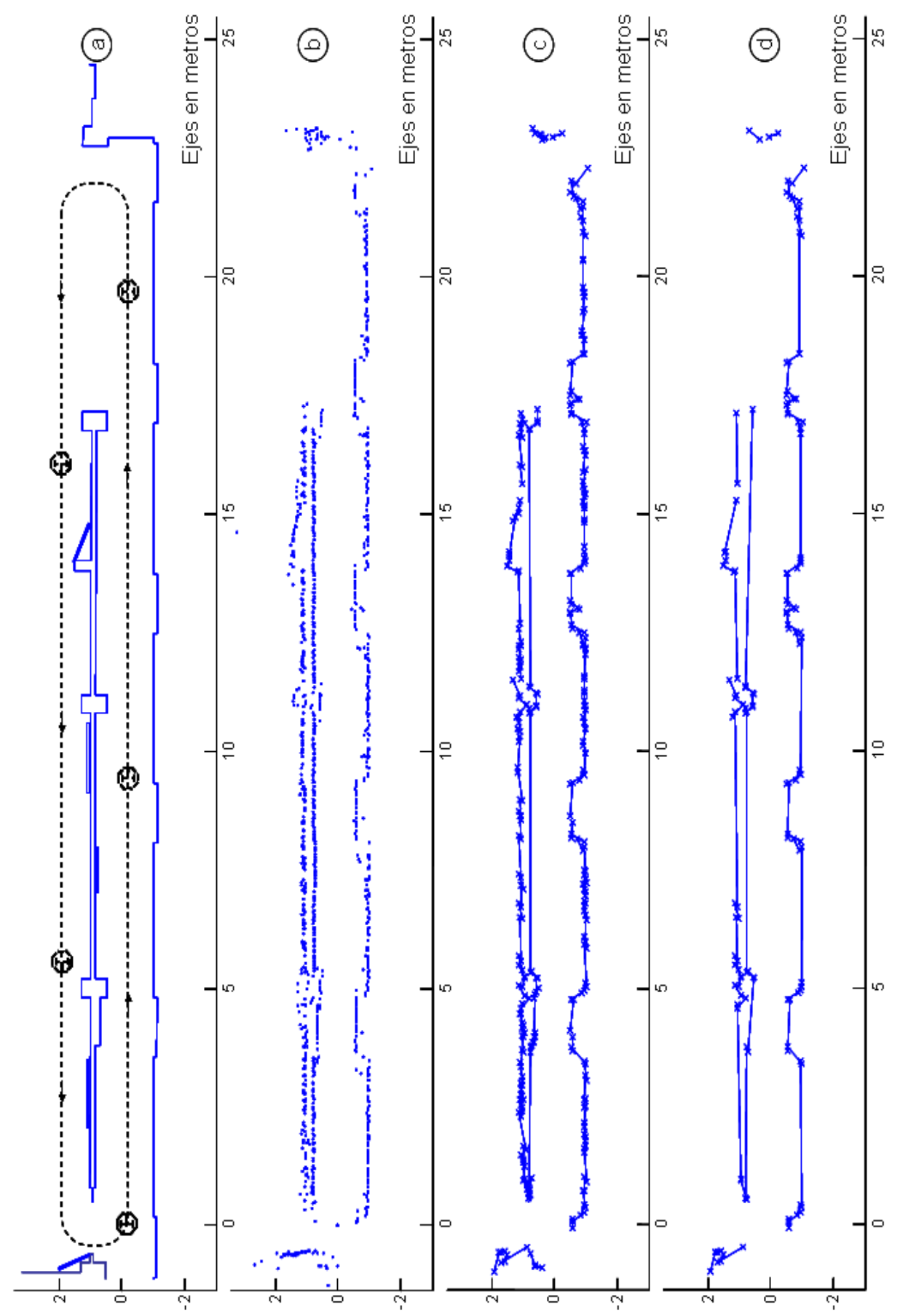

Figura 4.36: a) Mapa del escenario real donde se muestra el trayecto seguido por el robot durante el experimento realizado. b) Mapa de distancias extraídas mediante sensores de infrarrojos. c) Mapas de líneas después de la segmentación de los datos de infrarrojos. d) Mapa resultante después del proceso de fusión de líneas extraídas con sensores infrarrojos. 
CAPÍTULO 4. PERCEPCIÓN, FUSIÓN SENSORIAL Y CONSTRUCCIÓN DE MAPAS

ve que el algoritmo logra representar con pocos elementos y con buena precisión el entorno explorado. 


\section{Capítulo 5}

\section{Manejo de la incertidumbre en el sistema, en la medición y en la representación}

\subsection{Introducción}

La incertidumbre surge porque se tiene un conocimiento incompleto o incorrecto del mundo, o por limitaciones en la forma de representar dicho conocimiento. Existen varias causas de incertidumbre que tienen que ver con la información incompleta, poco fiable, o con ruido y distorsión. Otras fuentes de incertidumbre son el conocimiento impreciso y contradictorio, y la representación poco adecuada o con falta de poder descriptivo. Se han desarrollado diversas formas de representar y manejar la incertidumbre. Dentro de las técnicas numéricas, una de las más utilizadas son las probabilísticas: Cadenas de Markov, Redes Bayesianas, Procesos de Decisión de Markov, etc.

La teoría Bayesiana es un excelente marco de trabajo que permite tratar la incertidumbre en las aplicaciones de robótica móvil. Así por ejemplo, el problema general de localización de un robot móvil se puede describir como un problema de estimación Bayesiana[35, 57] en donde se desea estimar la localización de un robot dado un conjunto de medidas ruidosas. Observando el problema de forma probabilística, se puede decir que en base a toda la información disponible, el robot tiene cierto grado de confianza o certidumbre(Belief) sobre el lugar donde se encuentra localizado. Entonces el problema de la localización consiste en estimar la densidad de probabilidad de localización del robot sobre el espacio de todas las posibles localizaciones del mismo. Un algoritmo mediante el cual se puede estimar esta densidad es el conocido como Localización de Markov[77]. Este algoritmo combina la información proveniente de medidas sensoriales absolutas y relativas para obtener la densidad de probabilidad que describe la localización del robot; lo que se expresa como

$$
\operatorname{Bel}\left(x_{k}\right)=P\left(x_{k} \mid z_{0: k}\right)
$$




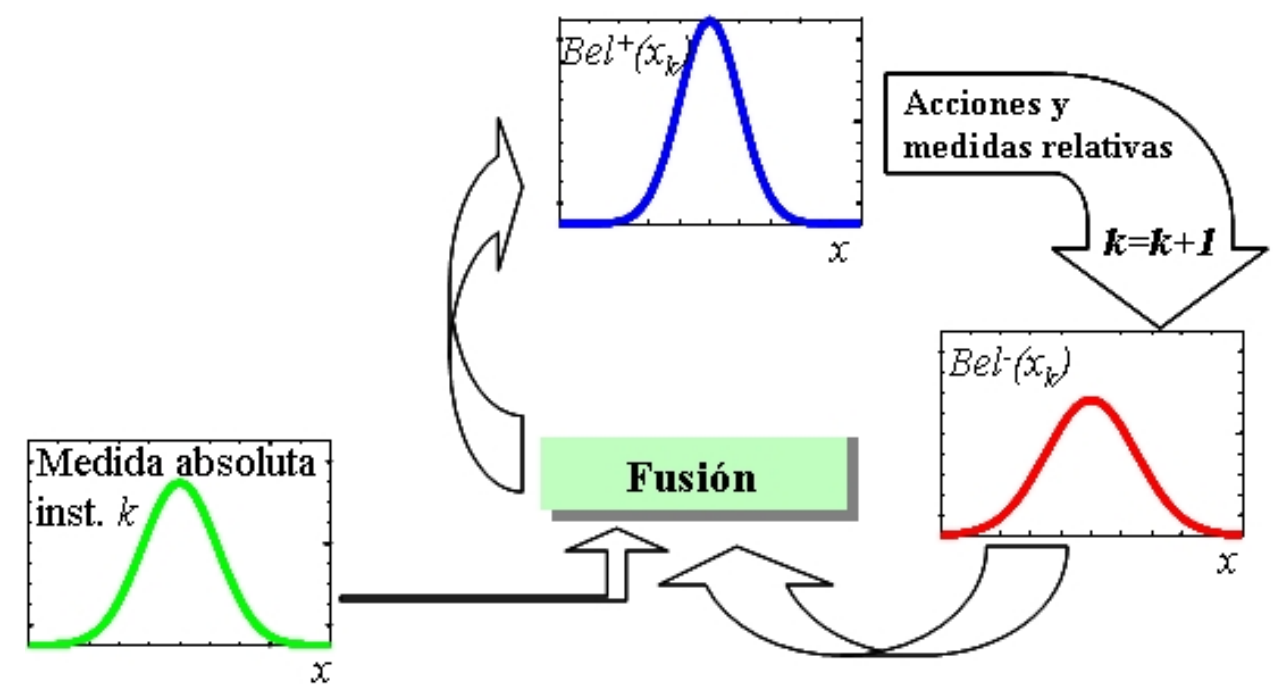

Figura 5.1: Ciclo de incremento y actualización de la confianza(Belief).

donde $\operatorname{Bel}(x)$ es la densidad de probabilidad sobre todas las localizaciones $x$ del robot, $\left\{x_{1}, x_{2}, \ldots, x_{k}\right\}$ representa los estados del robot en sucesivos instantes de tiempo $k, \mathrm{y}$ $z_{k}$ es la observación del robot en el mismo instante $k$.

La ecuación 5.1 representa la probabilidad de que el robot esté en $x_{k}$, dada toda la información o datos $z$ hasta el instante $k$. Esta información no sólo incluye los datos de los sensores propioceptivos y exteroceptivos sino que también puede incluir la información de un mapa a priori. Dada la distribución $\operatorname{Bel}(x)$, la localización $x_{k}$ que tenga la más alta confianza, es la localización en la que más probablemente se encuentre el robot.

La estimación de la confianza $\operatorname{Bel}(\cdot)$ en cada instante se realiza mediante una formulación recursiva que consta de dos componentes(ver figura 5.1): la confianza a priori $\mathrm{Bel}^{-}\left(x_{k}\right)$ la cual es la que resulta después de incorporar toda la información propioceptiva(relativa) y exteroceptiva(absoluta) hasta el instante $k$, sin incluir las medidas exteroceptivas de ese instante; y la confianza a posteriori $\mathrm{Bel}^{+}\left(x_{k}\right)$ que es la que resulta después de agregar justamente las medidas absolutas tomadas en el instante $k$.

La ecuación 5.2 muestra la fórmula general para la localización de Markov.

$$
\operatorname{Bel}^{+}\left(x_{k}\right)=\eta_{k} P\left(z_{k} / x_{k}\right) \int_{\Xi} P\left(x_{k} / x_{k-1}, u_{k}\right) \operatorname{Bel}^{+}\left(x_{k-1}\right) d x_{k-1}
$$

donde

$P\left(x_{k} / x_{k-1}, u_{k}\right)$ es el modelo de actuación, el cual representa a la densidad de probabilidad de terminar en la localización $x_{k}$ dado que estando en $x_{k-1}$ se realizó la acción $u_{k}$. Esta densidad se conoce como la densidad de transición. Normalmente modela la manera como cambia la localización del 
robot en función de las entradas de control, por lo que se suele extraer del modelo cinemático del móvil.

$P\left(z_{k} / x_{k}\right)$ es el modelo de percepción, el cual representa a la densidad de probabilidad de que estando en el estado $x_{k}$, se observe el vector de características del entorno $z_{k}$.

$\mathrm{Bel}^{+}\left(x_{k-1}\right)$ es la confianza en la localización estimada en el estado anterior, dada todas las evidencias hasta ese instante.

$\eta_{k} \quad$ es un normalizador que asegura que la densidad de probabilidad no sea mayor que uno.

La derivación de la fórmula de localización probabilística de Markov no es del todo sencilla. Los detalles de la misma, así como su implementación pueden encontrarse en $[75,198]$. No obstante, para propósitos de claridad de los conceptos, en el anexo A de esta tesis se detallan los fundamentos de la derivación de la mencionada fórmula.

Dentro de la implementación de la fórmula de localización de Markov, una manera de tratar la complejidad computacional de la incertidumbre sobre espacios continuos es mediante su representación como una función parametrizada y continua. El filtro de Kalman es una técnica mediante la cual se puede tratar este tipo de incertidumbre, siempre que la misma esté representada mediante densidades de probabilidad del tipo Normal o Gausiana. De aquí que en el resto de esta capítulo se desarrollen el modelo de transición y el modelo de percepción necesarios para implementar la localización del robot y representación probabilística del entorno mediante el Filtro de Kalman.

\subsection{Modelo cinemático estocástico de un vehículo de trac- ción diferencial}

Los robots móviles cuentan con sistemas de locomoción que le permiten moverse por su entorno. Comúnmente estos sistemas están basados en ruedas de tracción, pero también existen muchos robots con locomoción basada en patas, y otros menos típicos con desplazamiento tipo serpiente. Los vehículos con patas tienen el potencial de poder viajar sobre terrenos escarpados donde los robot con tracción a ruedas no pueden moverse. También los vehículos tipo serpiente pueden moverse por suelos con rocas y arena suelta por donde otros vehículos no pueden desplazarse. A pesar de esto, los vehículos basados en ruedas de tracción son los más comunes ya que los mismos son menos complejos y además son adecuados para navegar en la mayoría de los entornos interiores comunes a los humanos.

Los vehículos con locomoción basada en ruedas de tracción comúnmente cuentan con codificadores rotativos dispuestos en algunas de sus ruedas para medir el desplazamiento o la velocidad en la misma. No obstante, el interés no se centra en el desplazamiento relativo de cada rueda, sino en el desplazamiento del cuerpo del robot como acción conjunta de todas ellas. 
En la capítulo 3 ya se derivó el modelo cinemático para un robot diferencial, que es el tipo de vehículo en el cual se basan los experimentos realizados en esta tesis. Sin embargo, en ese modelado se asumió que el sistema de locomoción y el sistema sensorial asociado a él no estaban afectados por el ruido. Ahí se asumió que los codificadores trabajaban perfectamente y que las ruedas no sufrían derrapes; por lo que los pulsos emitidos por el codificador se podían transformar de manera perfecta a distancia recorrida por la rueda asociada a él. También, en ese mismo capítulo se desarrollaron métodos de calibración del sistema odométrico, con lo que se logró disminuir los errores en la localización odométrica. Pero a pesar de que los resultados obtenidos después de la calibración son bastante buenos, en los mismos también se verifica que en trayectos muy largos, la posición estimada difiere bastante de la posición real del robot. Este último resultado motiva a que en esta sección (5.2) se desarrollen el modelo estocástico de posicionamiento odométrico de un robot tipo diferencial. Este modelo además de representar la incertidumbre en la localización del robot, también permite incorporar el modelo de movimiento del robot a otros métodos de localización de mayor jerarquía como por ejemplo la localización mediante Filtros de Kalman.

\subsubsection{Modelo de ruido de un vehículo diferencial}

La ecuación 3.43 modela el caso ideal de un vehículo no afectado por el ruido. Es decir, en ella se ha asumido que no existen influencias del entorno sobre el sistema de navegación, y que los codificadores rotativos en las ruedas trabajan perfectamente, convirtiendo exactamente los pulsos recibidos a la respectiva distancia recorrida por cada rueda. Sin embargo, esta no es la situación más realista, por lo que es necesario modelar las influencias de estas perturbaciones en el sistema.

Ruido en el vector de excitación El ruido en el vector de excitación $v$, se puede asumir que esta normalmente distribuido con media igual a cero y con varianza igual a $\mathbf{U}$, lo que se expresa como,

$$
v_{k} \sim N(0, \mathbf{U})
$$

Con lo anterior, el ruido promedio que afecta al vector de excitación es

$$
\hat{v}_{k}=\left[\hat{v}_{\Delta S}, \hat{v}_{\Delta \theta}\right]_{k}^{\mathrm{T}}=[0,0]_{k}^{\mathrm{T}}
$$

También, dado que $\mathbf{U}$ es la matriz de covarianza de los desplazamientos relativos $\Delta S_{k}$ y $\Delta \theta_{k}$, entonces la misma corresponde a

$$
\mathbf{U}_{k}=\left[\begin{array}{cc}
\sigma_{S}^{2} & \sigma_{S \theta} \\
\sigma_{S \theta} & \sigma_{\theta}^{2}
\end{array}\right]
$$


Ruido aditivo en el sistema Este tipo de ruido es el que no esta directamente relacionado con el vector de excitación. Aunque este ruido es muy difícil de modelar, se puede asumir que el mismo es independiente, de distribución normal con media igual a cero, y de covarianza $\mathbf{Q}$.

$$
q_{k} \sim N(0, \mathbf{Q})
$$

De esta forma, el ruido y la matriz de covarianza serán de la forma

$$
\begin{gathered}
\hat{q}_{k}=\left[\hat{q}_{x}, \hat{q}_{y}, \hat{q}_{\theta}\right]_{k}^{\mathrm{T}}=[0,0,0]_{k}^{\mathrm{T}} \\
\mathbf{Q}_{k}=\left[\begin{array}{ccc}
\sigma_{x}^{2} & \sigma_{x y} & \sigma_{x \theta} \\
\sigma_{x y} & \sigma_{y}^{2} & \sigma_{y \theta} \\
\sigma_{x \theta} & \sigma_{y \theta} & \sigma_{\theta}^{2}
\end{array}\right]
\end{gathered}
$$

Finalmente, insertando en la ecuación 3.43 los elementos de ruido modelados, la ecuación que modela el sistema se convierte en

$$
\left[\begin{array}{l}
x_{k} \\
y_{k} \\
\theta_{k}
\end{array}\right]=\left[\begin{array}{c}
x_{k-1}+\left(\Delta S+v_{\Delta S}\right)_{k} \cos \left(\theta_{k-1}+\left(\Delta \theta+v_{\Delta \theta}\right)_{k} / 2\right) \\
y_{k-1}+\left(\Delta S+v_{\Delta S}\right)_{k} \sin \left(\theta_{k-1}+\left(\Delta \theta+v_{\Delta \theta}\right)_{k} / 2\right) \\
\theta_{k-1}+\left(\Delta \theta+v_{\Delta \theta}\right)_{k}
\end{array}\right]+\left[\begin{array}{c}
q_{x} \\
q_{y} \\
q_{\theta}
\end{array}\right]_{k}
$$

Un detalle que se pone de manifiesto en la ecuación 5.9, es que aunque de principio se asuma que todos los ruidos que afectan el sistema son de distribución normal, la distribución final del estado no lo será debido a los efectos no lineales que se introducen en el modelo del mismo.

\subsubsection{Estimación del error de estado}

El modelo odométrico de la ecuación 5.9 representa un sistema dinámico no lineal en variables de estados que puede ser representado en forma compacta según,

$$
\mathbf{x}_{k}=f\left(\mathbf{x}_{k-1}, \mathbf{u}_{k}, \mathbf{v}_{k}, \mathbf{q}_{k}\right)
$$

donde $\mathbf{x}=\langle x, y, \theta\rangle$ es el vector de estado, $\mathrm{y} \mathbf{u}=\langle\Delta S, \Delta \theta\rangle$ es la excitación de entrada.

El estimado del estado representado a través de la ecuación anterior, va acompañado de un estimado de la incertidumbre en el mismo, lo cual se representa mediante la matriz de covarianza $\mathbf{Q}$, la cual ya fue definida mediante la ecuación 5.8. El cálculo de esta matriz no es sencillo debido a la naturaleza no lineal de la ecuación de estimación del estado.

El estudio analítico de la propagación de la matriz de covarianza $\mathbf{Q}$ ha sido abordado por pocos investigadores. En este sentido hay pocos trabajos que tratan con estos modelos, y los que hay aplican sólo a una formas especifica de odometría o a una trayectoria particular. L. Kleeman[111] analiza los errores no sistemáticos y desarrolla un modelo estadístico para la estimación de la matriz de covarianza $\mathbf{Q}$ en 
plataformas móviles diferenciales. En particular desarrolla una forma cerrada de la covarianza del error para trayectos rectilíneos, para trayectos en forma de arco de curvatura constante, y para giros de la plataforma sobre su mismo eje. El enfoque o metodología de Kleeman consiste en la integración teórica de la ecuación del ruido a lo largo de la longitud total de la trayectoria que sigue el robot. De esta forma se consigue una expresión de forma cerrada para la matriz de covarianza, además de que se permite una actualización computacionalmente eficiente de la misma, después que se completa uno de los tipos de trayecto ya antes mencionado. Por ejemplo, para el caso de una trayectoria rectilínea, Kleeman aproxima una matriz de covarianza $\mathbf{Q}$ para un trayecto de longitud $s$ que esta dada por,

$$
\mathbf{Q}=\left[\begin{array}{ccc}
\frac{\left(\sigma_{R}^{2}+\sigma_{L}^{2}\right)}{4} & \frac{s\left(\sigma_{R}^{2}-\sigma_{L}^{2}\right)}{4 d} & \frac{\left(\sigma_{R}^{2}-\sigma_{L}^{2}\right)}{2 d} \\
\frac{s\left(\sigma_{R}^{2}-\sigma_{L}^{2}\right)}{4 d} & \frac{s^{2}\left(\sigma_{R}^{2}+\sigma_{L}^{2}\right)}{3 d^{2}} & \frac{s\left(\sigma_{R}^{2}+\sigma_{L}^{2}\right)}{2 d^{2}} \\
\frac{\left(\sigma_{R}^{2}-\sigma_{L}^{2}\right)}{2 d} & \frac{s\left(\sigma_{R}^{2}+\sigma_{L}^{2}\right)}{2 d^{2}} & \frac{\left(\sigma_{R}^{2}+\sigma_{L}^{2}\right)}{d^{2}}
\end{array}\right]
$$

Aquí $s$ es la distancia en metros totales recorrida a través del trayecto rectilíneo, siendo positiva cuando el robot avanza, y negativa cuando el robot retrocede; y $d$ es la distancia en metros entre las ruedas de odometría de la plataforma móvil diferencial. Igualmente, los parámetros de mayor importancia en el modelo de la matriz de covarianza anterior son $\sigma_{R}^{2}$ y $\sigma_{L}^{2}$, los cuales representan a la varianza del error en la medición del trayecto recorrido por las ruedas de odometría derecha e izquierda respectivamente.

Otro investigador que presenta una solución cerrada para la propagación del error sistemático o no sistemático, y válida para cualquier trayectoria y modelo de error es A. Kelly[104, 105]. A partir de la teoría de linealización de sistemas, el autor desarrolla un conjunto de ecuaciones integrales llamadas momentos, que luego puede aplicar a cualquier modelo y a cualquier trayectoria para particularizar la solución sobre este específico. Igualmente, A. Martinelli presenta una solución general para la propagación del error sistemático y no sistemático sobre cualquier trayectoria o modelo de error, y sugiere una posible estrategia para estimar los parámetros de odometría de un robot sincrónico[142, 144].

A pesar de que los resultados obtenidos con las propuestas anteriores son satisfactorias, el caso más general para estimar la matriz de covarianza ha sido el uso de aproximaciones lineales. Inicialmente $C$. M. Wang [201] verifica en forma analítica la validez de la aproximación en series de Taylor y describe los límites dentro los cuales puede ser aplicada la misma. En este trabajo, Wang se basa en un robot diferencial y analiza los errores no sistemáticos desde un punto de vista teórico y computa la matriz de covarianza del error odométrico Q. Así, este investigador introdujo un modelo de error basado en los parámetros físicos del robot. Este modelo fue desarrollado para un movimiento circular arbitrario pero limitado a pequeños ángulos de rotación. De esta manera hubo de dividir los trayectos en $\mathrm{N}$ pequeños trayectos elementales para que así el sistema conservara exactitud. Igualmente, dado que en la ecuación del estado aparece un factor no lineal que depende de los cambios en la orientación y la 
traslación medida por los codificadores, Wang introdujo otra aproximación: consideró este factor como una constante en el cálculo de la matriz de covarianza $\mathbf{Q}$, y también consideró la aproximación de este factor de ajuste mediante la expansión de series de Taylor de primer orden.

A partir del trabajo de Wang, la manera más usual de propagar el error del estado a través del sistema ha sido actualizar la matriz de covarianza $\mathbf{Q}$ de forma incremental a través de pequeños instantes de tiempo, de forma que el error sea pequeño y no se comentan errores significantes al hacer la expansión en series de Taylor de primer orden. La propagación de la covarianza del error en el estado se lleva a cabo mediante la linealización instantánea de la función $\mathbf{x}_{k}=f\left(\mathbf{x}_{k-1}, \mathbf{u}_{k}, \mathbf{v}_{k}, \mathbf{q}_{k}\right)$; donde se asume además que la varianza del error en el vector de excitación es independiente de la varianza en el error del vector de estado. De esta manera,

$$
\mathbf{Q}_{k}=\nabla_{\mathbf{x}} f(.) \mathbf{Q}_{k-1} \nabla_{\mathbf{x}} f(.)^{\mathrm{T}}+\nabla_{\mathbf{u}} f(.) \mathbf{U}_{k} \nabla_{\mathbf{u}} f(.)^{\mathrm{T}}
$$

Aquí, $\nabla_{\mathbf{x}} f($.$) representa la matriz Jacobiana de f\left(\mathbf{x}_{k-1}, \mathbf{u}_{k}, \mathbf{v}_{k}, \mathbf{q}_{k}\right)$ con respecto al estado $\mathbf{x}=\langle x, y, \theta\rangle$, mientras que $\nabla_{\mathbf{u}} f($.$) es la matriz Jacobiana de la misma función$ con respecto a la excitación de entrada $\mathbf{u}=\langle\Delta S, \Delta \theta\rangle$. Así, basándose en el modelo odométrico de la ecuación 5.9, la matrices Jacobianas corresponden a

$$
\begin{gathered}
\nabla_{\mathbf{x}} f(.)=\left[\begin{array}{ccc}
1 & 0 & -\Delta S_{k} \sin \left(\theta_{k-1}+\Delta \theta_{k} / 2\right) \\
0 & 1 & \Delta S_{k} \cos \left(\theta_{k-1}+\Delta \theta_{k} / 2\right) \\
0 & 0 & 1
\end{array}\right] \\
\nabla_{\mathbf{u}} f(.)=\left[\begin{array}{cc}
\cos \left(\theta_{k-1}+\Delta \theta_{k} / 2\right) & -\frac{1}{2} \Delta S_{k} \sin \left(\theta_{k-1}+\Delta \theta_{k} / 2\right) \\
\sin \left(\theta_{k-1}+\Delta \theta_{k} / 2\right) & \frac{1}{2} \Delta S_{k} \cos \left(\theta_{k-1}+\Delta \theta_{k} / 2\right) \\
0 & 1
\end{array}\right]
\end{gathered}
$$

Por otra parte, $\mathbf{Q}_{k-1}$ es la covarianza del estado que fue estimada en el instante anterior, mientras que $\mathbf{U}_{k}$ representa a la covarianza de la señal de entrada en el instante actual, que tiene la forma de la ecuación 5.12. Los elementos de la diagonal principal de $\mathbf{U}_{k}$ se derivan partiendo de las ecuaciones 3.6 y 3.7, las cuales se repiten a continuación.

$$
\begin{aligned}
\Delta \theta_{k} & =\frac{\Delta S_{k, R}-\Delta S_{k, L}}{d} \\
\Delta S_{k} & =\frac{\Delta S_{k, R}+\Delta S_{k, L}}{2}
\end{aligned}
$$

Así, tomando en cuenta la teoría general de propagación lineal del error, y considerando que la base de giro $d$ es una constante, el error en la estimación de la medida de desplazamiento de ángulo se calcula como,

$$
\varepsilon_{\Delta \theta}=\frac{\partial \Delta \theta}{\partial \Delta S_{R}} \varepsilon_{\Delta S_{R}}+\frac{\partial \Delta \theta}{\partial \Delta S_{L}} \varepsilon_{\Delta S_{L}}=\frac{\varepsilon_{\Delta S_{R}}+\varepsilon_{\Delta S_{L}}}{d}
$$


Aquí $\Delta S_{R}$ y $\Delta S_{L}$ son el arco o distancia diferencial registrada por el sensor odométrico de la rueda derecha e izquierda respectivamente; mientras que $\varepsilon_{\Delta S_{R}}$ y $\varepsilon_{\Delta S_{L}}$ son los errores aleatorios asociados a estos sensores. Con lo anterior se puede calcular la varianza según

$$
\begin{aligned}
\sigma_{\Delta \theta}^{2} & =E\left\langle\varepsilon_{\Delta \theta}, \varepsilon_{\Delta \theta}^{\mathrm{T}}\right\rangle \\
& =E\left\langle\frac{1}{d^{2}} \varepsilon_{\Delta S_{R}}^{2}+\frac{2}{d} \varepsilon_{\Delta S_{R}} \varepsilon_{\Delta S_{L}}+\frac{1}{d^{2}} \varepsilon_{\Delta S_{L}}^{2}\right\rangle \\
& =\frac{\left(\sigma_{R}^{2}+\sigma_{L}^{2}\right)}{d^{2}}
\end{aligned}
$$

donde $E\langle\cdot\rangle$ corresponde al operador del valor esperado, $\sigma_{R}^{2}$ y $\sigma_{L}^{2}$ son las varianzas que afectan a la medición del desplazamiento de la rueda derecha e izquierda respectivamente, y $E\left\langle\varepsilon_{\Delta S_{R}} \varepsilon_{\Delta S_{L}}\right\rangle=0$ debido a la independencia que existe entre el ruido que afecta a cada una de las ruedas.

La varianza para el desplazamiento relativo $\Delta S_{k}$ se obtiene de manera análoga a la anterior, pero tomando el valor esperado según $E\left\langle\varepsilon_{\Delta S}, \varepsilon_{\Delta S}^{\mathrm{T}}\right\rangle$, por lo que se obtiene,

$$
\sigma_{\Delta S}^{2}=\frac{\left(\sigma_{R}^{2}+\sigma_{L}^{2}\right)}{4}
$$

De igual manera, la covarianza entre el desplazamiento relativo $\Delta S_{k}$ y el cambio angular relativo $\Delta \theta_{k}$, se obtiene tomando la esperanza matemática según $E\left\langle\varepsilon_{\Delta S}, \varepsilon_{\Delta \theta}\right\rangle$, por lo que se obtiene,

$$
\sigma_{\Delta S}^{2}=\frac{\left(\sigma_{R}^{2}-\sigma_{L}^{2}\right)}{2 d}
$$

Con lo anterior, la matriz de covarianza $\mathbf{U}$, queda definida como

$$
\mathbf{U}=\left[\begin{array}{ll}
\frac{\left(\sigma_{R}^{2}+\sigma_{L}^{2}\right)}{4} & \frac{\left(\sigma_{R}^{2}-\sigma_{L}^{2}\right)}{2 d} \\
\frac{\left(\sigma_{R}^{2}-\sigma_{L}^{2}\right)}{2 d} & \frac{\left(\sigma_{R}^{2}+\sigma_{L}^{2}\right)}{d^{2}}
\end{array}\right]
$$

Basándose en los resultados de 5.19 y en la ecuación 5.12, se ve que la matriz de covarianza $\mathbf{Q}$ depende esencialmente de la varianza $\sigma_{R}^{2}$ y $\sigma_{L}^{2}$ en los codificadores.

\subsubsection{Modelado de la varianza en los sensores de odometría}

Modelar la varianza en los sensores de odometría es una labor muy compleja ya que la misma depende de varios factores que son de distinta naturaleza. En las aplicaciones de robótica, los sensores odométricos se usan casi exclusivamente para medir los desplazamientos lineales de las ruedas motrices del robot. De ahí que para obtener la varianza en estos sensores sea usual tratarlos como medidores electromecánicos de distancia, en los que se asume que el error en el que se incurre al medir una unidad de trayecto, está aleatoriamente distribuido en forma normal con 
media igual a cero. Igualmente también se asume que el error del trayecto actual es independiente del error cometido en el trayecto anterior, lo que conlleva a que la varianza a lo largo del trayecto total sea la suma de la varianza de cada uno de los trayectos registrados. Esto significa que la varianza será proporcional a la distancia total recorrida por la rueda de odometría a lo largo de un trayecto dado.

Una forma heurística que se utiliza usualmente para modelar la varianza en el sensor odométrico de distancias, consiste en modelarla a través de una función que varía linealmente con la distancia recorrida por la rueda de odometría[106, 111].

$$
\left\{\begin{array}{l}
\sigma_{R}^{2}=K_{R}^{2}\left|\Delta S_{R}\right| \\
\sigma_{L}^{2}=K_{L}^{2}\left|\Delta S_{L}\right|
\end{array}\right.
$$

donde $K_{R}^{2}$ y $K_{L}^{2}$ son constantes asociadas a la varianza de cada rueda.

Esta forma de modelar la varianza presupone que las ruedas giran siempre en contacto con la superficie de rodamiento; y establece que la varianza en el sensor odométrico de distancias depende esencialmente de la longitud del trayecto recorrido por la rueda, lo cual se corresponde con la naturaleza física del proceso. Por ejemplo, a mayor velocidad mayor es la posibilidad de que ocurran derrapes y patinazos en las ruedas de odometría; y para un período de muestreo constante, este efecto de ruido es modelado por la distancia recorrida por la rueda que también se ve aumentada, aumentando con ella la varianza. Igualmente, si el móvil no se mueve, la distancia recorrida es nula y por la tanto el error que se comete también será nulo.

Con esta forma de modelar la varianza basta con determinar o escoger apropiadamente el valor de $K^{2}$ para así obtenerla varianza o ruido en el sensor odométrico de distancias. Los valores típicos de $K_{R}^{2}$ y $K_{L}^{2}$ han mostrado encontrarse en el rango $\left[10^{-6}, 10^{-5}\right][51,6]$. Sin embargo, como este es un factor que es muy sensible a las condiciones del entorno de operación y a los mecanismos cinemáticos del móvil, a continuación se propone una estrategia para determinar este experimentalmente.

\subsubsection{Estrategia experimental para determinar la varianza en sen- sores de odometría}

Considere la variable aleatoria $\mathbf{x}=\langle x, y\rangle$ la cual puede ser representada mediante una función de densidad de probabilidad bivariante tipo Gaussiana,

$$
p(\mathbf{x})=\frac{1}{2 \pi \sqrt{\operatorname{det} \mathbf{Q}}} e^{-\frac{1}{2}\left[(\mathbf{x}-\overline{\mathbf{x}}) \mathbf{Q}^{-1}(\mathbf{x}-\overline{\mathbf{x}})^{\mathrm{T}}\right]}
$$

adonde $\mathbf{x}=\langle x, y\rangle$ es el vector de coordenadas las cuales son conjuntamente Gausianas, $\overline{\mathbf{x}}=\langle\bar{x}, \bar{y}\rangle$ es el vector de medias, y $\mathbf{Q}$ es la matriz de covarianza definida según,

$$
\mathbf{Q}=\left[\begin{array}{cc}
\sigma_{x}^{2} & \sigma_{x y} \\
\sigma_{x y} & \sigma_{y}^{2}
\end{array}\right]=\left[\begin{array}{cc}
\sigma_{x}^{2} & \rho \sigma_{x} \sigma_{y} \\
\rho \sigma_{x} \sigma_{y} & \sigma_{y}^{2}
\end{array}\right]
$$

La distribución bivariante $p(\mathbf{x})$ describe una superficie que al intersectarla con un plano de densidad de probabilidad constante, da lugar a elipsoides de probabilidad 


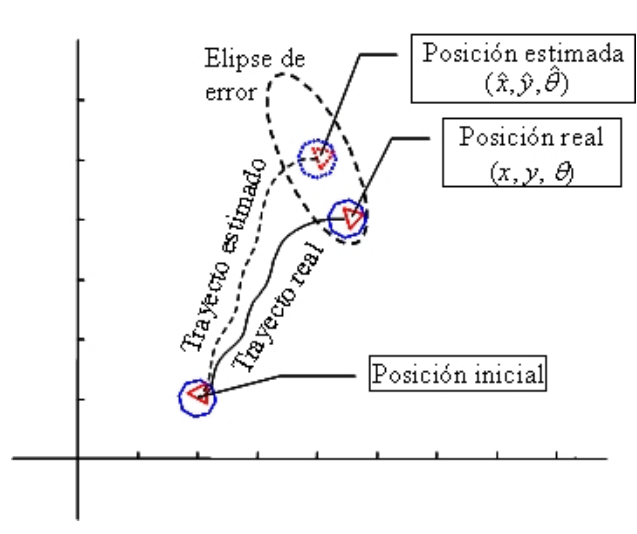

a)

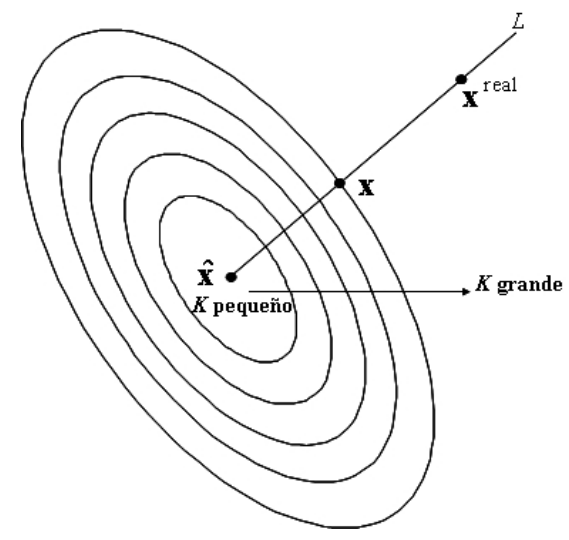

b)

Figura 5.2: a) Ejemplo de una elipse de error de localización de un móvil. b) Familia de elipses de error de acuerdo a $\mathrm{K}$

conocidas como elipsoides de concentración y que están definidas por el exponente de la función de verosimilitud

$$
\begin{gathered}
(\mathbf{x}-\overline{\mathbf{x}}) \mathbf{Q}^{-1}(\mathbf{x}-\overline{\mathbf{x}})^{\mathrm{T}}=\tau \\
\frac{(x-\bar{x})^{2}}{\sigma_{x}^{2}}-2 \rho \frac{(x-\bar{x})(y-\bar{y})}{\sigma_{x} \sigma_{y}}+\frac{(y-\bar{y})^{2}}{\sigma_{y}^{2}}=\tau(1-\rho)
\end{gathered}
$$

Cuando $\tau$ es igual a 1, la elipse resultante se conoce como elipse de error, y la misma especifica la región del espacio donde se concentran los componentes de la variable aleatoria $\mathbf{x}$.

En las aplicaciones de robótica móvil la elipse de error se utiliza para representar la incertidumbre en la estimación de la localización del robot. Esta elipse depende de la matriz de covarianza $\mathbf{Q}$ ya antes definida en la ecuación 5.8; y esta matriz depende de $\sigma_{R}^{2}$ y $\sigma_{L}^{2}$, que a su vez dependen de $K_{R}^{2}$ y $K_{L}^{2}$.

Bajo este contexto, una forma heurística de determinar la constante $K$ que modela la varianza en los sensores de odometría, es excitar al robot con trayectorias aleatorias y medir con alguna técnica la posición real del robot. Luego, a partir de la posición estimada por la odometría del robot, se hace una búsqueda exhaustiva a través de $K$ hasta que la elipse de error incluya el punto de la localización real del robot.

Basado en la figura 5.2b, un criterio de convergencia para asegurar que la elipse de incertidumbre incluya el punto de localización real del robot, es que la distancia euclidiana $\overline{p p_{2}}$ sea menor o igual que la distancia euclidiana $\overline{p p_{1}}$. El algoritmo 2 muestra una forma de implementar la búsqueda exhaustiva de $\mathrm{K}$.

Si se ejecutan $N$ ensayos y se aplica el algoritmo 2 , el valor de $K$ óptimo que garantiza que en el peor caso de ruido la elipse de error modelará la incertidumbre del robot, se obtiene maximizando los resultados. 

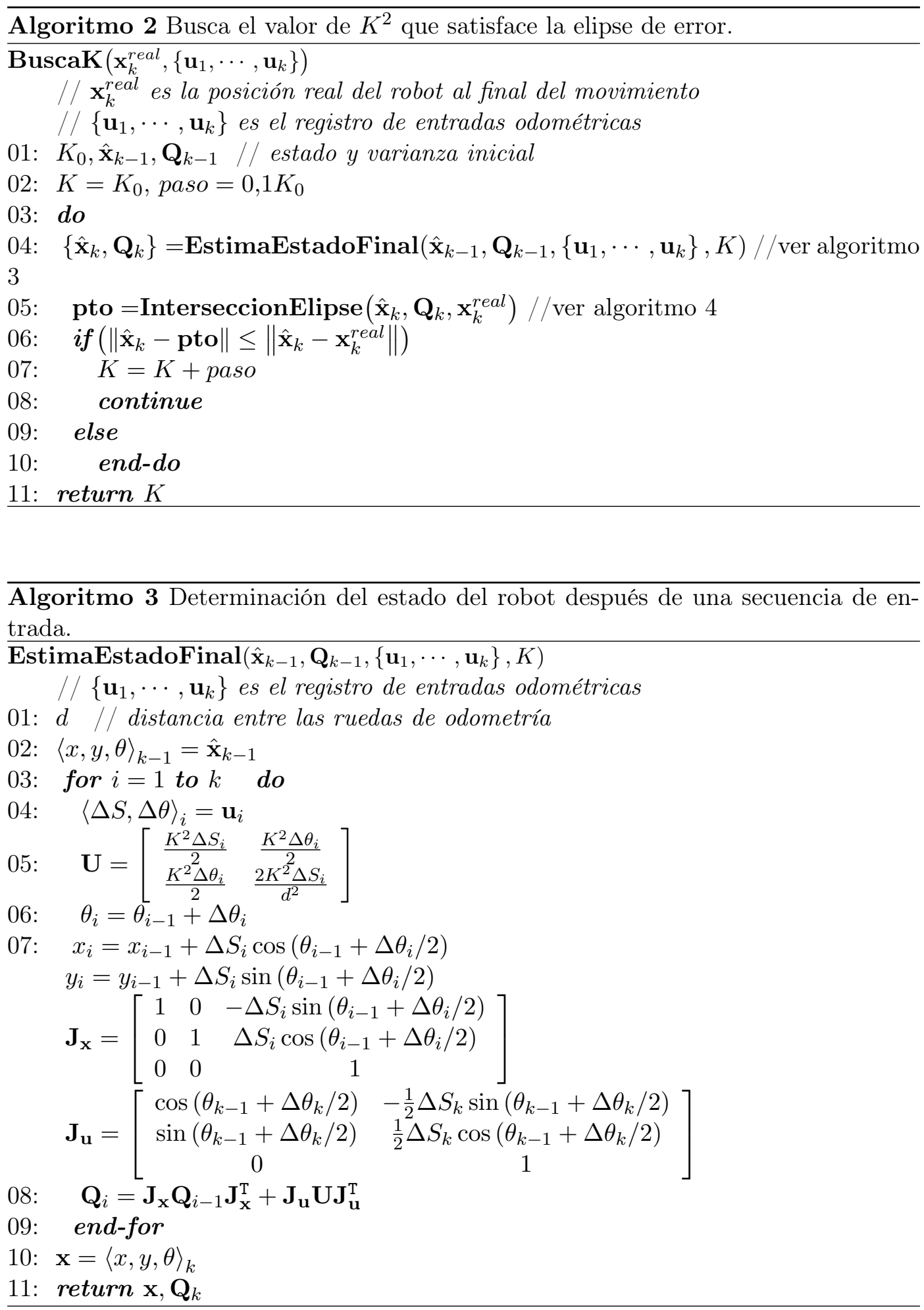


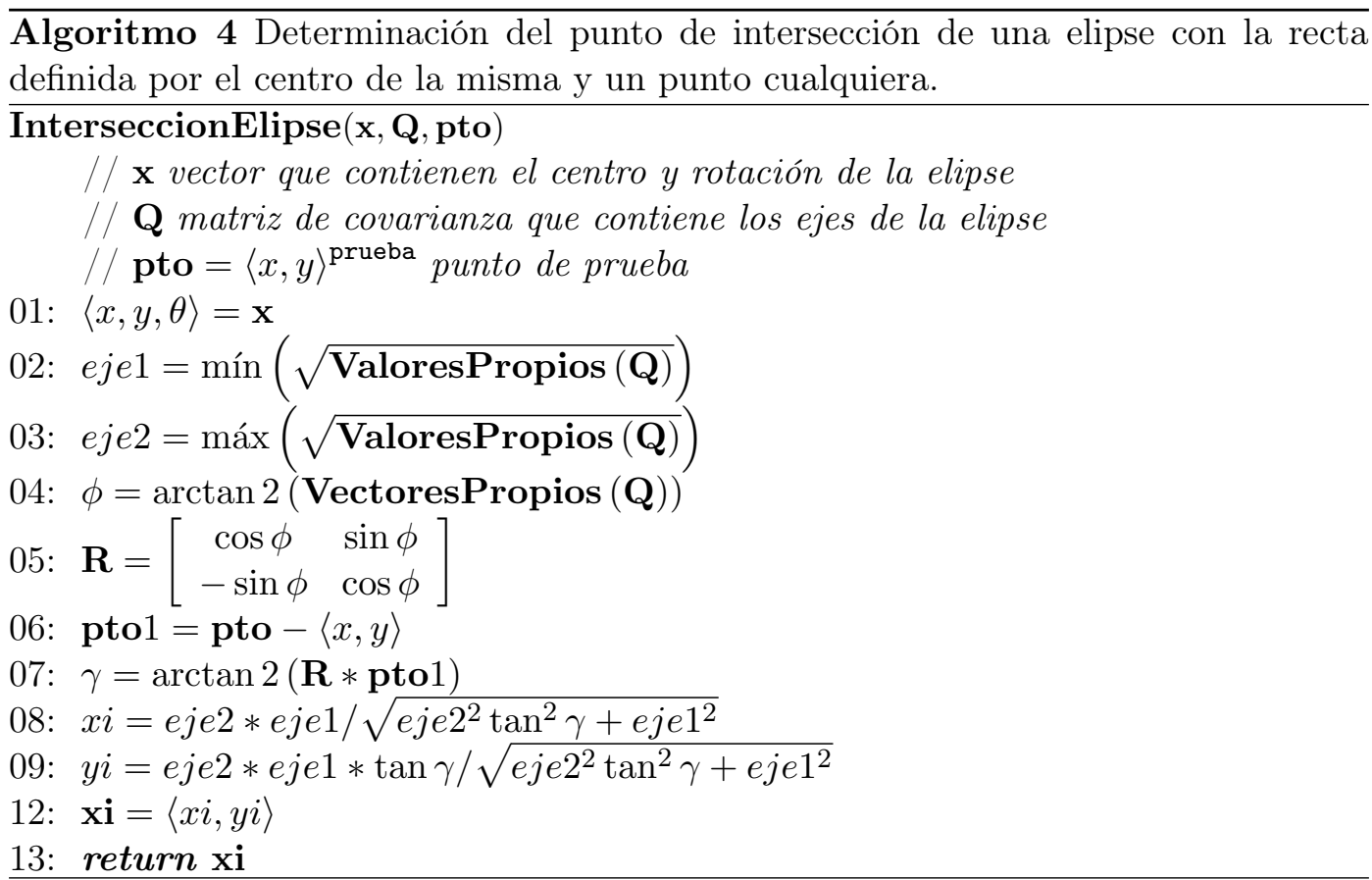

\subsubsection{Resultados experimentales en la determinación de la varianza en los sensores de odometría}

Para probar la validez del algoritmo de identificación del parámetro $K$ que modela la varianza en los sensores de odometría, se realizaron un conjunto de 25 pruebas donde el robot debía seguir una trayectoria aleatoria. La posición inicial y final del robot fueron medidas manualmente, mientras que los datos de odometría que generaron los sensores fueron registrados a lo largo de todo el trayecto. Asumiendo que el valor de $K$ en ambos sensores es el mismo, se aplicó el algoritmo 2, y se obtuvieron los valores de $K$ óptimo mostrados en la tabla 5.1.

La dispersión de los valores de $K$ óptimo, así como la media y la mediana de los mismos se muestran en la figura 5.3.

Considerando que se requiere modelar la varianza de tal forma que la elipse de incertidumbre incluya la localización real del robot en el máximo número de casos; al observar la distribución de los datos y después de filtrar los valores atípicos, se puede concluir que el valor de $K$ óptimo para el caso del robot YAIR resulta ser

$$
K_{\mathrm{opt}}=8,67 \times 10^{-5}
$$

De esta forma, la varianza en los sensores odométricos se puede modelar como,

$$
\left\{\begin{array}{l}
\sigma_{\mathrm{R}}^{2}=8,67 \times 10^{-5}\left|\Delta S_{\mathrm{R}}\right| \\
\sigma_{\mathrm{L}}^{2}=8,67 \times 10^{-5}\left|\Delta S_{\mathrm{L}}\right|
\end{array}\right.
$$

Para probar la validez del modelo estocástico del robot, se introdujo el modelo de 
Tabla 5.1: Valores de $K^{2}$ óptimo para la odometría del robot YAIR. Nótese que después de hacer un análisis estadístico de los datos se determinó que el resultado del ensayo número 8 es un valor espurio u outlier.

\begin{tabular}{|c|c|c|c|}
\hline $\mathrm{N}^{\circ}$ & $K^{2}$ óptimo & $\mathrm{N}^{\circ}$ & $K^{2}$ óptimo \\
\hline \hline 1 & $4.80 \mathrm{E}-06$ & 14 & $1.84 \mathrm{E}-05$ \\
\hline 2 & $7.70 \mathrm{E}-06$ & 15 & $2.44 \mathrm{E}-05$ \\
\hline 3 & $1.17 \mathrm{E}-05$ & 16 & $8.67 \mathrm{E}-05$ \\
\hline 4 & $7.85 \mathrm{E}-05$ & 17 & $5.20 \mathrm{E}-06$ \\
\hline 5 & $6.31 \mathrm{E}-05$ & 18 & $7.66 \mathrm{E}-05$ \\
\hline 6 & $3.74 \mathrm{E}-05$ & 19 & $2.00 \mathrm{E}-05$ \\
\hline 7 & $5.55 \mathrm{E}-05$ & 20 & $1.00 \mathrm{E}-06$ \\
\hline 8 & $1.41 \mathrm{E}-04$ & 21 & $1.22 \mathrm{E}-05$ \\
\hline 9 & $3.41 \mathrm{E}-05$ & 22 & $1.49 \mathrm{E}-05$ \\
\hline 10 & $3.62 \mathrm{E}-05$ & 23 & $7.24 \mathrm{E}-05$ \\
\hline 11 & $2.77 \mathrm{E}-05$ & 24 & $4.57 \mathrm{E}-05$ \\
\hline 12 & $4.40 \mathrm{E}-05$ & 25 & $2.14 \mathrm{E}-05$ \\
\hline 13 & $1.76 \mathrm{E}-05$ & 26 & \\
\hline
\end{tabular}

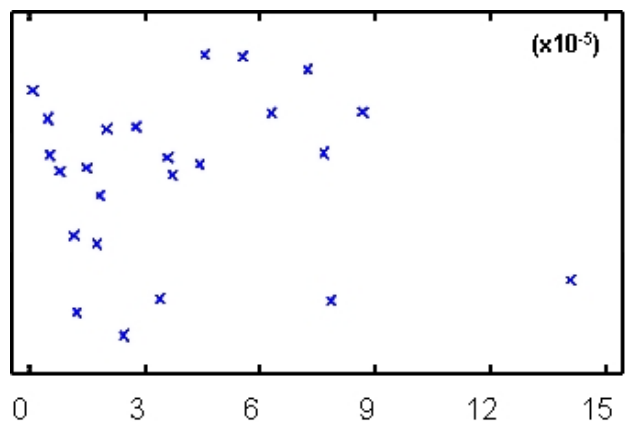

a)

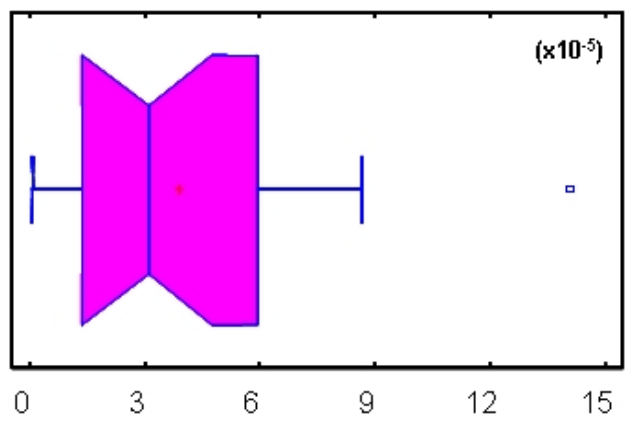

b)

Figura 5.3: Gráficos estadísticos valores de $K^{2}$ óptimo odometría robot YAIR. a) Dispersión de los valores de $K^{2}$ óptimo. b) Mediana(barra vertical) y media (cruz) de los valores de $K^{2}$ óptimo 


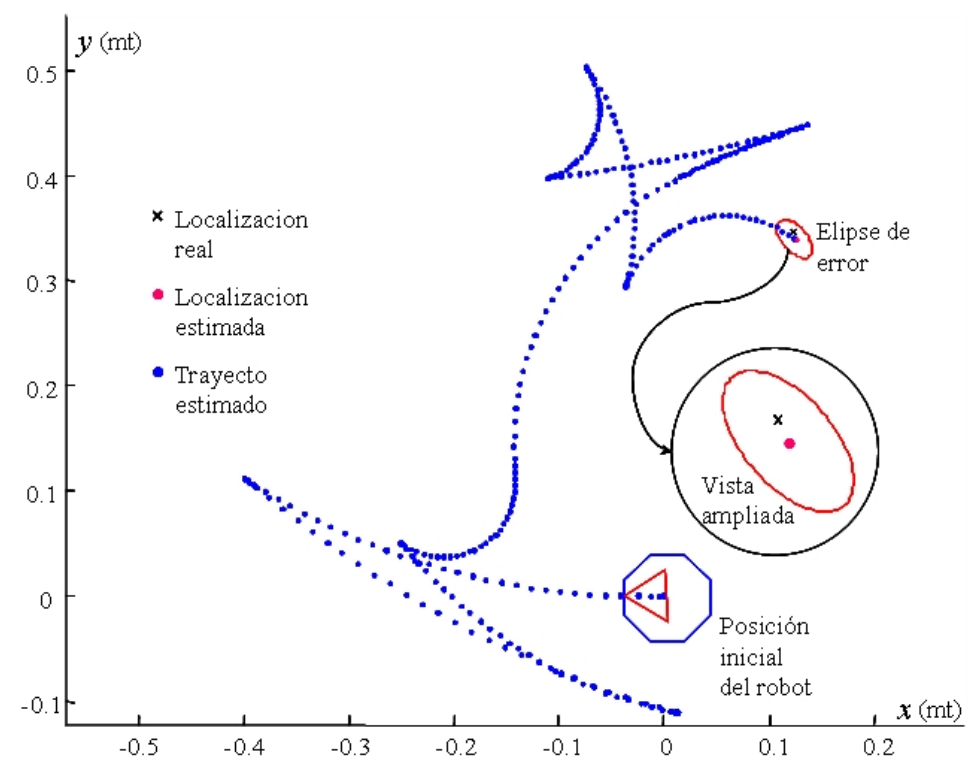

Figura 5.4: Representación de la incertidumbre de localización mediante elipse de error. Nótese en la vista ampliada como la elipse de incertidumbre rodea la localización real del robot.

varianza en el software operativo del robot YAIR, y se programó el mismo para que ejecutase un conjunto de trayectos arbitrarios. En estos trayectos se midió la posición real del robot y se comparó con la posición estimada, para verificar que la elipse de error modela la diferencia entre las dos.

En un primer experimento el robot ejecutó un trayecto aleatorio de aproximadamente 4 metros, a una velocidad máxima de $250 \mathrm{~mm} / \mathrm{seg}$. La visión gráfica del resultado obtenido se muestra en la figura 5.4.

En un segundo ensayo el robot se movió a través de un trayecto de aproximadamente 45 metros de longitud que incluye 6 giros de 90 grados cada uno. En la figura se muestra el resultado obtenido. Nótese como la elipse de error es capaz de modelar la incertidumbre en la posición del robot.

\subsection{Modelo estocástico del sensor de ultrasonidos}

Los sensores de ultrasonidos de Polaroid son ampliamente usados en la determinación de distancias basándose en la medición del tiempo de vuelo. Esta simple técnica convierte a la medición ultrasónica en una de los métodos más utilizados en robótica móvil por su relación costo-efectividad. Sin embargo, estos sensores tienen una desventaja importante: su amplio ancho de haz. Esto causa que el SONAR sea poco útil en el proceso de extracción de características del entorno. A pesar de esto, en nuestro laboratorio se ha desarrollado un SONAR que capaz de distinguir entre paredes y esquinas, basándose en la amplitud del eco ultrasónico recibido[20, 146]. En 


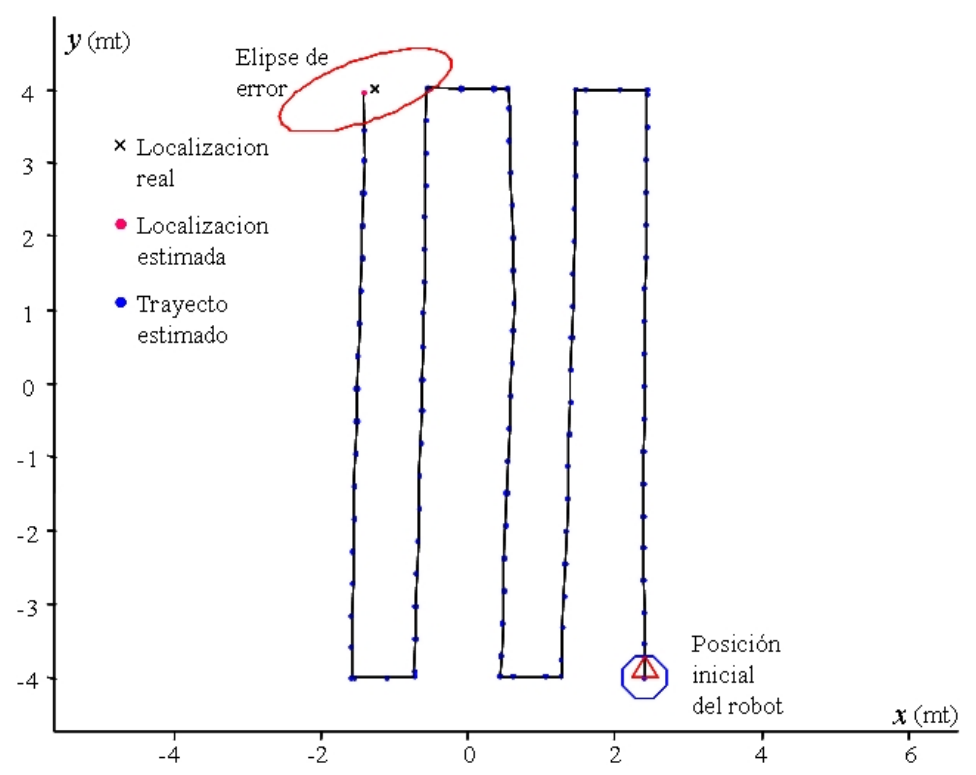

Figura 5.5: Elipse de error generada en un trayecto de aproximadamente 45 mts.

esta sección se introduce a este sensor, y se desarrolla el modelo de ruido del mismo.

\subsubsection{Clasificación y extracción de landmark a partir de datos de ultrasonidos}

En muchas aplicaciones de seguimiento e identificación de características geométricas, no es posible distinguir, a partir de un sólo barrido, el tipo de objeto que ha reflejado a la señal ultrasónica. Para poder hacer una clasificación sobre el origen de la misma, como mínimo es necesario disponer de dos observaciones, que a través del tiempo, haya realizado un mismo sensor[129]. En esta tesis se utiliza un SONAR rotativo que es capaz de diferenciar en un sólo barrido, y con una precisión cercana al $80 \%$, si se trata de una pared o de una esquina[145]. Dado que este sensor es el elemento básico de observación en los algoritmos de construcción de mapas y localización que se utilizan en este trabajo, a continuación se describe a el mismo.

El sensor que se usa en esta tesis posee un arreglo de transductores Tx/Rx que está instalado sobre un motor de pasos de 1.8 grados de resolución(ver figura 4.4). A cada paso angular, el emisor envía un tren de 16 pulsos de ultrasonidos, y luego registra la amplitud de los ecos que regresan del entorno. La amplitud del eco recibido se modela según,

$$
A=A_{0} C_{r}^{N} \frac{e^{-2 \alpha_{1} r}}{2 r} e^{-\left(4 \theta / \theta_{0}\right)^{2}}
$$

donde $A$ es el eco de amplitud obtenido en el receptor ultrasónico, $A_{0}$ es una constante del transductor, $\alpha_{1}$ es el coeficiente de atenuación del aire, $r$ es la distancia radial entre 
el par de transductores y la superficie reflectora, y $C_{r}$ es el coeficiente de reflexión del objeto, el cual varía entre 0 y 1 dependiendo del material que conforme al mismo. Igualmente, $N$ es un parámetro que depende de la forma del objeto reflector. Este parámetro puede tomar uno de dos valores: 1 para reflectores tipo pared y 2 para reflectores tipo esquina. El valor de este parámetro está directamente relacionado con el numero de reflexiones que sufre el eco antes de alcanzar el receptor.

Por otra parte, se sabe que la máxima amplitud del eco se recibe cuando el haz de ultrasonidos enfoca perpendicularmente a la superficie del reflector(ver figura 4.5). De esta manera, con $\theta=0$ se puede resolver para $N$ de acuerdo a,

$$
N=\frac{\ln \left(2 A r / A_{0}\right)+2 \alpha_{1} r}{\ln C_{r}}
$$

Si el resultado de evaluar la ecuación 5.28 está más próximo a 1 que a 2, el objeto reflector es una pared; mientras que si está más próximo a 2 , entonces el reflector es una esquina[146].

Los resultados experimentales han mostrado una efectividad que está entre el 80 y el $90 \%$ para la extracción de paredes, y alrededor del $80 \%$ para la extracción de esquinas.

\subsubsection{Modelo ideal de un SONAR tipo rotativo}

El SONAR rotativo que se acaba de presentar proporciona información geométrica y cualitativa del entorno que lo rodea. Las esquinas se representan mediate el modelo $\mathbf{z}=[x, y, i d]^{\mathrm{T}}$, mientras que las paredes o extructuras planas se representan mediante el modelo Hessiano $\mathbf{z}=[\rho, \varphi, i d]^{\mathrm{T}}$. Las variables $(x, y)$ representan las coordenadas cartesianas relativas al marco o sistema de coordenadas del sensor, mientras que $(\rho, \varphi)$ representan la distancia radial y el ángulo de acimut en coordenadas polares relacionadas también al sistema de coordenadas del sensor. La variable $i d$ contiene un identificador que clasifica el objeto detectado como una esquina o como una pared.

Un SONAR tipo rotativo, normalmente consiste de un sensor de ultrasonido ordinario que ha sido instalado en algún tipo de servomecanismo (ver figura 4.4). El servomecanismo suele ser construido en base a un motor de pasos cuya resolución puede estar entre los 50 y 200 pasos por revolución. Esta arquitectura confiere la propiedad de poder hacer un barrido de 360 grados de la escena con un sólo sensor, pero a cambio le resta mucha velocidad al proceso de observación del entorno. Una ventaja que no tiene contrapartida es que con este tipo de sensor se puede reducir la incertidumbre angular que introduce el sensor de ultrasonido. La técnica con la que se logra esta reducción ya se introdujo en la sección 4.1.3.

Idealmente, las coordenadas $\langle x, y\rangle^{\mathrm{S}}$ de un punto del entorno que actúa como reflector en el sistema de coordenadas del sensor pueden ser escritas como,

$$
\mathbf{z}^{\mathrm{S}}=h(\rho, \varphi)=\left[\begin{array}{l}
x^{\mathrm{S}} \\
y^{\mathrm{S}}
\end{array}\right]=\left[\begin{array}{l}
\rho \cos \varphi \\
\rho \sin \varphi
\end{array}\right]
$$


donde $\rho$ es la distancia radial desde el elemento transductor hasta el punto de reflexión, y $\varphi$ es el ángulo de vista acimutal contado a partir del eje $x$ positivo del sistema de referencia del sensor.

\subsubsection{Modelo de ruido del SONAR tipo rotativo}

Aunque la ecuación 5.29 expresa las coordenadas exactas del punto de reflexión del eco ultrasónico, en la práctica existe ruido que genera incertidumbre sobre esta lo-

calización. De aquí que la localización más realista de un punto reflector la modelemos como

$$
\mathbf{z}^{\mathrm{S}}=h(\rho, \varphi)=\left[\begin{array}{l}
x^{\mathrm{S}} \\
y^{\mathrm{S}}
\end{array}\right]=\left[\begin{array}{c}
\left(\rho+w_{\rho}\right) \cos \left(\varphi+w_{\varphi}\right) \\
\left(\rho+w_{\rho}\right) \sin \left(\varphi+w_{\varphi}\right)
\end{array}\right]
$$

donde $\left\langle w_{\rho}, w_{\varphi}\right\rangle$ es un vector ortogonal que corresponde al ruido que afecta al sensor.

Asumiendo que el ruido $w_{\varphi}$ es suficientemente pequeño de tal forma que,

$$
\left\{\begin{array}{l}
\cos \left(w_{\varphi}\right) \approx 1 \\
\sin \left(w_{\varphi}\right) \approx w_{\varphi}
\end{array}\right.
$$

y expandiendo las funciones trigonométricas de la ecuación 5.30; la localización de un punto reflector en el espacio cartesiano puede ser aproximada como

$$
\begin{aligned}
\mathbf{z}^{\mathrm{S}}=\left[\begin{array}{l}
x^{\mathrm{S}} \\
y^{\mathrm{S}}
\end{array}\right] & =\left[\begin{array}{l}
\left(\rho+w_{\rho}\right)\left(\cos \varphi-w_{\varphi} \sin \varphi\right) \\
\left(\rho+w_{\rho}\right)\left(\sin \varphi+w_{\varphi} \cos \varphi\right)
\end{array}\right] \\
& =\left[\begin{array}{c}
\rho \cos \varphi-\rho w_{\varphi} \sin \varphi+w_{\rho} \cos \varphi-w_{\rho} w_{\varphi} \sin \varphi \\
\rho \sin \varphi+\rho w_{\varphi} \cos \varphi+w_{\rho} \sin \varphi-w_{\rho} w_{\varphi} \cos \varphi
\end{array}\right]
\end{aligned}
$$

Por otra parte, de acuerdo a la naturaleza funcional del sensor, es de esperar que el vector de ruido que afecta al mismo sea ortogonal y que sus componentes se alineen en la dirección radial $\mathbf{u}_{\rho}$ y en la dirección angular $\mathbf{u}_{\varphi}$. Sin embargo, de acuerdo a los resultados experimentales que se verán en la sección 5.3.4, la correlación en el vector de ruido es significativamente distinta de cero. También, en la misma sección se verá que la distribución del ruido es del tipo Gausiano con media igual a cero. Así, el ruido sensorial puede modelarse según,

$$
\mathbf{w}^{\mathrm{S}} \sim N(\hat{\mathbf{w}}, \mathbf{R})=N\left(\left[\begin{array}{l}
0 \\
0
\end{array}\right],\left[\begin{array}{cc}
\sigma_{\rho}^{2} & \sigma_{\rho \varphi} \\
\sigma_{\rho \varphi} & \sigma_{\varphi}^{2}
\end{array}\right]\right)
$$

Ahora bien, considerando un conjunto de medidas $\Xi$ según,

$$
\Xi=\left[\left\{\rho_{1}, \rho, \ldots, \rho_{n}\right\},\left\{\varphi_{1}, \varphi_{2}, \ldots, \varphi_{n}\right\}\right]^{\mathrm{T}}
$$

Si se considera $\Xi$ como una variable aleatoria con distribución Gausiana, entonces se puede representar mediante su valor medio y su varianza de acuerdo a

$$
\Xi=N\left([\hat{\rho}, \hat{\varphi}]^{\mathrm{T}}, \mathbf{R}\right)
$$


donde $\hat{\rho}$ es el valor esperado de la magnigtud del vector que se extiende desde el origen del sistema de coordenadas del sensor hasta el punto reflector, y $\hat{\varphi}$ es el valor esperado del ángulo de acimut del mismo, y $\mathbf{R}$ es la matriz de covarianza del ruido sensorial ya definida en la ecuación 5.33 .

Con la presunción anterior, y sustituyendo $E\left\langle w_{\rho}\right\rangle=E\left\langle w_{\varphi}\right\rangle=E\left\langle w_{\rho} \cdot w_{\varphi}\right\rangle=0$, el valor promedio del vector de localización del punto reflector sería,

$$
E\left\langle\mathbf{z}^{\mathrm{S}}\right\rangle=\hat{\mathbf{z}}^{\mathrm{S}}=\left[\begin{array}{l}
\hat{x}^{\mathrm{S}} \\
\hat{y}^{\mathrm{S}}
\end{array}\right]=\left[\begin{array}{l}
\hat{\rho} \cos \hat{\varphi} \\
\hat{\rho} \sin \hat{\varphi}
\end{array}\right]
$$

donde $E\langle\cdot\rangle$ se refiere a la esperanza matemática.

Con el valor estimado en 5.36 y la expresión para la medición de la ecuación 5.32, se obtiene el error en la estimación, el cual será igual a

$$
\varepsilon_{\mathbf{z}}=\mathbf{z}^{\mathrm{S}}-\hat{\mathbf{z}}^{\mathrm{S}}=\left[\begin{array}{l}
-\rho w_{\varphi} \sin \varphi+w_{\rho} \cos \varphi-w_{\rho} w_{\varphi} \sin \varphi \\
\rho w_{\varphi} \cos \varphi+w_{\rho} \sin \varphi-w_{\rho} w_{\varphi} \cos \varphi
\end{array}\right]
$$

De aquí que la covarianza del error sea igual a,

$$
\begin{aligned}
\mathbf{Z} & =E\left\langle\varepsilon_{\mathbf{z}} \cdot \varepsilon_{\mathbf{z}}^{\mathrm{T}}\right\rangle \\
& =\left[\begin{array}{cc}
\sigma_{\rho}^{2} \cos ^{2} \varphi+\rho^{2} \sin ^{2} \varphi \sigma_{\varphi}^{2} & \left(\sigma_{\rho}^{2}-\rho^{2} \sigma_{\varphi}^{2}\right) \cos \varphi \sin \varphi \\
\left(\sigma_{\rho}^{2}-\rho^{2} \sigma_{\varphi}^{2}\right) \cos \varphi \sin \varphi & \sigma_{\rho}^{2} \sin ^{2} \varphi+\rho^{2} \cos ^{2} \varphi \sigma_{\varphi}^{2}
\end{array}\right]
\end{aligned}
$$

Esto mismo puede calcularse mediante aproximación lineal de la función de medición según,

$$
\begin{aligned}
\mathbf{Z} & =\left(\frac{\partial \hat{\mathbf{z}}}{\partial r \partial \varphi}\right) \mathbf{R}\left(\frac{\partial \hat{\mathbf{z}}}{\partial r \partial \varphi}\right)^{\mathrm{T}} \\
& =\left[\begin{array}{cc}
\cos \varphi & -\rho \sin \varphi \\
\sin \varphi & \rho \cos \varphi
\end{array}\right]\left[\begin{array}{cc}
\sigma_{\rho}^{2} & \sigma_{\rho \varphi} \\
\sigma_{\rho \varphi} & \sigma_{\varphi}^{2}
\end{array}\right]\left[\begin{array}{cc}
\cos \varphi & \sin \varphi \\
-\rho \sin \varphi & \rho \cos \varphi
\end{array}\right]
\end{aligned}
$$

\subsubsection{Resultados experimentales en la determinación del ruido sen- sorial}

Cuando se construyen mapas de ambientes interiores, las esquinas son excelentes puntos de referencia o landmark(véase figura 5.6). Independientemente del sensor utilizado (láser, infrarrojos, cámara de vídeo o SONAR) las esquinas se modelan mediante un punto de coordenadas $\langle x, y\rangle$.

Para determinar la matriz de varianza del sensor en la determinación de la distancia radial y del ángulo de vista a las esquinas, se programó al robot YAIR para que explorase un entorno típico de oficinas. En determinados lugares de la escena, se midió manualmente la distancia del sensor de ultrasonidos a las esquinas cercanas, así como también el ángulo de vista a la misma. La tabla 5.2 muestra el error entre la localización real y la registrada por el SONAR. 
Tabla 5.2: Registro del error en el modelado de las esquinas de un entorno de oficina usando el SONAR rotativo del robot YAIR.

\begin{tabular}{|c|c|c|c|c|c|}
\hline $\mathrm{N}^{\mathrm{O}}$ & $\varepsilon_{\text {Radio }}(\mathrm{m})$ & $\varepsilon_{\text {Angulo }}(\mathrm{rad})$ & $\mathrm{N}^{\mathrm{O}}$ & $\varepsilon_{\text {Radio }}(\mathrm{m})$ & $\varepsilon_{\text {Angulo }}(\mathrm{rad})$ \\
\hline \hline 01 & 0.030 & -0.0032 & 28 & -0.040 & -0.0244 \\
\hline 02 & 0.013 & 0.0107 & 29 & 0.029 & 0.0312 \\
\hline 03 & -0.019 & 0.0195 & 30 & -0.020 & -0.0067 \\
\hline 04 & 0.007 & 0.0151 & 31 & 0.007 & -0.0388 \\
\hline 05 & -0.017 & -0.0097 & 32 & -0.019 & 0.0326 \\
\hline 06 & -0.057 & 0.0086 & 33 & -0.020 & 0.0073 \\
\hline 07 & 0.020 & 0.0018 & 34 & 0.040 & -0.0001 \\
\hline 08 & 0.026 & -0.0546 & 35 & 0.015 & 0.0179 \\
\hline 09 & -0.028 & 0.0353 & 36 & 0.000 & -0.0020 \\
\hline 10 & 0.090 & -0.0272 & 37 & -0.032 & -0.0195 \\
\hline 11 & 0.016 & 0.0008 & 38 & -0.010 & 0.0162 \\
\hline 12 & -0.038 & 0.0068 & 39 & 0.083 & 0.0171 \\
\hline 13 & 0.018 & 0.0003 & 40 & 0.000 & -0.0328 \\
\hline 14 & 0.033 & -0.0041 & 41 & 0.016 & -0.0012 \\
\hline 15 & -0.012 & 0.0066 & 42 & 0.028 & -0.0726 \\
\hline 16 & -0.010 & 0.0152 & 43 & -0.004 & -0.0462 \\
\hline 17 & 0.020 & 0.0055 & 44 & 0.007 & -0.0262 \\
\hline 18 & 0.010 & 0.0317 & 45 & 0.069 & -0.0112 \\
\hline 19 & -0.023 & 0.0275 & 46 & 0.005 & -0.0339 \\
\hline 20 & -0.011 & 0.0014 & 47 & 0.054 & -0.0510 \\
\hline 21 & 0.015 & -0.0291 & 48 & 0.018 & -0.0261 \\
\hline 22 & 0.008 & 0.0308 & 49 & -0.062 & 0.0188 \\
\hline 23 & 0.056 & -0.0410 & 50 & -0.003 & -0.0297 \\
\hline 24 & -0.010 & 0.0743 & 51 & 0.033 & 0.0041 \\
\hline 25 & -0.011 & 0.0620 & 52 & 0.001 & 0.0262 \\
\hline 26 & 0.087 & -0.0076 & 53 & 0.051 & -0.0124 \\
\hline 27 & -0.020 & 0.0292 & 54 & -0.032 & -0.0061 \\
\hline & & & & & \\
\hline
\end{tabular}




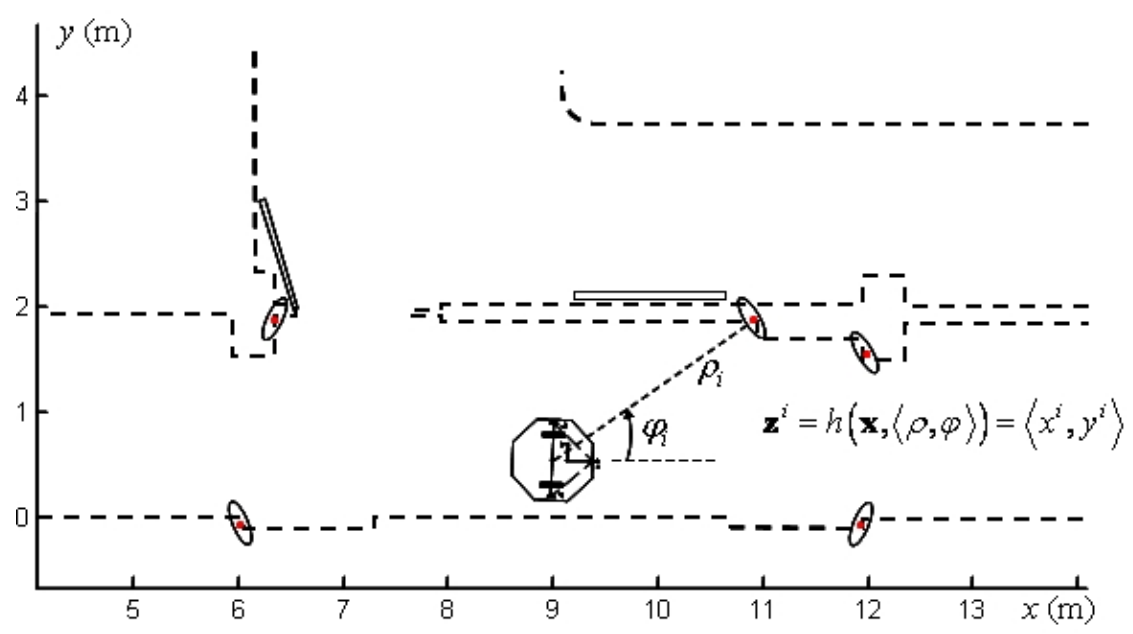

Figura 5.6: Mapa parcial de un entorno de oficinas. Los puntos indican Landmark puntuales(esquinas) y las elipses la incertidumbre en la localización de las mismas.

Tabla 5.3: Resumen de los estadísticos principales para el error en el radio y para el error en el ángulo al modelar esquinas con el SONAR del robot YAIR.

\begin{tabular}{|l|r|r|}
\hline & $\varepsilon_{\text {Radio }}(\mathrm{m})$ & $\varepsilon_{\text {Angulo }}(\mathrm{rad})$ \\
\hline \hline Media & 0,008 & $-0,0012$ \\
\hline Varianza & 0,001 & 0,0008 \\
\hline Desviación típica & 0,034 & 0,0285 \\
\hline Mínimo & $-0,062$ & $-0,0726$ \\
\hline Máximo & 0,090 & 0,0743 \\
\hline Asimetría estandarizada & 1,502 & $-0,1027$ \\
\hline Curtosis estandarizada & 0,481 & 0,6078 \\
\hline
\end{tabular}

El resumen estadístico de los datos de la tabla 5.2 se muestra en la tabla 5.3. Ahí, los coeficientes de asimetría y curtosis estandarizada indican que ambas poblaciones se distribuyen de manera normal; lo que también se puede apreciar en la figura 5.7.

Un contraste de hipótesis basado en la propuesta $E\left\langle\varepsilon_{\text {Radio }}\right\rangle=E\left\langle\varepsilon_{\text {Angulo }}\right\rangle=0$, muestra que la misma no se puede rechazar y por lo tanto se concluye que las medias de las distribuciones son igual a cero.

Un análisis de la covarianza del conjunto de datos, indica que el error radial y el angular están poco correlacionados(coeficiente de correlación $=-0.31$ ), pero que aun poseen una covarianza significativamente distinta de cero. Así, al no poder despreciar la covarianza entre los errores, la matriz de covarianza en la medición sensorial es la que se muestra a continuación. 


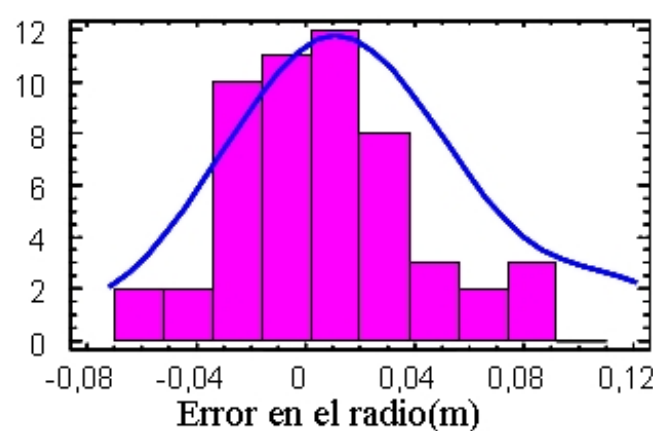

a)

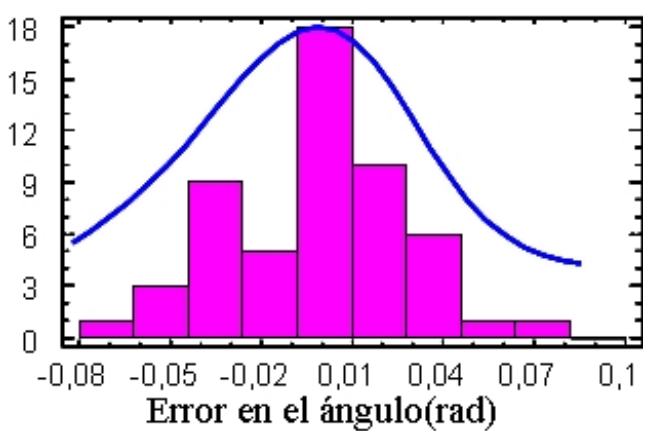

b)

Figura 5.7: Distribución del error en la medición de Landmark puntuales usando el SONAR rotativo del robot YAIR. a) Error en el radio. b) Error en el ángulo.

$$
\mathbf{R}=\left[\begin{array}{cc}
\sigma_{\rho}^{2} & \sigma_{\rho \varphi} \\
\sigma_{\rho \varphi} & \sigma_{\varphi}^{2}
\end{array}\right]=\left[\begin{array}{cc}
0,001139 \mathrm{~m}^{2} & -0,000295 \mathrm{~m} \cdot \mathrm{rad} \\
-0,000295 \mathrm{~m} \cdot \mathrm{rad} & 0,000815 \mathrm{rad}^{2}
\end{array}\right]
$$

Extraer líneas a partir de datos de ultrasonidos requiere de procesos adicionales de fusión de datos. Mandelbaum y Mintz[138] agregan datos de ultrasonidos tomados en diferentes estados para formar arcos y extraer líneas a partir de los arcos que comparten tangente común. Maksarov y Durrant-White[137] acumulan los datos proporcionados por un SONAR a través del tiempo, y los fusionan a través de un Filtro Extendido de Kalman, para obtener la localización y la identificación de los distintos objetos de un mapa geométrico de líneas y esquinas. En nuestro caso, el sensor que ya presentamos en la sección 5.3.1 es capaz de extraer líneas sin tener que moverse a otra poción. Lo anterior es posible gracias a una estrategia de seguimiento del máximo de amplitud que se recibe en el sensor.

Basándose en lo anterior, y aplicando un procedimiento experimental similar al aplicado en la determinación de la matriz de covarianza para la extracción de esquinas, se obtuvo que la matriz de covarianzas para el ruido sensorial en la extracción de líneas es aproximadamente igual a

$$
\mathbf{R}=\left[\begin{array}{cc}
\sigma_{\rho}^{2} & \sigma_{\rho \varphi} \\
\sigma_{\rho \varphi} & \sigma_{\varphi}^{2}
\end{array}\right]=\left[\begin{array}{cc}
0,0009 \mathrm{~m}^{2} & 0 \\
0 & 0,002741
\end{array}\right]
$$

\subsection{Representación del entorno mediante hitos o Land- marks}

En robótica móvil, la representación del entorno se ha sido dividido en dos grupos generales: los enfoques topológicos y los enfoques métricos[194]. Los mapas topológicos describen el entorno como un conjunto de lugares de características sobresalientes

o distintivas, que son conectadas a través de arcos que representan las rutas o posibles 
caminos que conectan estos lugares[119]. Por otra parte, los mapas métricos capturan las características geométricas del entorno, y los representa como un conjunto de objetos cuyas coordenadas están definidas en un plano cartesiano[72]. Dentro de los mapas métricos se consiguen 2 categorías principales: los mapas de ocupación y los mapas de hitos o landmark. Los mapas de ocupación son principalmente usados para tareas de navegación, ya que los mismos representan los espacios libres que son accesibles al robot[66]. Un inconveniente con este tipo de mapas es que requieren el uso de grandes cantidades de memoria para representar espacios de tamaño regular. Por su parte, los mapas de hitos resuelven este problema ya que ellos usan modelos paramétricos con los que se puede describir el entorno a costos de memoria muy bajos. De esta manera, usando un mapa de características se pueden modelar grandes áreas sin tener que usar importantes recursos de memoria; pero como contrapartida hay que utilizar sensores y algoritmos más avanzados y elaborados. Los mapas de características almacenan los objetos geométricos extraídos de los datos sensoriales del robot (i.e. líneas, esquinas, columnas) junto con su posición relativa a un sistemas de coordenadas fijas[130]. Este hecho hace que estos mapas sean especialmente convenientes para tareas de localización de robots móviles.

Los hitos o Landmarks son objetos del entorno que son fijos y que tienen características que los hacen sobresalientes o distintivos de otros objetos dentro del mismo entorno. En los entornos interiores, estos hitos pueden tener muchas formas físicas: esquinas, superficies planas, columnas, etc. Lo que constituye realmente un landmark estará marcado por las propiedades de observación del sensor que se use. Es decir, una landmark debe poseer una característica sobresaliente que el sensor pueda registrar.

Matemáticamente, un landmark se representa como un vector de parámetros que define la localización y otras propiedades del mismo. En esta tesis se consideran 2 tipos de landmark: esquinas, las cuales se representan mediante un punto de coordenadas cartesiana $\langle x, y\rangle$; y paredes o estructuras planas que se representan mediante segmentos de línea.

Igualmente, en esta tesis se considera que las Landmarks son del tipo estacionarias. Esto se representa como sigue: si $\mathbf{m}_{i}(k)$ es la representación de la $i$-esima landmark en el instante de tiempo $k$, entonces aplica la igualdad de la siguiente ecuación

$$
\mathbf{m}_{i}(k+1)=\mathbf{m}_{i}(k)=\mathbf{m}_{i}
$$

Finalmente, se considera que el modelo de un landmark no es un modelo dinámico, y por lo tanto no hay ruido aditivo al sistema. Así, la localización precisa del landmark puede ser incierta, pero esta incertidumbre no se incrementa con el tiempo. Esto se puede expresar como

$$
\mathbf{P}_{i}(k+1)=\mathbf{P}_{i}(k)=\mathbf{P}_{i}
$$

donde $\mathbf{P}_{i}$ representa la matriz de varianza-covarianza de la $i$-esima landmark en el instante de tiempo $k$.

En esta sección se desarrollan los modelos de observación y ruido asociados a la extracción de cada uno de los tipos de estructuras ya antes mencionados. 


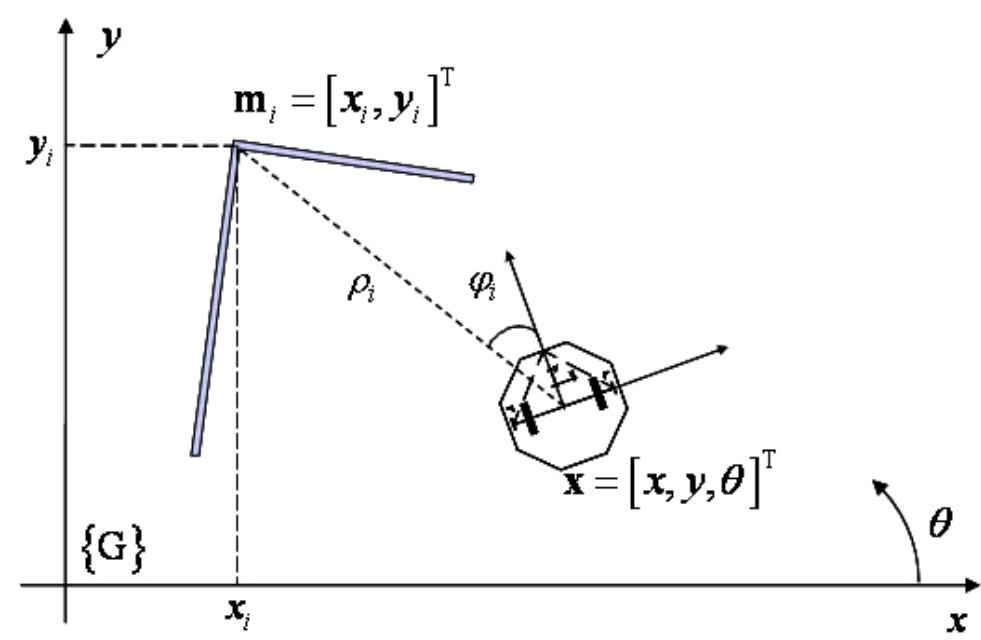

Figura 5.8: Modelo de una esquina y sistemas de coordenadas de un landmark tipo puntual.

\subsubsection{Modelado de esquinas como Landmarks puntuales}

Una esquina es un diedro cóncavo cuyo vector de estado está definido con las coordenadas de una característica tipo puntual,

$$
\mathbf{m}_{i}=\left[x_{i}, y_{i}\right]^{\mathrm{T}}
$$

donde $[x, y]^{\mathrm{T}}$ definen la localización de la esquina en el marco global $\{G\}$, según se muestra en la figura 5.8.

Ahora bien, la ecuación 5.36 y la ecuación 5.39, expresan las coordenadas y la matriz de covarianza de un landmark tipo puntual en el marco del sensor $\{S\}$, respectivamente. A continuación se desarrollan los mecanismos para expresar la medición del sensor como coordenadas puntuales del marco global.

\subsubsection{Sistemas coordenados de referencia}

En la figura 5.9 se muestra el conjunto de sistemas coordenados de referencia que se usa en esta tesis. El sistema de coordenadas globales es aquel donde finalmente se requiere determinar la posición $\langle x, y, \theta\rangle$ del vehículo. El sistema de coordenadas del robot $\langle x, y, \theta\rangle^{\mathrm{R}}$ se define dentro del cuerpo del móvil de tal manera que permanece solidario a él, y que la dirección del eje $x^{\mathrm{R}}$ se corresponda con la dirección del movimiento de avance del mismo. Asimismo, el sistema de referencia del sensor $\langle x, y, \theta\rangle^{\mathrm{S}}$ tiene su origen en el centro del elemento transductor o de percepción del entorno, y está orientado de manera similar al sistema de referencia del robot.

De acuerdo a la figura 5.10, para representar las coordenadas de una landmark puntual $\mathbf{m}=\left\langle m_{x}, m_{y}\right\rangle$ en el sistema de coordenadas global hay que realizar dos transformaciones: una transformación de coordenadas desde el marco sensorial al 
$\langle x, y\rangle$ : Coordenadas Globales

$\langle x, y\rangle^{\mathrm{R}}$ : Coordenadas Robot

$\langle x, y\rangle^{s}$ : Coordenadas Sensor

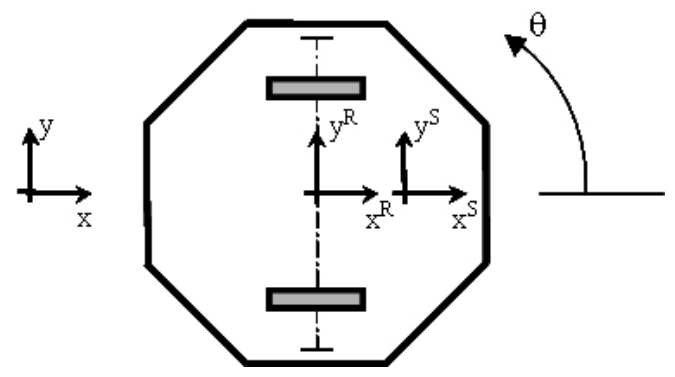

Figura 5.9: Sistemas de referencia en un vehículo diferencial típico.

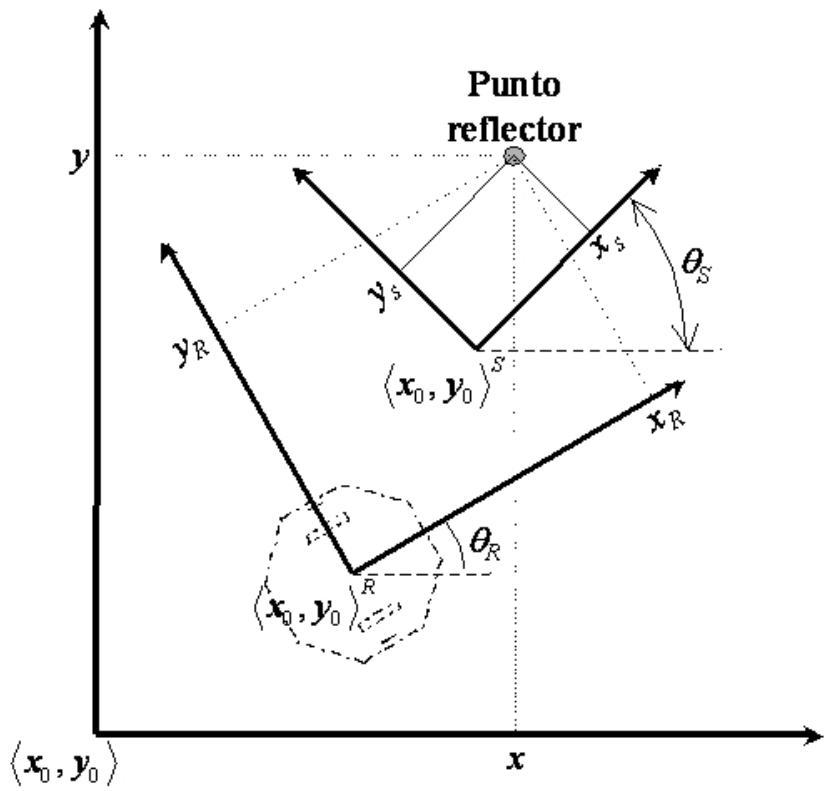

Figura 5.10: Esquema de relaciones entre los sistemas de referencia Global-RobotSensor. 
marco del móvil $\left(\mathbf{T}_{\mathrm{S}}^{\mathrm{R}}\right)$, y una transformación desde el marco del móvil al marco global $\left(\mathbf{T}_{R}^{G}\right)$. Asumiendo que el origen de coordenadas globales $\left\langle x_{0}, y_{0}, \theta_{0}\right\rangle^{G}$ está en $\langle 0,0,0\rangle$, las matrices de transformación necesarias para estas transformaciones son:

$$
\begin{gathered}
\mathbf{T}_{\mathrm{S}}^{\mathrm{R}}=\left[\begin{array}{ccc}
\cos \left(\theta_{0}^{\mathrm{S}}-\theta_{0}^{\mathrm{R}}\right) & -\sin \left(\theta_{0}^{\mathrm{S}}-\theta_{0}^{\mathrm{R}}\right) & \left(x_{0}^{\mathrm{S}}-x_{0}^{\mathrm{R}}\right) \\
\sin \left(\theta_{0}^{\mathrm{S}}-\theta_{0}^{\mathrm{R}}\right) & \cos \left(\theta_{0}^{\mathrm{S}}-\theta_{0}^{\mathrm{R}}\right) & \left(y_{0}^{\mathrm{S}}-y_{0}^{\mathrm{R}}\right) \\
0 & 0 & 1
\end{array}\right] \\
\mathbf{T}_{\mathrm{R}}^{\mathrm{G}}=\left[\begin{array}{ccc}
\cos \left(\theta_{0}^{\mathrm{R}}\right) & -\sin \left(\theta_{0}^{\mathrm{R}}\right) & \left(x_{0}^{\mathrm{R}}\right) \\
\sin \left(\theta_{0}^{\mathrm{R}}\right) & \cos \left(\theta_{0}^{\mathrm{R}}\right) & \left(y_{0}^{\mathrm{R}}\right) \\
0 & 0 & 1
\end{array}\right]
\end{gathered}
$$

donde $\left\langle x_{0}, y_{0}, \theta_{0}\right\rangle^{\mathrm{R}}$ y $\left\langle x_{0}, y_{0}, \theta_{0}\right\rangle^{\mathrm{S}}$ corresponden al origen de coordenadas del marco Robot y del marco Sensor respectivamente. De acuerdo a lo anterior, para modelar un landmark en el marco de coordenadas globales se utiliza la transformación,

$$
\mathbf{m}=\left[\begin{array}{l}
m_{x} \\
m_{y} \\
1
\end{array}\right]=\mathbf{T}_{\mathrm{R}}^{\mathrm{G}} \cdot\left[\begin{array}{l}
x^{\mathrm{R}} \\
y^{\mathrm{R}} \\
1
\end{array}\right]=\mathbf{T}_{\mathrm{R}}^{\mathrm{G}} \cdot \mathbf{T}_{\mathrm{S}}^{\mathrm{R}} \cdot\left[\begin{array}{l}
x^{\mathrm{S}} \\
y^{\mathrm{S}} \\
1
\end{array}\right]
$$

Aquí $\langle x, y\rangle^{\mathrm{R}}$ y $\langle x, y\rangle^{\mathrm{S}}$ son las coordenadas del landmark en el marco Robot y en el marco Sensor respectivamente.

\subsubsection{El modelo de representación: Transformación de hitos puntuales y propagación de su covarianza al marco Global}

Los hitos o landmark tipo puntuales son representados en un plano cartesiano global a través de sus coordenadas $\mathbf{m}=\left\langle m_{x}, m_{y}\right\rangle$. Para obtener estas coordenadas a partir de la medición del sensor, se asume que se conoce la posición $\langle x, y, \theta\rangle$ del vehículo portador del sensor, respecto al sistema de coordenadas global. También se asume que se conoce la matriz de covarianzas $\mathbf{Q}$ asociada a la incertidumbre del vehículo en esta posición y que las mediciones son realizadas con un SONAR tipo rotativo.

Una práctica común es que un SONAR tipo rotativo se instale haciendo coincidir su origen de coordenadas con el origen de coordenadas locales del vehículo donde se encuentra instalado. Esta condición reduce la matriz de transformación de la ecuación 5.29 a la matriz identidad. De esta forma, para modelar un landmark en el marco de coordenadas global se utiliza la siguiente ecuación,

$$
\mathbf{m}=\left[\begin{array}{l}
m_{x} \\
m_{y} \\
1
\end{array}\right]=\left[\begin{array}{ccc}
\cos \left(\theta_{0}^{\mathrm{R}}\right) & -\sin \left(\theta_{0}^{\mathrm{R}}\right) & \left(x_{0}^{\mathrm{R}}\right) \\
\sin \left(\theta_{0}^{\mathrm{R}}\right) & \cos \left(\theta_{0}^{\mathrm{R}}\right) & \left(y_{0}^{\mathrm{R}}\right) \\
0 & 0 & 1
\end{array}\right]\left[\begin{array}{l}
x^{\mathrm{S}} \\
y^{\mathrm{S}} \\
1
\end{array}\right]
$$

donde el vector $\left\langle x_{0}, y_{0}, \theta_{0}\right\rangle^{\mathrm{R}}$ en general representa el estado del robot. Así, el modelo de medición en el marco global es una función que depende de la función de medición en el marco sensor y de la posición del robot. Sustituyendo lo indicado, resulta 


$$
\begin{aligned}
\mathbf{m}=h\left(\mathbf{x}, \mathbf{z}^{\mathbf{S}}\right) & =\left[\begin{array}{l}
m_{x} \\
m_{y}
\end{array}\right] \\
& =\left[\begin{array}{l}
\rho \cos \varphi \cos \theta-\rho \sin \varphi \sin \theta+x \\
\rho \cos \varphi \sin \theta+\rho \sin \varphi \cos \theta+y
\end{array}\right] \\
& =\left[\begin{array}{l}
x+\rho \cos (\varphi+\theta) \\
y+\rho \sin (\varphi+\theta)
\end{array}\right]
\end{aligned}
$$

donde $\mathbf{x}=\langle x, y, \theta\rangle$ es el vector de estado del vehículo, $\mathrm{y} \mathbf{z}^{\mathrm{S}}=\langle\rho, \varphi\rangle$ es la medición en el marco sensorial.

Debido a la naturaleza no lineal de la función que modela las coordenadas del landmark en el marco global $\mathbf{m}=h\left(\mathbf{x}, \mathbf{z}^{\mathbf{S}}\right)$, la propagación del error a través de la misma se puede estimar mediante aproximación lineal en series de Taylor de primer orden. Igualmente, considerando que el error en la estimación del estado del robot es independiente del error en la medición a través del SONAR, entonces la covarianza de la medición en el marco global se puede estimar según,

$$
\mathbf{M}=\mathbf{J}_{\mathbf{z}} \mathbf{R} \mathbf{J}_{\mathbf{z}}^{\mathrm{T}}+\mathbf{J}_{\mathbf{x}} \mathbf{Q} \mathbf{J}_{\mathbf{x}}^{\mathrm{T}}
$$

donde $\mathbf{J}_{\mathbf{z}}$ es la matriz Jacobiana respecto al vector de medición $\mathbf{z}^{\mathbf{S}}$, y $\mathbf{J}_{\mathbf{x}}$ es la matriz Jacobiana respecto al estado $\mathbf{x}$. También, $\mathbf{Q}$ es la matriz de covarianza en el error de estado del vehículo, la cual ya fue definida en la ecuación 5.12. Igualmente, $\mathbf{R}$ es la varianza en el vector de medición, lo que ya fue definido en la ecuación 5.33.

Partiendo de la función que modela la landmark en el plano global(Eq. 5.49), las matrices Jacobianas se pueden derivar según,

$$
\begin{gathered}
\mathbf{J}_{\mathbf{z}}=\frac{\partial h\left(\mathbf{x}, \mathbf{z}^{\mathbf{S}}\right)}{\partial \mathbf{z}^{\mathbf{S}}}=\left[\begin{array}{cc}
\cos (\varphi+\theta) & -\rho \sin (\varphi+\theta) \\
\sin (\varphi+\theta) & \rho \cos (\varphi+\theta)
\end{array}\right] \\
\mathbf{J}_{\mathbf{x}}=\frac{\partial h\left(\mathbf{x}, \mathbf{z}^{\mathbf{S}}\right)}{\partial \mathbf{x}}=\left[\begin{array}{ccc}
1 & 0 & -\rho \sin (\varphi+\theta) \\
0 & 1 & \rho \cos (\varphi+\theta)
\end{array}\right]
\end{gathered}
$$

\subsubsection{El modelo de Observación: Transformación de hitos puntuales y propagación de su covarianza al marco Robot}

Para fusionar la información de Landmarks existentes, con la información de Landmarks que se van observando mediante el sensor, lo usual es utilizar las coordenadas homogéneas al vector de medición que en este caso corresponde a $\mathbf{z}^{\mathrm{S}}=\langle\rho, \varphi\rangle$. La función de transformación que permite homogenizar el vector de estado de una landmark puntual con el vector de medición, se conoce como modelo de observación. Esta función depende del vector de estado del robot en el instante $k, \mathbf{x}_{k}=\langle x, y, \theta\rangle_{k}$, y del vector de estado del landmark puntual $\mathbf{m}_{i}=\left[x_{i}, y_{i}\right]^{\mathrm{T}}$. Así, la función de observación en el instante $k$ se modela como 


$$
\begin{aligned}
\mathbf{z}_{k}=h\left(\mathbf{x}_{k}, \mathbf{m}_{i}\right) & =\left[\begin{array}{c}
\rho_{k} \\
\varphi_{k}
\end{array}\right] \\
& =\left[\begin{array}{c}
\sqrt{\left(x_{i}-x_{k}\right)^{2}+\left(y_{i}-y_{k}\right)^{2}} \\
\arctan \left(\frac{y_{i}-y_{k}}{x_{i}-x_{k}}\right)-\theta
\end{array}\right]
\end{aligned}
$$

donde la función $\arctan (\cdot)$ se refiere al arco-tangente del ángulo medido en los cuatro cuadrantes.

La propagación de la covarianza del landmark a través de la función de transformación, se consigue mediante aproximación lineal en series de Taylor de primer orden. Así, considerando que el error en la estimación del estado del robot es independiente del error en el mapa de Landmarks, la matriz de covarianza en los parámetros observados se puede estimar según,

$$
\mathbf{Z}_{k}=\mathbf{J}_{\mathbf{m}} \mathbf{M}_{i} \mathbf{J}_{\mathbf{m}}^{\mathrm{T}}+\mathbf{J}_{\mathbf{x}} \mathbf{Q} \mathbf{J}_{\mathbf{x}}^{\mathrm{T}}
$$

donde $\mathbf{J}_{\mathbf{m}}$ es la matriz Jacobiana respecto al vector de estado del landmark $\mathbf{m}_{i}$, y $\mathbf{J}_{\mathbf{x}}$ es la matriz Jacobiana respecto al estado del robot $\mathbf{x}_{k}$. También, $\mathbf{Q}$ es la matriz de covarianza en el error de estado del vehículo, la cual ya fue definida en la ecuación 5.12. Igualmente, $\mathbf{M}_{i}$ es la varianza en el vector de estado del landmark $\mathbf{m}_{i}$.

Partiendo de la función de observación(Eq. 5.53), las matrices Jacobianas se pueden derivar según,

$$
\begin{gathered}
\mathbf{J}_{\mathbf{m}}=\frac{\partial h\left(\mathbf{x}_{k}, \mathbf{m}_{i}\right)}{\partial \mathbf{m}_{i}}=\left[\begin{array}{cc}
\frac{\left(x_{i}-x_{k}\right)}{\sqrt{\left.\left(x_{i}-x_{k}\right)^{2}+y_{i}-y_{k}\right)^{2}}} & \frac{\left(y_{i}-y_{k}\right)}{\sqrt{\left.\left(x_{i}-x_{k}\right)^{2}+y_{i}-y_{k}\right)^{2}}} \\
\frac{-\left(y_{i}-y_{k}\right)}{\left(x_{i}-x_{k}\right)^{2}+\left(y_{i}-y_{k}\right)^{2}} & \frac{\left(x_{i}-x_{k}\right)}{\left(x_{i}-x_{k}\right)^{2}+\left(y_{i}-y_{k}\right)^{2}}
\end{array}\right] \\
\mathbf{J}_{\mathbf{x}}=\frac{\partial h\left(\mathbf{x}_{k}, \mathbf{m}_{i}\right)}{\partial \mathbf{x}_{k}}=\left[\begin{array}{lll}
\frac{-\left(x_{i}-x_{k}\right)}{\sqrt{\left(x_{i}-x_{k}\right)^{2}+\left(y_{i}-y_{k}\right)^{2}}} & \frac{-\left(y_{i}-y_{k}\right)}{\sqrt{\left(x_{i}-x_{k}\right)^{2}+\left(y_{i}-y_{k}\right)^{2}}} & 0 \\
\frac{\left(y_{i}-y_{k}\right)}{\left(x_{i}-x_{k}\right)^{2}+\left(y_{i}-y_{k}\right)^{2}} & \frac{-\left(x_{i}-x_{k}\right)}{\left(x_{i}-x_{k}\right)^{2}+\left(y_{i}-y_{k}\right)^{2}} & 1
\end{array}\right]
\end{gathered}
$$

\subsubsection{Modelado de paredes y estructuras planas como landmark tipo línea}

Una línea infinita se puede modelar de acuerdo a

$$
\mathbf{m}_{i}=\left[r_{i}, \alpha_{i}\right]^{\mathrm{T}}
$$

donde $r$ define la magnitud del vector que une perpendicularmente el origen de coordenadas con la recta $\mathbf{m}$, y $\alpha$ es la orientación acimutal de este vector(ver figura 5.11).

El modelo paramétrico para líneas infinitas es

$$
x \cos \alpha+y \sin \alpha-r=0
$$




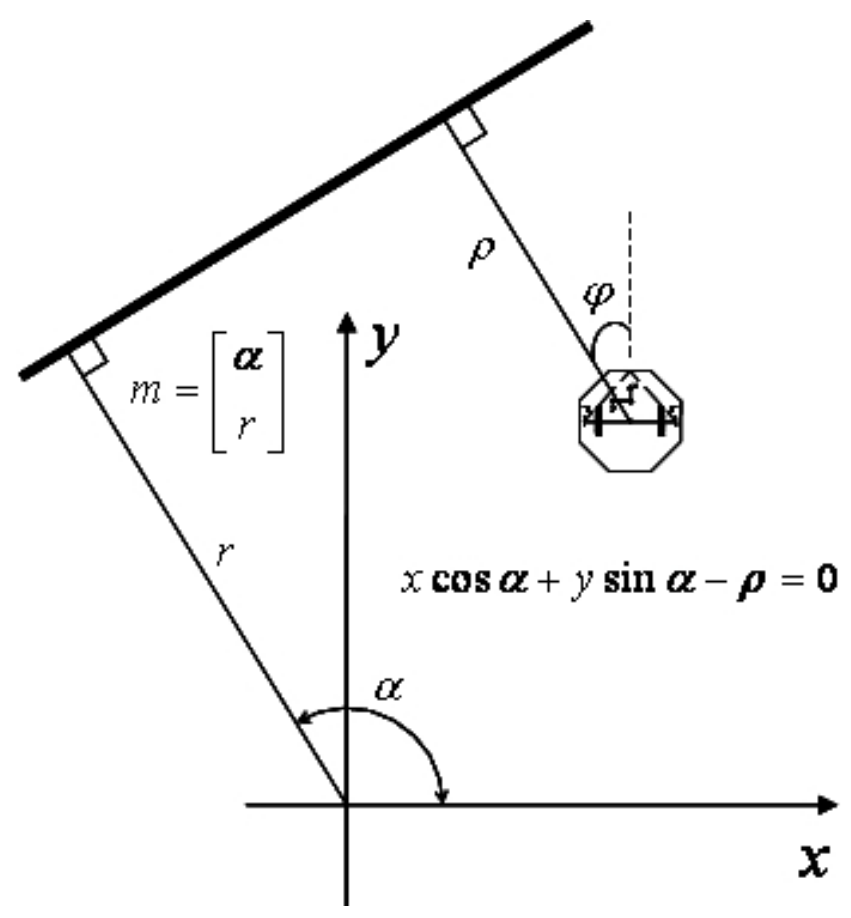

Figura 5.11: Definición del modelo Hessiano para una recta infinita en el sistema de coordenadas global.

En el modelo $\mathbf{m}_{i}=[r, \alpha]^{\mathrm{T}}$, el cual se conoce como modelo Hessiano, $r$ siempre es positiva; sin embargo existe un modelo alternativo $\mathbf{m}_{i}=[-r, \alpha+\pi]^{\mathrm{T}}$ donde $r$ es negativa debido a la posición relativa de observación de la recta[8].

\subsubsection{Transformación de coordenadas para el modelo Hessiano de línea infinita}

En las aplicaciones de robótica móvil, existen dos procesos básicos que requieren de la transformación del modelo Hessiano de la línea entre dos sistemas coordenados: la construcción de mapas de líneas y la localización basada en mapas de líneas. En el primer proceso, la operación de transformación no es muy usual, ya que normalmente las líneas se extraen a partir de conjuntos de datos que ya están representados en el marco global, que es donde se define finalmente el mapa de líneas. Sin embargo, en el caso de que el sensor sea capaz de extraer líneas en su propio sistema coordenado(como por ejemplo el sensor de ultrasonidos de YAIR), o en el caso que se utilicen algoritmos de extracción relativos al marco robot, está transformación sí que es de mucha utilidad. Por otra parte, como ya se dijo el conjunto de líneas que modelan el entorno usualmente son representadas en el marco de coordenadas global $\{G\}$. Sin embargo, debido a la operación de correspondencia que se debe realizar en los procesos de localización de robot móviles, estas líneas deben ser transformadas al marco del robot $\{R\}$. En [8] y en [168] existen amplios desarrollos en cuanto a este tema. No 


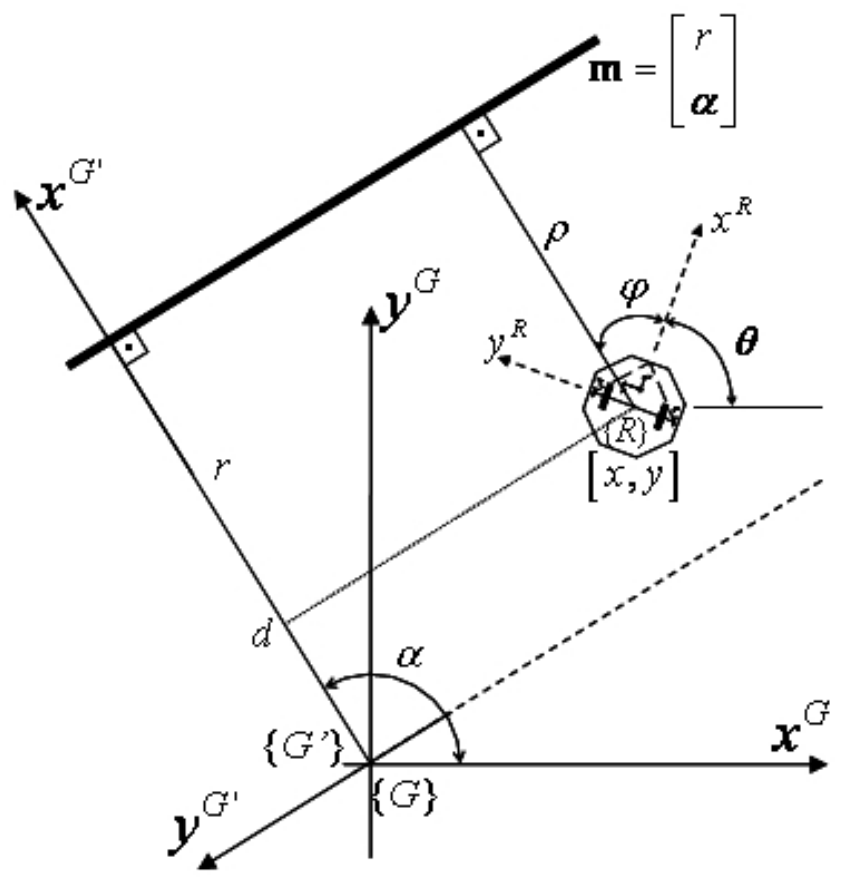

Figura 5.12: Esquema de derivación de las ecuaciones de transformacion del modelo Hessiano de la recta.

obstante, debido a la importancia de este procedimiento en el procesos relacionados a la localización y construcción de mapas de líneas mediante el SONAR rotativo del robot YAIR, a continuación se reproducen las ecuaciones principales implicadas en este tipo de transformación.

Dada una línea $\mathbf{m}=[r, \alpha]^{\mathrm{T}}$ definida en el marco global $\{G\}$, un vehículo posicionado en el estado $\mathbf{x}=[x, y, \theta]^{\mathrm{T}}$ referido también al marco global, $\mathrm{y}$ un sensor sólidamente anclado al cuerpo del vehículo; si se hace coincidir el sistema de coordenadas del vehículo $\{R\}$ y el sistema de coordenadas del sensor $\{S\}$, las ecuaciones de transformación que permiten representar la línea $\mathbf{m}$ en el marco del vehículo se desarrollan de la siguiente manera:

Supóngase un sistema de coordenadas $\left\{G^{\prime}\right\}$ que surge de rotar sobre su mismo eje al sistema de coordenadas $\{G\}$ hasta que el eje $x$ de este último coincida con el radio $r$. Esto es equivalente a rotar el sistema $\{G\}$ un ángulo $\alpha$. Esta operación se muestra en la figura 5.12. Así se puede considerar que el marco global $\{G\}$ está rotado un ángulo $-\alpha$ respecto al sistema coordenado $\left\{G^{\prime}\right\}$. Ahora bien, dado que el centro del $\operatorname{robot}[x, y]$ pertenece al marco global, la operación de rotación transforma la coordenada $x$ de dicho punto para hacerla equivalente a la distancia $d$ marcada sobre el eje $x^{G^{\prime}}$. Por lo tanto, esta distancia $d$ es igual a

$$
d=x \cos \alpha+y \sin \alpha
$$


De la figura 5.12 también se desprende la siguiente igualdad

$$
\begin{aligned}
r & =\rho+d \\
& =\rho+x \cos \alpha+y \sin \alpha
\end{aligned}
$$

Así mismo, en la misma figura se puede observar que

$$
\alpha=\varphi+\theta
$$

Así, mediante la ecuación 5.60 y la ecuación 5.61, se puede transformar una línea, representada mediante su modelo Hessiano, indistintamente del marco global al marco robot, o del marco robot al marco global.

De acuerdo a lo anterior, el modelo de representación de paredes y estructuras planas, en general depende del estado del vehículo que lleva el sensor $\mathbf{x}_{k}=\left[x_{k}, y_{k}, \theta_{k}\right]^{\mathrm{T}}$ y del vector de medición $\mathbf{z}^{\mathrm{S}}=\langle\rho, \varphi\rangle$.

$$
\begin{aligned}
\mathbf{m}_{i}=h\left(\mathbf{x}_{k}, \mathbf{z}_{i}^{\mathbf{S}}\right) & =\left[\begin{array}{l}
r_{i} \\
\alpha_{i}
\end{array}\right] \\
& =\left[\begin{array}{c}
\rho_{i}+x_{k} \cos \left(\varphi_{i}+\theta_{k}\right)+y_{k} \sin \left(\varphi_{i}+\theta_{k}\right) \\
\varphi_{i}+\theta_{k}
\end{array}\right]
\end{aligned}
$$

También, considerando que el error en la posición del vehículo no está correlacionado con el error en la medición, la matriz de covarianza de la línea representada en el marco global puede aproximarse mediante aproximación lineal de la función de representación de acuerdo a,

$$
\mathbf{M}=\mathbf{J}_{\mathbf{z}} \mathbf{R} \mathbf{J}_{\mathbf{z}}^{\mathrm{T}}+\mathbf{J}_{\mathbf{x}} \mathbf{Q} \mathbf{J}_{\mathbf{x}}^{\mathrm{T}}
$$

donde $\mathbf{J}_{\mathbf{z}}$ es la matriz Jacobiana respecto al vector de medición $\mathbf{z}^{\mathbf{S}}$, y $\mathbf{J}_{\mathbf{x}}$ es la matriz Jacobiana respecto al estado $\mathbf{x}$. También, $\mathbf{Q}$ es la matriz de covarianza en el error de estado del vehículo, la cual ya fue definida en la ecuación 5.12, e igualmente $\mathbf{R}$ es la varianza en el vector de medición, lo que ya fue definido en la ecuación 5.33 y cuyos valores extraídos experimentalmente se muestran en la ecuación 5.41.

Partiendo de la función que modela la línea Hessiana en el plano global(Eq. 5.62), las matrices Jacobianas se pueden derivar según,

$$
\begin{aligned}
& \mathbf{J}_{\mathbf{z}}=\frac{\partial h\left(\mathbf{x}, \mathbf{z}^{\mathbf{S}}\right)}{\partial \mathbf{z}^{\mathbf{S}}}=\left[\begin{array}{cc}
1 & -x_{k} \sin \left(\varphi_{i}+\theta_{k}\right)+y_{k} \cos \left(\varphi_{i}+\theta_{k}\right) \\
0 & 1
\end{array}\right] \\
\mathbf{J}_{\mathbf{x}} & =\frac{\partial h\left(\mathbf{x}, \mathbf{z}^{\mathbf{S}}\right)}{\partial \mathbf{x}} \\
& =\left[\begin{array}{ccc}
\cos \left(\varphi_{i}+\theta_{k}\right) & \sin \left(\varphi_{i}+\theta_{k}\right) & -x_{k} \sin \left(\varphi_{i}+\theta_{k}\right)+y_{k} \cos \left(\varphi_{i}+\theta_{k}\right) \\
0 & 0 & 1
\end{array}\right]
\end{aligned}
$$




\subsubsection{El modelo de observación para líneas Hessiana}

El vector de observación de un landmark modelado en el marco robot mediante una línea Hessiana consiste del radio y el ángulo acimutal medido desde el eje $x$ del marco robot.

$$
\mathbf{z}_{k}=\left[\begin{array}{c}
\rho_{k} \\
\varphi_{k}
\end{array}\right]
$$

De acuerdo a las ecuaciones 5.60 y 5.61, la función de observación depende del estado del robot $\mathbf{x}_{k}=\left[x_{k}, y_{k}, \theta_{k}\right]^{\mathrm{T}}$ en el instante $k$ y del vector que modela la landmark en el marco global $\mathbf{m}_{i}=\left[r_{i}, \alpha_{i}\right]^{\mathrm{T}}$.

$$
\begin{aligned}
\mathbf{z}_{k}=h\left(\mathbf{x}_{k}, \mathbf{m}_{i}\right) & =\left[\begin{array}{l}
\rho_{k} \\
\varphi_{k}
\end{array}\right] \\
& =\left[\begin{array}{c}
r_{i}-x_{k} \cos \alpha_{i}-y_{k} \sin \alpha_{i} \\
\alpha_{i}-\theta_{k}
\end{array}\right]
\end{aligned}
$$

Por otra parte, considerando que la covarianza del error en la posición del robot no está correlacionado con la covarianza asociada al modelo de la línea; entonces la covarianza de esta última en el marco robot se puede calcular por aproximación lineal en series de Taylor de primer orden de la función de observación, de acuerdo a

$$
\mathbf{Z}=\mathbf{J}_{\mathbf{m}} \mathbf{M}_{i} \mathbf{J}_{\mathbf{m}}^{\mathrm{T}}+\mathbf{J}_{\mathbf{x}} \mathbf{Q} \mathbf{J}_{\mathbf{x}}^{\mathrm{T}}
$$

donde $\mathbf{J}_{\mathbf{m}}$ es la matriz Jacobiana respecto al vector de estado del landmark $\mathbf{m}_{i}$, y $\mathbf{J}_{\mathbf{x}}$ es la matriz Jacobiana respecto al estado del robot $\mathbf{x}_{k}$. También, $\mathbf{Q}$ es la matriz de covarianza en el error de estado del vehículo, la cual ya fue definida en la ecuación 5.12 , e igualmente $\mathbf{M}_{i}$ es la varianza en el vector de estado del landmark $\mathbf{m}_{i}$.

Los dos Jacobianos necesarios para aproximar la covarianza mediante la ecuación 5.68 , se obtienen a partir de las siguientes ecuaciones

$$
\begin{gathered}
\mathbf{J}_{\mathbf{m}}=\frac{\partial h\left(\mathbf{x}_{k}, \mathbf{m}_{i}\right)}{\partial \mathbf{m}_{i}}=\left[\begin{array}{ccc}
1 & x_{k} \sin \alpha_{i}-y_{k} \cos \alpha_{i} \\
0 & 1
\end{array}\right] \\
\mathbf{J}_{\mathbf{x}}=\frac{\partial h\left(\mathbf{x}_{k}, \mathbf{m}_{i}\right)}{\partial \mathbf{x}_{k}}=\left[\begin{array}{ccc}
-\cos \alpha_{i} & -\sin \alpha_{i} & 0 \\
0 & 0 & -1
\end{array}\right]
\end{gathered}
$$

\subsection{Métodos de correspondencia}

Cuando se utiliza el Filtro Extendido de Kalman como método básico de fusión de datos en la construcción de mapas y la localización de robots, el método de correspondencia que usualmente se utiliza es el conocido como compuertas de validación(Validation Gates). Este método observa los residuales en la medición para detectar mediciones inesperadas o espurias[150]. Mediante el Filtro Extendido de Kalman se calcula la varianza en el vector de innovación para determinar si una medición 
tiene muy pocas probabilidades de corresponder al modelo que se está analizando. Esto se lleva a cabo determinando la Distancia de Mahalanobis de la medición respecto al modelo analizado, y comparándola con el valor probabilístico asignado por la tabla de distribución de probabilidad chi-cuadrado $\left(\chi^{2}\right)$. Debido a esto, esta prueba se conoce como la prueba chi-cuadrado.

La prueba de chi-cuadrado consiste en el cálculo de la variable aleatoria $D$ que posee una distribución tipo chi-cuadrado y que corresponde al error entre la medición y el modelo que está siendo probado. Este valor $D$ es entonces comparado con las tablas de probabilidad de una distribución chi-cuadrado, para determinar que el error resultante entre la medición y el modelo pueda ser generado por los efectos del ruido aleatorio sobre el mismo modelo. Normalmente se fija un límite por encima del cual se considera que la medición tiene poca probabilidad de haber sido generada por los efectos aleatorios que afectan al modelo, y en consecuencia se rechaza la hipótesis de que la medición pertenezca al conjunto de datos que generan el modelo.

Dada una variable aleatoria $n$-dimensional $\mathbf{u}$ y una medición de esta variable $\mathbf{u}_{i}$; asumiendo que esta variable se distribuye de manera normal con media igual a cero y matriz de covarianzas $n \times \mathbf{x} n$ igual a $\mathbf{C}$, entonces la distancia de Mahalanobis al cuadrado se calcula según,

$$
D^{2}=\left(\mathbf{u}-\mathbf{u}_{i}\right) \mathbf{C}^{-1}\left(\mathbf{u}-\mathbf{u}_{i}\right)^{\mathrm{T}}
$$

Nótese que bajo el supuesto de distribución normal de las variables involucradas, el valor de $D$ corresponde a la distancia de Mahalanobis. Esta es una medición adimensional que proporciona la distancia entre las variables aleatorias cuando son ponderadas por la incertidumbre relativa.

El límite para no rechazar la hipótesis de correspondencia entre la medición y el modelo, puede ser establecido en función del valor de la distribución $\chi^{2}(\alpha, n)$, donde $\alpha$ es el nivel de confianza que se tiene en que una medición $\mathbf{u}_{i}$ pueda haber sido generada por el modelo y $n$ son los grados de libertad del mismo. Si se desea ser restrictivo en cuanto a la posibilidad de falsos positivos, entonces el nivel de confianza $\alpha$ de be ser fijado a un valor bajo. Con todo lo anterior, se puede establecer que para rechazar la hipótesis de correspondencia, la distancia de Mahalanobis debe ser mayor que el límite fijado por la distribución chi-cuadrado, según

$$
D^{2}>\chi^{2}(\alpha, n)
$$

Este método de determinar la correspondencia presenta el inconveniente de no ser exhaustivo en la determinación de la misma. Por ejemplo, un alto valor de $D^{2}$ implica sin duda alguna que la medición no pertenece al modelo; sin embargo un bajo valor de $D^{2}$ no necesariamente implica que la medición pertenezca al modelo, sino más bien que existe un grado de confianza significativo que la medición pertenezca a dicho modelo.

La prueba de chi-cuadrado es suficiente para eliminar las mediciones que no pertenecen a un modelo determinado, pero sin embargo necesita de pruebas adicionales para validar la pertenencia de las mediciones al mismo. A continuación se desarrollan 


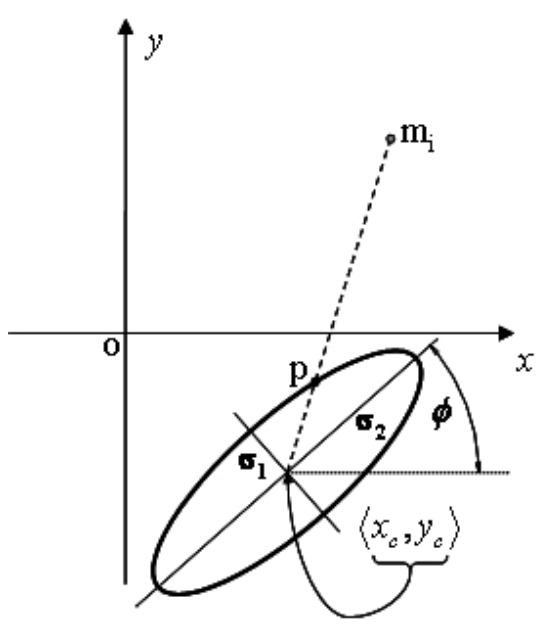

a)

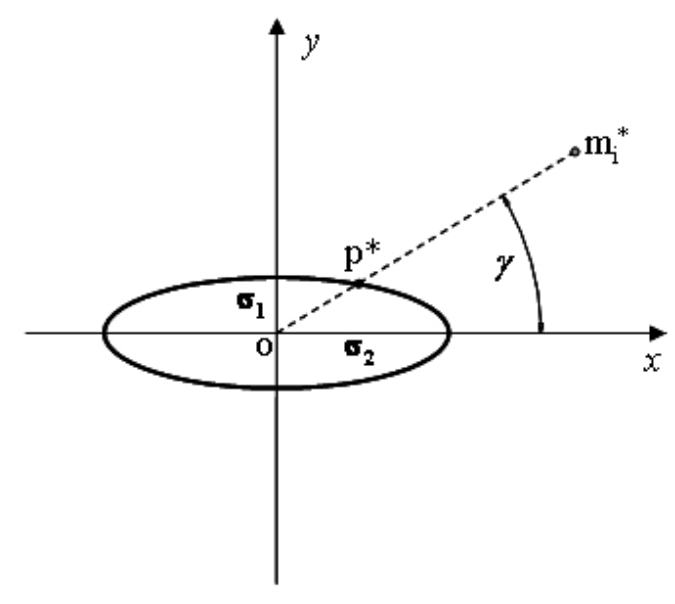

b)

Figura 5.13: a) Elipse de incertidumbre típica de una landmark tipo puntual. b) Traslación y rotación al origen de coordenadas de la elipse de incertidumbre y de un punto cualquiera solidario a su sistema de coordenadas local.

métodos de correspondencia que se particularizan sobre cada uno de los 2 tipos de landmark que puede detectar el SONAR rotativo de YAIR y que además se basan en un métrica asociada a la matriz de incertidumbre de estos landmark.

\subsubsection{Correspondencia entre landmark tipo puntuales}

El método de correspondencia entre landmark puntuales usado en esta tesis está basado en la característica de incertidumbre asociada a este tipo de característica del entorno. Distinto que la técnica basada en la prueba chi-cuadrado, este método determina inequívocamente si existe o no correspondencia, basándose en el criterio previamente establecido. La idea subyacente es la determinación si la medición está dentro o no de la elipse de incertidumbre que caracteriza al vector de observación.

En la figura 5.13b se puede observar que existe un punto de intersección $\mathbf{p} *$ definido por la intersección de la elipse con la recta que une al origen de coordenadas con el punto $\mathbf{m}_{i} *$. Las coordenadas del punto de intersección se pueden calcular según

$$
\mathbf{p} *=\left[\begin{array}{l}
x_{p} \\
y_{p}
\end{array}\right]=\left[\begin{array}{l}
\sigma_{1} \sigma_{2} / \sqrt{\sigma_{2}^{2} \tan ^{2} \gamma+\sigma_{1}^{2}} \\
\sigma_{1} \sigma_{2} \tan \gamma / \sqrt{\sigma_{2}^{2} \tan ^{2} \gamma+\sigma_{1}^{2}}
\end{array}\right]
$$

donde $\sigma_{1}$ y $\sigma_{2}$, indistitamente de la alineación con los ejes del sistema de coordenadas, representan al semieje menor y al semieje mayor respectivamente. También, $\gamma$ es el ángulo acimutal del vector que une al origen de coordenadas con el punto $\mathbf{m}_{i} *$. Las coordenadas de este punto se obtienen a partir de la secuencia de dos operaciones: anti-traslación de $\mathbf{m}_{i}$ de acuerdo al vector $\left\langle x_{c}, y_{c}\right\rangle$ formado por el centro de la elipse original , y anti-rotación de acuerdo al ángulo $\phi$ que mantiene el semieje mayor con 
el eje $x$ del sistema de coordenadas en la localización original de la elipse. Estas dos operaciones se expresan según,

$$
\mathbf{m}_{i}^{*}=\left[\begin{array}{l}
x_{i}^{*} \\
y_{i}^{*}
\end{array}\right]=\left[\begin{array}{cc}
\cos \phi & \sin \phi \\
-\sin \phi & \cos \phi
\end{array}\right]\left[\begin{array}{l}
x_{i}-x_{c} \\
y_{i}-y_{c}
\end{array}\right]
$$

A partir del modelo anterior, para determinar si el punto $\mathbf{m}_{i}^{*}$ está dentro del área de la elipse, y así aceptar la hipótesis de correspondencia, basta con comprobar que la norma de $\mathbf{p}^{*}$ es mayor que la norma de $\mathbf{m}_{i}^{*}$. Esto es,

$$
\left\|\mathbf{p}^{*}\right\|>\left\|\mathbf{m}_{i}^{*}\right\|
$$

Por otra parte, y al igual que en la metodología que utiliza la prueba chi-cuadrado, en esta propuesta el área de aceptación o de rechazo de la hipótesis de correspondencia puede hacerse adaptativa en función de la confianza que se tenga en los datos de la medición. Para ello basta con multiplicar el valor de los semiejes $\sigma_{1}$ y $\sigma_{2}$ por el valor correspondiente a la raíz cuadrada de la función $\chi^{2}(\alpha, n)$ evaluada en el nivel de confianza $\alpha$ que se desee.

Dado una elipse de incertidumbre cuyo centro $\mathbf{u}=\left\langle x_{c}, y_{c}\right\rangle$ pertenece al plano de coordenadas cartesianas, y cuyos semiejes están representados mediante una matriz de covarianza $\mathbf{C}$; entonces el procedimiento propuesto en esta tesis para determinar la mejor correspondencia del centro de la elipse con los elementos de un mapa de puntos $\mathbf{m}:\left\{\mathbf{m}_{1}, \mathbf{m}_{2}, \cdots, \mathbf{m}_{i}\right\}$ es el que se muestra en el algoritmo 5 . En esencia, el algoritmo inicialmente comprueba si existe correspondencia entre el punto $\mathbf{u}$ y alguno de los puntos perteneciente al mapa, mediante un procedimiento que va seleccionando los puntos $\mathbf{m}_{i}$ que están dentro de la elipse de error representada por C. Al mismo tiempo, se calcula la distancia de Mahalanobis correspondiente a cada uno de estos puntos. Si existe más de un punto $\mathbf{m}_{i}$ que cumpla con el criterio de estar dentro de la elipse de incertidumbre, entonces se decide cual de ellos ostenta la mayor probabilidad de corresponder a $\mathbf{u}$ siguiendo el criterio de la menor distancia de Mahalanobis.

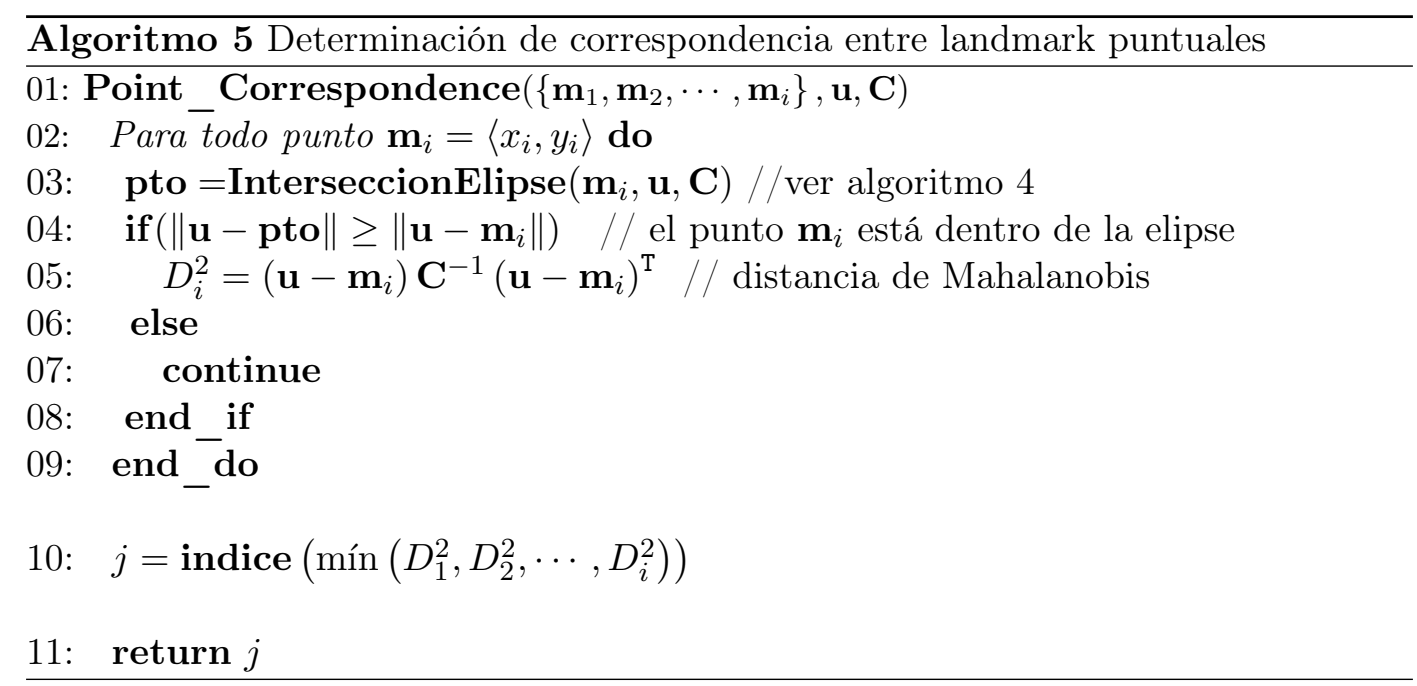




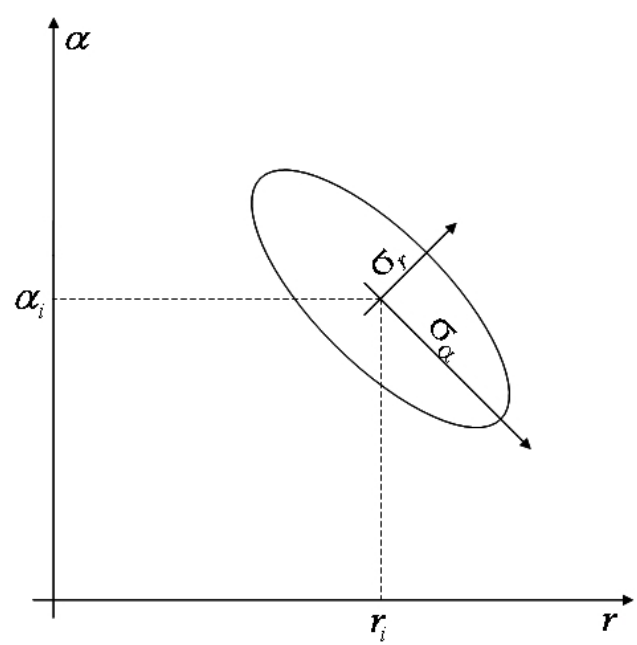

a)

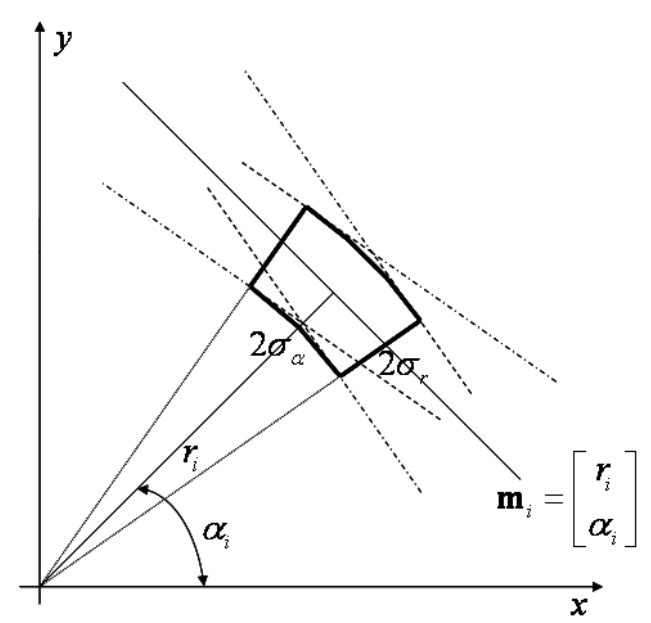

b)

Figura 5.14: Modelado del error en líneas infinitas. a) Elipse de incertidumbre de la función bivariante que modela el error en el plano polar. b) Representación de la región de error en el plano cartesiano.

\subsubsection{Correspondencia entre líneas}

El método de correspondencia entre segmentos de líneas ya se desarrollo en la sección 4.2.5.3, por lo que aquí sólo se desarrollan o se amplían algunos detalles vinculados al modelo Hessiano de líneas infinitas.

Una de las ventajas de la representación Hessiana de las líneas es que las mismas se puede tratar mediante las coordenadas ortogonales $\langle r, \alpha\rangle$. Esto permite modelar la incertidumbre como una distribución bivariante, donde a su vez se puede asumir que la variables son de distribución normal. Así, la incertidumbre en el modelo de la línea se representa mediante una elipse en el plano $\langle r, \alpha\rangle$, según como se muestra en la figura 5.14a. Esta incertidumbre se mapea en el plano cartesiano $\langle x, y\rangle$ según como se muestra en la figura 5.14b.

Esta forma de representar las líneas y la incertidumbre, permite aplicar el método de correspondencia clásico ya descrito en la sección 5.5 y que es conocido como compuertas de validación( Validation Gate); y reforzarlo con el método de correspondencia propuesto en esta tesis y que fué descrito en la sección 5.5.1. 



\section{Capítulo 6}

\section{Construcción de Mapas y Localización Estocástica}

\subsection{Introducción al enfoque estocástico en la robótica móvil}

Desde la década de los 90s, la robótica móvil ha estado dominada por las técnicas probabilísticas[193]. La razón de esto es que el movimiento del robot y el modelado del entorno están caracterizados por la incertidumbre y el ruido sensorial. El principio básico subyacente en todo algoritmo probabilístico es la regla de Bayes. Si $a$ es una variable que queremos inferir a partir de otra variable $b$, la probabilidad $p(a)$ se conoce como la distribución de probabilidad a priori, y la variable $b$ es conocida como los datos. La distribución $p(a / b)$ es conocida como la distribución de probabilidad $a$ posteriori. La regla de Bayes provee una manera conveniente de computar la probabilidad a posteriori en función de la probabilidad inversa $p(b / a)$ y la probabilidad a priori $p(a)$, de acuerdo a

$$
p(a / b)=\eta p(b / a) p(a)
$$

donde $\eta$ es un factor normalizador que asegura que la expresión se encuentre en el rango $[0,1]$.

La robótica probabilista buscar estimar la posición de un robot $s=\langle x, y, \theta\rangle$ y el modelo del entorno que lo rodea $\langle m\rangle$. En adelante, la composición de la posición del robot y del mapa, forma y se conoce como el estado $\mathbf{x}=\langle s, m\rangle$. La evolución del estado, el control y la sensorización de un robot móvil puede ser modelado mediante una red dinámica de Bayes. De acuerdo a la figura 6.1, el estado $\mathbf{x}_{k}$ es estocásticamente dependiente del estado anterior $\mathbf{x}_{k-1} \mathrm{y}$ del control $\mathbf{u}_{k}$, mientras que la medición $\mathbf{z}_{k}$ depende estocásticamente del estado $\mathbf{x}_{k}$.

El Filtro de Bayes extiende la regla de Bayes de la ecuación 6.1 para tratar problemas de estimación en el dominio del tiempo. Este filtro es un estimador recursivo que permite computar la secuencia de una distribución de probabilidad posterior que no 


\section{CAPÍTULO 6. CONSTRUCCIÓN DE MAPAS Y LOCALIZACIÓN} ESTOCÁSTICA

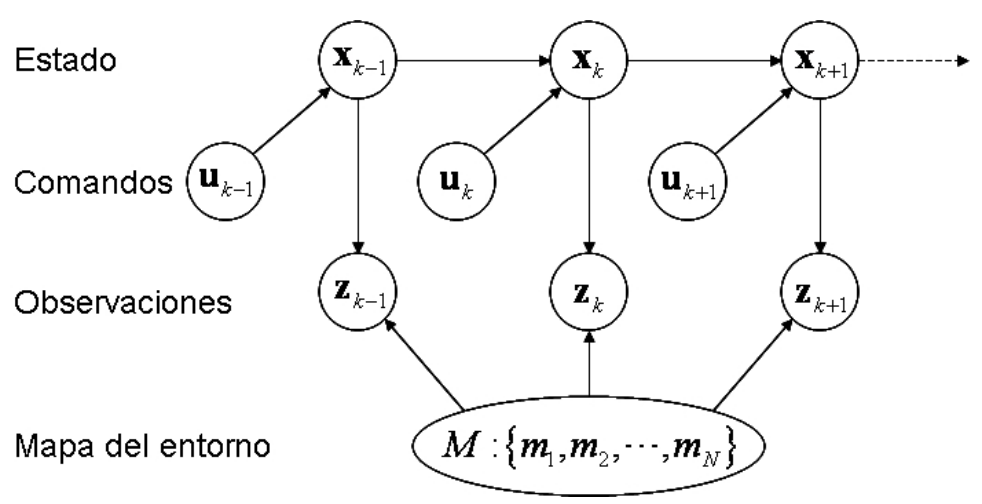

Figura 6.1: Red dinámica de Bayes que caracteriza la evolución del estado $\mathbf{x}$, la medición $\mathbf{z}$, y el control $\mathbf{u}$ en un robot móvil. El subíndice $k$ indica la dependencia temporal del modelo.

puede ser observada directamente. Así, con un Filtro de Bayes se puede calcular la probabilidad posterior sobre el estado $\mathbf{x}_{k}$ por medio de la siguiente ecuación.INSERCION

$$
\begin{aligned}
& p\left(s_{k}, m_{k} \mid z_{k}, u_{k}\right)= \\
& \eta p\left(z_{k} \mid s_{k}, m_{k}\right) \iint p\left(s_{k}, m_{k} \mid u_{k}, s_{k-1}, m_{k-1}\right) p\left(s_{k-1}, m_{k-1} \mid z_{k-1}, u_{k-1}\right) d s_{k-1} d m_{k-1}
\end{aligned}
$$

Asumiendo que el mapa del entorno es estático y que el movimiento del robot es independiente del mapa, la ecuación 6.2 se transforma en

$$
\begin{aligned}
& p\left(s_{k}, m \mid z_{k}, u_{k}\right)= \\
& \eta p\left(z_{k} \mid s_{k}, m\right) \int p\left(s_{k} \mid u_{k}, s_{k-1}\right) p\left(s_{k-1}, m \mid z_{k-1}, u_{k-1}\right) d s_{k-1}
\end{aligned}
$$

En la ecuación 6.3 , la distribución $p\left(s_{k}, m \mid z_{k}, u_{k}\right)$ se conoce como la confianza o certidumbre del estado $\mathbf{x}_{k}$. La misma se denota como $\operatorname{Bel}\left(x_{k}\right)$ y representa la probabilidad del que el sistema se encuentre en el estado $\mathbf{x}_{k}$, dado las mediciones $\mathbf{z}_{1: k}$ y comandos de control $\mathbf{u}_{1: k}$ ejecutados hasta el estado $k$. En la misma ecuación, el segundo factor dentro de la integral representa la confianza del estado anterior $\operatorname{Bel}\left(x_{k-1}\right)$, por lo que esta condición le da un carácter recursivo al filtro de Bayes. Una forma más compacta de expresar la ecuación 6.3, utilizando la forma recursiva del filtro de Bayes es,

$$
\operatorname{Bel}\left(x_{k}\right)=\eta p\left(z_{k} \mid x_{k}\right) \int p\left(x_{k} \mid x_{k-1}, u_{k}\right) \operatorname{Bel}\left(x_{k-1}\right) d x_{k-1}
$$

Para implementar o poner en funcionamiento este estimador, se deben especificar dos densidades generativas: la densidad $p\left(z_{k} \mid x_{k}\right)$ conocida como el modelo perceptual, y la densidad $p\left(x_{k} \mid x_{k-1}, u_{k}\right)$ conocido como el modelo de transición. La densidad $p\left(z_{k} \mid x_{k}\right)$ describe en términos probabilísticos la manera como se generan las distintas observaciones $\mathbf{z}$ para diferentes localizaciones y mapas del entorno. La 
densidad $p\left(x_{k} \mid x_{k-1}, u_{k}\right)$ describe como afecta el control $\mathbf{u}$ a la evolución de la posición del robot. Así, esta densidad describe la probabilidad de que estando el robot en el estado $x_{k-1}$, alcance el estado $x_{k}$ si le es aplicado el control $u_{k}$. Por eso este modelo también se conoce como el modelo de actuación. FININSERCION

El Filtro de Bayes, tal como aparece en la ecuación 6.4, no goza de sencilla implementación práctica, ya que la densidad posterior sobre todo el espacio del mapa y de las posiciones del robot, es una distribución sobre un espacio continuo que posee infinitas dimensiones. Por lo tanto, lo usual es usar aproximaciones que van a depender de la manera como se modelen las densidades de probabilidad. Así por ejemplo, representando el espacio de localización como una densidad de probabilidad paramétrizada, como por ejemplo una distribución Gausiana, la computación de la función de estimación se reduce enormemente.

Una de las implementaciones del Filtro de Bayes más utilizadas está basada en el Filtro de Kalman. Esta solución del Filtro de Bayes asume que el modelo de acción y el modelo de percepción están sujetos al efecto del ruido Gausiano, y también asume que la creencia puede ser representada por una función igualmente Gausiana. Con la asunción de ruido Gausiano, se puede usar el Filtro de Kalman para propagar la creencia a través del tiempo y para incorporar la información proveniente de los sensores. El desarrollo detallado de las ecuaciones que dan solución a este método de estimación se pueden conseguir en [198, 154].

El Filtro de Kalman es un estimador recursivo del método de mínimos cuadrados, que produce en el instante $k$ un estimado del estado cuya varianza es mínima. El estado estimado, denotado como $\hat{\mathbf{x}}(k \mid k)=\operatorname{Bel}\left(\mathbf{x}_{k}\right)=p\left(\mathbf{x}_{k} \mid \mathbf{z}_{k}, \mathbf{u}_{k}\right)$, se obtiene mediante la fusión de la predicción del estado estimado $\hat{\mathbf{x}}(k \mid k-1)$ con una observación $\mathbf{z}_{k}$ del vector de estado $\mathbf{x}_{k}$. Sobre el Filtro de Kalman existe abundante literatura. En el anexo B de esta tesis se establecen los principios y ecuaciones básicas del Filtro de Kalman y de su extensión para sistemas no lineales. Para un desarrollo más detallado se debe consultar [103, 102, 150, 82].

Utilizar funciones Gausianas para representar el estado de un robot y de su entorno puede ser algo restrictivo. Debido a que la funciones Gausianas son densidades unimodales, con ellas no se pueden representar múltiples hipótesis respecto a al estado del robot. Por esto último, el Filtro de Kalman se emplea más que nada en aplicaciones donde se conoce la localización inicial del robot. Este tipo de aplicación se conoce como seguimiento(Tracking).

En la figura 6.2 se muestra el rol del filtro de kalman en un problema de estimación de la posición de un robot móvil. El vehículo se mueve comandado por las señales $\mathbf{u}_{k}$, mientras que al mismo tiempo va observando el entorno mediante sus sensores. Estas dos operaciones están implícitamente afectadas por señales de ruido aleatorio que se asume son de distribución Gaussiana. Mediante la formulación del Filtro de Kalman, se predice el estado mediante el modelo de acción $p\left(\mathbf{x}_{k} \mid \mathbf{x}_{k-1}, \mathbf{u}_{k}\right)$ y con ello se predice la observación mediante el modelo $p\left(\mathbf{z}_{k} \mid \mathbf{x}_{k}, \mathbf{m}\right)$. En la siguiente etapa se utiliza la observación real para corregir el estado estimado. Aquí se utiliza un factor de corrección que surge de la relación entre la varianza estimada y la varianza en la medición. 


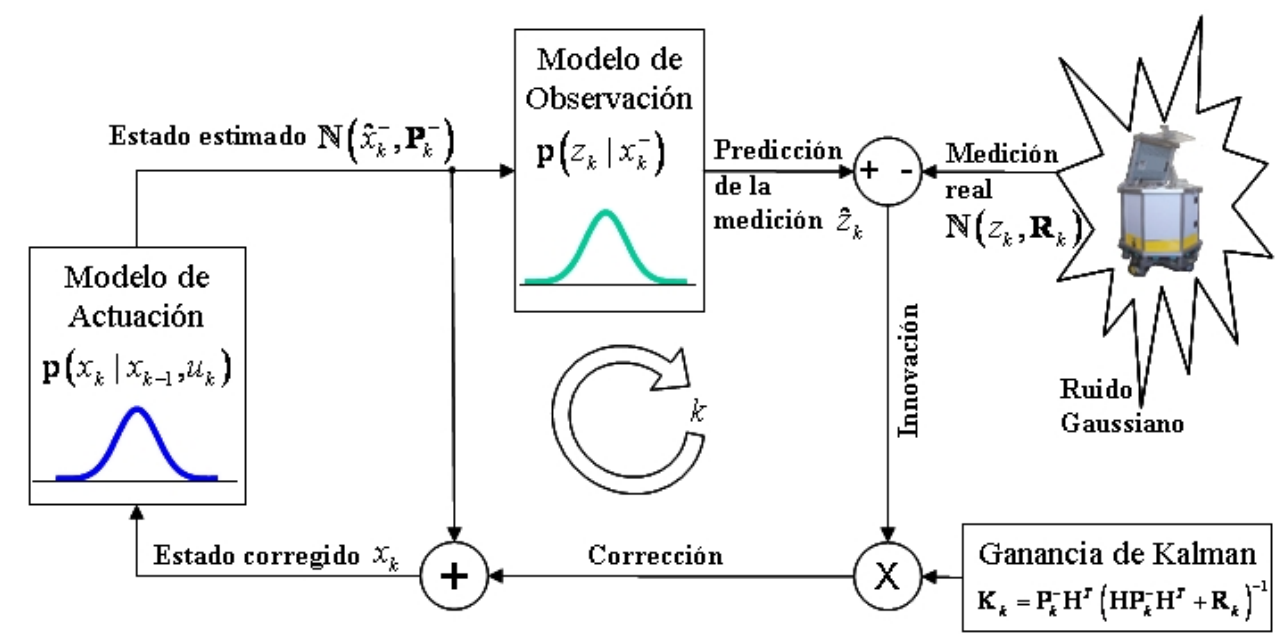

Figura 6.2: Esquema de estimación del estado de un robot móvil mediante el Filtro de Kalman. Nótese que la aplicación de este técnica de estimación requiere que los modelos puedan ser representados mediante una función Gaussiana.

En este capítulo se emplean los modelos probabilísticos del robot, de sus sensores y de su entorno, para junto con las ecuaciones del algoritmo del Filtro de Kalman, resolver tres problemas distintos de estimación: construcción de un mapa de entorno a partir de las posiciones perfectamente conocidas del móvil, localización del móvil asumiendo que el entorno es perfectamente conocido, construcción del mapa y localización del vehículo de forma simultanea. Los modelos estocásticos mencionados ya fueron introducidos en el capítulo 5, mientras que las ecuaciones del filtro de Kalman se desarrollan en el anexo B. De esta forma, el aporte fundamental de este capítulo es el desarrollo de una estrategia que permita integrar los sensores limitados con los que cuenta el robot YAIR, a las operaciones de estimación basada en el Filtro de Kalman.

\subsection{Construcción de mapas de esquinas}

Un mapa del entorno es una lista de objetos y de sus características o propiedades. Esto se expresa como,

$$
\mathbf{m}:\left\{\mathbf{m}_{1}, \mathbf{m}_{2}, \cdots, \mathbf{m}_{\mathbb{N}}\right\}
$$

donde cada $m_{i}$ es el modelo del objeto $i$-esimo del entorno, con $\mathrm{N}$ igual al número total de objetos del mismo. Las esquinas de un entorno usualmente se caracterizan mediante sus coordenadas de localización $(x, y)$ en el plano cartesiano. Esta forma de caracterizar las esquinas es especialmente útil cuando se quiere mejorar o ajustar su localización más probable, como resultado del proceso de sensorización donde se observe la misma esquina. 
En general, para construir un mapa del entorno se asume que se conoce la posición exacta $\mathbf{s}_{k}$ del vehículo que porta el mecanismo sensorial, y que se conocen también la secuencia de observaciones $\mathbf{z}_{k}$ que se van realizando a medida que el vehículo explora el entorno. Con estas condiciones lo que se requiere es encontrar la distribución de $\mathbf{m}$ condicionada sobre $\mathbf{z}_{k}$ y $\mathbf{s}_{k}$.

$$
\begin{aligned}
p\left(\mathbf{m} \mid \mathbf{s}_{k}, \mathbf{z}_{k}\right) & =\frac{p\left(\mathbf{z}_{k} \mid \mathbf{m}, \mathbf{s}_{k}\right) p\left(\mathbf{m}, \mathbf{s}_{k}\right)}{p\left(\mathbf{s}_{k}, \mathbf{z}_{k}\right)} \\
& =\frac{p\left(\mathbf{z}_{k} \mid \mathbf{m}, \mathbf{s}_{k}\right) p\left(\mathbf{m} \mid \mathbf{s}_{k}\right) p\left(\mathbf{s}_{k}\right)}{\int_{-\infty}^{-\infty} p\left(\mathbf{z}_{k} \mid \mathbf{m}, \mathbf{s}_{k}\right) p\left(\mathbf{m} \mid \mathbf{s}_{k}\right) p\left(\mathbf{s}_{k}\right) d \mathbf{m}}
\end{aligned}
$$

Sujeto a que se conoce la posición exacta del vehículo, $p\left(\mathbf{s}_{k}\right)=1$, entonces la ecuación 6.6 se puede reescribir como

$$
p\left(\mathbf{m} \mid \mathbf{s}_{k}, \mathbf{z}_{k}\right)=\eta p\left(\mathbf{z}_{k} \mid \mathbf{m}\right) p(\mathbf{m})
$$

donde $p\left(\mathbf{z}_{k} \mid \mathbf{m}\right)$ es el modelo de observación y $p(\mathbf{m})$ es la probabilidad a priori relacionada al estado del mapa. También, $\eta$ representa la probabilidad total que tiene el evento $\mathbf{z}_{k}$ sobre el espacio muestral, por lo que se convierte en un factor de normalización que garantiza que se cumpla el primer axioma de la probabilidad $\left(0 \leq p\left(\mathbf{m} \mid \mathbf{s}_{k}, \mathbf{z}_{k}\right) \leq 1\right)$. Si las densidades involucradas se parametrizan mediante el primero y segundo momento estadístico(media y varianza), entonces se puede utilizar el Filtro de Kalman para estimar el máximo a posteriori de la ecuación 6.7.

\subsubsection{El algoritmo de mapeado mediante el Filtro de Kalman Ex- tendido}

Para construir mapas de landmark puntuales basandose en el Filtro de Kalman Extendido, se asume que el vector de estado $\mathbf{x}_{k}$ va a estar formado por las coordenadas de las esquinas o hitos puntuales que conforman el mapa, las cuales se arreglan de la siguiente forma

$$
\mathbf{x}_{k}=\left[m_{1}^{x}, m_{1}^{y}, m_{2}^{x}, m_{2}^{y}, \cdots, m_{\mathrm{N}}^{x}, m_{\mathrm{N}}^{y}\right]_{k}^{\mathrm{T}}
$$

Aquí $m^{x}$ y $m^{y}$ representan las coordenadas de localización cartesianas de las esquinas pertenecientes al mapa, y $\mathrm{N}$ es el número de estos objetos que contiene el mapa en el instante $k$. También, asumiendo que los objetos del mapa son estáticos, entonces la ecuación de predicción del estado no tiene dinámica temporal, por lo que corresponde a

$$
\mathbf{x}_{k}=\mathbf{x}_{k-1}
$$

El algoritmo de construcción de mapas de esquinas se inicia asumiendo que el vector de estado está vacío, y que se va llenando según se vayan encontrando esquinas en el entorno explorado. De acuerdo a lo definido en el capítulo 5, las coordenadas 


\section{CAPÍTULO 6. CONSTRUCCIÓN DE MAPAS Y LOCALIZACIÓN ESTOCÁSTICA}

de localización de cada nuevo objeto puntual que se va encontrando, en este caso se obtienen mediante la ecuación

$$
\mathbf{m}_{i}=\left[\begin{array}{l}
m^{x} \\
m^{y}
\end{array}\right]_{i}=\left[\begin{array}{l}
x_{k}+\rho_{i} \cos \left(\theta_{k}+\varphi_{i}\right) \\
y_{k}+\rho_{i} \sin \left(\theta_{k}+\varphi_{i}\right)
\end{array}\right]
$$

donde $\mathbf{s}_{k}=\langle x, y, \theta\rangle_{k}$ representa la posición del robot en el instante $k$, y $\mathbf{z}_{i}=\langle\rho, \varphi\rangle_{i}$ representa el vector de medición (distancia y ángulo) desde la posición actual del robot hasta el objeto puntual $i$.

De esta forma, el vector de estado crecerá en forma de columna a medida que se van encontrando nuevos objetos según

$$
\mathbf{x}_{k}=\left[\begin{array}{c}
\mathbf{x}_{k} \\
\mathbf{m}_{i}
\end{array}\right]
$$

Igualmente, así como se va llenando el vector de estado, la matriz de covarianza del mismo también se va transformando según

$$
\mathbf{P}_{k}=\left[\begin{array}{cc}
\mathbf{P}_{k} & \mathbf{0}_{2 \mathrm{Nx} 2} \\
\mathbf{0}_{2 \mathrm{x} 2 \mathrm{~N}} & \mathbf{J}_{z} \mathbf{R} \mathbf{J}_{z}^{\mathrm{T}}
\end{array}\right]
$$

donde $\mathbf{R}$ es la matriz de covarianza que modela el ruido sensorial, y $\mathbf{J}_{z}$ es el Jacobiano de la función de mapeado con respecto al vector de medición. Este Jacobiano se obtiene a partir de la ecuación 6.10 de acuerdo a,

$$
\mathbf{J}_{\mathbf{z}}=\frac{\partial \mathbf{m}_{i}}{\partial \rho \partial \varphi}=\left[\begin{array}{cc}
\cos \left(\varphi_{i}+\theta_{k}\right) & -\rho_{i} \sin \left(\varphi_{i}+\theta_{k}\right) \\
\sin \left(\varphi_{i}+\theta_{k}\right) & \rho_{i} \cos \left(\varphi_{i}+\theta_{k}\right)
\end{array}\right]
$$

Por otra parte, si durante la exploración del entorno se detectan esquinas que ya forman parte del vector de estado, entonces se utiliza el Filtro de Kalman para fusionar la información y así reforzar el conocimiento que sobre la localización de las esquinas observadas se pueda tener. A continuación, por simplicidad se asumirá que se detecta una sola esquina, pero el procedimiento es extensible a la aplicación individual sobre cualquier número de esquinas detectadas en el ciclo de actualización $k$.

Considerando que se ha ejecutado el procedimiento de detección y de correspondencia de esquinas, el algoritmo de mapeado continua asumiendo que se conoce inequívocamente el objeto $\mathbf{m}_{i}$ al cual corresponde la observación actual $\mathbf{z}_{k}=\langle\rho, \varphi\rangle_{k}$. De esta forma el vector de observación se obtiene de acuerdo a

$$
\begin{aligned}
\mathbf{z}_{i}=h\left(\mathbf{s}_{k}, \mathbf{m}_{i}\right) & =\left[\begin{array}{l}
\rho_{i} \\
\varphi_{i}
\end{array}\right] \\
& =\left[\begin{array}{c}
\sqrt{\left(x_{i}-x_{k}\right)^{2}+\left(y_{i}-y_{k}\right)^{2}} \\
\arctan \left(\frac{y_{i}-y_{k}}{x_{i}-x_{k}}\right)-\theta_{k}
\end{array}\right]
\end{aligned}
$$


donde $\mathbf{s}_{k}=\langle x, y, \theta\rangle_{k}$ representa la posición del robot en el instante $k, \mathrm{y} \mathbf{m}_{i}=\langle x, y\rangle_{i}$ corresponde a las coordenadas de la esquina observada. De esta forma, la diferencia entre el vector de medición $\mathbf{z}_{k}$ y el vector de observación $\mathbf{z}_{i}$ proporciona lo que se conoce como la innovación $\Delta \mathbf{z}$.

$$
\Delta \mathbf{z}=\mathbf{z}_{k}-\mathbf{z}_{i}
$$

La covarianza en el vector de innovación puede obtenerse mediante aproximación lineal de acuerdo a

$$
\mathbf{Z}=\mathbf{R}+\mathbf{J}_{\mathrm{h}} \mathbf{P}_{i} \mathbf{J}_{\mathrm{h}}^{\mathrm{T}}
$$

donde $\mathbf{R}$ es la matriz de covarianza que modela el ruido sensorial, $\mathbf{J}_{\mathrm{h}}$ es el Jacobiano de la función de observación, y $\mathbf{P}_{i}$ es la submatriz perteneciente a la matriz de covarianza del estado $\mathbf{P}_{k}$, y que corresponde al objeto $i$. El Jacobiano $\mathbf{J}_{\mathrm{h}}$ se obtiene a partir de la ecuación 6.14 de acuerdo a,

$$
\mathbf{J}_{\mathrm{h}}=\frac{\partial h\left(\mathbf{s}_{k}, \mathbf{m}_{i}\right)}{\partial \mathbf{m}_{i}}=\left[\begin{array}{ll}
\frac{\left(x_{i}-x_{k}\right)}{\sqrt{\left(x_{i}-x_{k}\right)^{2}+\left(y_{i}-y_{k}\right)^{2}}} & \frac{\left(y_{i}-y_{k}\right)}{\sqrt{\left(x_{i}-x_{k}\right)^{2}+\left(y_{i}-y_{k}\right)^{2}}} \\
\frac{-\left(y_{i}-y_{k}\right)}{\left(x_{i}-x_{k}\right)^{2}+\left(y_{i}-y_{k}\right)^{2}} & \frac{\left(x_{i}-x_{k}\right)}{\left(x_{i}-x_{k}\right)^{2}+\left(y_{i}-y_{k}\right)^{2}}
\end{array}\right]
$$

Una forma de expresar la relación de 6.16 en función de la matriz de covarianza del estado $\mathbf{P}_{k}$, que a futuro es más conveniente porque toma en cuenta la relación de conjunto entre todos los componentes del vector de estado es

$$
\mathbf{Z}=\mathbf{R}+\mathbf{J P}_{k} \mathbf{J}^{\mathrm{T}}
$$

donde la matriz jacaobiana $\mathbf{J}$ del modelo de observación será de dimensiones $2 \times 2 \mathrm{~N}$, con todos sus componentes igual a cero excepto en los índices correspondiente al landmark observado, donde será igual a $\mathbf{J}_{\mathrm{h}}$. Esto es,

$$
\mathbf{J}=\left[\begin{array}{ccc}
\cdots 0 \cdots & \mathbf{J}_{\mathrm{h}} & \cdots 0 \cdots \\
\text { otros objetos } & \text { objeto observado } & \text { otros objetos }
\end{array}\right]
$$

A continuación, el algoritmo de construcción de mapas relaciona la covarianza en la observación y la covarianza en el vector de estado para ponderar la cantidad de corrección que se le va a aplicar al mismo. Esto se logra calculando un factor que realimenta la innovación y que se conoce como Ganancia de Kalman, la cual se obtiene según

$$
\mathbf{K}=\mathbf{P}_{k} \mathbf{J}^{\mathrm{T}} \mathbf{Z}^{-1}
$$

A partir de la Ganancia de Kalman se puede actualizar el vector de estado y su matriz de covarianza de acuerdo a las siguientes ecuaciones

$$
\mathbf{x}_{k}=\mathbf{x}_{k}+\mathbf{K} \Delta \mathbf{z}
$$




$$
\mathbf{P}_{k}=\mathbf{P}_{k}-\mathbf{K Z K} \mathbf{K}^{\mathrm{T}}
$$

El resumen del procedimiento para la construcción de mapas de entorno basándose en el Filtro de Kalman Extendido se muestra en el algoritmo 6.

\subsubsection{Resultados experimentales extracción y correspondencia de landmark puntuales en la construcción de mapas de esquinas.}

El robot experimental que se usa en esta tesis (YAIR) está equipado con un SONAR rotativo que suministra información cualitativa y cuantitativa del entorno que lo rodea. Este SONAR es capaz de extraer y clasificar en dos categorías(paredes y esquinas) las características del entorno basándose en el tiempo de vuelo y en la amplitud del eco ultrasónico recibido[19, 146]. El modelo del eco de ultrasonido que usa este sensor es

$$
A(r, \theta)=\mathrm{A}_{0} \mathrm{C}_{r}^{\mathrm{N}} \frac{e^{-2 \alpha r}}{2 r} e^{-4 \theta^{2} / \theta_{0}}
$$

donde $A$ es el pico de amplitud obtenido en el receptor de ultrasonidos, $\mathrm{A}_{0}$ es una constante del transductor, $\alpha$ es el coeficiente de atenuación en el aire, $r$ es la distancia desde el sensor al reflector, y $\mathrm{C}_{r}$ es el coeficiente de reflexión acústica de la superficie reflectora, el cual varia entre 0 y 1 . Adicionalmente, $\mathrm{N}$ es un parámetro que depende del tipo de reflector y representa el número de reflexiones antes de que el eco alcance el receptor. De esta manera, $\mathrm{N}=1$ para paredes, y $\mathrm{N}=2$ cuando se trata de esquinas. $\mathrm{Si}$ en la ecuación 6.23 se resuelve para $\mathrm{N}$, entonces es posible determinar que tipo de reflector fue el que devolvió el eco ultrasónico. Los resultados experimentales obtenidos para este estrategia de clasificación muestran un porcentaje de aciertos entre el 80 y el $90 \%$ cuando se trata de paredes, y del $80 \%$ cuando se trata de esquinas que forman ángulos rectos. Igualmente, los resultados mostraron que las esquinas pueden ser detectadas siempre y cuando el sensor no este apartado más allá de los 30 grados con respecto a la bisectriz del cuadrante que forman las paredes que constituyen la esquina[145].

Para probar la validez del método de construcción de mapas de hitos puntuales, y la del método de extracción y correspondencia entre esquinas, propuestos en esta tesis, se implementó el algoritmo de construcción de mapas mediante el Filtro Extendido de Kalman, y se programó al robot YAIR para explorar un entorno de oficinas . El robot se movió en tramos de aproximadamente 3 metros, deteniéndose para observar el entorno mediante su sensor de ultrasonidos. La posición del robot fue medida manualmente en cada uno de los lugares donde se observó el entorno mediante el SONAR. Posteriormente estas posiciones fueron cargadas en el algoritmo de construcción de mapas de esquinas de robot YAIR, obteniéndose como resultado el mapa mostrado en la figura 6.3.

En la figura 6.4 se muestra una ampliación del mapa obtenido mediante el algoritmo de mapeado estocástico usando el Filtro Extendido de Kalman. Ahí se puede observar que el sensor y el algoritmo de extracción de landmark puntuales funciona 
$\overline{\text { Algoritmo } 6 \text { Algoritmo de construcción de mapas de landmark puntuales mediante }}$ el Filtro de Kalman Extendido

01: EKF_Mapping $\left(\mathbf{x}_{k}, \mathbf{P}_{k}, \mathbf{z}_{k}, \mathbf{R}_{k}, \mathbf{s}_{k}\right)$

02: $\quad$ Para todas las observaciones $z_{k}^{i}=\left\langle\rho_{i}, \varphi_{i}\right\rangle_{k}$ do

03: $\quad \mathbf{m}_{i}=\left[\begin{array}{l}m^{x} \\ m^{y}\end{array}\right]_{i}=\left[\begin{array}{c}s_{k}^{x}+\rho_{i} \cos \left(s_{k}^{\theta}+\varphi_{i}\right) \\ s_{k}^{y}+\rho_{i} \sin \left(s_{k}^{\theta}+\varphi_{i}\right)\end{array}\right]$

04: $\quad \mathbf{M}_{i}=\mathbf{J}_{z} \mathbf{R}_{k}^{i} \mathbf{J}_{z}^{\mathrm{T}}$

$\operatorname{con} \quad \mathbf{J}_{\mathbf{z}}=\left[\begin{array}{cc}\cos \left(\varphi_{i}+s_{k}^{\theta}\right) & -\rho_{i} \sin \left(\varphi_{i}+s_{k}^{\theta}\right) \\ \sin \left(\varphi_{i}+s_{k}^{\theta}\right) & \rho_{i} \cos \left(\varphi_{i}+s_{k}^{\theta}\right)\end{array}\right]$

05: $\quad j=$ Point_Correspondence $\left(\mathbf{x}_{k}, \mathbf{m}_{i}, \mathbf{M}_{i}\right) / /$ ver algoritmo 5

06: if $j=\emptyset \quad / /$ se trata de una nueva landmark

07: $\quad \mathbf{x}_{k}=\left[\begin{array}{c}\mathbf{x}_{k} \\ \mathbf{m}_{i}\end{array}\right]$

08: $\quad \mathbf{P}_{k}=\left[\begin{array}{cc}\mathbf{P}_{k} & \mathbf{0}_{2 \mathrm{Nx} 2} \\ \mathbf{0}_{2 \times 2 \mathrm{~N}} & \mathbf{J}_{z} \mathbf{R}_{k}^{i} \mathbf{J}_{z}^{\mathrm{T}}\end{array}\right]$

09: else // existe correspondencia con la landmark $j$

10: $\quad \Delta \mathbf{z}=\left[\begin{array}{c}\rho_{i} \\ \varphi_{i}\end{array}\right]-\left[\begin{array}{c}\sqrt{\left(m_{j}^{x}-s_{k}^{x}\right)^{2}+\left(m_{j}^{y}-s_{k}^{y}\right)^{2}} \\ \arctan \left(\frac{m_{j}^{y}-s_{k}^{y}}{m_{j}^{x}-s_{k}^{x}}\right)-s_{k}^{\theta}\end{array}\right]$

11: $\quad \mathbf{Z}=\mathbf{R}_{k}^{i}+\mathbf{J P}_{k} \mathbf{J}^{\mathrm{T}}$

$\operatorname{con} \mathbf{J}=\left[\begin{array}{cccc}\cdots 0 \cdots & \frac{\left(m_{j}^{x}-s_{k}^{x}\right)}{\sqrt{\left(m_{j}^{x}-s_{k}^{x}\right)^{2}+\left(m_{j}^{y}-s_{k}^{y}\right)^{2}}} & \frac{\left(m_{j}^{y}-s_{k}^{y}\right)}{\sqrt{\left(m_{j}^{x}-s_{k}^{x}\right)^{2}+\left(m_{j}^{y}-s_{k}^{y}\right)^{2}}} & \cdots 0 \cdots \\ \cdots 0 \cdots & \frac{-\left(y_{i}-y_{k}\right)}{\left(m_{j}^{x}-s_{k}^{x}\right)^{2}+\left(m_{j}^{y}-s_{k}^{y}\right)^{2}} & \frac{\left(m_{j}^{x}-s_{k}^{x}\right)}{\left(m_{j}^{x}-s_{k}^{x}\right)^{2}+\left(m_{j}^{y}-s_{k}^{y}\right)^{2}} & \cdots 0 \cdots\end{array}\right]$

12: $\quad \mathbf{K}=\mathbf{P}_{k} \mathbf{J}^{\mathrm{T}} \mathbf{Z}^{-1}$

13: $\quad \mathbf{x}_{k}=\mathbf{x}_{k}+\mathbf{K} \Delta \mathbf{z}$

14: $\quad \mathbf{P}_{k}=\mathbf{P}_{k}-\mathbf{K Z K}^{\mathrm{T}}$

15: end if

16: $\operatorname{return} \mathbf{x}_{k}, \mathbf{P}_{k}$ 


\section{Trayectoria aproximada del robot}

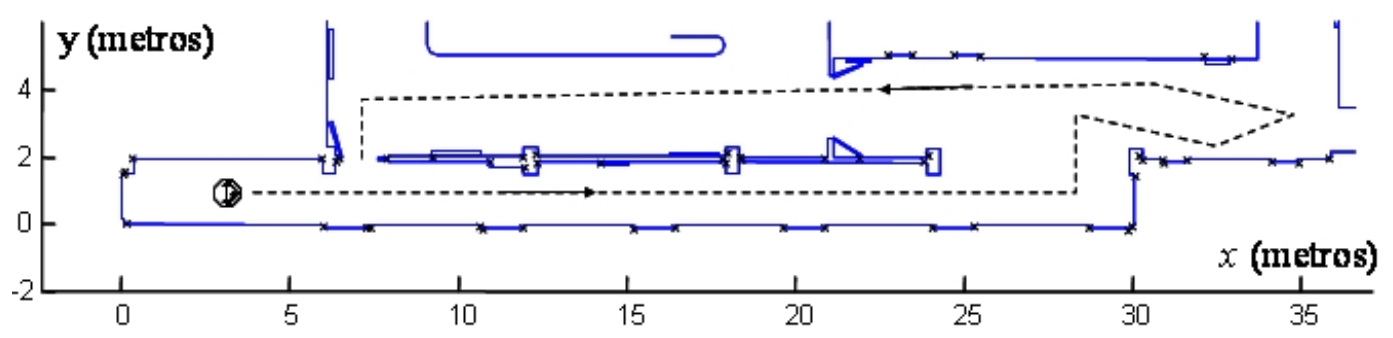

Figura 6.3: Mapas de esquinas resultante después de 2 secuencias de exploración del entorno de oficinas mostrado, en las que el robot siguió el trayecto aproximado mostrado mediante la línea a trazos discontinuos.

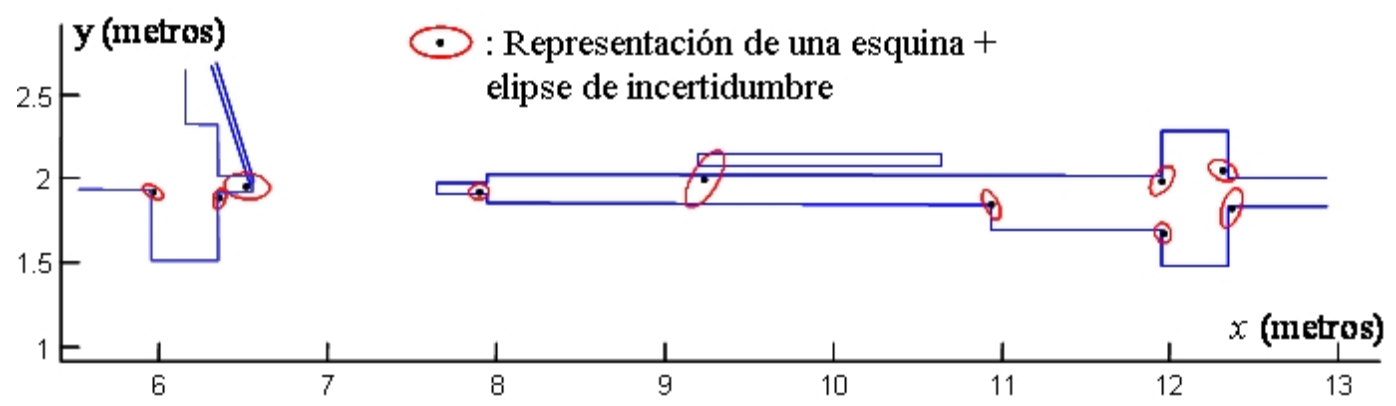

Figura 6.4: Ampliación de un área del mapa representado en la figura 6.3. Nótese como las esquinas son adecuadamente representadas mediante la elipse de incertidumbre correspondiente al punto mapeado.

correctamente mapeando las esquinas con una exactitud adecuada. También se observa que la incertidumbre en el mapa de esquinas es adecuadamente representado por las elipses provenientes de la matriz de covarianza resultante después de fusionar a través del tiempo las observaciones realizadas desde distintos lugares del entorno.

El método de fusión mediante el Filtro Extendido de Kalman muestra ser adecuado para ir refinando la localización de las distintas landmark puntuales. En la figura 6.5 se muestra la evolución de las coordenadas de localización de una de las esquinas observadas, junto a la evolución de la incertidumbre representada mediante la matriz de covarianza asociada a ella. Ahí se nota como la incertidumbre se va reduciendo a medida que se observa la esquina en repetidas ocasiones; y como la localización final de la esquina observada tiende a la localización real de la misma.

Finalmente, los resultados obtenidos mostraron que el método de correspondencia entre landmark puntuales propuesto en esta tesis logra discernir entre las distintas landmark del entorno, logrando determinar sin errores la correspondencia entre los objetos mapeados y los objetos observados. 

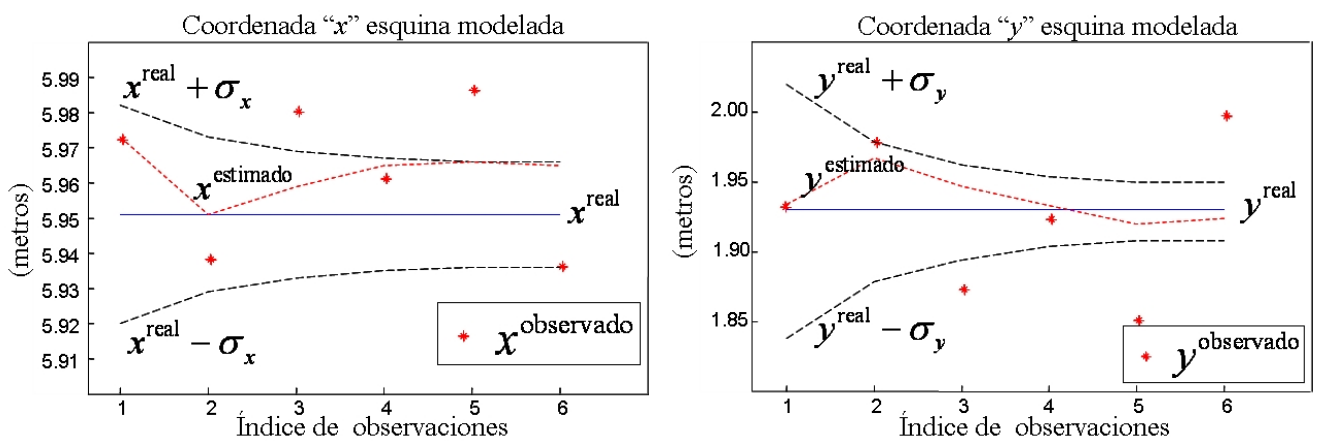

Figura 6.5: Evolución a través del tiempo del modelo de una landmark puntual cuando su repetida observación es fusionada mediante el Filtro Extendido de Kalman.

\subsection{Localización probabilística mediante landmark natu- rales tipo puntuales}

En robótica móvil, la localización se refiere al proceso de estimación de la posición relativa del robot, respecto a un sistema de coordenadas fijo. En general existen 2 tipos de localización: relativa y absoluta. La primera, conocida como Dead-Reckoning, se lleva a cabo mediante la medición provista por los sensores propioceptivos del robot. Esta técnica es impracticable o poco fiable para trayectos o recorridos muy largos, ya que el movimiento del robot está sometido a errores sistemáticos y no sistemáticos que hacen que el error en la estimación crezca sin límites en el tiempo[32]. Por esta razón, para que el robot pueda autolocalizarse, el mismo debe contar con sensores exteroceptivos (medidores de distancia láser, medidores basados en ultrasonido, cámaras de vídeo), que le permitan observar referencias externas(landmark) y así poder corregir su posición o localizarse de forma absoluta.

Otra forma de clasificar el problema de localización consiste en dividirla en: localización local(seguimiento o tracking), cuyo objetivo, dada una posición conocida del robot, es mantener su localización en el mapa; y localización global que consiste en determinar la posición del robot sin tener una referencia de su posición anterior o con un conocimiento erróneo relativo a la posición real del vehículo.

Desde el enfoque probabilístico, dada las lecturas sensoriales, existen varias posibles localizaciones para el robot. Bajo este enfoque, el problema de localización consiste en encontrar la configuración del robot que tiene mayor probabilidad de ser correcta. así, hay que determinar la configuración del robot en el espacio de posibles configuraciones, o lo que es lo mismo la probabilidad de cada posición $p(\mathbf{s})$, con $\mathbf{s}=\langle x, y, \theta\rangle$. Este problema puede ser tratado con el Filtro de Bayes(Localización de Markov), el cual se puede implementar de diferentes formas como por ejemplo mediante el Filtro de Kalman y mediante Filtros de partículas, entre otras. Mediante esta metodología, el robot va alternando sensado/movimiento hasta poder determinar su ubicación. Esta operación se ilustra en la figura 6.6, donde se ha asumido que las distintas densidades pueden ser representadas mediante funciones Gaussianas. 


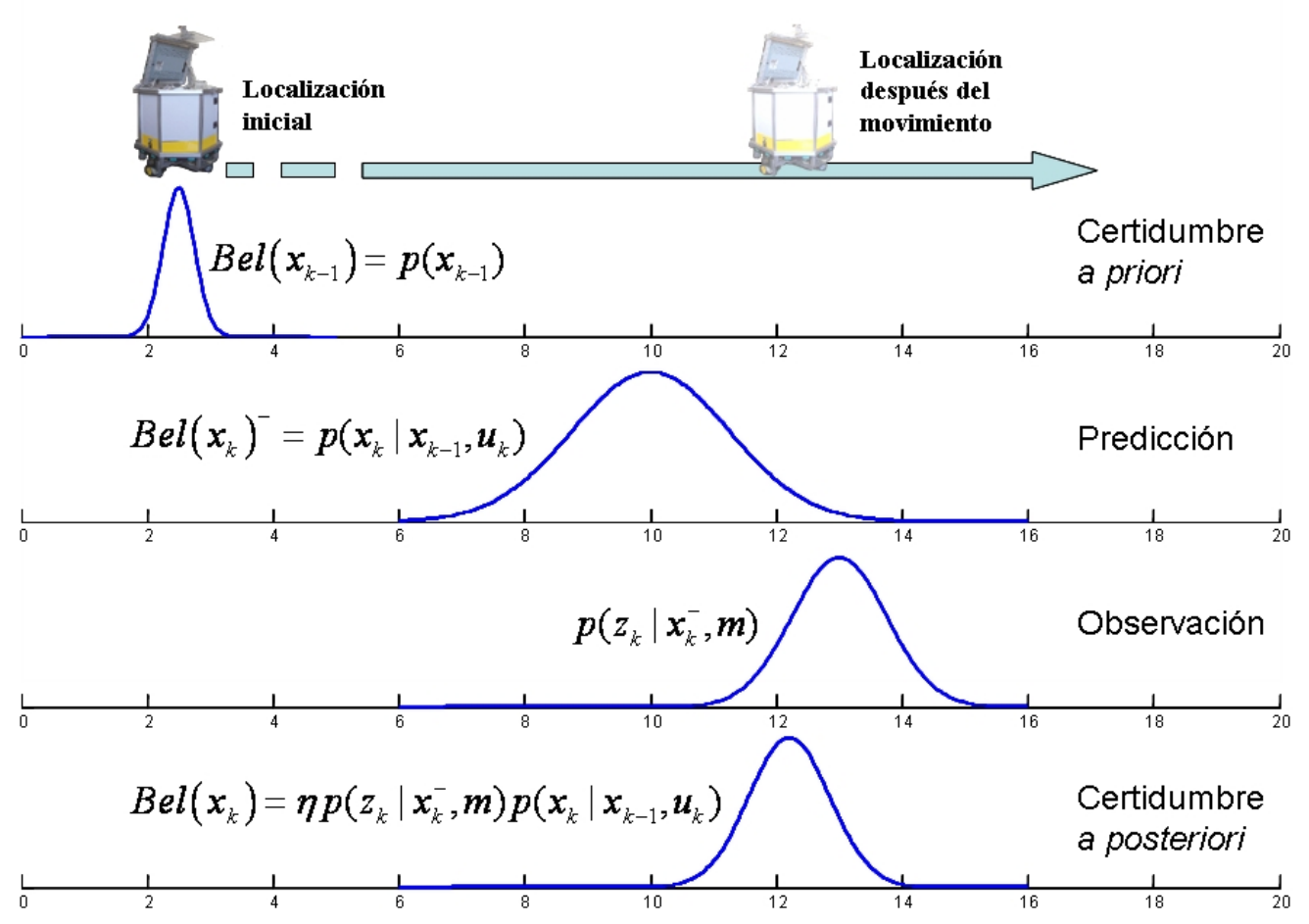

Figura 6.6: Esquema de la localización de Markov usando densidades Gaussianas. Nótese la secuencia de operaciones movimiento - predicción - observación - corrección - movimiento. 
También, tal como se describió en la sección 6.1, la composición de la posición del robot $\mathbf{s}=\langle x, y, \theta\rangle$ y del mapa $\mathbf{m}$, forma y se conoce como el estado $\mathbf{x}=\langle\mathbf{s}, \mathbf{m}\rangle$. Dado que en la localización se asume que el mapa del entorno se conoce perfectamente, entonces el vector de estado en el instante $k$ estaría definido sólo por el vector de posición según,

$$
\mathbf{x}_{k}=\langle x, y, \theta\rangle
$$

Este vector de estado evolucionará, manejado por el vector de control $\mathbf{u}_{k}$, de acuerdo al modelo cinemático del vehículo. Esto es,

$$
\mathbf{x}_{k}=f\left(\mathbf{x}_{k-1}, \mathbf{u}_{k}\right)
$$

donde $f(\cdot)$ en general es una función no lineal del estado del vehículo en el instante anterior $\mathbf{x}_{k-1}$, y del vector de control $\mathbf{u}_{k}$.

Con base en la figura 6.6, el algoritmo de localización de Markov puede describirse de la siguiente manera: Inicialmente se presume que se dispone de una certidumbre en la posición inicial $\operatorname{Bel}\left(\mathbf{x}_{k-1}\right)$, la cual debe ser muy próxima a la posición real del vehículo en ese mismo instante de tiempo. Según el vehículo se mueve, la certidumbre inicial se convoluciona con el modelo de movimiento, y resulta en una nueva distribución $\mathrm{Bel}^{-}\left(\mathbf{x}_{k}\right)=p\left(\mathbf{x}_{k} \mid \mathbf{x}_{k-1}, \mathbf{u}_{k}\right)$ que describe la posición estimada del vehículo antes de observar el entorno. Ahora, suponiendo que a través del sensor se observa una landmark $\mathbf{z}_{k}$ cuya características es descrita a través del modelo estocástico $p\left(\mathbf{z}_{k} \mid \mathbf{m}, \mathbf{x}_{k}\right)$, entonces la posición estimada del vehículo es corregida mediante la fusión de estas dos distribuciones de probabilidad, resultando en una distribución de probabilidad de menor varianza que es descrita mediante

$$
\operatorname{Bel}^{+}\left(\mathbf{x}_{k}\right)=\eta p\left(\mathbf{x}_{k} \mid \mathbf{x}_{k-1}, \mathbf{u}_{k}\right) p\left(\mathbf{z}_{k} \mid \mathbf{m}, \mathbf{x}_{k}\right)
$$

Aquí $\eta$ es un factor de normalización que garantiza que se cumpla el primer axioma de la probabilidad $\left(0 \leq \mathrm{Bel}^{+}\left(\mathbf{x}_{k}\right) \leq 1\right)$.

\subsubsection{El algoritmo de localización mediante hitos tipo puntuales usando el Filtro Extendido de Kalman}

En el ámbito de la localización probabilística, la tendencia actual es la de apoyarse en mapas de objetos que incluyen esquinas(puntos) y líneas[72, 83]. En general, en este proceso se asume que se conoce el mapa del entorno $\mathbf{m}:\left\{\mathbf{m}_{1}, \mathbf{m}_{2}, \cdots, \mathbf{m}_{\mathrm{N}}\right\}$ y que se conocen también la secuencia de observaciones $\mathbf{z}_{k}$ que va realizando el vehículo a medida que explora el entorno, las cuales son descritas por medio de la función de verosimilitud $p\left(\mathbf{z}_{k} \mid \mathbf{m}, \mathbf{x}_{k}\right)$. Con estas condiciones, el problema de localización de un robot se puede plantear como el estimar la distribución $p\left(\mathbf{x}_{k} \mid \mathbf{m}, \mathbf{z}_{k}\right)$, que es la función de verosimilitud de $\mathbf{x}_{k}$ condicionado a una serie de lecturas $\mathbf{z}_{k}$ sobre un entorno $\mathbf{m}$. 


$$
\begin{aligned}
p\left(\mathbf{x}_{k} \mid \mathbf{m}, \mathbf{z}_{k}\right) & =\frac{p\left(\mathbf{z}_{k} \mid \mathbf{m}, \mathbf{x}_{k}\right) p\left(\mathbf{m}, \mathbf{x}_{k}\right)}{p\left(\mathbf{m}, \mathbf{z}_{k}\right)} \\
& =\frac{p\left(\mathbf{z}_{k} \mid \mathbf{m}, \mathbf{x}_{k}\right) p\left(\mathbf{x}_{k} \mid \mathbf{m}\right) p(\mathbf{m})}{\int_{-\infty}^{-\infty} p\left(\mathbf{z}_{k} \mid \mathbf{m}, \mathbf{x}_{k}\right) p\left(\mathbf{x}_{k} \mid \mathbf{m}\right) p(\mathbf{m}) d \mathbf{x}_{k}}
\end{aligned}
$$

Sujeto a que se conoce el mapa del entorno de forma perfecta, $p(\mathbf{m})=1$, entonces la ecuación 6.27 se puede reescribir como

$$
p\left(\mathbf{x}_{k} \mid \mathbf{m}, \mathbf{z}_{k}\right)=\eta p\left(\mathbf{z}_{k} \mid \mathbf{x}_{k}\right) p\left(\mathbf{x}_{k}\right)
$$

donde $p\left(\mathbf{z}_{k} \mid \mathbf{x}_{k}\right)$ es el modelo de observación y $p\left(\mathbf{x}_{k}\right)$ es la probabilidad a priori relacionada al estado del vehículo. También, $\eta$ representa la probabilidad total que tiene el evento $\mathbf{z}_{k}$ sobre el espacio muestral, por lo que se convierte en un factor de normalización que garantiza que se cumpla el primer axioma de la $\operatorname{probabilidad}\left(0 \leq p\left(\mathbf{x}_{k} \mid \mathbf{m}, \mathbf{z}_{k}\right) \leq 1\right)$. Si las densidades involucradas se parametrizan mediante el primero y segundo momento estadístico(media y varianza), entonces se puede utilizar el Filtro de Kalman para estimar el máximo a posteriori de la ecuación 6.28 .

El algoritmo de localización usando el Filtro Extendido de Kalman o LocalizaciónEKF, es un caso especial de la localización de Markov donde la función de verosimilitud de la confianza o certidumbre en el estado $\operatorname{Bel}(\mathbf{x})$ se representa mediante su primer y segundo momento: la media $\hat{\mathbf{x}}$ y la covarianza $\mathbf{P}$. De aquí que el uso de esta técnica requiere que se deriven las representaciones estocásticas tanto del modelo de movimiento del robot, como del modelo de medición o de observación del entorno, para que puedan ser utilizadas en la etapa de predicción y de corrección, respectivamente

\subsubsection{Etapa de predicción. Modelado de la cinemática del vehículo}

Para un robot tipo diferencial, como el que se utiliza en los ensayos experimentales realizados en este trabajo de tesis, la posición puede ser estimada a partir de una posición conocida, mediante la integración de la información proveniente de los sensores de odometría acoplados a las ruedas motrices. De esta manera, dada la posición inicial del robot $\mathbf{x}_{k-1}=\langle x, y, \theta\rangle_{k-1}^{\mathrm{T}}$, y el vector de comandos de entrada $\mathbf{u}_{k}=\langle\Delta S, \Delta \theta\rangle_{k}^{\mathrm{T}}$, se puede predecir el estado estimado $\hat{\mathbf{x}}_{k}^{-}$del robot mediante la ecuación,

$$
\hat{\mathbf{x}}_{k}^{-}=\mathbf{x}_{k-1}^{+}+\left[\begin{array}{c}
\Delta S_{k} \cos \left(\theta_{k-1}+\Delta \theta_{k} / 2\right) \\
\Delta S_{k} \sin \left(\theta_{k-1}+\Delta \theta_{k} / 2\right) \\
\Delta \theta_{k}
\end{array}\right]
$$

donde $\Delta S_{k}$ y $\Delta \theta_{k}$ son la longitud y el ángulo diferencial recorrido por el centro del robot desde $\mathbf{x}_{k-1}$ hasta $\mathbf{x}_{k}$ (ver figura 6.7).

Por otra parte, el ruido Gaussiano que afecta al modelo de evolución del estado se modela mediante la matriz de covarianzas $\mathbf{P}$, la cual tiene la forma 


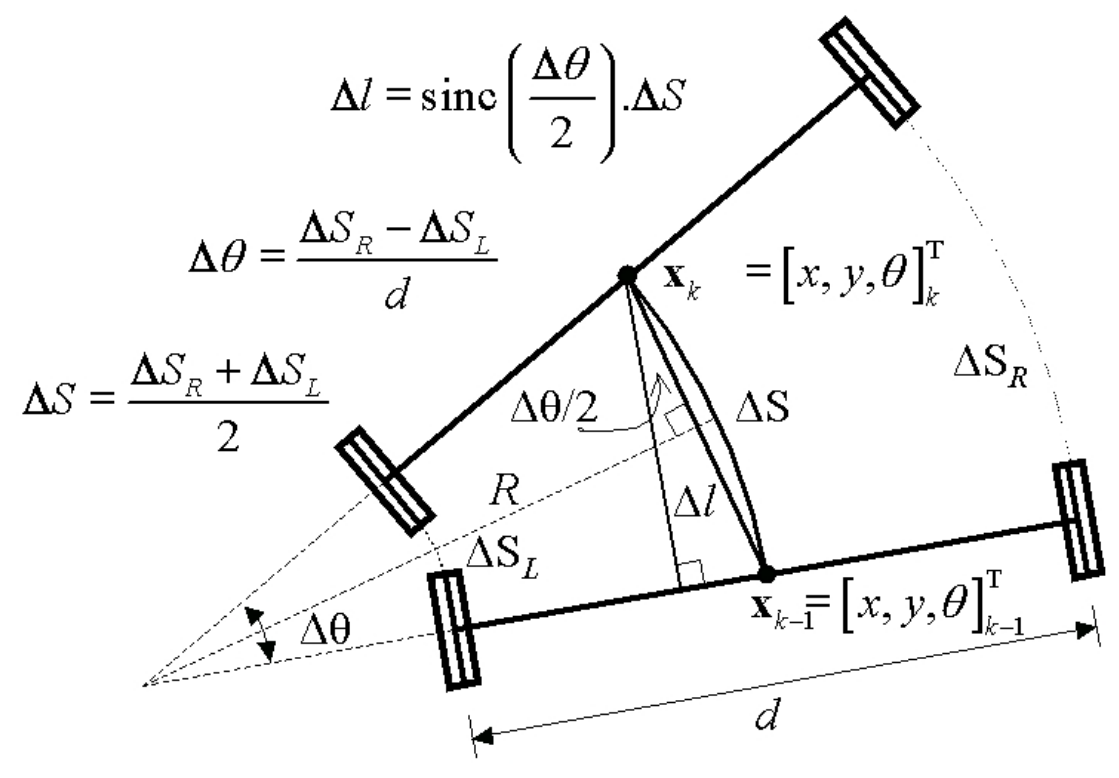

Figura 6.7: Relaciones geométricas involucradas en el modelo cinemático-odométrico de un móvil tipo diferencial.

$$
\mathbf{P}_{k}=\left[\begin{array}{ccc}
\sigma_{x}^{2} & \sigma_{x y} & \sigma_{x \theta} \\
\sigma_{x y} & \sigma_{y}^{2} & \sigma_{y \theta} \\
\sigma_{x \theta} & \sigma_{y \theta} & \sigma_{\theta}^{2}
\end{array}\right]
$$

donde los valores $\sigma_{x}^{2}, \sigma_{y}^{2}$ y $\sigma_{\theta}^{2}$ representan las varianzas en $x, y$ y $\theta$, respectivamente.

En la sección 5.2 .2 , ya se demostró que $\mathbf{P}_{k}$ se puede obtener por propagación lineal del ruido a través de la función que modela el movimiento del vehículo. Así, en el caso de un robot diferencial, $\mathbf{P}_{k}$ se obtiene según

$$
\mathbf{P}_{k}^{-}=\mathbf{J}_{\mathbf{x}} \mathbf{P}_{k-1}^{+} \mathbf{J}_{\mathbf{x}}^{\mathrm{T}}+\mathbf{J}_{\mathbf{u}} \mathbf{U}_{k} \mathbf{J}_{\mathbf{u}}^{\mathrm{T}}
$$

donde $\mathbf{J}_{\mathbf{x}}$ representa la matriz Jacobiana con respecto al estado $\mathbf{x}=\langle x, y, \theta\rangle$ de la ecuación de predicción representada en 6.29 , mientras que $\mathbf{J}_{\mathbf{u}}$ es la matriz Jacobiana de la misma función con respecto a la excitación de entrada $\mathbf{u}=\langle\Delta S, \Delta \theta\rangle$. Así, estos Jacobianos corresponden a

$$
\begin{gathered}
\mathbf{J}_{\mathbf{x}}=\left[\begin{array}{ccc}
1 & 0 & -\Delta S_{k} \sin \left(\theta_{k-1}+\Delta \theta_{k} / 2\right) \\
0 & 1 & \Delta S_{k} \cos \left(\theta_{k-1}+\Delta \theta_{k} / 2\right) \\
0 & 0 & 1
\end{array}\right] \\
\mathbf{J}_{\mathbf{u}}=\left[\begin{array}{cc}
\cos \left(\theta_{k-1}+\Delta \theta_{k} / 2\right) & -\frac{1}{2} \Delta S_{k} \sin \left(\theta_{k-1}+\Delta \theta_{k} / 2\right) \\
\sin \left(\theta_{k-1}+\Delta \theta_{k} / 2\right) & \frac{1}{2} \Delta S_{k} \cos \left(\theta_{k-1}+\Delta \theta_{k} / 2\right) \\
0 & 1
\end{array}\right]
\end{gathered}
$$


Por su parte, la matriz de covarianza $\mathbf{U}$ del vector de excitación ya se derivó en la sección 5.2.2. Sin embargo, para propositos de claridad de la metodología expuesta, a continuación se repite la formulación de la misma.

$$
\mathbf{U}=\left[\begin{array}{ll}
\frac{\left(\sigma_{R}^{2}+\sigma_{L}^{2}\right)}{4} & \frac{\left(\sigma_{R}^{2}-\sigma_{L}^{2}\right)}{2 d} \\
\frac{\left(\sigma_{R}^{2}-\sigma_{L}^{2}\right)}{2 d} & \frac{\left(\sigma_{R}^{2}+\sigma_{L}^{2}\right)}{d^{2}}
\end{array}\right]
$$

Aquí, $\sigma_{R}^{2}$ y $\sigma_{L}^{2}$ son la varianza del error odometrico de la rueda derecha e izquierda respectivamente. Estas varianzas pueden ser modeladas de la forma,

$$
\left\{\begin{array}{l}
\sigma_{R}^{2}=K_{R}^{2}\left|\Delta S_{R}\right| \\
\sigma_{L}^{2}=K_{L}^{2}\left|\Delta S_{L}\right|
\end{array}\right.
$$

donde $\Delta S_{R}$ y $\Delta S_{L}$ corresponden a lasa distancias viajadas por las ruedas derecha e izquierda, respectivamente. También $K_{R}^{2}$ y $K_{L}^{2}$ son constantes de error que modelan la interacción no determinística entre las ruedas y el acoplamiento con la superficie de rodamiento.

\subsubsection{Etapa de medición y correspondencia. Modelado de la función de observación}

En el proceso de localización se asume que el vehículo que navega por el entorno cuenta con un sensor que proporciona información relacionada a la distancia radial $\rho$ y al ángulo de vista $\varphi$ medidos en el sistema de coordenadas del mismo vehículo. Esta información estará representada en una matriz de medición indexada de acuerdo al instante de tiempo $k$ en el cual se produzca el ciclo de observación del entorno según,

$$
\mathbf{z}_{k}=\left[\begin{array}{cccc}
\rho^{1} & \rho^{2} & \cdots & \rho^{j} \\
\varphi^{1} & \varphi^{2} & \cdots & \varphi^{j}
\end{array}\right]_{k}
$$

donde el indicie $j$ se refiere al $j$-esimo objeto que es detectado por los sensores durante el proceso de observación $k$.

Por otra parte, en el caso de la localización en entornos interiores usando mapas de esquinas, se asume que el mapa es perfectamente conocido y que está representado mediante una colección de objetos puntuales $\mathbf{m}_{i}=\langle x, y\rangle_{i}$ pertenecientes a un sistema 2D fijo de coordenadas cartesianas, conocido como el sistema de referencias global. Por esto, el conjunto de objetos pertenecientes a la matriz de medición $\mathbf{z}_{k}$, junto a su matriz de covarianza $\mathbf{R}_{k}^{j}$, son transformados al marco de referencia Global para ejecutar así un proceso de pre-correspondencia, de acuerdo a la técnica que se propone en la sección 5.5.1 de esta tesis. No obstante, una vez que se ha ejecutado el proceso de pre-correspondencia, y debido a que el proceso de corrección del estado es llevado a cabo en el marco de referencia del vehículo (marco Robot), es necesario transformar a este marco todo objeto que pertenece al mapa global y que se corresponde con algún otro objeto perteneciente a la medición $\mathbf{z}_{k}$. Esto es llevado a cabo mediante la siguiente fórmula, la cual es conocida como la función de observación. 


$$
\begin{aligned}
\mathbf{z}_{i}=h\left(\mathbf{x}_{k}, \mathbf{m}_{i}\right) & =\left[\begin{array}{c}
\rho_{i} \\
\varphi_{i}
\end{array}\right] \\
& =\left[\begin{array}{c}
\sqrt{\left(x_{i}-x_{k}\right)^{2}+\left(y_{i}-y_{k}\right)^{2}} \\
\arctan \left(\frac{y_{i}-y_{k}}{x_{i}-x_{k}}\right)-\theta_{k}
\end{array}\right]
\end{aligned}
$$

donde $\mathbf{x}_{k}=\langle x, y, \theta\rangle_{k}$ representa la posición del robot o el estado en el instante $k, \mathrm{y}$ $\mathbf{m}_{i}=\langle x, y\rangle_{i}$ corresponde a las coordenadas de la esquina observada.

\subsubsection{Etapa de corrección y actualización. Cálculo de la ganancia de Kalman}

De esta forma, asumiendo que se ha ejecutado un proceso de correspondencia entre los objetos medidos y los objetos del mapa, la diferencia entre el vector de medición $\mathbf{z}_{k}^{j}$ y el vector de observación $\mathbf{z}_{i}$ proporciona lo que se conoce como la innovación $\Delta \mathbf{z}$.

$$
\Delta \mathbf{z}=\mathbf{z}_{k}^{j}-\mathbf{z}_{i}
$$

La covarianza en el vector de innovación puede obtenerse mediante aproximación lineal de acuerdo a

$$
\mathbf{Z}=\mathbf{R}_{j}+\mathbf{J}_{\mathrm{h}} \mathbf{P}_{k} \mathbf{J}_{\mathrm{h}}^{\mathrm{T}}
$$

donde $\mathbf{R}_{j}$ es la matriz de covarianza que modela el ruido sensorial en la medición del objeto $\mathbf{z}_{k}^{j}$, $\mathbf{J}_{\mathrm{h}}$ es el Jacobiano de la función de observación, y $\mathbf{P}_{k}$ es la matriz de covarianza del estado en el instante $k$. El Jacobiano $\mathbf{J}_{\mathrm{h}}$ se obtiene a partir de la ecuación 6.37 de acuerdo a,

$$
\mathbf{J}_{\mathrm{h}}=\frac{\partial h\left(\mathbf{x}_{k}, \mathbf{m}_{i}\right)}{\partial \mathbf{x}_{k}}=\left[\begin{array}{ccc}
\frac{-\left(x_{i}-x_{k}\right)}{\sqrt{\left(x_{i}-x_{k}\right)^{2}+\left(y_{i}-y_{k}\right)^{2}}} & \frac{-\left(y_{i}-y_{k}\right)}{\sqrt{\left(x_{i}-x_{k}\right)^{2}+\left(y_{i}-y_{k}\right)^{2}}} & 0 \\
\frac{\left(y_{i}-y_{k}\right)}{\left(x_{i}-x_{k}\right)^{2}+\left(y_{i}-y_{k}\right)^{2}} & \frac{-\left(x_{i}-x_{k}\right)}{\left(x_{i}-x_{k}\right)^{2}+\left(y_{i}-y_{k}\right)^{2}} & -1
\end{array}\right]
$$

A continuación, el algoritmo de localización relaciona la covarianza en el vector de innovación y la covarianza en el vector de estado para ponderar la cantidad de corrección que se le va a aplicar al mismo. Esto se logra calculando un factor que realimenta la innovación y que se conoce como Ganancia de Kalman, la cual se obtiene según

$$
\mathbf{K}=\mathbf{P}_{k}^{-} \mathbf{J}_{\mathrm{h}}^{\mathrm{T}} \mathbf{Z}^{-1}
$$

A partir de la Ganancia de Kalman se puede actualizar el vector de estado y su matriz de covarianza de acuerdo a las siguientes ecuaciones

$$
\mathbf{x}_{k}^{+}=\mathbf{x}_{k}^{-}+\mathbf{K} \Delta \mathbf{z}
$$




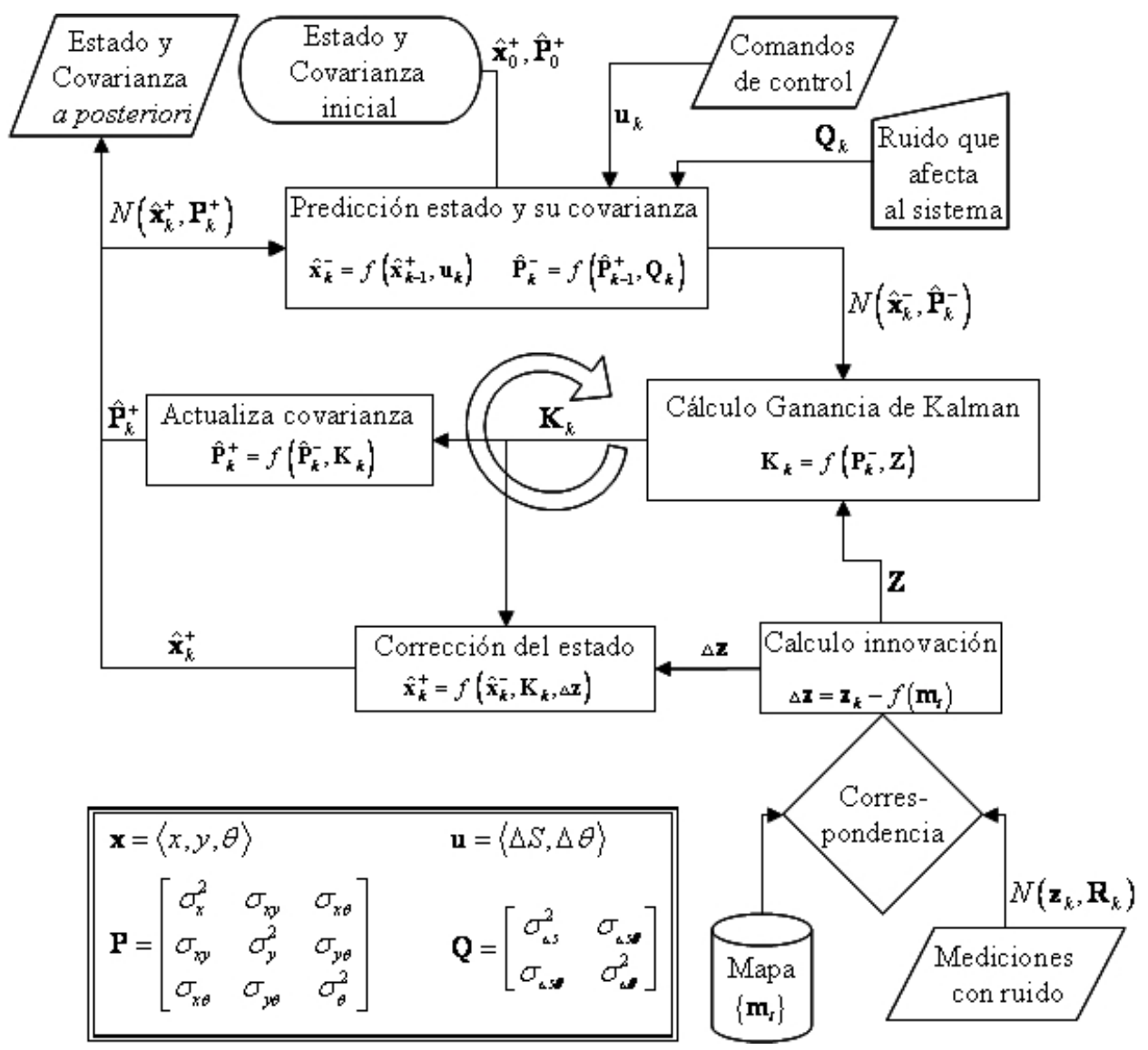

Figura 6.8: Algoritmo de localización usando el Filtro Extendido de Kalman. $N(\cdot)$ indica que es una densidad de distribución normal con media y varianza igual a los argumentos dentro del parentesis. $f(\cdot)$ indica que es una función no lineal de los argumentos entre paréntesis

$$
\mathbf{P}_{k}^{+}=\mathbf{P}_{k}^{-}-\mathbf{K Z K} \mathbf{K}^{\mathrm{T}}
$$

El resumen del procedimiento de localización basado en el Filtro Extendido de Kalman se muestra en la figura 6.8.

\subsubsection{Resultados experimentales en la localización usando esquinas extraídas con un SONAR rotativo.}

Los experimentos han sido realizados usando el robot YAIR, el cual cuenta con sendos codificadores óptico rotativos acoplados a sus ruedas motrices y con un sensor de ultrasonidos capaz de extraer las esquinas del entorno proporcionando así información cualitativa y cuantitativa del ambiente que lo rodea. Con la finalidad de demostrar la validez del método de localización usando esquinas como hitos naturales 
propuesto en esta tesis, se programó al robot para que navegara a través de un entorno típico de oficinas, mientras exploraba el mismo mediante su sensor de ultrasonidos. Durante el trayecto de aproximadamente 140 metros, el robot realizó paradas cada 3 metros con el objetivo de observar el entorno mediante su sensor de ultrasonidos. En cada una de estas paradas se medió manualmente la posición real de robot con la finalidad de compararla posteriormente con la posición estimada mediante la localización odométrica simple, así como también con los resultados de estimación de la posición obtenidos por el algoritmo de localización basado en el Filtro Extendido de Kalman.

En la figura 6.9 se muestra el trayecto real, así como también la trayectoria estimada usando sólo odometría y la estimada usando el Filtro Extendido de Kalman. Los resultados muestran el efecto pernicioso que tiene la acumulación del error en el caso de la localización odométrica; mientras que en el caso de la localización usando el EKF, la oportuna corrección de la posición en cada punto de observación permitió que el robot estimara adecuadamente la trayectoria seguida, así como también el lugar donde él se encontraba.

En la tabla 6.1 y en la tabla 6.2 se muestran el resumen estadístico del error de estimación de la posición usando sólo odometría y usando el EKF respectivamente. De especial interés es el gran rango del error $\varepsilon_{y}$ (que en este caso corresponde a la dirección penperdicular al movimiento del robot), que en el caso de la localización odométrica está significativamente correlacionado con el error de ángulo $\varepsilon_{\theta}$; mientras que en el caso del EKF no sólo se reduce drásticamente, sino que también se descorrelaciona del error en el ángulo. De menor interés pero aun importante es el error $\varepsilon_{x}$ (en este caso corresponde a la dirección del movimiento del robot), el cual mantiene en los dos casos una acusada correlación con el error perpendicular $\varepsilon_{y}$, mientras que su correlación con el error de ángulo no es significativa(ver tabla 6.3).

También, en la figura 6.10 se puede ver como en el caso de la localización odométrica el error en el ángulo de orientación crece sin límite a medida que crece la distancia recorrida por el robot. Esto a su vez causará que los errores de localización del robot $\left(\varepsilon_{x}, \varepsilon_{y}\right)$ crezcan también sin límites. Por otra parte, en la figura 6.11 se muestra la evolución del error en el estado cuando se utiliza el algoritmo de localización basado en las esquinas extraídas con el SONAR del robot YAIR. Como se aprecia en la mencionada figura, la fusión de la información mediante el algoritmo EKF, permite corregir el estimado en la posición del robot, manteniendo el error de estimación acotado en el tiempo.

Finalmente, a efectos de comparación, en la Tabla (6.4) se muestran el error RMS resultante en el punto final del trayecto. Como se puede observar, el error en la estimación del ángulo de orientación del robot es significativamente grande, lo que a su vez genera que el error en la componente transversal del vector de estado del robot, sea también significativamente alta. Igualmente, al comparar los resultados de la localización usando sólo el sistema odométrico con el sistema basado en el filtro de Kalman, se aprecia que el método aquí propuesto mejora significativamente el estimado que el robot tiene sobre su misma posición, reduciendo este error al rango de las decenas de milímetros. 

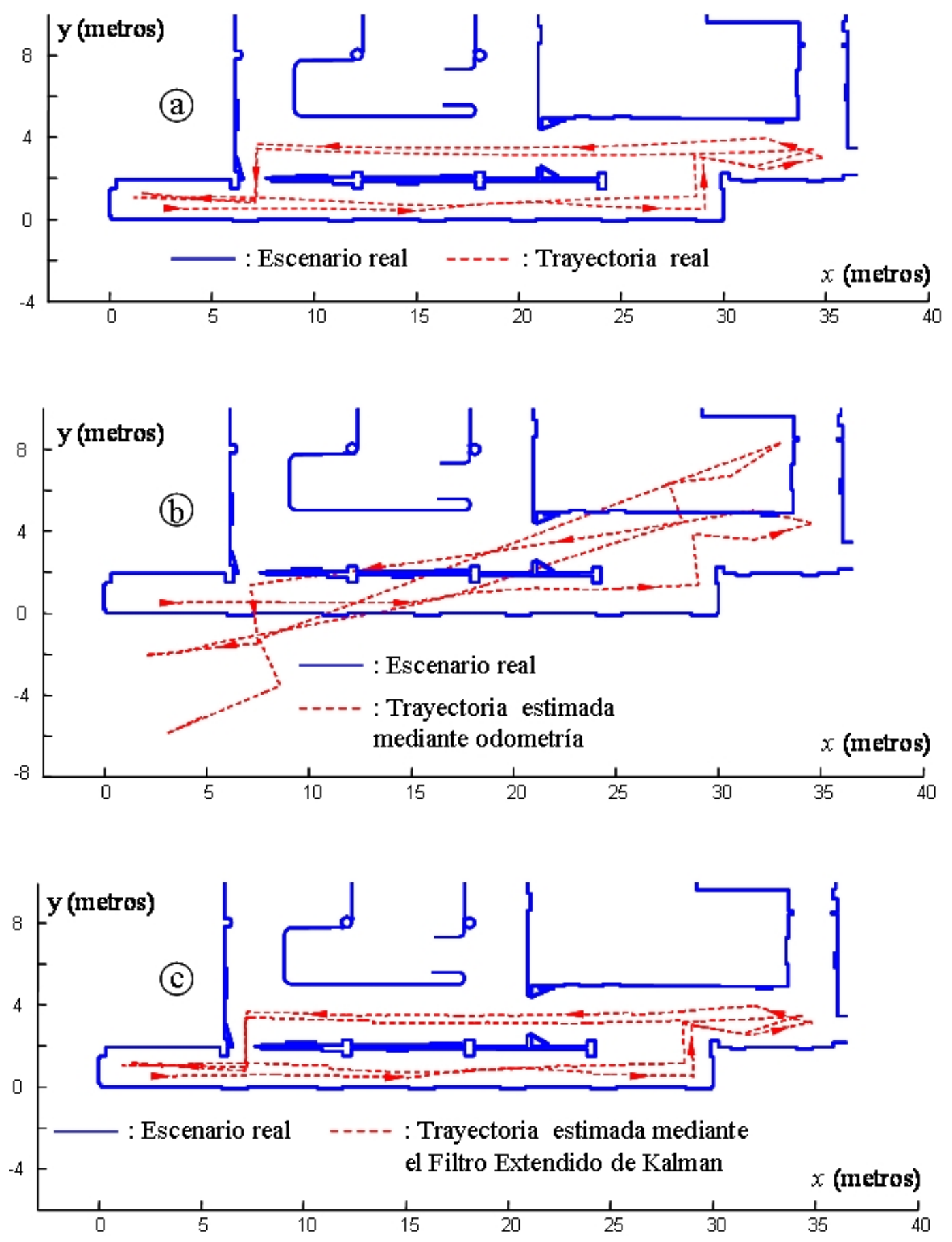

Figura 6.9: a) Trayecto real, el cual fue medido manualmente. b) Trayecto estimado mediante la integración de los datos de odometría. c) Trayecto estimado mediante el Filtro Extendido de Kalman. Para mayor comprensión de la figura, la flecha indica la dirección del trayecto ejecutado durante el primer ciclo de exploración. 


\subsection{LOCALIZACIÓN PROBABILÍSTICA MEDIANTE LANDMARK NATURALES TIPO PUNTUALES}

Tabla 6.1: Resumen estadístico del error de posicionamiento resultante al aplicar la técnica de localización que usa sólo odometría. De particular interés están la asimetría estandarizada y la curtosis estandarizada, las cuales pueden utilizarse para determinar si la muestra procede de una distribución normal. Valores de estos estadísticos fuera del rango de -2 a +2 indican una desviación significativa de la normalidad, que tendería a invalidar muchos de los procedimientos estadísticos aplicados habitualmente a estos datos. En este caso, el error $\varepsilon_{x}$ muestra una asimetría estandarizada y una curtosis estandarizada fuera del rango esperado.

\begin{tabular}{|l|c|c|c|}
\hline & $\varepsilon_{x}($ metros $)$ & $\varepsilon_{y}$ (metros) & $\varepsilon_{\theta}$ (radianes) \\
\hline \hline Frecuencia & 51 & 51 & 51 \\
\hline Media & -0.006 & 0.362 & -0.2080 \\
\hline Varianza & 0.406 & 6.794 & 0.0171 \\
\hline Desviación típica & 0.638 & 2.607 & 0.1310 \\
\hline Mínimo & -1.890 & -4.952 & -0.4303 \\
\hline Máximo & 1.314 & 6.934 & 0.0015 \\
\hline Rango & 3.204 & 11.886 & 0.4318 \\
\hline Asimetría estandarizada & -2.904 & 1.256 & -0.2450 \\
\hline Curtosis estandarizada & 3.393 & 0.324 & -1.7706 \\
\hline
\end{tabular}

Tabla 6.2: Resumen estadístico del error de posicionamiento resultante al aplicar la técnica de localización basada en el Filtro Extendido de Kalman. De particular interés están la asimetría estandarizada y la curtosis estandarizada, las cuales pueden utilizarse para determinar si la muestra procede de una distribución normal. Valores de estos estadísticos fuera del rango de -2 a +2 indican una desviación significativa de la normalidad, que tendería a invalidar muchos de los procedimientos estadísticos aplicados habitualmente a estos datos. En este caso, el error $\varepsilon_{x}$ muestra una curtosis estandarizada fuera del rango esperado.

\begin{tabular}{|l|c|c|c|}
\hline & $\varepsilon_{x}($ metros $)$ & $\varepsilon_{y}$ (metros) & $\varepsilon_{\theta}$ (radianes) \\
\hline \hline Frecuencia & 51 & 51 & 51 \\
\hline Media & 0.011 & -0.001 & -0.0155 \\
\hline Varianza & 0.000 & 0.005 & 0.0001 \\
\hline Desviación típica & 0.020 & 0.070 & 0.0101 \\
\hline Mínimo & -0.059 & -0.179 & -0.0471 \\
\hline Máximo & 0.053 & 0.150 & 0.0015 \\
\hline Rango & 0.112 & 0.329 & 0.0486 \\
\hline Asimetría estandarizada & -1.808 & -0.392 & -1.8373 \\
\hline Curtosis estandarizada & 3.105 & 0.403 & 0.7852 \\
\hline
\end{tabular}




\section{CAPÍTULO 6. CONSTRUCCIÓN DE MAPAS Y LOCALIZACIÓN ESTOCÁSTICA}

Tabla 6.3: Correlaciones, de acuerdo al momento de Pearson, entre los componentes del error de posición. a) Estimación de la posición usando sólo odometría. b) Estimación de la posición usando el Filtro Extendido de Kalman

\begin{tabular}{|c|c|c|c|}
\hline & $\varepsilon_{x}$ & $\varepsilon_{y}$ & $\varepsilon_{\theta}$ \\
\hline \hline$\varepsilon_{x}$ & 1 & -0.9110 & 0.2257 \\
\hline$\varepsilon_{y}$ & -0.9110 & 1 & -0.2761 \\
\hline$\varepsilon_{\theta}$ & 0.2257 & -0.2761 & 1 \\
\hline
\end{tabular}

a)

\begin{tabular}{|c|c|c|c|}
\hline & $\varepsilon_{x}$ & $\varepsilon_{y}$ & $\varepsilon_{\theta}$ \\
\hline \hline$\varepsilon_{x}$ & 1 & -0.3773 & -0.0351 \\
\hline$\varepsilon_{y}$ & -0.3773 & 1 & -0.0701 \\
\hline$\varepsilon_{\theta}$ & -0.0351 & -0.0701 & 1 \\
\hline
\end{tabular}

b)
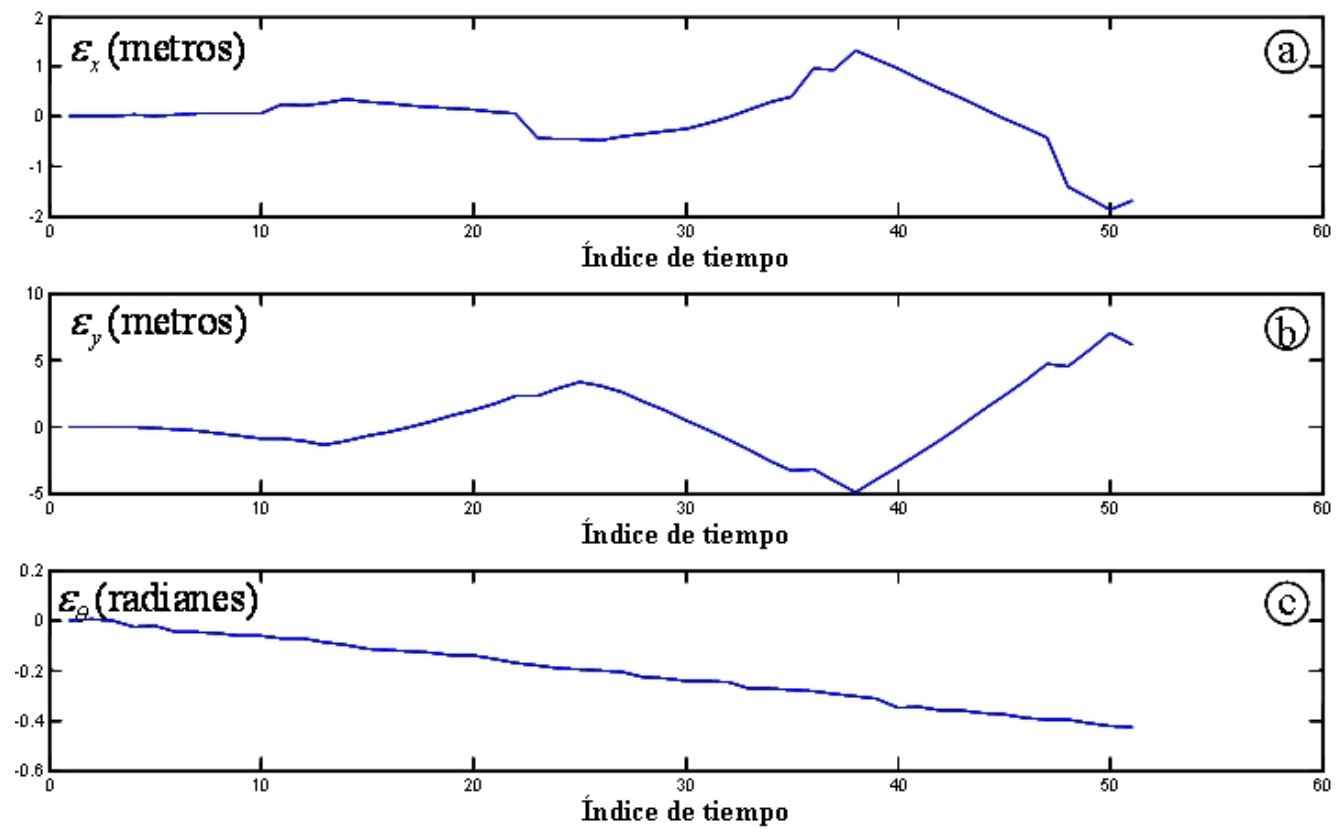

Figura 6.10: Errores de estimación en la posición del robot cuando se usa sólo los datos de odometría. a) Error en el eje $x$. b) Error en el eje $y$. c) Error en el ángulo de acimut $\mathrm{u}$ orientación del robot.

Tabla 6.4: Comparación del error RMS al final del trayecto de 140 metros programado en el robot.

\begin{tabular}{|c|c|c|}
\hline & Sólo odometría & Localización EKF \\
\hline \hline$\varepsilon_{x}$ & $0.631 \mathrm{~m}$ & $0.035 \mathrm{~m}$ \\
\hline$\varepsilon_{y}$ & $2.606 \mathrm{~m}$ & $0.053 \mathrm{~m}$ \\
\hline$\varepsilon_{\theta}$ & $14.06^{\circ}$ & $0.85^{\circ}$ \\
\hline
\end{tabular}



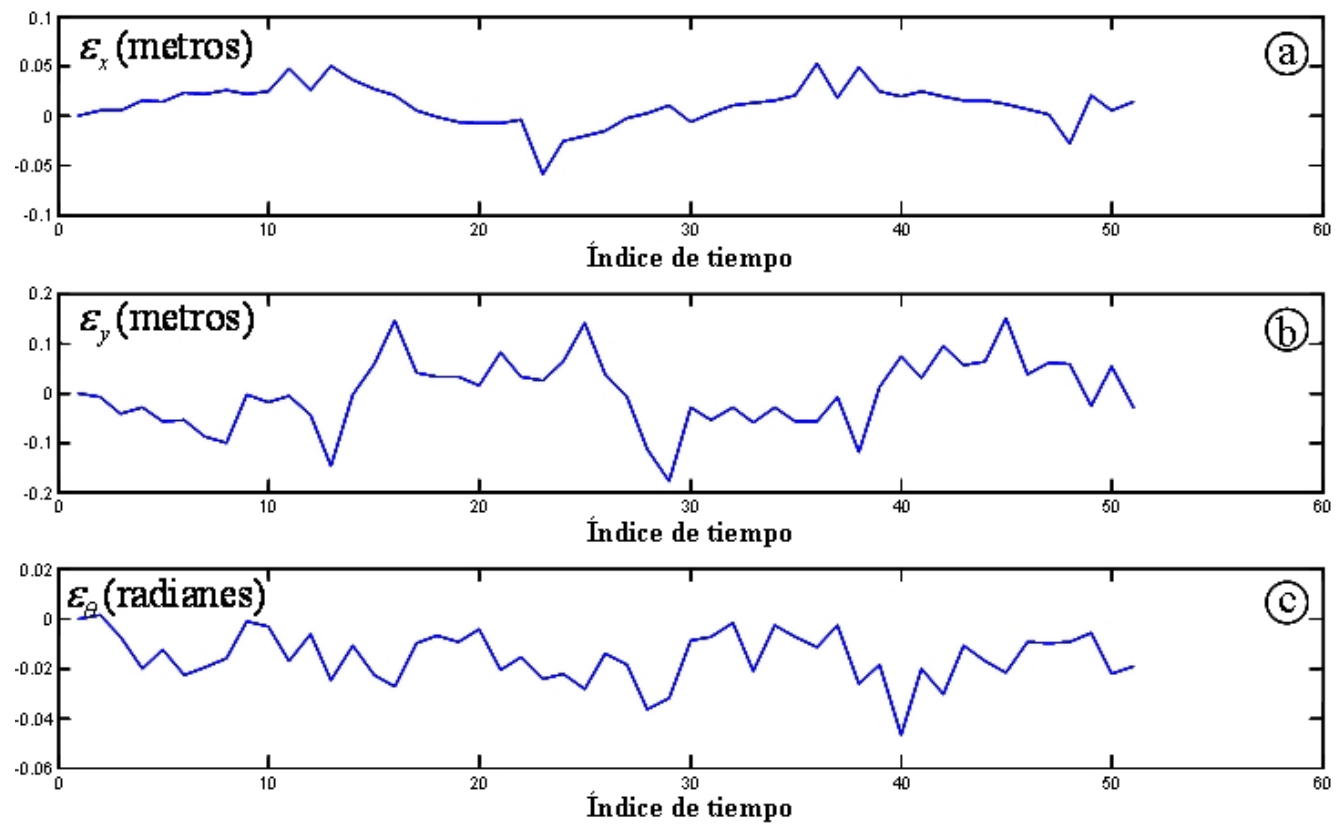

Figura 6.11: Errores de estimación en la posición del robot cuando se fusiona la información de la localización de las esquinas y la información odométrica usando el Filtro Extendido de Kalman. a) Error en el eje $x$. b) Error en el eje $y$. c) Error en el ángulo de acimut u orientación del robot. 


\subsection{Localización basada en mapas de líneas usando un SONAR y el EKF}

En robótica móvil, la auto localización es el proceso mediante el cual un robot móvil estima su posición respecto a un sistema de referencia fijo. Para que un robot se auto localice de manera absoluta, usualmente tiene que utilizar referencias externas captadas mediante sensores de ultrasonidos, de infrarrojos, láser, cámaras de vídeo, entre otros. Un buen número de algoritmos de localización, basados en mapas, han sido propuestos para resolver el problema de la localización absoluta. La tendencia actual es a usar algoritmos basados en mapas de características que incluyen líneas y puntos como hitos o landmark[72, 83]. Así, hay varias propuestas de localización basada en mapas de líneas, que generalmente requieren de sensores que suministren datos de alta densidad, al igual que requieren de algoritmos de extracción de características que sean muy precisos[33, 175]. De esta manera, el sensor que actualmente es el usado con más frecuencia en los procesos de localización basado en mapas de líneas, es el sensor de láser[168, 8, 43].

Por otra parte, los sistemas de localización basados en hitos extraídos con sensores de ultrasonido, son menos comunes. Esto se debe a que este tipo de sensor es poco preciso en los datos angulares que ofrece. Esto último dificulta la extracción, clasificación, localización y la asociación de datos de los objetos que conforman el entorno[112, 205, 97]. No obstante, en esta sección se desarrolla una metodología que fusiona los datos suministrados por los sensores de odometría, con los datos suministrados por un SONAR rotativo, para estimar la posición de un vehículo móvil haciendo uso de un Filtro de Kalman Extendido o EKF.

\subsubsection{Predicción de la posición del vehículo}

La posición del vehículo se puede predecir basándose en el mejor estimado de su última posición $\hat{\mathbf{x}}_{k-1}^{+} \mathrm{y}$ en la entrada de control actual $\mathbf{u}_{k}$. En el caso de un robot diferencial, como el que se usa en este trabajo de tesis, ya en la sección 6.3.1.1 se derivó el modelo de predicción del estado y de la covarianza del error. Por motivos de claridad, las principales relaciones se repiten a continuación.

$$
\begin{gathered}
\hat{\mathbf{x}}_{k}^{-}=\left[\begin{array}{c}
\hat{x}_{k}^{-} \\
\hat{y}_{k}^{-} \\
\hat{\theta}_{k}^{-}
\end{array}\right]=\left[\begin{array}{c}
\hat{x}_{k-1}^{+}+\Delta S_{k} \cos \left(\hat{\theta}_{k-1}^{+}+\Delta \theta_{k} / 2\right) \\
\hat{y}_{k-1}^{+}+\Delta S_{k} \sin \left(\hat{\theta}_{k-1}^{+}+\Delta \theta_{k} / 2\right) \\
\hat{\theta}_{k-1}^{+}+\Delta \theta_{k}
\end{array}\right] \\
\mathbf{P}_{k}^{-}=\mathbf{J}_{\mathbf{x}} \mathbf{P}_{k-1}^{+} \mathbf{J}_{\mathbf{x}}^{\mathrm{T}}+\mathbf{J}_{\mathbf{u}} \mathbf{U}_{k} \mathbf{J}_{\mathbf{u}}^{\mathrm{T}}
\end{gathered}
$$

En la ecuación $6.44,[x, y, \theta]^{\mathrm{T}}$ es el vector de estado que describe la posición del vehículo, mientras que $\Delta S_{k}$ y $\Delta \theta_{k}$ son el trayecto y giro diferencial del vehículo en el período $[k-1, k]$ (ver figura 6.7$)$. 


\subsubsection{Observación}

En la posición actual (instante $k$ ), el vehículo hace una observación del entorno mediante su SONAR, y entonces basándose en la estrategia para extracción de características descrita en la sección 5.4.2, se obtiene un conjunto de líneas características modeladas en el marco de referencia del sensor según,

$$
\mathbf{Z}_{j}^{\mathrm{S}}=\left\{\mathbf{z}_{j}\right\}_{k}^{\mathrm{S}}=\left\{\left[\rho_{j}, \varphi_{j}\right]^{\mathrm{T}}\right\}_{k}^{\mathrm{S}}
$$

donde el índice $j=1 \ldots N$ representa a cada una de los objetos extraídos. Igualmente, junto a cada una de las líneas extraídas, se modela también su matriz de incertidumbre la cual tiene la forma,

$$
\mathbf{R}_{j}=\left[\begin{array}{cc}
\sigma_{\rho}^{2} & \sigma_{\rho \varphi} \\
\sigma_{\rho \varphi} & \sigma_{\varphi}^{2}
\end{array}\right]_{j}
$$

En la sección 5.3.4 ya se demostró experimentalmente que la matriz de covarianza para la extracción de líneas es constante y aproximadamente igual a

$$
\mathbf{R}=\left[\begin{array}{cc}
0,0009 \mathrm{~m}^{2} & 0 \\
0 & 0,002741 \mathrm{rad}^{2}
\end{array}\right]
$$

\subsubsection{Predicción de las observaciones}

Basándose en un mapa de líneas previamente observadas o cargadas manualmente, y basándose en la posición que se ha estimado que el vehículo adoptará dentro del mapa global, se genera un vector de predicción con las características que se espera puedan ser observadas. No obstante, para reducir los requerimientos de computación que este procedimiento implica, como un primer paso se transforman las líneas observadas en el marco del sensor, al marco global, de acuerdo a las ecuaciones desarrolladas en la sección 5.4.2, y que aquí se repiten para propósitos de claridad. Así vemos que el modelo de representación de líneas, en general depende del estado del vehículo que lleva el sensor $\mathbf{x}_{k}=\left[x_{k}, y_{k}, \theta_{k}\right]^{\mathrm{T}}$ y del vector de medición $\mathbf{z}^{\mathrm{S}}=\langle\rho, \varphi\rangle$.

$$
\begin{aligned}
\hat{\mathbf{m}}_{j}=h\left(\mathbf{x}_{k}, \mathbf{z}_{j}^{\mathbf{S}}\right) & =\left[\begin{array}{l}
r_{j} \\
\alpha_{j}
\end{array}\right] \\
& =\left[\begin{array}{c}
\rho_{j}+x_{k} \cos \left(\varphi_{j}+\theta_{k}\right)+y_{k} \sin \left(\varphi_{j}+\theta_{k}\right) \\
\varphi_{j}+\theta_{k}
\end{array}\right]
\end{aligned}
$$

También, considerando que el error en la posición del vehículo no está correlacionado con el error en la medición, la matriz de covarianza de la línea representada en el marco global puede aproximarse mediante aproximación lineal de la función de representación de acuerdo a,

$$
\mathbf{M}_{j}=\mathbf{J}_{\mathbf{z}} \mathbf{R} \mathbf{J}_{\mathbf{z}}^{\mathrm{T}}+\mathbf{J}_{\mathbf{x}} \mathbf{P}_{k}^{-} \mathbf{J}_{\mathbf{x}}^{\mathrm{T}}=\left[\begin{array}{cc}
\sigma_{r}^{2} & \sigma_{r \alpha} \\
\sigma_{r \alpha} & \sigma_{\alpha}^{2}
\end{array}\right]_{j}
$$




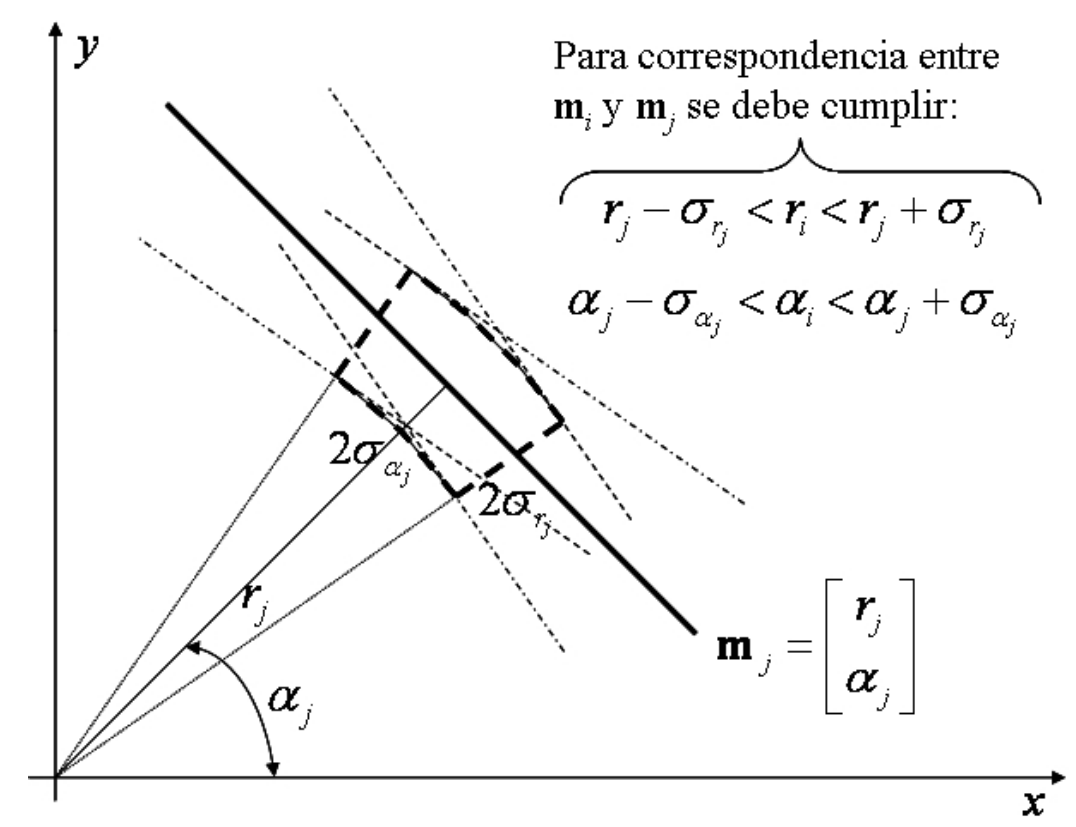

Figura 6.12: Representación de la región de correspondencia entre líneas representadas mediante el modelo Hessiano.

donde $\mathbf{J}_{\mathbf{z}}$ es la matriz Jacobiana respecto al vector de medición $\mathbf{z}^{\mathbf{S}}$, y $\mathbf{J}_{\mathbf{x}}$ es la matriz Jacobiana respecto al estado $\mathbf{x}_{k}$. También, $\mathbf{P}_{k}^{-}$es la matriz de covarianza en el error de estimación del estado del vehículo, la cual ya fue definida en la ecuación 6.45, e igualmente $\mathbf{R}$ es la varianza en el vector de medición, lo que ya fue definido en la ecuación 6.47 y cuyos valores extraídos experimentalmente se muestran en la ecuación 6.48 .

El procedimiento de pre-correspondencia consiste en comprobar cuales de las líneas $\mathbf{m}_{i}$ del mapa existente, se ubican dentro de la elipse de incertidumbre perteneciente a cada una de las líneas $\hat{\mathbf{m}}_{j}$ observadas en este instante $k$. Dado que la elipse de incertidumbre surge de la matriz de covarianzas $\mathbf{M}_{j}$, entoces la condición anterior se puede expresar como(ver figura 6.12)

$$
\mathbf{m}_{i} \sim \hat{\mathbf{m}}_{j} \text { si se cumple que }\left\{\begin{array}{l}
r_{j}-\sigma_{r_{j}}<r_{i}<r_{j}+\sigma_{r_{j}} \\
\varphi_{j}-\sigma_{\varphi_{j}}<\varphi_{i}<\varphi_{j}+\sigma_{\varphi_{j}}
\end{array}\right.
$$

De esta forma, sólo las líneas que satisfacen la condición de pre-correspondencia, son transformadas al marco de referencia del sensor para ejecutar ahí el procedimiento de correspondencia que se describe en la siguiente sección.

\subsubsection{Correspondencia o asociación de datos}

Se refiere al problema conocido también como asociación de datos. En este problema se requiere determinar cual de las líneas observadas se corresponde con cual 


\subsection{LOCALIZACIÓN BASADA EN MAPAS DE LÍNEAS USANDO UN SONAR}

Y EL EKF

de las líneas pertenecientes al mapa global. Aquí se debe identificar la mejor correspondencia entre pares de las siguientes líneas: $\mathbf{z}_{j}$, que son las extraídas u observadas mediante el sensor de ultrasonidos, y $\hat{\mathbf{z}}_{i}$ que son las líneas $\mathbf{m}_{i}$ que perteneciendo al mapa del entorno y que han superado la pre-correspondencia condicionada por la ecuación 6.51, han sido transformadas al marco de referencia de la observación.

Para transformar los objetos del mapa global al marco de referencia del sensor, se utiliza la metodología ya antes descrita en la sección 5.4.2.2. Para efectos de claridad, a continuación se repite la formulación fundamental de esta metodología.

$$
\begin{aligned}
\hat{\mathbf{z}}_{i}=h\left(\mathbf{x}_{k}, \mathbf{m}_{i}\right) & =\left[\begin{array}{l}
\rho_{i} \\
\varphi_{i}
\end{array}\right] \\
{\left[\begin{array}{c}
\rho_{i} \\
\varphi_{i}
\end{array}\right] } & =\left[\begin{array}{c}
r_{i}-x_{k} \cos \alpha_{i}-y_{k} \sin \alpha_{i} \\
\alpha_{i}-\theta_{k}
\end{array}\right]
\end{aligned}
$$

Aquí $\left[x_{k}, y_{k}, \theta_{k}\right]^{\mathrm{T}}$ es la posición del vehículo que ha sido estimada mediante la ecuación 6.44 .

La propagación del segundo momento o covarianza del error se consigue mediante la aproximación en series de Taylor de primer orden, según

$$
\mathbf{M}_{i}^{\mathrm{S}}=\mathbf{J}_{\mathbf{m}} \mathbf{M}_{i} \mathbf{J}_{\mathbf{m}}^{\mathrm{T}}+\mathbf{J}_{\mathbf{x}} \mathbf{P}_{k}^{-} \mathbf{J}_{\mathbf{x}}^{\mathrm{T}}
$$

donde $\mathbf{J}_{\mathbf{m}}$ es la matriz Jacobiana respecto al vector de estado del landmark $\mathbf{m}_{i}$, y $\mathbf{J}_{\mathbf{x}}$ es la matriz Jacobiana respecto al estado del robot $\mathbf{x}_{k}$. También, $\mathbf{P}_{k}^{-}$es la matriz de covarianza en el error de estado del vehículo, la cual ya fue definida en la ecuación 6.45. Igualmente $\mathbf{M}_{i}$ es la matriz de covarianza en el vector de estado del objeto $\mathbf{m}_{i}$, y $\mathbf{M}_{i}^{\mathrm{S}}$ es la covarianza del mismo objeto $\mathbf{m}_{i}$ pero visto en el marco de referencia del sensor. También, los dos Jacobianos necesarios para aproximar la covarianza mediante la ecuación 6.53 , se obtienen a partir de las siguientes ecuaciones

$$
\begin{gathered}
\mathbf{J}_{\mathbf{m}}=\frac{\partial h\left(\mathbf{x}_{k}, \mathbf{m}_{i}\right)}{\partial \mathbf{m}_{i}}=\left[\begin{array}{ccc}
1 & x_{k} \sin \alpha_{i}-y_{k} \cos \alpha_{i} \\
0 & 1
\end{array}\right] \\
\mathbf{J}_{\mathbf{x}}=\frac{\partial h\left(\mathbf{x}_{k}, \mathbf{m}_{i}\right)}{\partial \mathbf{x}_{k}}=\left[\begin{array}{ccc}
-\cos \alpha_{i} & -\sin \alpha_{i} & 0 \\
0 & 0 & -1
\end{array}\right]
\end{gathered}
$$

Así, dado un conjunto de predicciones vistas en el marco sensor, $\mathbf{Z}_{i}^{\mathbf{s}}=\left\{\hat{\mathbf{z}}_{i}\right\}, \mathrm{y}$ un conjunto de observaciones extraídas mediante un SONAR, $\mathbf{Z}_{j}^{\mathbf{s}}=\left\{\mathbf{z}_{j}\right\}$, el objetivo de la asociación de datos es encontrar la observación $\mathbf{z}_{j}$ que mejor se corresponde con la predicción $\hat{\mathbf{z}}_{i}$. Para resolver este problema, uno de los métodos más usados en el ámbito de la localización basada en el EKF, es el conocido como compuertas de validación (Validation Gates). Estas compuertas monitorean los residuales en el vector de observación(innovación) a fin de detectar mediciones inesperadas[150]. El vector de innovación surge cuando se comparan los vectores de observación y predicción según, 


$$
\Delta \mathbf{z}_{j i}=\mathbf{z}_{j}-\hat{\mathbf{z}}_{i}
$$

y la covarianza en la innovación resulta de la ecuación,

$$
\mathbf{S}=\mathbf{R}+\mathbf{M}_{i}^{\mathbf{S}}
$$

Así, para determinar cuando una observación $\mathbf{z}_{j}$ tiene poca probabilidad de corresponder a un objeto $\hat{\mathbf{z}}_{i}$ del mapa conocido, usualmente se calcula la distancia de Mahalanobis según,

$$
\left(\mathbf{z}_{j}-\hat{\mathbf{z}}_{i}\right) \mathbf{S}^{-1}\left(\mathbf{z}_{j}-\hat{\mathbf{z}}_{i}\right)^{\mathrm{T}}=D^{2}
$$

El límite para rechazar la hipótesis de correspondencia entre la observación $\mathbf{z}_{j}$ y la predicción $\hat{\mathbf{z}}_{i}$ se establece en función del valor de la distribución $\chi^{2}(\alpha, n)$, donde $\alpha$ es el nivel de confianza con el que se rechaza la hipótesis de correspondencia (se suele fijar en $95 \%$ ), y $n$ son los grados de libertad(en este caso $n=2)$. Si la distancia calculada en mayor que que el límite fijado por la distribución chi-cuadrado $\left(D^{2}>\chi^{2}(\alpha, n)\right)$, entonces se rechaza la hipótesis de correspondencia.

Así, el proceso de correspondencia que se usa en esta tesis para el algoritmo de localización usando el EKF, consiste en determinar cual es el objeto del mapa que presenta la menor distancia de Mahalanobis respecto a una observación en particular. De aquí que si $C_{i j}=i$ es la función que indica la correspondencia entre la observación $\mathbf{z}_{j}$ y la predicción $\hat{\mathbf{z}}_{i}$, entonces el índice de la línea perteneciente al mapa que maximiza la verosimilitud del modelo es,

$$
i=\arg \min \left(D^{2}\right)
$$

\subsubsection{Corrección del estado}

Mediante el EKF se calcula el mejor estimado de la posición del vehículo basándose en la predicción de la posición $\hat{\mathbf{x}}_{k}^{-}$y en el vector de innovación $\Delta \mathbf{z}_{j i}$. La formulación utilizada en bien conocida[150], por lo que aquí sólo se presentan las ecuaciones básicas. El eje básico de esta formulación lo constituye lo que se conoce como la Ganancia de Kalman, la cual se obtiene a partir de la covarianza en el estado y covarianza en la innovación de acuerdo a,

$$
\mathbf{K}_{k}=\mathbf{P}_{k}^{-} \cdot \mathbf{J}_{\mathbf{x}} \cdot \mathbf{S}_{k}^{-1}
$$

A partir de esta ganancia, que se calcula a cada instante $k$, se corrige el estado estimado previo $\hat{\mathbf{x}}_{k}^{-}$, y la covarianza del error del estado estimado previo $\mathbf{P}_{k}^{-}$, mediante el siguiente par de ecuaciones:

$$
\begin{array}{r}
\hat{\mathbf{x}}_{k}^{+}=\hat{\mathbf{x}}_{k}^{-}+\mathbf{K}_{k}\left(\mathbf{z}_{j}-\hat{\mathbf{z}}_{i}\right) \\
\mathbf{P}_{k}^{+}=\mathbf{P}_{k}^{-}-\mathbf{K}_{k} \cdot \mathbf{S}_{k} \cdot \mathbf{K}_{k}^{\mathrm{T}}
\end{array}
$$




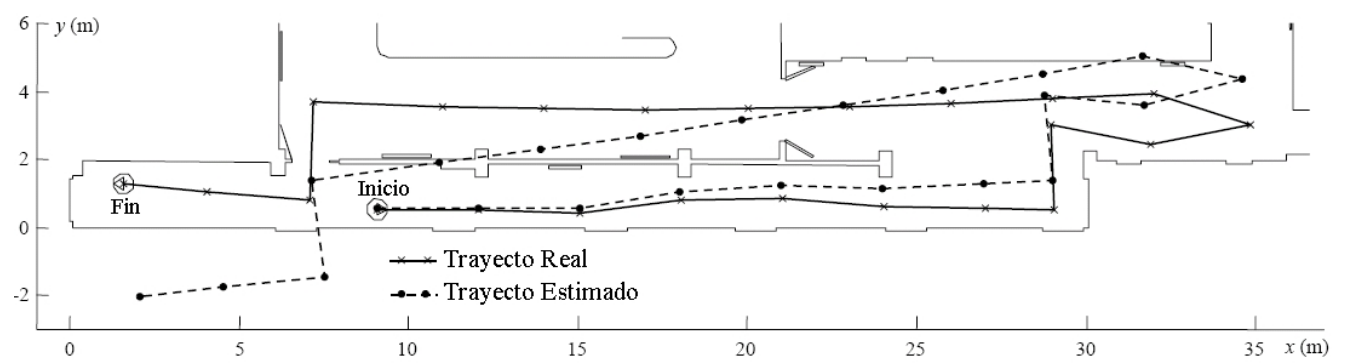

Figura 6.13: Aspecto del pasillo y trayecto experimental utilizado en las pruebas de localización, basada en mapas de líneas, realizadas con el robot YAIR.

\subsubsection{Experimentos}

Los experimentos se realizaron utilizando una plataforma robótica experimental de tracción diferencial llamada YAIR - Yet Another Intelligent Robot[25]. Con el objetivo de demostrar la utilidad de SONAR rotativo y de los algoritmos de extracción, asociación de datos y localización aquí propuesto, se programó el robot para que se moviera a través de un largo de un pasillo como el que se muestra en la figura 6.13.

A fin de completar un trayecto de aproximadamente 150 metros, el robot realizó dos vueltas moviéndose en tramos de 3 metros, al final de los cuales se detenía para observar el entorno mediante su SONAR. A cada parada del robot, se registró manualmente su posición real a fin de hacer comparaciones futuras con la posición que el robot puede estimar mediante su sistema de odometría, así como también para compararla con la posición que puede estimar con el sistema de localización basado en el filtro de Kalman que se ha propuesto en esta sección.

\subsubsection{Resultados y discusión}

La figura 6.13 muestra el trayecto estimado cuando el vehículo usa solamente su sistema de localización odométrico. Tal como se esperaba, la incertidumbre y el error en la localización crecen sin límites, sobretodo con mayor énfasis en la dirección transversa al movimiento. Por otra parte, la figura 6.14 muestra el mismo trayecto de prueba que el mostrado en la figura 6.13, pero esta vez usando el algoritmo de localización basado en mapas de líneas y en el algoritmo del EKF. En este caso el sistema de localización fue capaz de mejorar la exactitud en la estimación de la posición, y de mantener acotado el error de estimación. Además, la figura 6.14 muestra también que el trayecto estimado es justamente igual al trayecto real. Este hecho prueba que el robot está plenamente autolocalizado durante todo el trayecto ensayado.

Igualmente, en la figura 6.14, las líneas detectadas son representadas mediante trazos oscuros. A partir de esta figura se puede concluir que el corredor es un escenario adecuado para la localización basada en líneas, ya que existen muchas estructuras planas que le permiten al robot relacionarlas con las líneas del mapa que usa en su 


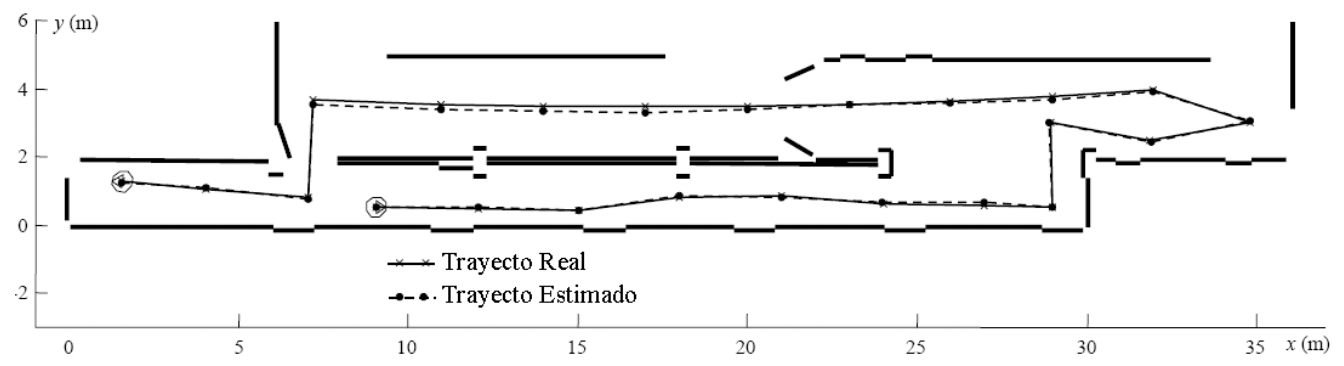

Figura 6.14: Corrección de la posición basándose en mapas de líneas y usando el Filtro de Kalman Extendido.

Tabla 6.5: Comparación del error de estimación de la posición al final del trayecto, obtenido usando dos estrategias de localización: Sólo odometría y localización basada en EKF.

\begin{tabular}{|c|c|c|}
\hline & Sólo con Odomatría & Localización EKF \\
\hline \hline$\varepsilon_{x}$ & $0.631 \mathrm{~m}$ & $0.055 \mathrm{~m}$ \\
\hline$\varepsilon_{y}$ & $2.606 \mathrm{~m}$ & $0.094 \mathrm{~m}$ \\
\hline$\varepsilon_{\theta}$ & $14.06^{\circ}$ & $1.05^{\circ}$ \\
\hline
\end{tabular}

sistema de autolocalización.

En la figura 6.15, se ha graficado el error de estimación del vector de estado $[x, y, \theta]^{\mathrm{T}}$ para dos casos: Localización basada en mapas de líneas y uso del EKF, y localización usando sólo odometría. Comparando ambas gráficas, se ve que la primera técnica reduce significativamente el error RMS en la estimación de la posición. También, la comparación de la exactitud de estimación del estado, al final del trayecto, es tabulado en la tabla 6.5. Ahí se puede ver que el error permaneció en el rango de los pocos centímetros.

\subsection{Localización y mapeado simultáneo basado en Datos de ultrasonido}

El problema de localización y mapeado simultáneo, conocido como SLAM - Simultaneous Localization And Mapping [58], busca darle respuesta a la posibilidad de que un robot que sea puesto en un entorno desconocido pueda construir un mapa del mismo, y que al mismo tiempo use este mapa para deducir o estimar su posición dentro del entorno, a medida que lo explora. Para describir mejor este problema, considérese la figura 6.16. En ella se definen las siguientes cantidades o variables:

$\mathbf{x}_{k}$ El vector de estado que describe la localización y la orientación del vehículo en el momento actual - $k$.

$\mathbf{u}_{k}$ El vector de control, aplicado en el instante $k-1$, para llevar el vehículo hasta el 

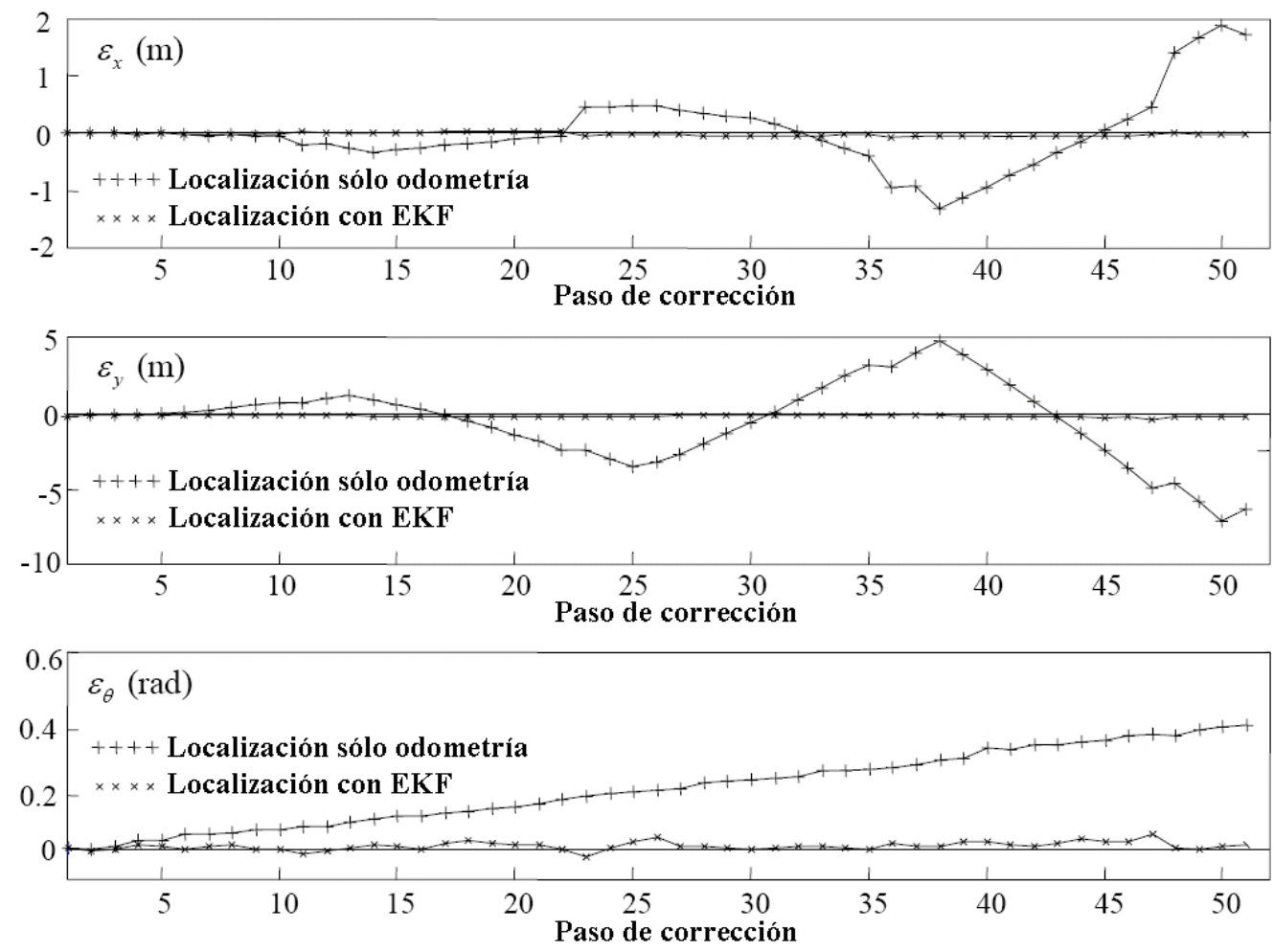

Figura 6.15: Comparación del error en la estimación del estado. 


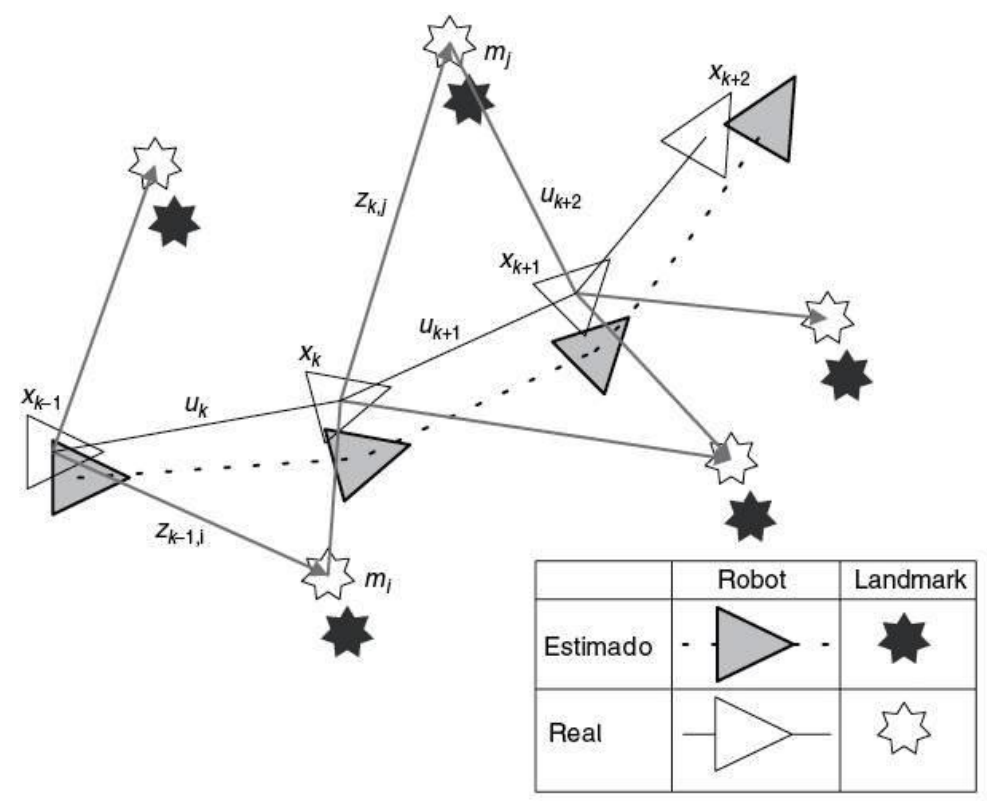

Figura 6.16: El problema básico del SLAM se refiere a la estimación simultánea de la posición del móvil y de la localizaciones de los objetos del mapas sin el previo conocimiento de la localización de los mismos. Este es un problema difícil de resolver debido a que las localización de los objetos del mapa no se conocen con exactitud, ya que son estimadas mediante sensores que están instalados en el móvil, y al mismo tiempo estos objetos son usados como landmark para la estimación de la posición del móvil[63].

estado $\mathbf{x}_{k}$ definido en el instante $k$.

$\mathbf{m}_{i}$ Un vector que describe la localización del i-esimo hito o landmark cuya localización real se supone que es invariante en el tiempo.

$\mathbf{z}_{k}$ El vector de observación actual, el cual usualmente está referido al sistema de coordenadas del vehículo.

Basado en lo anterior, el problema de SLAM consiste en determinar la distribución de probabilidad $p\left(\mathbf{x}_{k}, \mathbf{m} \mid \mathbf{Z}_{0: k}, \mathbf{U}_{0: k}, \mathbf{x}_{0}\right)$ para todo instante $k$. Aquí $\mathbf{m}$ es el conjunto de hitos que conforman el mapa del entorno, $\mathbf{Z}_{0: k}$ es el conjunto de observaciones realizadas hasta el instante $k, \mathbf{U}_{0: k}$ es la historia de las entradas de control aplicadas al vehículo, y $\mathbf{x}_{0}$ es la posición inicial del vehículo.

Este es un problema que en gran medida ha sido resuelto, y del que existe abundante literatura disponible. Hugh Durrant-White y Tim Bailey, en la primera parte de un tutorial sobre el tema de SLAM[63] introducen el problema como uno de estimación Bayesiana, y describen la solución a través de dos métodos: usando el Filtro de Kalman Extendido(EKF-SLAM) y usando un filtro de partículas tipo RaoBlackwellized(Fast-SLAM). En una segunda parte, estos mismos autores describen 
los principales problemas en la computación, convergencia y asociación de datos relacionados al SLAM[11]. Otro trabajo que hace una interesante revisión del estado de arte y de otros temas relacionados a la solución del problema, propiedades y variantes del algoritmo de SLAM, es la tesis doctoral de Diego Rodríguez-Losada[174].

La solución adoptada en esta tesis se basa en la metodología probabilista que usa al Filtro de Kalman Extendido como estimador recursivo óptimo del estado del vehículo. La localización básica del mismo se logra a través del sistema de odometría acoplada a las ruedas motrices, mientras que la corrección del estado del robot se basa en las observaciones de landmark naturales tipo puntuales, hechas mediante un SONAR. Uno de los trabajos más importante realizados en esta dirección, se llevo a cabo en la Universidad de Zaragoza por J. Tardós y sus colaboradores[189]. En este trabajo los investigadores desarrollan métodos para la fusión de datos sensoriales dispersos obtenidos con un SONAR, con los datos provenientes de sistemas odométricos. Este mecanismo de fusión lo incorporan a un método de localización y mapeado simultáneo basado sólo en medidas de distancia.

\subsubsection{El algoritmo de SLAM basado en el Filtro de Kalman Exten- dido}

Esta metodología surgió a partir de la contribución seminal de Smith, Self y Cheeseman [184, 185], quienes introdujeron el marco teórico estadístico para resolver el problema de localización y mapeado simultáneo. La aplicación del EKF al problema de SLAM, permite que un vehículo que inicie la navegación en un punto conocido, de un entorno desconocido, pueda construir un mapa de este entorno con el cual podrá autolocalizarse.

\subsubsection{El vector de estado}

El Filtro de Kalman Extendido estima el vector de estado que contiene tanto el estado del vehículo $\mathbf{x}_{\mathrm{V}}$ como el de los objetos $\mathbf{m}_{i}$ que componen el mapa goblal. De esta manera, para modelar el problema de SLAM hay que definir un vector de estado aumentado de acuerdo a

$$
\mathbf{x}_{k}=\left[\begin{array}{llll}
\mathbf{x}_{\mathrm{V}, k}^{\mathrm{T}} & \mathbf{m}_{1, k}^{\mathrm{T}} & \cdots & \mathbf{m}_{\mathrm{N}, k}^{\mathrm{T}}
\end{array}\right]^{\mathrm{T}}
$$

donde el estado del vehículo tiene la forma $\mathbf{x}_{\mathrm{V}, k}=[x, y, \theta]_{k}^{\mathrm{T}}$, mientras que el estado de los objetos del mapa tiene la forma $\mathbf{m}_{i, k}=\left[x_{i}, y_{i}\right]_{k}^{\mathrm{T}}$, con $i=1 \cdots \mathrm{N}$. Aquí $\mathrm{N}$ representa el número de objetos del mapa, y $k$ representa el índice o instante de tiempo discreto donde se evalúa el estado.

Asumiendo que el ruido que afecta al estado puede ser modelado como una distribución Normal multivariante de media igual a cero y varianza $\mathbf{P}$, entonces el estimado del estado y su covarianza se expresa como

$$
\hat{\mathbf{x}}_{k}=\left[\begin{array}{llll}
\hat{\mathbf{x}}_{\mathrm{V}, k}^{\mathrm{T}} & \hat{\mathbf{m}}_{1, k}^{\mathrm{T}} & \cdots & \hat{\mathbf{m}}_{\mathrm{N}, k}^{\mathrm{T}}
\end{array}\right]^{\mathrm{T}}
$$




$$
\mathbf{P}_{k}=\left[\begin{array}{cccc}
\mathbf{P}_{\mathrm{Vv}, k} & \mathbf{P}_{\mathrm{V} 1, k} & \cdots & \mathbf{P}_{\mathrm{vN}, k} \\
\mathbf{P}_{1 \mathrm{v}, k} & \mathbf{P}_{11, k} & \cdots & \mathbf{P}_{1 \mathrm{~N}, k} \\
\vdots & \vdots & \ddots & \vdots \\
\mathbf{P}_{\mathrm{NV}, k} & \mathbf{P}_{\mathrm{N} 1, k} & \cdots & \mathbf{P}_{\mathrm{NN}, k}
\end{array}\right]
$$

Aquí, $\mathbf{P}_{\mathrm{vv}, k}$ y $\mathbf{P}_{i i, k}$ para $i=1 \cdots \mathrm{N}$, son la varianza de el error en el estimado del estado del vehículo y la varianza en el error de estimación del estado del i-esimo objeto del mapa, respectivamente. También, el término $\mathbf{P}_{\mathrm{V} i, k}=\mathbf{P}_{i \mathrm{v}, k}$ corresponde a la covarianza entre el error en el estimado del estado del vehículo y el error en el estimado del estado del objeto $\mathbf{m}_{i, k}$ del mapa. Así mismo, el término $\mathbf{P}_{i j, k}=\mathbf{P}_{j i, k}$ representa la covarianza entre el error en la estimación del estado del objeto $\mathbf{m}_{i, k} \mathbf{y}$ el error en el estimado del estado del objeto $\mathbf{m}_{j, k}$ del mapa.

\subsubsection{Predicción del estado}

La predicción de la evolución del estado aumentado está formado a su vez por dos modelos: El modelo de movimiento del vehículo y el modelo de evolución de cada uno de los objetos del mapa.

La evolución del estado del vehículo, desde el instante $k-1$ hasta el instante $k$, puede expresarse como

$$
\mathbf{x}_{\mathrm{V}, k} \cong f\left(\mathbf{x}_{\mathrm{V}, k-1}, \mathbf{u}_{k}\right)+\mathbf{w}_{k}
$$

donde $\mathbf{x}_{\mathrm{V}, k-1}$ representa el estado del vehículo en el instante inmediatamente anterior al estado actual, $\mathbf{x}_{\mathrm{V}, k}$ representa el estado del vehículo en el instante actual - $k$-, $\mathbf{u}_{k}$ es la entrada de excitación en este mismo instante, $\mathrm{y} \mathbf{w}_{k}$ es el ruido que afecta el sistema y cuya distribución se asume que es gausiana, de media igual a cero y varianza igual a $\mathbf{Q}$.

Por otra parte, asumiendo que los objetos del mapa son estáticos y no evolucionan en el tiempo, entonces

$$
\mathbf{m}_{i, k}=\mathbf{m}_{i, k-1}
$$

con $i=1,2, \cdots$ N. Con la ecuación (6.66) y (6.67), el modelo del estado aumentado se puede expresar como

$$
\mathbf{x}_{k}=\left[\begin{array}{llll}
\mathbf{x}_{\mathrm{V}, k}^{\mathrm{T}} & \mathbf{m}_{1, k}^{\mathrm{T}} & \cdots & \mathbf{m}_{\mathrm{N}, k}^{\mathrm{T}}
\end{array}\right]^{\mathrm{T}}
$$

Luego, se puede mostrar que la predicción del estado estimado, y su covarianza se puede expresar según, 


$$
\begin{gathered}
\hat{\mathbf{x}}_{k}^{-} \cong f\left(\hat{\mathbf{x}}_{k-1}, \mathbf{u}_{k}\right) \\
\cong\left[\begin{array}{c}
f\left(\hat{\mathbf{x}}_{\mathrm{V}, k-1}, \mathbf{u}_{k}\right) \\
\hat{\mathbf{m}}_{1, k} \\
\vdots \\
\hat{\mathbf{m}}_{\mathrm{N}, k}
\end{array}\right] \\
\mathbf{P}_{k}^{-}=\left[\begin{array}{cccc}
\mathbf{J}_{\mathrm{V}} \mathbf{P}_{\mathrm{Vv}, k-1} \mathbf{J}_{\mathrm{V}}^{\mathrm{T}}+\mathbf{J}_{\mathbf{u}} \mathbf{Q}_{k} \mathbf{J}_{\mathbf{u}}^{\mathrm{T}} & \mathbf{J}_{\mathrm{V}} \mathbf{P}_{\mathrm{V} 1, k-1} & \cdots & \mathbf{J}_{\mathrm{V}} \mathbf{P}_{\mathrm{VN}, k-1} \\
\left(\mathbf{P}_{1 \mathrm{~V}, k-1} \mathbf{J}_{\mathrm{V}}\right)^{\mathrm{T}} & \mathbf{P}_{11, k-1} & \cdots & \mathbf{P}_{1 \mathrm{~N}, k-1} \\
\vdots & \vdots & \ddots & \vdots \\
\left(\mathbf{P}_{\mathrm{NV}, k-1} \mathbf{J}_{\mathrm{V}}\right)^{\mathrm{T}} & \mathbf{P}_{\mathrm{N} 1, k-1} & \cdots & \mathbf{P}_{\mathrm{NN}, k-1}
\end{array}\right]
\end{gathered}
$$

donde $\hat{\mathbf{x}}_{k}^{-}$es la estimación a priori del estado aumentado, es decir el estado predicho, y $\mathbf{P}_{k}^{-}$es la predicción de la covarianza del error en dicha estimación. También, $\mathbf{J}_{\mathrm{V}}$ corresponde al Jacobiano del modelo del vehículo respecto al estado del mismo $\left(\mathbf{x}_{\mathrm{V}, k}\right)$ en el instante $k$, y $\mathbf{J}_{\mathbf{u}}$ es el Jacobiano del modelo del vehículo respecto al vector de excitación de entrada $\mathbf{u}_{k}$ en el instante $k$. Como se sabe, estas funciones Jacobianas se calculan según,

$$
\begin{aligned}
& \mathbf{J}_{\mathrm{V}, k}=\left.\frac{\partial f\left(\hat{\mathbf{x}}_{k-1}, \mathbf{u}_{k}\right)}{\partial \mathbf{x}_{\mathrm{V}}}\right|_{\hat{\mathbf{x}}_{k}^{-}, \mathbf{u}_{k}} \\
& \mathbf{J}_{\mathbf{u}, k}=\left.\frac{\partial f\left(\hat{\mathbf{x}}_{k-1}, \mathbf{u}_{k}\right)}{\partial \mathbf{u}}\right|_{\hat{\mathbf{x}}_{k}^{-}, \mathbf{u}_{k}}
\end{aligned}
$$

\subsubsection{La observación}

El modelo de observación también tiene influencia sobre el vector de estado aumentado. En general, la función de observación se expresa como

$$
\mathbf{z}_{k}^{\text {obsv }}=h\left(\mathbf{x}_{k}\right)+\mathbf{v}_{k}=h\left(\mathbf{x}_{\mathrm{V}, k}, \mathbf{m}_{i, k}\right)+\mathbf{v}_{k}
$$

donde $\mathbf{v}_{k}$ es el ruido que introduce el proceso sensorial, y el cual se asume que se distribuye de forma Gausiana con media igual a cero y varianza igual a $\mathbf{R}$. Igualmente, $\mathbf{x}_{\mathrm{V}, k}$ es la posición del vehículo en el instante $k, \mathrm{y} \mathbf{m}_{i, k}$ es el $i$-esimo objeto del mapa que es observado en el mismo instante $k$. También, debido al carácter no lineal de la función de observación, para propagar el ruido a través de la mencionada función, lo usual es utilizar la aproximación lineal mediante series de Taylor de primer orden, para lo cual en general hará falta calcular el Jacobiano de la función de observación respecto al estado aumentado según,

$$
\mathbf{J}_{\mathbf{x}, k}=\left.\frac{\partial h\left(\mathbf{x}_{\mathrm{V}, k}, \mathbf{m}_{i, k}\right)}{\partial \mathbf{x}}\right|_{\mathbf{x}_{\mathrm{v}, k}, \mathbf{m}_{i, k}}=\left.\left[\begin{array}{lllll}
\mathbf{J}_{\mathbf{v m}, k} & \mathbf{J}_{\mathbf{m}_{1}, k} & \mathbf{J}_{\mathbf{m}_{2}, k} & \cdots & \mathbf{J}_{\mathbf{m}_{\mathbb{N}}, k}
\end{array}\right]\right|_{\mathbf{x}_{\mathbf{v}, k}, \mathbf{m}_{i, k}}
$$




\section{CAPÍTULO 6. CONSTRUCCIÓN DE MAPAS Y LOCALIZACIÓN ESTOCÁSTICA}

donde $\mathbf{J}_{\mathbf{x}_{\mathrm{V}}, k}$ es el Jacobiano de la función de observación (ecuación 6.73) respecto al estado del vehículo, y $\mathbf{J}_{\mathbf{m}_{i}, k}$ va a representar el Jacobiano de la función de observación respecto al estado estimado de cada uno de los objetos del mapa. Estos Jacobianos se calculan de acuerdo a,

$$
\begin{aligned}
& \mathbf{J}_{\mathbf{v m}, k}=\left.\frac{\partial h\left(\mathbf{x}_{\mathrm{V}, k}, \mathbf{m}_{i, k}\right)}{\partial \mathbf{x}_{\mathrm{V}}}\right|_{\mathbf{x}_{\mathrm{V}, k}, \mathbf{m}_{i, k}} \\
& \mathbf{J}_{\mathbf{m}_{i}, k}=\left.\frac{\partial h\left(\mathbf{x}_{\mathrm{V}, k}, \mathbf{m}_{i, k}\right)}{\partial \mathbf{m}_{i}}\right|_{\mathbf{x}_{\mathrm{V}, k}, \mathbf{m}_{i, k}}
\end{aligned}
$$

Ahora bien, considerando la observación simple de los objetos del mapa, es decir que existe capacidad para observar sólo un objeto a la vez, entonces el Jacobiano de la observación (ecuación 6.74) se expresaría como

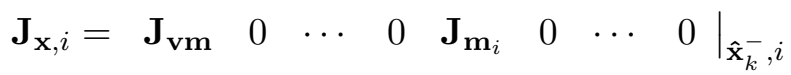

lo que muestra que todos los Jacobianos son cero excepto el que corresponde al vehículo y el que corresponde al objeto del mapa $\left(\mathbf{m}_{i}\right)$ que está siendo observado en este paso del algoritmo.

\subsubsection{La corrección del estado}

El estado aumentado $\mathbf{x}_{k}$ y su covarianza $\mathbf{P}_{k}$, permiten mantener la correlación entre el estimado del vehículo y los objetos del mapa, y entre los distintos objetos del mapa entre sí. Por esto, si en el instante $k$ y mediante un sensor externoceptivo, se puede detectar, clasificar y extraer las características de un objeto que ya esté formando parte del mapa que modela el entorno, entonces en general se podrá corregir el estimado del estado del vehículo y el de cada uno de los objetos que forman parte de este mapa.

Asumiendo que se cuenta con un mecanismo de asociación de datos que es perfecto, entonces la observación de un objeto conocido genera el siguiente vector de innovación,

$$
\Delta \mathbf{z}_{k}=\mathbf{z}_{j, k}-\mathbf{z}_{i}^{\text {obsv }}
$$

donde $\mathbf{z}_{j, k}$ es la medición u observación real hecha con el sensor en el instante $k, \mathrm{y}$

$\mathbf{z}_{i, k}^{\text {obsv }}$ es la predicción del objeto del mapa que se debería medir, dado que el vehículo se encuentra en el estado $\mathbf{x}_{k}$.

De acuerdo a la teoría de la propagación del error, la matriz de varianzas-covarianzas en la innovación corresponde a

$$
\mathbf{Z}_{j, k}=\mathbf{J}_{\mathbf{x}, i} \mathbf{P}_{k}^{-} \mathbf{J}_{\mathbf{x}, i}^{\mathrm{T}}+\mathbf{R}_{j}
$$

donde $\mathbf{P}_{k}^{-}$tiene la forma y se obtiene según lo que se muestra en la ecuación (6.70), y $\mathbf{J}_{\mathbf{x}, i}$ ya se definió en la ecuación (6.77). También, $\mathbf{R}_{j}$ es la matriz de varianzascovarianzas o el ruido introducido después de observar, con un sensor externoceptivo, el objeto $\mathbf{z}_{j}$ del entorno. 
Luego, el factor que pondera la cantidad de corrección que de manera óptima se debe agregar al estado estimado se conoce como la Ganancia de Kalman, y la misma puede ser calculada utilizando la siguiente ecuación

$$
\mathbf{K}_{k}=\mathbf{P}_{k}^{-} \mathbf{J}_{\mathbf{x}, i}^{\mathrm{T}} \mathbf{Z}_{j, k}^{-1}
$$

Con esta ganancia, el estado aumentado y su covarianza, pueden ser corregidos o actualizados de acuerdo a las siguientes ecuaciones

$$
\begin{gathered}
\hat{\mathbf{x}}_{k}^{+}=\hat{\mathbf{x}}_{k}^{-}+\mathbf{K}_{k}\left(\mathbf{z}_{j, k}-\mathbf{z}_{i}^{\text {obsv }}\right) \\
\mathbf{P}_{k}^{+}=\mathbf{P}_{k}^{-}-\mathbf{K}_{k} \mathbf{Z}_{j, k} \mathbf{K}_{k}^{\mathrm{T}}
\end{gathered}
$$

donde $\hat{\mathbf{x}}_{k}^{-}$es la predicción del estado antes de hacer la observación del objeto conocido, $\hat{\mathbf{x}}_{k}^{+}$es la estimación a posteriori del estado aumentado, es decir el estado corregido, y $\mathbf{P}_{k}^{+}$es la actualización de la matriz de varianzas-covarianzas del error en la estimación del estado aumentado.

\subsubsection{Integración de objetos al mapa}

Como se estableció en la sección 6.5.1.1, el vector de estado aumentado estará también formado por un conjunto de objetos, cuya localización, orientación, y geometría (respecto a un sistema de referencias fijo) pueden ser descritas por un conjunto de parámetros que se agrupan bajo el vector de características $\mathbf{m}_{i}$, con $i=1,2, \cdots \mathrm{N}$. Este vector de características surge a partir del modelo físico del sensor, y por ello lo llamamos el modelo de representación, el cual se puede expresar como

$$
\mathbf{m}_{i, k}^{\mathrm{rep}}=h\left(\mathbf{x}_{\mathrm{V}, k}, \mathbf{z}_{i, k}\right)+\mathbf{v}_{k}
$$

donde $\mathbf{v}_{k}$ es el ruido que introduce el proceso sensorial, y el cual se asume que se distribuye de forma Gausiana con media igual a cero y varianza igual a $\mathbf{R}$. Igualmente, $\mathbf{x}_{\mathrm{V}, k}$ es la posición del vehículo en el instante $k, \mathrm{y} \mathbf{z}_{i, k}$ es el $i$-esimo objeto del entorno que es observado en el mismo instante $k$. También, debido al carácter no lineal de la función de representación, para propagar el ruido a través de la mencionada función, lo usual es utilizar la aproximación lineal mediante series de Taylor de primer orden, para lo cual en general hará falta calcular el Jacobiano de la función de representación según,

$$
\begin{aligned}
& \mathbf{J}_{\mathbf{v} \mathbf{z}}=\left.\frac{\partial h\left(\mathbf{x}_{\mathrm{V}, k}, \mathbf{z}_{i, k}\right)}{\partial \mathbf{x}_{\mathrm{V}}}\right|_{\mathbf{x}_{\mathrm{V}, k}, \mathbf{z}_{i, k}} \\
& \mathbf{J}_{\mathbf{z}}=\left.\frac{\partial h\left(\mathbf{x}_{\mathrm{V}, k}, \mathbf{z}_{i, k}\right)}{\partial \mathbf{z}}\right|_{\mathbf{x}_{\mathrm{V}, k}, \mathbf{z}_{i, k}}
\end{aligned}
$$

El conjunto de los $\mathrm{N}$ vectores de características $\mathbf{m}_{i}$ que son extraídos del entorno, a su vez se agrupan en una mayor estructura que se conoce como mapa del entorno, y que puede ser modelado como el vector de estados

$$
\mathbf{x}_{\mathrm{M}}=\left[\begin{array}{llll}
\mathbf{m}_{1}^{\mathrm{T}} & \mathbf{m}_{2}^{\mathrm{T}} & \cdots & \mathbf{m}_{\mathrm{N}}^{\mathrm{T}}
\end{array}\right]^{\mathrm{T}}
$$


Con esto, el vector de estado aumentado puede expresar como

$$
\mathbf{x}_{k}=\left[\begin{array}{cc}
\mathbf{x}_{\mathrm{V}, k} ; & \mathbf{x}_{\mathrm{M}}
\end{array}\right]^{\mathrm{T}}
$$

Ahora bien, inicialmente el mapa $\mathbf{x}_{\mathrm{M}}$ estará vacío, pero a medida que se vayan observando objetos en el entorno, hay que ir agregándolos al mapa. Con esta operación, el vector de estado aumentado $\mathbf{x}_{k}$ también irá cambiando de dimensiones de acuerdo a la cantidad de objetos que se incorporen al mapa del entorno. Entonces, el vector de estado aumentado irá creciendo de la forma,

$$
\hat{\mathbf{x}}_{k}^{-}=\left[\begin{array}{c}
\hat{\mathbf{x}}_{\mathrm{v}, k}^{-} \\
\mathbf{m}_{1, k} \\
\vdots \\
\mathbf{m}_{\mathrm{N}, k}
\end{array}\right]
$$

Por otra parte, también hay que tomar en cuenta que cuando se agrega un nuevo objeto al mapa, y con ello al estado aumentado $\mathbf{x}_{k}$, la matriz de varianzas-covarianzas $\mathbf{P}_{k}$ del estado, también cambia. P. Newman[156], estudió esta problemática y estableció la siguiente estructura para la actualización de esta covarianza:

$$
\mathbf{P}_{k}^{+}=\mathbf{G}\left[\begin{array}{cc}
\mathbf{P}_{k}^{-} & 0 \\
0 & \mathbf{R}_{i}
\end{array}\right] \mathbf{G}^{\mathrm{T}}
$$

donde $\mathbf{P}_{k}^{-}$es la predicción de la covarianza del error en la predicción del estado, $\mathbf{P}_{k}^{+}$ es la actualización de la matriz de varianzas-covarianzas del error en la estimación del estado aumentado, y $\mathbf{R}_{i}$ es la matriz de varianzas-covarianzas o el ruido introducido después de observar, con un sensor externoceptivo, el objeto $\mathbf{m}_{i}$. La matriz de transformación $\mathbf{G}$, es una matriz Jacobiana compuesta por bloques, definida según,

$$
\mathbf{G}=\left[\begin{array}{ccc}
\mathbf{I}_{n \times n} & \cdots & \mathbf{0}_{n \times 2} \\
\mathbf{J}_{\mathbf{v z}} & \mathbf{0}_{2 \mathbf{x}(n-3)} & \mathbf{J}_{\mathbf{z}_{i}}
\end{array}\right]
$$

Aquí $\mathbf{I}_{n \times n}$ es la matriz identidad cuya dimensión $n$ corresponde al número de estados que contenga el vector de estado aumentado $\mathbf{x}_{k}$. Las matrices $\mathbf{0}_{n \times 2}$ y $\mathbf{0}_{2 \mathrm{x}(n-3)}$ son matrices nulas (todos sus elementos son cero), $\mathbf{J}_{\mathbf{v z}}$ es la matriz Jacobiana de la función de representación con respecto al estado del vehículo, y $\mathbf{J}_{\mathbf{z}_{i}}$ es la matriz Jacobiana de la función de representación con respecto a las variables de estado de los objetos del mapa. Estos dos últimos Jacobianos ya fueron definidos en la ecuaciones (6.84) y (6.85).

\subsubsection{SLAM basado en hitos puntuales extraídos con un sensor de ultrasonidos}

Los sensores de ultrasonido son populares debido a su bajo costo, sin embargo su uso en procesos de localización de robot móviles es poco común ya que este sensor es poco fiable en cuanto a la información que proporciona respecto a la posición angular 
de los objetos que con él se detectan. En adelante enfocamos el problema de la autolocalización de robots móviles y de la construcción simultánea de un mapa del entorno usando sensores de ultrasonido. Se propone el uso de un SONAR (SOund NAvigation and Ranging) que es capaz de reducir la incertidumbre angular basándose en una estrategia de seguimiento de la amplitud del eco reflejado. También, para mejorar el desempeño del proceso de mapeado y localización, proponemos usar un Filtro de Kalman Extendido para fusionar los datos de localización odométrica, con los datos de distancia proporcionado por el SONAR propuesto, y obtener así una mejor estimación de la posición que adopta el robot mientras explora el entorno.

\subsubsection{Predicción del estado aumentado y de su covarianza}

Como ya expresa la ecuación (6.69), la predicción del estado aumentado $\hat{\mathbf{x}}_{k}^{-}$estará formada por la predicción del estado o posición del vehículo $\hat{\mathbf{x}}_{\mathrm{V}, k}^{-}$, y por la predicción del estado o coordenadas de localización (en el marco Global) de cada uno de los objetos fijos que pertenecen al mapa.

La predicción de la posición del vehículo se obtiene a partir del modelo cinemático del mismo. Para un vehículo tipo diferencial, como el que se utiliza en los ensayos experimentales realizados en este trabajo, la posición puede ser estimada a partir de una posición conocida, mediante la integración de la información proveniente de los sensores de odometría acoplados a las ruedas motrices. De esta manera, dada la posición inicial del vehículo $\mathbf{x}_{\mathrm{V}, k-1}^{+}=\langle x, y, \theta\rangle_{k-1}^{\mathrm{T}}$, y el vector de comandos de entrada $\mathbf{u}_{k}=\langle\Delta S, \Delta \theta\rangle_{k}$, se puede predecir el estado estimado $\hat{\mathbf{x}}_{\mathrm{v}, k}^{-}$del vehículo mediante la ecuación,

$$
\hat{\mathbf{x}}_{\mathrm{v}, k}^{-}=\hat{\mathbf{x}}_{\mathrm{v}, k-1}^{+}+\left[\begin{array}{c}
\Delta S_{k} \cos \left(\theta_{k-1}+\Delta \theta_{k} / 2\right) \\
\Delta S_{k} \sin \left(\theta_{k-1}+\Delta \theta_{k} / 2\right) \\
\Delta \theta_{k}
\end{array}\right]
$$

donde $\Delta S_{k}$ y $\Delta \theta_{k}$ son la longitud y el ángulo diferencial recorrido por el centro del robot desde $\mathbf{x}_{\mathrm{V}, k-1}$ hasta $\mathbf{x}_{\mathrm{V}, k}$.

Por otra parte, el error en la estimación del estado se puede representar como una distribución Gausiana multivariante y de media igual a cero, que puede ser modelada mediante una matriz de covarianzas $\mathbf{P}$, la cual tendría la forma

$$
\mathbf{P}=\left[\begin{array}{ccc}
\sigma_{x}^{2} & \sigma_{x y} & \sigma_{x \theta} \\
\sigma_{x y} & \sigma_{y}^{2} & \sigma_{y \theta} \\
\sigma_{x \theta} & \sigma_{y \theta} & \sigma_{\theta}^{2}
\end{array}\right]
$$

donde los valores $\sigma_{x}^{2}, \sigma_{y}^{2}$ y $\sigma_{\theta}^{2}$ representan las varianzas en $x, y$, y $\theta$, respectivamente.

En la sección 5.2, ya se mostró que la predicción de la incertidumbre en el instante $k\left(\mathbf{P}_{\mathrm{v}, k}^{-}\right)$, se puede obtener por combinación lineal de la incertidumbre previa $\left(\mathbf{P}_{\mathrm{V}, k-1}^{+}\right)$y del ruido de entrada al sistema. Ahora bien, considerando que el ruido de entrada se debe principalmente a las fallas en el sistema de tracción del vehículo, cuyo resultado se mide mediante $\Delta S$ y $\Delta \theta$, entonces este ruido puede ser modelado 


\section{CAPÍTULO 6. CONSTRUCCIÓN DE MAPAS Y LOCALIZACIÓN ESTOCÁSTICA}

mediante una distribución Gausiana bivariante con media igual a cero y matriz de covarianzas igual a,

$$
\mathbf{U}=\left[\begin{array}{cc}
\sigma_{\Delta S}^{2} & \sigma_{\Delta S \Delta \theta} \\
\sigma_{\Delta S \Delta \theta} & \sigma_{\Delta \theta}^{2}
\end{array}\right]
$$

donde los valores $\sigma_{\Delta S}^{2}$ y $\sigma_{\Delta \theta}^{2}$ representan las varianzas en $\Delta S$ y $\Delta \theta$, respectivamente.

Así, propagando la incertidumbre $\left(\mathbf{P}_{\mathrm{v}, k-1}^{+}\right)$y $(\mathbf{U})$ a través de la aproximación lineal de la función que modela el movimiento del vehículo, se obtiene la incertidumbre en la predicción del estado estimado según,

$$
\mathbf{P}_{\mathrm{V}, k}^{-}=\mathbf{J}_{\mathrm{V}} \mathbf{P}_{\mathrm{V}, k-1}^{+} \mathbf{J}_{\mathrm{V}}^{\mathrm{T}}+\mathbf{J}_{\mathbf{u}} \mathbf{U}_{k} \mathbf{J}_{\mathbf{u}}^{\mathrm{T}}
$$

donde $\mathbf{J}_{\mathrm{V}}$ representa la matriz Jacobiana de la función que modela el movimiento del vehículo, con respecto al estado del mismo $\left(\mathbf{x}_{\mathrm{V}}=\langle x, y, \theta\rangle\right)$, mientras que $\mathbf{J}_{\mathbf{u}}$ es la matriz Jacobiana de la misma función con respecto a la excitación de entrada $\mathbf{u}=\langle\Delta S, \Delta \theta\rangle$. Así, estos Jacobianos corresponden a

$$
\begin{gathered}
\mathbf{J}_{\mathbf{v}}=\left[\begin{array}{ccc}
1 & 0 & -\Delta S_{k} \sin \left(\theta_{k-1}+\Delta \theta_{k} / 2\right) \\
0 & 1 & \Delta S_{k} \cos \left(\theta_{k-1}+\Delta \theta_{k} / 2\right) \\
0 & 0 & 1
\end{array}\right] \\
\mathbf{J}_{\mathbf{u}}=\left[\begin{array}{cc}
\cos \left(\theta_{k-1}+\Delta \theta_{k} / 2\right) & -\frac{1}{2} \Delta S_{k} \sin \left(\theta_{k-1}+\Delta \theta_{k} / 2\right) \\
\sin \left(\theta_{k-1}+\Delta \theta_{k} / 2\right) & \frac{1}{2} \Delta S_{k} \cos \left(\theta_{k-1}+\Delta \theta_{k} / 2\right) \\
0 & 1
\end{array}\right]
\end{gathered}
$$

Ahora bien, dado que en este trabajo se asume que el entorno es estático, entonces se cumple que $\mathbf{m}_{i, k}=\mathbf{m}_{i, k-1}$. Igualmente, como los objetos son modelados mediante su coordenadas de localización en el plano global; es decir modelados como $\mathbf{m}_{i}=$ $\left[x_{i}, y_{i}\right]^{\mathrm{T}}$, con $i=1 \cdots \mathrm{N}$, entonces el vector de estado aumentado queda dela forma

$$
\hat{\mathbf{x}}_{k}^{-}=\left[\begin{array}{c}
\mathbf{x}_{\mathrm{V}, k} \\
x_{1} \\
y_{1} \\
\vdots \\
x_{\mathrm{N}} \\
y_{\mathrm{N}}
\end{array}\right]
$$

También, dado que el estado de los objetos del mapa no es gobernado por ecuaciones dinámicas, ni está sujeto a ruido aditivo, entonces su matriz de covarianza no evoluciona en el tiempo. En este sentido, para cada uno de los objetos del mapa se puede establecer que $\mathbf{P}_{i, k}=\mathbf{P}_{i, k-1}$, lo cual queda como

$$
\mathbf{P}_{i, k}=\left[\begin{array}{cc}
\sigma_{x_{i}}^{2} & \sigma_{x y_{i}} \\
\sigma_{x y_{i}} & \sigma_{y_{i}}^{2}
\end{array}\right]
$$

donde $\sigma_{x_{i}}^{2}$ y $\sigma_{y_{i}}^{2}$ se corresponden con la varianza del error en la estimación de las coordenadas de localización de la $i$-esima landmark observada. Esto es, $\left\langle x_{i}, y_{i}\right\rangle$ respectivamente. 
También, de acuerdo a la ecuación (6.70), el estado del vehículo está correlacionado con el estado del mapa. De aquí que la predicción de la covarianza del estado aumentado $\mathbf{P}_{k}^{-}$, tenga la forma

$$
\mathbf{P}_{k}^{-}=\left[\begin{array}{llllllll}
\sigma_{x}^{2} & \sigma_{x y} & \sigma_{x \theta} & \sigma_{x x_{1}} & \sigma_{x y_{1}} & \cdots & \sigma_{x x_{\mathbb{N}}} & \sigma_{x y_{\mathbb{N}}} \\
\sigma_{x y} & \sigma_{y}^{2} & \sigma_{y \theta} & \sigma_{y x_{1}} & \sigma_{y y_{1}} & \cdots & \sigma_{y x_{\mathbb{N}}} & \sigma_{y y_{\mathbb{N}}} \\
\sigma_{x \theta} & \sigma_{y \theta} & \sigma_{\theta}^{2} & \sigma_{\theta x_{1}} & \sigma_{\theta y_{1}} & \cdots & \sigma_{\theta x_{\mathbb{N}}} & \sigma_{\theta y_{\mathbb{N}}} \\
\sigma_{x x_{1}} & \sigma_{y x_{1}} & \sigma_{\theta x_{1}} & \sigma_{x_{1}}^{2} & \sigma_{x_{1} y_{1}} & \cdots & \sigma_{x_{1} x_{\mathbb{N}}} & \sigma_{x_{1} y_{\mathbb{N}}} \\
\sigma_{x y_{1}} & \sigma_{y y_{1}} & \sigma_{\theta y_{1}} & \sigma_{x_{1} y_{1}} & \sigma_{y_{1}}^{2} & \cdots & \sigma_{y_{1} x_{\mathbb{N}}} & \sigma_{y_{1} y_{\mathbb{N}}} \\
\vdots & \vdots & \vdots & \vdots & \vdots & \ddots & \vdots & \vdots \\
\sigma_{x x_{\mathbb{N}}} & \sigma_{y x_{\mathbb{N}}} & \sigma_{\theta x_{\mathbb{N}}} & \sigma_{x_{1} x_{\mathbb{N}}} & \sigma_{y_{1} x_{\mathbb{N}}} & \cdots & \sigma_{x_{\mathbb{N}}}^{2} & \sigma_{x_{\mathbb{N}} y_{\mathbb{N}}} \\
\sigma_{x y_{\mathbb{N}}} & \sigma_{y y_{\mathbb{N}}} & \sigma_{\theta y_{\mathbb{N}}} & \sigma_{x_{1} y_{\mathbb{N}}} & \sigma_{y_{1} y_{\mathbb{N}}} & \cdots & \sigma_{x_{\mathbb{N}} y_{\mathbb{N}}} & \sigma_{y_{\mathbb{N}}}^{2}
\end{array}\right]
$$

donde los subíndices $\{x, y, \theta\}$ corresponden a las variables de estado que describen la posición del vehículo, mientras que los subíndices $\left\{x_{i}, y_{i}\right\}$ con $i=1 \cdots \mathrm{N}$, corresponden a las variables de estado que modelan la localización de la i-esima landmark observada.

\subsubsection{Representación del entorno}

En este trabajo se asume que el entorno está representado mediante una colección de objetos puntuales $\mathbf{m}_{i}=\langle x, y\rangle_{i}$ pertenecientes a un sistema 2D fijo de coordenadas cartesianas, conocido como el sistema de referencias Global(ver Figura 6.17).

Para el caso del proceso de autolocalización y mapeado simultáneo propuesto en esta tesis, se asume que el vehículo que navega por el entorno cuenta con un sensor que proporciona información relacionada a la distancia radial $\rho$ y al ángulo de vista $\varphi$ medidos desde el origen del sistema de coordenadas del vehículo hasta cada uno de los hitos observados. Esta información estará representada en una matriz de medición indexada de acuerdo al instante de tiempo $k$ en el cual se produzca el ciclo de observación del entorno según,

$$
\mathbf{z}_{k}=\left[\begin{array}{cccc}
\rho_{1} & \rho_{2} & \cdots & \rho_{j} \\
\varphi_{1} & \varphi_{2} & \cdots & \varphi_{j}
\end{array}\right]_{k}
$$

donde el índice $j$ se refiere al j-ésimo objeto que es detectado por los sensores durante el proceso de observación en el instante $k$. Ahora bien, esta matriz de medición tiene asociada una matriz de incertidumbre para cada columna del arreglo que tiene la forma

$$
\mathbf{R}_{j}=\left[\begin{array}{cc}
\sigma_{\rho_{j}}^{2} & \sigma_{\rho_{j} \varphi_{j}} \\
\sigma_{\rho_{j} \varphi_{j}} & \sigma_{\varphi_{j}}^{2}
\end{array}\right]
$$

Dada la medición de un objeto $\mathbf{z}=\langle\rho, \varphi\rangle^{\mathrm{T}}$ en el marco Sensor, el mismo se ve en el marco Global de acuerdo a la siguiente transformación,

$$
\mathbf{m}=h\left(\hat{\mathbf{x}}_{\mathrm{V}, k}, \mathbf{z}\right)=\left[\begin{array}{l}
m_{x} \\
m_{y}
\end{array}\right]=\left[\begin{array}{l}
x+\rho \cos (\varphi+\theta) \\
y+\rho \sin (\varphi+\theta)
\end{array}\right]
$$




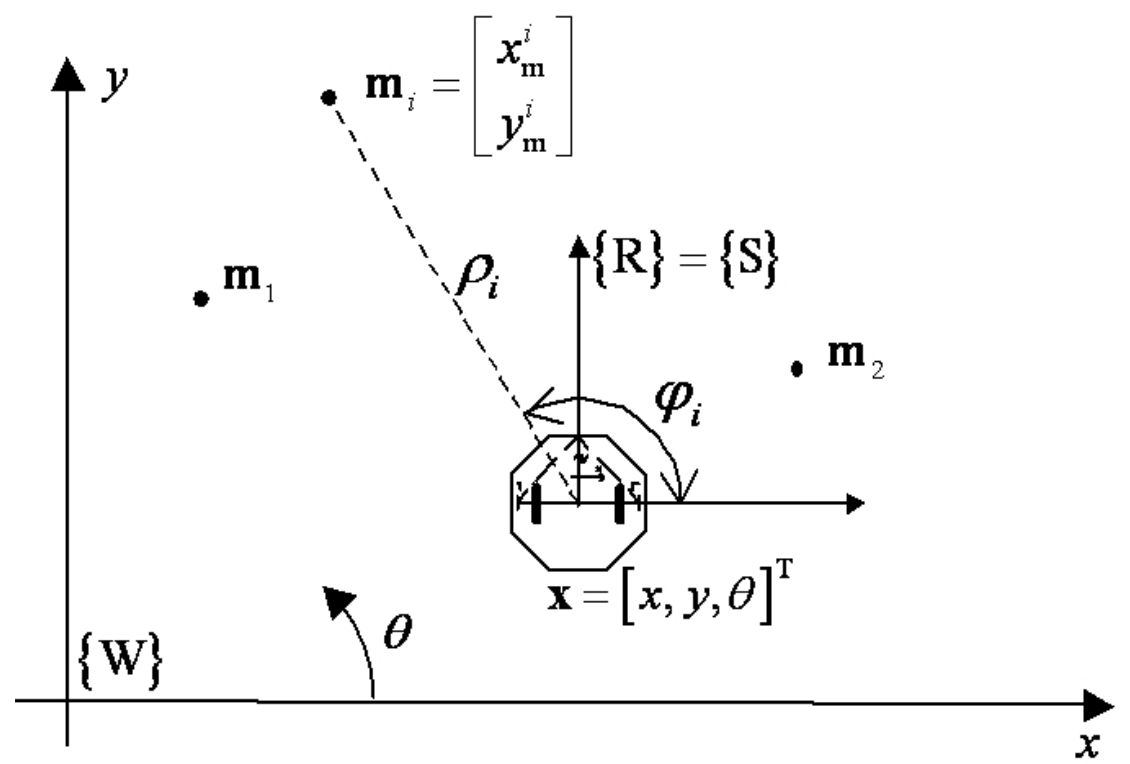

Figura 6.17: Representación del entorno mediante características puntuales $\mathbf{m}_{i}$. $\{\mathrm{W}\}$ : Marco Global, $\{\mathrm{R}\}$ : Marco Robot, $\{\mathrm{S}\}$ : Marco sensor.

Por otra parte, la matriz de covarianzas en la medición se puede transformar al marco Global usando la ecuación,

$$
\mathbf{M}=\mathbf{J}_{\mathbf{v z}} \mathbf{P}_{\mathrm{v}, k}^{-} \mathbf{J}_{\mathbf{v z}}^{\mathrm{T}}+\mathbf{J}_{\mathbf{z}} \mathbf{R} \mathbf{J}_{\mathbf{z}}^{\mathrm{T}}
$$

donde $\mathbf{P}_{\mathrm{V}, k}^{-}$es la predicción de la covarianza del error en la predicción del estado del vehículo que porta al sensor, y $\mathbf{R}$ es la matriz de varianzas-covarianzas o el ruido introducido por el sensor al extraer del entorno el objeto $\mathbf{z}$. También, $\mathbf{J}_{\mathbf{z}}$ es la matriz Jacobiana de la función de representación dada en la ecuación (6.102), respecto al vector de medición $\mathbf{z}$; mientras que $\mathbf{J}_{\mathbf{v z}}$ es la matriz Jacobiana de la misma función de representación pero respecto al vector de estado del vehículo $\mathbf{x}_{\mathrm{V}}=\langle x, y, \theta\rangle$.

Entonces, partiendo de la función dada en (6.102), los Jacobianos se pueden calcular según,

$$
\begin{array}{r}
\mathbf{J}_{\mathbf{v z}}=\left.\frac{\partial h\left(\mathbf{x}_{\mathrm{V}, k}, \mathbf{z}_{j, k}\right)}{\partial \mathbf{x}_{\mathbf{V}}}\right|_{\mathbf{x}_{\mathrm{V}, k}, \mathbf{z}_{j, k}}=\left[\begin{array}{ccc}
1 & 0 & -\rho \sin (\varphi+\theta) \\
0 & 1 & \rho \cos (\varphi+\theta)
\end{array}\right] \\
\mathbf{J}_{\mathbf{z}}=\left.\frac{\partial h\left(\mathbf{x}_{\mathrm{V}, k}, \mathbf{z}_{j, k}\right)}{\partial \mathbf{z}}\right|_{\mathbf{x}_{\mathrm{V}, k}, \mathbf{z}_{j, k}}=\left[\begin{array}{cc}
\cos (\varphi+\theta) & -\rho \sin (\varphi+\theta) \\
\sin (\varphi+\theta) & \rho \cos (\varphi+\theta)
\end{array}\right]
\end{array}
$$

Ahora bien, de las ecuaciones (6.92), (6.101), (6.103), (6.104) y (6.105), se extrae que la matriz de covarianza que representa la incertidumbre en la localización de un 
objeto $\mathbf{z}_{j}$, pero visto en el marco Global, tiene la forma

$$
\mathbf{M}^{j}=\left[\begin{array}{cc}
\sigma_{x_{j}}^{2} & \sigma_{x_{j} y_{j}} \\
\sigma_{x_{j} y_{j}} & \sigma_{y_{j}}^{2}
\end{array}\right]
$$

\subsubsection{Asociación de datos o correspondencia entre hitos puntuales}

El método de correspondencia que se usa con mayor frecuencia es el conocido como compuertas de validación [150]. Sin embargo, consideramos que esta metodología no es del todo exhaustiva y que además representa una alta carga computacional en el proceso de autolocalización. Esto último se debe a que esta metodología requiere el transformar al marco del sensor, el estado de todos y cada uno de los objetos del mapa, incluyendo las matrices de covarianza asociada a los mismos. En esta tesis se propone un método de pre-correspondencia que se lleva a cabo en el marco Global, y que sólo requiere la transformación del estado del objeto $\mathbf{z}_{j}$ y de su matriz de covarianza $\mathbf{R}_{j}$. En la sección 5.5.1 ya se expusieron los detalles del método, por lo que en adelante sólo se consideran los dos posibles resultados: sin correspondencia, en cuyo caso es un objeto nuevo que hay que agregar al mapa y al vector de estado aumentado, y correspondencia positiva, que se trata del caso cuando el objeto observado ya existe en el mapa y se utiliza para corregir el estimado de la posición del vehículo, así como también el estimado de localización del resto de los objetos que forman el estado aumentado.

\subsubsection{Objeto nuevo: incorporación al vector de estado aumentado}

Si durante el proceso de asociación de datos, no se detecta ninguna correspondencia con el objeto $\mathbf{z}_{j}=\langle\rho, \varphi\rangle^{\mathrm{T}}$ que actualmente ha sido extraído con el sensor, entonces se trata de un objeto nuevo que hay que incorporar al mapa, o lo que es lo mismo, al vector de estado aumentado. Para ello hay que hacer uso de la ecuación de representación, que para el caso de objetos puntuales, ya se formuló a través de la ecuación (6.102). Así, el nuevo estado aumentado del sistema sería

$$
\hat{\mathbf{x}}_{k}^{+}=\left[\begin{array}{c}
\hat{\mathbf{x}}_{k}^{+} \\
\hat{x}+\rho \cos (\varphi+\hat{\theta}) \\
\hat{y}+\rho \sin (\varphi+\hat{\theta})
\end{array}\right]
$$

donde $\hat{\mathbf{x}}_{k}^{+}=\left[\begin{array}{llll}\mathbf{x}_{\mathrm{V}, k}^{\mathrm{T}} & \mathbf{m}_{1, k}^{\mathrm{T}} & \cdots & \mathbf{m}_{\mathrm{N}, k}^{\mathrm{T}}\end{array}\right]^{\mathrm{T}}$ representa la mejor estimación que se tiene en relación al estado del sistema. Aquí $\mathbf{x}_{\mathrm{V}, k}^{\mathrm{T}}=\langle\hat{x}, \hat{y}, \hat{\theta}\rangle$ es la mejor estimación que se tiene respecto al estado o posición del vehículo, $\mathrm{y}\left[\begin{array}{llll}\mathbf{m}_{1, k}^{\mathrm{T}} & \cdots & \mathbf{m}_{\mathrm{N}, k}^{\mathrm{T}}\end{array}\right]^{\mathrm{T}}$ es el vector que contiene el estado de los elementos que ya forman parte del mapa que el vehículo va construyendo.

La tranformación de la medición $\mathbf{z}_{j}=\langle\rho, \varphi\rangle^{\mathrm{T}}$ desde el marco Sensor al marco Global, amerita que también se transforme el efecto de la incertidumbre en la medición 
y en el estimado de posición del vehículo que porta al sensor. Esto ya se expresó a través de la ecuación (6.103). Aunque esta ecuación en esencia recoge el mecanismo de propagación de la incertidumbre en el estado del nuevo objeto, nosotros usamos la formulación de la ecuación (6.89), la cual permite manejar el estado aumentado como un todo y no como piezas sueltas. En este sentido, el cambio en la matriz de covarianzas del estado aumentado se debe a la introducción del bloque de covarianzas correspondiente al nuevo objeto que se incorporó al vector de estado aumentado. De acuerdo a lo anterior, la actualización de la matriz de covarianzas del estado sería,

$$
\mathbf{P}_{k}^{+}=\mathbf{G}_{j}\left[\begin{array}{cc}
\mathbf{P}_{k}^{-} & 0 \\
0 & \mathbf{R}_{j}
\end{array}\right] \mathbf{G}_{j}^{\mathrm{T}}
$$

donde $\mathbf{P}_{k}^{-}$es la matriz de covarianzas del estado estimado antes de incorporar la nueva observación, mientras que $\mathbf{P}_{k}^{+}$corresponde a la actualización de la matriz de varianzas-covarianzas del error en la estimación del estado aumentado después de incorporar el nuevo objeto. Por su parte, $\mathbf{R}_{j}$ es la matriz de varianzas-covarianzas o el ruido introducido después de extraer el objeto $\mathbf{z}_{j}=\langle\rho, \varphi\rangle^{\mathrm{T}}$ en el marco Sensor (ver ecuación 6.101), y $\mathbf{G}_{j}$ es la matriz que permite transformar este ruido al marco Global. La forma y composición de la matriz $\mathbf{G}_{j}$ ya se introdujo en la ecuación (6.90), pero por propósitos de claridad se repite a continuación.

$$
\mathbf{G}_{j}=\left[\begin{array}{ccc}
\mathbf{I}_{n \mathbf{x} n} & \cdots & \mathbf{0}_{n \times 2} \\
\mathbf{J}_{\mathbf{v z}} & \mathbf{0}_{2 \mathbf{x}(n-3)} & \mathbf{J}_{\mathbf{z}_{j}}
\end{array}\right]
$$

Aquí $\mathbf{I}_{n \times n}$ es la matriz identidad cuya dimensión $n$ corresponde al número de estados que contenga el vector de estado aumentado $\mathbf{x}_{k}$, mientras que las matrices $\mathbf{0}_{n \times 2} \mathrm{y}$ $\mathbf{0}_{2 \mathrm{x}(n-3)}$ son matrices nulas (todos sus elementos son cero). Por su parte, $\mathbf{J}_{\mathbf{v z}}$ es la matriz Jacobiana de la función de representación con respecto al estado del vehículo, y $\mathbf{J}_{\mathbf{z}_{j}}$ es la matriz Jacobiana de la función de representación con respecto a las variables de estado de los objetos del mapa. Estos dos últimos ya fueron definidos en la ecuaciones (6.104) y (6.105).

\subsubsection{Objeto existente: Corrección del estado estimado del vehículo}

Asúmase que se ha ejecutado en forma perfecta un proceso de correspondencia entre los objetos medidos y los objetos contenidos en un mapa ya conocido. Asúmase también que por ejemplo, el mismo arrojó como resultado que el objeto $\mathbf{m}_{i}$ del mapa se corresponde con el objeto $\mathbf{z}_{j}$ que ha sido extraído del entorno mediante un medidor de distancia y ángulo ubicado en el centro del vehículo. De esta forma, sólo es necesario transformar al marco Sensor el objeto $\mathbf{m}_{i}$. Este requerimiento se debe a que el proceso de corrección del estado es llevado a cabo en el marco de referencia del vehículo (marco Robot), que por conveniencia suele hacerse coincidir con el marco Sensor. La ecuación que permite representar el objeto $\mathbf{m}_{i}$ del marco Global, en el marco Robot, se conoce como el modelo de observación. En nuestro caso, dado que el sensor que se usa es capaz de medir la distancia y el ángulo de vista al objeto detectado, entonces la 
siguiente formulación cubre los requerimientos de transformación necesarios.

$$
\begin{aligned}
& \mathbf{z}_{i}=h\left(\hat{\mathbf{x}}_{\mathrm{V}, k}, \mathbf{m}_{i}\right)=\left[\begin{array}{l}
\rho_{i} \\
\varphi_{i}
\end{array}\right] \\
& \mathbf{z}_{i}=\left[\begin{array}{c}
\sqrt{\left(x_{i}-x_{k}\right)^{2}+\left(y_{i}-y_{k}\right)^{2}} \\
\arctan \left(\frac{y_{i}-y_{k}}{x_{i}-x_{k}}\right)-\theta_{k}
\end{array}\right]
\end{aligned}
$$

donde $\hat{\mathbf{x}}_{\mathrm{V}, k}=\langle x, y, \theta\rangle_{k}$ representa la posición del vehículo o el estado en el instante $k, \mathrm{y} \mathbf{m}_{i}=\langle x, y\rangle_{i}$ corresponde a las coordenadas del objeto observado.

A continuación, la diferencia entre el vector de medición $\mathbf{z}_{j}$ (lo realmente medido por el sensor) y el vector de observación $\mathbf{z}_{i}$ (la predicción de lo que el sensor debería medir), proporciona lo que se conoce como la innovación $\Delta \mathbf{z}$.

$$
\Delta \mathbf{z}=\mathbf{z}_{j}-\mathbf{z}_{i}=\left[\begin{array}{c}
\rho_{j} \\
\varphi_{j}
\end{array}\right]-\left[\begin{array}{c}
\sqrt{\left(x_{i}-x_{k}\right)^{2}+\left(y_{i}-y_{k}\right)^{2}} \\
\arctan \left(\frac{y_{i}-y_{k}}{x_{i}-x_{k}}\right)-\theta_{k}
\end{array}\right]
$$

También, la covarianza en el vector de innovación puede obtenerse mediante aproximación lineal de acuerdo a

$$
\mathbf{Z}_{i}=\mathbf{J}_{\mathbf{x}, i} \mathbf{P}_{k}^{-} \mathbf{J}_{\mathbf{x}, i}^{\mathrm{T}}+\mathbf{R}_{j}
$$

donde $\mathbf{R}_{j}$ es la matriz de covarianza que modela el ruido sensorial en la medición del objeto $\mathbf{z}_{j}, \mathbf{P}_{k}^{-}$es la matriz de covarianza de la predicción del estado aumentado en el instante $k$, la cual ya fue formulada en la ecuación (6.70), resultando la forma de la ecuación (6.99). Por otra parte, $\mathbf{J}_{\mathbf{x}, i}$ es el Jacobiano compuesto de la función de observación, la cual se puede calcular de acuerdo a,

$$
\mathbf{J}_{\mathbf{x}, i}=\left.\frac{\partial h\left(\mathbf{x}_{\mathrm{V}, k}, \mathbf{m}_{i, k}\right)}{\partial \mathbf{x}}\right|_{\mathbf{x}_{\mathbf{V}, k}, \mathbf{m}_{i, k}}=\left.\left[\begin{array}{lllll}
\mathbf{J}_{\mathbf{v m}} & \mathbf{J}_{\mathbf{m}_{1}} & \mathbf{J}_{\mathbf{m}_{2}} & \cdots & \mathbf{J}_{\mathbf{m}_{\mathbb{N}}}
\end{array}\right]\right|_{\mathbf{x}_{\mathbf{V}, k}, \mathbf{m}_{i, k}}
$$

donde $\mathbf{J}_{\mathbf{v m}}$ es el Jacobiano de la función de observación (ecuación 6.109) respecto al estado del vehículo $\mathbf{x}_{\mathrm{V}, k}$, y $\mathbf{J}_{\mathbf{m}_{i}}$ va a representar el Jacobiano de la función de observación respecto al estado estimado de cada uno de los objetos del mapa. Ahora bien, considerando la observación simple de los objetos del mapa, es decir que existe capacidad para observar sólo un objeto a la vez, entonces el Jacobiano compuesto de la función de observación se puede reescribir como

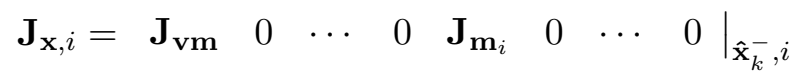

lo que muestra que todos los Jacobianos son cero excepto el que corresponde al vehículo y el que corresponde al objeto del mapa $\left(\mathbf{m}_{i}\right)$ que está siendo observado en este paso del algoritmo. 


\section{CAPÍTULO 6. CONSTRUCCIÓN DE MAPAS Y LOCALIZACIÓN ESTOCÁSTICA}

Los Jacobianos $\mathbf{J}_{\mathbf{v m}}$ y $\mathbf{J}_{\mathbf{m}_{i}}$ se obtienen a partir de la función de observación de acuerdo a,

$$
\begin{aligned}
\mathbf{J}_{\mathbf{v m}} & =\left.\frac{\partial h\left(\mathbf{x}_{\mathrm{V}, k}, \mathbf{m}_{i, k}\right)}{\partial \mathbf{x}_{\mathrm{V}}}\right|_{\mathbf{x}_{\mathrm{V}, k}, \mathbf{m}_{i, k}} \\
= & {\left[\begin{array}{lll}
\frac{-\left(x_{i}-x_{k}\right)}{\sqrt{\left(x_{i}-x_{k}\right)^{2}+\left(y_{i}-y_{k}\right)^{2}}} & \frac{\left(y_{i}-y_{k}\right)}{\sqrt{\left(x_{i}-x_{k}\right)^{2}+\left(y_{i}-y_{k}\right)^{2}}} & 0 \\
\frac{\left.x_{i}-x_{k}\right)^{2}+\left(y_{i}-y_{k}\right)^{2}}{\left(x_{i}-x_{k}\right)^{2}+\left(y_{i}-y_{k}\right)^{2}} & -1
\end{array}\right] } \\
\mathbf{J}_{\mathbf{m}_{i}} & =\left.\frac{\partial h\left(\mathbf{x}_{\mathrm{V}, k}, \mathbf{m}_{i, k}\right)}{\mathbf{m}_{i}}\right|_{\mathbf{x}_{\mathrm{V}, k}, \mathbf{m}_{i, k}} \\
& =\left[\begin{array}{cc}
\frac{\left(x_{i}-x_{k}\right)}{\sqrt{\left(x_{i}-x_{k}\right)^{2}+\left(y_{i}-y_{k}\right)^{2}}} & \frac{-\left(y_{i}-y_{k}\right)}{\sqrt{\left(x_{i}-x_{k}\right)^{2}+\left(y_{i}-y_{k}\right)^{2}}} \\
\frac{\left(x_{i}-x_{k}\right)}{\left(x_{i}-x_{k}\right)^{2}+\left(y_{i}-y_{k}\right)^{2}} & \frac{}{\left(x_{i}-x_{k}\right)^{2}+\left(y_{i}-y_{k}\right)^{2}}
\end{array}\right]
\end{aligned}
$$

A partir de este estado, el algoritmo del Filtro de Kalman Extendido calcula la ganancia que pondera la cantidad de corrección que se debe aplicar al estado estimado previo $\hat{\mathbf{x}}_{k}^{-}$, para obtener así el estimado óptimo del mismo $\hat{\mathbf{x}}_{k}^{+}$. La ganancia del Filtro de Kalman depende de la matriz de covarianzas del estimado previo, y de la matriz de covarianzas en la medición. Una forma de calcularla es mediante

$$
\mathbf{K}_{k}=\mathbf{P}_{k}^{-} \mathbf{J}_{\mathbf{x}, i}^{\mathrm{T}} \mathbf{Z}_{i}^{-1}
$$

Con la ganancia $\mathbf{K}_{k}$, el estado aumentado y su covarianza, pueden ser corregidos o actualizados de forma óptima de acuerdo a las siguientes ecuaciones

$$
\begin{gathered}
\hat{\mathbf{x}}_{k}^{+}=\hat{\mathbf{x}}_{k}^{-}+\mathbf{K}_{k}\left(\mathbf{z}_{j}-\mathbf{z}_{i}\right) \\
\mathbf{P}_{k}^{+}=\mathbf{P}_{k}^{-}-\mathbf{K}_{k} \mathbf{Z}_{i} \mathbf{K}_{k}^{\mathrm{T}}
\end{gathered}
$$

donde $\hat{\mathbf{x}}_{k}^{+}$es la estimación a posteriori del estado aumentado, es decir el estado corregido, y $\mathbf{P}_{k}^{+}$es la actualización de la matriz de varianzas-covarianzas del error en la estimación del estado aumentado.

\subsubsection{Resultados experimentales del SLAM usando esquinas como hitos puntuales}

Con la finalidad de demostrar la validez del método de autolocalización y mapeado simultáneo basado en hitos puntuales extraídos con un SONAR, se programó al robot YAIR para que navegara a través de un entorno típico de oficinas, mientras que exploraba el mismo mediante su SONAR rotativo. En la figura 6.18 se puede observar una representación CAD del entorno, así como el trayecto de dos ciclos seguido por el robot. Durante el trayecto de aproximadamente 140 metros, el robot realizó paradas sistemáticas cada 3 metros con el objetivo último de observar el entorno mediante su 


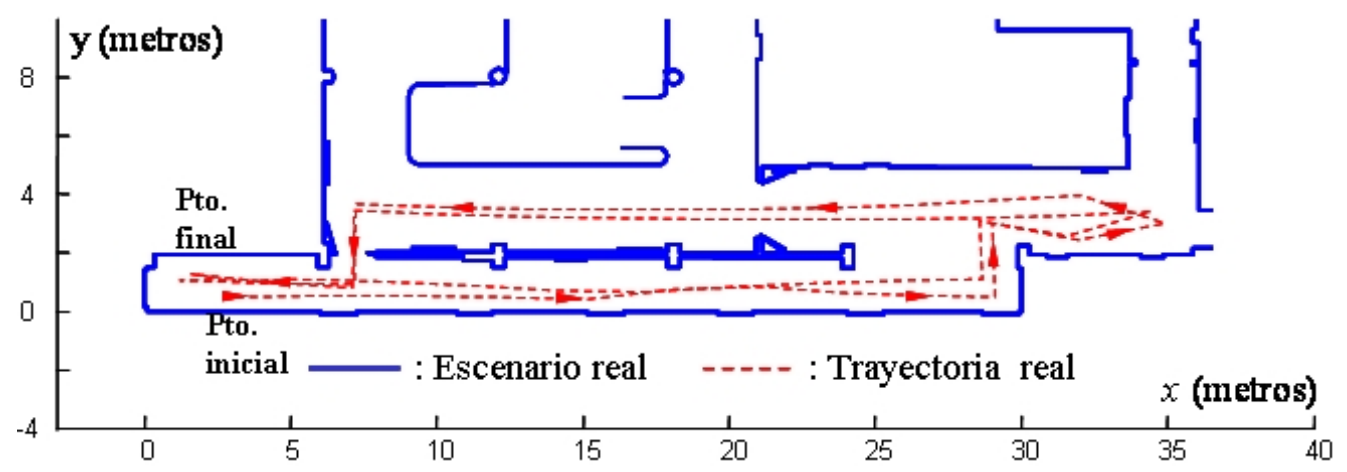

Figura 6.18: Escenario donde se realizó el ensayo mostrando el trayecto real que ejecutó el robot. Nótese que el trayecto consiste de dos ciclos aproximadamente iguales, que se inician y terminan en las cercanías del punto de coordenadas $(0,0)$.

SONAR (51 paradas en total). Este sensor es capaz de detectar las esquinas del entorno y de suministrar datos de distancia y ángulo de vista a ellas[145]. También, en cada una de estas paradas se medió manualmente la posición real de robot, para compararla posteriormente con la posición estimada mediante la localización odométrica simple, así como también con los resultados de estimación de la posicion obtenidos por el algoritmo de localización basado en el Filtro de Kalman Extendido.

Para realizar este ensayo se supuso que la posición inicial del robot era perfectamente conocida. También, dadas las condiciones particulares del escenario y del trayecto ejecutado, es necesario que el robot complete un trayecto de aproximadamente 40 metros antes de poder observar una referencia por él conocida. Por esta razón, para lograr que el robot mantuviera durante la fase inicial de exploración, una estimación razonablemente acotada en cuanto a su posición real, se asumió que se contaba con un mapa del entorno con 3 landmark perfectamente conocidas. De esta forma, las condiciones iniciales del estado aumentado fueron,

$$
\begin{aligned}
& \mathbf{x}_{0}= {\left[\begin{array}{c}
x \\
y \\
\theta \\
x_{1} \\
y_{1} \\
x_{2} \\
y_{2} \\
x_{3} \\
y_{3}
\end{array}\right]=\left[\begin{array}{c}
3,100 \\
0,513 \\
0,0000 \\
11,950 \\
1,695 \\
20,870 \\
-0,120 \\
29,950 \\
-0,120
\end{array}\right] } \\
& \mathbf{P}_{0}=\operatorname{zeros}[9 \times 9]
\end{aligned}
$$

Como resultado general relacionado a la localización, en la figura 6.19 se muestra el trayecto estimado y corregido usando el algoritmo del Filtro de Kalman Extendido. Como se puede observar, el trayecto se ajusta con una aceptable precisión a 


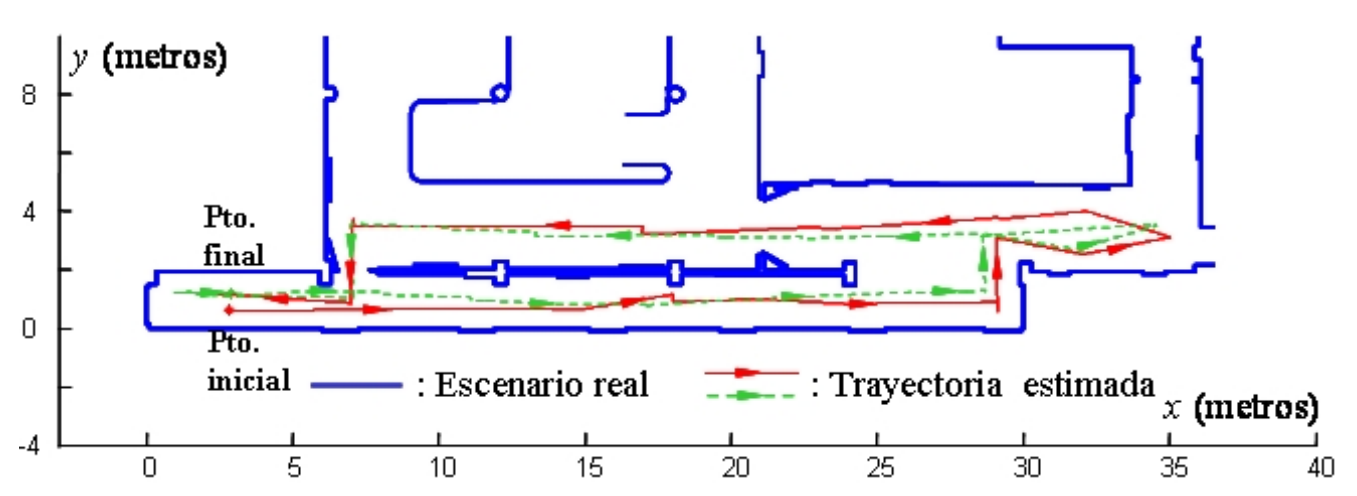

Figura 6.19: Trayectoria estimada mediante el Filtro de Kalman Extendido. Obsérvese que el robot ejecutó dos vueltas. La primera en color rojo continuo, se trata principalmente de una fase de exploración. La segunda en color verde a trazos, se trata de la fase de navegación autónoma.

la trayectoria real seguida por el robot durante la exploración y navegación por el entorno. También se observa que durante la primera vuelta (fase de exploración), las correcciones son más abruptas que en la segunda vuelta (fase de navegación). Esto claramente se debe a que en la primera vuelta el robot desconoce casi por completo el entorno que lo rodea, y por lo tanto encuentra muy pocas referencias con las cuales corregir su estimado de posición.

La figura 6.20 muestra el error en la estimación de la posición del vehículo como una función del tiempo. A simple vista se ve que los errores se mantuvieron acotados en el tiempo. También se aprecia que el error preponderante es el que se alínea en la dirección transversal al movimiento del vehículo. En este caso particular, dado que la dirección de navegación estuvo alineada más que todo en el eje $x$, el error más preponderante es al que se acumula en la dirección del eje $y$. También, la figura 6.21 muestra como evoluciona la desviación estándar en el estimado del estado de vehículo. Por lo que muestra esta figura, se puede concluir que en general el error en la estimación del estado del vehículo permaneció dentro de los límites de $\pm 1 \sigma$. Esto último valida el modelo estocástico que se ha propuesto para el vehículo, ya que el mismo fue capaz de modelar la incertidumbre de posición de manera conservadora.

Por otra parte, en la figura 6.20 también se aprecia que a excepción del error de orientación, el error promedio en la estimación del estado del vehículo se aproxima a cero. Esto último tiende a validar parcialmente la presunción de ruido Gausiano de media igual a cero, que es condición necesaria para la aplicación del Filtro de Kalman Extendido. Para explorar más esta presunción, se hizo un análisis estadístico del error del estado estimado, obteniéndose los estadísticos mostrados en la tabla (6.6). Uno de los aspecto relevantes que se pueden ver en la mencionada tabla, es que la asimetría y la curtosis muestran valores que indican o confirman que la distribución del error en la estimación del estado proviene de poblaciones normales. También se confirma que la mayor cantidad de error se introduce en la dirección transversal al movimiento del vehículo, lo que se evidencia al comparar las desviación estándar del error en $x$ y 

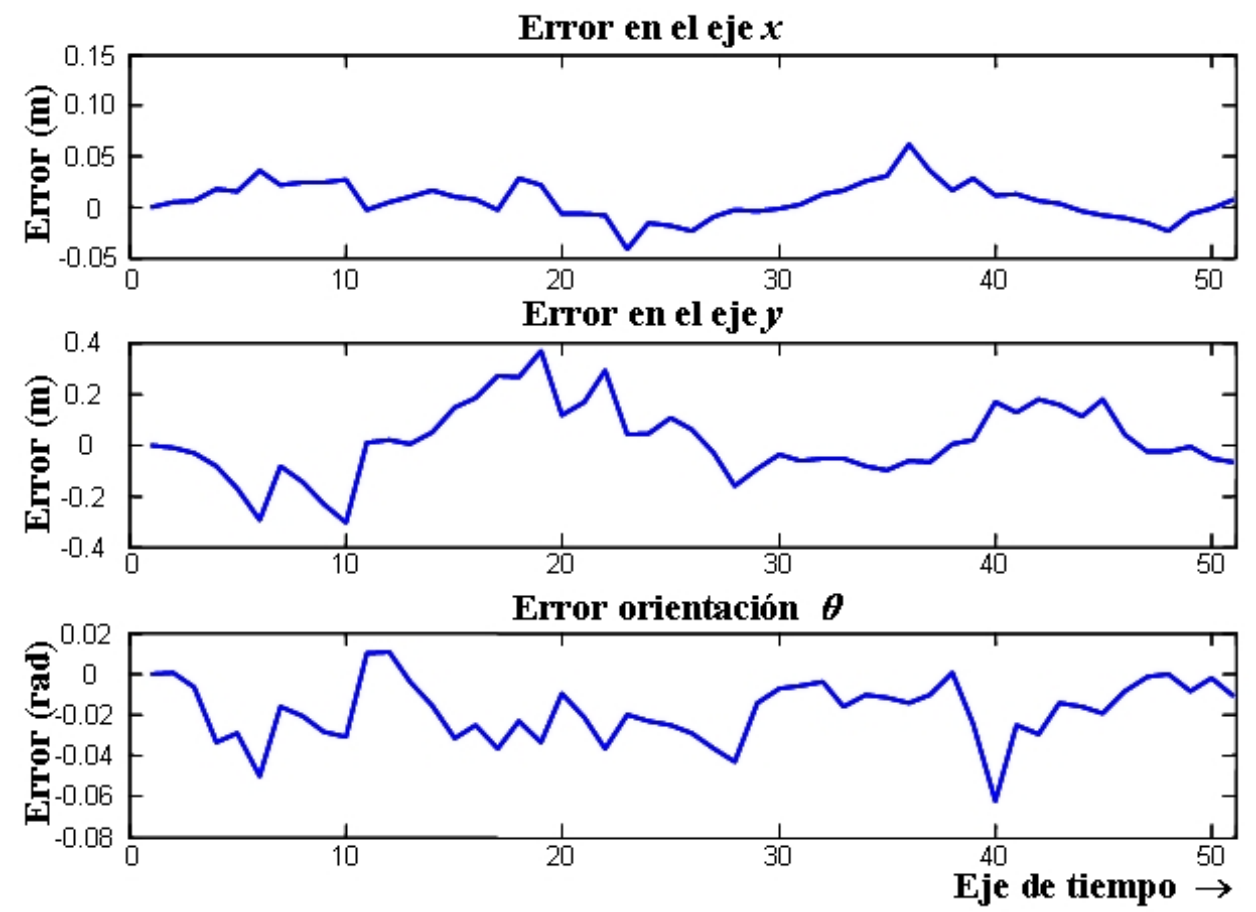

Figura 6.20: Error resultante en la estimación de la posición del robot usando EKF
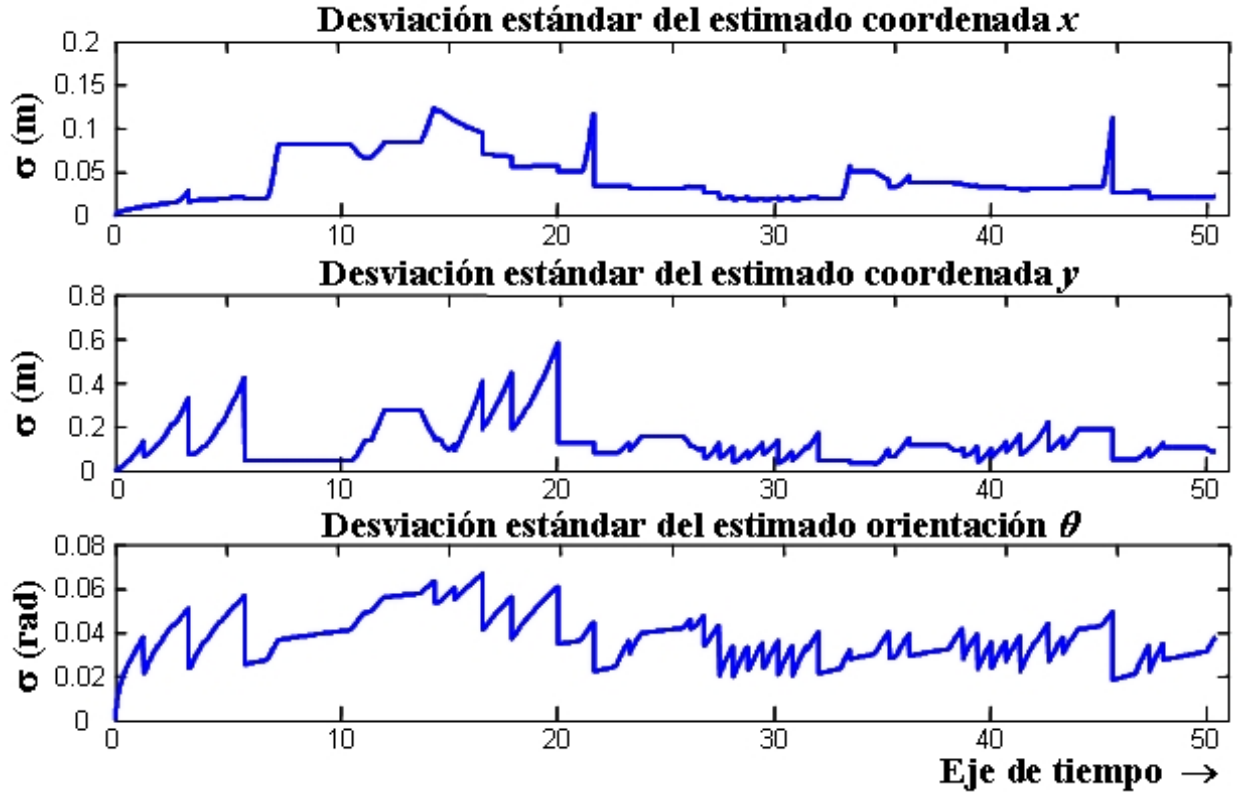

Figura 6.21: Evolución a través del tiempo de la desviación estándar en el estimado del estado del robot 
Tabla 6.6: Resumen de estadísticos del error en la estimación del estado. $\varepsilon_{x}, \varepsilon_{y}$, y $\varepsilon_{\theta}$ es el error en la coordenada $x, y, \mathrm{y} \theta$, respectivamente

\begin{tabular}{|c|c|c|c|}
\hline & $\varepsilon_{x}(\mathrm{~m})$ & $\varepsilon_{y}(\mathrm{~m})$ & $\varepsilon_{\theta}(\mathrm{rad})$ \\
\hline \hline Frecuencia & 51 & 51 & 51 \\
\hline Media & 0,007 & 0.014 & -0.0181 \\
\hline Varianza & 0,000 & 0.020 & 0.0002 \\
\hline Desv. Estándar & 0.018 & 0.141 & 0.0148 \\
\hline Mínimo & -0.042 & -0.304 & -0.0624 \\
\hline Máximo & 0.062 & 0.369 & 0.0109 \\
\hline Asimetría & 0.612 & 0.549 & -1.411 \\
\hline Curtosis & 1.432 & 0.411 & 0.715 \\
\hline
\end{tabular}

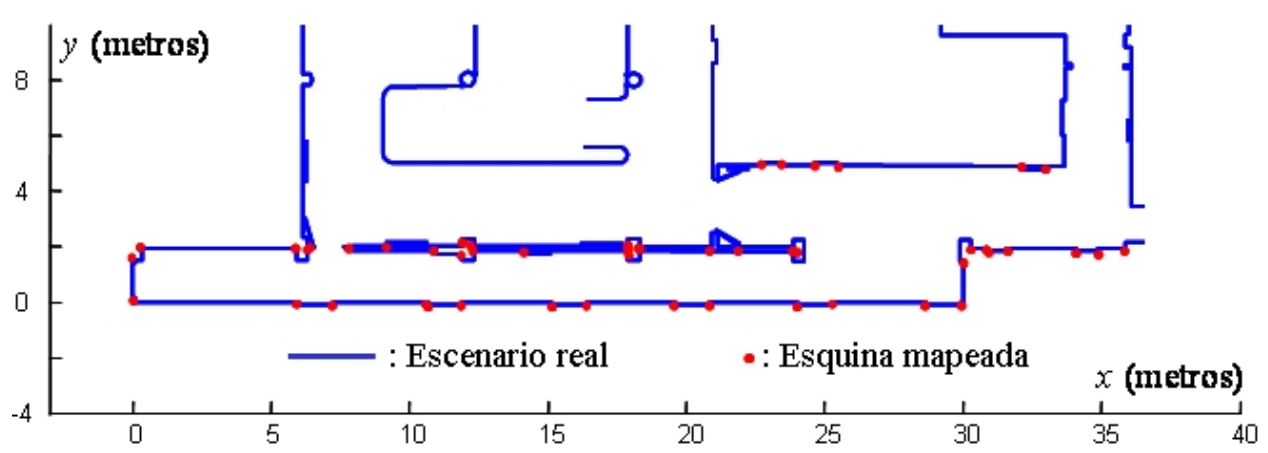

Figura 6.22: Mapa de esquinas extraídas mediante un SONAR rotativo, usando el algoritmo de localización y mapeado simultáneo basado en el EKF

en $y$.

En cuanto a la representación del entorno, el resultado general es el que se muestra en la figura (6.22). Ahí se puede observar el mapa de hitos puntuales (esquinas) que el robot pudo extraer mediante su SONAR rotativo. Con este mapa se demuestra que el algoritmo propuesto es capaz de representar el entorno con una exactitud adecuada, permitiendo así su uso en procesos de localización de robots móviles.

Una imperfección que se observa en el mapa obtenido es que existen algunas esquinas mapeadas con dos hitos distintos (ver figura 6.23). Esto se debe a que el algoritmo de asociación de datos fue ajustado de forma bastante restrictiva a fin de evitar al máximo que surgieran asociaciones de datos erróneas. Una asociación de datos errónea conduce a la pérdida de estabilidad del algoritmo de localización y mapeado simultáneo, mientras que un hito que se agregue demás, siempre podrá ser absorbido por un proceso posterior de fusión de datos a nivel de características.

En la tabla 6.7 se muestra el conjunto completo de los hitos puntuales extraídos durante el proceso de localización y mapeado simultáneo usando el Filtro de Kalman Extendido. También se muestra el error de la localización estimada para cada hito extraído, respecto a la localización real del hito o esquina mapeada. En general, esta 


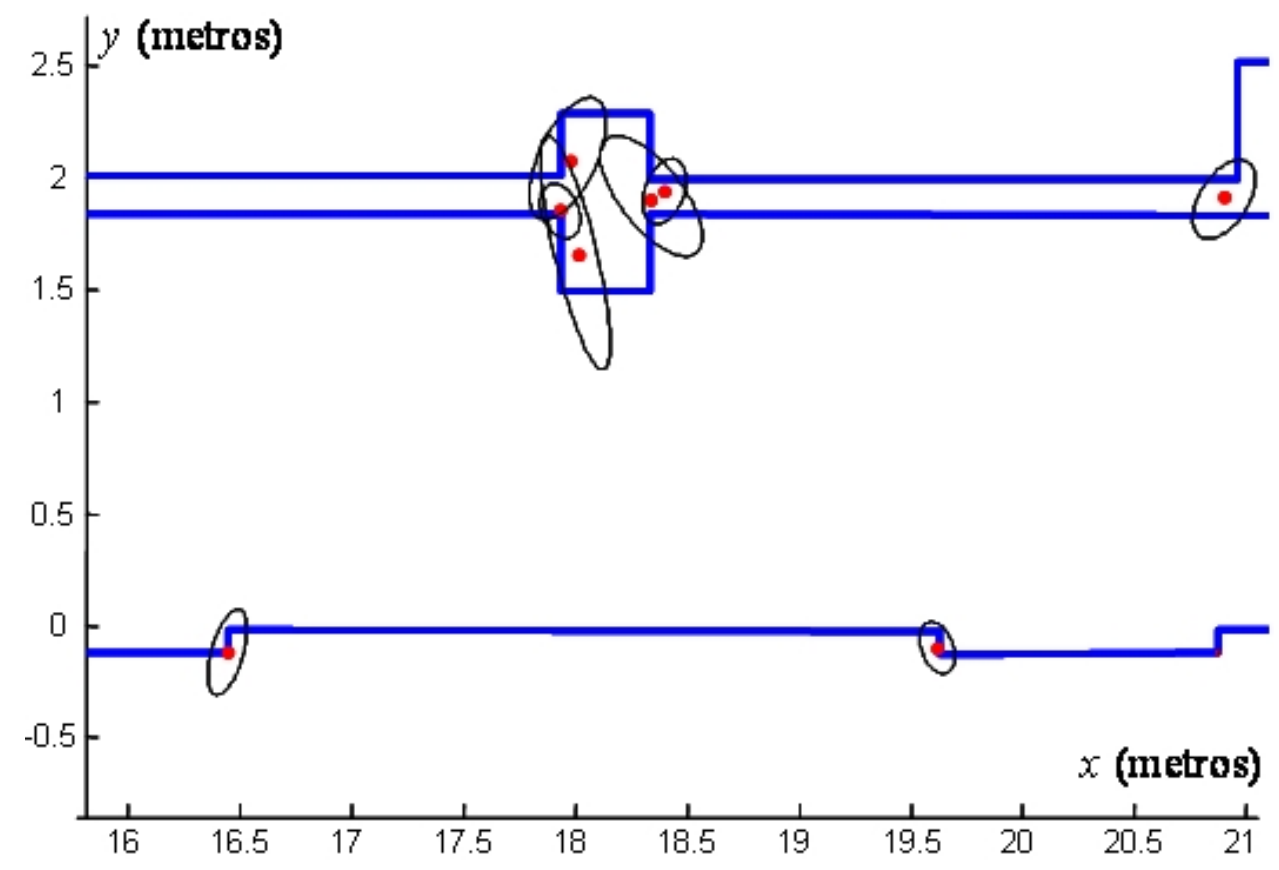

Figura 6.23: Ampliación de una zona del mapa de hitos puntuales extraídas mediante un SONAR rotativo. Nótese que la elipse de error permite representar la incertidumbre en la localización de la esquina. También se puede observar que hay esquinas que pueden ser representadas por más de un hito.

tabla muestra que los resultados obtenidos son muy adecuados para futuros procesos de autolocalización, ya que los errores de estimación se sitúan alrededor de los pocos centímetros. También, en la mencionada tabla se pone de manifiesto una vez más lo marcado del error en la dirección transversal al movimiento del robot(en este caso el eje $y$ ), cuando se compara con el error en la dirección longitudinal al movimiento(en este caso el eje $x$ ).

\subsection{Limitaciones en los métodos ensayados}

A la luz de los resultados obtenidos, se puede afirmar que el Filtro de Kalman es adecuado para el seguimiento de la posición de un robot móvil. No obstante hay situaciones particulares donde el Filtro de Kalman falla. En particular se ha detectado que en los casos de baja frecuencia en la observación de landmark y en el caso del traslado súbito del robot (kidnapping), el filtro no es capaz de realizar una adecuada correspondencia y en consecuencia estima erróneamente su estado.

En los ensayos realizados, el robot observó el entorno aproximadamente cada 3 metros de recorrido y a veces hasta menos. Esto permitió un balance entre la varianza del estimado previo y la varianza en la medición, de forma que la ganancia del filtro permitió la realimentación de una parte prudente de los residuales (innovación), 


\section{CAPÍTULO 6. CONSTRUCCIÓN DE MAPAS Y LOCALIZACIÓN}

ESTOCÁSTICA

Tabla 6.7: Error de localización en varias de las esquinas extraídas en el proceso de SLAM

\begin{tabular}{|r|r|r|r|r|r|}
\hline \multicolumn{2}{|c|}{ Esquinas pertenecientes al entorno } & & \\
\hline Extraídas en el SLAM & Medidas manualmente & Error estimación estado \\
\hline$X$ (metros) & $Y$ (metros) & X (metros) & $Y$ (metros) & Error en X & Error en $Y$ \\
\hline 0.095 & 1.589 & 0.100 & 1.520 & -0.006 & 0.069 \\
\hline 0.153 & 0.050 & 0.100 & 0.000 & 0.053 & 0.050 \\
\hline 0.377 & 1.998 & 0.375 & 1.940 & 0.002 & 0.058 \\
\hline 5.970 & 1.948 & 5.951 & 1.930 & 0.019 & 0.018 \\
\hline 6.014 & -0.062 & 6.050 & -0.110 & -0.037 & 0.048 \\
\hline 6.386 & 1.891 & 6.351 & 1.915 & 0.035 & -0.024 \\
\hline 7.308 & -0.122 & 7.300 & -0.110 & 0.008 & -0.012 \\
\hline 7.882 & 1.930 & 7.945 & 1.942 & -0.063 & -0.012 \\
\hline 10.723 & -0.163 & 10.700 & -0.105 & 0.023 & -0.058 \\
\hline 10.947 & 1.840 & 10.945 & 1.850 & 0.002 & -0.010 \\
\hline 11.931 & -0.104 & 11.950 & -0.120 & -0.019 & 0.016 \\
\hline 11.964 & 2.147 & 11.950 & 2.015 & 0.014 & 0.132 \\
\hline 12.293 & 2.052 & 12.350 & 2.005 & -0.057 & 0.047 \\
\hline 12.359 & 1.853 & 12.350 & 1.840 & 0.009 & 0.013 \\
\hline 15.207 & -0.134 & 15.200 & -0.125 & 0.007 & -0.009 \\
\hline 16.444 & -0.118 & 16.450 & -0.120 & -0.006 & 0.002 \\
\hline 17.931 & 1.850 & 17.935 & 1.840 & -0.004 & 0.010 \\
\hline 17.965 & 2.085 & 17.935 & 2.005 & 0.030 & 0.080 \\
\hline 18.000 & 1.667 & 18.335 & 1.840 & -0.335 & -0.173 \\
\hline 18.339 & 1.917 & 18.335 & 1.995 & 0.003 & -0.078 \\
\hline 19.617 & -0.098 & 19.630 & -0.125 & -0.013 & 0.027 \\
\hline 20.889 & 1.851 & 20.960 & 1.995 & -0.071 & -0.144 \\
\hline 22.779 & 4.957 & 22.789 & 5.005 & -0.010 & -0.048 \\
\hline 23.471 & 4.952 & 23.489 & 5.005 & -0.018 & -0.053 \\
\hline 23.856 & 1.870 & 23.885 & 1.823 & -0.029 & 0.047 \\
\hline 24.044 & -0.161 & 24.070 & -0.120 & -0.026 & -0.041 \\
\hline 24.054 & 1.818 & 23.895 & 1.970 & 0.159 & -0.152 \\
\hline 24.690 & 4.920 & 24.689 & 5.005 & 0.001 & -0.085 \\
\hline 25.310 & -0.065 & 25.310 & -0.120 & 0.000 & 0.055 \\
\hline 25.519 & 4.864 & 25.489 & 5.004 & 0.030 & -0.140 \\
\hline 28.661 & -0.109 & 28.710 & -0.120 & -0.049 & 0.011 \\
\hline 30.067 & 1.406 & 30.055 & 1.449 & 0.012 & -0.043 \\
\hline 30.301 & 1.903 & 30.310 & 1.951 & -0.009 & -0.048 \\
\hline 30.855 & 1.890 & 30.910 & 1.851 & -0.055 & 0.039 \\
\hline 31.631 & 1.837 & 31.610 & 1.851 & 0.021 & -0.014 \\
\hline 34.116 & 1.764 & 34.110 & 1.851 & 0.006 & -0.087 \\
\hline 34.910 & 1.724 & 34.910 & 1.851 & 0.000 & -0.127 \\
\hline 35.836 & 1.838 & 35.900 & 1.951 & -0.064 & -0.113 \\
\hline & & & & & \\
\hline
\end{tabular}


suficiente para mantener la posición del robot acotada alrededor del valor verdadero. Sin embargo, en un caso real es posible que el robot no disponga de mediciones a landmark conocidas en tramos cortos, sino que es posible que pase más de 4 o 5 periodos de actualización para que el mismo pueda encontrar una referencia conocida. En este caso el robot seguirá estimando su trayectoria sólo con el modelo cinemático del mismo, y por lo tanto la incertidumbre irá creciendo sin límites. Así, cuando el robot detecte una landmark del entorno, es probable que el algoritmo no realice una adecuada correspondencia produciéndose un error tipo I (falsos negativos) o un error tipo II(falsos positivos).

El error tipo I se refiere a que se observa una landmark que ya pertenece al mapa, pero que al no lograr la correspondencia, el algoritmo la trata como una nueva landmark y la agrega al mapa existente. Obviamente, este tipo de fallo lleva a una falta de calidad en la representación del entorno, que a la postre influirá perniciosamente en la autolocalización del móvil.

El error tipo II es especialmente perjudicial en la correcta estimación del estado del robot, ya que se refiere a que una landmark observada es asociada de forma incorrecta con una de las landmark ya existentes en el modelo que el robot ha ido construyendo. En este caso de baja frecuencia de observación, se presume que la varianza en el estimado previo sea mucho mayor que la varianza en la medición (que es aproximadamente constante). Así, debido a que en estas condiciones la ganancia del filtro realimentará gran parte de los residuales de la observación, entonces la operación de corrección en el estado del robot alejará la posición estimada de la posición real. Al mismo tiempo, la varianza en este estado corregido caerá a un valor pequeño, impidiendo que a futuro el robot se recupere de este error, aunque en los pasos sucesivos consiga landmark conocidas y haga con ellas una correcta asociación de datos o correspondencia.

Como se comentó anteriormente, el traslado súbito o secuestro, es otra de las situaciones donde el Filtro de Kalman Extendido falla. El problema del secuestro del robot consiste en que el robot "sabiendo donde él está" (incertidumbre pequeña), es trasladado súbitamente a otro lugar pero sin que él se entere. En este caso pueden presentarse dos escenarios: El primero es que cuando lleguen nuevas observaciones de Hitos se comentan errores del tipo I o del tipo II, con lo que los resultados estarían en concordancia con lo descrito en los párrafos inmediatos anteriores. El segundo es que ante nuevas observaciones de Hitos se logre establecer una perfecta correspondencia, con lo que resultarán altos residuales, ya que las mediciones diferirán significativamente de las predicciones en las mismas. En este caso se puede analizar el escenario de acuerdo a lo siguiente:

- Asumiendo que el Filtro de Kalman tenía baja incertidumbre en el estado que ha estimado, la posición estimada después del traslado súbito será incorrecta e inconsistente. Cuando haya observaciones disponibles, el Filtro de Kalman incorporará los altos residuales de la medición en la corrección del estimado del estado, lo que llevará a que la posición estimada se oriente hacia la nueva y real posición que tiene el robot. Sin embargo, puede tomar algún tiempo 
antes de que el estado estimado se ajuste a su correcta posición, ya que eso va a depender de la varianza acumulada en el estimado previo y su relación con la varianza en la medición. Si la varianza en la incertidumbre del estimado previo es relativamente baja, la Ganancia de Kalman incorporará sólo una pequeña proporción de los residuales de la observación. De esta manera el estado estimado permanecerá inconsistente, y la varianza en el mismo permanecerá igualmente en un valor relativamente bajo. A la postre, esto indica que la corrección del estado con suerte se logrará, pero a pasos muy pequeños. Por otro lado, si el robot es capaz de detectar que ha sido trasladado, se pueden tomar las acciones apropiadas para re-localizarse correctamente y de forma rápida. Un posible mecanismo para detectar un traslado súbito, es llevar un registro de los residuales, y en el caso de que se observen unos valores inusualmente altos, se puede inferir que se ha producido un traslado súbito. En este caso se debería modificar externamente la varianza en el estimado previo para hacerla comparable con la varianza en la medición, y de esta forma lograr una ponderación conveniente de las observaciones que se están haciendo. 


\section{Capítulo 7}

\section{Conclusiones, trabajos futuros, $\mathrm{y}$ publicaciones}

\subsection{Sumario y Conclusiones}

La autolocalización de un robot móvil tiene un puntal muy importante que es el sistema de localización odométrica. A pesar de que la experiencia demuestra que la odometría es insuficiente para la localización fiable, la misma es necesaria para obtener una primera aproximación, computacionalmente ligera y a costos reducidos, de la estimación de la posición de un vehículo móvil.

La principal fuente de error en los sistemas de localización odométrica, que están basados en codificadores rotativos acoplados a las ruedas motrices, lo constituye la fallas en el modelado de los parámetros que describen la cinemática del vehículo. Por supuesto el error no surge de la identificación de los valores iniciales de estos parámetros, sino que surgen con los cambios que sufren los mismos a través del tiempo o debido también a los cambios dinámicos que se manifiestan durante la operación normal del vehículo.

La mejora en el hardware de los sistemas de localización odométrica, constituye un avance insuficiente hacia la solución de la problemática de los errores de localización de un vehículo móvil. Por eso en las investigaciones más recientes se registran propuestas de sistemas automáticos para la identificación y calibración de sistemas odométricos.

Parte de las investigaciones realizadas en esta tesis, estuvieron orientadas a diseñar e implementar un sistema de identificación y calibración automática para plataformas móviles de tracción diferencial. De este estudio se ha podido extraer las siguientes conclusiones:

- En un vehículo tipo diferencial, la correcta determinación de la base de giro es un factor fundamental en la reducción tanto de los errores sistemáticos como de los no sistemáticos. La incertidumbre en el conocimiento del diámetro real de las ruedas, tiene influencia más que todo en los errores sistemáticos.

- Los métodos de identificación y calibración automática actualmente propuestos, 
CAPÍTULO 7. CONCLUSIONES, TRABAJOS FUTUROS, Y PUBLICACIONES

incluyendo el UMBmark que no es un método automático, no desacoplan los efectos de la incertidumbre del diámetro de las ruedas y de la base de giro. Esto conduce a que estos métodos arrojen resultados sustentados en aproximaciones. La mayor exactitud en la identificación de los parámetros odométricos se obtiene seleccionando trayectos de excitación que desacoplan los efectos entre los mismos. Los movimientos rectilíneos cortos son trayectorias muy adecuadas para la calibración de un robot diferencial, ya que en el proceso de calibración se desacopla la influencia de la incertidumbre en la base de giro cuando se trata de calibrar la sensibilidad odométrica. Igualmente, la longitud relativamente corta del trayecto favorece el uso de velocidades de bajas a moderadas, lo que a su vez reduce la aparición de valores atípicos producidos por el derrapes de las ruedas motrices. Para identificar el diámetro de las ruedas el trayecto debe ser puramente rectilíneo y con una longitud aproximadamente igual a 2 veces la longitud de la base de giro. Por otra parte, para identificar la base de giro, el trayecto de excitación debe ser puramente angular y aproximadamente igual a $360^{\circ}$.

- La mejor calibración de la base de giro en un vehículo diferencial se obtiene realizando ensayos de operación típica del vehículo. Los trayectos aleatorios utilizados en la calibración de la base de giro, permiten recoger las condiciones reales en las cuales operará el robot, lo que garantiza que los resultados de la calibración estén más ajustados a la realidad. Durante el ensayo se deben registrar la posición inicial y final del vehículo, así como también los pulsos de odometría generado por los sensores. Posteriormente, se debe realizar la identificación aplicando un método de optimización(i.e. mínimos cuadrados) sobre los datos registrados.

- Los valores de sensibilidad odométrica y de la base de giro, muestran corresponder a distribuciones Gausianas. Esto último, a futuro es muy conveniente al momento de representar la incertidumbre en la estimación de la posición de un robot móvil.

El principal problema de la sensorización mediante ultrasonidos es la falta de fiabilidad relacionada a la información angular que ofrecen este tipo de sensores. Estrategias como la propuesta en esta tesis, donde se hace un seguimiento de la amplitud del eco ultrasónico, se convierten en paliativos que combaten el problema de la incertidumbre angular. Sin embargo la técnica más usada por su excelencia, se basa en la fusión de múltiples lecturas provenientes del mismo sensor o en la fusión multisensorial.

Las técnicas de fusión sensorial a nivel de señal, generalmente requieren de modelos sensoriales que ponderen la información que registran los sensores. Esta ponderación suele darse dentro de un marco probabilístico o también dentro de un marco posibilístico. Es por ello que las investigaciones se han orientado hacia la construcción de modelos probabilísticos del sensor de ultrasonidos. El problema con estos modelos ha sido su débil vinculación con la realidad física sensorial del proceso de medición 
de distancia basado en ultrasonidos. Esta falencia causa que la mayoría de los algoritmos de construcción de mapas de ocupación por medio de celdas de grid, originen fallas de representación del entorno. Así, una falla típica surge cuando el sensor está enfocando una esquina, y a consecuencia de la aplicación del modelo típico del sensor, las paredes que conforman la esquina desaparecen, ya que se les asigna como áreas vacías o con muy pocas probabilidades de estar ocupadas.

Los hechos anteriores conducen a la conclusión general de que un modelo sensorial que pondere probabilísticamente las mediciones de distancia hechas con un sensor de ultrasonidos, y que además pueda aplicar un modelo particular al observar las esquinas del entorno, sería uno de lo modelos más adecuados para construir mapas de ocupación de buena calidad.

Además de la conclusión general antes expuesta, de las investigaciones realizadas en este ámbito, se pueden extraer las siguientes conclusiones más particulares:

- Con un SONAR rotativo que tenga capacidad para extraer la amplitud del eco ultrasónico, es posible reducir la incertidumbre angular a valores próximos a los $3^{\mathrm{o}}$.

- Los resultados obtenidos mostraron la utilidad tanto de la fusión Bayesiana como de la fusión de Dempster-Shafer, en el mapeado incremental de grandes áreas. No obstante, a pesar de que los mapas obtenidos son satisfactorios en cuanto al mapeado de las paredes o bordes de los espacios explorados, las celdas que conforman las esquinas son afectadas adversamente por el modelo típico del SONAR. Esto se resuelve integrando al modelo del SONAR, una máscara de esquinas para que la aplicación del mismo no elimine las paredes que forman a éstas.

- Cuando ocurren falsos positivos en la construcción de mapas de ocupación, la fusión Bayesiana tiene efectos más perniciosos que la fusión basada en la teoría de la evidencia(Dempster-Shafer). Lo mismo se debe a que ante el evento de un falso positivo, mediante el modelo sensorial se asignará una probabilidad de ocupación igual a cero, y al fusionar esta información mediante el algoritmo de Bayes, el resultado será cero en ésta y en cualquier iteración que le suceda. En este mismo caso, el algoritmo de fusión basado en la teoría de la evidencia sólo dejará de acumular evidencia relacionada a la zona de ocupación, lo cual podrá ser compensado en una iteración posterior donde la detección sea correcta.

- Mejorar significativamente el modelo sensorial, adicionándole funciones que introduzcan límites pertinentes a la realidad física del proceso de medición sensorial, también mejora la calidad de los mapas obtenidos. Pero en contrapartida a este beneficio, los requerimientos computacionales se ven también significativamente incrementados, lo que hace que se pierda interés en desarrollar estos modelos tan elaborados.

El sensor de infrarrojos es adecuado para procesos de navegación con evitación de obstáculos, debido a que él ofrece altas prestaciones de rapidez y aceptables presta- 
ciones de precisión en lo que se refiere a medición de distancia. Sin embargo, en los procesos de construcción de mapas, el sensor de infrarrojos es poco adecuado debido a su pequeño alcance(aproximadamente 1 metro). Una solución a este problema es la construcción de mapas de infrarrojos en forma incremental, lo que a su vez requiere que se desarrollen esquemas de fusión de datos que sean adecuados para tal fin.

Por otra parte, los mapas de ocupación obtenidos con sensores de infrarrojos son adecuados para navegación, pero para efectos de los procesos de localización, no son adecuados y es necesario construir mapas de mayor abstracción, como por ejemplo los mapas de líneas. Los algoritmos más adecuados para la extracción de líneas a partir de datos de infrarrojos son: Mínimos Cuadrados Recursivos(RLS) y estimación mediante Filtro de Kalman Extendido(EKF). Estos dos algoritmos tienen en común que producen una sobre segmentación del modelo del entorno, lo que sugiere que posterior a su uso, se apliquen procesos de fusión de mayor abstracción a fin de agrupar características que pertenezcan al mismo modelo. En este sentido, las técnicas de agrupamiento y correspondencia propuestas en esta tesis, junto al algoritmo de Mínimos Cuadrados Totales, mostraron ser adecuados para el fin antes descrito.

Las investigaciones alrededor de la temática de los sensores infrarrojos que han sido realizadas en esta tesis, también aportan otras conclusiones más particulares como:

- En la medición fiable de distancias por infrarrojo, conocer el ángulo de incidencia y el coeficiente de reflectividad de la superficie reflectora es fundamental. No obstante, en la construcción de mapas de entorno, el desconocimiento del valor real del coeficiente de reflectividad no es muy fundamental, ya que su aproximación no distorsiona significativamente el entorno modelado, a no ser que se consigan cambios muy abruptos de reflectividad(i.e. superficies brillantes y tipo espejo).

- Los tres algoritmos de extracción de líneas estudiados en esta tesis(RLS, EKF, TRLS), permiten fusionar datos imprecisos de forma óptima y tienen igual desempeño en cuanto a la precisión que ofrecen. Sin embargo, cuando las pendientes son muy elevadas, el método de Mínimos Cuadrados Totales es más robusto.

- Considerando que el período de actualización de datos de una aplicación típica en robótica móvil es de 100 mseg, el tiempo de cómputo de los tres métodos aquí estudiados es adecuado para tal tipo de aplicaciones. Sin embargo es de hacer notar que en cuanto a la velocidad o tiempo de cómputo, el método de Mínimos Cuadrados Generalizados mostró tener mejor índice de desempeño.

- Debido a la gran imprecisión de las mediciones de distancia mediante el sensor de infrarrojos, los mapas de líneas que se extraen con ellos, siempre resultan estar sobre segmentados. El uso de procesos de agrupación y fusión de segmentos de líneas logra reducir significativamente este problema. Un mecanismo que demostró ser muy adecuado para llevar a cabo la fusión, es el de Mínimos Cuadrados Totales. 
Los procesos altamente no lineales involucrados en la autolocalización de un vehículo móvil son muy difíciles de modelar. También, los procesos aleatorios inherentes a la navegación de este tipo de vehículos, le adiciona dificultad al modelado del error en la estimación de la posición del mismo. De aquí que la forma más adecuada para modelar la incertidumbre en los procesos involucrados con la robótica móvil sea usando técnicas probabilísticas.

Cuando se requiere modelar procesos de hipótesis única, como el seguimiento de la posición de un robot móvil, el Filtro de Kalman es una herramienta muy adecuada ya que produce un estimado óptimo del estado del proceso. Para ello, este filtro requiere que se modele la incertidumbre de los procesos involucrados, mediante funciones de distribución Normal o Gausiana.

Otro proceso importante dentro de la operación de un vehículo móvil es la representación del entorno. Gracias a ello es posible corregir la estimación en la localización de este tipo de vehículos. Ahora bien, la observación del entorno, y en algunos casos su modelado, está afectado por un proceso de ruido que es necesario representar. Nuevamente, la representación Gausiana de la incertidumbre, ofrece una manera conveniente de discriminar entre los diferentes objetos del mapa, al momento de ejecutar el proceso de asociación de datos. Este último proceso es fundamental en la autolocalización de robots móviles, cuando se usan Filtros de Kalman.

De las investigaciones realizadas alrededor de esta temática, se extrajeron las siguientes conclusiones:

- En la robótica móvil, la representación Gausiana es adecuada para modelar procesos de hipótesis única. De esta forma se puede representar el estado de un robot o de un mapa del entorno, mediante el primer y segundo momento estadístico. Esto trae la ventaja de que la propagación del estado y su covarianza, así como su combinación con otras funciones del mismo tipo, darán como resultado otra distribución Gausiana.

- A pesar de que el modelo vehicular de un robot móvil es altamente no lineal, la propagación del ruido a través de la linealización del modelo cinemático del mismo, logra representar adecuadamente la incertidumbre en la posición del vehículo. Esta incertidumbre es representada mediante una distribución Normal bivariante.

- Cuando se utilizan codificadores rotativos como fuente de datos para la estimación de la posición de un vehículo de tracción diferencial, el modelo de varianza en los codificadores $\left(\sigma^{2}=k^{2}|\Delta S|\right)$ permite representar el ruido en el vector de excitación, lo que a la postre representará la incertidumbre en la posición del vehículo. Un valor de $k^{2}$ que permite representar esta incertidumbre de forma muy prudente es $1 \times 10^{-4}$.

- El ruido sensorial en la determinación de la localización de paredes y esquinas extraídas con un SONAR tipo rotativo, es perfectamente modelado mediante distribuciones Normales bivariantes en las coordenadas $\langle\rho, \varphi\rangle$. 
- Los entornos interiores son adecuadamente representados mediante hitos puntuales(esquinas) y características tipo lineales(estructuras planas y paredes), teniendo así la ventaja de requerir menos memoria que un mapa de ocupación, al igual que la ventaja de no sufrir del problema de discretización que sufren los mapas de rejilla.

- Los modelos de observación de paredes y esquinas están íntimamente relacionados con la posición del vehículo que porta al sensor, y esto a su vez es muy conveniente para la corrección de la localización del mismo.

- La representación del entorno mediante el modelo Hessiano para líneas infinitas, simplifica el proceso de localización de un robot móvil, al mismo tiempo que reduce las operaciones computacionales requeridas.

- El método de correspondencia propuesto en esta tesis es muy adecuado para completar la operación de asociación de datos, porque la misma se basa en la evidencia estadística que modela incertidumbre en la medición del sensor.

- Cuando los hitos o landmark están muy cercanos entre sí y existen desviaciones significativas en la estimación de la posición del vehículo que porta el sensor, es muy factible que se cometa un error en la asociación de datos . El aumento en la frecuencia de corrección de la posición estimada del mismo, evita considerablemente este tipo de error en la asociación de datos.

Las imperfecciones en los actuadores y sensores, y las diferentes fuentes de ruido que afectan a un vehículo móvil, convierten a las distintas técnicas de estimación en la herramienta por excelencia para resolver el problema de localización y de representación del entorno en las aplicaciones de robótica móvil. Debido a su precisión, robustez y alta practicabilidad computacional, el Filtro de Kalman Extendido - EKF, es uno de los métodos más adecuados para la estimación de la posición del móvil, así como también para la consolidación de un modelo del entorno donde se encuentra el mismo.

Dentro de las aplicaciones del Filtro de Kalman en la robótica móvil, se destacan 3 casos particulares: Construcción de mapas, Localización de un móvil, Construcción de mapas y localización en forma simultanea. El primer caso corresponde a estimar las localizaciones de cada uno de los objetos $\mathbf{m}_{i}$ que conforman un mapa $\mathbf{M}$, dando por hecho que se conoce perfectamente la localización del vehículo que porta el sensor. La Localización corresponde al problema de estimar el estado $\langle x, y, \theta\rangle$ de un vehículo móvil, dando por hecho que se conoce perfectamente el mapa $\mathbf{M}$ del entorno. El tercer caso, conocido como SLAM, es un problema de estimación más complejo ya que no se conoce de manera perfecta el estado del vehículo, ni el estado de los objetos del mapa.

En general, en este trabajo se demostró que la localización basada en hitos puntuales y también en segmentos de líneas, usando el EKF, ofrece muy buena precisión. No obstante, otras conclusiones que se pudieron extraer son: 
- La capacidad de construcción y mantenimiento de una representación del entorno es fundamental para dotar de autonomía a un robot móvil. Cuando se utliiza el EKF como método de mapeado, la incertidumbre en cada uno de los objetos que constituyen el mapa disminuye monotonicamente con cada observación que de ellos se realice.

- En los ensayos de localización realizados, el hecho de que el móvil se mantuviera localizado durante todo el trayecto ensayado, confirmó que las asunciones básicas de independencia, linealidad aproximada, ruido y errores Gausianos; son adecuados para la localización basada en el EKF. También se pudo confirmar que el método EKF es robusto ante las ligeras violaciones de estas condiciones.

- La exactitud de la localización basada en el EKF estuvo dentro del rango de los $4 \mathrm{~cm}$ para la dirección longitudinal al eje del robot(eje $x$ ), dentro del rango de los $6 \mathrm{~cm}$ para la dirección transversal al eje del robot(eje y), y dentro del rango de $1^{\circ}$ para el ángulo de acimut u orientación del mismo. Pero a pesar de estos buenos resultados, también se determinó que la baja discriminación espacial entre los objetos del mapa, causa errores de asociación de datos, y con ello surgen fallas en la corrección de la localización del móvil.

- Las principales causas de fallas en la asociación de datos son:

1. Fuerte violación de los modelos del sistema o de la medición. Por ejemplo los derrapes y colisiones del robot afectan de manera muy severa a la estimación odométrica del estado, y como usualmente esto no es observable, entonces el error en la estimación del estado puede causar fallas severas en la asociación de datos.

2. La poca discriminación espacial de los objetos del entorno. Por ejemplo, cuando la distancia entre los objetos del entorno es comparable con el error de estimación acumulado, pueden surgir fallas en la asociación de datos.

- Con la metodología básica del SLAM se elimina la restricción general de tener que conocer el mapa del entorno, pero a costa de una elevada carga computacional. Esto hace que el SLAM básico sea poco practicable en sistemas de tiempo real estrictos

\subsection{Trabajos futuros}

En el capítulo 3 se demostró la utilidad del método para la identificación y calibración automática de vehículos diferenciales propuesto en esta tesis. Como se expuso, este método integra una serie de sensores entre los que se encuentran un medidor por ultrasonidos, un medidor de distancia por infrarrojo, una brújula o compás, y un par de codificadores rotativos. Estos sensores participan de manera coordinada en la identificación y la calibración de los parámetros cinemáticos que conforman el 
modelo de movimiento de un vehículo de tracción diferencial. Como trabajo futuro se propone extender esta metodología para aplicarla en otro tipo de configuración de vehículo con ruedas, como por ejemplo triciclos y vehículos de ackerman. Para otras configuraciones con locomoción a patas, como robot bípedos o cuadrúpedos, se propone utilizar métodos de identificación paramétricos, como el basado en el Filtro de Kalman Extendido, para identificar el modelo cinemático aproximado de los mismos.

En la sección 4.1.4 y 4.1.5, se propusieron modelos que se adaptan muy bien al proceso físico de medición de distancias usando un sensor de ultrasonidos tipo rotativo, como el usado por el robot YAIR. El problema con estos modelos tan elaborados es que la computación de las funciones de asignación de probabilidad representa una carga computacional importante debido sobre todo a las funciones exponenciales que se deben evaluar. Considerando que esto puede ralentizar las demás operaciones del robot, como trabajo futuro se propone pre-computar el modelo sensorial, evaluándolo a varias distancias y ángulos típicos, y luego aplicar el modelo a cada una de las celdas del mapa de rejilla que se ven afectada por el cono de detección. Así, la asignación básica de probabilidad de cada celda se llevará a cabo mediante tablas de interpolación o mapas de funciones(Look Up Tables).

La diversidad de materiales que componen las superficies de un entorno típico de oficinas, disminuye la fiabilidad de las mediciones de distancia realizadas con el sensor de infrarrojos. Para disminuir los efectos de este problema, se propone desarrollar un algoritmo que fusione los datos de distancia obtenidos con un sensor de ultrasonidos, con los datos de distancia obtenidos con un sensor de infrarrojos, para determinar dinámicamente el coeficiente de reflectividad de las superficie reflectora que está siendo observada por los sensores. Esta propuesta conducirá a un algoritmo adaptativo que tendrá una capacidad mejorada para la extracción de distancia mediante técnicas de infrarrojos.

En la sección 4.2.5 se demostró que los mapas de líneas obtenidos con los sensores de infrarrojos del robot YAIR, representan fiablemente el entorno, lo que los convierte en modelos adecuados para procesos de localización de vehículos móviles. Como trabajo futuro se pretende implementar un algoritmo basado en el Filtro Kalman Extendido, para la extracción de líneas y localización simultánea a partir de los datos suministrados por este tipo de sensores.

La principal causa para que un robot se encuentre "perdido" dentro de un entorno modelado por un mapa perfectamente conocido, es la incorrecta asociación de datos. Ya se concluyó que las fallas en la asociación de datos se deben principalmente a la baja discriminación espacial entre los objetos que modelan el entorno. Como trabajo futuro se pretende desarrollar métodos de correspondencia con nivel de abstracción más alto, como por ejemplo uno que use redes de decisión Bayesianas y que al mismo tiempo pueda tratar problemas de múltiples hipótesis, de forma tal que se pueda disminuir este tipo de problemas.

Los métodos de seguimiento de una hipótesis única, como el basado en el EKF, frecuentemente pueden re-localizar un vehículo que se encuentra "perdido", siempre que su estimado de posición se encuentre muy cerca de su posición real. A futuro es fundamental dotar al vehículo con un sistema de posicionamiento global o absoluto, 
como por ejemplo uno que esté basado en la triangulación usando hitos puntuales naturales. Ahora bien, dado que en cada ciclo de corrección, usualmente no se suelen observar más de 2 de estos hitos puntuales, se pretende desarrollar un algoritmo multi-hipótesis que pueda resolver recursivamente el problema de localización global en el término de dos ciclos de corrección.

\subsection{Publicaciones relacionadas a esta tesis}

En esta sección se expone el resumen de los trabajos relacionados con esta tesis, y que ya han sido publicados, expuestos o aceptados en revistas y congresos especializados en la temática tratada.

\section{Artículos en revistas}

- Danilo Navarro, Ginés Benet y Félix García. A direct odometric calibration method focused on improving differential-drive robots localization. Aceptado para ser publicado en la revista Ciencia e Ingeniería. ISSN 1316-7081. Universidad de los Andes. Venezuela.

In spite of disadvantages of the odometric localization, it is widely used in mobile robots positioning system. Accuracy on the parameters of the kinematics model improves the localization results and contributes to reduce the operational costs. This paper proposes a simple method for differential-drive robot odometry calibration, where no special instruments are required. This is a two steps method: odometric sensibility and wheel-base calibration. Aimed to sensibility determination, we propose a straight-line path which uncouples the effect of the wheel-base uncertainty in this test. Aimed to wheel-base calibration, we propose a random path which takes into account the not-modelled dynamic effects and realistic conditions for robots operation. Particularly, this wheel-base is determined by mean of a optimization technique of the localization error applied to the executed path. The statistics procedure used in the proposed method, allows the estimation of the kinematics parameters variances, which ones are necessary in the stochastic robot localization processes. After we ran the calibration method on a differential-drive robot, the results shows that the localization system already calibrated, has a good capacity to estimate actual robot position, with errors below $10 \%$ in path 40 meters long including 90degrees turns on the spot.

- Danilo Navarro, Ginés Benet y Luis Ríos. Stochastic modelling of rotating SONAR: a study case on point-type landmark probabilistic mapping. Scientia et Técnica. año XV, No 41.pp. 197-202. Mayo 2009.

Ultrasonic sensing is a well suited cost-benefit technique used in mobile robotics. Nevertheless, this sensor type has a key drawback: its very Wide beam. This fact causes that this sensor becomes useless in the environment map building process. Another problem attached with this sensor is that it offers very 
little facilities for landmark extraction and object recognition. This problem is known as Matching. In this paper we introduce an ultrasonic sensor capable to extract wall or corner from an indoor environment, as well as to reduce the angle uncertainty, on the based of echo amplitude reaching the transducer. Moreover, we developed the stochastic model for this sensor as well as a matching algorithm for using this device as main observation element for probabilistic SLAM (Simultaneous Localization And Mapping).

- Danilo Navarro, Ginés Benet y Luis Ríos. Improving odometry accuracy in a differential-drive robot by correcting systematic errors. Scientia et Técnica, año XIII, No 37. pp 257-262. Diciembre 2007.

In spite of disadvantages of the odometric localization, it is widely used in mobile robots positioning system. Accuracy on the parameters of the kinematics model improves the localization results and contributes to reduce the operational costs. This paper proposes a simple method for differential-drive robot odometry calibration, where no special instruments are required. The localization system thus calibrated, showed a good capacity to estimate actual robot position, with errors below $10 \%$ in path 40 meters long including 90-degrees turns on the spot.

- Danilo Navarro, Hernando Parra y Luis Ríos: Ultrasound sensors used in mobile robotics for range measurement. Scientia et Técnica, año $\mathrm{X}, \mathrm{N}^{\mathrm{o}}$ 25. pp 35-40. Octubre 2004.

In this paper we describe the use of ultrasonic sensors for range measurements in mobile robotics application and for the construction of environment maps. Also we show the results obtained for the rotating sonar module we designed.

\section{Artículos de ámbito internacional}

- Danilo Navarro y Ginés Benet. Magnetic Map Building for Mobile Robot Localization Purpose. Aceptado en the 14th IEEE Conference on Emerging Technologies and Factory Automation (ETFA2009). September 22-26, 2008. Palma de Mallorca, España.

Robotic mapping addresses the problem of modeling environments through mobile robot's sensors. The Resulting maps are commonly used for robot localization and navigation. Using a compass in mobile robotics is not frequently considered because it has a significant disadvantage: the data it provides can be easily contaminated by surrounding electromagnetic noise or by large ferrous structures. This makes the compass unreliable for heading determination in indoor environments. This paper addresses construction of an environmental magnetic map with a mobile robot, and how to use such map to determine the robot's local heading. We present a novel approach that characterize indoor environments as a magnetic field function. A mobile robot gathers data from a low cost compass as it moves around. This method models the robot's working 
area by means of a simple representation which is time persistent and does not require specialized sensors. Thus, we propose a methodology based on 2D interpolation aimed at determining the robot's heading on the working area. Real test results showed the method is suitable for the partial correction of the robot's position.

- Danilo Navarro, Ginés Benet y Félix García. Auto-Localización de Robots Móviles Usando el Filtro de Kalman: Un Caso Basado en Hitos Puntuales Extraidos con un SONAR. XIII Congreso Latinoamericano de Control Automático CLCA2008. Noviembre 25-28, 2008. Mérida - Venezuela.

La auto-localización, lo cual es el proceso mediante el cual un robot estima su posición respecto a un sistema de coordenadas fijo, es una de las capacidades básicas que debe poseer un robot móvil. Para auto-localizarse, un robot debe buscar referencias externas usando medios tales como sensores de ultrasonido, sensores láser, cámaras de video, etc. Dentro de estos, los sensores de ultrasonido son populares debido a su bajo costo, sin embargo su uso en procesos de localización de robot móviles es poco común ya que este sensor es poco fiable en cuanto a la información que proporciona respecto a la posición angular de los objetos que con él se detectan. En este trabajo enfocamos el problema de la auto-localización de robots móviles usando sensores de ultrasonido, y proponemos el uso de un SONAR (SOund NAvigation and Ranging) que es capaz de reducir la incertidumbre angular basándose en una estrategia de seguimiento de la amplitud del eco reflejado. También, para mejorar el desempeño del proceso de localización en robots móviles, proponemos usar un Filtro Extendido de Kalman para fusionar los datos odométricos con los datos del SONAR propuesto . Los ensayos realizados en un entorno de oficinas mostraron que el sistema de localización propuesto reduce significativamente la incertidumbre en la estimación de la posición del robot. Los errores totales obtenidos estuvieron por debajo de los $5 \mathrm{~cm}$ en cuanto a la estimación de las coordenadas $\langle x, y\rangle$ del centro robot, mientras que el error en la estimación de la orientación angular del mismo estuvo por debajo de 1 grado.

- Danilo Navarro, Ginés Benet y Francisco Blanes. Line-based Incremental Map Building Using Infrared Sensor Ring. Proceedings of the 13th IEEE Conference on Emerging Technologies and Factory Automation (ETFA2008). September 15-18, 2008. Hamburg, Germany.

Geometric representation of the environment, known as mapping, plays an important role in mobile robotics as it support various tasks such as localization, path planning and motion control. Line models represent a popular approach to represent the geometric features of the environment. The mapping problem gives rise to a variety of solutions for using different exteroceptive sensors (sonar, infrared, laser, vision, etc.). One of the possible choices is to use an infrared sensor (IR sensor). These devices are inexpensive, practical and widely available. 
Infrared sensors are largely used in obstacle avoiding processes because they are fast and cheap, and require only simple signal processing. Although the collision avoidance routine has received good attention for many years, the infrared feature mapping has been neglected. A problem with this kind of sensor is its short range (about one meter). This problem forces to an incremental mapping approach, so the robot has to move and concurrently model the environment. In this paper we present an IR sensor able to measure distances based on the light that is back-scattered from objects. This IR sensor is used for line-based map building purposes, so we also present a methodology for line extraction, range data clustering and line segmentation. The experimental test has been carried out in a long corridor, and results show the usefulness of the IR sensor and the methodology we propose for feature extraction and line-based mapping processes.

- Danilo Navarro, Ginés Benet y Félix García. Phisically-Based Ultrasonic Modelling for Improving Dempster-Shafer fusión Results in SONAR Map Building. Proceedings of the IX International Congress on Numerical Methods in Engineering and Applied Sciences. pp. EC 61 - 66 . Marzo, 2008. Isla de Margarita - Venezuela.

Grid-type representations for sensor based map-building are popular in mobile robot applications because they are computationally easy to maintain and allow for integration of data from different sensors. In this paper we consider the problem of building the map of an unknown environment by using range readings obtained through ultrasonic sensors. Ultrasonic mapping is nontrivial due to several sources of uncertainty. Main sources are spurious reading due to specular reflections and obstacle location uncertainty due to the beam width of the sensor. In order to cope with this problem, we use a Dempster-Shafer evidential reasoning approach to fuse the information provided by a rotating SONAR. Although the underlying theory has been applied and reasonable results have been obtained, to improve the map through the efficient use of the sensory information have been neglected. We propose a physically-based SONAR model aimed to improve the results of the Dempster-Shafer data fusión process. This process is applied on a two-dimensional occupancy grid where each grid has an inherent uncertainty that is modelled in a possibilistic way. The sensor model we propose is especially suitable to estimate de basic probability assignment required by the Dempster-Shafer data fusión because in this model we consider the sensibility limit obtained experimentally. The map building system is experimentally evaluated using sonar data from real environment obtained from SONAR in a long corridor. Result shows maps highly precise.

- Danilo Navarro y Ginés Benet. Evaluating Line Extraction Algorithms for Infrared Feature Mapping in Mobile Robotics. Proceedings of the IX International Congress on Numerical Methods in Engineering and Applied Sciences. pp. EC 25 - 30 . Marzo, 2008. Isla de 


\section{Margarita - Venezuela.}

Geometric representation of the environment, known as mapping, plays an important role in mobile robotics as it support various tasks such as localization, path planning and motion control. A popular approach to represent the geometric features of the environment is by mean of line models. The mapping problem gives rise to variety solutions of using different exteroceptive sensors (sonar, infrared, laser, vision, etc.). One of the possible choices is to use infrared sensor. These devices are inexpensive, practical and widely available. Infrared sensors are largely used in obstacle avoiding processes because they are fast and cheap devices that require simple signal processing. Although the collision avoidance routine has been focused for many years, the infrared feature mapping has been neglected. A problem with this kind of sensor is its short range (about 1 meter). This forces to an incremental mapping problem, so the robot has to move and concurrently model the environment. Since Kalman filtering enables to fuse sensor data of a single sensor measured at successive time intervals, in order to cope with the problem above, we use a Kalman Filter approach to line extraction and following. Furthermore, this paper presents an experimental evaluation of two different techniques for line extraction on 2D point obtained from infrared measurement in indoor environment. Several comparison criteria are proposed and discussed to highlight the advantages and drawbacks of each algorithm, including speed, complexity, correctness and precision. The results of the algorithms are compared with the ground truth using standard statistical methods. The applicability of line mapping algorithm was demonstrate by experiments performed on real data collected in a long corridor in an office environment. The resulting maps are highly accurate and suitable for robot localization.

- Danilo Navarro, Ginés Benet y Milagros Martinez. Line Based Robot Localization Using a Rotary Sonar. Proceedings of the 12th IEEE Conference on Emerging Technologies and Factory Automation (ETFA2007). September 25-28, 2007. Patras - Greece.

Ultrasound sensors, despite their low cost, are less common than other sensors used for robot localization. This is because its main drawback: The lack of reliable bearing information. We introduce a rotary ultrasonic sensor able to extract features in a $360^{\circ}$ scan and classify them as wall or corner. For robot localization purposes, these features are matched with a set of linefeatures stored on a priori known map. The localization process is carried out through an Extended Kalman Filter that fuses data provided by a dead-reckoning system with measurements data provided by the rotary sonar. This paper also presents techniques we use to classify the detected features. The experimental test carried out in a long corridor shows the usefulness of the rotary sensor for feature extraction and robot localization process. The results show that localization error is in subcentimeter range. 
CAPÍTULO 7. CONCLUSIONES, TRABAJOS FUTUROS, Y PUBLICACIONES

- Danilo Navarro y Ginés Benet. An automatic identification-calibration method for differential-drive robot odometry. Proceedings of the 2nd International Conference on Automation, Control and Instrumentation. IADAT-aci2006. pp. 63-68. 5-7 July, 2006. Valencia-Spain.

Mobile robot position can be determined by absolute or relative measurements. The relative positioning method more frequently used is the odometric dead reckoning, which is provided by integration of the information given by -usuallytwo wheel's encoders. This operation results in a drift in the robot position data that is strongly influenced by wrong parameters estimates in the odometric model. Therefore, the odometric parameters have to be identified and calibrated with suitable precision. Approaches to the calibration process are diverse: geometric-based calibration methods, which need a special excitation path; methods that rely on multisensory data fusión, offline and online calibration methods. This paper proposes a low cost automatic approach for identification and calibration of kinematic parameters in a differential-driven robot. The approach is a two-step process: identification and calibration. As a first step, wheel's encoder sensitivity ( $\mathrm{mm} / \mathrm{pulse}$ ) and wheelbase are identified. The second step is a calibration process that takes the previous results and combines them with the differencial-drive geometric model set to determine the parameters's most probable values. This process is carried out through a set of systematic tests that include robot movement and reading of its propioceptive and exteroceptive sensors. In addition, the algorithm filters out the outliers by means of a statistical filter based on the error covariance observation. An important innovation that the proposed method introduces is the capability to identify odometric parameters without having prior knowledge of their nominal value. Parameters values obtained by means of the identification process are very near to real values of the robot's odometric parameters. The resulting error obtained in real tests was smaller than $2 \%$.

- Danilo Navarro y Ginés Benet. Mobile Robots Localisation Using Magnetic Field Modelling. Proceedings of the 2nd International Conference on Automation, Control and Instrumentation. IADAT-aci2006. pp. 84- 88. 5-7 July, 2006. Valencia-Spain.

Robotic mapping addresses the problem of modelling environments through mobile robot's sensors. Resulting maps are commonly used for robot localization and navigation. Using compass in mobile robotics is not much considered because it has an important disadvantage: Data that it provides can easily be contaminated by surrounding electromagnetic noise or by big ferrous structures. In practice, this causes compass becomes unreliable for heading determination in indoor environment. This paper addresses the task of constructing an environmental magnetic map with a mobile robot, and how to use such map to determinate robot's local heading. We present a novel approach that characterize indoor environment as a magnetic field function. To do this, a mobile robot 
gathers data from a low cost compass while it is moving around. By applying this method, the robot's working area is modelled by means of a simple representation that is time persistent and does not require of specialized sensors. Thus, we propose a methodology based on $2 \mathrm{D}$ interpolation that is aimed to determine the robot's heading into the working area. Real test results showed the suitability of the method for the partial correction of the robot's position.

\section{Artículos de ámbito nacional}

- Danilo Navarro y Abelardo Rodríguez. Estimador de Estado de Bajo Costo para la Auto-Localización Relativa de Robots Móviles. XXIII Congreso Nacional de Instrumentación - SOMI XXIII. Octubre, 2008. Xalapa, Veracruz - México.

La auto localización absoluta de un vehículo autónomo requiere de hardware costoso o de algoritmos computacionales complejos. La auto localización relativa es generalmente más económica pero en contrapartida es menos precisa. Una forma usual de implementar la localización relativa es observando las velocidades de las ruedas mediante codificadores ópticos acoplados a las mismas, y estimando la posición del móvil a partir del modelo cinemático del mismo. Esta operación conocida como dead-reckoning, es simple, de bajo costo computacional y fácil de implementar en tiempo real; pero presenta la desventaja de que acumula error sin límites debido a factores como las no linealidades no contempladas en el modelo del sistema, los derrapes y el imperfecto acoplamiento de las ruedas con la superficie del plano del movimiento. En este trabajo se propone un sistema de localización odométrico de bajo costo que logra disminuir sensiblemente el efecto de los factores que causan los errores en la odometría. El sistema está basado en 2 codificadores incrementales que van acoplados al cuerpo del móvil, de forma independiente a las ruedas motrices del mismo. También, un controlador programable de circuito integrado(PIC) realiza la estimación del estado completo del móvil a partir de los datos proporcionados por estos codificadores, y además ofrece el soporte para la comunicación e integración con otros sistemas. El sistema propuesto fué implantado en una plataforma robótica móvil cuyo error de estimación odométrica original estaba alrededor de 5\%; lográndose reducir el mismo a un valor por debajo del $1 \%$. 



\section{Apéndice A}

\section{Localización de Markov}

El problema general de localización se puede describir como un problema de estimación Bayesiana $[35,57]$ en donde se desea estimar la localización de un robot dado un conjunto de medidas ruidosas. Observando el problema de forma probabilística, se puede decir que en base a toda la información disponible el robot tiene cierto grado de confianza o certidumbre(Belief) sobre el lugar donde se encuentra localizado. Entonces el problema de la localización consiste en estimar la densidad de probabilidad de localización del robot sobre el espacio de todas las posibles localizaciones del mismo. Un marco Bayesiano que estima esta densidad es el de la Localización de Markov[75], el cual combina la información proveniente de medidas sensoriales absolutas y relativas para obtener la densidad de probabilidad ya antes mencionada.

\section{A.1. Certidumbre o Confianza(Belief $)$}

El robot tiene cierta confianza acerca del lugar donde se encuentra. Ésta es descrita por la densidad de probabilidad sobre todas las localizaciones $x$ del robot, lo cual usualmente se conoce como confianza o certidumbre y se denota como $\operatorname{Bel}(x)$. Sea $\mathbf{X}$ una variable aleatoria que puede tomar diferentes valores $x$, caracterizando cada uno de ellos un posible estado (posición) del robot. De esta forma, la secuencia $\left\{x_{1}, x_{2}, \ldots, x_{k}\right\}$ representa los estados del robot en sucesivos instantes de tiempo. En cada instante $k$, el robot obtiene una observación $z_{k}$ del entorno. Así, la localización del robot en un instante $k$ se expresa como,

$$
\operatorname{Bel}\left(x_{k}\right)=P\left(x_{k} \mid z_{0: k}\right)
$$

La ecuación A.1 representa la probabilidad de que el robot esté en $x_{k}$, dada toda la información o datos $z$ hasta el instante $k$. Esta información no sólo incluye los datos de los sensores propioceptivos y exteroceptivos sino que también puede incluir la información de un mapa a priori. Dada la distribución $\operatorname{Bel}(x)$, la localización $x_{k}$ que tenga la más alta confianza, es la localización en la que más probablemente se encuentre el robot. Así, el método de Localización de Markov (o Bayesiano) consiste en 


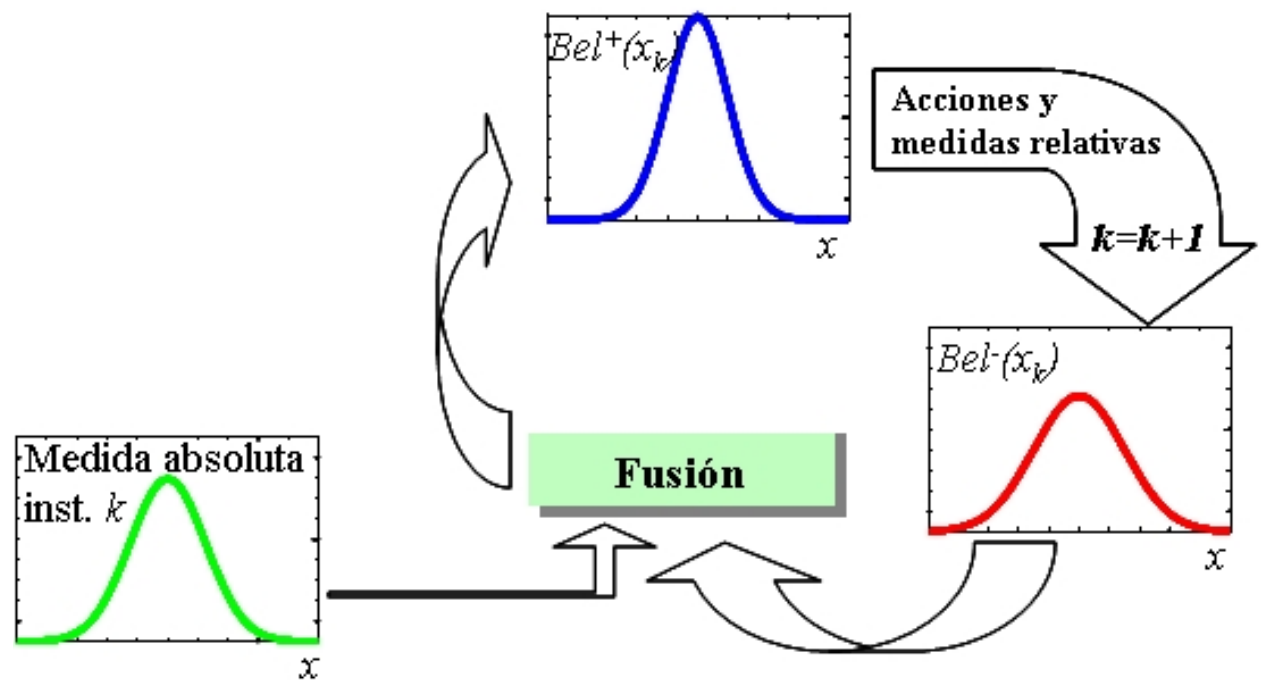

Figura A.1: Ciclo de incremento y actualización de la confianza(Belief).

obtener, para cada instante $k$, una distribución de probabilidad sobre la variable aleatoria $\mathbf{X}$ que caracterice, para cada valor particular $x$ de la variable, su probabilidad de corresponder al estado actual del robot.

La estimación de la confianza $\operatorname{Bel}(\mathbf{X})$ en cada instante se realiza mediante una formulación recursiva que consta de dos componentes: la confianza a priori $\mathrm{Bel}^{-}\left(x_{k}\right)$ la cual es la que resulta después de incorporar toda la información propioceptiva(relativa) y exteroceptiva(absoluta) hasta el instante $k$, sin incluir las medidas exteroceptivas de ese instante; y la confianza a posteriori $\mathrm{Bel}^{+}\left(x_{k}\right)$ que es la que resulta después de agregar justamente las medidas absolutas tomadas en el instante $k$.

\section{A.2. Modelo probabilístico de actuación del robot}

Un robot que navega en su entorno va realizando acciones para cambian su posición dentro del mismo. Si se define $u_{k}$ como la acción realizada por el robot en el instante $k$, un modelo probabilístico que expresa la manera como cambia la localización del robot es la densidad de transición,

$$
p\left(x_{k} / x_{k-1}, u_{k-1}\right)
$$

Esta densidad modela la probabilidad de que el robot alcance el estado $x_{k}$, dado que estando en el estado anterior $x_{k-1}$, se realizó la acción $u_{k-1}$. Es decir, la densidad de transición describe cómo las acciones del robot cambian su localización. Esta densidad por lo tanto se conoce como el modelo de actuación o del movimiento, y en la práctica, la misma se puede aproximar a partir de la cinemática del robot. 


\section{A.3. Modelo probabilístico de medición}

Sea $Z$ el conjunto de todas las medidas posibles provenientes de un sensor, y sea $z_{k}$ un elemento de $Z$ observado en el instante $k$. Se puede describir la probabilidad que un sensor observe $z_{k}$ desde una cierta localización $x_{k}$ mediante la densidad[199],

$$
p\left(z_{k} / x_{k}\right)
$$

la cual se conoce como el modelo perceptivo.

Esta densidad de probabilidad es difícil de calcular debido a la alta dimensionalidad de las medidas sensoriales. Sin embargo una posible solución es propuesta por el mismo Thrun[191], quien usa un extractor de características basado en el algoritmo de $k$ - nearest neighbor para proyectar el conjunto de datos sensoriales brutos del espacio $S$ a un vector de características de menor dimensionalidad en un espacio $Z(\sigma: S \rightarrow Z)$. De esta forma la densidad de probabilidad representada en la ecuación A.3 se toma en base a los vectores de características $Z$ en lugar que sobre los datos en bruto del sensor $Z$. Un ejemplo de la técnica propuesta se encuentra en los métodos de localización basados en Landmarks[95], donde un vector de características que es extraído de los datos sensoriales puede contener sólo la ausencia o presencia de Landmarks, omitiendo así el resto de la información contenida en el flujo de datos sensoriales. Otro ejemplo es el de los métodos de localización basados en la correspondencia de modelos (Model Matching)[22], donde a partir de los datos sensoriales se extraen modelos parciales tales como los mapas de características, los cuales son entonces comparados con un modelo de los existentes en el entorno, para en lo siguiente usar sólo el resultado único de esta comparación en el proceso de localización. De lo anterior se ve que la densidades así obtenidas no relacionan el flujo de datos en bruto del sensor con las diversas localizaciones del entorno, sino que más bien relacionan a los vectores de característica con las mismas.

\section{A.4. Algoritmo de Localización de Markov}

Para iniciar el algoritmo, se supone que al inicio el robot cuenta con una confianza inicial respecto a su localización $\mathrm{Bel}^{+}\left(x_{0}\right)$. A cada instante $k$, el robot realiza una acción $u_{k}$ que finaliza cuando se inicia el próximo espacio temporal. Esta acción cambia el estado del robot de acuerdo a la ecuación A.2, por lo que su confianza evoluciona hasta $\mathrm{Bel}^{-}\left(x_{k}\right)$. Cuando el robot se mueve, la distribución de probabilidad se actualiza para tomar en cuenta la incertidumbre adicionada en la posición del robot debido por ejemplo a los errores de odometría. La confianza que el robot tiene después de haber incorporado la acción $u_{k-1}$ y antes de que incorpore un nuevo vector de medidas $z_{k}$, es lo que se conoce como confianza a priori.

$$
\operatorname{Bel}^{-}\left(x_{k}\right)=P\left(x_{k} / z_{1}, u_{1}, z_{2}, u_{2}, \ldots, z_{k-1}, u_{k-1}\right)
$$

Además de lo anterior, el robot también puede adquirir información al observar el entorno con sus sensores exteroceptivos. Aquí quizás extraiga características de esta 
información para formar un vector $z_{k}=\sigma\left(s_{k}\right)$, el cual se distribuye según la distribución de la probabilidad de la ecuación A.3. Lo siguiente es que el robot actualice su confianza $\mathrm{Bel}^{+}\left(x_{k}\right)$ con la nueva información para conseguir así una mejor estimación de su localización. Es decir, una vez que el robot reciba una medida absoluta $z_{k}$ en el instante $k$, incorpora esta medida para obtener la confianza a posteriori,

$$
\operatorname{Bel}^{+}\left(x_{k}\right)=P\left(x_{k} / z_{1}, u_{1}, z_{2}, u_{2}, \ldots, z_{k-1}, u_{k-1}, z_{k}\right)
$$

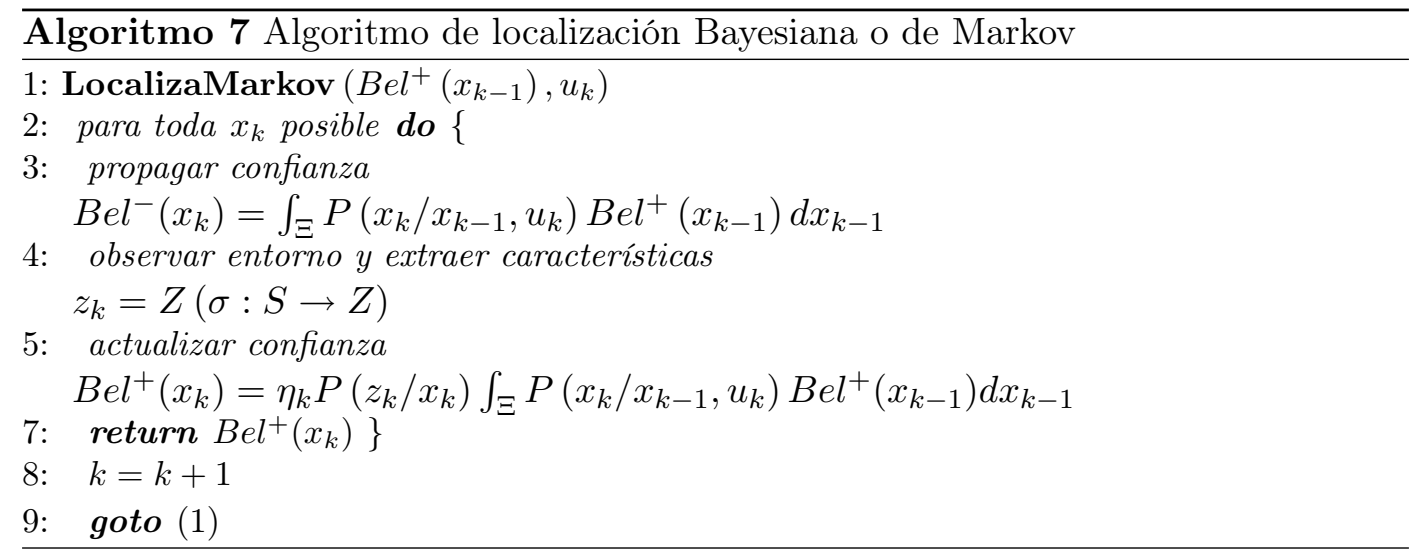

\section{A.4.1. Confianza inicial}

Antes de que el robot comience a navegar por el entorno, tiene una certidumbre inicial sobre el lugar donde él se encuentra localizado. Esta certidumbre se modela mediante la confianza del estado inicial $\mathrm{Bel}^{+}\left(x_{k-1}\right)$. Si el robot conoce su localización inicial, entonces $\mathrm{Bel}^{+}\left(x_{k-1}\right)$ es una distribución con un pico bien definido en las coordenadas donde él se encuentra. Cuando el robot no conoce su posición inicial, la confianza inicial $\mathrm{Bel}^{+}\left(x_{k-1}\right)$ es una distribución uniforme o aplanada. En el primer caso, el objetivo de un sistema de localización será el mantener acotado o seguir la posición del robot. Este problema se conoce como seguimiento o pose-tracking. En el segundo caso, el objetivo principal del sistema de localización es lograr determinar la posición del robot respecto a un sistema de coordenadas global. Este problema se conoce como localización global o también como wake-up. Existe un caso poco usual que es cuando el robot crea estar en una determinada localización, pero realmente no está ahí. En este caso la confianza $\mathrm{Bel}^{+}\left(x_{k-1}\right)$ se inicializa con un pico en la localización en donde el robot cree estar, pero como no se encuentra localizado realmente ahí, el robot debe detectar esta anomalía y corregir la misma. A este problema se le conoce como secuestro o kidnapping.

\section{A.4.2. Predicción de la certidumbre}

Durante la etapa de predicción, el modelo de propagación $p\left(x_{k} / x_{k-1}, u_{k-1}\right)$, también conocido como modelo de movimiento o de actuación, se usa para incorporar 
el efecto de la acción sobre el estimado o certidumbre previa $\mathrm{Bel}^{+}\left(x_{k-1}\right)$. Asúmase que el robot ha realizado una acción y se desea incluir la medida relativa de la posición para actualizar su confianza. Aplicando a la ecuación A.4 el teorema de la probabilidad total y haciendo uso de la asunción de Markov[193], se puede reescribir esta ecuación de manera que sea computacionalmente más eficiente.

Teorema de la probabilidad total El teorema de la probabilidad total indica que la probabilidad de un evento es igual a la suma de las probabilidad de cada uno de los eventos que forman parte del mismo. Con esta definición se puede reescribir A.4 como

$\operatorname{Bel}^{-}\left(x_{k}\right)=\int_{\Xi} P\left(x_{k} / x_{k-1}, z_{1}, u_{1}, \ldots, z_{k-1}, u_{k-1}\right) P\left(x_{k-1} / z_{1}, u_{1}, \ldots, z_{k-1}, u_{k-1}\right) d x_{k-1}$

En esta ecuación, $P\left(x_{k} / x_{k-1}, z_{1}, u_{1}, \ldots, z_{k-1}, u_{k-1}\right)$ expresa la probabilidad de moverse del estado $x_{k-1}$ al estado $x_{k}$, dado todas las acciones y las medidas anteriores al instante $k$; mientras que $P\left(x_{k-1} / z_{1}, u_{1}, \ldots, z_{k-1}, u_{k-1}\right)$ es la probabilidad de estar en el estado $x_{k-1}$, dado todas las medidas y las acciones anteriores al instante $k$. Así, la ecuación A.6 expresa que la confianza que a priori se tiene de estar en el estado $x_{k}$, es la suma de las probabilidades de haberse movido del estado $x_{k-1}$ al estado $x_{k}$, multiplicado por la probabilidad de haber estado realmente en el estado $x_{k-1}$.

El segundo término del integral en A.6 es la probabilidad de estar en la localización $x_{k-1}$ dada toda la información hasta el instante $k-1$; y en particular la acción realizada en este mismo período. Sin embargo, la localización física del robot en el instante $k-1$ no depende de la acción que se realiza en ese período, y por lo tanto no se debe tomar en cuenta al expresar esta probabilidad. Con esto, y con la definición de confianza $a$ posteriori dada en la ecuación A.5, se puede reescribir la ecuación A.6 como,

$$
\operatorname{Bel}^{-}\left(x_{k}\right)=\int_{\Xi} P\left(x_{k} / x_{k-1}, z_{1}, u_{1}, \ldots, z_{k-1}, u_{k-1}\right) \operatorname{Bel}^{+}\left(x_{k-1}\right) d x_{k-1}
$$

Asunción de Markov Los procesos Markovianos son aquellos procesos estocásticos donde sólo el valor presente es relevante para predecir el valor futuro. Así, mediante la Asunción de Markov se establece que dado el conocimiento del estado actual, el estado pasado es independiente del estado futuro y viceversa. De aquí que conociendo la localización anterior $x_{k-1}$, es irrelevante lo que el robot medió con sus sensores, o el como llegó hasta ahí. Con esta asunción se tiene que,

$$
P\left(x_{k} / x_{k-1}, z_{1}, u_{1}, \ldots, z_{k-1}, u_{k-1}\right)=P\left(x_{k} / x_{k-1}, u_{k-1}\right)
$$

Nótese que el lado derecho de esta ecuación es la probabilidad condicional de estar en el estado $x_{k}$ dado el conocimiento del estado anterior $x_{k-1}$ y de la acción $u_{k-1}$ 
realizada. La ecuación A.8 se conoce como el modelo de actuación. Sustituyendo A.8 en A.7 se obtiene la ecuación necesaria para incorporar las acciones al modelo de la confianza a priori.

$$
\operatorname{Bel}^{-}\left(x_{k}\right)=\int_{\Xi} P\left(x_{k} / x_{k-1}, u_{k-1}\right) \operatorname{Bel}^{+}\left(x_{k-1}\right) d x_{k-1}
$$

La ecuación anterior indica que la confianza a priori de estar en el estado $x_{k}$, después de haber realizado una acción $u_{k-1}$, es el resultado de integrar, sobre todas las localizaciones posibles $x_{k-1}$, la probabilidad de que realizando la acción $u_{k-1}$ el robot evolucione desde el estado $x_{k-1}$ hasta el estado $x_{k}$, multiplicado por la confianza a posteriori de que el robot estuviera en el estado $x_{k-1}$.

\section{A.4.3. Corrección de la confianza}

Asúmase que el robot conoce la confianza a priori $\mathrm{Bel}^{-}\left(x_{k}\right)$, y que hace una observación del entorno del cual extrae un vector de características $z_{k}$. Si se desea incorporar esta medida absoluta a fin de obtener la confianza a posteriori $\mathrm{Bel}^{+}\left(x_{k}\right)$, entonces se puede aplicar a la ecuación A.5 la Regla de Bayes y la Asunción de Markov con el objetivo final de reescribir esta ecuación de manera que sea computacionalmente más eficiente.

Regla de Bayes La regla de Bayes es una técnica para calcular probabilidades condicionales. A partir de un conjunto de probabilidades a priori, mediante la regla de Bayes se calcula un conjunto de probabilidades a posteriori, basadas en la evidencia de que un determinado suceso ha ocurrido[152].

En la técnica probabilística de localización de robots, mediante la Regla de Bayes se explica matemáticamente la manera como el robot cambiará su confianza a medida que consigue nuevas evidencias de su posición. Así, usando la Regla de Bayes y la definición de la confianza a priori dada en A.4, se puede reescribir A.5 como,

$$
\begin{aligned}
\operatorname{Bel}^{+}\left(x_{k}\right) & =\frac{P\left(z_{k} / x_{k}, z_{1}, u_{1}, \ldots, z_{k-1}, u_{k-1}\right) P\left(x_{k} / z_{1}, u_{1}, \ldots, z_{k-1}, u_{k-1}\right)}{P\left(z_{k} / z_{1}, u_{1}, \ldots, z_{k-1}, u_{k-1}\right)} \\
& =\frac{P\left(z_{k} / x_{k}, z_{1}, u_{1}, \ldots, z_{k-1}, u_{k-1}\right) B e l^{-}\left(x_{k}\right)}{P\left(z_{k} / z_{1}, u_{1}, \ldots, z_{k-1}, u_{k-1}\right)}
\end{aligned}
$$

Es decir, la confianza a posteriori de estar localizado en $x_{k}$ es igual a la confianza a priori de estar en ese estado, escalado por la evidencia que ofrece el observar $z_{k}$ desde esa misma localización $x_{k}$.

Asunción de Markov Aquí se utiliza la Asunción de Markov para establecer que la lectura del sensor depende solamente del estado actual y que por lo tanto es independiente de las acciones y las observaciones que fueron hechas antes de que el robot 
alcanzara su estado actual. Utilizando esta asunción se puede reescribir el primer término del numerador de A.10 como,

$$
P\left(z_{k} / x_{k}, z_{1}, u_{1}, \ldots, z_{k-1}, u_{k-1}\right)=P\left(z_{k} / x_{k}\right)
$$

Sustituyendo A.11 en A.10 se obtiene la ecuación básica de corrección de la confianza.

$$
\mathrm{Bel}^{+}\left(x_{k}\right)=\frac{P\left(z_{k} / x_{k}\right) \mathrm{Bel}^{-}\left(x_{k}\right)}{P\left(z_{k} / z_{1}, u_{1}, \ldots, z_{k-1}, u_{k-1}\right)}
$$

El denominador de esta ecuación es una constante de normalización para asegurar que la densidad de probabilidad $\mathrm{Bel}^{+}\left(x_{k}\right)$ como máximo sea igual a 1. Esta constante es calculada integrando el numerador sobre todos los estados $x_{k}$ posibles,

$$
P\left(z_{k} / z_{1}, u_{1}, \ldots, z_{k-1}, u_{k-1}\right)=\int_{\Xi} P\left(z_{k} / x_{k}\right) \operatorname{Bel}^{-}\left(x_{k}\right) d x_{k}
$$

\section{A.4.4. Fórmula de localización de Markov}

Combinando los resultados anteriores se consigue una fórmula de localización la cual considera la información absoluta proveniente de las mediciones con los sensores externos, al igual que también considera la información relativa que está relacionada con las acciones ejecutadas por el robot. Así, substituyendo la ecuación A.9 en la ecuación A.12, la confianza a posteriori $\mathrm{Bel}^{+}\left(x_{k}\right)$ se convierte en,

$$
\begin{aligned}
\mathrm{Bel}^{+}\left(x_{k}\right) & =\frac{P\left(z_{k} / x_{k}\right) B e l^{-}\left(x_{k}\right)}{P\left(z_{k} / z_{1}, u_{1}, \ldots, z_{k-1}, u_{k-1}\right)} \\
& =\frac{P\left(z_{k} / x_{k}\right) \int_{\Xi} P\left(x_{k} / x_{k-1}, u_{k-1}\right) B e l^{+}\left(x_{k-1}\right) d x_{k-1}}{P\left(z_{k} / z_{1}, u_{1}, \ldots, z_{k-1}, u_{k-1}\right)} \\
& =\eta_{k} P\left(z_{k} / x_{k}\right) \int_{\Xi} P\left(x_{k} / x_{k-1}, u_{k-1}\right) B e l^{+}\left(x_{k-1}\right) d x_{k-1}
\end{aligned}
$$

donde

$P\left(x_{k} / x_{k-1}, u_{k-1}\right)$ es el modelo de actuación, el cual representa a la densidad de probabilidad de terminar en la localización $x_{k}$ dado que estando en $x_{k-1}$ se realizó la acción $u_{k-1}$.

$P\left(z_{k} / x_{k}\right)$ es el modelo de percepción, el cual representa a la densidad de probabilidad de que estando en el estado $x_{k}$, se observe el vector de características del entorno $z_{k}$.

$\mathrm{Bel}^{+}\left(x_{k-1}\right)$ es la confianza en la localización estimada en el estado anterior, dada todas las evidencias hasta ese instante. Dada la forma recursiva de la ecuación A.14, lo usual es especificar solamente la confianza inicial $\mathrm{Bel}^{+}\left(x_{0}\right)$. 
APÉNDICE A. LOCALIZACIÓN DE MARKOV

$\eta_{k} \quad$ es el normalizador de la densidad de probabilidad ya antes definido en A.13. 


\section{Apéndice B}

\section{Filtro de Kalman}

Una manera de tratar la complejidad computacional de la incertidumbre sobre espacios continuos, es mediante su representación como una función parametrizada. El filtro de Kalman es una técnica mediante la cual se puede tratar este tipo de incertidumbre, siempre que la misma este representada mediante densidades de probabilidad del tipo Normal o Gausiana. Un filtro de Kalman[103, 150, 204] es un algoritmo recursivo de procesamiento de datos que estima el estado de un sistema dinámico contaminado por ruido Gausiano aditivo. En efecto, el Filtro de Kalman es un estimador de estado que trabaja sobre la base de la predicción y la posterior corrección. Esto significa que primero calcula la incertidumbre en cierto estado, estimado mediante un mecanismo de predicción basado en la dinámica del sistema, para luego corregir esta predicción usando las mediciones que entran al sistema. La idea fundamental de estas operaciones se muestran en la figura B.1.

El Filtro de Kalman ha sido usado en un amplio rango de aplicaciones, pero su mayor uso es en el área de control y predicción de sistemas dinámicos. Cuando un filtro de Kalman es usado para controlar un sistema dinámico, tal como la localización en robótica móvil, su objetivo general es estimar el estado del mismo. Para ello, el Filtro de Kalman hace uso del conocimiento de la dinámica del sistema y de los sensores, de la descripción probabilística del ruido aditivo en el sistema y en la medición, de los estados iniciales disponibles, y de toda las mediciones disponibles bien sean precisas o imprecisas. Si el ruido que contamina el sistema o las mediciones están distribuidos en forma Gausiana, entonces el Filtro de Kalman produce una estimación que es estadísticamente óptima en el sentido que se minimiza la covarianza en la estimación del vector de estados.

\section{B.1. Conceptos y asunciones básicas del Filtro de Kalman}

La idea principal del filtro de Kalman es estimar el estado más probable de algún sistema como por ejemplo la localización de un robot. En particular el filtro estima el estado y también suministra una medida de la confianza o certidumbre de ese estado estimado en relación al estado verdadero. Lo que hace difícil este estimado del 


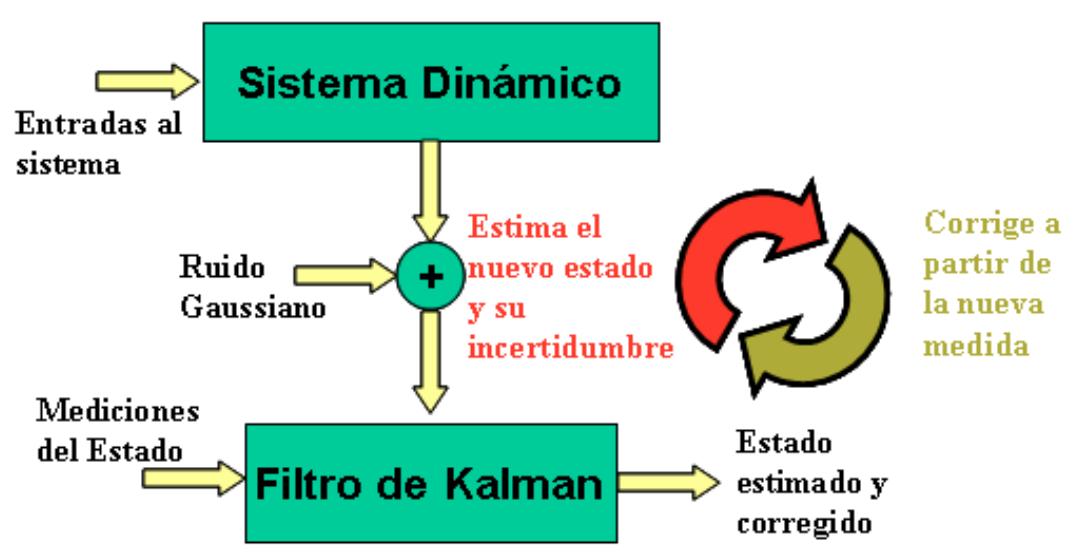

Figura B.1: Operacion cíclica básica del Filtro de Kalman.

estado es que el estado puede cambiar a lo largo del tiempo y además está sujeto a la influencia del ruido.

El filtro de Kalman asume que tanto las mediciones como el estado del sistema, pueden ser descritos mediante un sistema dinámico lineal que es afectado por ruido blanco. El conjunto de ecuaciones que modela la evolución del estado del sistema en el tiempo, y que describe el cómo las mediciones están relacionadas con el mismo, pueden ser modeladas en el espacio de estado discreto de acuerdo a

$$
\left\{\begin{array}{l}
\text { a) } \mathbf{x}_{k}=\mathbf{A} \mathbf{x}_{k-1}+\mathbf{B} \mathbf{u}_{k}+\mathbf{w}_{k} \\
\text { b) } \mathbf{z}_{k}=\mathbf{H} x_{k}+\mathbf{v}_{k}
\end{array}\right.
$$

donde la ecuación B.1a representa el modelo del sistema, la cual es necesaria a fin de hacer las predicciones del estado. También, la ecuación B.1b representa el modelo de la medición, el cual será útil en la corrección del estado que se predice anticipadamente a la medición.

El estado real en el instante $k, \mathbf{x}_{k} \in \Re^{n}$ depende del estado anterior $\mathbf{x}_{k-1}$, de la excitación de entrada al sistema $\mathbf{u}_{k}$, y de algún tipo de ruido $\mathbf{w}_{k}$. Si $n$ es el orden del sistema, la matriz A de tamaño $n \times n$ es la matriz de coeficientes que modelan al sistema y que relacionan el estado actual en el instante $k$, con el estado anterior en el instante $k-1$. El vector $\mathbf{w}_{k} \in \Re^{n}$ modela el ruido de entrada al sistema y toma en cuenta los efectos no modelados en el vector de estado $\mathbf{x}_{k}$.

La medida verdadera en el instante $k, \mathbf{z}_{k} \in \Re^{m}$ depende linealmente del estado $\mathbf{x}_{k}$. La matriz $\mathbf{H}$ de tamaño $m \times \mathbf{x} n$ relaciona el estado actual $\mathbf{x}_{k}$ con la medición $\mathbf{z}_{k}$. Conocido el estado $\mathbf{x}_{k}$, la matriz $\mathbf{H}$ modela la medición que realmente debería existir si no existiera a su vez ruido en los sensores. Sin embargo, lo común es que exista ruido en los sensores el cual es modelado por el vector $\mathbf{v}_{k} \in \Re^{m}$.

En la formulación del Filtro de Kalman se asume que el ruido aditivo en el sistema $\mathbf{w}_{k}$ y en la medición $\mathbf{v}_{k}$, son variables aleatorias de ruido blanco, independientes entre sí, de media igual a cero y con probabilidad de distribución Gausiana. Esta 
última asunción trae consigo importantes simplificaciones que se deben a que las distribuciones gausianas $N(\mu, \Sigma)$ son completamente descritas a través de su media $\mu$ y matriz de covarianza $\Sigma$. Otra importante simplificación es que la operación lineal sobre distribuciones normales da lugar a otra distribución normal, con lo que es suficiente con describir el resultado mediante su media y covarianza. Igualmente, la asunción de independencia simplifica los cálculos. En general es razonable asumir que el ruido en el sistema y en la medición son independientes, lo que significa que una cantidad de ruido no tiene ninguna influencia sobre la otra. La asunción de la presencia de ruido blanco trae implícito que su media sea cero. El ruido puede ser clasificado como sistemático, y no sistemático o aleatorio. La asunción de que la media es cero implica que los errores en el sistema y en la medición son completamente aleatorios. Esto ultimo se denota matemáticamente mediante el calculo del primer momento estadístico o media de forma

$$
\begin{gathered}
\mu_{w}=\hat{\mathbf{w}}=E\left\langle\mathbf{w}_{k}\right\rangle=0 \\
\mu_{v}=\hat{\mathbf{v}}=E\left\langle\mathbf{v}_{k}\right\rangle=0
\end{gathered}
$$

donde $E\langle\cdot\rangle$ representa la experanza matemática del argumento de la función.

\section{B.2. Algoritmo del Filtro del Kalman lineal}

Bajo el supuesto de linealidad en el sistema y en la medición del estado del mismo, el algoritmo de Filtrado de Kalman (KF-Kalman Filtering) consiste en un paso de inicialización, después del cual, alternativamente se realizan pasos de predicción y corrección del estado. En la inicialización, el KF debe ser alimentado con el supuesto posterior del estado en el instante inicial, es decir con $\mathrm{Bel}^{+}\left(\mathbf{x}_{0}^{+}\right)=N\left(\hat{\mathbf{x}}_{0}^{+}, \mathbf{P}_{0}^{+}\right)$. Por esto al inicio del algoritmo se debe especificar el estimado del estado inicial $\hat{\mathbf{x}}_{0}^{+} \mathrm{y}$ su incertidumbre $\mathbf{P}_{0}^{+}$.

\section{B.2.1. Predicción}

A cada instante de tiempo $k$, el KF estima o predice el nuevo estado $\mathbf{x}_{k}^{-}$del sistema y su incertidumbre $\mathbf{P}_{k}^{-}$. La combinación de estos dos estimados es el supuesto previo $\mathrm{Bel}^{-}\left(\mathbf{x}_{k}\right)=N\left(\hat{\mathbf{x}}_{k}^{-}, \mathbf{P}_{k}^{-}\right)$, la cual se calcula propagando el sistema a través del tiempo mediante las ecuaciones

$$
\begin{gathered}
\hat{\mathbf{x}}_{k}^{-}=\mathbf{A} \hat{\mathbf{x}}_{k-1}^{+}+\mathbf{B} \hat{\mathbf{u}}_{k} \\
\mathbf{P}_{k}^{-}=\mathbf{A P}_{k-1}^{+} \mathbf{A}^{\mathrm{T}}+\mathbf{B U}_{k} \mathbf{B}^{\mathrm{T}}+\mathbf{Q}_{k}
\end{gathered}
$$

En la ecuación B.4 se ha considerado que el promedio del ruido de entrada al sistema es cero $\left(E\left\langle\mathbf{w}_{k}\right\rangle=0\right)$. Como se aprecia en esta misma ecuación, el mejor estimado $\hat{\mathbf{x}}_{k}^{-}$que el KF puede hacer del sistema antes de que el mismo progrese en 


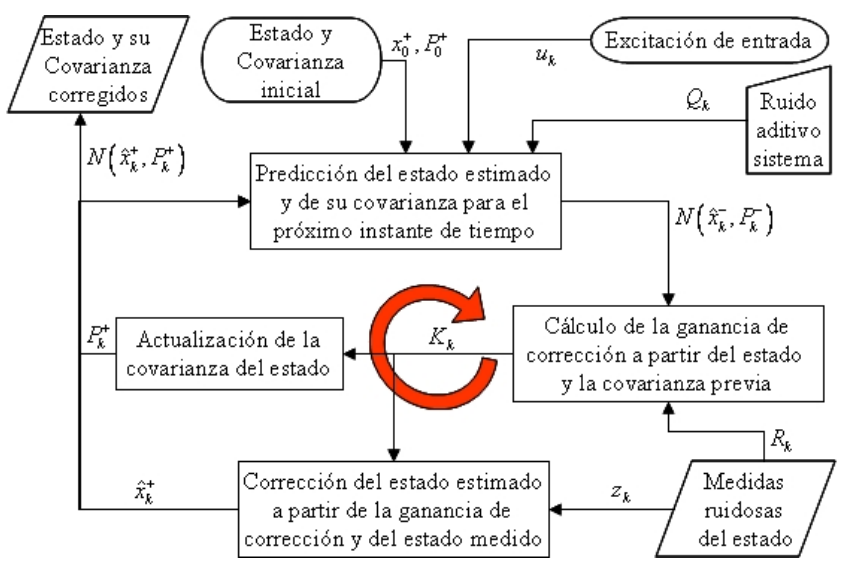

Figura B.2: Esquema del algoritmo cíclico del Filtro de Kalman lineal.

un instante de tiempo $k$, es el que hace propagando el último estado conocido $\hat{\mathbf{x}}_{k-1}^{+}$ a través del modelo del sistema A. También, si el sistema esta sometido a entradas externas $\mathbf{u}_{k}$, estas serán tomadas en cuentas a través del modelo de acción $\mathbf{B}$.

Por otra parte, según la ecuación B.1a, la evolución del sistema está sujeta al ruido aditivo $\mathbf{w}_{k}$ lo que tiende a incrementar la incertidumbre $\mathbf{P}_{k}^{-}$del estado estimado. El KF actualiza esta incertidumbre mediante la ecuación B.5, donde el primer término propaga a través del sistema la ultima incertidumbre conocida $\mathbf{P}_{k-1}^{+}$, el segundo término toma en cuenta la incertidumbre que pudiera existir en el conocimiento que se tiene respecto a las entradas(i.e. la covarianza de la excitación externa $\mathbf{U}_{k}$ ), y el tercer término $\mathbf{Q}_{k}=E\left\langle\mathbf{w}_{k}, \mathbf{w}_{k}\right\rangle$ es la covarianza del ruido aditivo que contamina el sistema a cada instante de tiempo. En resumen, la ecuación B.5 cuantifica la incertidumbre que existe de que el estado estimado a priori sea el verdadero estado, y en su simplificación se utiliza la presunción de independencia entre los errores del estado, la entrada externa y el ruido.

\section{B.2.2. Corrección}

Cuando hay información disponible acerca del estado real del sistema, esta se usa para corregir el estimado más reciente que se tiene del estado; es decir para corregir a el supuesto previo $\mathrm{Bel}^{-}\left(\mathbf{x}_{k}\right)=N\left(\hat{\mathbf{x}}_{k}^{-}, \mathbf{P}_{k}^{-}\right)$. Esto se logra mediante la incorporación de la información que proporcionan las mediciones a través de la ecuación

$$
\hat{\mathbf{x}}_{k}^{+}=\hat{\mathbf{x}}_{k}^{-}+\mathbf{K}_{k}\left(\mathbf{z}_{k}-\mathbf{H} \hat{\mathbf{x}}_{k}^{-}\right)
$$

donde

$$
\mathbf{K}_{k}=\mathbf{P}_{k}^{-} \mathbf{H}^{\mathrm{T}}\left(\mathbf{H P}_{k}^{-} \mathbf{H}^{\mathrm{T}}-\mathbf{R}_{k}\right)^{-1}
$$

Mediante la ecuación B.6 se actualiza y corrige al estado estimado. En esta misma ecuación, el factor $\left(\mathbf{z}_{k}-\mathbf{H} \hat{\mathbf{x}}_{k}^{-}\right)$es conocido como la innovación, el cual representa 
junto a la Ganancia de Kalman $K$, el factor de corrección del estado previamente estimado. Analíticamente hablando, la innovación es la diferencia entre la medida sensorial $\mathbf{z}_{k}$ y la medida $\hat{\mathbf{z}}_{k}$ pronosticada para ese mismo instante de tiempo, por lo que es igual a

$$
\tilde{\mathbf{z}}=\mathbf{z}_{k}-\hat{\mathbf{z}}_{k}=\mathbf{z}_{k}-\mathbf{H} \hat{x}_{k}^{-}
$$

De lo anterior se puede afirmar que la innovación expresa cuanto difiere la medida pronosticada de la medida verdadera. Si la innovación es igual a cero, entonces la medida pronosticada refleja exactamente a la medida real, lo que implica a su vez que el estado estimado está muy cerca del verdadero valor del estado del sistema desde donde se hizo esa medición, y por lo tanto no necesita corrección. Lo contrario, si la innovación es grande, implica que el estado estimado necesita de algún tipo o cantidad de corrección.

Por otra parte, el factor $\mathbf{K}_{k}$ en la ecuación B.7 se conoce como la Ganancia de Kalman. Este factor de ganancia es tal, que con él se garantiza que la covarianza del error en el estado estimado sea mínima. Cuantitativamente, la Ganancia de Kalman determina la cantidad de innovación que se tomará en cuenta para la corrección del estado estimado. Esta cantidad surge de la relación entre la incertidumbre propagada a través del modelo del sistema y la incertidumbre en la medición. Por ejemplo, es fácil mostrar que si el ruido en la medición se aproxima a cero, a través de la Ganancia de Kalman se le da más confianza a la innovación y a las mediciones, y menos confianza al estado al estimado obtenido a través del modelo del sistema. Esto se demuestra mediante la siguiente ecuación.

$$
\lim _{\mathbf{R}_{k} \rightarrow 0}\left(\mathbf{K}_{k}=\mathbf{P}_{k}^{-} \mathbf{H}^{\mathrm{T}}\left(\mathbf{H} \mathbf{P}_{k}^{-} \mathbf{H}^{\mathrm{T}}-\mathbf{R}_{k}\right)^{-1}\right)=\mathbf{H}^{-1}
$$

En el caso contrario, cuando la covarianza del error previo se aproxima a cero, la Ganancia de Kalman le da menos peso a la innovación y de esa manera se le da más confianza al modelo del sistema que a las mediciones. Lo expuesto se puede ver en la siguiente ecuación.

$$
\lim _{\mathbf{P}_{k}^{-} \rightarrow 0}\left(\mathbf{K}_{k}=\mathbf{P}_{k}^{-} \mathbf{H}^{\mathrm{T}}\left(\mathbf{H} \mathbf{P}_{k}^{-} \mathbf{H}^{\mathrm{T}}-\mathbf{R}_{k}\right)^{-1}\right)=0
$$

\section{B.2.3. Actualización de la covarianza}

La actualización de la incertidumbre en la estimación del estado posterior también es llevada a cabo a través del la Ganancia de Kalman, mediante la ecuación

$$
\mathbf{P}_{k}^{+}=\left(\mathbf{I}-\mathbf{K}_{k} \mathbf{H}\right)^{-1} \mathbf{P}_{k}^{-}
$$

Si la ganancia de Kalman esta cercana a $\mathbf{H}^{-1}$ entonces la innovación es tomada en cuenta casi en su totalidad. Esto significa que la innovación contienen relativamente mucha más información que el estado estimado previo. Esto a su vez implica un máximo decrecimiento de la incertidumbre en el estado estimado. En particular, si 


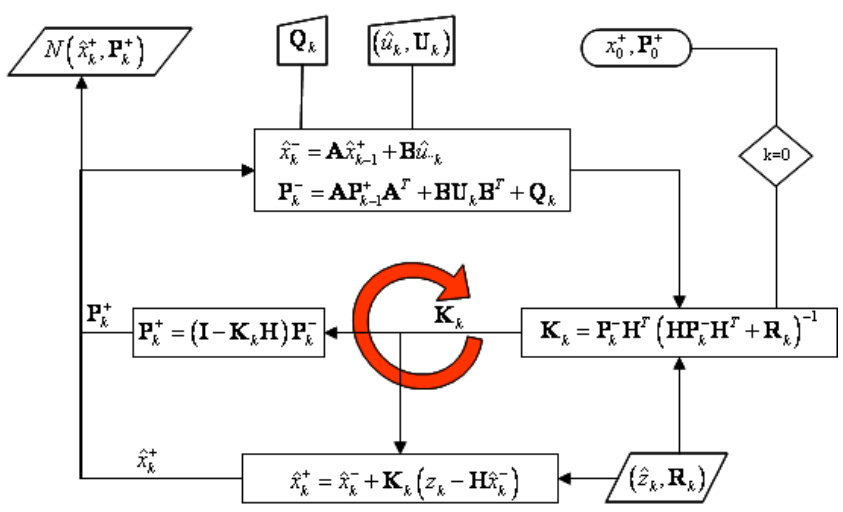

Figura B.3: Ecuaciones de recurrencia del algoritmo del Filtro de Kalman Lineal

hay una gran cantidad de incertidumbre en el estado estimado previo en relación a las mediciones, entonces la Ganancia de Kalman estará cercana a $\mathbf{H}^{-1}$ y el estimado del error posterior estará cercano a cero. Esto tiene como consecuencia que la ganancia de Kalman no tomará muy en cuenta la siguientes mediciones ya que la incertidumbre en el estado es muy pequeña y con esto la Ganancia de Kalman tiende a bajar. El tiempo que toma para que la ganancia de Kalman tome nueva y significativamente en cuenta a la innovación depende de la cantidad de ruido adicionada en cada instante de tiempo a la incertidumbre del estado estimado previo.

\section{B.2.4. Parámetros y ajuste del Filtro de Kalman}

El Filtro de Kalman requiere de una estimación del estado inicial $\mathbf{x}_{0}^{+}$y de la covarianza del error inicial $\mathbf{P}_{0}^{+}$. Si se tiene el absoluto conocimiento del estado inicial, entonces el error inicial es nulo y por tanto también lo es la estimación de la covarianza del error inicial. Sin embargo si se tiene incertidumbre en la estimación de estado inicial, no es conveniente elegir la covarianza del error de estimación inicial nula ya que esto causaría, en caso de suponer una covarianza del error de proceso muy pequeña, que el filtro tuviera problemas de convergencia. Por tanto si el estado inicial no es conocido o se tiene una elevada incertidumbre en cuanto a su estimación debemos elegir una covarianza del error inicial distinta de cero para garantizar la convergencia del filtro. Lo usual es elegir $\mathbf{x}_{0}^{+}=0$ y $\mathbf{P}_{0}^{+}=\alpha \mathbf{I}_{n \times n}$, donde $\alpha$ es un valor muy grande y $n$ es el numero de variables de estado que modelan al sistema.

La covarianza del ruido de medida $\mathbf{R}_{k}$ es normalmente determinada a partir de medidas tomadas previamente, de modo que su valor puede ser calculado con relativa facilidad antes de poner en funcionamiento el filtro.

\section{B.3. Filtro de Kalman Extendido - EKF}

Debido a que muchos sistemas dinámicos o sus modelos de observación no son completamente lineales; a través de los años se han propuesto un conjunto de exten- 
siones del Filtro de Kalman lineal que abordan justamente los sistemas no lineales mediante técnicas de linealización. La primera de estas extensiones es la conocida como Filtro de Kalman para pequeñas perturbaciones(PKF, Perturbation Kalman Filter), en la cual la idea general es definir una trayectoria nominal tanto para el estado como para las observaciones, linealizar así el sistema alrededor de esta trayectoria, y entonces aplicar el algoritmo general del Filtro de Kalman. Rápidamente esta extensión fue desplazada por el Filtro de Kalman Extendido(EKF, Extended Kalman Filter) que resuelve la limitación que para grandes perturbaciones presenta el PKF, mediante la incorporación dinámica del nuevo estado estimado a la trayectoria nominal que se usa en la linealización del sistema. Sin embargo esta extensión todavía presenta limitación en cuanto al tratamiento de sistemas altamente no lineales.

Las técnicas de linealización simulan localmente el comportamiento lineal del sistema en un punto dado. La técnica más usada para simular este comportamiento es la aproximación mediante series de Taylor de primer orden. Un problema con este procedimiento es que si el punto de linealización está lejos del estado real del sistema, entonces los resultados son inadecuados. Por ello, el EKF incorpora el último estado estimado al proceso de linealización para así obtener una mejor aproximación del sistema.

En el caso de los sistemas no lineales, el sistema se puede modelar mediante una ecuación no lineal del tipo

$$
\mathbf{x}_{k}=f\left(\mathbf{x}_{k-1}, \mathbf{u}_{k}\right)+\mathbf{w}_{k}
$$

donde $f(\cdot)$ es la función no lineal del sistema que relaciona al estado actual $\mathbf{x}_{k}$ con el estado anterior $\mathbf{x}_{k-1}$, con la entrada o excitación actual $\mathbf{u}_{k}$, y con el ruido aditivo al sistema $\mathbf{w}_{k}$.

Igualmente, el modelo de observación esta gobernado por una ecuación no lineal de la forma,

$$
\mathbf{z}_{k}=h\left(\mathbf{x}_{k}\right)+\mathbf{v}_{k}
$$

donde $h(\cdot)$ es la función no lineal que relaciona al estado con la observación o medición actual, y donde $\mathbf{v}_{k}$ representa el ruido aditivo al sistema de medición.

$\mathrm{Al}$ igual que el Filtro de Kalman Lineal, el algoritmo del EKF trabaja de forma cíclica en dos pasos básicos: predicción y corrección.

Predicción A cada instante de tiempo $k$, el EKF propaga el estado y su covarianza mediante la siguiente formulación

$$
\begin{gathered}
\hat{\mathbf{x}}_{k}^{-}=f\left(\hat{\mathbf{x}}_{k-1}^{+}, \mathbf{u}_{k}\right) \\
\mathbf{P}_{k}^{-}=\mathbf{A}_{k} \mathbf{P}_{k-1} \mathbf{A}^{\mathrm{T}}+\mathbf{B}_{k} \mathbf{U}_{k} \mathbf{B}^{\mathrm{T}}+\mathbf{Q}_{k}
\end{gathered}
$$

donde A y $\mathbf{B}$ son matrices Jacobianas que contienen las derivadas parciales del sistema no lineal con respecto al estado $x$ y a la excitación $u$, respectivamente. Estas matrices 
permiten la aproximación lineal del sistema no lineal $f(\mathbf{x}, \mathbf{u})$, por lo que para su mejor desempeño deben ser evaluadas en el ultimo estado conocido o estimado del sistema. Esto es,

$$
\begin{aligned}
\mathbf{A}_{k} & =\left.\frac{\partial f(\mathbf{x}, \mathbf{u})}{\partial \mathbf{x}}\right|_{\mathbf{x}_{k-1}^{+}, \mathbf{u}_{k}} \\
\mathbf{B}_{k} & =\left.\frac{\partial f(\mathbf{x}, \mathbf{u})}{\partial \mathbf{u}}\right|_{\mathbf{x}_{k-1}^{+}, \mathbf{u}_{k}}
\end{aligned}
$$

Corrección Una vez que hay datos $\mathbf{z}_{k}$ disponibles, el estimado previo del estado se corrige haciendo uso de las siguientes ecuaciones:

$$
\begin{gathered}
\mathbf{K}_{k}=\mathbf{P}_{k}^{-} \mathbf{H}_{k}^{\mathrm{T}}\left(\mathbf{H}_{k} \mathbf{P}_{k}^{-} \mathbf{H}_{k}^{\mathrm{T}}-\mathbf{R}_{k}\right)^{-1} \\
\hat{\mathbf{x}}_{k}^{+}=\hat{\mathbf{x}}_{k}^{-}+\mathbf{K}_{k}\left(\mathbf{z}_{k}-h\left(\hat{\mathbf{x}}_{k}^{-}\right)\right) \\
\mathbf{P}_{k}^{+}=\left(\mathbf{I}-\mathbf{K}_{k} \mathbf{H}_{k}\right)^{-1} \mathbf{P}_{k}^{-}
\end{gathered}
$$

donde $H_{k}$ es la matriz Jacobiana que contiene las derivadas parciales de la función de observación $h(\mathbf{x})$ con respecto al estado $\mathbf{x}$. Al igual que para la aproximación lineal del estado estimado, este caso también require que la matriz se evalúe en el punto más próximo al estado real del sistema. Esto es,

$$
\mathbf{H}_{k}=\left.\frac{\partial h(\mathbf{x})}{\partial \mathbf{x}}\right|_{\hat{\mathbf{x}}_{k}^{-}}
$$




\section{Apéndice $\mathrm{C}$}

\section{Estimación de parámetros mediante Mínimos Cuadrados}

El método básico para la estimación de parámetros de un modelo consiste en minimizar la diferencia entre los datos de salida del modelo del sistema y los datos de salida real en el sistema que se pretende modelar. Esto se lleva a cabo mediante técnicas de optimización, de tal forma que los valores de los parámetros estimados se ajusten lo mejor posible a los datos recolectados. Un aspecto a resaltar es que los datos medidos son ruidosos y por lo tanto difícilmente se ajustan perfectamente al modelo. Para determinar si el modelo es apropiado, hace falta un criterio de prueba para evaluar la bondad del mismo. Normalmente este test estará basado en algún estadístico extraído del error medido. De esta forma, para que un procedimiento de estimación sea útil debe proveer

1. Los parámetros estimados extraídos de los datos reales

2. El error de estimación de los parámetros

3. Una medida estadística de la bondad del ajuste realizado

Un método ampliamente usado en la estimación de parámetros es el conocido como Minimos Cuadrados. Este método fue desarrollado por K.F Gauss como instrumento estadístico que daba un tratamiento probabilístico a los errores de observación ligados con la estimación de parámetros del modelo de la órbita elíptica de un cuerpo planetario, cuando las observaciones disponibles excedian el número de parámetros a estimar. En una serie de trabajos[81], Gauss demuestra que dentro de los estimadores lineales insesgados, el estimador de mínimos cuadrados es el que conduce a la obtención del menor error cuadrático medio. En estos mismos trabajos, Gauss también estableció las bases para la estimación recursiva demostrando que es posible detectar los cambios mas probables de un evento desconocido cuando una nueva ecuación es incorporada. Este algoritmo recursivo fue ignorado por años hasta que R.E Kalman[103] y Kalman\&Bucy[102] lo utilizaron, en el contexto de la teoría de control, como solución recursiva para el problema de filtrado lineal de datos discretos. 
El algoritmo propuesto por estos investigadores se conoce como el Filtro de Kalman, y el mismo es un conjunto de ecuaciones que proveen la solución recursiva eficiente del método de mínimos cuadrados. En adelante se describe la implementación del método de Mínimos Cuadrados como estimador de parámetros en el modelado de funciones.

\section{C.1. Mínimos Cuadrados Generalizados}

Un sistema lineal se puede modelar mediante una representación en variables de estados según,

$$
y=a_{1} x_{1}+a_{2} x_{2}+\cdots+a_{n} x_{n}
$$

donde $y$ representa la salida del sistema, $\mathbf{X}=\left[x_{1}, x_{2}, \cdots, x_{n}\right]$ corresponde al vector de estados, y $\mathbf{A}=\left[a_{1}, a_{2}, \cdots, a_{n}\right]$ son los parámetros que caracterizan al sistema.

Si se realiza una serie de ensayos controlados donde las variables de estado serán las entradas al sistema (por eso se considerarán conocidas), y donde $\hat{\mathbf{A}}$ representa los valores estimados de los parámetros del sistema, las distintas salidas de los ensayos se pueden ordenar de acuerdo a,

$$
\left[\begin{array}{c}
\hat{y}(1) \\
\hat{y}(2) \\
\vdots \\
\hat{y}(k)
\end{array}\right]=\left[\begin{array}{l}
\hat{a}_{1} x_{1}(1)+\hat{a}_{2} x_{2}(1)+\cdots+\hat{a}_{n} x_{n}(1) \\
\hat{a}_{1} x_{1}(2)+\hat{a}_{2} x_{2}(2)+\cdots+\hat{a}_{n} x_{n}(2) \\
\vdots \\
\vdots \\
\hat{a}_{1} x_{1}(k)+\hat{a}_{2} x_{2}(k)+\cdots+\hat{a}_{n} x_{n}(k)
\end{array}\right]
$$

Considerando que los parámetros que caracterizan al sistema no varían con el tiempo, se puede volver a escribir la ecuación C.2 en la forma

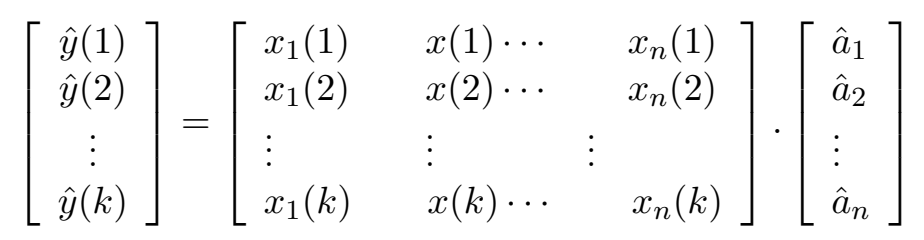

que en forma compacta se puede escribir como

$$
\hat{\mathbf{Y}}=\mathbf{X} \hat{\mathbf{A}}
$$

Habiendo medido la salida real del sistema, la podemos representar según

$$
\mathbf{Y}=[y(1), y(2), \cdots, y(k)]^{\mathrm{T}}
$$

A partir de aquí se puede expresar el error en forma matricial de acuedo a,

$$
\mathbf{E}=\mathbf{Y}-\hat{\mathbf{Y}}=\mathbf{Y}-\mathbf{X} \hat{\mathbf{A}}
$$

Se puede demostrar que un conjunto de parámetros $\hat{\mathbf{A}}=\left[\hat{a}_{1}, \hat{a}_{2} \cdots, \hat{a}_{m}\right]^{\mathrm{T}}$ que minimiza el error cuadrático $\mathbf{E}^{2}$ entre la salida real y la estimada es 


$$
\hat{\mathbf{A}}=\left[\mathbf{X}^{\mathrm{T}} \mathbf{X}\right]^{-1} \mathbf{X}^{\mathrm{T}} \mathbf{Y}
$$

Así por ejemplo, en el caso de que el sistema pueda ser representado por una simple línea recta, se conoce que una función paramétrica que describe el sistema sería $y=\mathrm{m} x+\mathrm{c}$, que se puede escribir como

$$
\hat{y}=\hat{a}_{1} x_{1}+\hat{a}_{2} x_{2}^{0}
$$

de aquí que el vector de estado, conocido en este caso como el vector de regresión, sea igual a

$$
\mathbf{X}=\left[\begin{array}{ll}
x_{1} & 1
\end{array}\right]
$$

por lo que si se toman $k$ mediciones de entrada $(\mathbf{X})$ y salida $(y)$ del sistema, los parámetros estimados que minimizan el error se calculan de acuerdo a

$$
\left.\hat{\mathbf{A}}=\left[\begin{array}{l}
\hat{a}_{1} \\
\hat{a}_{2}
\end{array}\right]=\left[\begin{array}{lll}
\mathbf{X}(1) & \cdots & \mathbf{X}(k)
\end{array}\right]\left[\begin{array}{c}
\mathbf{X}(1) \\
\vdots \\
\mathbf{X}(k)
\end{array}\right]\right]^{-1}\left[\begin{array}{lll}
\mathbf{X}(1) & \cdots & \mathbf{X}(k)
\end{array}\right]\left[\begin{array}{c}
y(1) \\
\vdots \\
y(k)
\end{array}\right]
$$

\section{Validación del modelo}

Para saber si el error en el modelo se debe a efectos aleatorios en los datos o a que los mismos realmente no se ajustan al modelo propuesto, se requiere tener una medida de cuán bueno es el ajuste de las mediciones al modelo estimado. Cuando se asume que los errores de observación son independientes entre sí y están normalmente distribuidos, entonces se puede usar el test estadístico chi-cuadrado $\left(\chi^{2}\right)$. Este estadístico se obtiene dividiendo la suma del error mínimo $\mathbf{E}_{\min }$ entre la varianza $\sigma_{i}$ de los datos observados. Esto es,

$$
\chi^{2}=\sum_{i=1}^{N}\left(\frac{y(i)-\mathbf{X}(i) \hat{\mathbf{A}}}{\sigma_{i}}\right)^{2}
$$

Si el modelo es correcto, el error debe distribuirse de acuerdo a la distribución chicuadrado con $N-M$ grados de libertad, con $N$ igual al número de observaciones y $M$ el número de parámetros estimados. El valor de $\chi^{2}$ obtenido para el mejor estimado de los parámetros se puede observar en una tabla la cual suministra la probabilidad de que este valor haya sido obtenido a causa del error aleatorio en la medición. Esta probabilidad da una medida cuantitativa de cuán bueno es el ajuste al modelo. Si el valor de $\chi^{2}$ calculado es menor que el valor $\chi_{\alpha, N-M}^{2}$ tabulado, entonces es muy probable que el error en el modelo se deba a los efectos aleatorios en las observaciones. Si por el contrario, el $\chi^{2}$ calculado es mayor que el valor $\chi_{\alpha, N-M}^{2}$ tabulado, entonces hay que rechazar el modelo porque los datos no se ajustan al mismo. El parámetro 
$\alpha$ se refiere al grado de confianza en los datos observados, el cual se suele establecer a $95 \%$.

\section{C.2. Mínimos Cuadrados Recursivos}

Un problema con esta forma de estimar parámetros de modelos funcionales es que los datos se deben procesar por lotes u off-line, lo que puede representar un inconveniente en ciertas aplicaciones. Igualmente, también presenta el inconveniente de requerir de procedimientos de inversión de grandes matrices, lo que puede ser computacionalmente costoso. Una solución inmediata es modificar el algoritmo para que pueda ir modificando los parámetros estimados a medida que se vayan obteniendo nuevos datos. Este proceso, conocido como Mínimos Cuadrados Recursivos (RLS Recursive Least Square), puede realizarse on-line, por lo que los parámetros a estimar serán dinámicos, adaptándose en cada instante para que el error entre el modelo estimado y el modelo real sea mínimo.

El RLS ha sido suficientemente estudiado[88, 89], por eso aquí sólo se presentan las ecuaciones que componen el algoritmo. Así, partiendo del resultado que se obtuvo mediante la ecuación C.10, con la cual se estimaban los parámetros hasta el instante $k$, la idea consiste en que si se agrega una nueva medida $\mathbf{X}(k+1)$, los parámetros $\hat{\mathbf{A}}$ pueden estimarse mejor al incluir esta nueva medición. Se trata entonces de obtener un procedimiento recursivo que proporcione los valores de los parámetros $\hat{\mathbf{A}}(k+$ 1) partiendo de los parámetros $\hat{\mathbf{A}}(k)$ y considerando la última medida obtenida. El conjunto de ecuaciones necesarias para implementar el algoritmo son las que se resumen a continuación:

$$
\begin{gathered}
\mathbf{W}(k+1)=\frac{\mathbf{P}(k) \mathbf{X}(k+1)}{1+\mathbf{X}(k+1)^{\mathrm{T}} \mathbf{P}(k) \mathbf{X}(k+1)} \\
\mathbf{E}(k+1)=\mathbf{Y}(k+1)-\mathbf{X}(k+1)^{\mathrm{T}} \hat{\mathbf{A}}(k) \\
\hat{\mathbf{A}}(k+1)=\hat{\mathbf{A}}(k)+\mathbf{W}(k+1) \mathbf{E}(k+1) \\
\mathbf{P}(k+1)=\mathbf{P}(k)-\frac{\mathbf{P}(k) \mathbf{X}(k+1) \mathbf{X}(k+1)^{\mathrm{T}} \mathbf{P}(k)}{1+\mathbf{X}(k+1)^{\mathrm{T}} \mathbf{P}(k) \mathbf{X}(k+1)}
\end{gathered}
$$

donde

$\mathbf{X}(k+1)$ es el vector de regresión en el instante de la estimación

$\mathbf{P}(\cdot)$ es la matriz de covarianzas de los parámetros estimados

$\mathbf{W}(k+1)$ es la matriz de ganancias que corrige los parámetros estimados

$\hat{\mathbf{A}}(k)$ es el vector de parámetros estimados 
$\mathbf{Y}(k+1)$ es la salida real del sistema en el instante de la estimación

$\mathbf{E}(k+1)$ es el error de predicción

Como se puede observar, se necesita el conocimiento a priori de la matriz inicial de covarianzas y de los valores iniciales de los parámetros a estimar. Usualmente se suele inicializar $\mathbf{P}(k)=\alpha \mathbf{I}_{n \times n}$, donde $\mathbf{I}$ es la matriz identidad y $\alpha$ una constante muy grande. Por otra parte, si no se tiene ningún conocimiento acerca del valor de los parámetros a estimar, lo más frecuente es inicializarlos en cero.

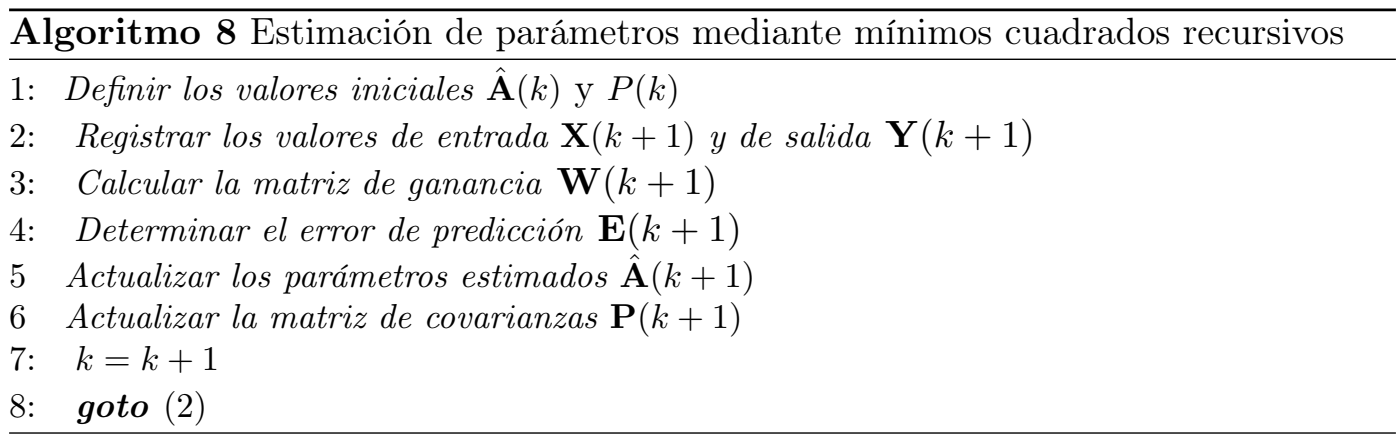

El algoritmo 8 describe los pasos a seguir para estimación de los parámetros de una función mediante el método de mínimos cuadrados recursivos. El problema que presenta este algoritmo es la pérdida de actividad o "apagado" del algoritmo (incapacidad para detectar cambios en los parámetros del modelo) a lo largo del tiempo, debido a que la traza de la matriz $\mathbf{P}(k)$ se hace muy pequeña. La capacidad adaptativa del algoritmo RLS va desapareciendo a medida que la estimación converge a su verdadero valor y la diagonal principal de la matriz de covarianza del error de estimación va tomando valores cada vez más pequeños. A partir de esos momentos, la sensibilidad del algoritmo para detectar posibles cambios en los parámetros es muy reducida. Si se desea utilizar el RLS para detectar esas posibles variaciones, la estructura original no es adecuada, y en consecuencia se deben introducir modificaciones orientadas a evitar la disminución excesiva de la varianza del error de estimación. Una de las técnicas más populares consiste en introducir al algoritmo un Factor de Olvido $\lambda$, de tal forma que se pondere más a las muestras más recientes, permitiendo así detectar un posible cambio de parámetros cuando el algoritmo lleva un tiempo considerable funcionando y la traza de la matriz $\mathbf{P}(k)$ se ha hecho muy pequeña.

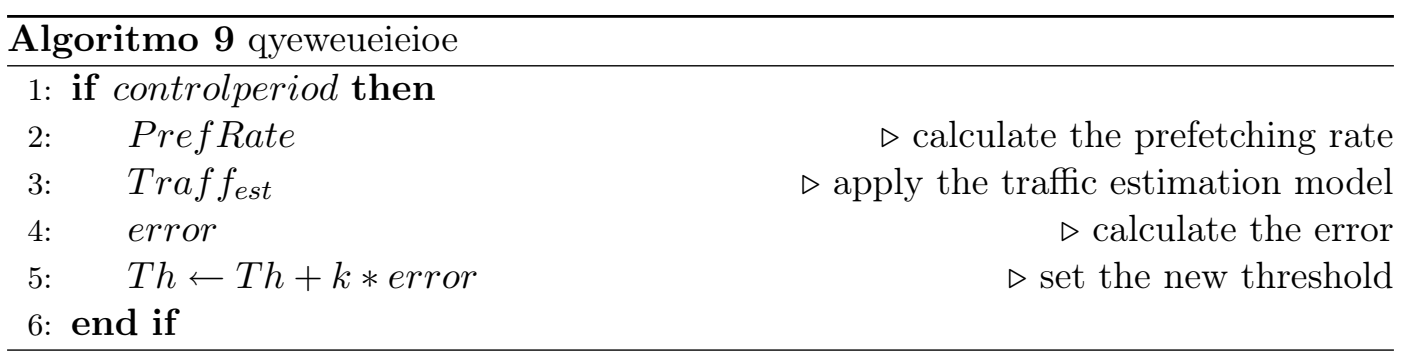


Matemáticamente, el algoritmo RLS con factor de olvido se puede resumir en las

siguientes fórmulas, siendo su procedimiento de aplicación el mismo que el descrito en el algoritmo 8.

$$
\begin{gathered}
\mathbf{W}(k+1)=\frac{\mathbf{P}(k) \mathbf{X}(k+1)}{\lambda+\mathbf{X}(k+1)^{\mathrm{T}} \mathbf{P}(k) \mathbf{X}(k+1)} \\
\mathbf{E}(k+1)=\mathbf{Y}(k+1)-\mathbf{X}(k+1)^{\mathrm{T}} \hat{\mathbf{A}}(k) \\
\hat{\mathbf{A}}(k+1)=\hat{\mathbf{A}}(k)+\mathbf{W}(k+1) \mathbf{E}(k+1) \\
\mathbf{P}(k+1)=\frac{\mathbf{1}}{\lambda}\left(\mathbf{P}(\mathbf{k})-\frac{\mathbf{P}(\mathbf{k}) \mathbf{X}(\mathbf{k}+\mathbf{1}) \mathbf{X}(\mathbf{k}+\mathbf{1})^{\mathrm{T}} \mathbf{P}(\mathbf{k})}{\lambda+\mathbf{X}(\mathbf{k}+\mathbf{1})^{\mathrm{T}} \mathbf{P}(\mathbf{k}) \mathbf{X}(\mathbf{k}+\mathbf{1})}\right)
\end{gathered}
$$

Dependiendo del valor de $\lambda, 0<\lambda<1$, se obtienen los siguientes comportamientos:

- Un factor de olvido $\lambda \approx 1$ implica que las matrices $\mathbf{P}(k)$ y $\mathbf{W}(k)$ tenderán a cero y el algoritmo de estimación no responderá a cambios de parámetros o no estimará adecuadamente parámetros variantes en el tiempo (pérdida de actividad del algoritmo). Para evitarlo se puede utilizar $\lambda<1$ o bien reinicializar la matriz de covarianza $\mathbf{P}(k)$.

- Un factor de olvido pequeño (i.e. $\lambda \approx 0,9$ ) puede originar parámetros muy variables, ya que los datos pasados se descartan demasiado pronto. Debido a esto, se suele elegir $\lambda$ entre 0.9 y 0.98 , con lo que los elementos de las matrices $\mathbf{P}(k)$ y $\mathbf{W}(k)$ se hacen grandes, lo que a su vez supone la posibilidad de detección de posibles cambios en parámetros. Otra solución es utilizar un factor de olvido variable, por ejemplo como lo propone el método de Fortescue[74]. 


\section{Bibliografía}

[1] T. Abbas, M. Arif, and W. Ahmed. Measurement and correction of systematic odometry errors caused by kinematics imperfections in mobile robots. In Proc. International Joint Conference SICE-ICASE, pages 2073-2078, 18-21 Oct. 2006.

[2] C. Adams. Is "dead reckoning"short for "deduced reckoningi http://www.straightdope.com/mailbag/mdeadreckoning.html, Noviembre 2002. Accesado el 24 de Julio de 2007.

[3] M. Adams. Sensor modelling, design and data processing for autonomous navigation. In Christopher J Harris, editor, World Scientific Series in Robotics and Intelligent Systems, volume 13. World Scientific Publishing Co. Ptd. Ltd., 1999.

[4] G. Antonelli and S. Chiaverini. Experimental odometry calibration of the mobile robot khepera ii based on the least-squares technique. In Robotics and Automation, 2005. ICRA 2005. Proceedings of the 2005 IEEE International Conference on, pages 1465-1470, 18-22 April 2005.

[5] G. Antonelli and S. Chiaverini. Linear estimation of the odometric parameters for differential-drive mobile robots. In Intelligent Robots and Systems, 2006 IEEE/RSJ International Conference on, pages 3287-3292, Oct. 2006.

[6] K. Arras, N. Tomatis, B. Jensen, and R. Siegwart. Multisensor on-the-fly localization: Precision and reliability for applications. Robotics and Autonomous Systems, 34:131-143, 2001.

[7] Kai O. Arras and Roland Siegwart. Feature extraction and scene interpretation for map-based navigation and map building. In Proc. of SPIE, vol. 3210, Mobile Robotics XII, p. 42-53, Pittsburgh, USA, 1997.

[8] Kai Oliver Arras. Feature-Based Robot Navigation in Known and Unknown Environments. PhD thesis, École Polytechnique Fédérale de Lausanne, Switzerland, 2003.

[9] K.O. Arras and S.J. Vestli. Hybrid, high-precision localisation for the mail distributing mobile robot system mops. In Robotics and Automation, 1998. 
Proceedings. 1998 IEEE International Conference on, volume 4, pages 31293134vol.4, 16-20 May 1998.

[10] N. Ayache and O.D. Faugeras. Maintaining representations of the environment of a mobile robot. Robotics and Automation, IEEE Transactions on, 5(6):804819, Dec. 1989.

[11] T. Bailey and H. Durrant-Whyte. Simultaneous localization and mapping(slam): Part ii. IEEE Robotics \&6 Automation Magazine, 13(3):108-117, September 2006.

[12] H. Baltzakis and P. Trahanias. Hybrid mobile robot localization using switching state-space models. In Robotics and Automation, 2002. Proceedings. ICRA '02. IEEE International Conference on, volume 1, pages 366-373vol.1, 11-15 May 2002.

[13] Y. Bar-Shalom and T. Fortmann. Tracking and Data Association. Academic Press, 1988.

[14] Y. Bar-Shalom and X. Rong Li. Estimation and Tracking: Principles, Techniques, and Software. Artech House., 1993.

[15] V. Barnett and T. Lewis. Outliers in Statistical Data. John Wiley \& Sons, New York, 1984.

[16] Billur Barshan and Birsel Ayrulu. Comparative analysis of different approaches to target differentiation and localization with sonar. Pattern Recognition, 36:1213-1231, 2003.

[17] G. Benet, F. Blanes, J. Simó, and P. Perez. Using infrared sensors for distance measurement in mobile robots. Robotics and Autonomous Systems, 40:255-266, 2002.

[18] G. Benet, F. Blanes, J. Simó, and P. Pérez. New Developments in Robotics Research, chapter Map building using infrared sensors in mobile robots, pages 73-103. Nova Science Publishers, Inc., 2005.

[19] G. Benet, M. Martínez, F. Blanes, P. Perez, and J. Simó. Differentiating between walls and corners using the amplitud of ultrasonic echoes. In 11th International Conference on Advance Robotics, Coimbra, July 2003.

[20] Ginés Benet, Milagros Martínez, Francisco Blanes, Pascual Pérez, and Jose Simó. Differentiating wall from corners using the amplitude of ultrasonic echoes. Robotic and Autonomous Systems, 50:13-25, 2005.

[21] O. Bengtsson. Robot localization in changing environments using a laser range scanner. Master's thesis, Computer Systems Engineering. University of Halmstad, 1998. 
[22] O. Bengtsson and A-J Baerveldt. Robot localization based on scanmatching:estimating the covariance matrix for the idc algorithm. Robotics and Autonomous Systems, 44:29-40, 2003.

[23] M. Betke and L. Gurvits. Mobile robot localization using landmarks. Robotics and Automation, IEEE Transactions on, 13(2):251-263, April 1997.

[24] F. Blanes, G. Benet, J.F. Simo, and P. Perez. Enhancing the real-time response of an ultrasonic sensor for map building tasks. In Industrial Electronics, 1999. ISIE '99. Proceedings of the IEEE International Symposium on, volume 3, pages 990-995vol.3, 12-16 July 1999.

[25] Francisco Blanes. Percepción y representación del entorno en robótica móvil. $\mathrm{PhD}$ thesis, Universidad Politécnica de Valencia, Valencia.España, 2000.

[26] I. Bloch. Information combination operators for data fusion: A comparative review with classification. IEEE Transactions on Systems, Man, and Cibernetics, 26(1):52-67, 1996.

[27] J. Borenstein and L. Feng. Umbmark: A benchmark test for measuring odometry errors in mobile robots. In Proceedings of the 1995 SPIE Conference on Mobile Robots, Philadelphia, October 1995.

[28] J. Borenstein and Liqiang Feng. Correction of systematic odometry errors in mobile robots. In Intelligent Robots and Systems 95. 'Human Robot Interaction and Cooperative Robots', Proceedings. 1995 IEEE/RSJ International Conference on, volume 3, pages 569-574vol.3, 5-9 Aug. 1995.

[29] J. Borenstein and Y. Koren. Obstacle avoindance with ultrasonics sensors. IEEE journal on Robotics and Automatics, 4:213-218, june 1988.

[30] J. Borenstein and Y. Koren. Real-time obstacle avoidance for fact mobile robots. Systems, Man and Cybernetics, IEEE Transactions on, 19(5):1179-1187, Sept.Oct. 1989.

[31] J. Borenstein and Y. Koren. Histogramic in-motion mapping for mobile robot obstacle avoidance. Robotics and Automation, IEEE Transactions on, 7(4):535539, Aug. 1991.

[32] Johann Borenstein and Liquiang Feng. Measurement and correction of systematic odometry errors in mobile robots. IEEE Transactions on Robotic and Automation, 12:869-880, 1996.

[33] G. Borges and M. Aldon. A split-and-merge segmentation algoritm for line extraction en $2 \mathrm{~d}$ range images. In 15th International Conference on Pattern Recognition, Barcelona, Spain, 2000. 
[34] A. Bostani, A. Vakili, and T. A. Denidni. A novel method to measure and correct the odometry errors in mobile robots. In Proc. Canadian Conference on Electrical and Computer Engineering CCECE 2008, pages 000897-000900, 4-7 May 2008.

[35] H. Bruyninkx. Bayesian probability theory. The Robotics WEBook. http://www.roble.info/basicST/stat/bayes-index.php, 2005. Accesado el 02 de Agosto de 2007.

[36] W Burgard, A. Cremers, D. Fox, D. Hähnel, G. Lakemeyer, D. Schulz, W. Steiner, and S. Thrun. The interactive museum tour-guide robot. In Fifteenth National Conference on Artificial Intelligence (AAAI-98), 1998.

[37] A. Burguera, Y. Gonzalez, and G. Oliver. Probabilistic sonar scan matching for robust localization. In Proc. IEEE International Conference on Robotics and Automation, pages 3154-3160, 10-14 April 2007.

[38] M.J. Caruso. Applications of magnetic sensors for low cost compass systems. In Position Location and Navigation Symposium, IEEE 2000, pages 177-184, 13-16 March 2000.

[39] A Cassandra. Optimal policies for partially observable markov decision processes. Technical Report CS-94-14, Brown University, Department of Computer Science,Providence, RI., 1994.

[40] A. Cassandra. Pomdps for dummies. http://www.pomdp.org/pomdp/tutorial/index.shtml, Octubre 2003. Accesado el 08 de Agosto de 2007.

[41] A.R. Cassandra, L.P. Kaelbling, and J.A. Kurien. Acting under uncertainty: discrete bayesian models for mobile-robot navigation. In Intelligent Robots and Systems '96, IROS 96, Proceedings of the 1996 IEEE/RSJ International Conference on, volume 2, pages 963-972vol.2, 4-8 Nov. 1996.

[42] J. Castellanos, J. Martinez, J. Neira, and J. Tardos. Simultaneous map building and localization for mobile robots: A multisensor fusion approach. In Proceedings of IEEE International Conference on Robotics and Automation, pages 1244-1249, Leuven, Belgium., May 1998.

[43] J.A. Castellanos, J.M.M. Montiel, J. Neira, and J.D. Tardos. The spmap: a probabilistic framework for simultaneous localization and map building. Robotics and Automation, IEEE Transactions on, 15(5):948-952, Oct. 1999.

[44] J.A. Castellanos and J.D. Tardos. Mobile Robot Localization and Map Building: A Multisensor Fusión Approach. Kluwer Academic Publishers, 1999.

[45] R. Chatila and J. Laumond. Position referencing and consistent world modeling for mobile robots. In Robotics and Automation. Proceedings. 1985 IEEE International Conference on, volume 2, pages 138-145, Mar 1985. 
[46] A. Chella, I. Macaluso, and L. Riano. Automatic place detection and localization in autonomous robotics. In Proc. IEEE/RSJ International Conference on Intelligent Robots and Systems IROS 2007, pages 741-746, Oct. 29 2007-Nov. 22007.

[47] W. Cho. Certainty grid representation for robot navigation by a bayesian method. Robotica, 8:159-165, 1990.

[48] Jinwoo Choi, Sunghwan Ahn, Minyong Choi, and Wan Kyun Chung. Metric slam in home environment with visual objects and sonar features. In Intelligent Robots and Systems, 2006 IEEE/RSJ International Conference on, pages 40484053, Oct. 2006.

[49] Won-Seok Choi and Se-Young Oh. Range sensor-based robot localization using neural network. In Proc. International Conference on Control, Automation and Systems ICCAS '07, pages 230-234, 17-20 Oct. 2007.

[50] K. Chong and L. Kleeman. Sonar based map building for mobile robot. In IEEE International Conference on Robotics and Automation, volume 2, pages 1700-1705, Alburquerque, New Mexico, April 1997.

[51] Kok Seng Chong and L. Kleeman. Accurate odometry and error modelling for a mobile robot. In Robotics and Automation, 1997. Proceedings., 1997 IEEE International Conference on, volume 4, pages 2783-2788vol.4, 20-25 April 1997.

[52] C. Cohen and F. Koss. A comprehensive study of three object triangulation. In Proceedings of the 1993 SPIE Conference on Mobile Robots, Boston, Ma., Nov. 1993.

[53] J. Crowley. Dynamic world modeling for an intelligent mobile robot using a rotating ultra-sonic ranging device. In Robotics and Automation. Proceedings. 1985 IEEE International Conference on, volume 2, pages 128-135, Mar 1985.

[54] A. Curran and K. Kyriakopoulos. Sensor-based self-localization for wheeled mobile robots. Journal of Robotics and Autonomous Systems, 12(3):163-176, 1995.

[55] M. De Cecco, L. Baglivo, and M. Pertile. Real-time uncertainty estimation of odometric trajectory as a function of the actual manoeuvres of autonomous guided vehicles. In Proc. IEEE International Workshop on Advanced Methods for Uncertainty Estimation in Measurement (AMUEM 2006), pages 80-85, 2021 April 2006.

[56] F. Dellaert, D. Fox, W. Burgard, and S. Thrun. Monte carlo localization for mobile robots. In Robotics and Automation, 1999. Proceedings. 1999 IEEE International Conference on, volume 2, pages 1322-1328vol.2, 10-15 May 1999. 
[57] J. Diard. The Bayesian map: A hierarchical probabilistic model for mobile robot navigation. PhD thesis, Institut National Polytechnique de Grenoble, 2003.

[58] M.W.M.G. Dissanayake, P. Newman, S. Clark, H.F. Durrant-Whyte, and M. Csorba. A solution to the simultaneous localization and map building (slam) problem. Robotics and Automation, IEEE Transactions on, 17(3):229-241, June 2001.

[59] J. Doebbler, J. J. Davis, J. L. Junkins, and J. Valasek. Odometry and calibration methods for multi-castor vehicles. In Proc. IEEE International Conference on Robotics and Automation ICRA 2008, pages 2110-2115, 19-23 May 2008.

[60] Tom Duckett and Ulrich Nehmzow. Mobile robot self-localisation using occupancy histograms and a mixture of gaussian location hypotheses. Robotics and Autonomous Systems, 34(34):117-129, 2001.

[61] R Duda and P. Hart. Pattern Classification and Scene Analysis. John Wiley and Sons, 1973.

[62] G. Dudek and M. Jenkin. Computational principles of mobile robotics. Cambridge University Press, 2000.

[63] H Durrant-Whyte and T Bailey. Simultaneous localization and mapping: Part i. IEEE Robotics $\&$ Automation Magazine, 13(2):99-108, June 2006.

[64] M. Egerstedt and P. Jensfelt. A control theoretic formulation of the generalized slam problem in robotics. In Proc. American Control Conference, pages 24092414, 11-13 June 2008.

[65] A. Elfes. Sonar-based real-world mapping and navigation. IEEE journal on Robotics and Automatics, 3:249-265, june 1987.

[66] A Elfes. A tesselated probabilistic representation for spatial robot perception and navigation. In NASA conference on space telerobotics, 1989.

[67] A Elfes. Data fusion in robotics and machine intelligence, chapter Multi-source spatial data fusion using Bayesian reasoning. Academics Press, New York, 1992.

[68] S. Engelson. Passive Map Learning and Visual Place Recognition. PhD thesis, Department of Computer Science.Yale University, 1994.

[69] H. R. Everett. Sensors for Mobile Robots: Theory and Application. A. K. Peters, Ltd, 1995.

[70] E. Fabrizi and A. Saffiotti. Extracting topology-based maps from gridmaps. In Robotics and Automation, 2000. Proceedings. ICRA '00. IEEE International Conference on, volume 3, pages 2972-2978vol.3, 24-28 April 2000. 
[71] L. Feng, J. Borenstein, and B. Everett. Where am i? sensors and methods for autonomous mobile robot localization. Technical Report UM-MEAM-94-21, The University of Michigan, Diciembre 1994.

[72] David Filliat and Jean-Arcady Meyer. Map-based navigation in mobile robot: A review of localization strategies. Cognitive systems Research, 4:243-282, 2003.

[73] A. Flynn. Combining sonar and infrared sensors for mobile robot navigation. International Journal of Robotics Research, 7(6):5-14, December 1988.

[74] T. R. Fortescue, L. S. Kershenbaum, and B. E. Ydstie. Implementation of self tuning regulators with variable forgetting factors. Automatica, 17(6):831-835, 1981.

[75] D. Fox, W Burgard, and S. Thrun. Active markov localization for mobile robots in dynamic environments. Journal of Artificial Intelligence Research, 11:391427, 1999.

[76] D. Fox, W. Burgard, S. Thrun, and A. Cremers. Position estimation for mobile robots in dynamic environments. In Artificial Intelligence, Proceedings. AAAI Fifteenth National Conference on, 1998.

[77] Dieter Fox. Markov Localization: A probabilistic Framework for Robot Localization and Navigation. PhD thesis, Institute of computer science III. University of Bonn. Germany, 1998.

[78] F. Gambino, G. Ulivi, and M. Vendittelli. The transferable belief model in ultrasonic map building. In Sixth IEEE International Conference on Fuzzy Systems,199\%., volume 1, pages 601 - 608, July 1997.

[79] J. García. Sistema de posicionamiento y autolocalización para sillas de ruedas autónomas. PhD thesis, Departamento de Electrónica. Universidad de Alcalá, 2001.

[80] J. Gasós and A. Rosetti. Uncertainty representation for mobile robots: Perception, modeling and navigation in unknown environments. Fuzzy Sets and Systems, 107:1-24, 1999.

[81] K.F. Gauss. Theoria combinationis observationum erroribus minimis obnoxiae, 1823.

[82] A. Gelb. Applied Optimal Estimation. MIT Press, 1996.

[83] Jang Gijeong, Kim Sungho, Lee Wangheon, and Kweon Inso. Robust selflocalization of mobile robots using artificial and natural landmarks. In IEEE International Symposium on Computational Intelligence in Robotics and Automation., 2003. 
[84] P. Goel, S.I. Roumeliotis, and G.S. Sukhatme. Robust localization using relative and absolute position estimates. In Intelligent Robots and Systems, 1999. IROS '99. Proceedings. 1999 IEEE/RSJ International Conference on, volume 2, pages 1134-1140vol.2, 17-21 Oct. 1999.

[85] F. Grubbs. Procedures for detecting outlying observations in samples. Technometrics, 11:1-21, 1969.

[86] E. Guglielmelli, V. Genovese, P. Dario, and G. Morana. Avoiding obstacles by using a proximity us/ir sensitive skin. In Intelligent Robots and Systems '93, IROS '93. Proceedings of the 1993 IEEE/RSJ International Conference on, volume 3, pages 2207-2214vol.3, 26-30 July 1993.

[87] J. Gutmann and C. Schlegel. Amos: Comparison of scan matching approaches for self-localization in indoor environments. In 1st Euromicro Workshop on Advanced Mobile Robots (Eurobot'96), pages 61-67, 1996.

[88] Monson Hayes. Statistical Digital Signal Processing and Modeling. John Wiley \& Sons, 1996.

[89] Simon Haykin. Adaptive Filter Theory. Prentice Hall, 2002.

[90] R.W. Hogg, A.L. Rankin, S.I. Roumeliotis, M.C. McHenry, D.M. Helmick, C.F. Bergh, and L. Matthies. Algorithms and sensors for small robot path following. In Robotics and Automation, 2002. Proceedings. ICRA '02. IEEE International Conference on, volume 4, pages 3850-3857vol.4, 11-15 May 2002.

[91] Shoudong Huang and Gamini Dissanayake. Convergence and consistency analysis for extended kalman filter based slam. 23(5):1036-1049, Oct. 2007.

[92] Kyung-Hun Hwang, Do-Eun Kim, Dong-Hun Lee, and Tae-Young Kuc. A simple ultrasonic gps system for indoor mobile robot system using kalman filtering. In Proc. International Joint Conference SICE-ICASE, pages 2915-2918, Oct. 2006.

[93] B. Iglewicz and D. C Hoaglin. How to detect and handle outliers. In $A S Q C$ basic references in quality control. ASQC Quality Press, 1993.

[94] A.K Jain. Fundamentals of Digital Image Processing. Prentice Hall, 1989.

[95] Gijeong Jang, Sungho Kim, Wangheon Lee, and Inso Kweon. Robust selflocalization of mobile robots using artificial and natural landmarks. In Computational Intelligence in Robotics and Automation, 2003. Proceedings. 2003 IEEE International Symposium on, volume 1, pages 412-417vol.1, 16-20 July 2003.

[96] P. Jensfelt and H.I. Christensen. Pose tracking using laser scanning and minimalistic environmental models. Robotics and Automation, IEEE Transactions on, 17(2):138-147, April 2001. 
[97] Lepoldo Jetto, Sauro Longhi, and Giuseppe Venturini. Development and experimental validation of an adaptive extended kalman filter for the localization of mobile robots. IEEE Transactions on Robotics and Automation, 15(2):219-229, April 1999.

[98] Xiucai Ji, Hui Zhang, Dan Hai, and Zhiqiang Zheng. Incremental slam with backtracking data association for mobile robots. In Proc. International Conference on Information and Automation ICIA 2008, pages 634-639, 20-23 June 2008.

[99] Hao Jifei, Li Xiang, and Yan Dashun. A multisensor fusing system on ultrasonic sensors. In Vehicle Electronics Conference, 1999. (IVEC '99) Proceedings of the IEEE International, pages 140-144vol.1, 6-9 Sept. 1999.

[100] Jong jin Yu, Sung sub Park, and Woong Keun Hyun. A correction system of odometry error for simultaneous map building based on sensor fusion. In Proc. International Conference on Smart Manufacturing Application ICSMA 2008, pages 393-396, 9-11 April 2008.

[101] L. Kaelbling, M. Littman, and A. Cassandra. Planning and acting in partially observable stochastic domains. Artificial Intelligence, 101:99-134, 1998.

[102] R.E Kalman and R.S Bucy. New results in linear filtering and prediction theory. Transactions of the ASME - Journal of Basic Engineering, 83:95-107, 1961.

[103] Rudolf E. Kalman. A new approach to linear filtering and prediction problems. Transactions ASME, Journal of Basic Engineering, 82:35-45, 1960.

[104] A. Kelly. General solution for linearized systematic error propagation in vehicle odometry. In Intelligent Robots and Systems, 2001. Proceedings. 2001 IEEE/RSJ International Conference on, volume 4, pages 1938-1945vol.4, 29 Oct.-3 Nov. 2001.

[105] A. Kelly. Fast and easy systematic and stochastic odometry calibration. In Intelligent Robots and Systems, 2004. (IROS 2004). Proceedings. 2004 IEEE/RSJ International Conference on, volume 4, pages 3188-3194vol.4, 28 Sept.-2 Oct. 2004.

[106] Alonzo Kelly. Some useful results for closed-form propagation of error in vehicle odometry. Technical Report CMU-RI-TR-00-20, The Robotics Institute,Carnegie Mellon University, Pittsburgh, PA, December 2000.

[107] Ho-Duck Kim, Dae-Wook Kim, and Kwee-Bo Sim. Simultaneous localization and map building using vision camera and electrical compass. In SICE-ICASE, 2006. International Joint Conference, pages 5915-5918, Oct. 2006.

[108] Ho-Duck Kim, Sang-Wook Seo, In hun Jang, and Kwee-Bo Sim. Slam of mobile robot in the indoor environment with digital magnetic compass and ultrasonic 
sensors. In Proc. International Conference on Control, Automation and Systems ICCAS 'O7, pages 87-90, 17-20 Oct. 2007.

[109] J. Kim and P. Seong. Experiments on orientation recovery and steering of autonomous mobile robot using encoded magnetic compass disc. IEEE Transactions on Instrumentation and Measurements, 45(1):271-273, 1996.

[110] P. Klarer. Simple 2d navigation for wheeled vehicles. Technical report, Sandia National Laboratories, Albuquerque,NM., 1998.

[111] L. Kleeman. Odometry error covariance estimation for two wheel robot vehicles. Technical Report MECSE-95-1, Intelligent Robotics Research Centre. Department of Electrical and Computer Systems Engineering, Monash University, 1995.

[112] L. Kleeman. Advanced sonar and odometry error modeling for simultaneous localisation and map building. In Intelligent Robots and Systems, 2003. (IROS 2003). Proceedings. 2003 IEEE/RSJ International Conference on, volume 1, pages 699-704vol.1, 27-31 Oct. 2003.

[113] Kurt Konolige. Improved occupancy grids for map building. Autonomous Robots, 4:351-367, 1997.

[114] L. Korba, S. Elgazzar, and T. Welch. Active infrared sensors for robotics. In Instrumentation and Measurement Technology Conference, 1993. IMTC/93. Conference Record., IEEE, pages 589-594, 18-20 May 1993.

[115] D. Kortenkamp and T. Weymouth. Topological mapping for mobile robots using a combination of sonar and vision sensing. In National Conference on Artificial Intelligence(AAAI-94), 1994.

[116] P. Krammer and H. Schweinzer. Localization of object edges in arbitrary spatial positions based on ultrasonic data. 6(1):203-210, Feb. 2006.

[117] R. Kuc and V. viard. A physically based navigation strategy for sonar-guided vehicles. The international journal of robotics research, 10(2):75-87, April 1991.

[118] B.J Kuipers. Representing Knowledge of Large-Scale Space. PhD thesis, Mathematics Department, Massachusetts Institute of Technology, Cambridge, Massachusetts, June 1977.

[119] B.J. Kuipers and Y. Byun. A robot exploration and mapping strategy based on a semantic hierarchy of spatial representations. Journal of Robotics and Autonomous Systems, 8:47-63, 1991.

[120] A. Kurz. Building maps based on a learned clasification of ultrasonic range data. In D Charnley, editor, Intelligent autonomous Vehicle, pages 193-198. Elsevier Science, 1993. 
[121] N. M. Kwok, Q. P. Ha, and G. Fang. Data association in bearing-only slam using a cost function-based approach. In Proc. IEEE International Conference on Robotics and Automation, pages 4108-4113, 10-14 April 2007.

[122] SangJoo Kwon, KwangWoong Yang, and Sangdeok Park. An effective kalman filter localization method for mobile robots. In Proc. IEEE/RSJ International Conference on Intelligent Robots and Systems, pages 1524-1529, Oct. 2006.

[123] Choon-Young Lee, Ho-Gun Choi, Jun-Sik Park, Keun-Young Park, and SangRyong Lee. Collision avoidance by the fusion of different beam-width ultrasonic sensors. In Proc. IEEE Sensors, pages 985-988, 28-31 Oct. 2007.

[124] David Lee. The Map-Building and Exploration of a Simple Sonar-Equipped Robot. Cambridge University Press, 1996.

[125] Jeong-Hyeong Lee and Seul Jung. Global position tracking control of an omnidirectional mobile robot using fusion of a magnetic compass and encoders. In Proc. IEEE International Conference on Multisensor Fusion and Integration for Intelligent Systems MFI 2008, pages 246-251, 20-22 Aug. 2008.

[126] Sooyong Lee and Jae-Bok Song. Use of coded infrared light as artificial landmarks for mobile robot localization. In Proc. IEEE/RSJ International Conference on Intelligent Robots and Systems IROS 2007, pages 1731-1736, Oct. 29 2007-Nov. 22007.

[127] J. Leonard and H. Durrant-Whyte. Directed sonar sensing for mobile robot navigation. Kluwer Academic Publishers, Cambridge, MA, 1992.

[128] J. Leonard, P. Newman, R. Rikoski, J. Neira, and J Tardós. Towards robust data association and feature modeling for concurrent mapping and localization. In Tenth International Symposium on Robotics Research., Lorne, Victoria, Australia., November 2001.

[129] J.J. Leonard and H.F. Durrant-Whyte. Mobile robot localization by tracking geometric beacons. Robotics and Automation, IEEE Transactions on, 7(3):376382, June 1991.

[130] John J. Leonard and Hugh F. Durrant-Whyte. Directed Sonar Sensing for Mobile Robot Navigation, volume 175 of The Springer International Series in Engineering and Computer Science. Springer, 1992.

[131] J. Lim and D. Cho. Physically based sensor modelling for sonar map in specular environment. In IEEE international conference on Robotics and Automatics, pages $727-733,1992$.

[132] Maria Lopez. Sistema de navegación global basado en procesos de decisión de Markov parcialmente observables. Aplicación a un ronot de asistencia personal. $\mathrm{PhD}$ thesis, Departamento de Electrónica. Escuela Politecnica. Universidad de Alcalá, 2004. 
[133] F. Lu and E. Milios. Globally consistent range scan alignment for environment mapping. Autonomous Robot, 4:333-349, 1997.

[134] F. Lu and E. Milios. Robot pose estimation in unknown environments by matching 2d range scans. Journal of Intelligent Robotic Systems. Kluwer Academic Publishers, 18:249-275, 1997.

[135] V. Lumelsky and E. Cheung. Towards safe real-time robot teleoperation: automatic whole-sensitive arm collision avoidance frees the operator for global control. In Robotics and Automation, 1991. Proceedings., 1991 IEEE International Conference on, pages 797-802vol.1, 9-11 April 1991.

[136] R.C. Luo, Tse Min Chen, and Meng Hsien Lin. Automatic guided intelligent wheelchair system using hierarchical grey-fuzzy motion decision-making algorithms. In Intelligent Robots and Systems, 1999. IROS '99. Proceedings. 1999 IEEE/RSJ International Conference on, volume 2, pages 900-905vol.2, 17-21 Oct. 1999.

[137] D. Maksarov and H. Durrant-Whyte. Mobile vehicle navigation in unknown environments: a multiple hypothesis approach. In IEE proceedings in Control Theory and Applications, volume 142, pages 385-400, July 1995.

[138] Robert Maldelbaum and Max Mintz. Sonar signal processing using tangent cluster. In Ocean Engineering for Today's Technology and Tomorrow's Preservation, volume 2, pages 544 - 549, Brest, France, September 1994.

[139] R. Malik and H. Yu. The infrared detector ring: Obstacle detection for an autonomous mobile robot. In IEEE 35th Midwest Symposium on Circuit and Systems., 1992.

[140] D. Marioli, C. Narduzzi, C. Offelli, D. Petri, E. Sardini, and A. Taroni. Digital time of flight measurement for ultrasonic sensors. In Instrumentation and Measurement Technology Conference, 1991. IMTC-91. Conference Record., 8th IEEE, pages 198-201, 14-16 May 1991.

[141] M Martin and H. Moravec. Robot evidence grids. Technical Report CMURI-TR-96-06, The Robotics Institute. Carnegie Mellon University, Pittsburgh, Pennsylvania, March 1996.

[142] A. Martinelli. A possible strategy to evaluate the odometry error of a mobile robot. In Intelligent Robots and Systems, 2001. Proceedings. 2001 IEEE/RSJ International Conference on, volume 4, pages 1946-1951vol.4, 29 Oct.-3 Nov. 2001.

[143] A. Martinelli. The accuracy on the parameter estimation of an odometry system of a mobile robot. In Robotics and Automation, 2002. Proceedings. ICRA '02. IEEE International Conference on, volume 2, pages 1378-1383vol.2, 11-15 May 2002. 
[144] A. Martinelli. Evaluating the odometry error of a mobile robot. In Intelligent Robots and System, 2002. IEEE/RSJ International Conference on, volume 1, pages 853-858vol.1, 30 Sept.-5 Oct. 2002.

[145] M. Martinez. Contribuciones a la construcción de mapas para la navegación de robots, con información procedente de sensores de ultrasonidos. PhD thesis, Departamento de Informática de Sistemas y Computadores. Universidad Politecnica de Valencia, Valencia-España, 2004.

[146] Milagros Martínez, Ginés Benet, Francisco Blanes, Pascual Pérez, and Jose simó. Using the amplitude of the ultrasonic echoes to classify detected objects in a scene. In IEEE International Conference on Advanced Robotics, volume 1, pages 1136-1142, Coimbra, Portugal, June 2003.

[147] S. Mascaro and H.H. Asada. Docking control of holonomic omnidirectional vehicles with applications to a hybrid wheelchair/bed system. In Robotics and Automation, 1998. Proceedings. 1998 IEEE International Conference on, volume 1, pages 399-405vol.1, 16-20 May 1998.

[148] M. Mataric. Distributed model for mobile robot environment-learning and navegation. Technical Report AI-TR 1228, MIT Artificial Intelligence Laboratory, 545, Technology Square, Cambridge, MA, 02139, USA, 1990.

[149] L. Mathies and A. Elfes. Integration of sonar and stereo range data using a grid-based representation. In IEEE international conference on Robotics and Automatics, pages 727-733, 1988.

[150] Peter S. Maybeck. Stochastic models, estimation, and control, volume 141. Academic Press, 1979.

[151] H. Moravec and A. Elfes. High resolution maps from wide angle sonar. In Robotics and Automation. Proceedings. 1985 IEEE International Conference on, volume 2, pages 116-121, Mar 1985.

[152] K. Murphy. Dynamic Bayesian Networks: Representation, Inference and Learning. PhD thesis, University of California, Berkeley, 2002.

[153] D. Navarro and G. Benet. Evaluating line extraction algorithms for infrared feature mapping in mobile robotics. In IX International Congress on Numerical Methods in Engineering and Applied Sciences, pages EC 25-30, Margarita Venezuela, March 2008.

[154] R. Negenborn. Robot localization and kalman filters. on finding your position in a noisy world. Master's thesis, Institute of Information and Computing Sciences. Utrecht University, 2003.

[155] J. Neira, J. Horn, J. Tardos, and G. Schmidt. Multisensor mobile robot localization. In IEEE International Conference on Robotics and Automation, pages 673-679, Minneapolis, USA, April 1996. 
[156] Pau Newman. On the Structure and Solution of the Simultaneous Localisation and Map Building. PhD thesis, Australian Centre for Field Robotics. The University of Sydney, 1999.

[157] V. Nguyen, A. Martinelli, N. Tomatis, and R. Siegwart. A comparison of line extraction algorithms using $2 \mathrm{~d}$ laser rangefinder for indoor mobile robotics. In the IEEE/RSJ intenational conference on intelligent robots and systems, IROS, Edmonton, Canada, 2005.

[158] Illah Nourbakhsh. Artificial Intelligence and Mobile Robots, chapter Dervish. The MIT Press, 1998.

[159] P.M. Novotny and N.J. Ferrier. Using infrared sensors and the phong illumination model to measure distances. In Robotics and Automation, 1999. Proceedings. 1999 IEEE International Conference on, volume 2, pages 1644-1649vol.2, 10-15 May 1999.

[160] G. Oriolo, G. Ulivi, and M. Vendittelli. Real-time map building and navigation for autonomous robots in unknown environments. IEEE trans. on Systems, Man, and Cybernetics, 3(3):316-333, June 1998.

[161] G. Oriolo, M. Vendittelli, and G. Ulivi. On-line map building and navigation for autonomous mobile robots. In Robotics and Automation, 1995. Proceedings., 1995 IEEE International Conference on, volume 3, pages 2900-2906vol.3, 21-27 May 1995.

[162] R. Ouellette and K. Hirasawa. A comparison of slam implementations for indoor mobile robots. In Proc. IEEE/RSJ International Conference on Intelligent Robots and Systems IROS 200\%, pages 1479-1484, Oct. 29 2007-Nov. 22007.

[163] D. Pagac, E.M. Nebot, and H. Durrant-Whyte. An evidential approach to map-building for autonomous vehicles. IEEE Transactions on Robotics and Automation, Volume 14(4):623-629, Aug. 1998.

[164] M. Parrilla and C Anaya, J.and Fritsch. Digital signal processing techniques for high accuracy ultrasonic range measurements. IEEE Transactions on Instrumentation and Measurement, 40(4):759-763, aug. 1991.

[165] L. M. Paz, P. Jensfelt, J. D. Tardos, and J. Neira. Ekf slam updates in o(n) with divide and conquer slam. In Proc. IEEE International Conference on Robotics and Automation, pages 1657-1663, 10-14 April 2007.

[166] Carla Penedo, Joao Pavao, Pedro Lima, and M. I. Ribeiro. Markov localization in the robocup simulation league. Robotica, 4:16-21, 2003.

[167] Pascual Perez. Miceu:módulo inteligente para la captura del entorno mediante ultrasonidos en robots autónomos. Proyecto fin de carrera. Facultad de informática. Universidad politecnica de Valencia, 1998. 
[168] Samuel Pfister. Algoritms for Mobile Robot Localization and Mapping, Incorporating Detailed Noise Modeling and Multi-Scale Feature Extraction. PhD thesis, California Institute of Technology, Pasadena, California, 2006.

[169] Piedrahita, Giovanny Andres, Guayacundo, and Diana Marcela. Evaluation of accelerometers as inertial navigation system for mobile robots. In Proc. IEEE 3rd Latin American Robotics Symposium LARS '06, pages 84-90, 26-27 Oct. 2006.

[170] P. Pong and S. Challa. Empirical analysis of generalised uncertainty measures with dempster shafer fusion. In Proc. 10th International Conference on Information Fusion, pages 1-9, 9-12 July 2007.

[171] V. Raghavan and M. Jamshidi. Sensor fusion based autonomous mobile robot navigation. In Proc. IEEE International Conference on System of Systems Engineering SoSE '07, pages 1-6, 16-18 April 2007.

[172] Francesco M. Raimondi and Maurizio Melluso. Fuzzy ekf control for wheeled nonholonomic vehicles. In Proc. IECON 2006 - 32nd Annual Conference on IEEE Industrial Electronics, pages 43-48, Nov. 2006.

[173] M. Ribo and A. Pinz. A comparison of three uncertainty calculi for building sonar-based occupancy grid. Robotics and Autonomous Systems, 35:201-209, 2001.

[174] Diego Rodriguez-Losada. SLAM geometrico en tiempo real para robots moviles en interiores basado en EKF. PhD thesis, Departamento de Automática, Ingeniería Industrial e informática industrial. Universidad Politecnica de Madrid., Madrid-España, 2004.

[175] Stergios Roumeliotis and G. Bekey. Segments: A layered, dual kalman filter algorithm for indoor feature extration. In IEEE Internatinal Conference on Robotics and Automation, pages 454-461, Takmatsu, Japan, 2000.

[176] N. Roy and S. Thrun. Online self-calibration for mobile robots. In Robotics and Automation, 1999. Proceedings. 1999 IEEE International Conference on, volume 3, pages 2292-2297vol.3, 10-15 May 1999.

[177] D. Sack and W Burgard. A comparison of methods for line extraction from range data. In 5th IFAC symposium on intelligent autonomous vehicles, 2004.

[178] J. Z. Sasiadek, A. Monjazeb, and D. Necsulescu. Navigation of an autonomous mobile robot using ekf-slam and fastslam. In Proc. 16th Mediterranean Conference on Control and Automation, pages 517-522, 25-27 June 2008.

[179] B. Schiele and J.L. Crowley. A comparison of position estimation techniques using occupancy grids. In Robotics and Automation, 1994. Proceedings., 1994 IEEE International Conference on, pages 1628-1634vol.2, 8-13 May 1994. 
[180] M. Seyr and S. Jakubek. Proprioceptive navigation, slip estimation and slip control for autonomous wheeled mobile robots. In Proc. IEEE Conference on Robotics, Automation and Mechatronics, pages 1-6, Dec. 2006.

[181] Glenn Shafer. A matemathical theory of evidence. Princenton University Press, Princenton, New Jersey - USA, 1976.

[182] M. Skolnik. Introduction to Radar Systems. McGraw-Hill., New York, 1962.

[183] V. Y. Skvortzov, Hyoung-Ki Lee, SeokWon Bang, and YongBeom Lee. Application of electronic compass for mobile robot in an indoor environment. In Proc. IEEE International Conference on Robotics and Automation, pages 2963-2970, 10-14 April 2007.

[184] R. Smith and P. Cheeseman. On the representation and estimation of spatial uncertainty. Technical Report TR 4760 \& 7239,, SRI, 1985.

[185] R. Smith, M. Self, and P. Cheeseman. Autonomous Robot Vehnicles, chapter Estimating uncertain spatial relationships in robotics., pages 167-193. SpringerVerlag, 1990.

[186] Xiaokang Song and Yuechao Wang. A novel model-based method for odometry calculation of all-terrain mobile robots. In Proc. 7th World Congress on Intelligent Control and Automation WCICA 2008, pages 581-586, 25-27 June 2008.

[187] Rongchuan Sun, Shugen Ma, Bin Li, and Yuechao Wang. Improving consistency of ekf-based slam algorithms by using accurate linear approximation. In Proc. IEEE/ASME International Conference on Advanced Intelligent Mechatronics AIM 2008, pages 619-624, 2-5 July 2008.

[188] L. Tamas, G. Lazea, R. Robotin, C. Marcu, S. Herle, and Z. Szekely. State estimation based on kalman filtering techniques in navigation. In Proc. IEEE International Conference on Automation, Quality and Testing, Robotics AQTR 2008, volume 2, pages 147-152, 22-25 May 2008.

[189] J. Tardos, J. Neira, P. Newman, and J. Leonard. Robust mapping and localization in indoor environments using sonar data. The International Journal of Robotics Research, 21(4):311 - 330, April 2002.

[190] F. Thomas and L. Ros. Revisiting trilateration for robot localization. IEEE Transaction on Robotics, 21(1):93-101, February 2005.

[191] S. Thrun. Bayesian landmark learning for mobile robot localization. Machine Learning, 33(1):41-76, 1998.

[192] S. Thrun. Learning maps for indoor mobile robot navigation. Artificial Intelligence, 99(1):21-71, 1998. 
[193] S. Thrun. Probabilistic algorithms in robotics. Technical Report CMU-CS-00126, Computer Science Department. Carnegie Mellon University, Pittsburgh, PA., 2000.

[194] S. Thrun. Robotic mapping: A survey. In G. Lakemeyer and B. Nebel, editors, Exploring Artificial Intelligence in the New Millenium, chapter 1, pages 1-36. Morgan Kaufmann publishers, 2002.

[195] S Thrun. Learning occupancy grid maps with forward sensor models. Autonomous Robots, 15:111-127, 2003.

[196] S. Thrun, M. Beetz, M. Bennewitz, W. Burgard, A. Cremers, F. Dellaert, D. Fox, D. Hähnel, C. Rosenberg, N. Roy, J. Schulte, and D. Schulz. Probabilistic algorithms and the interactive museum tour-guide robot minerva. international Journal of Robotics Research, 19(11):972-999, 2000.

[197] S. Thrun, A. Bucken, W. Burgard, D. Fox, T. Froehlinghaus, D. Henning, T. Hofmann, M. Krell, and T. Schmidt. Map learning and high-speed navigation in rhino. In D. Kortenkamp, R.P. Bonasso, and R Murphy, editors, Artificial Intelligence and Mobile Robots, pages 21-52. MIT Press, 1998.

[198] S. Thrun, W. Burgard, and D. Fox. Probabilistic Robotic. The MIT Press, 2005.

[199] sebastian Thrun. Robotic mapping: A survey. Technical Report CMU-CS-02111, School of Computer Science. Carnegie Mellon University, Pittsburgh, PA, February 2002.

[200] M. Veeck and W. Burgard. Learning polyline maps from range scan data acquired with mobile robots. In Intelligent Robots and Systems, 2004. (IROS 2004). Proceedings. 2004 IEEE/RSJ International Conference on, volume 2, pages 1065-1070vol.2, 28 Sept.-2 Oct. 2004.

[201] M Wang. Location estimation and uncertainty analysis for mobile robots. In IEEE International Conference on Robotics and Automation, pages 1230-1235, Philadelphia, PA., 1988.

[202] Niu Wang, Zu shu Li, Yong long Li, Ya Pan, and Heng Wang. Identify the motion-actuator states of two wheeled robot with dfdcms. In Proc. 7th World Congress on Intelligent Control and Automation WCICA 2008, pages 16631668, 25-27 June 2008.

[203] G. Weiss, C. Wetzler, and E. von Puttkamer. Keeping track of position and orientation of moving indoor systems by correlation of range-finder scans. In Intelligent Robots and Systems '94. 'Advanced Robotic Systems and the Real World', IROS '94. Proceedings of the IEEE/RSJ/GI International Conference on, volume 1, pages 595-601vol.1, 12-16 Sept. 1994. 
[204] G. Welch and G. Bishop. An introduction to the kalman filter. Technical Report TR 95-041, Deparment of Computer Science. University of North Carolina, 1995.

[205] O. Wijk and H.I. Christensen. Triangulation-based fusion of sonar data with application in robot pose tracking. Robotics and Automation, IEEE Transactions on, 16(6):740-752, Dec. 2000.

[206] Baiyu Xi, Rui Guo, Fengchi Sun, and Yalou Huang. Simulation research for active simultaneous localization and mapping based on extended kalman filter. In Proc. IEEE International Conference on Automation and Logistics ICAL 2008, pages 2443-2448, 1-3 Sept. 2008.

[207] JaeMu Yun, Jae-Pyung Ko, and JangMyung Lee. An inexpensive and accurate absolute position sensor for driving assistance. 57(4):864-873, April 2008.

[208] Youngmok Yun, Byungjae Park, and Wan Kyun Chung. Odometry calibration using home positioning function for mobile robot. In Proc. IEEE International Conference on Robotics and Automation ICRA 2008, pages 2116-2121, 19-23 May 2008. 